\title{
Certified Programming with Dependent Types
}

\author{
Adam Chlipala
}

April 21, 2019 
A print version of this book is available from the MIT Press. For more information, see the book's home page:

$$
\text { http://adam.chlipala.net/cpdt/ }
$$

Copyright Adam Chlipala 2008-2013, 2015, 2017.

This work is licensed under a Creative Commons Attribution-Noncommercial-No

Derivative Works 3.0 Unported License. The license text is available at:

http://creativecommons.org/licenses/by-nc-nd/3.0/ 


\section{Contents}

1 Introduction $\quad 6$

1.1 Whence This Book? . . . . . . . . . . . . . . . . . . . 6

1.2 Why Coq? . . . . . . . . . . . . . . . . . . . 7

1.2.1 Based on a Higher-Order Functional Programming Language . . . . . 8

1.2.2 Dependent Types . . . . . . . . . . . . . . . . . . . . 8

1.2.3 An Easy-to-Check Kernel Proof Language . . . . . . . . . . . . . . . 9

1.2.4 Convenient Programmable Proof Automation . . . . . . . . . . . . . . 9

1.2.5 Proof by Reflection . . . . . . . . . . . . . . . . . . . 10

1.3 Why Not a Different Dependently Typed Language? . . . . . . . . . . . . . 10

1.4 Engineering with a Proof Assistant . . . . . . . . . . . . . . . . 11

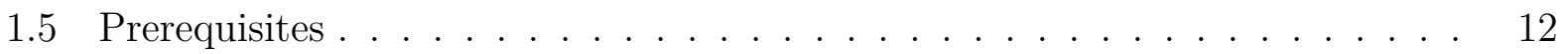

1.6 Using This Book . . . . . . . . . . . . . . . . . . . . . . . 12

1.6.1 Reading This Book . . . . . . . . . . . . . . . . . 13

1.6.2 On the Tactic Library . . . . . . . . . . . . . . . . . . . . . . . . . . . . . . . 14

1.6.3 Installation and Emacs Set-Up . . . . . . . . . . . . . . 14

1.7 Chapter Source Files . . . . . . . . . . . . . . . . . . . . . 16

2 Some Quick Examples $\quad 17$

2.1 Arithmetic Expressions Over Natural Numbers . . . . . . . . . . . . . . 17

2.1 .1 Source Language . . . . . . . . . . . . . . . . . . . 18

2.1.2 Target Language . . . . . . . . . . . . . . . . . . . . . . . . . . . . . . . . . .

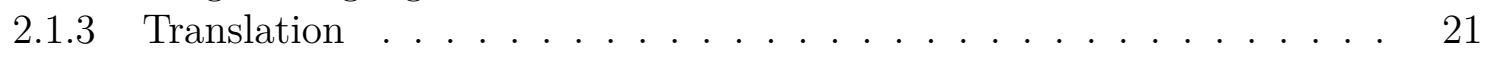

2.1.4 Translation Correctness . . . . . . . . . . . . . . . . . . . 22

2.2 Typed Expressions . . . . . . . . . . . . . . . . . . . . . 30

2.2 .1 Source Language . . . . . . . . . . . . . . . . . . . . . . . . . . . . . . . . . . . . . . . 30

2.2 .2 Target Language . . . . . . . . . . . . . . . . . . . . . . . . . . . . . . . . . . . . . . . . 32

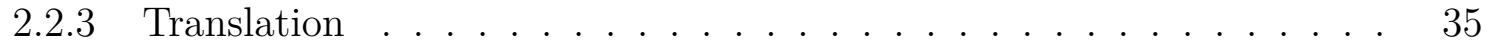

2.2.4 Translation Correctness ................... 36

$\begin{array}{lll}\text { I Basic Programming and Proving } & 39\end{array}$

3 Introducing Inductive Types 440 
3.1 Proof Terms . . . . . . . . . . . . . . . . . . . . . . . . 40

3.2 Enumerations . . . . . . . . . . . . . . . . . . . . . . . . . . 42

3.3 Simple Recursive Types . . . . . . . . . . . . . . . . . . . . . . . 45

3.4 Parameterized Types . . . . . . . . . . . . . . . . . . . . . . . . . . . . . . . 49

3.5 Mutually Inductive Types . . . . . . . . . . . . . . . . . . . . . . . 51

3.6 Reflexive Types . . . . . . . . . . . . . . . . . . . . . . . . 54

3.7 An Interlude on Induction Principles . . . . . . . . . . . . . . . 56

3.8 Nested Inductive Types . . . . . . . . . . . . . . . . . . . . . . . 60

3.9 Manual Proofs About Constructors . . . . . . . . . . . . . . . . . 65

$\begin{array}{lll}4 & \text { Inductive Predicates } & 68\end{array}$

4.1 Propositional Logic . . . . . . . . . . . . . . . . . . . . . . . . . . . 69

4.2 What Does It Mean to Be Constructive? . . . . . . . . . . . . . . . . . . 74

4.3 First-Order Logic . . . . . . . . . . . . . . . . . . . . . . 75

4.4 Predicates with Implicit Equality . . . . . . . . . . . . . . . 76

4.5 Recursive Predicates . . . . . . . . . . . . . . . . . . . . . . . 79

5 Infinite Data and Proofs $\quad 86$

5.1 Computing with Infinite Data . . . . . . . . . . . . . . . . 87

5.2 Infinite Proofs . . . . . . . . . . . . . . . . . . . . . . . . . . . . 90

5.3 Simple Modeling of Non-Terminating Programs . . . . . . . . . . . . . . 98

II Programming with Dependent Types 102

6 Subset Types and Variations 103

6.1 Introducing Subset Types . . . . . . . . . . . . . . . . . . 103

6.2 Decidable Proposition Types . . . . . . . . . . . . . . . . . . . . . 110

6.3 Partial Subset Types . . . . . . . . . . . . . . . . . . . . . . . . . 113

6.4 Monadic Notations . . . . . . . . . . . . . . . . . . . . . . . . 115

6.5 A Type-Checking Example . . . . . . . . . . . . . . . . . . . 116

7 General Recursion $\quad 121$

7.1 Well-Founded Recursion . . . . . . . . . . . . . . . . . . . . 122

7.2 A Non-Termination Monad Inspired by Domain Theory . . . . . . . . . . 128

7.3 Co-Inductive Non-Termination Monads . . . . . . . . . . . . . . . 133

7.4 Comparing the Alternatives . . . . . . . . . . . . . . . 137

8 More Dependent Types 139

8.1 Length-Indexed Lists . . . . . . . . . . . . . . . . . . . . . 139

8.2 The One Rule of Dependent Pattern Matching in Coq . . . . . . . . . . . . . 143

8.3 A Tagless Interpreter . . . . . . . . . . . . . . . . . . . . . . . . 144

8.4 Dependently Typed Red-Black Trees . . . . . . . . . . . . . . . . . 150

8.5 A Certified Regular Expression Matcher . . . . . . . . . . . . . . . 159 
9 Dependent Data Structures 165

9.1 More Length-Indexed Lists . . . . . . . . . . . . . . . . . . . . . . . . . . . . 165

9.2 Heterogeneous Lists . . . . . . . . . . . . . . . . . . . . . 169

9.2.1 A Lambda Calculus Interpreter . . . . . . . . . . . . . 171

9.3 Recursive Type Definitions . . . . . . . . . . . . . . . . . . . . . 173

9.4 Data Structures as Index Functions . . . . . . . . . . . . . . . . . . . 175

9.4.1 Another Interpreter Example . . . . . . . . . . . . . . . . 179

9.5 Choosing Between Representations . . . . . . . . . . . . . . 183

10 Reasoning About Equality Proofs $\quad 185$

10.1 The Definitional Equality . . . . . . . . . . . . . . . . . . 185

10.2 Heterogeneous Lists Revisited . . . . . . . . . . . . . . . . . . . . . . 189

10.3 Type-Casts in Theorem Statements . . . . . . . . . . . . . . . . . . . . . . 194

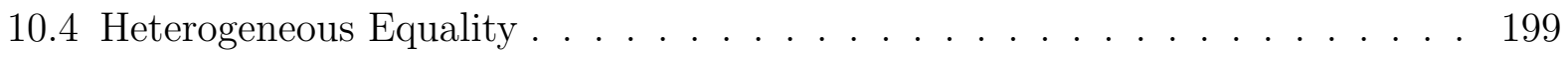

10.5 Equivalence of Equality Axioms . . . . . . . . . . . . . . . . . 203

10.6 Equality of Functions . . . . . . . . . . . . . . . . . . . . . . . 205

11 Generic Programming $\quad 207$

11.1 Reifying Datatype Definitions . . . . . . . . . . . . . . . 207

11.2 Recursive Definitions . . . . . . . . . . . . . . . . . . . . 209

11.2.1 Pretty-Printing . . . . . . . . . . . . . . . 212

11.2.2 Mapping . . . . . . . . . . . . . . . . . . 215

11.3 Proving Theorems about Recursive Definitions . . . . . . . . . . . . . . . 216

12 Universes and Axioms 223

12.1 The Type Hierarchy . . . . . . . . . . . . . . . . . . . . . . . . . . . . . . . . . . . . . . . . . . . .

12.1.1 Inductive Definitions . . . . . . . . . . . . . . . . 226

12.1.2 Deciphering Baffling Messages About Inability to Unify . . . . . . . . 230

12.2 The Prop Universe . . . . . . . . . . . . . . . . . . . . . . . . . . . . . . . . . . . . . . . . . . . . . . .

12.3 Axioms . . . . . . . . . . . . . . . . . . . . 235

12.3.1 The Basics . . . . . . . . . . . . . . . . 236

12.3.2 Axioms of Choice . . . . . . . . . . . . . . . . . . . . . . . . . . . . . . . . . . . . . . .

12.3.3 Axioms and Computation . . . . . . . . . . . . . . . . . 242

12.3.4 Methods for Avoiding Axioms . . . . . . . . . . . . . . . . . 243

III Proof Engineering 252

13 Proof Search by Logic Programming 253

13.1 Introducing Logic Programming . . . . . . . . . . . . . . . . . . . . . . . 253

13.2 Searching for Underconstrained Values . . . . . . . . . . . . . . . 260

13.3 Synthesizing Programs . . . . . . . . . . . . . . . . . . 263

13.4 More on auto Hints . . . . . . . . . . . . . . . . . . . . . . . . . . . 267 
13.5 Rewrite Hints . . . . . . . . . . . . . . . . . . . . . . . 269

14 Proof Search in Ltac $\quad 272$

14.1 Some Built-In Automation Tactics . . . . . . . . . . . . . . . . . . . . 272

14.2 Ltac Programming Basics . . . . . . . . . . . . . . . . . . 273

14.3 Functional Programming in Ltac . . . . . . . . . . . . . . . . . . . . . 279

14.4 Recursive Proof Search . . . . . . . . . . . . . . . . . . . . . 284

14.5 Creating Unification Variables . . . . . . . . . . . . . . . . . 291

15 Proof by Reflection $\quad 297$

15.1 Proving Evenness . . . . . . . . . . . . . . . . . . . . . . . 297

15.2 Reifying the Syntax of a Trivial Tautology Language . . . . . . . . . . . . 300

15.3 A Monoid Expression Simplifier . . . . . . . . . . . . . . . . . . . . 302

15.4 A Smarter Tautology Solver . . . . . . . . . . . . . . . . . 305

15.4.1 Manual Reification of Terms with Variables . . . . . . . . . . . . . . 311

15.5 Building a Reification Tactic that Recurses Under Binders . . . . . . . . . . 313

$\begin{array}{ll}\text { IV The Big Picture } & 317\end{array}$

16 Proving in the Large $\quad 318$

16.1 Ltac Anti-Patterns . . . . . . . . . . . . . . . . . . . . . 318

16.2 Debugging and Maintaining Automation . . . . . . . . . . . . . . 325

16.3 Modules . . . . . . . . . . . . . . . . . . . . . . . . . . . . . . . . . . . . . . . . . . . . . . .

16.4 Build Processes . . . . . . . . . . . . . . . . . . . . 336

17 A Taste of Reasoning About Programming Language Syntax 340

17.1 Dependent de Bruijn Indices . . . . . . . . . . . . . . . . . . . . . 341

17.2 Parametric Higher-Order Abstract Syntax . . . . . . . . . . . . . . . 347

17.2.1 Functional Programming with PHOAS . . . . . . . . . . . . . 348

17.2.2 Verifying Program Transformations . . . . . . . . . . . . . . . 351

17.2.3 Establishing Term Well-Formedness . . . . . . . . . . . . . . . 356

17.2.4 A Few More Remarks . . . . . . . . . . . . . . . . . . . . 357

$\begin{array}{lr}\text { Conclusion } & 359\end{array}$

$\begin{array}{ll}\text { Bibliography } & 360\end{array}$

$\begin{array}{ll}\text { Index } & 364\end{array}$ 


\section{Chapter 1}

\section{Introduction}

\subsection{Whence This Book?}

We would all like to have programs check that our programs are correct. Due in no small part to some bold but unfulfilled promises in the history of computer science, today most people who write software, practitioners and academics alike, assume that the costs of formal program verification outweigh the benefits. The purpose of this book is to convince you that the technology of program verification is mature enough today that it makes sense to use it in a support role in many kinds of research projects in computer science. Beyond the convincing, I also want to provide a handbook on practical engineering of certified programs with the Coq proof assistant. Almost every subject covered is also relevant to interactive computer theorem-proving in general, such as for traditional mathematical theorems. In fact, I hope to demonstrate how verified programs are useful as building blocks in all sorts of formalizations.

Research into mechanized theorem proving began in the second half of the 20th century, and some of the earliest practical work involved Nqthm [3], the "Boyer-Moore Theorem Prover," which was used to prove such theorems as correctness of a complete hardware and software stack [25]. ACL2 [17], Nqthm's successor, has seen significant industry adoption, for instance, by AMD to verify correctness of floating-point division units [26].

Around the beginning of the 21st century, the pace of progress in practical applications of interactive theorem proving accelerated significantly. Several well-known formal developments have been carried out in Coq, the system that this book deals with. In the realm of pure mathematics, Georges Gonthier built a machine-checked proof of the four-color theorem [13], a mathematical problem first posed more than a hundred years before, where the only previous proofs had required trusting ad-hoc software to do brute-force checking of key facts. In the realm of program verification, Xavier Leroy led the CompCert project to produce a verified C compiler back-end [19] robust enough to use with real embedded software.

Many other recent projects have attracted attention by proving important theorems using computer proof assistant software. For instance, the L4.verified project [18] led by Gerwin 
Klein has given a mechanized proof of correctness for a realistic microkernel, using the Isabelle/HOL proof assistant [29]. The amount of ongoing work in the area is so large that I cannot hope to list all the recent successes, so from this point I will assume that the reader is convinced both that we ought to want machine-checked proofs and that they seem to be feasible to produce. (To readers not yet convinced, I suggest a Web search for "machine-checked proof"!)

The idea of certified program features prominently in this book's title. Here the word "certified" does not refer to governmental rules for how the reliability of engineered systems may be demonstrated to sufficiently high standards. Rather, this concept of certification, a standard one in the programming languages and formal methods communities, has to do with the idea of a certificate, or formal mathematical artifact proving that a program meets its specification. Government certification procedures rarely provide strong mathematical guarantees, while certified programming provides guarantees about as strong as anything we could hope for. We trust the definition of a foundational mathematical logic, we trust an implementation of that logic, and we trust that we have encoded our informal intent properly in formal specifications, but few other opportunities remain to certify incorrect software. For compilers and other programs that run in batch mode, the notion of a certifying program is also common, where each run of the program outputs both an answer and a proof that the answer is correct. Any certifying program can be composed with a proof checker to produce a certified program, and this book focuses on the certified case, while also introducing principles and techniques of general interest for stating and proving theorems in Coq.

There are a good number of (though definitely not "many") tools that are in wide use today for building machine-checked mathematical proofs and machine-certified programs. The following is my attempt at an exhaustive list of interactive "proof assistants" satisfying a few criteria. First, the authors of each tool must intend for it to be put to use for softwarerelated applications. Second, there must have been enough engineering effort put into the tool that someone not doing research on the tool itself would feel his time was well spent using it. A third criterion is more of an empirical validation of the second: the tool must have a significant user community outside of its own development team.

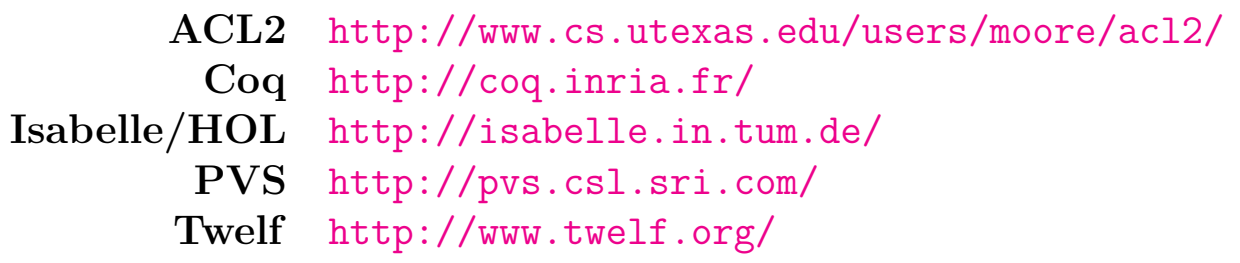

Isabelle/HOL, implemented with the "proof assistant development framework" Isabelle [32], is the most popular proof assistant for the HOL logic. The other implementations of HOL can be considered equivalent for purposes of the discussion here. 


\subsection{Why Coq?}

This book is going to be about certified programming using Coq, and I am convinced that it is the best tool for the job. Coq has a number of very attractive properties, which I will summarize here, mentioning which of the other candidate tools lack which properties.

\subsubsection{Based on a Higher-Order Functional Programming Language}

There is no reason to give up the familiar comforts of functional programming when you start writing certified programs. All of the tools I listed are based on functional programming languages, which means you can use them without their proof-related features to write and run regular programs.

ACL2 is notable in this field for having only a first-order language at its foundation. That is, you cannot work with functions over functions and all those other treats of functional programming. By giving up this facility, ACL2 can make broader assumptions about how well its proof automation will work, but we can generally recover the same advantages in other proof assistants when we happen to be programming in first-order fragments.

\subsubsection{Dependent Types}

A language with dependent types may include references to programs inside of types. For instance, the type of an array might include a program expression giving the size of the array, making it possible to verify absence of out-of-bounds accesses statically. Dependent types can go even further than this, effectively capturing any correctness property in a type. For instance, later in this book, we will see how to give a compiler a type that guarantees that it maps well-typed source programs to well-typed target programs.

ACL2 and HOL lack dependent types outright. Each of PVS and Twelf supports a different strict subset of Coq's dependent type language. Twelf's type language is restricted to a bare-bones, monomorphic lambda calculus, which places serious restrictions on how complicated computations inside types can be. This restriction is important for the soundness argument behind Twelf's approach to representing and checking proofs.

In contrast, PVS's dependent types are much more general, but they are squeezed inside the single mechanism of subset types, where a normal type is refined by attaching a predicate over its elements. Each member of the subset type is an element of the base type that satisfies the predicate. Chapter 6 of this book introduces that style of programming in Coq, while the remaining chapters of Part II deal with features of dependent typing in Coq that go beyond what PVS supports.

Dependent types are useful not only because they help you express correctness properties in types. Dependent types also often let you write certified programs without writing anything that looks like a proof. Even with subset types, which for many contexts can be used to express any relevant property with enough acrobatics, the human driving the proof assistant usually has to build some proofs explicitly. Writing formal proofs is hard, so we want to avoid it as far as possible. Dependent types are invaluable for this purpose. 


\subsubsection{An Easy-to-Check Kernel Proof Language}

Scores of automated decision procedures are useful in practical theorem proving, but it is unfortunate to have to trust in the correct implementation of each procedure. Proof assistants satisfy the "de Bruijn criterion" when they produce proof terms in small kernel languages, even when they use complicated and extensible procedures to seek out proofs in the first place. These core languages have feature complexity on par with what you find in proposals for formal foundations for mathematics (e.g., ZF set theory). To believe a proof, we can ignore the possibility of bugs during search and just rely on a (relatively small) proof-checking kernel that we apply to the result of the search.

Coq meets the de Bruijn criterion, while ACL2 does not, as it employs fancy decision procedures that produce no "evidence trails" justifying their results. PVS supports strategies that implement fancier proof procedures in terms of a set of primitive proof steps, where the primitive steps are less primitive than in Coq. For instance, a propositional tautology solver is included as a primitive, so it is a question of taste whether such a system meets the de Bruijn criterion. The HOL implementations meet the de Bruijn criterion more manifestly; for Twelf, the situation is murkier.

\subsubsection{Convenient Programmable Proof Automation}

A commitment to a kernel proof language opens up wide possibilities for user extension of proof automation systems, without allowing user mistakes to trick the overall system into accepting invalid proofs. Almost any interesting verification problem is undecidable, so it is important to help users build their own procedures for solving the restricted problems that they encounter in particular theorems.

Twelf features no proof automation marked as a bona fide part of the latest release; there is some automation code included for testing purposes. The Twelf style is based on writing out all proofs in full detail. Because Twelf is specialized to the domain of syntactic metatheory proofs about programming languages and logics, it is feasible to use it to write those kinds of proofs manually. Outside that domain, the lack of automation can be a serious obstacle to productivity. Most kinds of program verification fall outside Twelf's forte.

Of the remaining tools, all can support user extension with new decision procedures by hacking directly in the tool's implementation language (such as OCaml for Coq). Since ACL2 and PVS do not satisfy the de Bruijn criterion, overall correctness is at the mercy of the authors of new procedures.

Isabelle/HOL and Coq both support coding new proof manipulations in ML in ways that cannot lead to the acceptance of invalid proofs. Additionally, Coq includes a domain-specific language for coding decision procedures in normal Coq source code, with no need to break out into ML. This language is called Ltac, and I think of it as the unsung hero of the proof assistant world. Not only does Ltac prevent you from making fatal mistakes, it also includes a number of novel programming constructs which combine to make a "proof by decision procedure" style very pleasant. We will meet these features in the chapters to come. 


\subsubsection{Proof by Reflection}

A surprising wealth of benefits follows from choosing a proof language that integrates a rich notion of computation. Coq includes programs and proof terms in the same syntactic class. This makes it easy to write programs that compute proofs. With rich enough dependent types, such programs are certified decision procedures. In such cases, these certified procedures can be put to good use without ever running them! Their types guarantee that, if we did bother to run them, we would receive proper "ground" proofs.

The critical ingredient for this technique, many of whose instances are referred to as proof by reflection, is a way of inducing non-trivial computation inside of logical propositions during proof checking. Further, most of these instances require dependent types to make it possible to state the appropriate theorems. Of the proof assistants I listed, only Coq really provides support for the type-level computation style of reflection, though PVS supports very similar functionality via refinement types.

\subsection{Why Not a Different Dependently Typed Language?}

The logic and programming language behind Coq belongs to a type-theory ecosystem with a good number of other thriving members. $\mathrm{Agda}^{1}$ and Epigram ${ }^{2}$ are the most developed tools among the alternatives to Coq, and there are others that are earlier in their lifecycles. All of the languages in this family feel sort of like different historical offshoots of Latin. The hardest conceptual epiphanies are, for the most part, portable among all the languages. Given this, why choose Coq for certified programming?

I think the answer is simple. None of the competition has well-developed systems for tactic-based theorem proving. Agda and Epigram are designed and marketed more as programming languages than proof assistants. Dependent types are great, because they often help you prove deep theorems without doing anything that feels like proving. Nonetheless, almost any interesting certified programming project will benefit from some activity that deserves to be called proving, and many interesting projects absolutely require semi-automated proving, to protect the sanity of the programmer. Informally, proving is unavoidable when any correctness proof for a program has a structure that does not mirror the structure of the program itself. An example is a compiler correctness proof, which probably proceeds by induction on program execution traces, which have no simple relationship with the structure of the compiler or the structure of the programs it compiles. In building such proofs, a mature system for scripted proof automation is invaluable.

On the other hand, Agda, Epigram, and similar tools have less implementation baggage associated with them, and so they tend to be the default first homes of innovations in practical type theory. Some significant kinds of dependently typed programs are much easier to write in Agda and Epigram than in Coq. The former tools may very well be superior choices for projects that do not involve any "proving." Anecdotally, I have gotten the impression that

\footnotetext{
${ }^{1}$ http://appserv.cs.chalmers.se/users/ulfn/wiki/agda.php

${ }^{2}$ https://code.google.com/p/epigram/
} 
manual proving is orders of magnitudes more costly than manual coping with Coq's lack of programming bells and whistles. In this book, I will devote significant space to patterns for programming with dependent types in Coq as it is today. We can hope that the type theory community is tending towards convergence on the right set of features for practical programming with dependent types, and that we will eventually have a single tool embodying those features.

\subsection{Engineering with a Proof Assistant}

In comparisons with its competitors, Coq is often derided for promoting unreadable proofs. It is very easy to write proof scripts that manipulate proof goals imperatively, with no structure to aid readers. Such developments are nightmares to maintain, and they certainly do not manage to convey "why the theorem is true" to anyone but the original author. One additional (and not insignificant) purpose of this book is to show why it is unfair and unproductive to dismiss Coq based on the existence of such developments.

I will go out on a limb and guess that the reader is a fan of some programming language and may even have been involved in teaching that language to undergraduates. I want to propose an analogy between two attitudes: coming to a negative conclusion about Coq after reading common Coq developments in the wild, and coming to a negative conclusion about Your Favorite Language after looking at the programs undergraduates write in it in the first week of class. The pragmatics of mechanized proving and program verification have been under serious study for much less time than the pragmatics of programming have been. The computer theorem proving community is still developing the key insights that correspond to those that programming texts and instructors impart to their students, to help those students get over that critical hump where using the language stops being more trouble than it is worth. Most of the insights for Coq are barely even disseminated among the experts, let alone set down in a tutorial form. I hope to use this book to go a long way towards remedying that.

If I do that job well, then this book should be of interest even to people who have participated in classes or tutorials specifically about Coq. The book should even be useful to people who have been using Coq for years but who are mystified when their Coq developments prove impenetrable by colleagues. The crucial angle in this book is that there are "design patterns" for reliably avoiding the really grungy parts of theorem proving, and consistent use of these patterns can get you over the hump to the point where it is worth your while to use Coq to prove your theorems and certify your programs, even if formal verification is not your main concern in a project. We will follow this theme by pursuing two main methods for replacing manual proofs with more understandable artifacts: dependently typed functions and custom Ltac decision procedures. 


\subsection{Prerequisites}

I try to keep the required background knowledge to a minimum in this book. I will assume familiarity with the material from usual discrete math and logic courses taken by undergraduate computer science majors, and I will assume that readers have significant experience programming in one of the ML dialects, in Haskell, or in some other, closely related language. Experience with only dynamically typed functional languages might lead to befuddlement in some places, but a reader who has come to understand Scheme deeply will probably be fine.

My background is in programming languages, formal semantics, and program verification. I sometimes use examples from that domain. As a reference on these topics, I recommend Types and Programming Languages [36], by Benjamin C. Pierce; however, I have tried to choose examples so that they may be understood without background in semantics.

\subsection{Using This Book}

This book is generated automatically from Coq source files using the wonderful coqdoc program. The latest PDF version, with hyperlinks from identifier uses to the corresponding definitions, is available at:

$$
\text { http://adam.chlipala.net/cpdt/cpdt.pdf }
$$

There is also an online HTML version available, which of course also provides hyperlinks:

$$
\text { http://adam.chlipala.net/cpdt/html/toc.html }
$$

The source code to the book is also freely available at:

$$
\text { http://adam.chlipala.net/cpdt/cpdt.tgz }
$$

There, you can find all of the code appearing in this book, with prose interspersed in comments, in exactly the order that you find in this document. You can step through the code interactively with your chosen graphical Coq interface. The code also has special comments indicating which parts of the chapters make suitable starting points for interactive class sessions, where the class works together to construct the programs and proofs. The included Makefile has a target templates for building a fresh set of class template files automatically from the book source.

A traditional printed version of the book is slated to appear from MIT Press in the future. The online versions will remain available at no cost even after the printed book is released, and I intend to keep the source code up-to-date with bug fixes and compatibility changes to track new Coq releases.

I believe that a good graphical interface to Coq is crucial for using it productively. I use the Proof General ${ }^{3}$ mode for Emacs, which supports a number of other proof assistants

\footnotetext{
${ }^{3}$ http://proofgeneral.inf.ed.ac.uk/
} 
besides Coq. There is also the standalone CoqIDE program developed by the Coq team. I like being able to combine certified programming and proving with other kinds of work inside the same full-featured editor. In the initial part of this book, I will reference Proof General procedures explicitly, in introducing how to use Coq, but most of the book will be interface-agnostic, so feel free to use CoqIDE if you prefer it. The one issue with CoqIDE before version 8.4, regarding running through the book source, is that I will sometimes begin a proof attempt but cancel it with the Coq Abort or Restart commands, which CoqIDE did not support until recently. It would be bad form to leave such commands lying around in a real, finished development, but I find these commands helpful in writing single source files that trace a user's thought process in designing a proof.

\subsubsection{Reading This Book}

For experts in functional programming or formal methods, learning to use Coq is not hard, in a sense. The Coq manual [7], the textbook by Bertot and Castéran [1], and Pierce et al.'s Software Foundations ${ }^{4}$ have helped many people become productive Coq users. However, I believe that the best ways to manage significant Coq developments are far from settled. In this book, I mean to propose my own techniques, and, rather than treating them as advanced material for a final chapter or two, I employ them from the very beginning. After a first chapter showing off what can be done with dependent types, I retreat into simpler programming styles for the first part of the book. I adopt the other main thrust of the book, Ltac proof automation, more or less from the very start of the technical exposition.

Some readers have suggested that I give multiple recommended reading orders in this introduction, targeted at people with different levels of Coq expertise. It is certainly true that Part I of the book devotes significant space to basic concepts that most Coq users already know quite well. However, as I am introducing these concepts, I am also developing my preferred automated proof style, so I think even the chapters on basics are worth reading for experienced Coq hackers.

Readers with no prior Coq experience can ignore the preceding discussion! I hope that my heavy reliance on proof automation early on will seem like the most natural way to go, such that you may wonder why others are spending so much time entering sequences of proof steps manually.

Coq is a very complex system, with many different commands driven more by pragmatic concerns than by any overarching aesthetic principle. When I use some construct for the first time, I try to give a one-sentence intuition for what it accomplishes, but I leave the details to the Coq reference manual [7]. I expect that readers interested in complete understanding will be consulting that manual frequently; in that sense, this book is not meant to be completely standalone. I often use constructs in code snippets without first introducing them at all, but explanations should always follow in the prose paragraphs immediately after the offending snippets.

Previous versions of the book included some suggested exercises at the ends of chapters.

\footnotetext{
${ }^{4}$ http://www.cis.upenn.edu/ bcpierce/sf/
} 
Since then, I have decided to remove the exercises and focus on the main book exposition. A database of exercises proposed by various readers of the book is available on the $\mathrm{Web}^{5}$. I do want to suggest, though, that the best way to learn Coq is to get started applying it in a real project, rather than focusing on artificial exercises.

\subsubsection{On the Tactic Library}

To make it possible to start from fancy proof automation, rather than working up to it, I have included with the book source a library of tactics, or programs that find proofs, since the built-in Coq tactics do not support a high enough level of automation. I use these tactics even from the first chapter with code examples.

Some readers have asked about the pragmatics of using this tactic library in their own developments. My position there is that this tactic library was designed with the specific examples of the book in mind; I do not recommend using it in other settings. Part III should impart the necessary skills to reimplement these tactics and beyond. One generally deals with undecidable problems in interactive theorem proving, so there can be no tactic that solves all goals, though the crush tactic that we will meet soon may sometimes feel like that! There are still very useful tricks found in the implementations of crush and its cousins, so it may be useful to examine the commented source file CpdtTactics.v. I implement a new tactic library for each new project, since each project involves a different mix of undecidable theories where a different set of heuristics turns out to work well; and that is what I recommend others do, too.

\subsubsection{Installation and Emacs Set-Up}

At the start of the next chapter, I assume that you have installed Coq and Proof General. The code in this book is tested with Coq version 8.9.0. Though parts may work with other versions, it is expected that the book source will fail to build with many earlier versions.

To set up your Proof General environment to process the source to the next chapter, a few simple steps are required.

1. Get the book source from

$$
\text { http://adam.chlipala.net/cpdt/cpdt.tgz }
$$

2. Unpack the tarball to some directory DIR.

3. Run make in DIR (ideally with a $-\mathrm{j}$ flag to use multiple processor cores, if you have them).

4. There are some minor headaches associated with getting Proof General to pass the proper command line arguments to the coqtop program, which provides the interactive

\footnotetext{
${ }^{5}$ http://adam.chlipala.net/cpdt/ex/
} 
Coq toplevel. One way to add settings that will be shared by many source files is to add a custom variable setting to your . emacs file, like this:

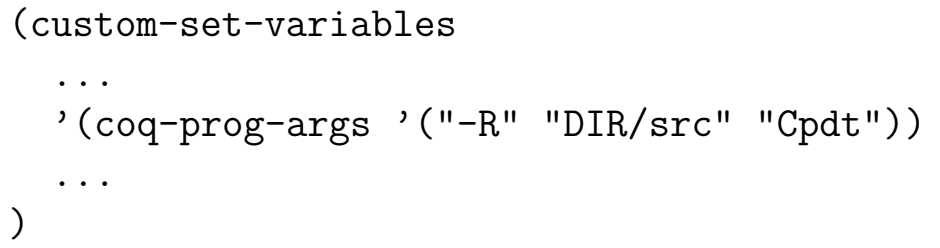

The extra arguments demonstrated here are the proper choices for working with the code for this book. The ellipses stand for other Emacs customization settings you may already have. It can be helpful to save several alternate sets of flags in your .emacs file, with all but one commented out within the custom-set-variables block at any given time.

Alternatively, Proof General configuration can be set on a per-directory basis, using a .dir-locals.el file in the directory of the source files for which you want the settings to apply. Here is an example that could be written in such a file to enable use of the book source. Note the need to include an argument that starts Coq in Emacs support mode.

$(($ coq-mode . ((coq-prog-args . ("-emacs-U" "-R" "DIR/src" "Cpdt")))))

Every chapter of this book is generated from a commented Coq source file. You can load these files and run through them step-by-step in Proof General. Be sure to run the Coq binary coqtop with the command-line argument -R DIR/src Cpdt. If you have installed Proof General properly, the Coq mode should start automatically when you visit a . v buffer in Emacs, and the above advice on .emacs settings should ensure that the proper arguments are passed to coqtop by Emacs.

With Proof General, the portion of a buffer that Coq has processed is highlighted in some way, like being given a blue background. You step through Coq source files by positioning the point at the position you want Coq to run to and pressing C-C C-RET. This can be used both for normal step-by-step coding, by placing the point inside some command past the end of the highlighted region; and for undoing, by placing the point inside the highlighted region. 


\subsection{Chapter Source Files}

\begin{tabular}{|r|l|}
\hline Chapter & Source \\
\hline Some Quick Examples & StackMachine.v \\
\hline Introducing Inductive Types & InductiveTypes.v \\
\hline Inductive Predicates & Predicates.v \\
\hline Infinite Data and Proofs & Coinductive.v \\
\hline Subset Types and Variations & Subset.v \\
\hline General Recursion & GeneralRec.v \\
\hline More Dependent Types & MoreDep.v \\
\hline Dependent Data Structures & DataStruct.v \\
\hline Reasoning About Equality Proofs & Equality.v \\
\hline Generic Programming & Generic.v \\
\hline Universes and Axioms & Universes.v \\
\hline Proof Search by Logic Programming & LogicProg.v \\
\hline Proof Search in Ltac & Match.v \\
\hline Proof by Reflection & Reflection.v \\
\hline Proving in the Large & Large.v \\
\hline A Taste of Reasoning About Programming Language Syntax & ProgLang.v \\
\hline
\end{tabular}




\section{Chapter 2}

\section{Some Quick Examples}

I will start off by jumping right in to a fully worked set of examples, building certified compilers from increasingly complicated source languages to stack machines. We will meet a few useful tactics and see how they can be used in manual proofs, and we will also see how easily these proofs can be automated instead. This chapter is not meant to give full explanations of the features that are employed. Rather, it is meant more as an advertisement of what is possible. Later chapters will introduce all of the concepts in bottom-up fashion. In other words, it is expected that most readers will not understand what exactly is going on here, but I hope this demo will whet your appetite for the remaining chapters!

As always, you can step through the source file StackMachine.v for this chapter interactively in Proof General. Alternatively, to get a feel for the whole lifecycle of creating a Coq development, you can enter the pieces of source code in this chapter in a new. $\mathrm{v}$ file in an Emacs buffer. If you do the latter, include these three lines at the start of the file.

Require Import Bool Arith List Cpdt.CpdtTactics.

Set Implicit Arguments.

Set Asymmetric Patterns.

In general, similar commands will be hidden in the book rendering of each chapter's source code, so you will need to insert them in from-scratch replayings of the code that is presented. To be more specific, every chapter begins with the above three lines, with the import list tweaked as appropriate, considering which definitions the chapter uses. The second command above affects the default behavior of definitions regarding type inference, and the third allows for more concise pattern-matching syntax in Coq versions 8.5 and higher (having no effect in earlier versions).

\subsection{Arithmetic Expressions Over Natural Numbers}

We will begin with that staple of compiler textbooks, arithmetic expressions over a single type of numbers. 


\subsubsection{Source Language}

We begin with the syntax of the source language.

Inductive binop : Set $:=$ Plus | Times.

Our first line of Coq code should be unsurprising to ML and Haskell programmers. We define an algebraic datatype binop to stand for the binary operators of our source language. There are just two wrinkles compared to ML and Haskell. First, we use the keyword Inductive, in place of data, datatype, or type. This is not just a trivial surface syntax difference; inductive types in Coq are much more expressive than garden variety algebraic datatypes, essentially enabling us to encode all of mathematics, though we begin humbly in this chapter. Second, there is the : Set fragment, which declares that we are defining a datatype that should be thought of as a constituent of programs. Later, we will see other options for defining datatypes in the universe of proofs or in an infinite hierarchy of universes, encompassing both programs and proofs, that is useful in higher-order constructions.

Inductive exp : Set :=

| Const : nat $\rightarrow$ exp

| Binop : binop $\rightarrow \exp \rightarrow \exp \rightarrow$ exp.

Now we define the type of arithmetic expressions. We write that a constant may be built from one argument, a natural number; and a binary operation may be built from a choice of operator and two operand expressions.

A note for readers following along in the PDF version: coqdoc supports pretty-printing of tokens in $\mathrm{AT}_{\mathrm{E}} \mathrm{X}$ or HTML. Where you see a right arrow character, the source contains the ASCII text $->$. Other examples of this substitution appearing in this chapter are a double right arrow for $=>$, the inverted 'A' symbol for forall, and the Cartesian product ' $\mathrm{X}$ ' for *. When in doubt about the ASCII version of a symbol, you can consult the chapter source code.

Now we are ready to say what programs in our expression language mean. We will do this by writing an interpreter that can be thought of as a trivial operational or denotational semantics. (If you are not familiar with these semantic techniques, no need to worry: we will stick to "common sense" constructions.)

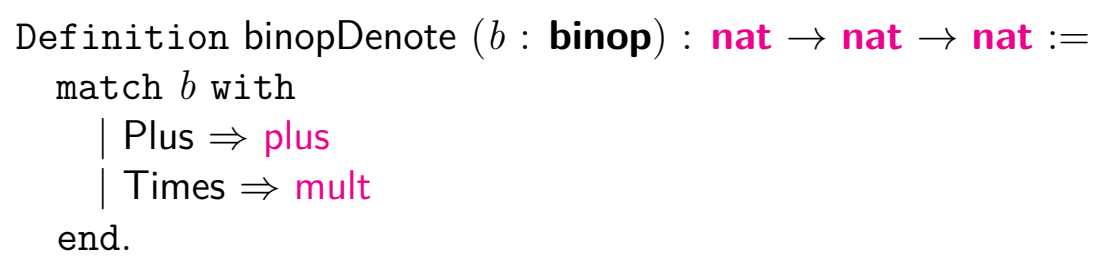

The meaning of a binary operator is a binary function over naturals, defined with patternmatching notation analogous to the case and match of ML and Haskell, and referring to the functions plus and mult from the Coq standard library. The keyword Definition is Coq's all-purpose notation for binding a term of the programming language to a name, with some associated syntactic sugar, like the notation we see here for defining a function. That sugar could be expanded to yield this definition: 


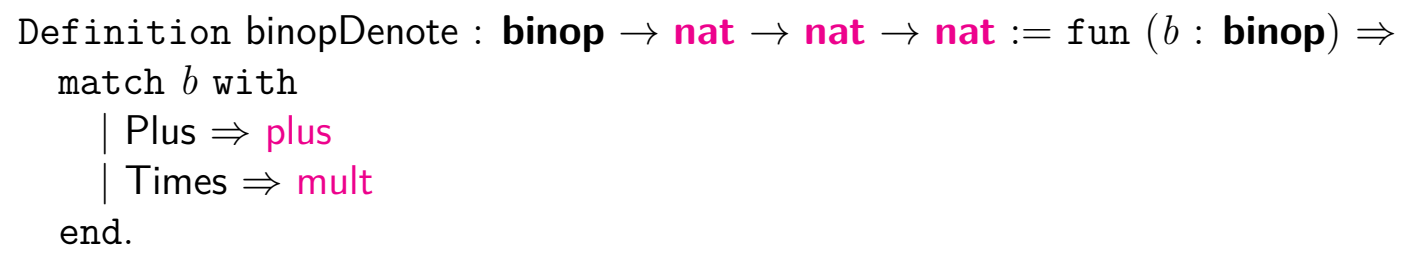

In this example, we could also omit all of the type annotations, arriving at:

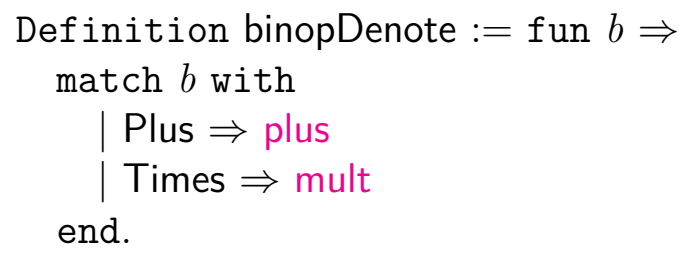

Languages like Haskell and ML have a convenient principal types property, which gives us strong guarantees about how effective type inference will be. Unfortunately, Coq's type system is so expressive that any kind of "complete" type inference is impossible, and the task even seems to be hard in practice. Nonetheless, Coq includes some very helpful heuristics, many of them copying the workings of Haskell and ML type-checkers for programs that fall in simple fragments of Coq's language.

This is as good a time as any to mention the profusion of different languages associated with Coq. The theoretical foundation of Coq is a formal system called the Calculus of Inductive Constructions (CIC) [31], which is an extension of the older Calculus of Constructions (CoC) [9]. CIC is quite a spartan foundation, which is helpful for proving metatheory but not so helpful for real development. Still, it is nice to know that it has been proved that CIC enjoys properties like strong normalization [31], meaning that every program (and, more importantly, every proof term) terminates; and relative consistency [48] with systems like versions of Zermelo-Fraenkel set theory, which roughly means that you can believe that Coq proofs mean that the corresponding propositions are "really true," if you believe in set theory.

Coq is actually based on an extension of CIC called Gallina. The text after the := and before the period in the last code example is a term of Gallina. Gallina includes several useful features that must be considered as extensions to CIC. The important metatheorems about CIC have not been extended to the full breadth of the features that go beyond the formalized language, but most Coq users do not seem to lose much sleep over this omission.

Next, there is Ltac, Coq's domain-specific language for writing proofs and decision procedures. We will see some basic examples of Ltac later in this chapter, and much of this book is devoted to more involved Ltac examples.

Finally, commands like Inductive and Definition are part of the Vernacular, which includes all sorts of useful queries and requests to the Coq system. Every Coq source file is a series of vernacular commands, where many command forms take arguments that are Gallina or Ltac programs. (Actually, Coq source files are more like trees of vernacular commands, thanks to various nested scoping constructs.)

We can give a simple definition of the meaning of an expression: 


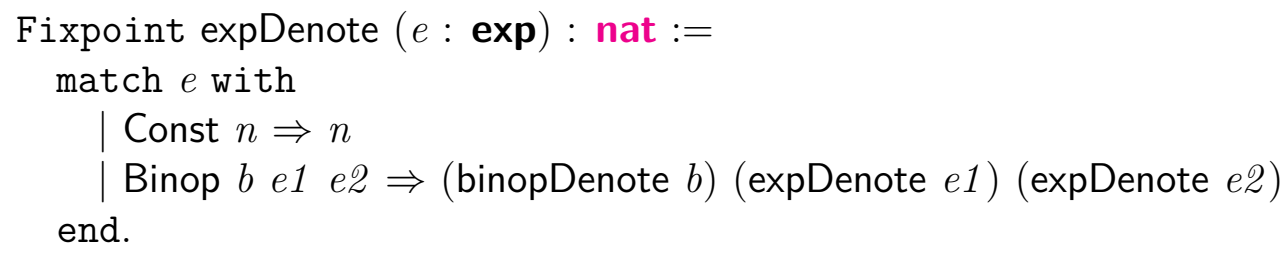

We declare explicitly that this is a recursive definition, using the keyword Fixpoint. The rest should be old hat for functional programmers.

It is convenient to be able to test definitions before starting to prove things about them. We can verify that our semantics is sensible by evaluating some sample uses, using the command Eval. This command takes an argument expressing a reduction strategy, or an "order of evaluation." Unlike with ML, which hardcodes an eager reduction strategy, or Haskell, which hardcodes a lazy strategy, in Coq we are free to choose between these and many other orders of evaluation, because all Coq programs terminate. In fact, Coq silently checked termination of our Fixpoint definition above, using a simple heuristic based on monotonically decreasing size of arguments across recursive calls. Specifically, recursive calls must be made on arguments that were pulled out of the original recursive argument with match expressions. (In Chapter 7, we will see some ways of getting around this restriction, though simply removing the restriction would leave Coq useless as a theorem proving tool, for reasons we will start to learn about in the next chapter.)

To return to our test evaluations, we run the Eval command using the simpl evaluation strategy, whose definition is best postponed until we have learned more about Coq's foundations, but which usually gets the job done.

Eval simpl in expDenote (Const 42 ).

$=42$ : nat

Eval simpl in expDenote (Binop Plus (Const 2) (Const 2)).

$=4:$ nat

Eval simpl in expDenote (Binop Times (Binop Plus (Const 2) (Const 2)) (Const 7)).

$=28:$ nat

Nothing too surprising goes on here, so we are ready to move on to the target language of our compiler.

\subsubsection{Target Language}

We will compile our source programs onto a simple stack machine, whose syntax is:

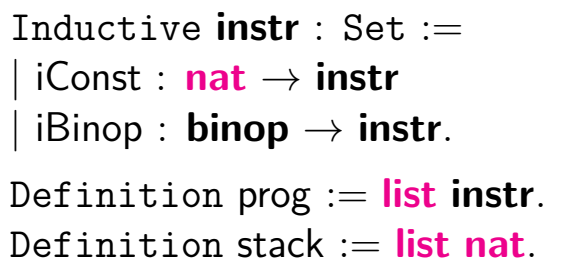

An instruction either pushes a constant onto the stack or pops two arguments, applies 
a binary operator to them, and pushes the result onto the stack. A program is a list of instructions, and a stack is a list of natural numbers.

We can give instructions meanings as functions from stacks to optional stacks, where running an instruction results in None in case of a stack underflow and results in Some $s$ ' when the result of execution is the new stack $s$ '. The infix operator :: is "list cons" from the Coq standard library.

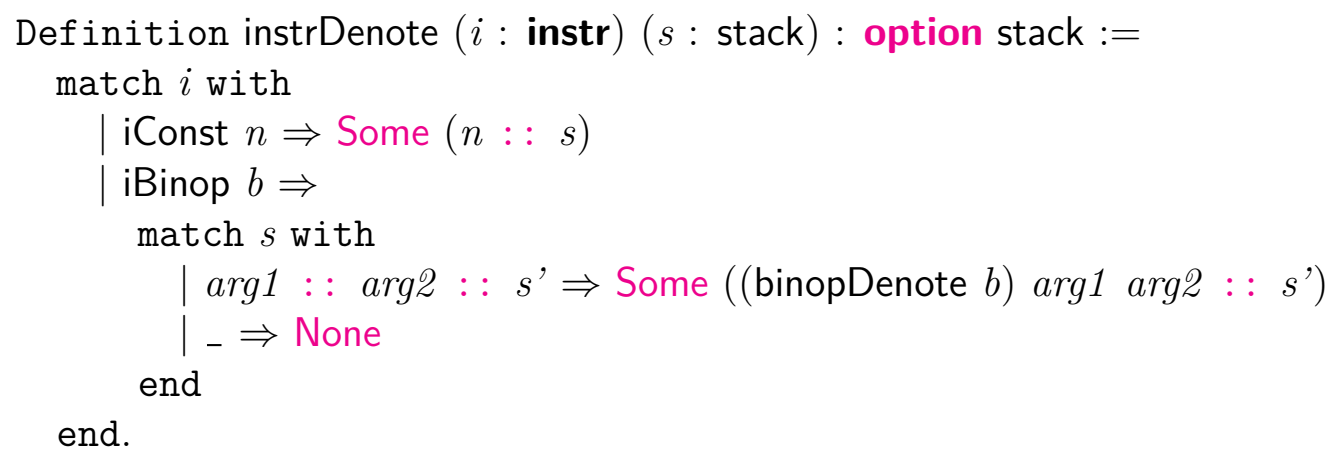

With instrDenote defined, it is easy to define a function progDenote, which iterates application of instrDenote through a whole program.

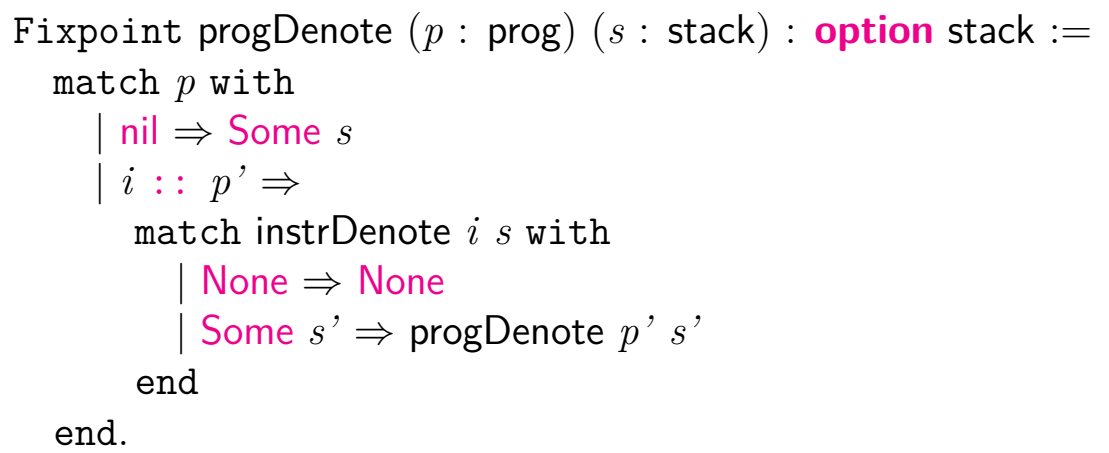

With the two programming languages defined, we can turn to the compiler definition.

\subsubsection{Translation}

Our compiler itself is now unsurprising. The list concatenation operator ++ comes from the Coq standard library.

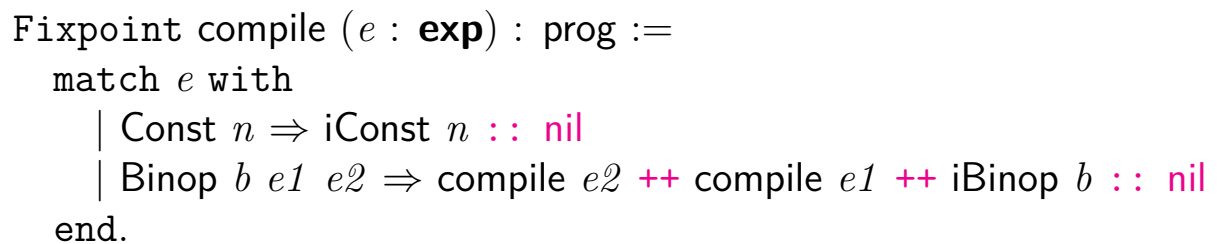

Before we set about proving that this compiler is correct, we can try a few test runs, using our sample programs from earlier.

Eval simpl in compile (Const 42).

$=$ iConst 42 :: nil : prog 
Eval simpl in compile (Binop Plus (Const 2) (Const 2)).

$=$ iConst $2::$ iConst $2::$ iBinop Plus :: nil : prog

Eval simpl in compile (Binop Times (Binop Plus (Const 2) (Const 2)) (Const 7)).

$=$ iConst $7::$ iConst $2:$ iConst $2::$ iBinop Plus :: iBinop Times :: nil : prog

We can also run our compiled programs and check that they give the right results.

Eval simpl in progDenote (compile (Const 42)) nil.

$=$ Some $(42::$ nil) : option stack

Eval simpl in progDenote (compile (Binop Plus (Const 2) (Const 2))) nil.

$=$ Some $(4::$ nil $)$ : option stack

Eval simpl in progDenote (compile (Binop Times (Binop Plus (Const 2) (Const 2))

(Const 7))) nil.

= Some $(28::$ nil $)$ : option stack

So far so good, but how can we be sure the compiler operates correctly for all input programs?

\subsubsection{Translation Correctness}

We are ready to prove that our compiler is implemented correctly. We can use a new vernacular command Theorem to start a correctness proof, in terms of the semantics we defined earlier:

Theorem compile_correct : $\forall e$, progDenote (compile $e$ ) nil = Some (expDenote $e:$ : nil).

Though a pencil-and-paper proof might clock out at this point, writing "by a routine induction on $e$," it turns out not to make sense to attack this proof directly. We need to use the standard trick of strengthening the induction hypothesis. We do that by proving an auxiliary lemma, using the command Lemma that is a synonym for Theorem, conventionally used for less important theorems that appear in the proofs of primary theorems.

Abort.

Lemma compile_correct' : $\forall$ e p s, progDenote (compile $e++p) s=$ progDenote $p$ (expDenote $e:: s$ ).

After the period in the Lemma command, we are in the interactive proof-editing mode. We find ourselves staring at this ominous screen of text:

1 subgoal

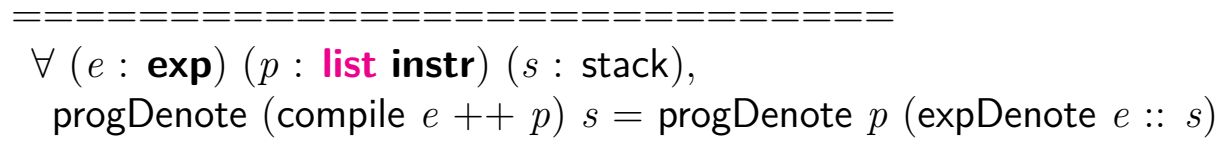

Coq seems to be restating the lemma for us. What we are seeing is a limited case of a more general protocol for describing where we are in a proof. We are told that we have 
a single subgoal. In general, during a proof, we can have many pending subgoals, each of which is a logical proposition to prove. Subgoals can be proved in any order, but it usually works best to prove them in the order that Coq chooses.

Next in the output, we see our single subgoal described in full detail. There is a doubledashed line, above which would be our free variables and hypotheses, if we had any. Below the line is the conclusion, which, in general, is to be proved from the hypotheses.

We manipulate the proof state by running commands called tactics. Let us start out by running one of the most important tactics:

induction $e$.

We declare that this proof will proceed by induction on the structure of the expression $e$. This swaps out our initial subgoal for two new subgoals, one for each case of the inductive proof:

2 subgoals

$$
\begin{aligned}
& n: \text { nat } \\
& =================== \\
& \forall(s: \text { stack })(p: \text { list instr }), \\
& \quad \text { progDenote }(\text { compile }(\text { Const } n)++p) s= \\
& \quad \text { progDenote } p(\text { expDenote }(\text { Const } n):: s)
\end{aligned}
$$

subgoal 2 is

$$
\begin{aligned}
& \forall(s: \text { stack })(p \text { : list instr }) \\
& \quad \text { progDenote }(\text { compile (Binop } b \text { el } 1 \text { e2) }++p) s= \\
& \quad \text { progDenote } p(\operatorname{expDenote}(\text { Binop } b \text { e1 } 1 \text { e2) }:: s)
\end{aligned}
$$

The first and current subgoal is displayed with the double-dashed line below free variables and hypotheses, while later subgoals are only summarized with their conclusions. We see an example of a free variable in the first subgoal; $n$ is a free variable of type nat. The conclusion is the original theorem statement where $e$ has been replaced by Const $n$. In a similar manner, the second case has $e$ replaced by a generalized invocation of the Binop expression constructor. We can see that proving both cases corresponds to a standard proof by structural induction.

We begin the first case with another very common tactic.

intros.

The current subgoal changes to:

$n$ : nat

$s$ : stack

$p$ : list instr 


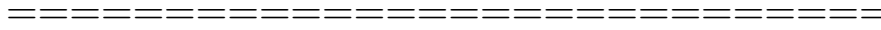

progDenote (compile (Const $n)++p) s=$

progDenote $p$ (expDenote (Const $n):: s$ )

We see that intros changes $\forall$-bound variables at the beginning of a goal into free variables.

To progress further, we need to use the definitions of some of the functions appearing in the goal. The unfold tactic replaces an identifier with its definition.

unfold compile.

$n$ : nat

$s:$ stack

$p$ : list instr

$==================$

progDenote ((iConst $n::$ nil $)++p) s=$

progDenote $p$ (expDenote (Const $n):: s$ )

unfold expDenote.

$n$ : nat

$s:$ stack

$p$ : list instr

$==================$

progDenote $(($ iConst $n::$ nil $)++p) s=\operatorname{progDenote~} p(n:: s)$

We only need to unfold the first occurrence of progDenote to prove the goal. An at clause used with unfold specifies a particular occurrence of an identifier to unfold, where we count occurrences from left to right.

unfold progDenote at 1 .

$n$ : nat

$s:$ stack

$p$ : list instr

$==================$

(fix progDenote $(p 0:$ prog $)(s 0$ : stack) \{struct $p 0\}$ :

option stack $:=$

match $p 0$ with

| nil $\Rightarrow$ Some $s 0$

| $i: p^{\prime} \Rightarrow$

match instrDenote $i s 0$ with 


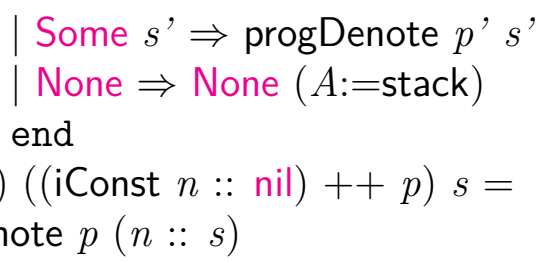

This last unfold has left us with an anonymous recursive definition of progDenote (similarly to how fun or "lambda" constructs in general allow anonymous non-recursive functions), which will generally happen when unfolding recursive definitions. Note that Coq has automatically renamed the fix arguments $p$ and $s$ to $p 0$ and $s 0$, to avoid clashes with our local free variables. There is also a subterm None $(A:=$ stack), which has an annotation specifying that the type of the term ought to be option stack. This is phrased as an explicit instantiation of a named type parameter $A$ from the definition of option.

Fortunately, in this case, we can eliminate the complications of anonymous recursion right away, since the structure of the argument (iConst $n::$ nil) $++p$ is known, allowing us to simplify the internal pattern match with the simpl tactic, which applies the same reduction strategy that we used earlier with Eval (and whose details we still postpone).

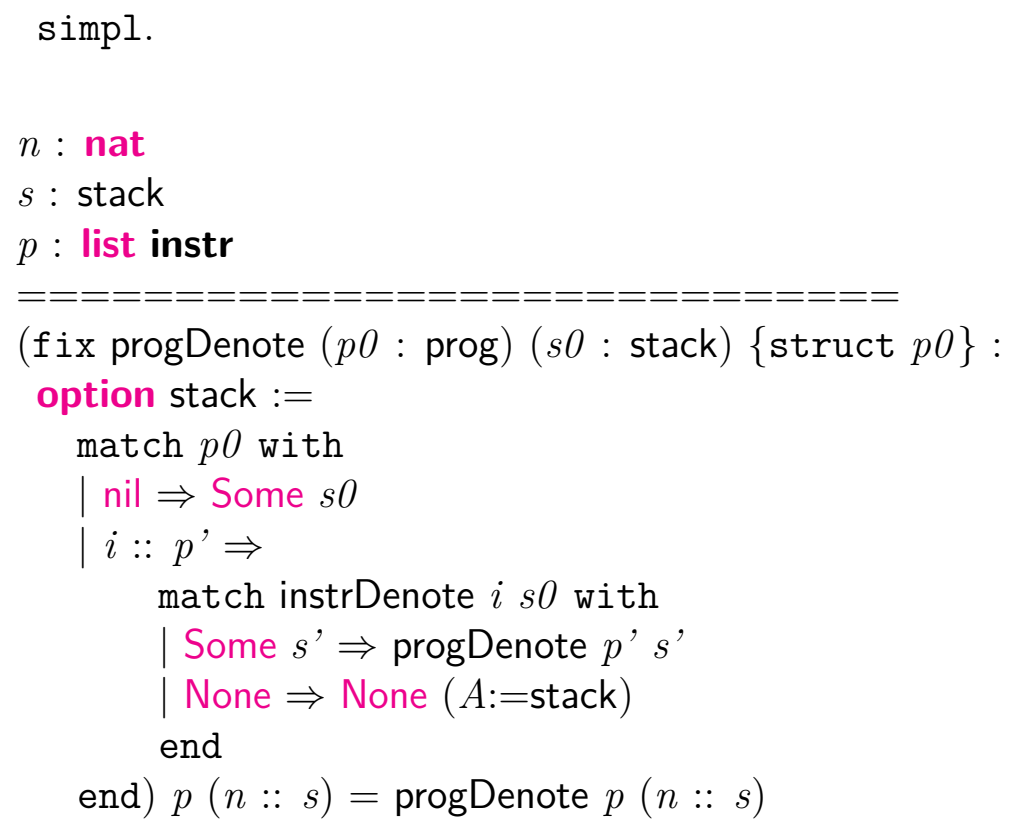

Now we can unexpand the definition of progDenote: fold progDenote.

$n$ : nat

$s$ : stack

$p$ : list instr

$==\mathrm{=}=\mathrm{=}=\mathrm{=}=\mathrm{=}=\mathrm{=}=\mathrm{=}=\mathrm{=}=\mathrm{=}=\mathrm{=}$ 
progDenote $p(n:: s)=$ progDenote $p(n:: s)$

It looks like we are at the end of this case, since we have a trivial equality. Indeed, a single tactic finishes us off:

reflexivity.

On to the second inductive case:

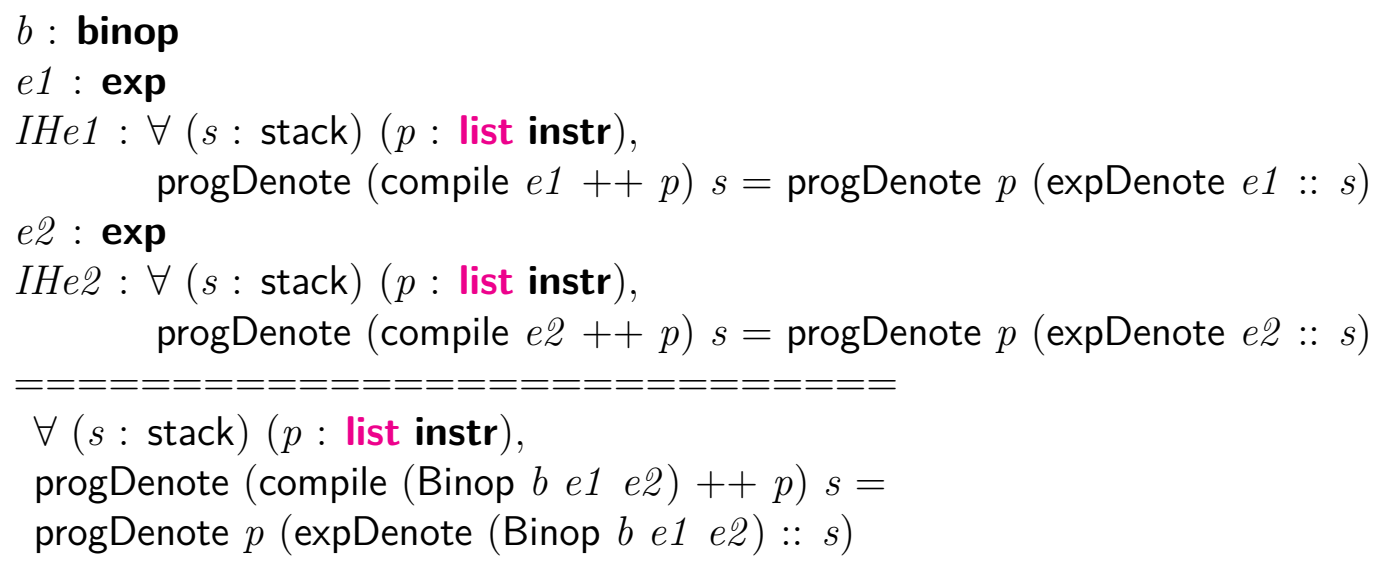

We see our first example of hypotheses above the double-dashed line. They are the inductive hypotheses $I H e 1$ and IHe2 corresponding to the subterms $e 1$ and $e \mathscr{2}$, respectively.

We start out the same way as before, introducing new free variables and unfolding and folding the appropriate definitions. The seemingly frivolous unfold/fold pairs are actually accomplishing useful work, because unfold will sometimes perform easy simplifications.

intros.

unfold compile.

fold compile.

unfold expDenote.

fold expDenote.

Now we arrive at a point where the tactics we have seen so far are insufficient. No further definition unfoldings get us anywhere, so we will need to try something different.

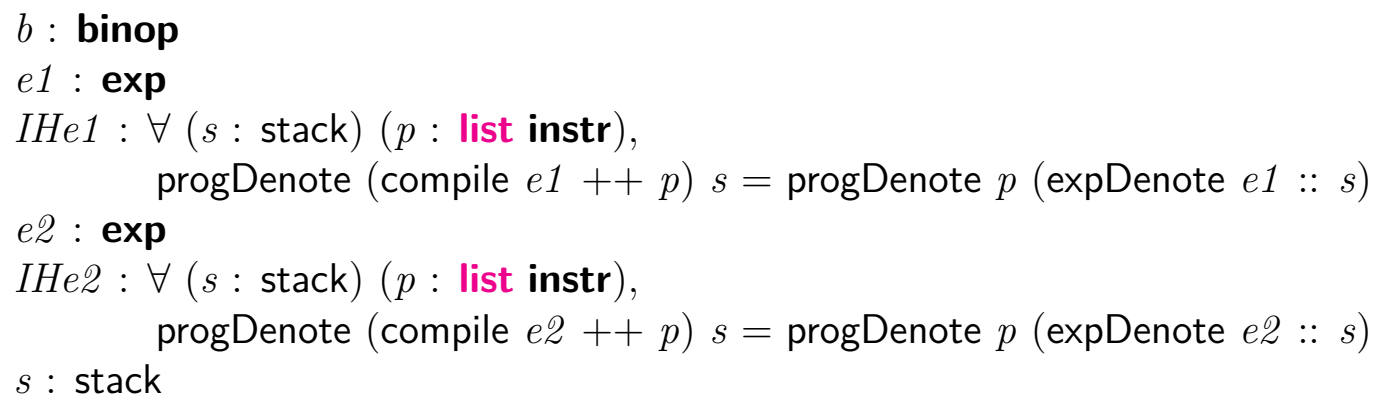




\section{$p$ : list instr}

$==================$

progDenote ((compile $e \mathscr{L}++$ compile $e 1++$ iBinop $b::$ nil $)++p) s=$ progDenote $p$ (binopDenote $b(\operatorname{expDenote} e 1)(\operatorname{expDenote} e \mathscr{2}):: s)$

What we need is the associative law of list concatenation, which is available as a theorem app_assoc_reverse in the standard library. (Here and elsewhere, it is possible to tell the difference between inputs and outputs to Coq by periods at the ends of the inputs.)

Check app_assoc_reverse.

app_assoc_reverse

$: \forall(A:$ Type $)(l m n:$ list $A),(l++m)++n=l++m++n$

If we did not already know the name of the theorem, we could use the SearchRewrite command to find it, based on a pattern that we would like to rewrite:

SearchRewrite $\left(\left(_{-}++_{-}\right)++{ }_{-}\right)$.

app_assoc_reverse:

$\forall(A:$ Type $)(l m n:$ list $A),(l++m)++n=l++m++n$

app_assoc: $\forall(A$ : Type $)(l m n$ : list $A), l++m++n=(l++m)++n$

We use app_assoc_reverse to perform a rewrite:

rewrite app_assoc_reverse.

changing the conclusion to:

progDenote (compile $e^{\mathscr{L}}++($ compile $e 1++$ iBinop $b::$ nil $\left.)++p\right) s=$ progDenote $p$ (binopDenote $b(\operatorname{expDenote} e 1)(\operatorname{expDenote} e 2):: s)$

Now we can notice that the lefthand side of the equality matches the lefthand side of the second inductive hypothesis, so we can rewrite with that hypothesis, too.

rewrite $I H$ e.

progDenote $(($ compile $e 1++\operatorname{iBinop} b::$ nil $)++p)($ expDenote $e \mathscr{2}:: s)=$ progDenote $p$ (binopDenote $b(\operatorname{expDenote} e 1)(\operatorname{expDenote} e \mathscr{2}):: s)$

The same process lets us apply the remaining hypothesis.

rewrite app_assoc_reverse.

rewrite $I H e 1$. 
progDenote $(($ iBinop $b::$ nil $)++p)\left(\operatorname{expDenote~} e 1\right.$ : expDenote $\left.e^{2}:: s\right)=$

progDenote $p$ (binopDenote $b(\operatorname{expDenote} e 1)(\operatorname{expDenote} e 2):: s)$

Now we can apply a similar sequence of tactics to the one that ended the proof of the first case.

unfold progDenote at 1.

simpl.

fold progDenote.

reflexivity.

And the proof is completed, as indicated by the message:

Proof completed.

And there lies our first proof. Already, even for simple theorems like this, the final proof script is unstructured and not very enlightening to readers. If we extend this approach to more serious theorems, we arrive at the unreadable proof scripts that are the favorite complaints of opponents of tactic-based proving. Fortunately, Coq has rich support for scripted automation, and we can take advantage of such a scripted tactic (defined elsewhere) to make short work of this lemma. We abort the old proof attempt and start again.

Abort.

Lemma compile_correct' : $\forall$ e $s p$, progDenote (compile $e++p) s=$ progDenote $p$ (expDenote $e:: s)$.

induction $e$; crush.

Qed.

We need only to state the basic inductive proof scheme and call a tactic that automates the tedious reasoning in between. In contrast to the period tactic terminator from our last proof, the semicolon tactic separator supports structured, compositional proofs. The tactic $t 1$; t2 has the effect of running $t 1$ and then running $t 2$ on each remaining subgoal. The semicolon is one of the most fundamental building blocks of effective proof automation. The period terminator is very useful for exploratory proving, where you need to see intermediate proof states, but final proofs of any serious complexity should have just one period, terminating a single compound tactic that probably uses semicolons.

The crush tactic comes from the library associated with this book and is not part of the Coq standard library. The book's library contains a number of other tactics that are especially helpful in highly automated proofs.

The Qed command checks that the proof is finished and, if so, saves it. The tactic commands we have written above are an example of a proof script, or a series of Ltac programs; while Qed uses the result of the script to generate a proof term, a well-typed term of Gallina. To believe that a theorem is true, we only need to trust that the (relatively simple) checker for proof terms is correct; the use of proof scripts is immaterial. Part I of this book will introduce the principles behind encoding all proofs as terms of Gallina. 
The proof of our main theorem is now easy. We prove it with four period-terminated tactics, though separating them with semicolons would work as well; the version here is easier to step through.

Theorem compile_correct : $\forall e, \operatorname{progDenote}(\operatorname{compile} e)$ nil $=$ Some $(\operatorname{expDenote} e::$ nil). intros.

$e: \exp$

$==\overline{=}=\overline{=}=\overline{=}=\overline{=}=\overline{=}=\overline{=}==$

$\operatorname{progDenote}($ compile $e)$ nil $=$ Some $(\operatorname{expDenote} e::$ nil $)$

At this point, we want to massage the lefthand side to match the statement of compile_correct'. A theorem from the standard library is useful:

Check app_nil_end.

app_nil_end

$: \forall(A:$ Type $)(l:$ list $A), l=l++$ nil

rewrite (app_nil_end (compile $e)$ ).

This time, we explicitly specify the value of the variable $l$ from the theorem statement, since multiple expressions of list type appear in the conclusion. The rewrite tactic might choose the wrong place to rewrite if we did not specify which we want.

$e: \exp$

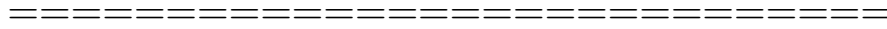

$\operatorname{progDenote}($ compile $e++$ nil) nil $=$ Some $(\operatorname{expDenote} e::$ nil $)$

Now we can apply the lemma.

rewrite compile_correct'.

$e: \exp$

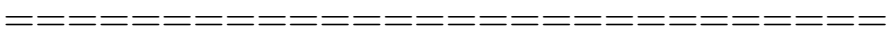

$\operatorname{progDenote}$ nil $(\operatorname{expDenote} e::$ nil $)=$ Some $(\operatorname{expDenote} e::$ nil $)$

We are almost done. The lefthand and righthand sides can be seen to match by simple symbolic evaluation. That means we are in luck, because Coq identifies any pair of terms as equal whenever they normalize to the same result by symbolic evaluation. By the definition of progDenote, that is the case here, but we do not need to worry about such details. A simple invocation of reflexivity does the normalization and checks that the two results are syntactically equal.

reflexivity. 
Qed.

This proof can be shortened and automated, but we leave that task as an exercise for the reader.

\subsection{Typed Expressions}

In this section, we will build on the initial example by adding additional expression forms that depend on static typing of terms for safety.

\subsubsection{Source Language}

We define a trivial language of types to classify our expressions:

Inductive type : Set := Nat $\mid$ Bool.

Like most programming languages, Coq uses case-sensitive variable names, so that our user-defined type type is distinct from the Type keyword that we have already seen appear in the statement of a polymorphic theorem (and that we will meet in more detail later), and our constructor names Nat and Bool are distinct from the types nat and bool in the standard library.

Now we define an expanded set of binary operators.

Inductive tbinop : type $\rightarrow$ type $\rightarrow$ type $\rightarrow$ Set $:=$

| TPlus : tbinop Nat Nat Nat

TTimes : tbinop Nat Nat Nat

| TEq : $\forall t$, tbinop $t t$ Bool

| TLt : tbinop Nat Nat Bool.

The definition of tbinop is different from binop in an important way. Where we declared that binop has type Set, here we declare that tbinop has type type $\rightarrow$ type $\rightarrow$ type $\rightarrow$ Set. We define tbinop as an indexed type family. Indexed inductive types are at the heart of Coq's expressive power; almost everything else of interest is defined in terms of them.

The intuitive explanation of tbinop is that a tbinop $t 1$ t2 $t$ is a binary operator whose operands should have types $t 1$ and $t 2$, and whose result has type $t$. For instance, constructor TLt (for less-than comparison of numbers) is assigned type tbinop Nat Nat Bool, meaning the operator's arguments are naturals and its result is Boolean. The type of TEq introduces a small bit of additional complication via polymorphism: we want to allow equality comparison of any two values of any type, as long as they have the same type.

ML and Haskell have indexed algebraic datatypes. For instance, their list types are indexed by the type of data that the list carries. However, compared to Coq, ML and Haskell 98 place two important restrictions on datatype definitions.

First, the indices of the range of each data constructor must be type variables bound at the top level of the datatype definition. There is no way to do what we did here, where we, for instance, say that TPlus is a constructor building a tbinop whose indices are all fixed at 
Nat. Generalized algebraic datatypes (GADTs) [50] are a popular feature in GHC Haskell, OCaml 4, and other languages that removes this first restriction.

The second restriction is not lifted by GADTs. In ML and Haskell, indices of types must be types and may not be expressions. In Coq, types may be indexed by arbitrary Gallina terms. Type indices can live in the same universe as programs, and we can compute with them just like regular programs. Haskell supports a hobbled form of computation in type indices based on multi-parameter type classes, and recent extensions like type functions bring Haskell programming even closer to "real" functional programming with types, but, without dependent typing, there must always be a gap between how one programs with types and how one programs normally.

We can define a similar type family for typed expressions, where a term of type texp $t$ can be assigned object language type $t$. (It is conventional in the world of interactive theorem proving to call the language of the proof assistant the meta language and a language being formalized the object language.)

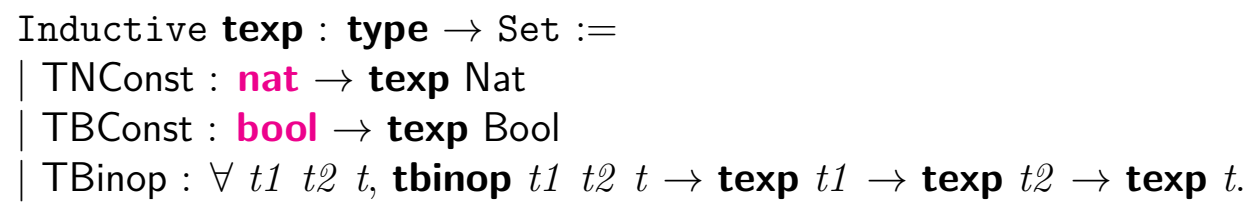

Thanks to our use of dependent types, every well-typed texp represents a well-typed source expression, by construction. This turns out to be very convenient for many things we might want to do with expressions. For instance, it is easy to adapt our interpreter approach to defining semantics. We start by defining a function mapping the types of our object language into Coq types:

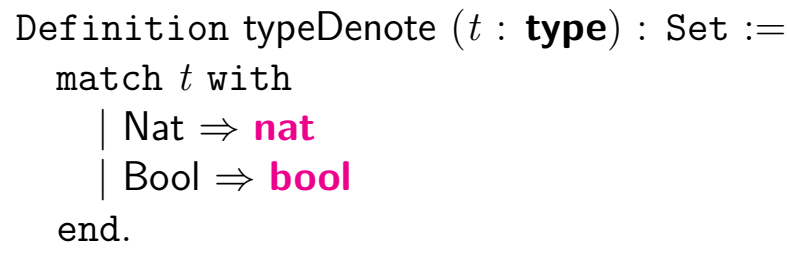

It can take a few moments to come to terms with the fact that Set, the type of types of programs, is itself a first-class type, and that we can write functions that return Sets. Past that wrinkle, the definition of typeDenote is trivial, relying on the nat and bool types from the Coq standard library. We can interpret binary operators by relying on standard-library equality test functions eqb and beq_nat for Booleans and naturals, respectively, along with a less-than test leb:

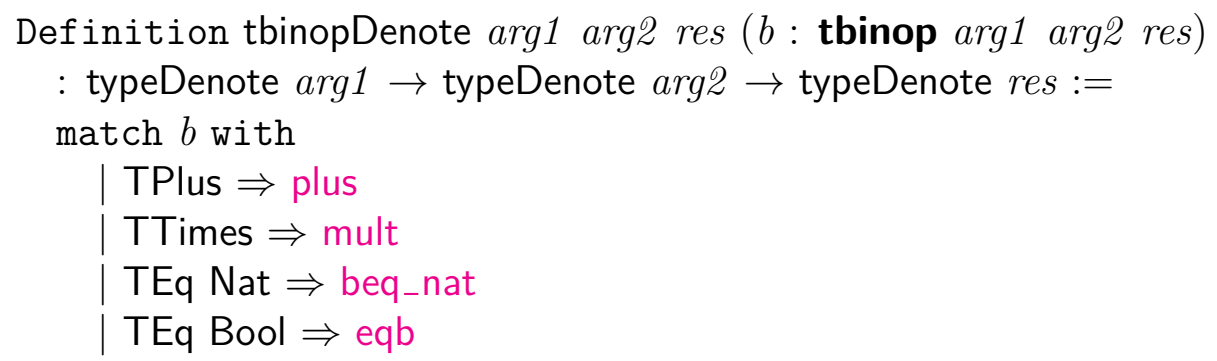




$$
\underset{\text { end. }}{\mid \mathrm{TLt}} \Rightarrow \text { leb }
$$

This function has just a few differences from the denotation functions we saw earlier. First, tbinop is an indexed type, so its indices become additional arguments to tbinopDenote. Second, we need to perform a genuine dependent pattern match, where the necessary type of each case body depends on the value that has been matched. At this early stage, we will not go into detail on the many subtle aspects of Gallina that support dependent patternmatching, but the subject is central to Part II of the book.

The same tricks suffice to define an expression denotation function in an unsurprising way. Note that the type arguments to the TBinop constructor must be included explicitly in pattern-matching, but here we write underscores because we do not need to refer to those arguments directly.

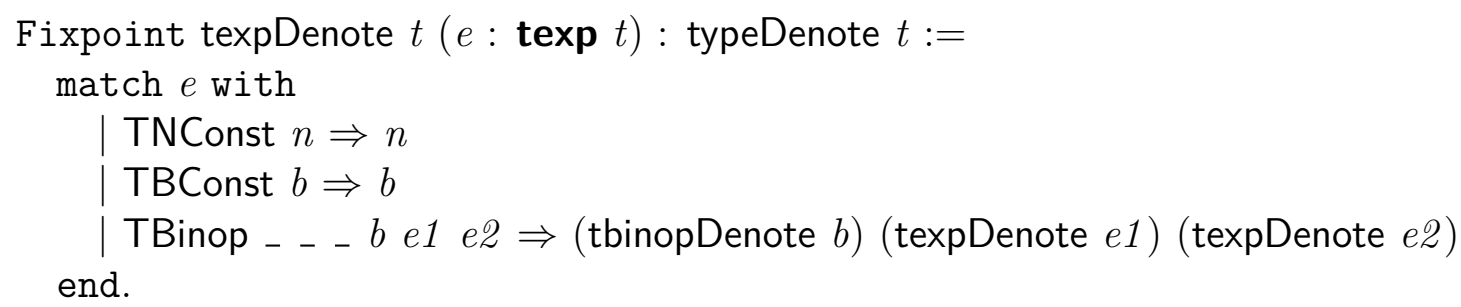

We can evaluate a few example programs to convince ourselves that this semantics is correct.

Eval simpl in texpDenote (TNConst 42).

$$
=42: \text { typeDenote Nat }
$$

Eval simpl in texpDenote (TBConst true).

$=$ true : typeDenote Bool

Eval simpl in texpDenote (TBinop TTimes (TBinop TPlus (TNConst 2) (TNConst 2))

(TNConst 7)).

$=28:$ typeDenote Nat

Eval simpl in texpDenote (TBinop (TEq Nat) (TBinop TPlus (TNConst 2) (TNConst 2))

(TNConst 7)).

$=$ false : typeDenote Bool

Eval simpl in texpDenote (TBinop TLt (TBinop TPlus (TNConst 2) (TNConst 2))

(TNConst 7)).

$=$ true : typeDenote Bool

Now we are ready to define a suitable stack machine target for compilation.

\subsubsection{Target Language}

In the example of the untyped language, stack machine programs could encounter stack underflows and "get stuck." This was unfortunate, since we had to deal with this complication even though we proved that our compiler never produced underflowing programs. We could have used dependent types to force all stack machine programs to be underflow-free. 
For our new languages, besides underflow, we also have the problem of stack slots with naturals instead of bools or vice versa. This time, we will use indexed typed families to avoid the need to reason about potential failures.

We start by defining stack types, which classify sets of possible stacks.

Definition tstack := list type.

Any stack classified by a tstack must have exactly as many elements, and each stack element must have the type found in the same position of the stack type.

We can define instructions in terms of stack types, where every instruction's type tells us what initial stack type it expects and what final stack type it will produce.

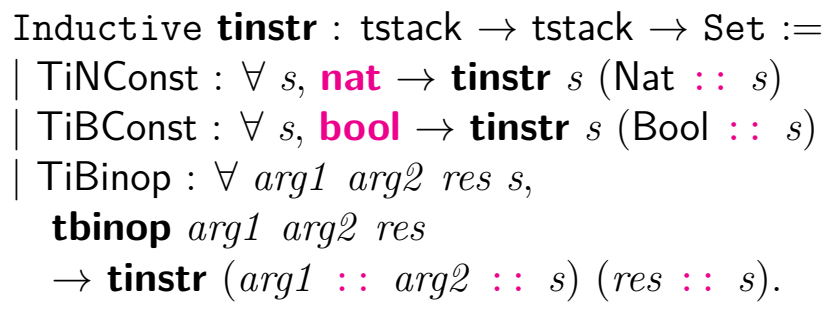

Stack machine programs must be a similar inductive family, since, if we again used the list type family, we would not be able to guarantee that intermediate stack types match within a program.

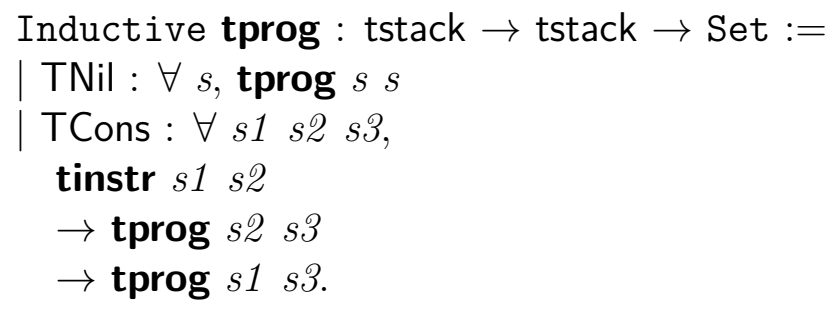

Now, to define the semantics of our new target language, we need a representation for stacks at runtime. We will again take advantage of type information to define types of value stacks that, by construction, contain the right number and types of elements.

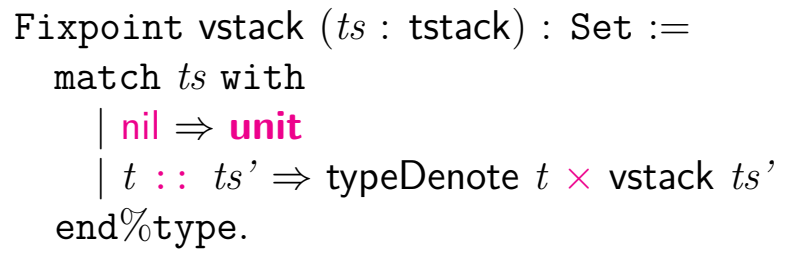

This is another Set-valued function. This time it is recursive, which is perfectly valid, since Set is not treated specially in determining which functions may be written. We say that the value stack of an empty stack type is any value of type unit, which has just a single value, tt. A nonempty stack type leads to a value stack that is a pair, whose first element has the proper type and whose second element follows the representation for the remainder of the stack type. We write \%type as an instruction to Coq's extensible parser. In particular, this directive applies to the whole match expression, which we ask to be parsed as though it were a type, so that the operator $\times$ is interpreted as Cartesian product instead of, say, 
multiplication. (Note that this use of type has no connection to the inductive type type that we have defined.)

This idea of programming with types can take a while to internalize, but it enables a very simple definition of instruction denotation. Our definition is like what you might expect from a Lisp-like version of ML that ignored type information. Nonetheless, the fact that tinstrDenote passes the type-checker guarantees that our stack machine programs can never go wrong. We use a special form of let to destructure a multi-level tuple.

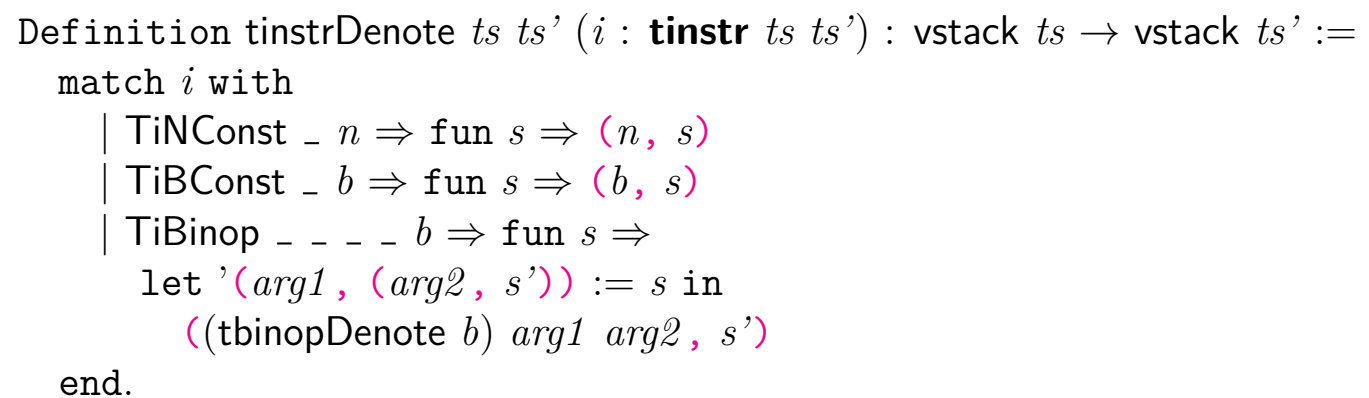

Why do we choose to use an anonymous function to bind the initial stack in every case of the match? Consider this well-intentioned but invalid alternative version:

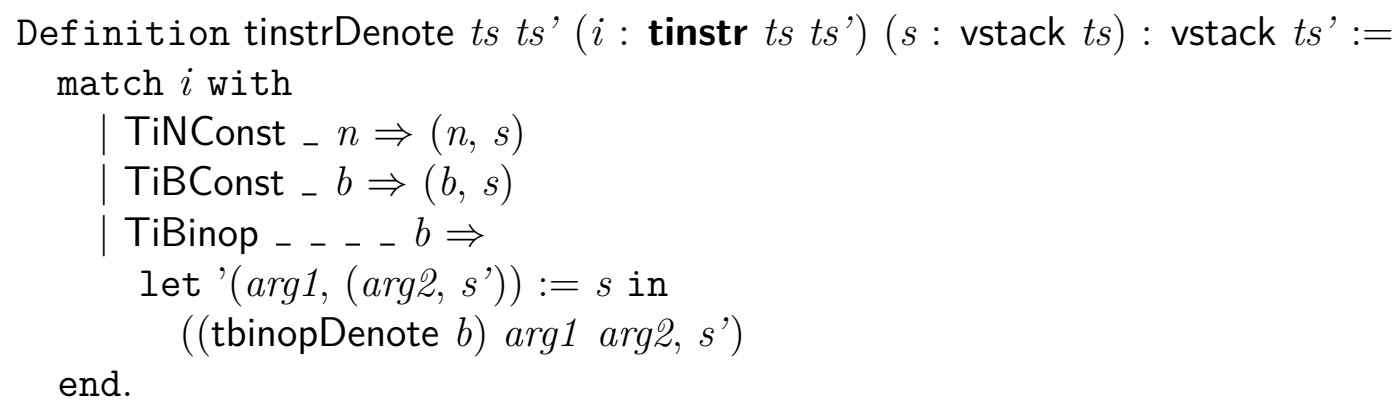

The Coq type checker complains that:

The term " $(\mathrm{n}, \mathrm{s})$ " has type "(nat * vstack ts)\%type"

while it is expected to have type "vstack ?119".

This and other mysteries of Coq dependent typing we postpone until Part II of the book. The upshot of our later discussion is that it is often useful to push inside of match branches those function parameters whose types depend on the type of the value being matched. Our later, more complete treatment of Gallina's typing rules will explain why this helps.

We finish the semantics with a straightforward definition of program denotation.

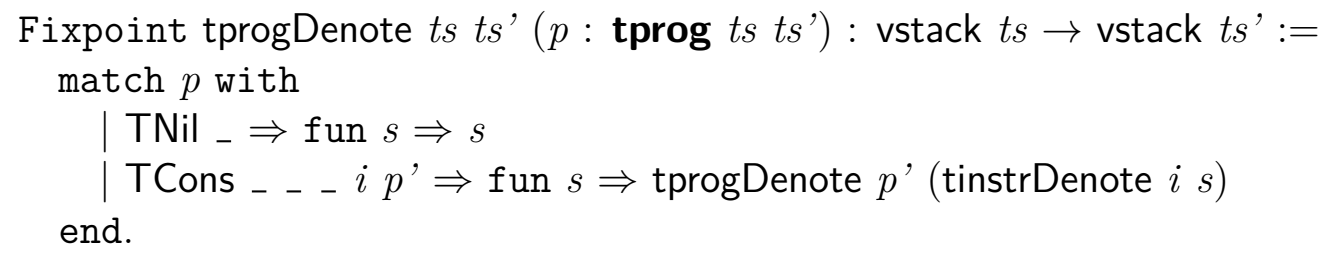

The same argument-postponing trick is crucial for this definition. 


\subsubsection{Translation}

To define our compilation, it is useful to have an auxiliary function for concatenating two stack machine programs.

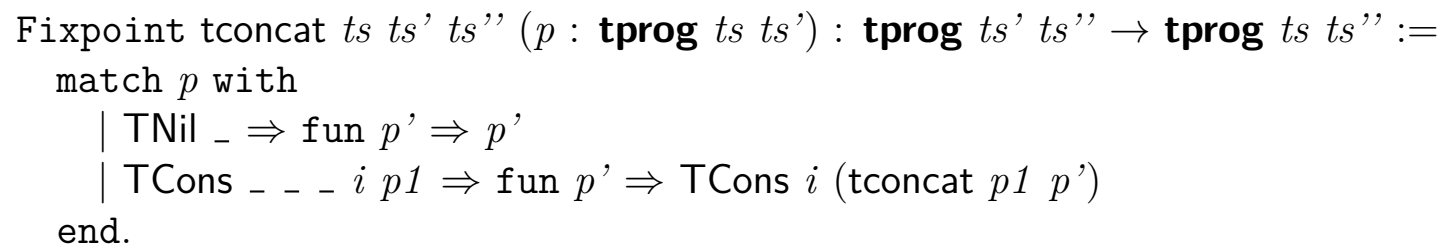

With that function in place, the compilation is defined very similarly to how it was before, modulo the use of dependent typing.

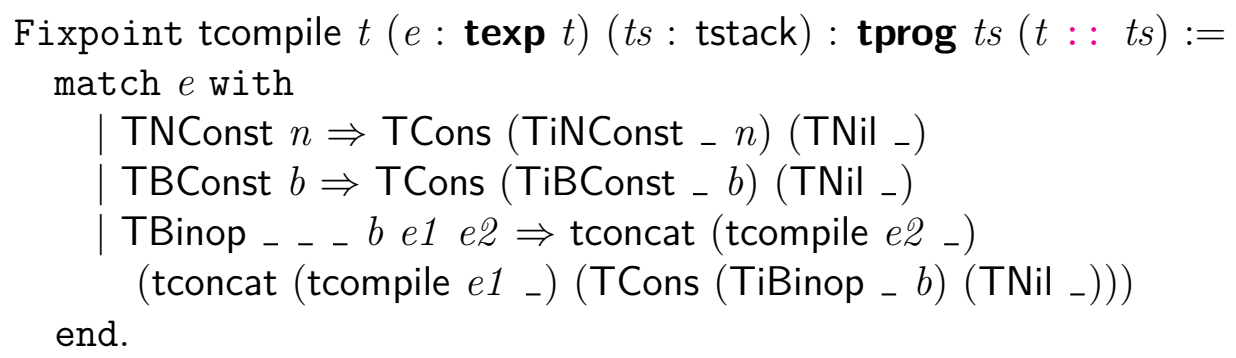

One interesting feature of the definition is the underscores appearing to the right of $\Rightarrow$ arrows. Haskell and ML programmers are quite familiar with compilers that infer type parameters to polymorphic values. In Coq, it is possible to go even further and ask the system to infer arbitrary terms, by writing underscores in place of specific values. You may have noticed that we have been calling functions without specifying all of their arguments. For instance, the recursive calls here to tcompile omit the $t$ argument. Coq's implicit argument mechanism automatically inserts underscores for arguments that it will probably be able to infer. Inference of such values is far from complete, though; generally, it only works in cases similar to those encountered with polymorphic type instantiation in Haskell and ML.

The underscores here are being filled in with stack types. That is, the Coq type inferencer is, in a sense, inferring something about the flow of control in the translated programs. We can take a look at exactly which values are filled in:

Print tcompile.

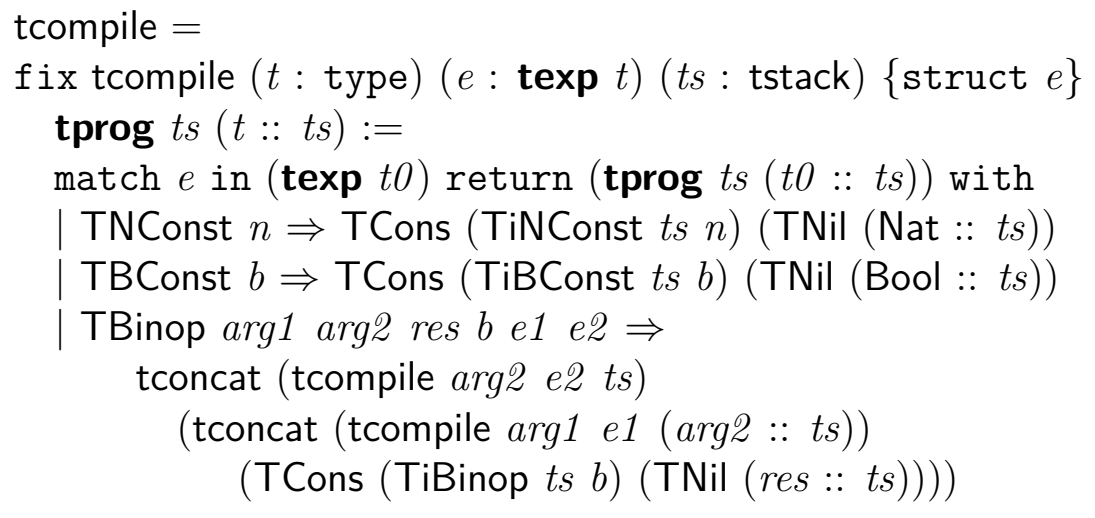


end

$: \forall t:$ type, texp $t \rightarrow \forall t s:$ tstack, tprog $t s(t:: t s)$

We can check that the compiler generates programs that behave appropriately on our sample programs from above:

Eval simpl in tprogDenote (tcompile (TNConst 42) nil) tt.

$=(42, \mathrm{tt}): \operatorname{vstack}($ Nat :: nil)

Eval simpl in tprogDenote (tcompile (TBConst true) nil) tt.

$=($ true, $\mathrm{tt}):$ vstack $($ Bool :: nil)

Eval simpl in tprogDenote (tcompile (TBinop TTimes (TBinop TPlus (TNConst 2)

(TNConst 2)) (TNConst 7)) nil) tt.

$=(28, \mathrm{tt}): \operatorname{vstack}($ Nat :: nil)

Eval simpl in tprogDenote (tcompile (TBinop (TEq Nat) (TBinop TPlus (TNConst 2)

(TNConst 2)) (TNConst 7)) nil) tt.

$=($ false, $\mathrm{tt})$ : vstack (Bool :: nil)

Eval simpl in tprogDenote (tcompile (TBinop TLt (TBinop TPlus (TNConst 2) (TNConst 2))

(TNConst 7)) nil) tt.

$=($ true, $\mathrm{tt})$ : vstack (Bool :: nil)

The compiler seems to be working, so let us turn to proving that it always works.

\subsubsection{Translation Correctness}

We can state a correctness theorem similar to the last one.

Theorem tcompile_correct : $\forall t(e: \operatorname{texp} t)$, tprogDenote (tcompile $e$ nil) $\mathrm{tt}=($ texpDenote $e, \mathrm{tt})$.

Again, we need to strengthen the theorem statement so that the induction will go through. This time, to provide an excuse to demonstrate different tactics, I will develop an alternative approach to this kind of proof, stating the key lemma as:

Lemma tcompile_correct' : $\forall t(e: \operatorname{texp} t) t s(s:$ vstack $t s)$, tprogDenote (tcompile $e$ ts) $s=($ texpDenote $e, s)$.

While lemma compile_correct' quantified over a program that is the "continuation" [39] for the expression we are considering, here we avoid drawing in any extra syntactic elements. In addition to the source expression and its type, we also quantify over an initial stack type and a stack compatible with it. Running the compilation of the program starting from that stack, we should arrive at a stack that differs only in having the program's denotation pushed onto it.

Let us try to prove this theorem in the same way that we settled on in the last section.

induction $e$; crush.

We are left with this unproved conclusion: 
tprogDenote

(tconcat (tcompile e2 $t s$ )

(tconcat (tcompile e1 $(\arg 2:: t s)$ )

(TCons (TiBinop $t s t)($ TNil $(r e s:: t s))))) s=$

(tbinopDenote $t$ (texpDenote $e 1)$ (texpDenote $e 2), s$ )

We need an analogue to the app_assoc_reverse theorem that we used to rewrite the goal in the last section. We can abort this proof and prove such a lemma about tconcat.

Abort.

Lemma tconcat_correct : $\forall t s t s^{\prime} t s^{\prime \prime}\left(p: \operatorname{tprog} t s t s^{\prime}\right)\left(p^{\prime}: \operatorname{tprog} t s^{\prime} t s^{\prime \prime}\right)$

( $s$ : vstack $t s$,

tprogDenote (tconcat $\left.p p^{\prime}\right) s$

$=$ tprogDenote $p^{\prime}($ tprogDenote $p s)$.

induction $p$; crush.

Qed.

This one goes through completely automatically.

Some code behind the scenes registers app_assoc_reverse for use by crush. We must register tconcat_correct similarly to get the same effect:

Hint Rewrite tconcat_correct.

Here we meet the pervasive concept of a hint. Many proofs can be found through exhaustive enumerations of combinations of possible proof steps; hints provide the set of steps to consider. The tactic crush is applying such brute force search for us silently, and it will consider more possibilities as we add more hints. This particular hint asks that the lemma be used for left-to-right rewriting.

Now we are ready to return to tcompile_correct', proving it automatically this time.

Lemma tcompile_correct' : $\forall t(e: \operatorname{texp} t) t s(s:$ vstack $t s)$,

tprogDenote (tcompile $e$ ts) $s=($ texpDenote $e, s)$.

induction $e$; crush.

Qed.

We can register this main lemma as another hint, allowing us to prove the final theorem trivially.

Hint Rewrite tcompile_correct'.

Theorem tcompile_correct : $\forall t(e: \operatorname{texp} t)$,

tprogDenote (tcompile $e$ nil) $\mathrm{tt}=($ texpDenote $e, \mathrm{tt})$.

crush.

Qed.

It is probably worth emphasizing that we are doing more than building mathematical models. Our compilers are functional programs that can be executed efficiently. One strategy for doing so is based on program extraction, which generates OCaml code from Coq developments. 
To set up the feature properly in recent versions of Coq, we must run the command Require Extraction.. However, this book PDF is still built with a patched old version of Coq that neither requires nor allows that command, so it is commented out in this rendering!

Now we run a command to output the OCaml version of tcompile:

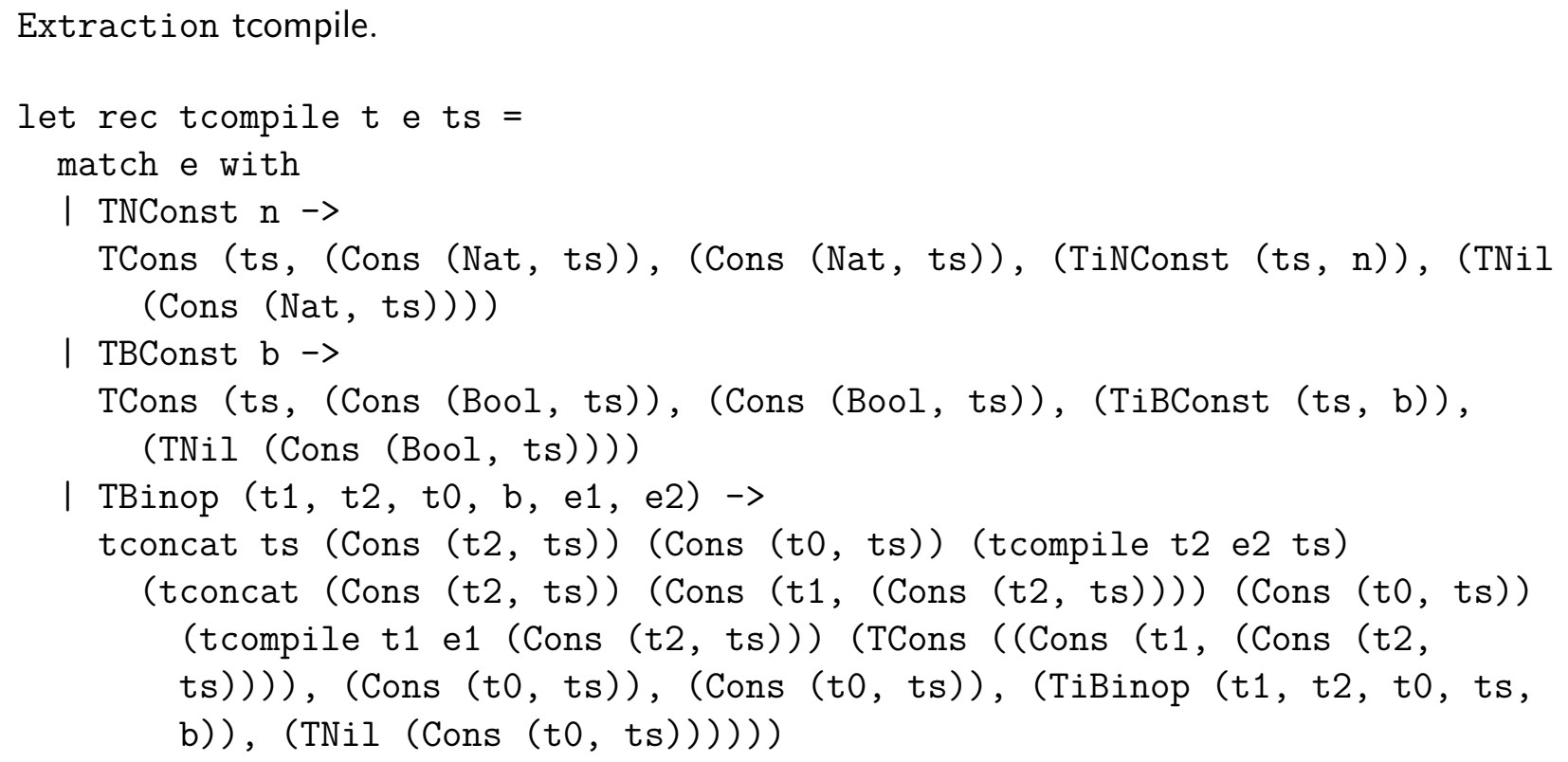

We can compile this code with the usual OCaml compiler and obtain an executable program with halfway decent performance.

This chapter has been a whirlwind tour through two examples of the style of Coq development that I advocate. Parts II and III of the book focus on the key elements of that style, namely dependent types and scripted proof automation, respectively. Before we get there, we will spend some time in Part I on more standard foundational material. Part I may still be of interest to seasoned Coq hackers, since I follow the highly automated proof style even at that early stage. 


\section{Part I}

\section{Basic Programming and Proving}




\section{Chapter 3}

\section{Introducing Inductive Types}

The logical foundation of Coq is the Calculus of Inductive Constructions, or CIC. In a sense, CIC is built from just two relatively straightforward features: function types and inductive types. From this modest foundation, we can prove essentially all of the theorems of math and carry out effectively all program verifications, with enough effort expended. This chapter introduces induction and recursion for functional programming in Coq. Most of our examples reproduce functionality from the Coq standard library, and I have tried to copy the standard library's choices of identifiers, where possible, so many of the definitions here are already available in the default Coq environment.

The last chapter took a deep dive into some of the more advanced Coq features, to highlight the unusual approach that I advocate in this book. However, from this point on, we will rewind and go back to basics, presenting the relevant features of Coq in a more bottom-up manner. A useful first step is a discussion of the differences and relationships between proofs and programs in Coq.

\subsection{Proof Terms}

Mainstream presentations of mathematics treat proofs as objects that exist outside of the universe of mathematical objects. However, for a variety of reasoning tasks, it is convenient to encode proofs, traditional mathematical objects, and programs within a single formal language. Validity checks on mathematical objects are useful in any setting, to catch typos and other uninteresting errors. The benefits of static typing for programs are widely recognized, and Coq brings those benefits to both mathematical objects and programs via a uniform mechanism. In fact, from this point on, we will not bother to distinguish between programs and mathematical objects. Many mathematical formalisms are most easily encoded in terms of programs.

Proofs are fundamentally different from programs, because any two proofs of a theorem are considered equivalent, from a formal standpoint if not from an engineering standpoint. However, we can use the same type-checking technology to check proofs as we use to validate our programs. This is the Curry-Howard correspondence [10, 14], an approach for relating 
proofs and programs. We represent mathematical theorems as types, such that a theorem's proofs are exactly those programs that type-check at the corresponding type.

The last chapter's example already snuck in an instance of Curry-Howard. We used the token $\rightarrow$ to stand for both function types and logical implications. One reasonable conclusion upon seeing this might be that some fancy overloading of notations is at work. In fact, functions and implications are precisely identical according to Curry-Howard! That is, they are just two ways of describing the same computational phenomenon.

A short demonstration should explain how this can be. The identity function over the natural numbers is certainly not a controversial program.

Check ( un $x$ : nat $\Rightarrow x$ ).

$:$ nat $\rightarrow$ nat

Consider this alternate program, which is almost identical to the last one.

Check (fun $x$ : True $\Rightarrow x$ ).

$:$ True $\rightarrow$ True

The identity program is interpreted as a proof that True, the always-true proposition, implies itself! What we see is that Curry-Howard interprets implications as functions, where an input is a proposition being assumed and an output is a proposition being deduced. This intuition is not too far from a common one for informal theorem proving, where we might already think of an implication proof as a process for transforming a hypothesis into a conclusion.

There are also more primitive proof forms available. For instance, the term I is the single proof of True, applicable in any context.

Check I.

: True

With I, we can prove another simple propositional theorem.

Check (fun _ : False $\Rightarrow I$ ).

$:$ False $\rightarrow$ True

No proofs of False exist in the top-level context, but the implication-as-function analogy gives us an easy way to, for example, show that False implies itself.

Check ( fun $x:$ False $\Rightarrow x$ ).

$:$ False $\rightarrow$ False

Every one of these example programs whose type looks like a logical formula is a proof term. We use that name for any Gallina term of a logical type, and we will elaborate shortly on what makes a type logical.

In the rest of this chapter, we will introduce different ways of defining types. Every example type can be interpreted alternatively as a type of programs or proofs.

One of the first types we introduce will be bool, with constructors true and false. Newcomers to Coq often wonder about the distinction between True and true and the distinction between False and false. One glib answer is that True and False are types, but true and false are not. A more useful answer is that Coq's metatheory guarantees that any term of 
type bool evaluates to either true or false. This means that we have an algorithm for answering any question phrased as an expression of type bool. Conversely, most propositions do not evaluate to True or False; the language of inductively defined propositions is much richer than that. We ought to be glad that we have no algorithm for deciding our formalized version of mathematical truth, since otherwise it would be clear that we could not formalize undecidable properties, like almost any interesting property of general-purpose programs.

\subsection{Enumerations}

Coq inductive types generalize the algebraic datatypes found in Haskell and ML. Confusingly enough, inductive types also generalize generalized algebraic datatypes (GADTs), by adding the possibility for type dependency. Even so, it is worth backing up from the examples of the last chapter and going over basic, algebraic-datatype uses of inductive datatypes, because the chance to prove things about the values of these types adds new wrinkles beyond usual practice in Haskell and ML.

The singleton type unit is an inductive type:

Inductive unit : Set :=

| tt.

This vernacular command defines a new inductive type unit whose only value is tt. We can verify the types of the two identifiers we introduce:

Check unit.

unit : Set

Check tt.

tt : unit

We can prove that unit is a genuine singleton type.

Theorem unit_singleton : $\forall x:$ unit, $x=$ tt.

The important thing about an inductive type is, unsurprisingly, that you can do induction over its values, and induction is the key to proving this theorem. We ask to proceed by induction on the variable $x$.

induction $x$.

The goal changes to:

$\mathrm{tt}=\mathrm{tt}$

...which we can discharge trivially.

reflexivity.

Qed.

It seems kind of odd to write a proof by induction with no inductive hypotheses. We could have arrived at the same result by beginning the proof with:

destruct $x$. 
...which corresponds to "proof by case analysis" in classical math. For non-recursive inductive types, the two tactics will always have identical behavior. Often case analysis is sufficient, even in proofs about recursive types, and it is nice to avoid introducing unneeded induction hypotheses.

What exactly is the induction principle for unit? We can ask Coq:

Check unit_ind.

unit_ind : $\forall P$ : unit $\rightarrow$ Prop, $P$ tt $\rightarrow \forall u:$ unit, $P u$

Every Inductive command defining a type $T$ also defines an induction principle named T_ind. Recall from the last section that our type, operations over it, and principles for reasoning about it all live in the same language and are described by the same type system. The key to telling what is a program and what is a proof lies in the distinction between the type Prop, which appears in our induction principle; and the type Set, which we have seen a few times already.

The convention goes like this: Set is the type of normal types used in programming, and the values of such types are programs. Prop is the type of logical propositions, and the values of such types are proofs. Thus, an induction principle has a type that shows us that it is a function for building proofs.

Specifically, unit_ind quantifies over a predicate $P$ over unit values. If we can present a proof that $P$ holds of $\mathrm{tt}$, then we are rewarded with a proof that $P$ holds for any value $u$ of type unit. In our last proof, the predicate was (fun $u:$ unit $\Rightarrow u=\mathrm{tt}$ ).

The definition of unit places the type in Set. By replacing Set with Prop, unit with True, and tt with I, we arrive at precisely the definition of True that the Coq standard library employs! The program type unit is the Curry-Howard equivalent of the proposition True. We might make the tongue-in-cheek claim that, while philosophers have expended much ink on the nature of truth, we have now determined that truth is the unit type of functional programming.

We can define an inductive type even simpler than unit:

Inductive Empty_set : Set := .

Empty_set has no elements. We can prove fun theorems about it:

Theorem the_sky_is_falling : $\forall x$ : Empty_set, $2+2=5$.

destruct 1 .

Qed.

Because Empty_set has no elements, the fact of having an element of this type implies anything. We use destruct 1 instead of destruct $x$ in the proof because unused quantified variables are relegated to being referred to by number. (There is a good reason for this, related to the unity of quantifiers and implication. At least within Coq's logical foundation of constructive logic, which we elaborate on more in the next chapter, an implication is just a quantification over a proof, where the quantified variable is never used. It generally makes more sense to refer to implication hypotheses by number than by name, and Coq treats our quantifier over an unused variable as an implication in determining the proper behavior.)

We can see the induction principle that made this proof so easy: 
Check Empty_set_ind.

Empty_set_ind : $\forall$ ( $P$ : Empty_set $\rightarrow$ Prop) $(e$ : Empty_set $), P e$

In other words, any predicate over values from the empty set holds vacuously of every such element. In the last proof, we chose the predicate (fun _ : Empty_set $\Rightarrow 2+2=5$ ).

We can also apply this get-out-of-jail-free card programmatically. Here is a lazy way of converting values of Empty_set to values of unit:

Definition $\mathrm{e} 2 \mathrm{u}(e$ : Empty_set) : unit := match $e$ with end.

We employ match pattern matching as in the last chapter. Since we match on a value whose type has no constructors, there is no need to provide any branches. It turns out that Empty_set is the Curry-Howard equivalent of False. As for why Empty_set starts with a capital letter and not a lowercase letter like unit does, we must refer the reader to the authors of the Coq standard library, to which we try to be faithful.

Moving up the ladder of complexity, we can define the Booleans:

Inductive bool : Set :=

| true

| false.

We can use less vacuous pattern matching to define Boolean negation.

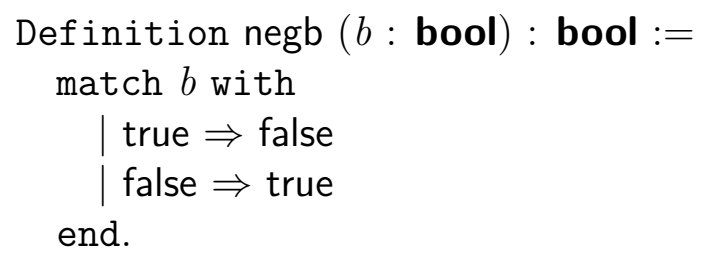

An alternative definition desugars to the above, thanks to an if notation overloaded to work with any inductive type that has exactly two constructors:

Definition negb' ( $b$ : bool) : bool :=

if $b$ then false else true.

We might want to prove that negb is its own inverse operation.

Theorem negb_inverse : $\forall b$ : bool, negb (negb $b)=b$.

destruct $b$.

After we case-analyze on $b$, we are left with one subgoal for each constructor of bool.

2 subgoals

$$
\begin{aligned}
& ==\mathrm{=}=\mathrm{=}=\mathrm{=}=\mathrm{=}=\mathrm{=}=\mathrm{=}=\mathrm{=}=\mathrm{=}=\mathrm{=}=\mathrm{=} \\
& \text { negb }(\text { negb true })=\text { true }
\end{aligned}
$$

subgoal 2 is

negb $($ negb false $)=$ false

The first subgoal follows by Coq's rules of computation, so we can dispatch it easily: 
reflexivity.

Likewise for the second subgoal, so we can restart the proof and give a very compact justification.

Restart.

destruct $b$; reflexivity.

Qed.

Another theorem about Booleans illustrates another useful tactic.

Theorem negb_ineq : $\forall b$ : bool, negb $b \neq b$.

destruct $b$; discriminate.

Qed.

The discriminate tactic is used to prove that two values of an inductive type are not equal, whenever the values are formed with different constructors. In this case, the different constructors are true and false.

At this point, it is probably not hard to guess what the underlying induction principle for bool is.

Check bool_ind.

bool_ind : $\forall P$ : bool $\rightarrow$ Prop, $P$ true $\rightarrow P$ false $\rightarrow \forall b:$ bool, $P b$

That is, to prove that a property describes all bools, prove that it describes both true and false.

There is no interesting Curry-Howard analogue of bool. Of course, we can define such a type by replacing Set by Prop above, but the proposition we arrive at is not very useful. It is logically equivalent to True, but it provides two indistinguishable primitive proofs, true and false. In the rest of the chapter, we will skip commenting on Curry-Howard versions of inductive definitions where such versions are not interesting.

\subsection{Simple Recursive Types}

The natural numbers are the simplest common example of an inductive type that actually deserves the name.

Inductive nat : Set :=

O : nat

S : nat $\rightarrow$ nat.

The constructor $\mathrm{O}$ is zero, and $\mathrm{S}$ is the successor function, so that 0 is syntactic sugar for $\mathrm{O}, 1$ for $\mathrm{S} \mathrm{O}, 2$ for $\mathrm{S}(\mathrm{S} \mathrm{O})$, and so on.

Pattern matching works as we demonstrated in the last chapter:

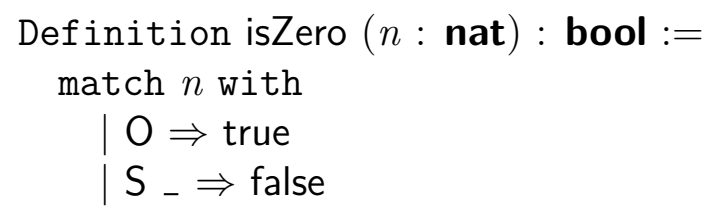


end.

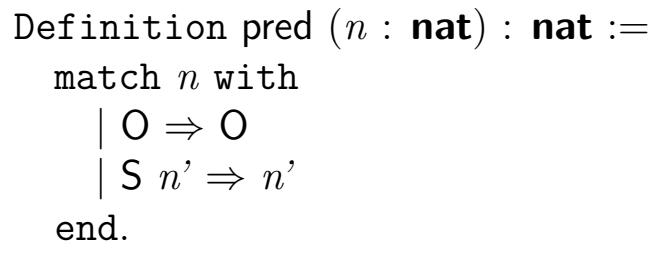

We can prove theorems by case analysis with destruct as for simpler inductive types, but we can also now get into genuine inductive theorems. First, we will need a recursive function, to make things interesting.

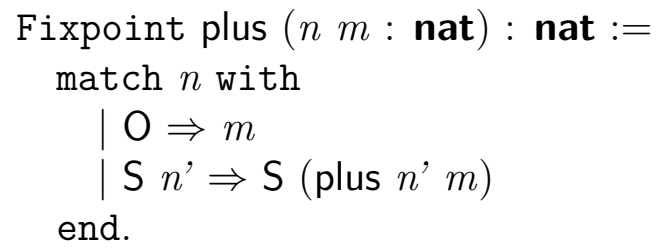

Recall that Fixpoint is Coq's mechanism for recursive function definitions. Some theorems about plus can be proved without induction.

Theorem O_plus_n : $\forall n:$ nat, plus $\mathrm{O} n=n$.

intro; reflexivity.

Qed.

Coq's computation rules automatically simplify the application of plus, because unfolding the definition of plus gives us a match expression where the branch to be taken is obvious from syntax alone. If we just reverse the order of the arguments, though, this no longer works, and we need induction.

Theorem n_plus_O $: \forall n:$ nat, plus $n \mathrm{O}=n$.

induction $n$.

Our first subgoal is plus $\mathrm{O} \mathrm{O}=\mathrm{O}$, which is trivial by computation.

reflexivity.

Our second subgoal requires more work and also demonstrates our first inductive hypothesis.

$n$ : nat

IHn : plus $n \mathrm{O}=n$

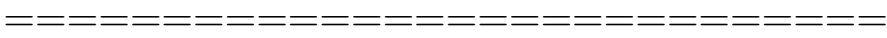

plus $(\mathrm{S} n) \mathrm{O}=\mathrm{S} n$

We can start out by using computation to simplify the goal as far as we can.

simpl.

Now the conclusion is $\mathrm{S}$ (plus $n \mathrm{O}$ ) $=\mathrm{S} n$. Using our inductive hypothesis: 
rewrite $I H n$.

...we get a trivial conclusion $\mathrm{S} n=\mathrm{S} n$.

reflexivity.

Not much really went on in this proof, so the crush tactic from the CpdtTactics module can prove this theorem automatically.

Restart.

induction $n$; crush.

Qed.

We can check out the induction principle at work here:

Check nat_ind.

$$
\text { nat_ind : } \begin{aligned}
\forall & P: \text { nat } \rightarrow \text { Prop, } \\
& P \mathrm{O} \rightarrow(\forall n: \text { nat, } P n \rightarrow P(\mathrm{~S} n)) \rightarrow \forall n: \text { nat, } P n
\end{aligned}
$$

Each of the two cases of our last proof came from the type of one of the arguments to nat_ind. We chose $P$ to be ( un $n$ : nat $\Rightarrow$ plus $n \mathrm{O}=n$ ). The first proof case corresponded to $P \mathrm{O}$ and the second case to $(\forall n:$ nat, $P n \rightarrow P(\mathrm{~S} n))$. The free variable $n$ and inductive hypothesis $I H n$ came from the argument types given here.

Since nat has a constructor that takes an argument, we may sometimes need to know that that constructor is injective.

Theorem S_inj : $\forall n m:$ nat, $\mathbf{S} n=\mathrm{S} m \rightarrow n=m$.

$$
\text { injection 1; trivial. }
$$

Qed.

The injection tactic refers to a premise by number, adding new equalities between the corresponding arguments of equated terms that are formed with the same constructor. We end up needing to prove $n=m \rightarrow n=m$, so it is unsurprising that a tactic named trivial is able to finish the proof. This tactic attempts a variety of single proof steps, drawn from a user-specified database that we will later see how to extend.

There is also a very useful tactic called congruence that can prove this theorem immediately. The congruence tactic generalizes discriminate and injection, and it also adds reasoning about the general properties of equality, such as that a function returns equal results on equal arguments. That is, congruence is a complete decision procedure for the theory of equality and uninterpreted functions, plus some smarts about inductive types.

We can define a type of lists of natural numbers.

Inductive nat_list : Set :=

| NNil : nat_list

| NCons : nat $\rightarrow$ nat_list $\rightarrow$ nat_list.

Recursive definitions over nat_list are straightforward extensions of what we have seen before.

Fixpoint nlength $(l s:$ nat_list $):$ nat $:=$ 


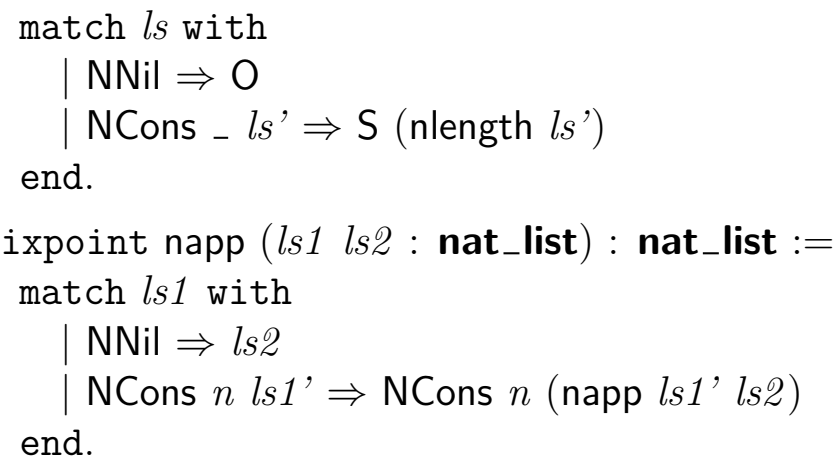

Inductive theorem proving can again be automated quite effectively.

Theorem nlength_napp : $\forall l s 1$ ls2 : nat_list, nlength (napp ls1 ls2)

$=$ plus (nlength $l s 1)$ (nlength ls2).

induction $l s 1$; crush.

Qed.

Check nat_list_ind.

nat_list_ind

$: \forall P:$ nat_list $\rightarrow$ Prop,

$P$ NNil $\rightarrow$

$(\forall(n:$ nat $)(n 0:$ nat_list $), P n 0 \rightarrow P($ NCons $n n 0)) \rightarrow$

$\forall n$ : nat_list, $P n$

In general, we can implement any "tree" type as an inductive type. For example, here are binary trees of naturals.

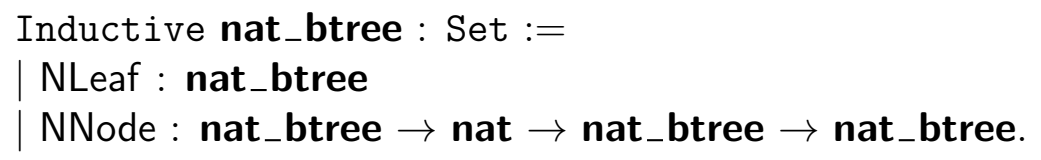

Here are two functions whose intuitive explanations are not so important. The first one computes the size of a tree, and the second performs some sort of splicing of one tree into the leftmost available leaf node of another.

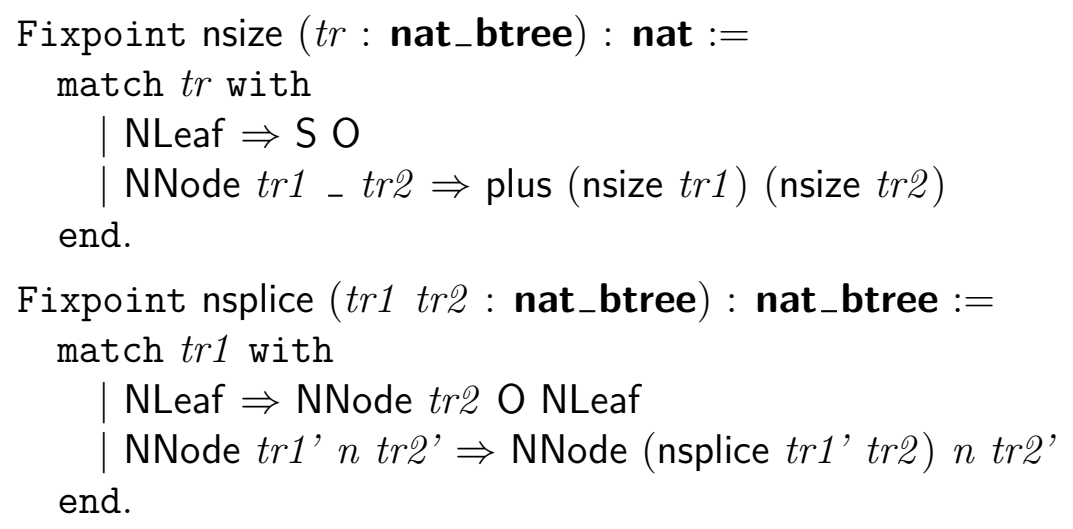

Theorem plus_assoc: $\forall n 1$ n2 $n 3$ : nat, plus (plus $n 1$ n2) n3= plus $n 1$ (plus n2 n3). 
induction $n 1$; crush.

Qed.

Hint Rewrite n_plus_O plus_assoc.

Theorem nsize_nsplice : $\forall \operatorname{tr} 1$ tr2 : nat_btree, nsize (nsplice tr1 tr2)

= plus (nsize tr2) (nsize tr1).

induction $\operatorname{tr} 1$; crush.

Qed.

It is convenient that these proofs go through so easily, but it is still useful to look into the details of what happened, by checking the statement of the tree induction principle.

Check nat_btree_ind.

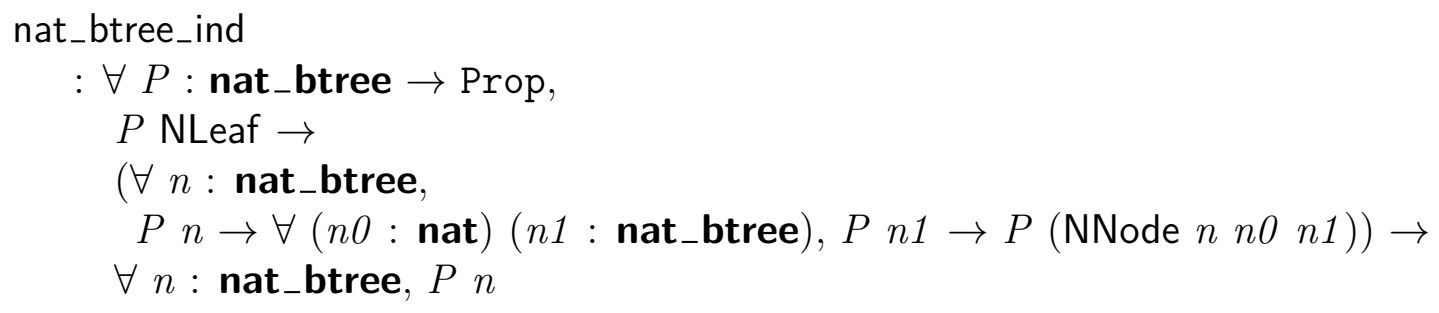

We have the usual two cases, one for each constructor of nat_btree.

\subsection{Parameterized Types}

We can also define polymorphic inductive types, as with algebraic datatypes in Haskell and ML.

Inductive list $(T:$ Set $):$ Set $:=$

| Nil : list $T$

Cons : $T \rightarrow$ list $T \rightarrow$ list $T$.

Fixpoint length $T(l s:$ list $T):$ nat $:=$ match $l s$ with

Nil $\Rightarrow \mathrm{O}$

| Cons_ $l s^{\prime} \Rightarrow \mathrm{S}$ (length $l s^{\prime}$ )

end.

Fixpoint app $T(l s 1$ ls2 : list $T)$ : list $T:=$ match $l s 1$ with

$\mathrm{Nil} \Rightarrow$ ls2

| Cons $x l s 1^{\prime} \Rightarrow$ Cons $x$ (app ls1'ls2)

end.

Theorem length_app : $\forall T(l s 1$ ls2 : list $T)$, length (app ls1 ls2)

= plus (length $l s 1$ ) (length $l s 2)$.

induction ls1; crush.

Qed. 
There is a useful shorthand for writing many definitions that share the same parameter, based on Coq's section mechanism. The following block of code is equivalent to the above:

\section{Section list.}

Variable $T$ : Set.

Inductive list : Set :=

Nil : list

Cons : $T \rightarrow$ list $\rightarrow$ list.

Fixpoint length $(l s:$ list $):$ nat $:=$

match $l s$ with

$\mid \mathrm{Nil} \Rightarrow \mathrm{O}$

| Cons_ $l s^{\prime} \Rightarrow \mathrm{S}$ (length $\left.l s^{\prime}\right)$

end.

Fixpoint app (ls1 ls2 : list) : list := match ls1 with

$\mathrm{Nil} \Rightarrow l s 2$

| Cons $x l s 1^{\prime} \Rightarrow$ Cons $x$ (app ls1'ls2)

end.

Theorem length_app : $\forall l s 1$ ls2 : list, length (app ls1 ls2)

$=$ plus (length $l s 1$ ) (length ls2).

induction $l s 1$; crush.

Qed.

End list.

Arguments Nil $[T]$.

After we end the section, the Variables we used are added as extra function parameters for each defined identifier, as needed. With an Arguments command, we ask that $T$ be inferred when we use Nil; Coq's heuristics already decided to apply a similar policy to Cons, because of the Set Implicit Arguments command elided at the beginning of this chapter. We verify that our definitions have been saved properly using the Print command, a cousin of Check which shows the definition of a symbol, rather than just its type.

Print list.

Inductive list $(T:$ Set $):$ Set $:=$

Nil : list $T \mid$ Cons : $T \rightarrow$ list $T \rightarrow$ list $T$

The final definition is the same as what we wrote manually before. The other elements of the section are altered similarly, turning out exactly as they were before, though we managed to write their definitions more succinctly.

Check length.

length

$: \forall T:$ Set, list $T \rightarrow$ nat 
The parameter $T$ is treated as a new argument to the induction principle, too.

Check list_ind.

list_ind

$$
\begin{aligned}
& : \forall(T: \text { Set })(P: \text { list } T \rightarrow \text { Prop }), \\
& \quad P(\text { Nil } T) \rightarrow \\
& \quad(\forall(t: T)(l: \text { list } T), P l \rightarrow P(\text { Cons } t l)) \rightarrow \\
& \quad \forall l: \text { list } T, P l
\end{aligned}
$$

Thus, despite a very real sense in which the type $T$ is an argument to the constructor Cons, the inductive case in the type of list_ind (i.e., the third line of the type) includes no quantifier for $T$, even though all of the other arguments are quantified explicitly. Parameters in other inductive definitions are treated similarly in stating induction principles.

\subsection{Mutually Inductive Types}

We can define inductive types that refer to each other:

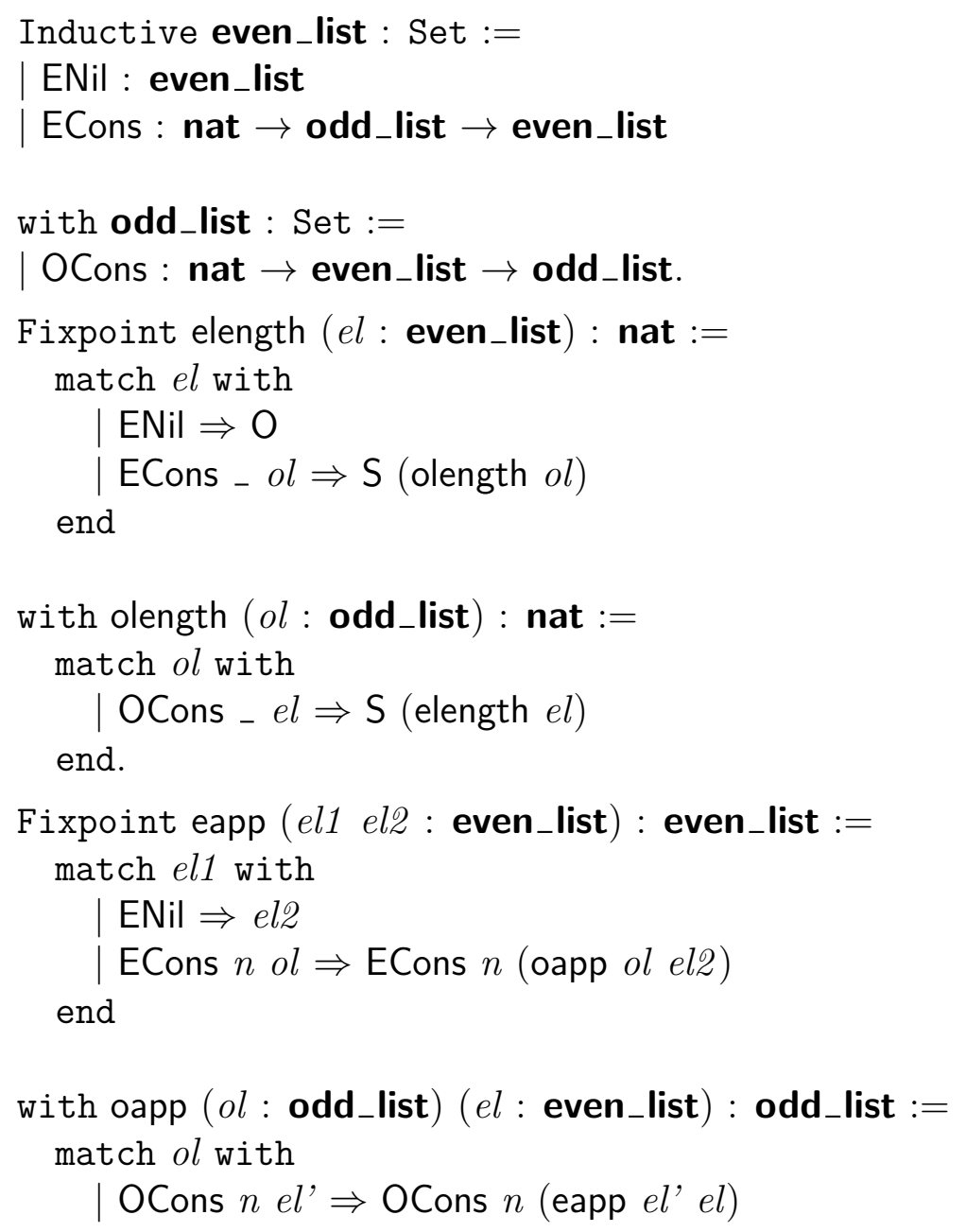


end.

Everything is going roughly the same as in past examples, until we try to prove a theorem similar to those that came before.

Theorem elength_eapp : $\forall$ el1 el2 : even_list, elength $($ eapp el1 el2) $=$ plus $($ elength el1 $)($ elength el2 $)$.

induction el1; crush.

One goal remains:

\section{$n$ : nat \\ $o:$ odd_list \\ el2 : even_list}

$\mathrm{S}($ olength $($ oapp o el2) $)=\mathrm{S}($ plus $($ olength $o)($ elength el2 $))$

We have no induction hypothesis, so we cannot prove this goal without starting another induction, which would reach a similar point, sending us into a futile infinite chain of inductions. The problem is that Coq's generation of $T_{-}$ind principles is incomplete. We only get non-mutual induction principles generated by default.

Abort.

Check even_list_ind.

even_list_ind

$: \forall P:$ even_list $\rightarrow$ Prop,

$P$ ENil $\rightarrow$

$(\forall(n:$ nat $)(o:$ odd_list $), P($ ECons $n o)) \rightarrow$

$\forall e$ : even_list, $P e$

We see that no inductive hypotheses are included anywhere in the type. To get them, we must ask for mutual principles as we need them, using the Scheme command.

Scheme even_list_mut := Induction for even_list Sort Prop

with odd_list_mut := Induction for odd_list Sort Prop.

This invocation of Scheme asks for the creation of induction principles even_list_mut for the type even_list and odd_list_mut for the type odd_list. The Induction keyword says we want standard induction schemes, since Scheme supports more exotic choices. Finally, Sort Prop establishes that we really want induction schemes, not recursion schemes, which are the same according to Curry-Howard, save for the Prop/Set distinction.

Check even_list_mut.

even_list_mut

$: \forall(P:$ even_list $\rightarrow$ Prop $)(P 0:$ odd_list $\rightarrow$ Prop $)$,

$P$ ENil $\rightarrow$

$(\forall(n:$ nat $)(o:$ odd_list $), P 0 \quad o \rightarrow P($ ECons $n o)) \rightarrow$

$(\forall(n:$ nat $)(e:$ even_list $), P e \rightarrow P 0$ (OCons $n e)) \rightarrow$ 
$\forall e$ : even_list, $P e$

This is the principle we wanted in the first place.

The Scheme command is for asking Coq to generate particular induction schemes that are mutual among a set of inductive types (possibly only one such type, in which case we get a normal induction principle). In a sense, it generalizes the induction scheme generation that goes on automatically for each inductive definition. Future Coq versions might make that automatic generation smarter, so that Scheme is needed in fewer places. In a few sections, we will see how induction principles are derived theorems in Coq, so that there is not actually any need to build in any automatic scheme generation.

There is one more wrinkle left in using the even_list_mut induction principle: the induction tactic will not apply it for us automatically. It will be helpful to look at how to prove one of our past examples without using induction, so that we can then generalize the technique to mutual inductive types.

Theorem n_plus_O' $: \forall n:$ nat, plus $n \mathrm{O}=n$.

apply nat_ind.

Here we use apply, which is one of the most essential basic tactics. When we are trying to prove fact $P$, and when thm is a theorem whose conclusion can be made to match $P$ by proper choice of quantified variable values, the invocation apply thm will replace the current goal with one new goal for each premise of thm.

This use of apply may seem a bit too magical. To better see what is going on, we use a variant where we partially apply the theorem nat_ind to give an explicit value for the predicate that gives our induction hypothesis.

Undo.

apply (nat_ind (fun $n \Rightarrow$ plus $n \mathrm{O}=n)$ ); crush. Qed.

From this example, we can see that induction is not magic. It only does some bookkeeping for us to make it easy to apply a theorem, which we can do directly with the apply tactic.

This technique generalizes to our mutual example:

Theorem elength_eapp : $\forall$ el1 el2 : even_list,

elength $($ eapp el1 el2) $=$ plus $($ elength el1 $)($ elength el2 $)$.

apply (even_list_mut

(fun $e l 1$ : even_list $\Rightarrow \forall$ el2 : even_list, elength $($ eapp el1 el2) $=$ plus $($ elength el1 $)($ elength el2 $))$

(fun $o l$ : odd_list $\Rightarrow \forall$ el : even_list, olength $($ oapp ol el) $=$ plus $($ olength ol) $($ elength $e l))) ;$ crush .

Qed.

We simply need to specify two predicates, one for each of the mutually inductive types. In general, it is not a good idea to assume that a proof assistant can infer extra predicates, so this way of applying mutual induction is about as straightforward as we may hope for. 


\subsection{Reflexive Types}

A kind of inductive type called a reflexive type includes at least one constructor that takes as an argument a function returning the same type we are defining. One very useful class of examples is in modeling variable binders. Our example will be an encoding of the syntax of first-order logic. Since the idea of syntactic encodings of logic may require a bit of acclimation, let us first consider a simpler formula type for a subset of propositional logic. We are not yet using a reflexive type, but later we will extend the example reflexively.

Inductive pformula : Set :=

| Truth : pformula

| Falsehood : pformula

| Conjunction : pformula $\rightarrow$ pformula $\rightarrow$ pformula.

A key distinction here is between, for instance, the syntax Truth and its semantics True. We can make the semantics explicit with a recursive function. This function uses the infix operator $\wedge$, which desugars to instances of the type family and from the standard library. The family and implements conjunction, the Prop Curry-Howard analogue of the usual pair type from functional programming (which is the type family prod in Coq's standard library).

Fixpoint pformulaDenote $(f$ : pformula) : Prop :=

match $f$ with

Truth $\Rightarrow$ True

Falsehood $\Rightarrow$ False

| Conjunction $f 1$ f2 $\Rightarrow$ pformulaDenote $f 1 \wedge$ pformulaDenote $f \mathscr{2}$

end.

This is just a warm-up that does not use reflexive types, the new feature we mean to introduce. When we set our sights on first-order logic instead, it becomes very handy to give constructors recursive arguments that are functions.

Inductive formula : Set :=

| Eq : nat $\rightarrow$ nat $\rightarrow$ formula

And : formula $\rightarrow$ formula $\rightarrow$ formula

Forall $:$ (nat $\rightarrow$ formula) $\rightarrow$ formula.

Our kinds of formulas are equalities between naturals, conjunction, and universal quantification over natural numbers. We avoid needing to include a notion of "variables" in our type, by using Coq functions to encode the syntax of quantification. For instance, here is the encoding of $\forall x$ : nat, $x=x$ :

Example forall_refl : formula $:=$ Forall ( fun $x \Rightarrow$ Eq $x x$ ).

We can write recursive functions over reflexive types quite naturally. Here is one translating our formulas into native Coq propositions.

Fixpoint formulaDenote $(f$ : formula) : Prop := match $f$ with

| Eq $n 1 n 2 \Rightarrow n 1=n 2$ 


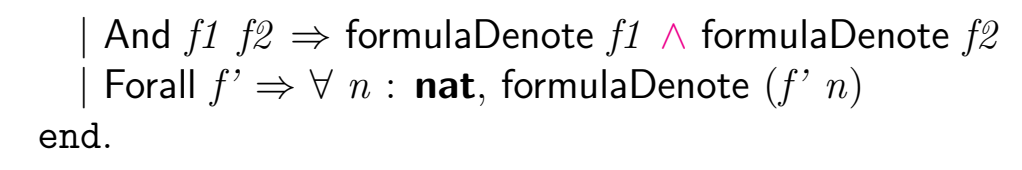

We can also encode a trivial formula transformation that swaps the order of equality and conjunction operands.

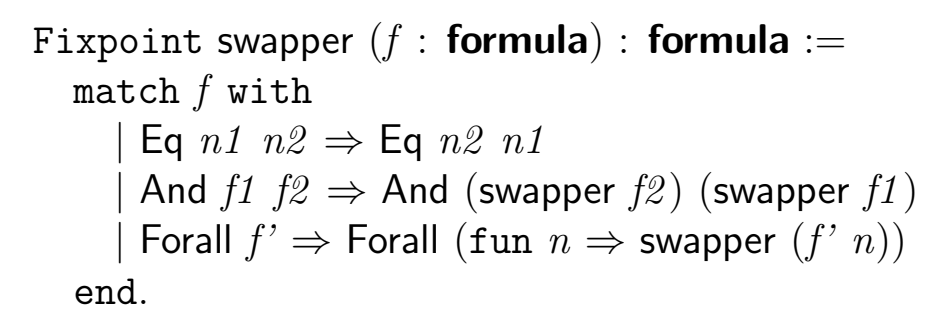

It is helpful to prove that this transformation does not make true formulas false.

Theorem swapper_preserves_truth : $\forall f$, formulaDenote $f \rightarrow$ formulaDenote (swapper $f$ ). induction $f$; crush.

Qed.

We can take a look at the induction principle behind this proof.

Check formula_ind.

formula_ind

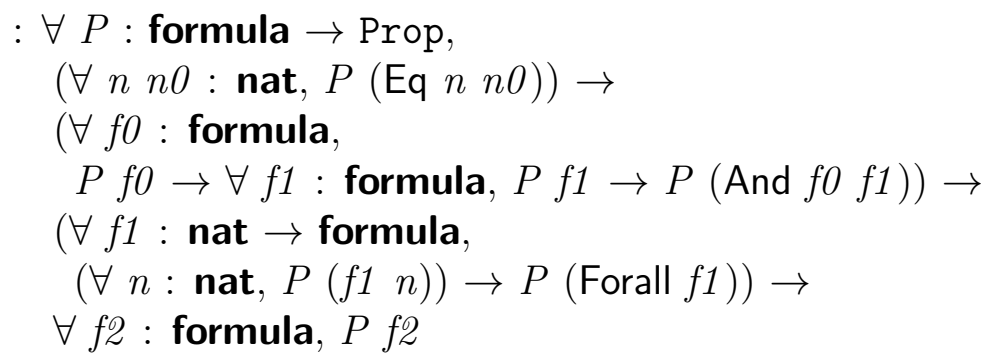

Focusing on the Forall case, which comes third, we see that we are allowed to assume that the theorem holds for any application of the argument function f1. That is, Coq induction principles do not follow a simple rule that the textual representations of induction variables must get shorter in appeals to induction hypotheses. Luckily for us, the people behind the metatheory of Coq have verified that this flexibility does not introduce unsoundness.

Up to this point, we have seen how to encode in Coq more and more of what is possible with algebraic datatypes in Haskell and ML. This may have given the inaccurate impression that inductive types are a strict extension of algebraic datatypes. In fact, Coq must rule out some types allowed by Haskell and ML, for reasons of soundness. Reflexive types provide our first good example of such a case; only some of them are legal.

Given our last example of an inductive type, many readers are probably eager to try encoding the syntax of lambda calculus. Indeed, the function-based representation technique that we just used, called higher-order abstract syntax (HOAS) [35], is the representation of choice for lambda calculi in Twelf and in many applications implemented in Haskell and ML. Let us try to import that choice to Coq: 
Inductive term : Set :=

App : term $\rightarrow$ term $\rightarrow$ term

Abs : (term $\rightarrow$ term) $\rightarrow$ term.

Error: Non strictly positive occurrence of "term" in "(term $\rightarrow$ term) -> term"

We have run afoul of the strict positivity requirement for inductive definitions, which says that the type being defined may not occur to the left of an arrow in the type of a constructor argument. It is important that the type of a constructor is viewed in terms of a series of arguments and a result, since obviously we need recursive occurrences to the lefts of the outermost arrows if we are to have recursive occurrences at all. Our candidate definition above violates the positivity requirement because it involves an argument of type term $\rightarrow$ term, where the type term that we are defining appears to the left of an arrow. The candidate type of App is fine, however, since every occurrence of term is either a constructor argument or the final result type.

Why must Coq enforce this restriction? Imagine that our last definition had been accepted, allowing us to write this function:

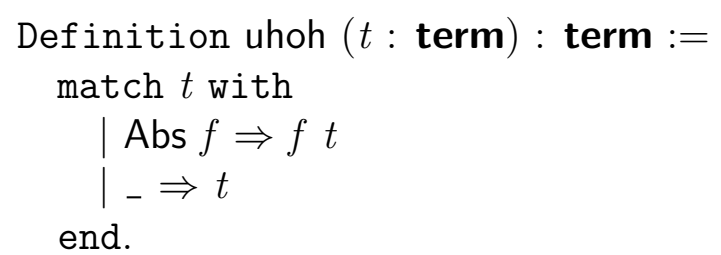

Using an informal idea of Coq's semantics, it is easy to verify that the application uhoh (Abs uhoh) will run forever. This would be a mere curiosity in OCaml and Haskell, where non-termination is commonplace, though the fact that we have a non-terminating program without explicit recursive function definitions is unusual.

For Coq, however, this would be a disaster. The possibility of writing such a function would destroy all our confidence that proving a theorem means anything. Since Coq combines programs and proofs in one language, we would be able to prove every theorem with an infinite loop.

Nonetheless, the basic insight of HOAS is a very useful one, and there are ways to realize most benefits of HOAS in Coq. We will study a particular technique of this kind in the final chapter, on programming language syntax and semantics.

\subsection{An Interlude on Induction Principles}

As we have emphasized a few times already, Coq proofs are actually programs, written in the same language we have been using in our examples all along. We can get a first sense of what this means by taking a look at the definitions of some of the induction principles we have used. A close look at the details here will help us construct induction principles manually, which we will see is necessary for some more advanced inductive definitions.

Print nat_ind. 


$$
\begin{aligned}
& \text { nat_ind }= \\
& \text { fun } P: \text { nat } \rightarrow \text { Prop } \Rightarrow \text { nat_rect } P \\
& \qquad: \forall P: \text { nat } \rightarrow \text { Prop, } \\
& \quad P \mathrm{O} \rightarrow(\forall n: \text { nat, } P n \rightarrow P(\mathrm{~S} n)) \rightarrow \forall n: \text { nat, } P n
\end{aligned}
$$

We see that this induction principle is defined in terms of a more general principle, nat_rect. The rec stands for "recursion principle," and the t at the end stands for Type.

Check nat_rect.

nat_rect

$$
\begin{aligned}
& : \forall P: \text { nat } \rightarrow \text { Type, } \\
& \quad P \mathrm{O} \rightarrow(\forall n: \text { nat, } P n \rightarrow P(\mathrm{~S} n)) \rightarrow \forall n: \text { nat, } P n
\end{aligned}
$$

The principle nat_rect gives $P$ type nat $\rightarrow$ Type instead of nat $\rightarrow$ Prop. This Type is another universe, like Set and Prop. In fact, it is a common supertype of both. Later on, we will discuss exactly what the significances of the different universes are. For now, it is just important that we can use Type as a sort of meta-universe that may turn out to be either Set or Prop. We can see the symmetry inherent in the subtyping relationship by printing the definition of another principle that was generated for nat automatically:

Print nat_rec.

$$
\begin{aligned}
& \text { nat_rec }= \\
& \text { fun } P: \text { nat } \rightarrow \text { Set } \Rightarrow \text { nat_rect } P \\
& \quad: \quad \forall P: \text { nat } \rightarrow \text { Set, } \\
& \quad P \mathrm{O} \rightarrow(\forall n: \text { nat, } P n \rightarrow P(\mathrm{~S} n)) \rightarrow \forall n: \text { nat, } P n
\end{aligned}
$$

This is identical to the definition for nat_ind, except that we have substituted Set for Prop. For most inductive types $T$, then, we get not just induction principles $T$ _ ind, but also recursion principles $T_{-}$rec. We can use $T_{-}$rec to write recursive definitions without explicit Fixpoint recursion. For instance, the following two definitions are equivalent:

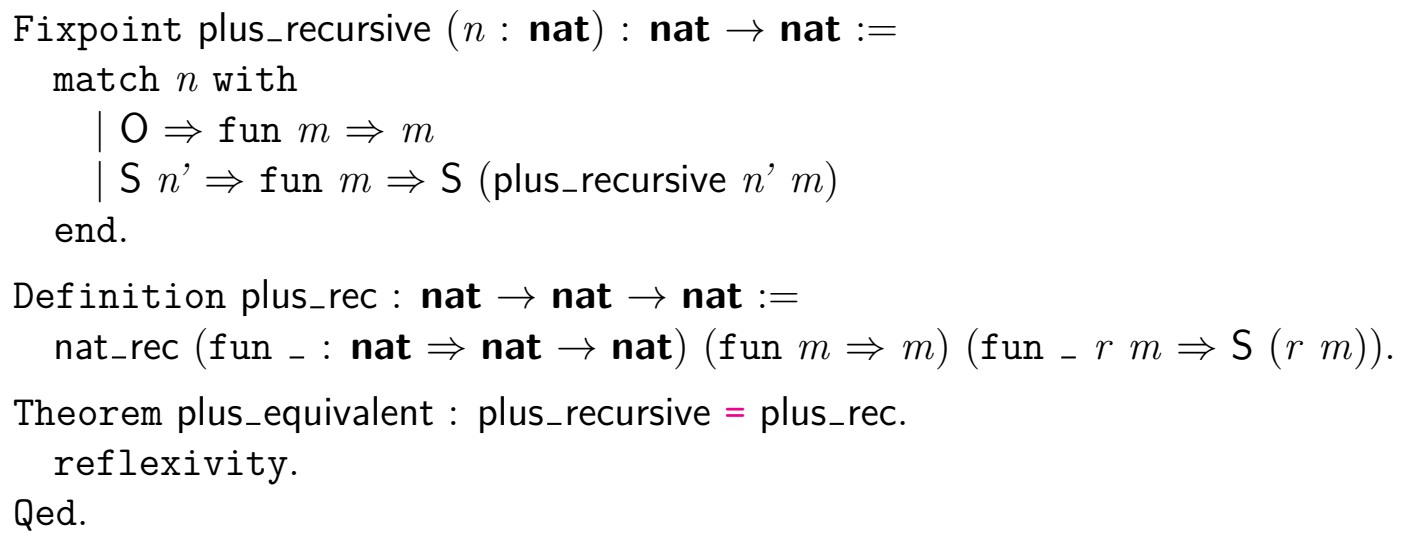

Going even further down the rabbit hole, nat_rect itself is not even a primitive. It is a functional program that we can write manually. 


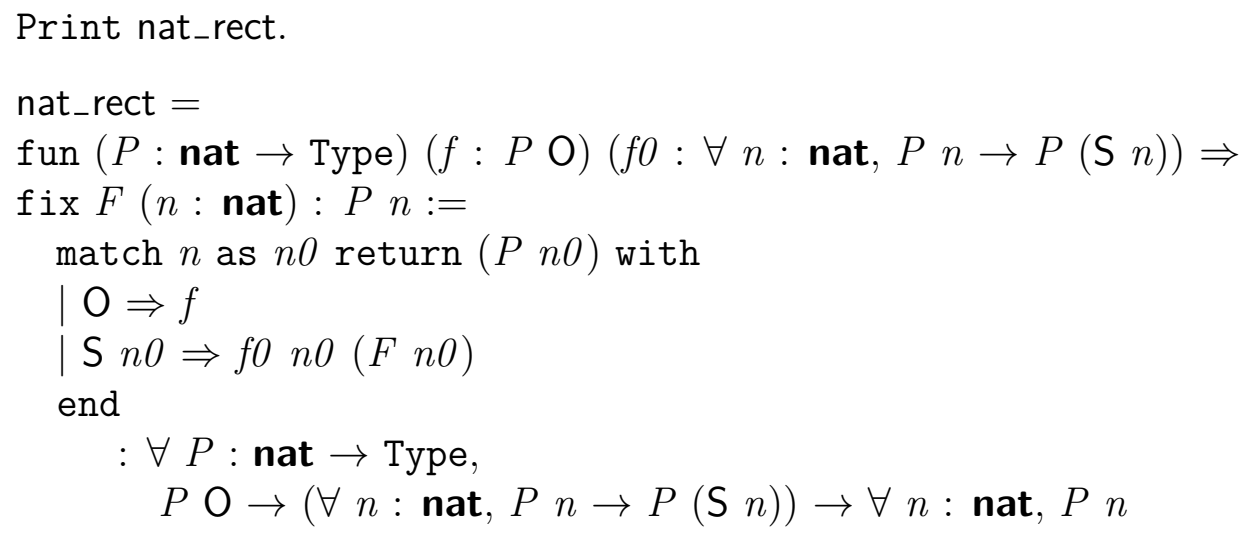

The only new wrinkles here are, first, an anonymous recursive function definition, using the $\mathrm{fix}$ keyword of Gallina (which is like fun with recursion supported); and, second, the annotations on the match expression. This is a dependently typed pattern match, because the type of the expression depends on the value being matched on. We will meet more involved examples later, especially in Part II of the book.

Type inference for dependent pattern matching is undecidable, which can be proved by reduction from higher-order unification [15]. Thus, we often find ourselves needing to annotate our programs in a way that explains dependencies to the type checker. In the example of nat_rect, we have an as clause, which binds a name for the discriminee; and a return clause, which gives a way to compute the match result type as a function of the discriminee.

To prove that nat_rect is nothing special, we can reimplement it manually.

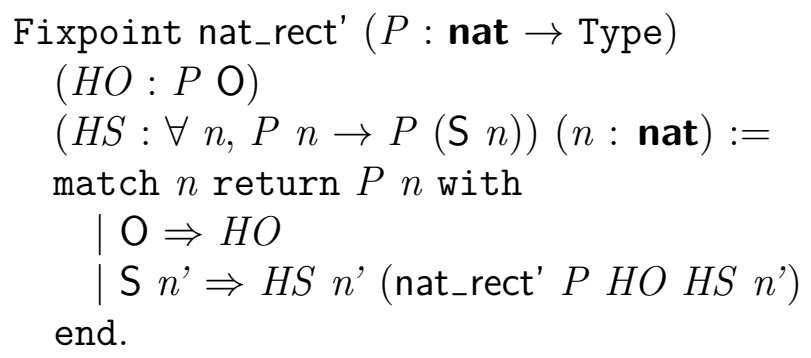

We can understand the definition of nat_rect better by reimplementing nat_ind using sections.

Section nat_ind'.

First, we have the property of natural numbers that we aim to prove.

Variable $P$ : nat $\rightarrow$ Prop.

Then we require a proof of the $\mathrm{O}$ case, which we declare with the command Hypothesis, which is a synonym for Variable that, by convention, is used for variables whose types are propositions.

Hypothesis $O_{-}$case : $P \mathrm{O}$.

Next is a proof of the $\mathrm{S}$ case, which may assume an inductive hypothesis.

Hypothesis $S_{-}$case $: \forall n:$ nat, $P n \rightarrow P(\mathrm{~S} n)$. 
Finally, we define a recursive function to tie the pieces together.

Fixpoint nat_ind' ( $n:$ nat) $: P n:=$ match $n$ with

$$
\begin{aligned}
& \mathrm{O} \Rightarrow O_{-} \text {case } \\
& \left.\mathrm{S} n^{\prime} \Rightarrow S_{-} \text {case (nat_ind' } n^{\prime}\right)
\end{aligned}
$$

end.

End nat_ind'.

Closing the section adds the Variables and Hypothesises as new fun-bound arguments to nat_ind', and, modulo the use of Prop instead of Type, we end up with the exact same definition that was generated automatically for nat_rect.

We can also examine the definition of even_list_mut, which we generated with Scheme for a mutually recursive type.

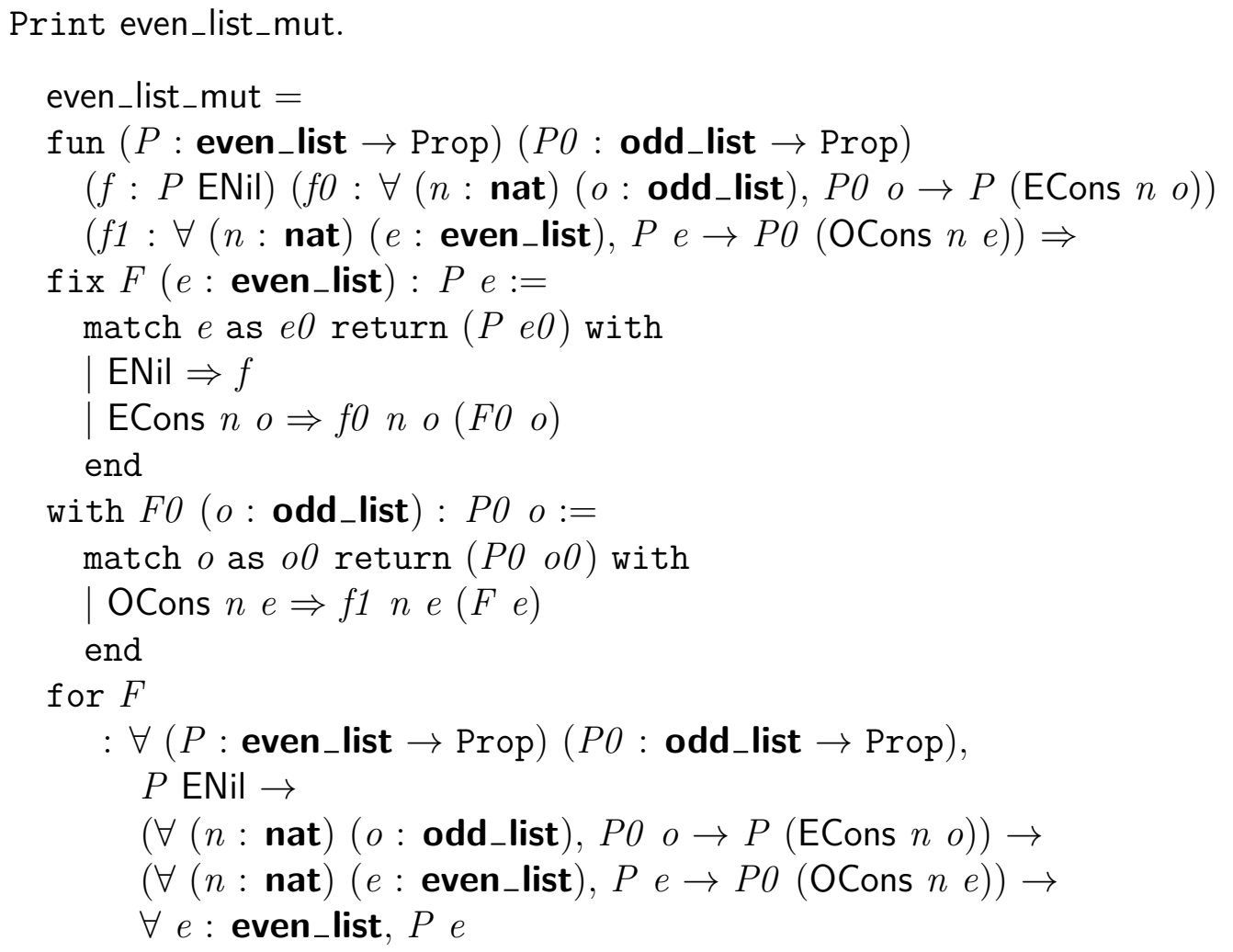

We see a mutually recursive fix, with the different functions separated by with in the same way that they would be separated by and in ML. A final for clause identifies which of the mutually recursive functions should be the final value of the $\mathrm{fix}$ expression. Using this definition as a template, we can reimplement even_list_mut directly.

Section even_list_mut'.

First, we need the properties that we are proving.

Variable Peven : even_list $\rightarrow$ Prop.

Variable Podd : odd_list $\rightarrow$ Prop. 
Next, we need proofs of the three cases.

Hypothesis ENil_case: Peven ENil.

Hypothesis ECons_case $: \forall(n:$ nat) ( $o$ : odd_list), Podd $o \rightarrow$ Peven (ECons $n o$ ).

Hypothesis OCons_case $: \forall$ ( $n$ : nat) ( $e$ : even_list), Peven $e \rightarrow$ Podd (OCons $n e$ ).

Finally, we define the recursive functions.

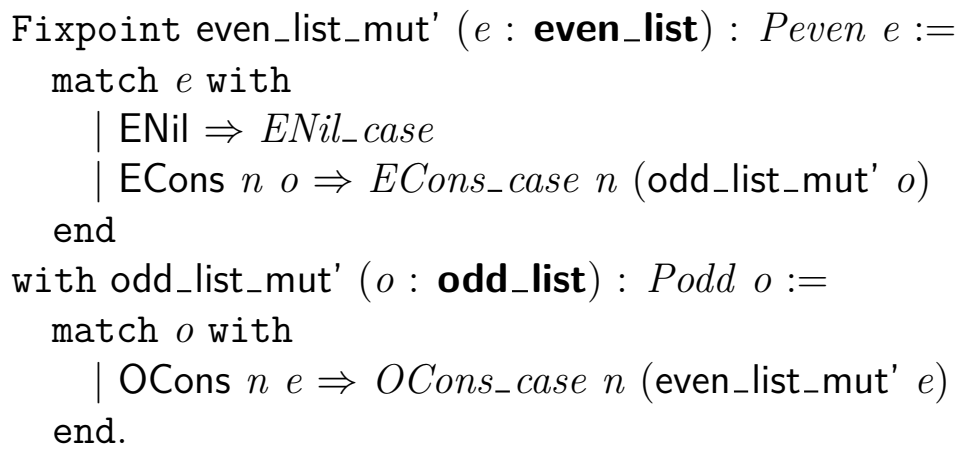

End even_list_mut'.

Even induction principles for reflexive types are easy to implement directly. For our formula type, we can use a recursive definition much like those we wrote above.

Section formula_ind'.

Variable $P$ : formula $\rightarrow$ Prop.

Hypothesis Eq-case: $\forall n 1$ n2 : nat, $P$ (Eq $n 1$ n2).

Hypothesis And_case : $\forall f 1$ f2 : formula,

$P f 1 \rightarrow P f 2 \rightarrow P($ And $f 1$ f2 $)$.

Hypothesis Forall_case $: \forall f:$ nat $\rightarrow$ formula,

$(\forall n:$ nat, $P(f n)) \rightarrow P$ (Forall $f)$.

Fixpoint formula_ind' $(f:$ formula) $: P f:=$ match $f$ with

I Eq $n 1$ n2 $\Rightarrow E q_{-}$case $n 1$ n2

And $f 1$ f2 $\Rightarrow$ And_case (formula_ind' f1) (formula_ind' f2)

| Forall $f^{\prime} \Rightarrow$ Forall_case f' (fun $n \Rightarrow$ formula_ind' $\left(f^{\prime} n\right)$ )

end.

End formula_ind'.

It is apparent that induction principle implementations involve some tedium but not terribly much creativity.

\subsection{Nested Inductive Types}

Suppose we want to extend our earlier type of binary trees to trees with arbitrary finite branching. We can use lists to give a simple definition.

Inductive nat_tree : Set :=

$\mid$ NNode' $:$ nat $\rightarrow$ list nat_tree $\rightarrow$ nat_tree. 
This is an example of a nested inductive type definition, because we use the type we are defining as an argument to a parameterized type family. Coq will not allow all such definitions; it effectively pretends that we are defining nat_tree mutually with a version of list specialized to nat_tree, checking that the resulting expanded definition satisfies the usual rules. For instance, if we replaced list with a type family that used its parameter as a function argument, then the definition would be rejected as violating the positivity restriction.

As we encountered with mutual inductive types, we find that the automatically generated induction principle for nat_tree is too weak.

Check nat_tree_ind.

nat_tree_ind

$$
\begin{aligned}
& : \forall P: \text { nat_tree } \rightarrow \text { Prop, } \\
& \left(\forall(n: \text { nat })(l: \text { list nat_tree }), P\left(\text { NNode' }^{\prime} l\right)\right) \rightarrow
\end{aligned}
$$

$\forall n$ : nat_tree, $P n$

There is no command like Scheme that will implement an improved principle for us. In general, it takes creativity to figure out good ways to incorporate nested uses of different type families. Now that we know how to implement induction principles manually, we are in a position to apply just such creativity to this problem.

Many induction principles for types with nested uses of list could benefit from a unified predicate capturing the idea that some property holds of every element in a list. By defining this generic predicate once, we facilitate reuse of library theorems about it. (Here, we are actually duplicating the standard library's Forall predicate, with a different implementation, for didactic purposes.)

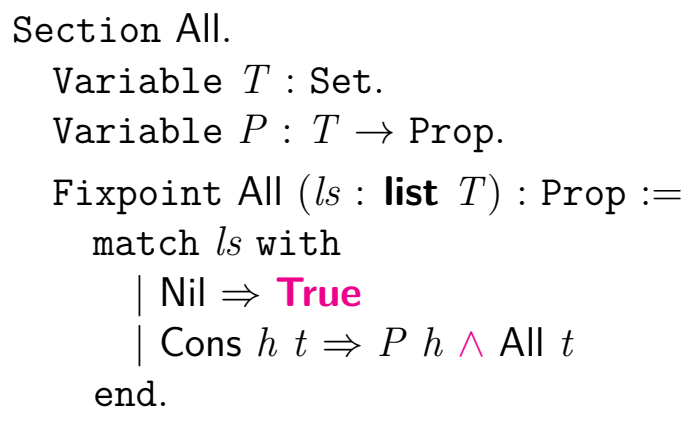

It will be useful to review the definitions of True and $\wedge$, since we will want to write manual proofs of them below.

Print True.

Inductive True : Prop := I : True

That is, True is a proposition with exactly one proof, I, which we may always supply trivially.

Finding the definition of $\wedge$ takes a little more work. Coq supports user registration of arbitrary parsing rules, and it is such a rule that is letting us write $\wedge$ instead of an application 
of some inductive type family. We can find the underlying inductive type with the Locate command, whose argument may be a parsing token.

Locate " $\bigwedge "$.

"A $\bigwedge \mathrm{B}^{\prime}:=$ and $A B:$ type_scope (default interpretation)

Print and.

Inductive and ( $A$ : Prop) ( $B:$ Prop) : Prop := conj: $A \rightarrow B \rightarrow A \wedge B$

For conj: Arguments A, B are implicit

In addition to the definition of and itself, we get information on implicit arguments (and some other information that we omit here). The implicit argument information tells us that we build a proof of a conjunction by calling the constructor conj on proofs of the conjuncts, with no need to include the types of those proofs as explicit arguments.

Now we create a section for our induction principle, following the same basic plan as in the previous section of this chapter.

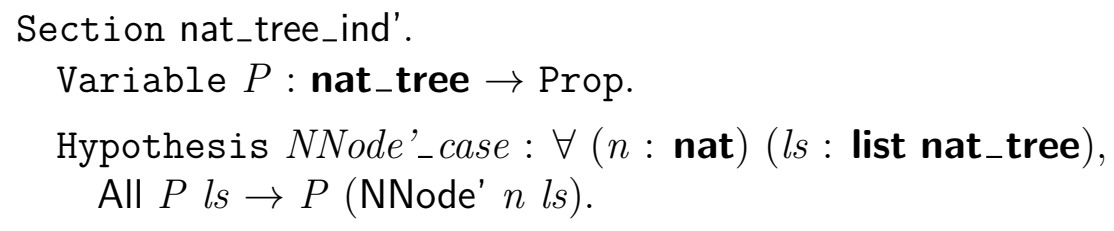

A first attempt at writing the induction principle itself follows the intuition that nested inductive type definitions are expanded into mutual inductive definitions.

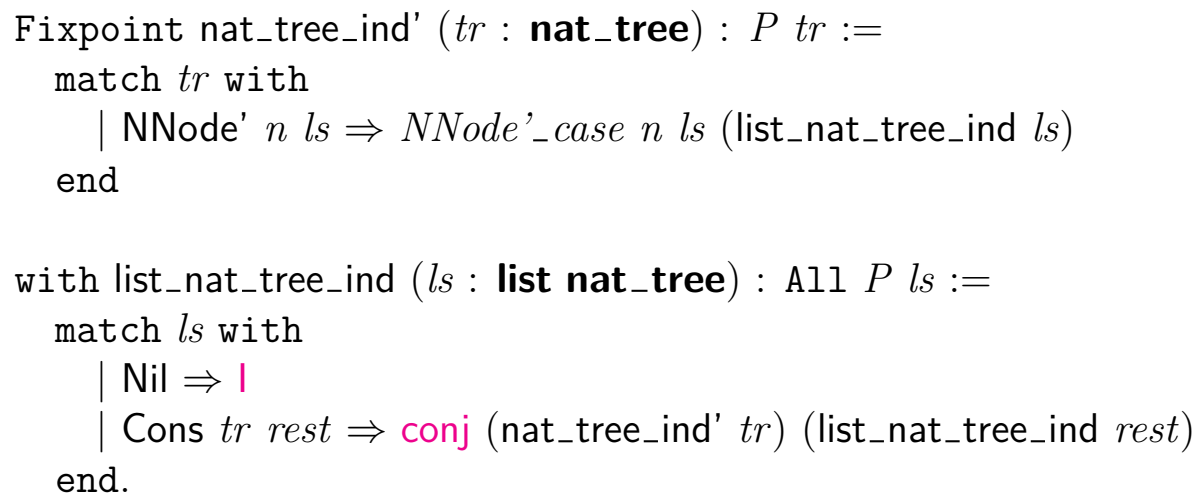

Coq rejects this definition, saying

Recursive call to nat_tree_ind' has principal argument equal to "tr" instead of rest.

There is no deep theoretical reason why this program should be rejected; Coq applies incomplete termination-checking heuristics, and it is necessary to learn a few of the most 
important rules. The term "nested inductive type" hints at the solution to this particular problem. Just as mutually inductive types require mutually recursive induction principles, nested types require nested recursion.

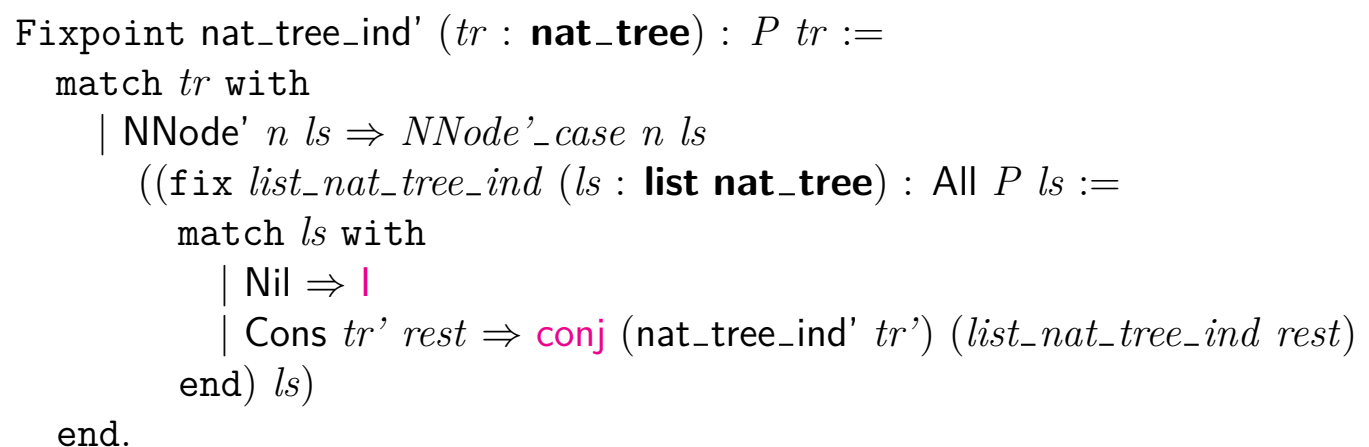

We include an anonymous $\mathrm{f} i \mathrm{x}$ version of list_nat_tree_ind that is literally nested inside the definition of the recursive function corresponding to the inductive definition that had the nested use of list.

End nat_tree_ind'.

We can try our induction principle out by defining some recursive functions on nat_tree and proving a theorem about them. First, we define some helper functions that operate on lists.

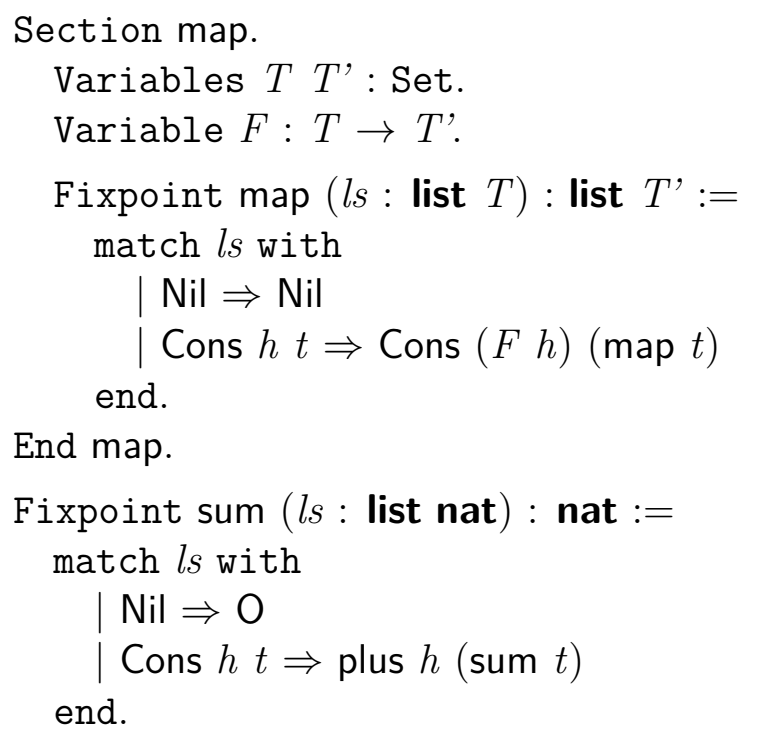

Now we can define a size function over our trees.

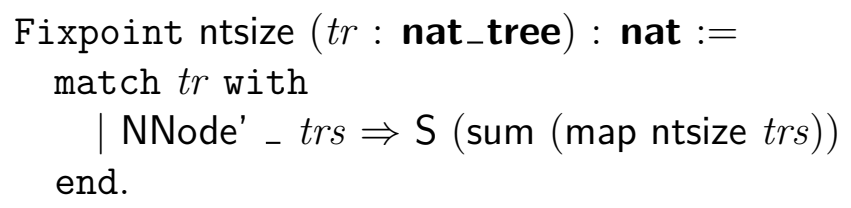

Notice that Coq was smart enough to expand the definition of map to verify that we are using proper nested recursion, even through a use of a higher-order function. 


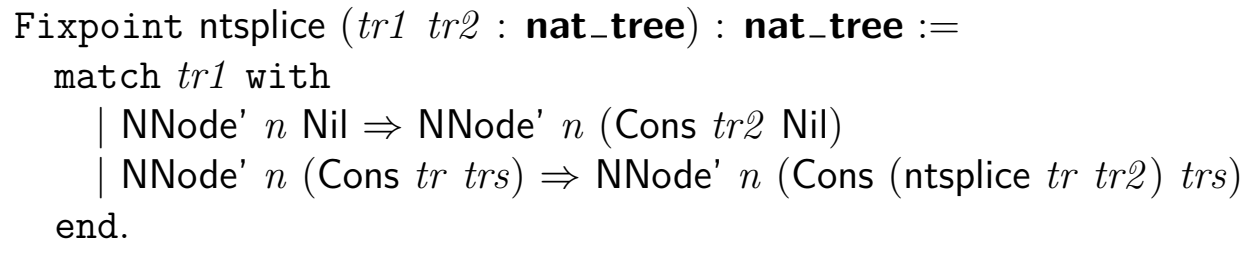

We have defined another arbitrary notion of tree splicing, similar to before, and we can prove an analogous theorem about its relationship with tree size. We start with a useful lemma about addition.

Lemma plus_S : $\forall n 1 n 2:$ nat, plus $n 1(\mathrm{~S} n 2)=\mathrm{S}$ (plus $n 1$ n2).

induction $n 1$; crush. Qed.

Now we begin the proof of the theorem, adding the lemma plus_S as a hint.

Hint Rewrite plus_S.

Theorem ntsize_ntsplice : $\forall \operatorname{tr} 1$ tr2 : nat_tree, ntsize (ntsplice $\operatorname{tr} 1$ tr2)

= plus (ntsize $\operatorname{tr} 2)$ (ntsize $\operatorname{tr} 1$ ).

We know that the standard induction principle is insufficient for the task, so we need to provide a using clause for the induction tactic to specify our alternate principle.

induction $\operatorname{tr} 1$ using nat_tree_ind'; crush.

One subgoal remains:

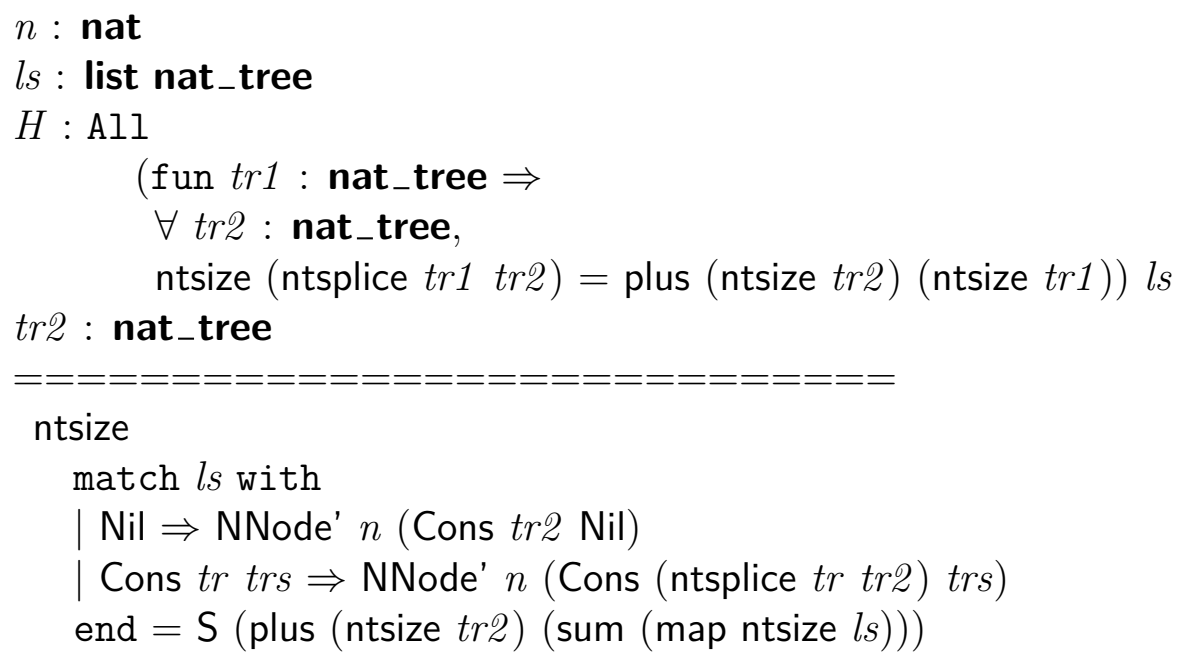

After a few moments of squinting at this goal, it becomes apparent that we need to do a case analysis on the structure of $l s$. The rest is routine.

destruct $l s$; crush.

We can go further in automating the proof by exploiting the hint mechanism.

Restart. 


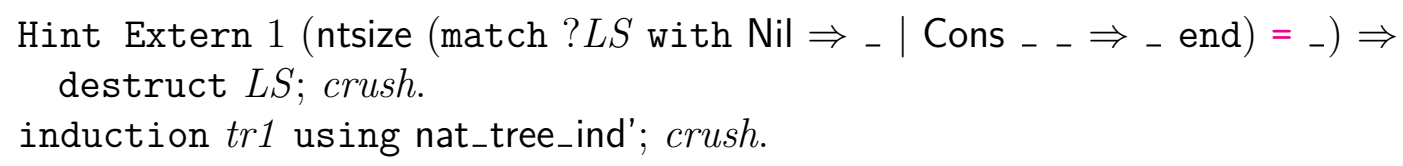

We will go into great detail on hints in a later chapter, but the only important thing to note here is that we register a pattern that describes a conclusion we expect to encounter during the proof. The pattern may contain unification variables, whose names are prefixed with question marks, and we may refer to those bound variables in a tactic that we ask to have run whenever the pattern matches.

The advantage of using the hint is not very clear here, because the original proof was so short. However, the hint has fundamentally improved the readability of our proof. Before, the proof referred to the local variable $l s$, which has an automatically generated name. To a human reading the proof script without stepping through it interactively, it was not clear where $l s$ came from. The hint explains to the reader the process for choosing which variables to case analyze, and the hint can continue working even if the rest of the proof structure changes significantly.

\subsection{Manual Proofs About Constructors}

It can be useful to understand how tactics like discriminate and injection work, so it is worth stepping through a manual proof of each kind. We will start with a proof fit for discriminate.

Theorem true_neq_false : true $\neq$ false.

We begin with the tactic red, which is short for "one step of reduction," to unfold the definition of logical negation.

red.

$=====================$
true $=$ false $\rightarrow$ False

The negation is replaced with an implication of falsehood. We use the tactic intro $H$ to change the assumption of the implication into a hypothesis named $H$.

intro $H$.

$H:$ true $=$ false

$==\mathrm{=}=\mathrm{=}=\mathrm{=}=\mathrm{=}=\mathrm{=}=\mathrm{=}=\mathrm{=}=\mathrm{=}=\mathrm{=}=\mathrm{=}$

False

This is the point in the proof where we apply some creativity. We define a function whose utility will become clear soon.

Definition toProp $(b$ : bool) $:=$ if $b$ then True else False.

It is worth recalling the difference between the lowercase and uppercase versions of truth and falsehood: True and False are logical propositions, while true and false are Boolean 
values that we can case-analyze. We have defined toProp such that our conclusion of False is computationally equivalent to toProp false. Thus, the change tactic will let us change the conclusion to toProp false. The general form change $e$ replaces the conclusion with $e$, whenever Coq's built-in computation rules suffice to establish the equivalence of $e$ with the original conclusion.

change (toProp false).

$H:$ true $=$ false

$===================$

toProp false

Now the righthand side of $H$ 's equality appears in the conclusion, so we can rewrite, using the notation $\leftarrow$ to request to replace the righthand side of the equality with the lefthand side.

$$
\begin{aligned}
& \text { rewrite } \leftarrow H . \\
& H: \text { true }=\text { false } \\
& ====================== \\
& \text { toProp true }
\end{aligned}
$$

We are almost done. Just how close we are to done is revealed by computational simplification.

simpl.

$H:$ true $=$ false

$=================$

True

trivial.

Qed.

I have no trivial automated version of this proof to suggest, beyond using discriminate or congruence in the first place.

We can perform a similar manual proof of injectivity of the constructor S. I leave a walkthrough of the details to curious readers who want to run the proof script interactively.

Theorem S_inj' : $\forall n m:$ nat, $\mathbf{S} n=\mathrm{S} m \rightarrow n=m$.

intros $n m H$.

change $(\operatorname{pred}(\mathrm{S} n)=\operatorname{pred}(\mathrm{S} m))$.

rewrite $H$.

reflexivity.

Qed.

The key piece of creativity in this theorem comes in the use of the natural number predecessor function pred. Embodied in the implementation of injection is a generic recipe for writing such type-specific functions. 
The examples in this section illustrate an important aspect of the design philosophy behind Coq. We could certainly design a Gallina replacement that built in rules for constructor discrimination and injectivity, but a simpler alternative is to include a few carefully chosen rules that enable the desired reasoning patterns and many others. A key benefit of this philosophy is that the complexity of proof checking is minimized, which bolsters our confidence that proved theorems are really true. 


\section{Chapter 4}

\section{Inductive Predicates}

The so-called "Curry-Howard correspondence" [10, 14] states a formal connection between functional programs and mathematical proofs. In the last chapter, we snuck in a first introduction to this subject in Coq. Witness the close similarity between the types unit and True from the standard library:

Print unit.

Inductive unit : Set $:=\mathrm{tt}:$ unit

Print True.

Inductive True : Prop := I : True

Recall that unit is the type with only one value, and True is the proposition that always holds. Despite this superficial difference between the two concepts, in both cases we can use the same inductive definition mechanism. The connection goes further than this. We see that we arrive at the definition of True by replacing unit by True, tt by I, and Set by Prop. The first two of these differences are superficial changes of names, while the third difference is the crucial one for separating programs from proofs. A term $T$ of type Set is a type of programs, and a term of type $T$ is a program. A term $T$ of type Prop is a logical proposition, and its proofs are of type $T$. Chapter 12 goes into more detail about the theoretical differences between Prop and Set. For now, we will simply follow common intuitions about what a proof is.

The type unit has one value, tt. The type True has one proof, I. Why distinguish between these two types? Many people who have read about Curry-Howard in an abstract context but who have not put it to use in proof engineering answer that the two types in fact should not be distinguished. There is a certain aesthetic appeal to this point of view, but I want to argue that it is best to treat Curry-Howard very loosely in practical proving. There are Coqspecific reasons for preferring the distinction, involving efficient compilation and avoidance of paradoxes in the presence of classical math, but I will argue that there is a more general principle that should lead us to avoid conflating programming and proving.

The essence of the argument is roughly this: to an engineer, not all functions of type $A \rightarrow$ $B$ are created equal, but all proofs of a proposition $P \rightarrow Q$ are. This idea is known as proof 
irrelevance, and its formalizations in logics prevent us from distinguishing between alternate proofs of the same proposition. Proof irrelevance is compatible with, but not derivable in, Gallina. Apart from this theoretical concern, I will argue that it is most effective to do engineering with Coq by employing different techniques for programs versus proofs. Most of this book is organized around that distinction, describing how to program, by applying standard functional programming techniques in the presence of dependent types; and how to prove, by writing custom Ltac decision procedures.

With that perspective in mind, this chapter is sort of a mirror image of the last chapter, introducing how to define predicates with inductive definitions. We will point out similarities in places, but much of the effective Coq user's bag of tricks is disjoint for predicates versus "datatypes." This chapter is also a covert introduction to dependent types, which are the foundation on which interesting inductive predicates are built, though we will rely on tactics to build dependently typed proof terms for us for now. A future chapter introduces more manual application of dependent types.

\subsection{Propositional Logic}

Let us begin with a brief tour through the definitions of the connectives for propositional logic. We will work within a Coq section that provides us with a set of propositional variables. In Coq parlance, these are just variables of type Prop.

\section{Section Propositional.}

Variables $P Q R$ : Prop.

In Coq, the most basic propositional connective is implication, written $\rightarrow$, which we have already used in almost every proof. Rather than being defined inductively, implication is built into Coq as the function type constructor.

We have also already seen the definition of True. For a demonstration of a lower-level way of establishing proofs of inductive predicates, we turn to this trivial theorem.

Theorem obvious: True.

$$
\text { apply I. }
$$

Qed.

We may always use the apply tactic to take a proof step based on applying a particular constructor of the inductive predicate that we are trying to establish. Sometimes there is only one constructor that could possibly apply, in which case a shortcut is available:

Theorem obvious' : True.

constructor.

Qed.

There is also a predicate False, which is the Curry-Howard mirror image of Empty_set from the last chapter.

Print False. 
Inductive False : Prop :=

We can conclude anything from False, doing case analysis on a proof of False in the same way we might do case analysis on, say, a natural number. Since there are no cases to consider, any such case analysis succeeds immediately in proving the goal.

Theorem False_imp : False $\rightarrow 2+2=5$.

destruct 1 .

Qed.

In a consistent context, we can never build a proof of False. In inconsistent contexts that appear in the courses of proofs, it is usually easiest to proceed by demonstrating the inconsistency with an explicit proof of False.

Theorem arith_neq : $2+2=5 \rightarrow 9+9=835$.

intro.

At this point, we have an inconsistent hypothesis $2+2=5$, so the specific conclusion is not important. We use the elimtype tactic. For a full description of it, see the Coq manual. For our purposes, we only need the variant elimtype False, which lets us replace any conclusion formula with False, because any fact follows from an inconsistent context.

elimtype False.

$H: 2+2=5$

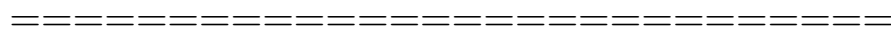

False

For now, we will leave the details of this proof about arithmetic to crush.

crush.

Qed.

A related notion to False is logical negation.

Print not.

$$
\begin{aligned}
& \text { not }=\text { fun } A: \text { Prop } \Rightarrow A \rightarrow \text { False } \\
& \quad: \text { Prop } \rightarrow \text { Prop }
\end{aligned}
$$

We see that not is just shorthand for implication of False. We can use that fact explicitly in proofs. The syntax $\neg P$ (written with a tilde in ASCII) expands to not $P$.

Theorem arith_neq' : $\neg(2+2=5)$.

unfold not.

$\begin{aligned}= & == \\ 2 & +2=5 \rightarrow \text { False } \\ \text { crush. } & \end{aligned}$


Qed.

We also have conjunction, which we introduced in the last chapter.

Print and.

Inductive and ( $A$ : Prop) ( $B$ : Prop) : Prop := conj : $A \rightarrow B \rightarrow A \wedge B$

The interested reader can check that and has a Curry-Howard equivalent called prod, the type of pairs. However, it is generally most convenient to reason about conjunction using tactics. An explicit proof of commutativity of and illustrates the usual suspects for such tasks. The operator $\wedge$ is an infix shorthand for and.

Theorem and_comm : $P \wedge Q \rightarrow Q \wedge P$.

We start by case analysis on the proof of $P \wedge Q$.

destruct 1 .

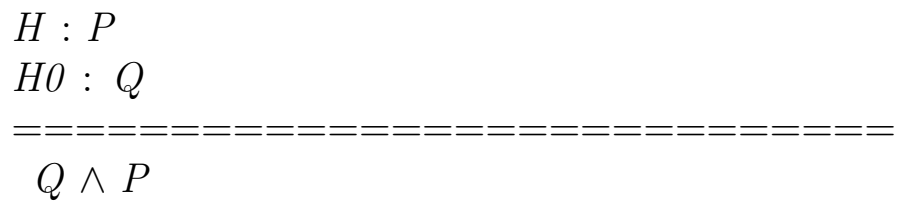

Every proof of a conjunction provides proofs for both conjuncts, so we get a single subgoal reflecting that. We can proceed by splitting this subgoal into a case for each conjunct of $Q$ $\wedge P$.

split.

2 subgoals

$H: P$

HO : Q

$====================$

$Q$

subgoal 2 is

$P$

In each case, the conclusion is among our hypotheses, so the assumption tactic finishes the process.

assumption.

assumption.

Qed. 
Coq disjunction is called or and abbreviated with the infix operator $\vee$.

Print or.

Inductive or ( $A$ : Prop) ( $B$ : Prop) : Prop :=

or_introl : $A \rightarrow A \vee B \mid$ or_intror : $B \rightarrow A \vee B$

We see that there are two ways to prove a disjunction: prove the first disjunct or prove the second. The Curry-Howard analogue of this is the Coq sum type. We can demonstrate the main tactics here with another proof of commutativity.

Theorem or_comm : $P \vee Q \rightarrow Q \vee P$.

As in the proof for and, we begin with case analysis, though this time we are met by two cases instead of one.

destruct 1 .

2 subgoals

$H: P$

$Q \vee P$

subgoal 2 is

$Q \vee P$

We can see that, in the first subgoal, we want to prove the disjunction by proving its second disjunct. The right tactic telegraphs this intent.

right; assumption.

The second subgoal has a symmetric proof.

1 subgoal

$H: Q$

$===\overline{=}=\overline{=}=\overline{=}=\overline{=}=\overline{=}=\overline{=}=\overline{=}=\overline{=}=$

$Q \vee P$

left; assumption.

Qed.

It would be a shame to have to plod manually through all proofs about propositional logic. Luckily, there is no need. One of the most basic Coq automation tactics is tauto, which is a complete decision procedure for constructive propositional logic. (More on what 
"constructive" means in the next section.) We can use tauto to dispatch all of the purely propositional theorems we have proved so far.

Theorem or_comm' : $P \vee Q \rightarrow Q \vee P$.

tauto.

Qed.

Sometimes propositional reasoning forms important plumbing for the proof of a theorem, but we still need to apply some other smarts about, say, arithmetic. The tactic intuition is a generalization of tauto that proves everything it can using propositional reasoning. When some further facts must be established to finish the proof, intuition uses propositional laws to simplify them as far as possible. Consider this example, which uses the list concatenation operator ++ from the standard library.

Theorem arith_comm : $\forall l s 1$ ls2 : list nat, length $l s 1=$ length $l s 2 \vee$ length $l s 1+$ length $l s 2=6$

$\rightarrow$ length $(l s 1++l s 2)=6 \vee$ length $l s 1=$ length $l s 2$.

intuition.

A lot of the proof structure has been generated for us by intuition, but the final proof depends on a fact about lists. The remaining subgoal hints at what cleverness we need to inject.

$$
\begin{aligned}
& l s 1: \text { list nat } \\
& l s 2: \text { list nat } \\
& H 0: \text { length } l s 1+\text { length } l s 2=6 \\
& ======================== \\
& \text { length }(l s 1++l s 2)=6 \vee \text { length } l s 1=\text { length } l s 2
\end{aligned}
$$

We can see that we need a theorem about lengths of concatenated lists, which we proved last chapter and is also in the standard library.

rewrite app_length.

$$
\begin{aligned}
& l s 1: \text { list nat } \\
& l s \mathscr{2}: \text { list nat } \\
& H 0: \text { length } l s 1+\text { length } l s \mathscr{2}=6 \\
& ================================= \\
& \text { length } l s 1+\text { length } l s \mathscr{2}=6 \vee \text { length } l s 1=\text { length } l s 2
\end{aligned}
$$

Now the subgoal follows by purely propositional reasoning. That is, we could replace length $l s 1+$ length $l s 2=6$ with $P$ and length $l s 1=$ length $l s 2$ with $Q$ and arrive at a tautology of propositional logic.

tauto.

Qed. 
The intuition tactic is one of the main bits of glue in the implementation of crush, so, with a little help, we can get a short automated proof of the theorem.

Theorem arith_comm' : $\forall l s 1$ ls2 : list nat,

length $l s 1=$ length $l s 2 \vee$ length $l s 1+$ length $l s 2=6$

$\rightarrow$ length $(l s 1++l s 2)=6 \vee$ length $l s 1=$ length $l s 2$.

Hint Rewrite app_length.

crush.

Qed.

End Propositional.

Ending the section here has the same effect as always. Each of our propositional theorems becomes universally quantified over the propositional variables that we used.

\subsection{What Does It Mean to Be Constructive?}

One potential point of confusion in the presentation so far is the distinction between bool and Prop. The datatype bool is built from two values true and false, while Prop is a more primitive type that includes among its members True and False. Why not collapse these two concepts into one, and why must there be more than two states of mathematical truth, True and False?

The answer comes from the fact that Coq implements constructive or intuitionistic logic, in contrast to the classical logic that you may be more familiar with. In constructive logic, classical tautologies like $\neg \neg P \rightarrow P$ and $P \vee \neg P$ do not always hold. In general, we can only prove these tautologies when $P$ is decidable, in the sense of computability theory. The Curry-Howard encoding that Coq uses for or allows us to extract either a proof of $P$ or a proof of $\neg P$ from any proof of $P \vee \neg P$. Since our proofs are just functional programs which we can run, a general law of the excluded middle would give us a decision procedure for the halting problem, where the instantiations of $P$ would be formulas like "this particular Turing machine halts."

A similar paradoxical situation would result if every proposition evaluated to either True or False. Evaluation in Coq is decidable, so we would be limiting ourselves to decidable propositions only.

Hence the distinction between bool and Prop. Programs of type bool are computational by construction; we can always run them to determine their results. Many Props are undecidable, and so we can write more expressive formulas with Props than with bools, but the inevitable consequence is that we cannot simply "run a Prop to determine its truth."

Constructive logic lets us define all of the logical connectives in an aesthetically appealing way, with orthogonal inductive definitions. That is, each connective is defined independently using a simple, shared mechanism. Constructivity also enables a trick called program extraction, where we write programs by phrasing them as theorems to be proved. Since our proofs are just functional programs, we can extract executable programs from our final proofs, which we could not do as naturally with classical proofs. 
We will see more about Coq's program extraction facility in a later chapter. However, I think it is worth interjecting another warning at this point, following up on the prior warning about taking the Curry-Howard correspondence too literally. It is possible to write programs by theorem-proving methods in Coq, but hardly anyone does it. It is almost always most useful to maintain the distinction between programs and proofs. If you write a program by proving a theorem, you are likely to run into algorithmic inefficiencies that you introduced in your proof to make it easier to prove. It is a shame to have to worry about such situations while proving tricky theorems, and it is a happy state of affairs that you almost certainly will not need to, with the ideal of extracting programs from proofs being confined mostly to theoretical studies.

\subsection{First-Order Logic}

The $\forall$ connective of first-order logic, which we have seen in many examples so far, is built into Coq. Getting ahead of ourselves a bit, we can see it as the dependent function type constructor. In fact, implication and universal quantification are just different syntactic shorthands for the same Coq mechanism. A formula $P \rightarrow Q$ is equivalent to $\forall x: P, Q$, where $x$ does not appear in $Q$. That is, the "real" type of the implication says "for every proof of $P$, there exists a proof of $Q . "$

Existential quantification is defined in the standard library.

Print ex.

Inductive ex $(A:$ Type $)(P: A \rightarrow$ Prop) : Prop :=

ex_intro: $\forall x: A, P x \rightarrow$ ex $P$

(Note that here, as always, each $\forall$ quantifier has the largest possible scope, so that the type of ex_intro could also be written $\forall x: A,(P x \rightarrow$ ex $P)$.)

The family ex is parameterized by the type $A$ that we quantify over, and by a predicate $P$ over $A$ s. We prove an existential by exhibiting some $x$ of type $A$, along with a proof of $P$ $x$. As usual, there are tactics that save us from worrying about the low-level details most of the time.

Here is an example of a theorem statement with existential quantification. We use the equality operator $=$, which, depending on the settings in which they learned logic, different people will say either is or is not part of first-order logic. For our purposes, it is.

Theorem exist1 : $\exists x$ : nat, $x+1=2$.

We can start this proof with a tactic exists, which should not be confused with the formula constructor shorthand of the same name. In the version of this document that you are reading, the reverse "E" appears instead of the text "exists" in formulas.

exists 1 .

The conclusion is replaced with a version using the existential witness that we announced.

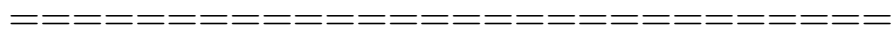




$$
1+1=2
$$

reflexivity.

Qed.

We can also use tactics to reason about existential hypotheses.

Theorem exist2: $\forall n m$ : nat, $(\exists x:$ nat, $n+x=m) \rightarrow n \leq m$.

We start by case analysis on the proof of the existential fact.

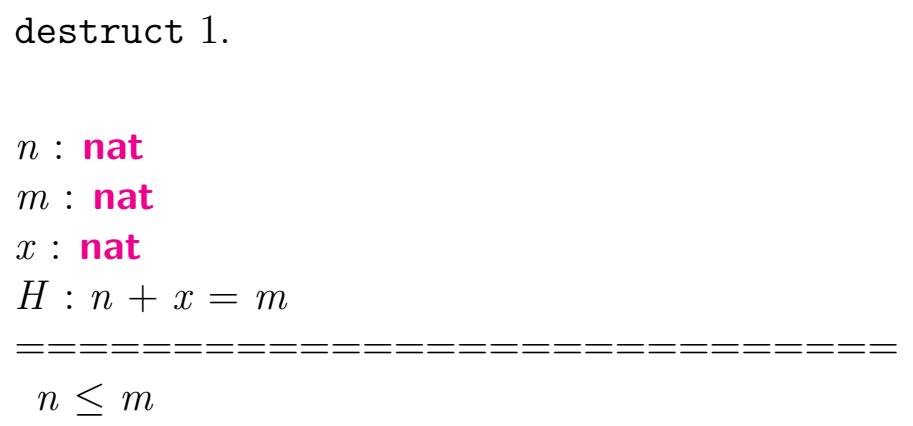

The goal has been replaced by a form where there is a new free variable $x$, and where we have a new hypothesis that the body of the existential holds with $x$ substituted for the old bound variable. From here, the proof is just about arithmetic and is easy to automate.

crush.

Qed.

The tactic intuition has a first-order cousin called firstorder, which proves many formulas when only first-order reasoning is needed, and it tries to perform first-order simplifications in any case. First-order reasoning is much harder than propositional reasoning, so firstorder is much more likely than intuition to get stuck in a way that makes it run for long enough to be useless.

\subsection{Predicates with Implicit Equality}

We start our exploration of a more complicated class of predicates with a simple example: an alternative way of characterizing when a natural number is zero.

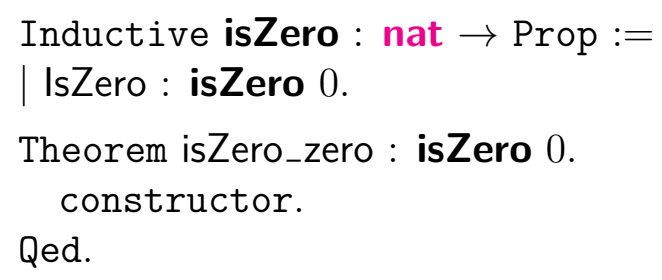

We can call isZero a judgment, in the sense often used in the semantics of programming languages. Judgments are typically defined in the style of natural deduction, where we write a number of inference rules with premises appearing above a solid line and a conclusion 
appearing below the line. In this example, the sole constructor IsZero of isZero can be thought of as the single inference rule for deducing isZero, with nothing above the line and isZero 0 below it. The proof of isZero_zero demonstrates how we can apply an inference rule. (Readers not familiar with formal semantics should not worry about not following this paragraph!)

The definition of isZero differs in an important way from all of the other inductive definitions that we have seen in this and the previous chapter. Instead of writing just Set or Prop after the colon, here we write nat $\rightarrow$ Prop. We saw examples of parameterized types like list, but there the parameters appeared with names before the colon. Every constructor of a parameterized inductive type must have a range type that uses the same parameter, whereas the form we use here enables us to choose different arguments to the type for different constructors.

For instance, our definition isZero makes the predicate provable only when the argument is 0 . We can see that the concept of equality is somehow implicit in the inductive definition mechanism. The way this is accomplished is similar to the way that logic variables are used in Prolog (but worry not if not familiar with Prolog), and it is a very powerful mechanism that forms a foundation for formalizing all of mathematics. In fact, though it is natural to think of inductive types as folding in the functionality of equality, in Coq, the true situation is reversed, with equality defined as just another inductive type!

Print eq.

Inductive eq $(A:$ Type $)(x: A): A \rightarrow$ Prop $:=$ eq_refl $: x=x$

Behind the scenes, uses of infix $=$ are expanded to instances of eq. We see that eq has both a parameter $x$ that is fixed and an extra unnamed argument of the same type. The type of eq allows us to state any equalities, even those that are provably false. However, examining the type of equality's sole constructor eq_refl, we see that we can only prove equality when its two arguments are syntactically equal. This definition turns out to capture all of the basic properties of equality, and the equality-manipulating tactics that we have seen so far, like reflexivity and rewrite, are implemented treating eq as just another inductive type with a well-chosen definition. Another way of stating that definition is: equality is defined as the least reflexive relation.

Returning to the example of isZero, we can see how to work with hypotheses that use this predicate.

Theorem isZero_plus : $\forall n m$ : nat, isZero $m \rightarrow n+m=n$.

We want to proceed by cases on the proof of the assumption about isZero.

destruct 1 .

$n$ : nat

$==================$

$n+0=n$

Since isZero has only one constructor, we are presented with only one subgoal. The 
argument $m$ to isZero is replaced with that type's argument from the single constructor IsZero. From this point, the proof is trivial.

crush.

Qed.

Another example seems at first like it should admit an analogous proof, but in fact provides a demonstration of one of the most basic gotchas of Coq proving.

Theorem isZero_contra : isZero $1 \rightarrow$ False.

Let us try a proof by cases on the assumption, as in the last proof.

destruct 1 .

\section{False}

It seems that case analysis has not helped us much at all! Our sole hypothesis disappears, leaving us, if anything, worse off than we were before. What went wrong? We have met an important restriction in tactics like destruct and induction when applied to types with arguments. If the arguments are not already free variables, they will be replaced by new free variables internally before doing the case analysis or induction. Since the argument 1 to isZero is replaced by a fresh variable, we lose the crucial fact that it is not equal to 0 .

Why does Coq use this restriction? We will discuss the issue in detail in a future chapter, when we see the dependently typed programming techniques that would allow us to write this proof term manually. For now, we just say that the algorithmic problem of "logically complete case analysis" is undecidable when phrased in Coq's logic. A few tactics and design patterns that we will present in this chapter suffice in almost all cases. For the current example, what we want is a tactic called inversion, which corresponds to the concept of inversion that is frequently used with natural deduction proof systems. (Again, worry not if the semantics-oriented terminology from this last sentence is unfamiliar.)

Undo.

inversion 1.

Qed.

What does inversion do? Think of it as a version of destruct that does its best to take advantage of the structure of arguments to inductive types. In this case, inversion completed the proof immediately, because it was able to detect that we were using isZero with an impossible argument.

Sometimes using destruct when you should have used inversion can lead to confusing results. To illustrate, consider an alternate proof attempt for the last theorem.

Theorem isZero_contra' : isZero $1 \rightarrow 2+2=5$.

destruct 1 .

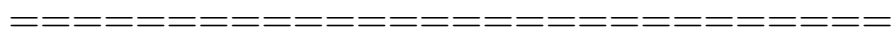




$$
1+1=4
$$

What on earth happened here? Internally, destruct replaced 1 with a fresh variable, and, trying to be helpful, it also replaced the occurrence of 1 within the unary representation of each number in the goal. Then, within the $\mathrm{O}$ case of the proof, we replace the fresh variable with $\mathrm{O}$. This has the net effect of decrementing each of these numbers.

Abort.

To see more clearly what is happening, we can consider the type of isZero's induction principle.

Check isZero_ind.

isZero_ind

$: \forall P:$ nat $\rightarrow$ Prop, $P 0 \rightarrow \forall n:$ nat, isZero $n \rightarrow P n$

In our last proof script, destruct chose to instantiate $P$ as fun $n \Rightarrow \mathrm{S} n+\mathrm{S} n=\mathrm{S}$ (S $(S(S n)))$. You can verify for yourself that this specialization of the principle applies to the goal and that the hypothesis $P 0$ then matches the subgoal we saw generated. If you are doing a proof and encounter a strange transmutation like this, there is a good chance that you should go back and replace a use of destruct with inversion.

\subsection{Recursive Predicates}

We have already seen all of the ingredients we need to build interesting recursive predicates, like this predicate capturing even-ness.

Inductive even : nat $\rightarrow$ Prop :=

| EvenO : even $\mathrm{O}$

| EvenSS : $\forall n$, even $n \rightarrow$ even (S (S $n)$ ).

Think of even as another judgment defined by natural deduction rules. The rule EvenO has nothing above the line and even $\mathrm{O}$ below the line, and EvenSS is a rule with even $n$ above the line and even ( $\mathrm{S}(\mathrm{S} n)$ ) below.

The proof techniques of the last section are easily adapted.

Theorem even_0 : even 0 .

constructor.

Qed.

Theorem even_4 : even 4.

constructor; constructor; constructor.

Qed.

It is not hard to see that sequences of constructor applications like the above can get tedious. We can avoid them using Coq's hint facility, with a new Hint variant that asks 
to consider all constructors of an inductive type during proof search. The tactic auto performs exhaustive proof search up to a fixed depth, considering only the proof steps we have registered as hints.

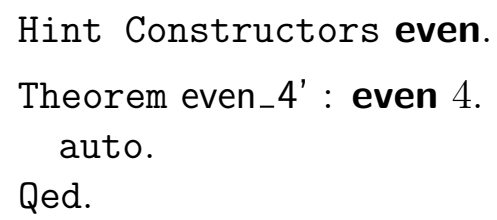

We may also use inversion with even.

Theorem even_1_contra : even $1 \rightarrow$ False. inversion 1.

Qed.

Theorem even_3_contra : even $3 \rightarrow$ False. inversion 1.

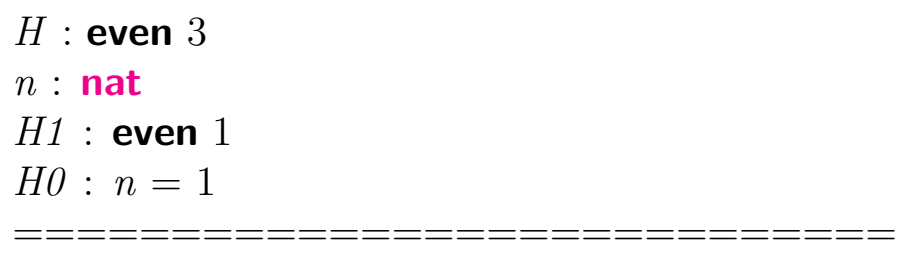

False

The inversion tactic can be a little overzealous at times, as we can see here with the introduction of the unused variable $n$ and an equality hypothesis about it. For more complicated predicates, though, adding such assumptions is critical to dealing with the undecidability of general inversion. More complex inductive definitions and theorems can cause inversion to generate equalities where neither side is a variable.

inversion $H 1$.

Qed.

We can also do inductive proofs about even.

Theorem even_plus : $\forall n m$, even $n \rightarrow$ even $m \rightarrow$ even $(n+m)$.

It seems a reasonable first choice to proceed by induction on $n$.

induction $n$; crush.

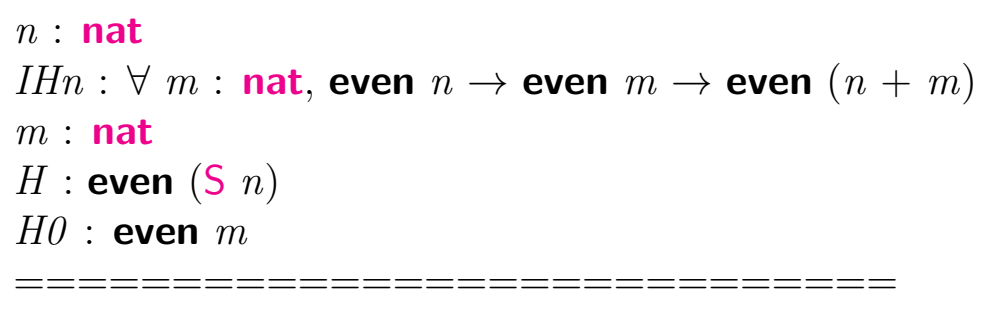


even $(S(n+m))$

We will need to use the hypotheses $H$ and $H O$ somehow. The most natural choice is to invert $H$.

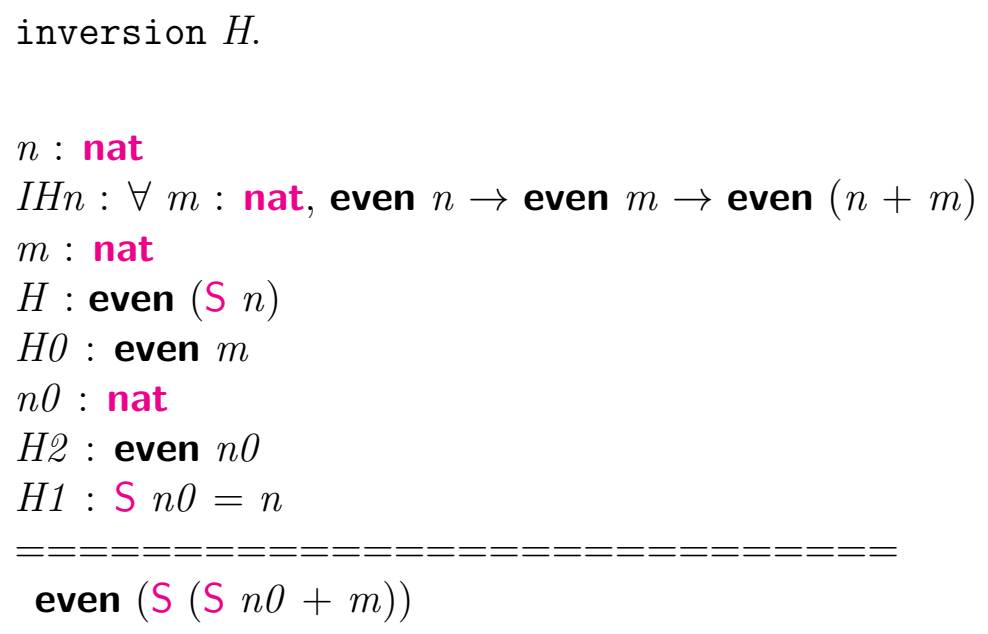

Simplifying the conclusion brings us to a point where we can apply a constructor. simpl.

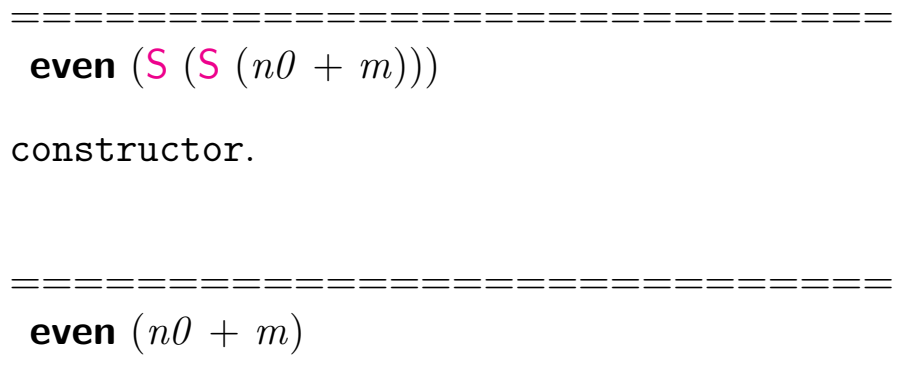

At this point, we would like to apply the inductive hypothesis, which is:

IHn $: \forall m:$ nat, even $n \rightarrow$ even $m \rightarrow$ even $(n+m)$

Unfortunately, the goal mentions $n 0$ where it would need to mention $n$ to match $I H n$. We could keep looking for a way to finish this proof from here, but it turns out that we can make our lives much easier by changing our basic strategy. Instead of inducting on the structure of $n$, we should induct on the structure of one of the even proofs. This technique is commonly called rule induction in programming language semantics. In the setting of Coq, we have already seen how predicates are defined using the same inductive type mechanism as datatypes, so the fundamental unity of rule induction with "normal" induction is apparent. 
Recall that tactics like induction and destruct may be passed numbers to refer to unnamed lefthand sides of implications in the conclusion, where the argument $n$ refers to the $n$th such hypothesis.

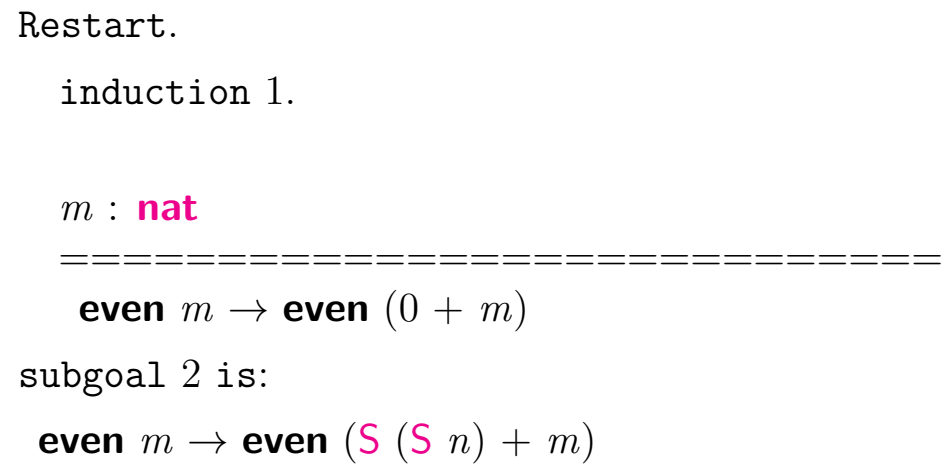

The first case is easily discharged by crush, based on the hint we added earlier to try the constructors of even.

crush.

Now we focus on the second case:

intro.

$m$ : nat

$n$ : nat

$H$ : even $n$

IHeven : even $m \rightarrow$ even $(n+m)$

$H O$ : even $m$

$==\overline{=}=\overline{=}=\overline{=}=\overline{=}=\overline{=}=\overline{=}=\overline{=}=\overline{=}$

even $(S(S n)+m)$

We simplify and apply a constructor, as in our last proof attempt.

simpl; constructor.

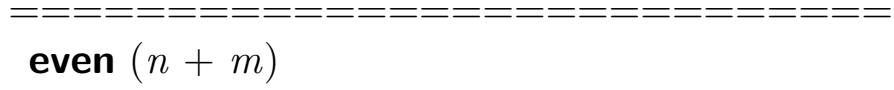

Now we have an exact match with our inductive hypothesis, and the remainder of the proof is trivial.

apply IHeven; assumption.

In fact, crush can handle all of the details of the proof once we declare the induction strategy. 
Restart.

induction 1 ; crush.

Qed.

Induction on recursive predicates has similar pitfalls to those we encountered with inversion in the last section.

Theorem even_contra : $\forall n$, even $(\mathrm{S}(n+n)) \rightarrow$ False.

induction 1.

$n$ : nat

False

subgoal 2 is:

False

We are already sunk trying to prove the first subgoal, since the argument to even was replaced by a fresh variable internally. This time, we find it easier to prove this theorem by way of a lemma. Instead of trusting induction to replace expressions with fresh variables, we do it ourselves, explicitly adding the appropriate equalities as new assumptions.

Abort.

Lemma even_contra' : $\forall n^{\prime}$, even $n^{\prime} \rightarrow \forall n, n^{\prime}=\mathrm{S}(n+n) \rightarrow$ False.

induction 1 ; crush.

At this point, it is useful to consider all cases of $n$ and $n 0$ being zero or nonzero. Only one of these cases has any trickiness to it.

destruct $n$; destruct $n 0 ;$ crush.

$n$ : nat

$H$ : even $(S n)$

IHeven : $\forall n 0:$ nat, $\mathrm{S} n=\mathrm{S}(n 0+n 0) \rightarrow$ False

n0 : nat

$H 0: \mathrm{S} n=n 0+\mathrm{S} n 0$

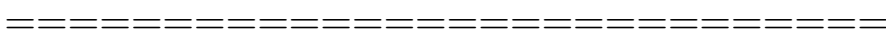

False

At this point it is useful to use a theorem from the standard library, which we also proved with a different name in the last chapter. We can search for a theorem that allows us to rewrite terms of the form $x+\mathrm{S} y$.

$$
\begin{aligned}
& \text { SearchRewrite }\left({ }_{-}+\mathrm{S}_{-}\right) \text {. } \\
& \text { plus_n_Sm : } \forall n m: \text { nat, } \mathrm{S}(n+m)=n+\mathrm{S} m
\end{aligned}
$$


rewrite $\leftarrow$ plus_n_Sm in $H O$.

The induction hypothesis lets us complete the proof, if we use a variant of apply that has a with clause to give instantiations of quantified variables.

apply IHeven with $n 0$; assumption.

As usual, we can rewrite the proof to avoid referencing any locally generated names, which makes our proof script more readable and more robust to changes in the theorem statement. We use the notation $\leftarrow$ to request a hint that does right-to-left rewriting, just like we can with the rewrite tactic.

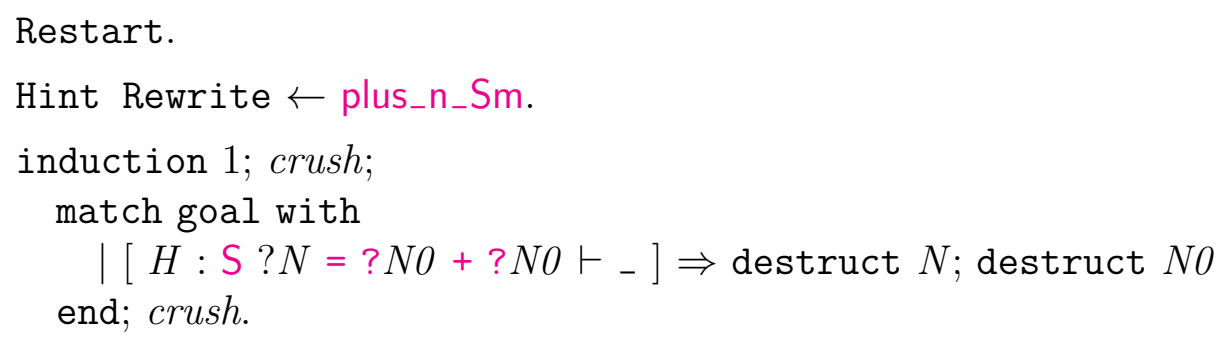

Qed.

We write the proof in a way that avoids the use of local variable or hypothesis names, using the match tactic form to do pattern-matching on the goal. We use unification variables prefixed by question marks in the pattern, and we take advantage of the possibility to mention a unification variable twice in one pattern, to enforce equality between occurrences. The hint to rewrite with plus_n_Sm in a particular direction saves us from having to figure out the right place to apply that theorem.

The original theorem now follows trivially from our lemma, using a new tactic eauto, a fancier version of auto whose explanation we postpone to Chapter 13.

Theorem even_contra : $\forall n$, even $(\mathrm{S}(n+n)) \rightarrow$ False.

intros; eapply even_contra'; eauto.

Qed.

We use a variant eapply of apply which has the same relationship to apply as eauto has to auto. An invocation of apply only succeeds if all arguments to the rule being used can be determined from the form of the goal, whereas eapply will introduce unification variables for undetermined arguments. In this case, eauto is able to determine the right values for those unification variables, using (unsurprisingly) a variant of the classic algorithm for unification [41].

By considering an alternate attempt at proving the lemma, we can see another common pitfall of inductive proofs in Coq. Imagine that we had tried to prove even_contra' with all of the $\forall$ quantifiers moved to the front of the lemma statement.

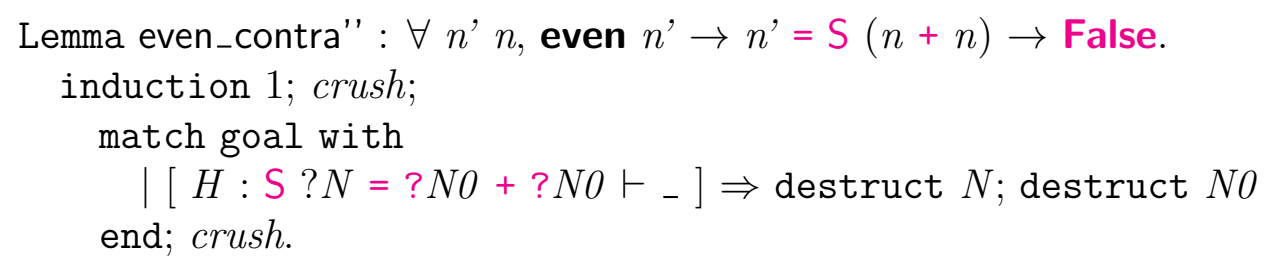


One subgoal remains:

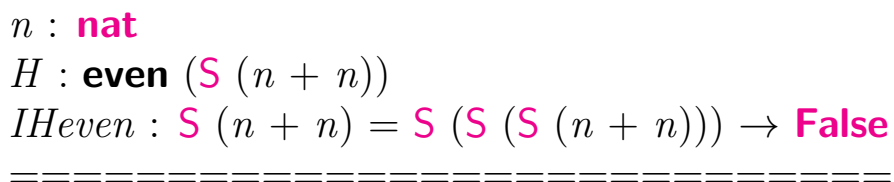

False

We are out of luck here. The inductive hypothesis is trivially true, since its assumption is false. In the version of this proof that succeeded, IHeven had an explicit quantification over $n$. This is because the quantification of $n$ appeared after the thing we are inducting on in the theorem statement. In general, quantified variables and hypotheses that appear before the induction object in the theorem statement stay fixed throughout the inductive proof. Variables and hypotheses that are quantified after the induction object may be varied explicitly in uses of inductive hypotheses.

Abort.

Why should Coq implement induction this way? One answer is that it avoids burdening this basic tactic with additional heuristic smarts, but that is not the whole picture. Imagine that induction analyzed dependencies among variables and reordered quantifiers to preserve as much freedom as possible in later uses of inductive hypotheses. This could make the inductive hypotheses more complex, which could in turn cause particular automation machinery to fail when it would have succeeded before. In general, we want to avoid quantifiers in our proofs whenever we can, and that goal is furthered by the refactoring that the induction tactic forces us to do. 


\section{Chapter 5}

\section{Infinite Data and Proofs}

In lazy functional programming languages like Haskell, infinite data structures are everywhere [16]. Infinite lists and more exotic datatypes provide convenient abstractions for communication between parts of a program. Achieving similar convenience without infinite lazy structures would, in many cases, require acrobatic inversions of control flow.

Laziness is easy to implement in Haskell, where all the definitions in a program may be thought of as mutually recursive. In such an unconstrained setting, it is easy to implement an infinite loop when you really meant to build an infinite list, where any finite prefix of the list should be forceable in finite time. Haskell programmers learn how to avoid such slip-ups. In Coq, such a laissez-faire policy is not good enough.

We spent some time in the last chapter discussing the Curry-Howard isomorphism, where proofs are identified with functional programs. In such a setting, infinite loops, intended or otherwise, are disastrous. If Coq allowed the full breadth of definitions that Haskell did, we could code up an infinite loop and use it to prove any proposition vacuously. That is, the addition of general recursion would make CIC inconsistent. For an arbitrary proposition $P$, we could write:

Fixpoint bad ( $u$ : unit) $: P:=$ bad $u$.

This would leave us with bad tt as a proof of $P$.

There are also algorithmic considerations that make universal termination very desirable. We have seen how tactics like reflexivity compare terms up to equivalence under computational rules. Calls to recursive, pattern-matching functions are simplified automatically, with no need for explicit proof steps. It would be very hard to hold onto that kind of benefit if it became possible to write non-terminating programs; we would be running smack into the halting problem.

One solution is to use types to contain the possibility of non-termination. For instance, we can create a "non-termination monad," inside which we must write all of our generalrecursive programs; several such approaches are surveyed in Chapter 7 . This is a heavyweight solution, and so we would like to avoid it whenever possible.

Luckily, Coq has special support for a class of lazy data structures that happens to contain most examples found in Haskell. That mechanism, co-inductive types, is the subject 
of this chapter.

\subsection{Computing with Infinite Data}

Let us begin with the most basic type of infinite data, streams, or lazy lists.

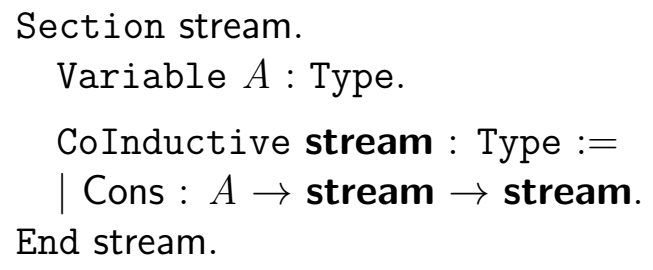

The definition is surprisingly simple. Starting from the definition of list, we just need to change the keyword Inductive to CoInductive. We could have left a Nil constructor in our definition, but we will leave it out to force all of our streams to be infinite.

How do we write down a stream constant? Obviously, simple application of constructors is not good enough, since we could only denote finite objects that way. Rather, whereas recursive definitions were necessary to use values of recursive inductive types effectively, here we find that we need co-recursive definitions to build values of co-inductive types effectively.

We can define a stream consisting only of zeroes.

CoFixpoint zeroes : stream nat $:=$ Cons 0 zeroes.

We can also define a stream that alternates between true and false.

CoFixpoint trues_falses : stream bool := Cons true falses_trues

with falses_trues : stream bool := Cons false trues_falses.

Co-inductive values are fair game as arguments to recursive functions, and we can use that fact to write a function to take a finite approximation of a stream.

Fixpoint approx $A(s:$ stream $A)(n:$ nat $):$ list $A:=$ match $n$ with

$\mathrm{O} \Rightarrow$ nil

$\mathrm{S} n^{\prime} \Rightarrow$

match $s$ with

| Cons $h t \Rightarrow h:$ : approx $t n^{\prime}$

end

end.

Eval simpl in approx zeroes 10 .

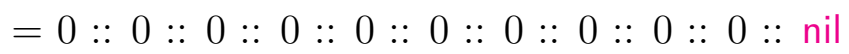

: list nat

Eval simpl in approx trues_falses 10 .

$=$ true 


$$
\begin{aligned}
& \text { :: false } \\
& :: \text { true } \\
& \text { :: false } \\
& :: \text { true }:: \text { false }:: \text { true }:: \text { false }:: \text { true }:: \text { false }:: \text { nil }
\end{aligned}
$$

So far, it looks like co-inductive types might be a magic bullet, allowing us to import all of the Haskeller's usual tricks. However, there are important restrictions that are dual to the restrictions on the use of inductive types. Fixpoints consume values of inductive types, with restrictions on which arguments may be passed in recursive calls. Dually, co-fixpoints produce values of co-inductive types, with restrictions on what may be done with the results of co-recursive calls.

The restriction for co-inductive types shows up as the guardedness condition. First, consider this stream definition, which would be legal in Haskell.

CoFixpoint looper: stream nat := looper.

Error:

Recursive definition of looper is ill-formed.

In environment

looper : stream nat

unguarded recursive call in "looper"

The rule we have run afoul of here is that every co-recursive call must be guarded by a constructor; that is, every co-recursive call must be a direct argument to a constructor of the co-inductive type we are generating. It is a good thing that this rule is enforced. If the definition of looper were accepted, our approx function would run forever when passed looper, and we would have fallen into inconsistency.

Some familiar functions are easy to write in co-recursive fashion.

Section map.

Variables $A B$ : Type.

Variable $f: A \rightarrow B$.

CoFixpoint map $(s:$ stream $A)$ : stream $B:=$ match $s$ with

| Cons $h t \Rightarrow$ Cons $(f h)(\operatorname{map} t)$ end.

End map.

This code is a literal copy of that for the list map function, with the nil case removed and Fixpoint changed to CoFixpoint. Many other standard functions on lazy data structures can be implemented just as easily. Some, like filter, cannot be implemented. Since the predicate passed to filter may reject every element of the stream, we cannot satisfy the guardedness condition. 
The implications of the condition can be subtle. To illustrate how, we start off with another co-recursive function definition that is legal. The function interleave takes two streams and produces a new stream that alternates between their elements.

Section interleave.

Variable $A$ : Type.

CoFixpoint interleave $(s 1$ s2 : stream $A)$ : stream $A:=$ match $s 1$, s2 with

| Cons h1 t1, Cons h2 t2 $\Rightarrow$ Cons h1 (Cons h2 (interleave t1 t2)) end.

End interleave.

Now say we want to write a weird stuttering version of map that repeats elements in a particular way, based on interleaving.

Section map'.

Variables $A B$ : Type.

Variable $f: A \rightarrow B$.

CoFixpoint map' $(s:$ stream $A)$ : stream $B:=$ match $s$ with

| Cons $h t \Rightarrow$ interleave (Cons $(f h)\left(\right.$ map' $\left.\left.^{\prime}\right)\right)$ (Cons $(f h)\left(\right.$ map' $\left.\left.^{\prime}\right)\right)$ end.

We get another error message about an unguarded recursive call.

End map'.

What is going wrong here? Imagine that, instead of interleave, we had called some other, less well-behaved function on streams. Here is one simpler example demonstrating the essential pitfall. We start by defining a standard function for taking the tail of a stream. Since streams are infinite, this operation is total.

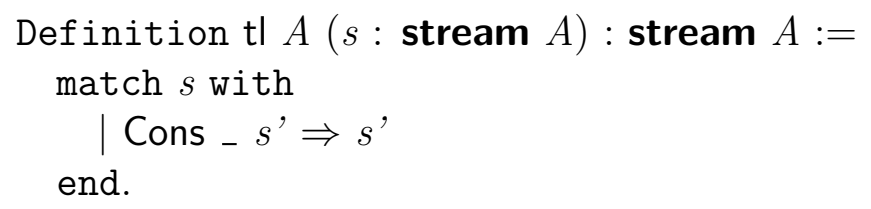

Coq rejects the following definition that uses $\mathrm{tl}$.

CoFixpoint bad : stream nat $:=\mathrm{tl}$ (Cons 0 bad).

Imagine that Coq had accepted our definition, and consider how we might evaluate approx bad 1 . We would be trying to calculate the first element in the stream bad. However, it is not hard to see that the definition of bad "begs the question": unfolding the definition of $\mathrm{tl}$, we see that we essentially say "define bad to equal itself"! Of course such an equation admits no single well-defined solution, which does not fit well with the determinism of Gallina reduction.

Coq's complete rule for co-recursive definitions includes not just the basic guardedness condition, but also a requirement about where co-recursive calls may occur. In particular, 
a co-recursive call must be a direct argument to a constructor, nested only inside of other constructor calls or fun or match expressions. In the definition of bad, we erroneously nested the co-recursive call inside a call to $t l$, and we nested inside a call to interleave in the definition of map'.

Coq helps the user out a little by performing the guardedness check after using computation to simplify terms. For instance, any co-recursive function definition can be expanded by inserting extra calls to an identity function, and this change preserves guardedness. However, in other cases computational simplification can reveal why definitions are dangerous. Consider what happens when we inline the definition of $\mathrm{tl}$ in bad:

CoFixpoint bad : stream nat := bad.

This is the same looping definition we rejected earlier. A similar inlining process reveals an alternate view on our failed definition of map':

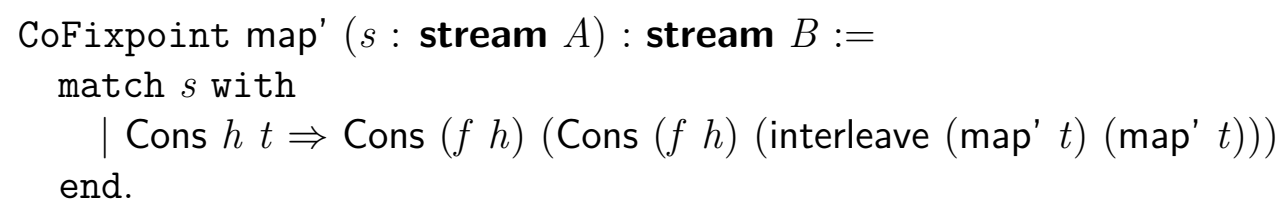

Clearly in this case the map' calls are not immediate arguments to constructors, so we violate the guardedness condition.

A more interesting question is why that condition is the right one. We can make an intuitive argument that the original map' definition is perfectly reasonable and denotes a well-understood transformation on streams, such that every output would behave properly with approx. The guardedness condition is an example of a syntactic check for productivity of co-recursive definitions. A productive definition can be thought of as one whose outputs can be forced in finite time to any finite approximation level, as with approx. If we replaced the guardedness condition with more involved checks, we might be able to detect and allow a broader range of productive definitions. However, mistakes in these checks could cause inconsistency, and programmers would need to understand the new, more complex checks. Coq's design strikes a balance between consistency and simplicity with its choice of guard condition, though we can imagine other worthwhile balances being struck, too.

\section{$5.2 \quad$ Infinite Proofs}

Let us say we want to give two different definitions of a stream of all ones, and then we want to prove that they are equivalent.

CoFixpoint ones : stream nat $:=$ Cons 1 ones.

Definition ones' $:=$ map $S$ zeroes.

The obvious statement of the equality is this:

Theorem ones_eq : ones = ones'.

However, faced with the initial subgoal, it is not at all clear how this theorem can be proved. In fact, it is unprovable. The eq predicate that we use is fundamentally limited to 
equalities that can be demonstrated by finite, syntactic arguments. To prove this equivalence, we will need to introduce a new relation.

Abort.

Co-inductive datatypes make sense by analogy from Haskell. What we need now is a co-inductive proposition. That is, we want to define a proposition whose proofs may be infinite, subject to the guardedness condition. The idea of infinite proofs does not show up in usual mathematics, but it can be very useful (unsurprisingly) for reasoning about infinite data structures. Besides examples from Haskell, infinite data and proofs will also turn out to be useful for modelling inherently infinite mathematical objects, like program executions.

We are ready for our first co-inductive predicate.

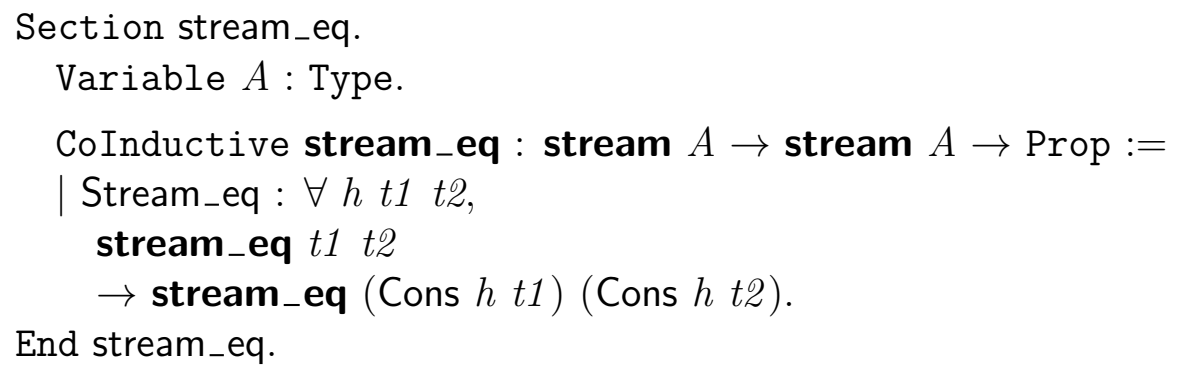

We say that two streams are equal if and only if they have the same heads and their tails are equal. We use the normal finite-syntactic equality for the heads, and we refer to our new equality recursively for the tails.

We can try restating the theorem with stream_eq.

Theorem ones_eq : stream_eq ones ones'.

Coq does not support tactical co-inductive proofs as well as it supports tactical inductive proofs. The usual starting point is the cofix tactic, which asks to structure this proof as a co-fixpoint.

cofix ones_eq.

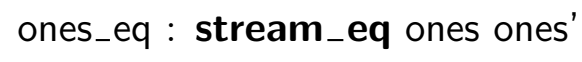

It looks like this proof might be easier than we expected!

assumption.

Proof completed.

Unfortunately, we are due for some disappointment in our victory lap. Qed.

Error: 
Recursive definition of ones_eq is ill-formed.

In environment

ones_eq : stream_eq ones ones'

unguarded recursive call in "ones_eq"

Via the Curry-Howard correspondence, the same guardedness condition applies to our co-inductive proofs as to our co-inductive data structures. We should be grateful that this proof is rejected, because, if it were not, the same proof structure could be used to prove any co-inductive theorem vacuously, by direct appeal to itself!

Thinking about how Coq would generate a proof term from the proof script above, we see that the problem is that we are violating the guardedness condition. During our proofs, Coq can help us check whether we have yet gone wrong in this way. We can run the command Guarded in any context to see if it is possible to finish the proof in a way that will yield a properly guarded proof term.

Guarded.

Running Guarded here gives us the same error message that we got when we tried to run Qed. In larger proofs, Guarded can be helpful in detecting problems before we think we are ready to run Qed.

We need to start the co-induction by applying stream_eq's constructor. To do that, we need to know that both arguments to the predicate are Conses. Informally, this is trivial, but simpl is not able to help us.

Undo.

simpl.

ones_eq : stream_eq ones ones'

$=========================$

stream_eq ones ones'

It turns out that we are best served by proving an auxiliary lemma.

Abort.

First, we need to define a function that seems pointless at first glance.

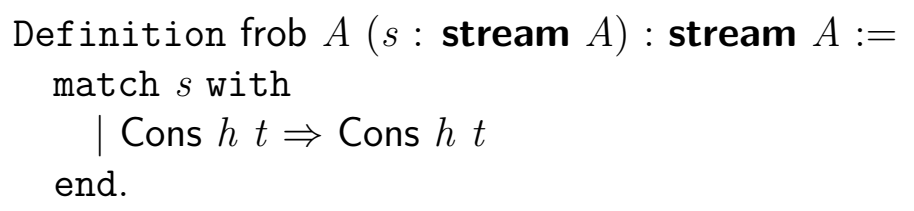

Next, we need to prove a theorem that seems equally pointless.

Theorem frob_eq : $\forall A(s:$ stream $A), s=$ frob $s$.

destruct $s$; reflexivity. 
Qed.

But, miraculously, this theorem turns out to be just what we needed.

Theorem ones_eq : stream_eq ones ones'.

cofix ones_eq.

We can use the theorem to rewrite the two streams.

rewrite (frob_eq ones).

rewrite (frob_eq ones').

ones_eq : stream_eq ones ones'

$==================$

stream_eq (frob ones) (frob ones')

Now simpl is able to reduce the streams.

simpl.

ones_eq : stream_eq ones ones'

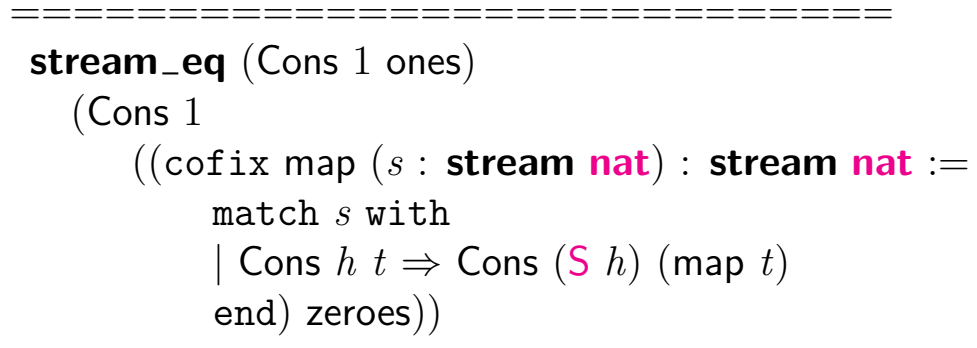

Note the cofix notation for anonymous co-recursion, which is analogous to the $\mathrm{fix}$ notation we have already seen for recursion. Since we have exposed the Cons structure of each stream, we can apply the constructor of stream_eq.

constructor.

ones_eq : stream_eq ones ones'

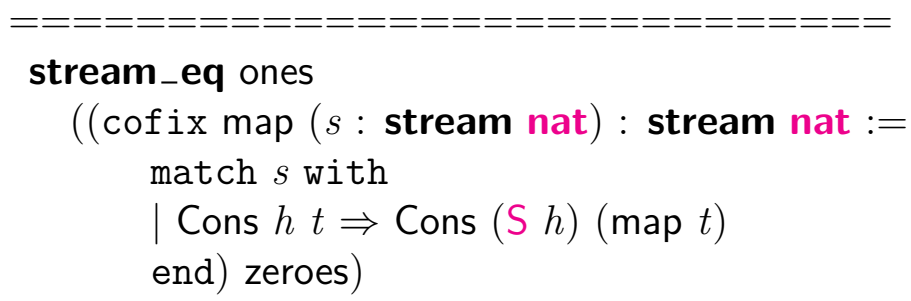

Now, modulo unfolding of the definition of map, we have matched our assumption. assumption. 
Qed.

Why did this silly-looking trick help? The answer has to do with the constraints placed on Coq's evaluation rules by the need for termination. The cofix-related restriction that foiled our first attempt at using simpl is dual to a restriction for fix. In particular, an application of an anonymous fix only reduces when the top-level structure of the recursive argument is known. Otherwise, we would be unfolding the recursive definition ad infinitum.

Fixpoints only reduce when enough is known about the definitions of their arguments. Dually, co-fixpoints only reduce when enough is known about how their results will be used. In particular, a cofix is only expanded when it is the discriminee of a match. Rewriting with our superficially silly lemma wrapped new matches around the two cofixes, triggering reduction.

If cofixes reduced haphazardly, it would be easy to run into infinite loops in evaluation, since we are, after all, building infinite objects.

One common source of difficulty with co-inductive proofs is bad interaction with standard Coq automation machinery. If we try to prove ones_eq' with automation, like we have in previous inductive proofs, we get an invalid proof.

Theorem ones_eq' : stream_eq ones ones'.

cofix one_eq'; crush.

Guarded.

Abort.

The standard auto machinery sees that our goal matches an assumption and so applies that assumption, even though this violates guardedness. A correct proof strategy for a theorem like this usually starts by destructing some parameter and running a custom tactic to figure out the first proof rule to apply for each case. Alternatively, there are tricks that can be played with "hiding" the co-inductive hypothesis.

Must we always be cautious with automation in proofs by co-induction? Induction seems to have dual versions of the same pitfalls inherent in it, and yet we avoid those pitfalls by encapsulating safe Curry-Howard recursion schemes inside named induction principles. It turns out that we can usually do the same with co-induction principles. Let us take that tack here, so that we can arrive at an induction $x$; crush-style proof for ones_eq'.

An induction principle is parameterized over a predicate characterizing what we mean to prove, as a function of the inductive fact that we already know. Dually, a co-induction principle ought to be parameterized over a predicate characterizing what we mean to prove, as a function of the arguments to the co-inductive predicate that we are trying to prove.

To state a useful principle for stream_eq, it will be useful first to define the stream head function.

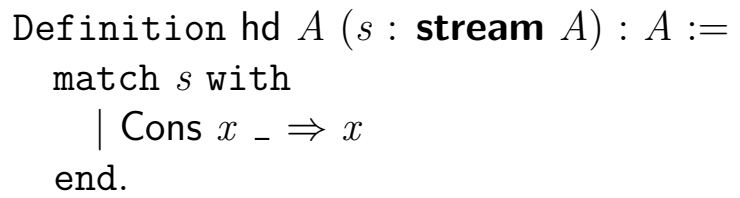

Now we enter a section for the co-induction principle, based on Park's principle as intro- 
duced in a tutorial by Giménez [12].

Section stream_eq_coind.

Variable $A$ : Type.

Variable $R:$ stream $A \rightarrow$ stream $A \rightarrow$ Prop.

This relation generalizes the theorem we want to prove, defining a set of pairs of streams that we must eventually prove contains the particular pair we care about.

Hypothesis Cons_case_hd: $\forall s 1$ s2, $R$ s1 s2 $\rightarrow$ hd $s 1=$ hd $s 2$.

Hypothesis Cons_case_tl : $\forall s 1$ s2, $R$ s1 s2 $\rightarrow R(\mathrm{tl} s 1)(\mathrm{tl} s 2)$.

Two hypotheses characterize what makes a good choice of $R$ : it enforces equality of stream heads, and it is "hereditary" in the sense that an $R$ stream pair passes on " $R$-ness" to its tails. An established technical term for such a relation is bisimulation.

Now it is straightforward to prove the principle, which says that any stream pair in $R$ is equal. The reader may wish to step through the proof script to see what is going on.

Theorem stream_eq_coind : $\forall s 1$ s2, $R$ s1 s2 $\rightarrow$ stream_eq $s 1$ s2.

cofix stream_eq_coind; destruct $s 1$; destruct $s 2$; intro.

generalize (Cons_case_hd H); intro Heq; simpl in Heq; rewrite Heq.

constructor.

apply stream_eq_coind.

apply (Cons_case_tl $H$ ).

Qed.

End stream_eq_coind.

To see why this proof is guarded, we can print it and verify that the one co-recursive call is an immediate argument to a constructor.

Print stream_eq_coind.

We omit the output and proceed to proving ones_eq" again. The only bit of ingenuity is in choosing $R$, and in this case the most restrictive predicate works.

Theorem ones_eq" : stream_eq ones ones'.

apply (stream_eq_coind (fun $s 1$ s2 $\Rightarrow s 1=$ ones $\wedge$ s2 = ones')); crush. Qed.

Note that this proof achieves the proper reduction behavior via hd and tl, rather than frob as in the last proof. All three functions pattern match on their arguments, catalyzing computation steps.

Compared to the inductive proofs that we are used to, it still seems unsatisfactory that we had to write out a choice of $R$ in the last proof. An alternate is to capture a common pattern of co-recursion in a more specialized co-induction principle. For the current example, that pattern is: prove stream_eq $s 1$ s2 where $s 1$ and $s 2$ are defined as their own tails.

Section stream_eq_loop.

Variable $A$ : Type.

Variables $s 1$ s2 : stream $A$. 
Hypothesis Cons_case_hd : hd $s 1=$ hd $s 2$.

Hypothesis loop1: tl $s 1=s 1$.

Hypothesis loop2: $\mathrm{tl}$ s2 = s2.

The proof of the principle includes a choice of $R$, so that we no longer need to make such choices thereafter.

Theorem stream_eq_loop : stream_eq $s 1$ s2.

apply (stream_eq_coind (fun $\left.s 1^{\prime} s 2^{\prime} \Rightarrow s 1^{\prime}=s 1 \wedge s 2^{\prime}=s 2\right)$ ); crush. Qed.

End stream_eq_loop.

Theorem ones_eq"' : stream_eq ones ones'.

apply stream_eq_loop; crush.

Qed.

Let us put stream_eq_coind through its paces a bit more, considering two different ways to compute infinite streams of all factorial values. First, we import the fact factorial function from the standard library.

Require Import Arith.

Print fact.

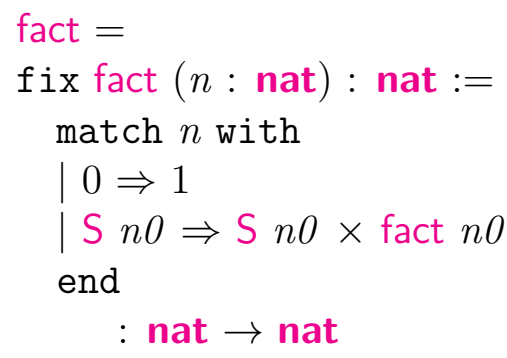

The simplest way to compute the factorial stream involves calling fact afresh at each position.

CoFixpoint fact_slow' ( $n$ : nat) $:=$ Cons (fact $n$ ) (fact_slow' $(\mathrm{S} n)$ ).

Definition fact_slow := fact_slow' 1 .

A more clever, optimized method maintains an accumulator of the previous factorial, so that each new entry can be computed with a single multiplication.

CoFixpoint fact_iter' (cur acc : nat) := Cons acc (fact_iter' (S cur) $($ acc $\times$ cur $)$ ). Definition fact_iter := fact_iter' 21 .

We can verify that the streams are equal up to particular finite bounds.

Eval simpl in approx fact_iter 5 .

$=1:: 2:: 6:: 24:: 120$ : 2 nil

: list nat

Eval simpl in approx fact_slow 5.

$=1:: 2:: 6:: 24:: 120$ :: nil 


\section{: list nat}

Now, to prove that the two versions are equivalent, it is helpful to prove (and add as a proof hint) a quick lemma about the computational behavior of fact. (I intentionally skip explaining its proof at this point.)

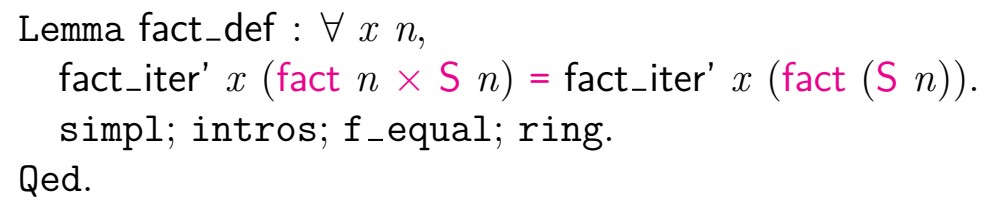

Hint Resolve fact_def.

With the hint added, it is easy to prove an auxiliary lemma relating fact_iter' and fact_slow'. The key bit of ingenuity is introduction of an existential quantifier for the shared parameter $n$.

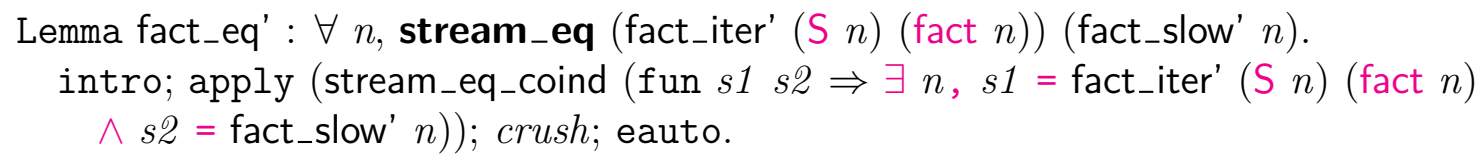
Qed.

The final theorem is a direct corollary of fact_eq'.

Theorem fact_eq : stream_eq fact_iter fact_slow. apply fact_eq'. Qed.

As in the case of ones_eq', we may be unsatisfied that we needed to write down a choice of $R$ that seems to duplicate information already present in a lemma statement. We can facilitate a simpler proof by defining a co-induction principle specialized to goals that begin with single universal quantifiers, and the strategy can be extended in a straightforward way to principles for other counts of quantifiers. (Our stream_eq_loop principle is effectively the instantiation of this technique to zero quantifiers.)

\section{Section stream_eq_onequant.}

Variables $A B$ : Type.

We have the types $A$, the domain of the one quantifier; and $B$, the type of data found in the streams.

Variables $f g: A \rightarrow$ stream $B$.

The two streams we compare must be of the forms $f x$ and $g x$, for some shared $x$. Note that this falls out naturally when $x$ is a shared universally quantified variable in a lemma statement.

Hypothesis Cons_case_hd: $\forall x$, hd $(f x)=$ hd $(g x)$.

Hypothesis Cons_case_tl: $\forall x, \exists y, \mathrm{tl}(f x)=f y \wedge \mathrm{tl}(g x)=g y$.

These conditions are inspired by the bisimulation requirements, with a more general version of the $R$ choice we made for fact_eq' inlined into the hypotheses of stream_eq_coind.

Theorem stream_eq_onequant : $\forall x$, stream_eq $(f x)(g x)$. 
intro; apply (stream_eq_coind (fun $s 1$ s2 $\Rightarrow \exists x, s 1=f x \wedge s \mathscr{2}=g x$ )); crush; eauto. Qed.

End stream_eq_onequant.

Lemma fact_eq" : $\forall n$, stream_eq (fact_iter' (S $n)$ (fact $n)$ ) (fact_slow' $n$ ).

apply stream_eq_onequant; crush; eauto.

Qed.

We have arrived at one of our customary automated proofs, thanks to the new principle.

\subsection{Simple Modeling of Non-Terminating Programs}

We close the chapter with a quick motivating example for more complex uses of co-inductive types. We will define a co-inductive semantics for a simple imperative programming language and use that semantics to prove the correctness of a trivial optimization that removes spurious additions by 0 . We follow the technique of co-inductive big-step operational semantics [20].

We define a suggestive synonym for nat, as we will consider programs over infinitely many variables, represented as nats.

Definition var $:=$ nat.

We define a type vars of maps from variables to values. To define a function set for setting a variable's value in a map, we use the standard library function beq_nat for comparing natural numbers.

Definition vars $:=$ var $\rightarrow$ nat.

Definition set (vs: vars) ( $v:$ var) ( $n:$ nat) : vars :=

fun $v^{\prime} \Rightarrow$ if beq_nat $v v^{\prime}$ then $n$ else $v s v^{\prime}$.

We define a simple arithmetic expression language with variables, and we give it a semantics via an interpreter.

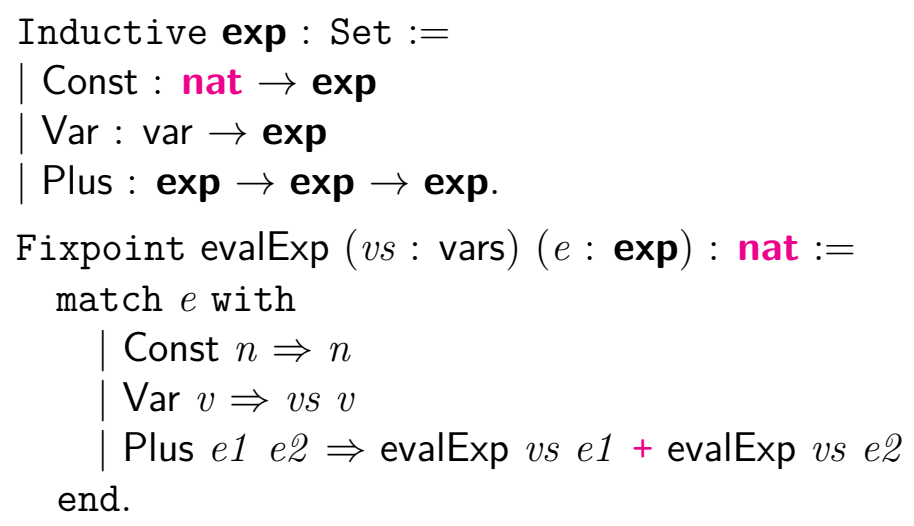

Finally, we define a language of commands. It includes variable assignment, sequencing, and a while form that repeats as long as its test expression evaluates to a nonzero value.

Inductive cmd : Set :=

| Assign : var $\rightarrow \exp \rightarrow$ cmd 


\section{Seq : cmd $\rightarrow$ cmd $\rightarrow$ cmd}

While : $\exp \rightarrow$ cmd $\rightarrow$ cmd.

We could define an inductive relation to characterize the results of command evaluation. However, such a relation would not capture nonterminating executions. With a co-inductive relation, we can capture both cases. The parameters of the relation are an initial state, a command, and a final state. A program that does not terminate in a particular initial state is related to any final state. For more realistic languages than this one, it is often possible for programs to crash, in which case a semantics would generally relate their executions to no final states; so relating safely non-terminating programs to all final states provides a crucial distinction.

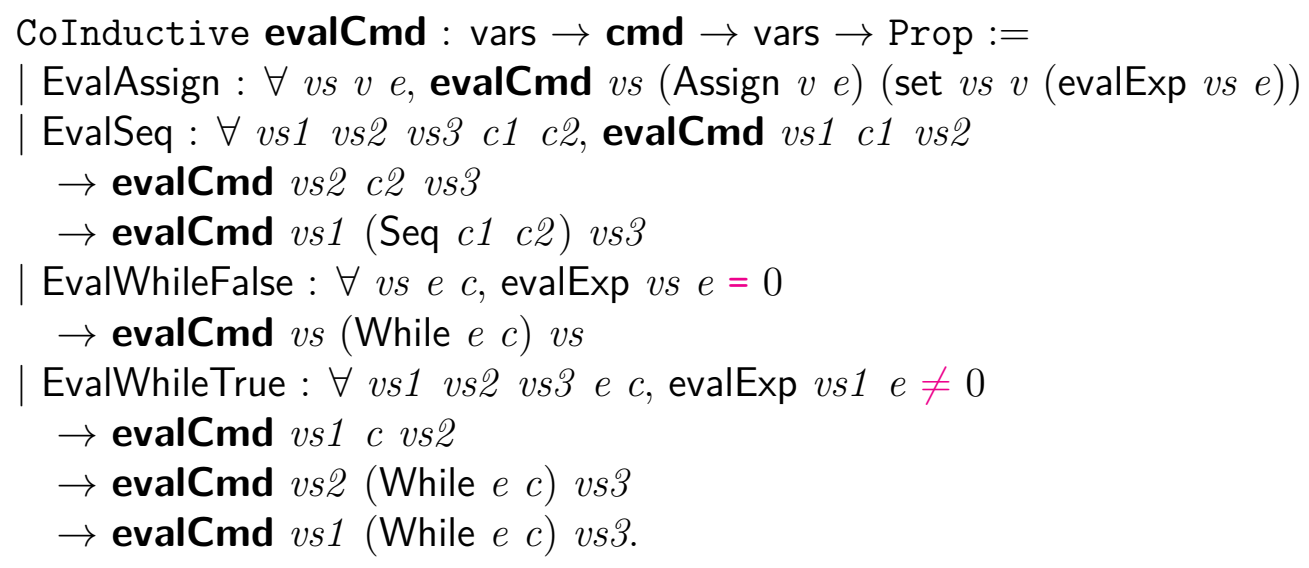

Having learned our lesson in the last section, before proceeding, we build a co-induction principle for evalCmd.

\section{Section evalCmd_coind.}

Variable $R:$ vars $\rightarrow$ cmd $\rightarrow$ vars $\rightarrow$ Prop.

Hypothesis AssignCase : $\forall$ vs1 vs2 $v$ e, $R$ vs1 (Assign $v e$ ) vs2

$\rightarrow v s 2=\operatorname{set} v s 1 \quad v(\operatorname{evalExp} v s 1 e)$.

Hypothesis SeqCase : $\forall$ vs 1 vs3 c1 c2, $R$ vs1 (Seq c1 c2) vs3

$\rightarrow \exists v s_{2}, R$ vs1 c1 vs2 $\wedge R$ vs2 c2 vs3.

Hypothesis WhileCase : $\forall$ vs1 vs3 e c, $R$ vs1 (While e c) vs3

$\rightarrow($ evalExp vs1 $e=0 \wedge v s 3=v s 1)$

$\vee \exists v s 2$, evalExp vs1 $e \neq 0 \wedge R$ vs1 c vs2 $\wedge R$ vs2 (While e c) vs3.

The proof is routine. We make use of a form of destruct that takes an intro pattern in an as clause. These patterns control how deeply we break apart the components of an inductive value, and we refer the reader to the Coq manual for more details.

Theorem evalCmd_coind : $\forall$ vs1 c vs2, $R$ vs1 $c$ vs2 $\rightarrow$ evalCmd vs1 c vs2.

cofix evalCmd_coind; intros; destruct $c$.

rewrite (AssignCase $H)$; constructor.

destruct (SeqCase $H$ ) as [? [? ?]]; econstructor; eauto.

destruct (WhileCase $H$ ) as [[? ?] | [? [? [? ?]]]]; subst; econstructor; eauto. 
Qed.

End evalCmd_coind.

Now that we have a co-induction principle, we should use it to prove something! Our example is a trivial program optimizer that finds places to replace $0+e$ with $e$.

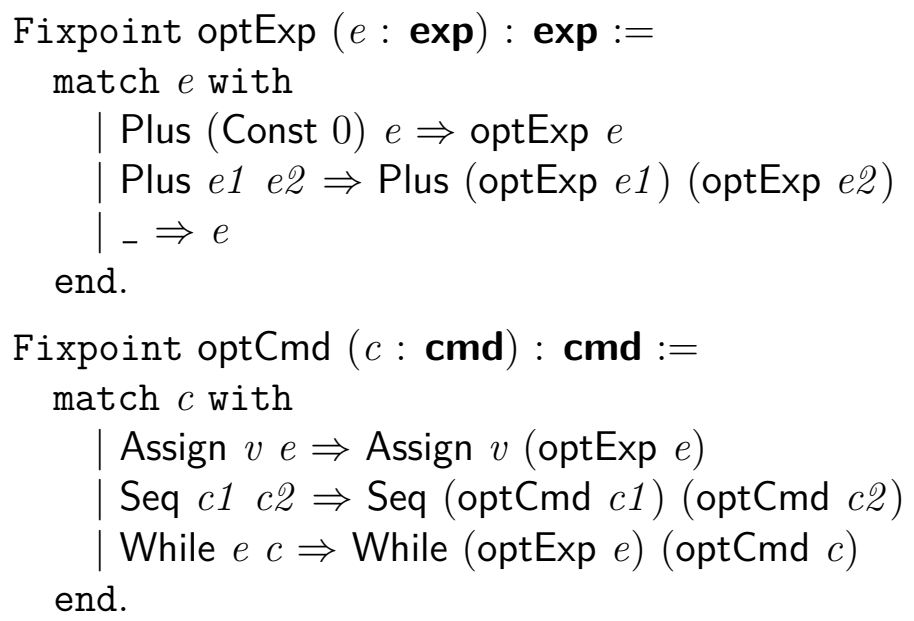

Before proving correctness of optCmd, we prove a lemma about optExp. This is where we have to do the most work, choosing pattern match opportunities automatically.

Lemma optExp_correct : $\forall$ vs e, evalExp vs (optExp $e)=\operatorname{evalExp}$ vs $e$.

induction $e$; crush;

repeat (match goal with

$$
\begin{aligned}
& \quad \mid\left[\vdash \text { context }\left[\text { match ? E with Const }{ }_{-} \Rightarrow-\mid{ }_{-} \Rightarrow \text { - end }\right]\right] \Rightarrow \operatorname{destruct~} E \\
& \mid\left[\vdash \text { context }\left[\text { match ? E with } O \Rightarrow{ }_{-} \mid S_{-} \Rightarrow{ }_{-} \text {end }\right]\right] \Rightarrow \operatorname{destruct~} E \\
& \text { end; crush }) \text {. }
\end{aligned}
$$

Qed.

Hint Rewrite optExp_correct.

The final theorem is easy to establish, using our co-induction principle and a bit of Ltac smarts that we leave unexplained for now. Curious readers can consult the Coq manual, or wait for the later chapters of this book about proof automation. At a high level, we show inclusions between behaviors, going in both directions between original and optimized programs.

Ltac finisher := match goal with

$$
\begin{aligned}
& \quad \mid\left[H \text { : evalCmd } \ldots+\vdash{ }_{-}\right] \Rightarrow((\text { inversion } H ;[]) \\
& \quad \|(\text { inversion } H ;[\mid])) \text {; subst } \\
& \text { end; crush; eauto } 10 \text {. }
\end{aligned}
$$

Lemma optCmd_correct1 : $\forall$ vs1 c vs2, evalCmd vs1 c vs2

$\rightarrow$ evalCmd vs1 (optCmd c) vs2.

intros; apply (evalCmd_coind (fun vs1 c'vs2 $\Rightarrow \exists c$, evalCmd vs1 c vs2

$\wedge c^{\prime}=\operatorname{opt}(\mathrm{md} c)$ ); eauto; crush;

match goal with 
| [H: $:_{-}=$optCmd ?E $\vdash$ - ] destruct $E$; simpl in *; discriminate

II injection $H$; intros; subst

end; finisher.

Qed.

Lemma optCmd_correct2 : $\forall$ vs1 c vs2, evalCmd vs1 (optCmd c) vs2

$\rightarrow$ evalCmd vs1 c vs2.

intros; apply (evalCmd_coind (fun vs1 c vs2 $\Rightarrow$ evalCmd vs1 (optCmd c) vs2)); crush; finisher.

Qed.

Theorem optCmd_correct : $\forall$ vs1 c vs2, evalCmd vs1 (optCmd c) vs2

$\leftrightarrow$ evalCmd vs1 c vs2.

intuition; apply optCmd_correct1 || apply optCmd_correct2; assumption.

Qed.

In this form, the theorem tells us that the optimizer preserves observable behavior of both terminating and nonterminating programs, but we did not have to do more work than for the case of terminating programs alone. We merely took the natural inductive definition for terminating executions, made it co-inductive, and applied the appropriate co-induction principle. Curious readers might experiment with adding command constructs like if; the same proof script should continue working, after the co-induction principle is extended to the new evaluation rules. 


\section{Part II}

\section{Programming with Dependent Types}




\section{Chapter 6}

\section{Subset Types and Variations}

So far, we have seen many examples of what we might call "classical program verification." We write programs, write their specifications, and then prove that the programs satisfy their specifications. The programs that we have written in Coq have been normal functional programs that we could just as well have written in Haskell or ML. In this chapter, we start investigating uses of dependent types to integrate programming, specification, and proving into a single phase. The techniques we will learn make it possible to reduce the cost of program verification dramatically.

\subsection{Introducing Subset Types}

Let us consider several ways of implementing the natural number predecessor function. We start by displaying the definition from the standard library:

Print pred.

pred $=$ fun $n:$ nat $\Rightarrow$ match $n$ with

$$
\begin{aligned}
& \mid 0 \Rightarrow 0 \\
& \mid \mathrm{S} u \Rightarrow u \\
& \text { end }
\end{aligned}
$$

$:$ nat $\rightarrow$ nat

We can use a new command, Extraction, to produce an OCaml version of this function. Extraction pred.

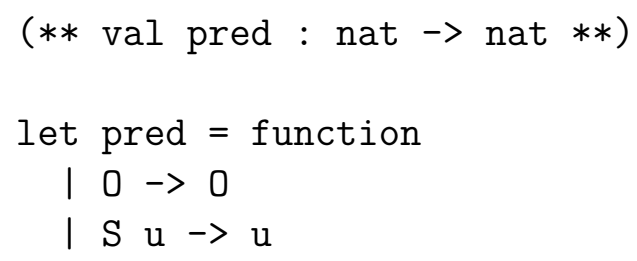


Returning 0 as the predecessor of 0 can come across as somewhat of a hack. In some situations, we might like to be sure that we never try to take the predecessor of 0 . We can enforce this by giving pred a stronger, dependent type.

\section{Lemma zgtz : $0>0 \rightarrow$ False.}

crush.

Qed.

Definition pred_strong1 $(n:$ nat $): n>0 \rightarrow$ nat $:=$ match $n$ with

$\mathrm{O} \Rightarrow$ fun $p f: 0>0 \Rightarrow$ match zgtz $p f$ with end

$\mathrm{S} n^{\prime} \Rightarrow \mathrm{fun}_{-} \Rightarrow n^{\prime}$

end.

We expand the type of pred to include a proof that its argument $n$ is greater than 0 . When $n$ is 0 , we use the proof to derive a contradiction, which we can use to build a value of any type via a vacuous pattern match. When $n$ is a successor, we have no need for the proof and just return the answer. The proof argument can be said to have a dependent type, because its type depends on the value of the argument $n$.

Coq's Eval command can execute particular invocations of pred_strong1 just as easily as it can execute more traditional functional programs. Note that Coq has decided that argument $n$ of pred_strong1 can be made implicit, since it can be deduced from the type of the second argument, so we need not write $n$ in function calls.

Theorem two_gt0 : $2>0$.

crush.

Qed.

Eval compute in pred_strong1 two_gt0.

$$
=1
$$$$
\text { : nat }
$$

One aspect in particular of the definition of pred_strong1 may be surprising. We took advantage of Definition's syntactic sugar for defining function arguments in the case of $n$, but we bound the proofs later with explicit fun expressions. Let us see what happens if we write this function in the way that at first seems most natural.

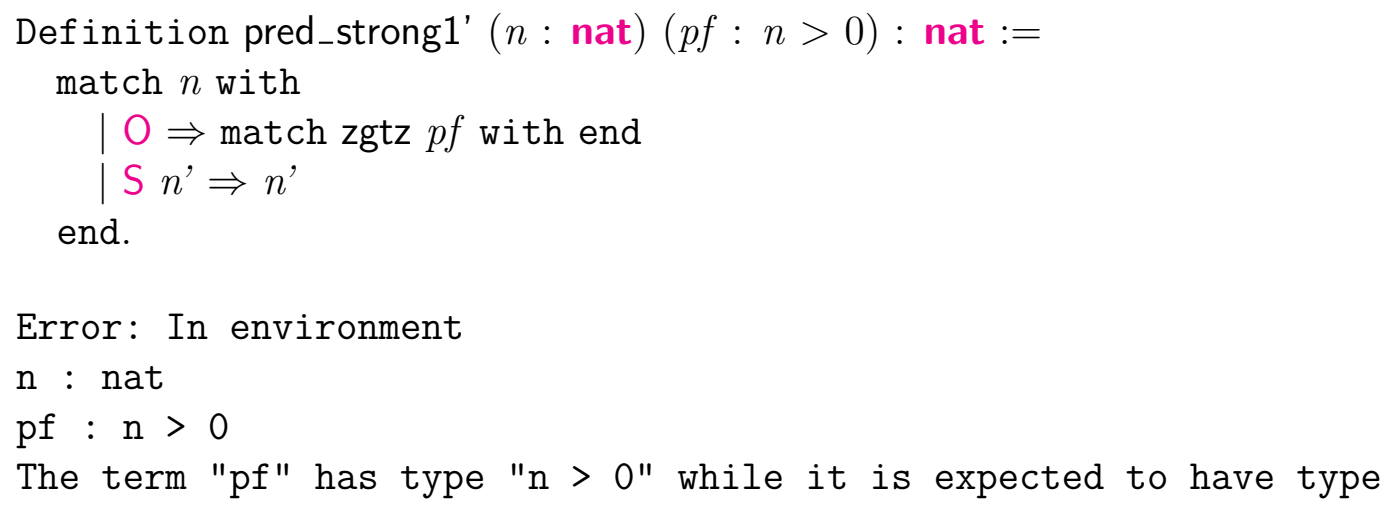


$" 0>0 "$

The term zgtz of fails to type-check. Somehow the type checker has failed to take into account information that follows from which match branch that term appears in. The problem is that, by default, match does not let us use such implied information. To get refined typing, we must always rely on match annotations, either written explicitly or inferred.

In this case, we must use a return annotation to declare the relationship between the value of the match discriminee and the type of the result. There is no annotation that lets us declare a relationship between the discriminee and the type of a variable that is already in scope; hence, we delay the binding of $p f$, so that we can use the return annotation to express the needed relationship.

We are lucky that Coq's heuristics infer the return clause (specifically, return $n>0 \rightarrow$ nat) for us in the definition of pred_strong1, leading to the following elaborated code:

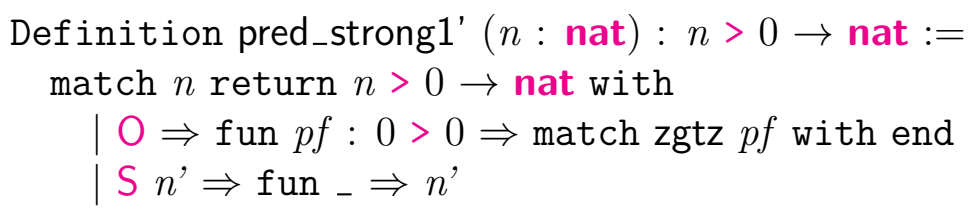

end.

By making explicit the functional relationship between value $n$ and the result type of the match, we guide Coq toward proper type checking. The clause for this example follows by simple copying of the original annotation on the definition. In general, however, the match annotation inference problem is undecidable. The known undecidable problem of higher-order unification [15] reduces to the match type inference problem. Over time, Coq is enhanced with more and more heuristics to get around this problem, but there must always exist matches whose types Coq cannot infer without annotations.

Let us now take a look at the OCaml code Coq generates for pred_strong1.

Extraction pred_strong1.

(** val pred_strong1 : nat $\rightarrow$ nat $* *$ )

let pred_strong1 $=$ function

| $0 \rightarrow$ assert false ( $*$ absurd case $*$ )

| $\mathrm{S} \mathrm{n}^{\prime} \rightarrow \mathrm{n}^{\prime}$

The proof argument has disappeared! We get exactly the OCaml code we would have written manually. This is our first demonstration of the main technically interesting feature of Coq program extraction: proofs are erased systematically.

We can reimplement our dependently typed pred based on subset types, defined in the standard library with the type family sig.

Print sig.

Inductive sig $(A:$ Type $)(P: A \rightarrow$ Prop) : Type $:=$ 


$$
\text { exist : } \forall x: A, P x \rightarrow \operatorname{sig} P
$$

The family sig is a Curry-Howard twin of ex, except that sig is in Type, while ex is in Prop. That means that sig values can survive extraction, while ex proofs will always be erased. The actual details of extraction of sigs are more subtle, as we will see shortly.

We rewrite pred_strong1, using some syntactic sugar for subset types.

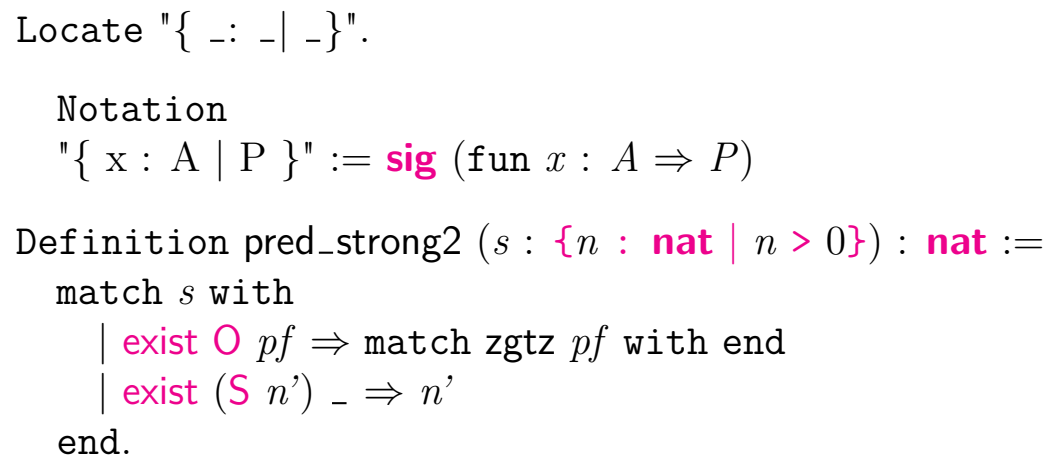

To build a value of a subset type, we use the exist constructor, and the details of how to do that follow from the output of our earlier Print sig command, where we elided the extra information that parameter $A$ is implicit. We need an extra - here and not in the definition of pred_strong2 because parameters of inductive types (like the predicate $P$ for sig) are not mentioned in pattern matching, but are mentioned in construction of terms (if they are not marked as implicit arguments).

Eval compute in pred_strong2 (exist _ 2 two_gt0).

$$
\begin{aligned}
& =1 \\
& \text { : nat }
\end{aligned}
$$

Extraction pred_strong2.

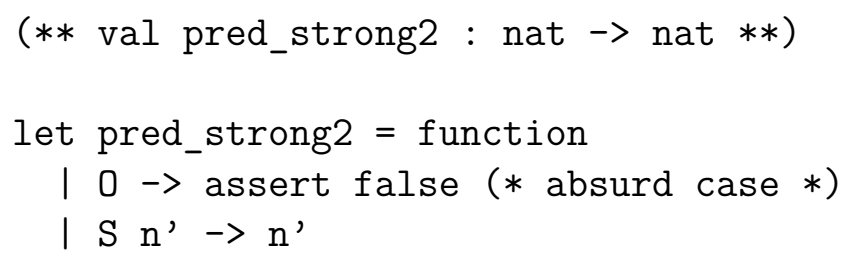

We arrive at the same OCaml code as was extracted from pred_strong1, which may seem surprising at first. The reason is that a value of sig is a pair of two pieces, a value and a proof about it. Extraction erases the proof, which reduces the constructor exist of sig to taking just a single argument. An optimization eliminates uses of datatypes with single constructors taking single arguments, and we arrive back where we started.

We can continue on in the process of refining pred's type. Let us change its result type to capture that the output is really the predecessor of the input.

Definition pred_strong3 $(s:\{n:$ nat $\mid n>0\}):\{m:$ nat $\mid$ proj1_sig $s=\mathrm{S} m\}:=$ match $s$ return $\{m$ : nat $\mid$ proj1_sig $s=\mathrm{S} m\}$ with 


$$
\begin{aligned}
& \mid \text { exist } 0 p f \Rightarrow \text { match zgtz } p f \text { with end } \\
& \mid \text { exist }(\mathrm{S} n \text { ') } p f \Rightarrow \text { exist__n' (eq_refl_) } \\
& \text { end. }
\end{aligned}
$$

Eval compute in pred_strong3 (exist _ 2 two_gt0).

$$
\begin{aligned}
& =\text { exist (fun } m: \text { nat } \Rightarrow 2=\mathrm{S} m) 1 \text { (eq_refl } 2) \\
& :\{m: \text { nat } \mid \text { proj1_sig (exist (lt } 0) 2 \text { two_gt0) }=\mathrm{S} m\}
\end{aligned}
$$

A value in a subset type can be thought of as a dependent pair (or sigma type) of a base value and a proof about it. The function proj1_sig extracts the first component of the pair. It turns out that we need to include an explicit return clause here, since Coq's heuristics are not smart enough to propagate the result type that we wrote earlier.

By now, the reader is probably ready to believe that the new pred_strong leads to the same OCaml code as we have seen several times so far, and Coq does not disappoint.

\section{Extraction pred_strong3.}

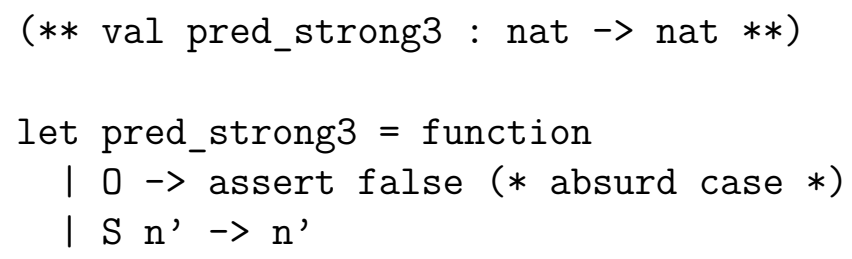

We have managed to reach a type that is, in a formal sense, the most expressive possible for pred. Any other implementation of the same type must have the same input-output behavior. However, there is still room for improvement in making this kind of code easier to write. Here is a version that takes advantage of tactic-based theorem proving. We switch back to passing a separate proof argument instead of using a subset type for the function's input, because this leads to cleaner code. (Recall that False_rec is the Set-level induction principle for False, which can be used to produce a value in any Set given a proof of False.)

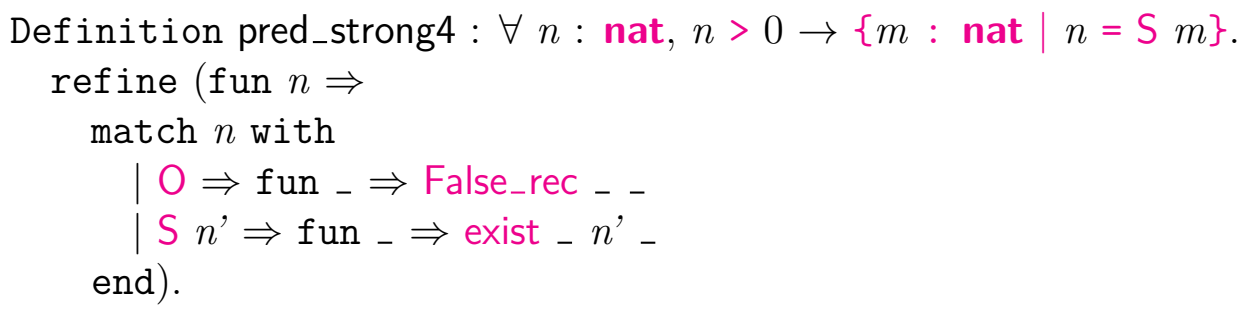

We build pred_strong4 using tactic-based proving, beginning with a Definition command that ends in a period before a definition is given. Such a command enters the interactive proving mode, with the type given for the new identifier as our proof goal. It may seem strange to change perspective so implicitly between programming and proving, but recall that programs and proofs are two sides of the same coin in Coq, thanks to the Curry-Howard correspondence.

We do most of the work with the refine tactic, to which we pass a partial "proof" of the type we are trying to prove. There may be some pieces left to fill in, indicated by 
underscores. Any underscore that Coq cannot reconstruct with type inference is added as a proof subgoal. In this case, we have two subgoals:

2 subgoals

$n:$ nat
$-: 0>0$
$============================1$

False

subgoal 2 is

$\mathrm{S} n^{\prime}=\mathrm{S} n^{\prime}$

We can see that the first subgoal comes from the second underscore passed to False_rec, and the second subgoal comes from the second underscore passed to exist. In the first case, we see that, though we bound the proof variable with an underscore, it is still available in our proof context. It is hard to refer to underscore-named variables in manual proofs, but automation makes short work of them. Both subgoals are easy to discharge that way, so let us back up and ask to prove all subgoals automatically.

Undo.

refine (fun $n \Rightarrow$

match $n$ with

$\mathrm{O} \Rightarrow$ fun $_{-} \Rightarrow$ False_rec _ -

| S $n^{\prime} \Rightarrow$ fun _ $\Rightarrow$ exist _ n' -

end); crush.

Defined.

We end the "proof" with Defined instead of Qed, so that the definition we constructed remains visible. This contrasts to the case of ending a proof with Qed, where the details of the proof are hidden afterward. (More formally, Defined marks an identifier as transparent, allowing it to be unfolded; while Qed marks an identifier as opaque, preventing unfolding.) Let us see what our proof script constructed.

Print pred_strong4.

pred_strong4 $=$

fun $n$ : nat $\Rightarrow$

match $n$ as $n 0$ return $(n 0>0 \rightarrow\{m:$ nat $\mid n 0=\mathrm{S} m\})$ with

$0 \Rightarrow$

fun $_{-}: 0>0 \Rightarrow$

False_rec $\{m$ : nat $\mid 0=\mathrm{S} m\}$

(Bool.diff_false_true

(Bool.absurd_eq_true false

(Bool.diff_false_true 
(Bool.absurd_eq_true false (pred_strong__subproof $\left.\left.\left.n_{-}\right)\right)\right)$))

| S $n^{\prime} \Rightarrow$

fun $_{-}: \mathrm{S}^{\prime}>0 \Rightarrow$

exist (fun $m$ : nat $\left.\Rightarrow \mathrm{S} n^{\prime}=\mathrm{S} m\right) n^{\prime}\left(\right.$ eq_refl $\left(\mathrm{S} n^{\prime}\right)$ )

end

$: \forall n:$ nat, $n>0 \rightarrow\{m:$ nat $\mid n=\mathrm{S} m\}$

We see the code we entered, with some proofs filled in. The first proof obligation, the second argument to False_rec, is filled in with a nasty-looking proof term that we can be glad we did not enter by hand. The second proof obligation is a simple reflexivity proof.

Eval compute in pred_strong4 two_gt0.

= exist (fun $m$ : nat $\Rightarrow 2=\mathrm{S} m$ ) 1 (eq_refl 2)

$:\{m:$ nat $\mid 2=\mathrm{S} m\}$

A tactic modifier called abstract can be helpful for producing shorter terms, by automatically abstracting subgoals into named lemmas.

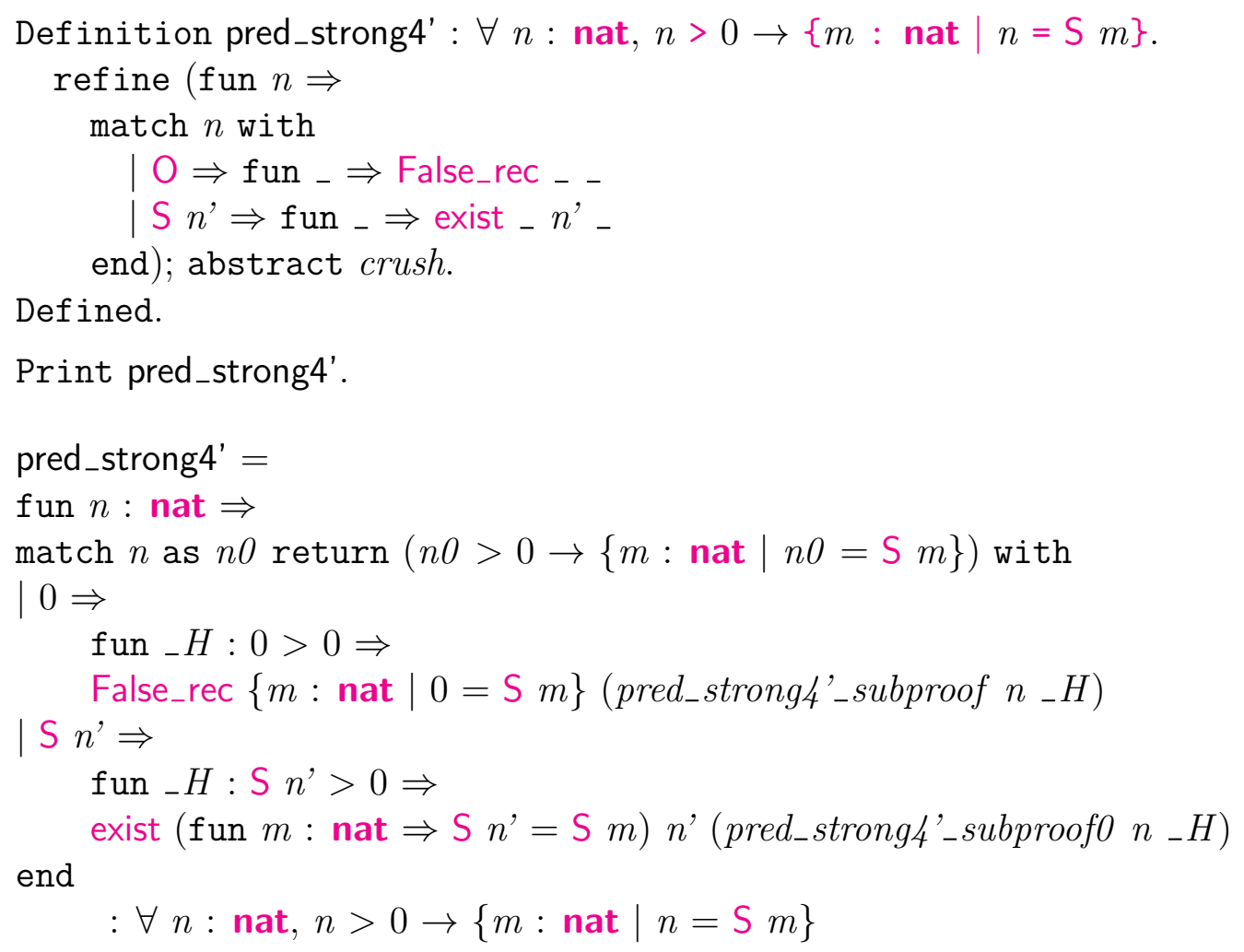

We are almost done with the ideal implementation of dependent predecessor. We can use Coq's syntax extension facility to arrive at code with almost no complexity beyond a Haskell or ML program with a complete specification in a comment. In this book, we will not dwell on the details of syntax extensions; the Coq manual gives a straightforward introduction to them.

Notation "!" := (False_rec _ - ). 


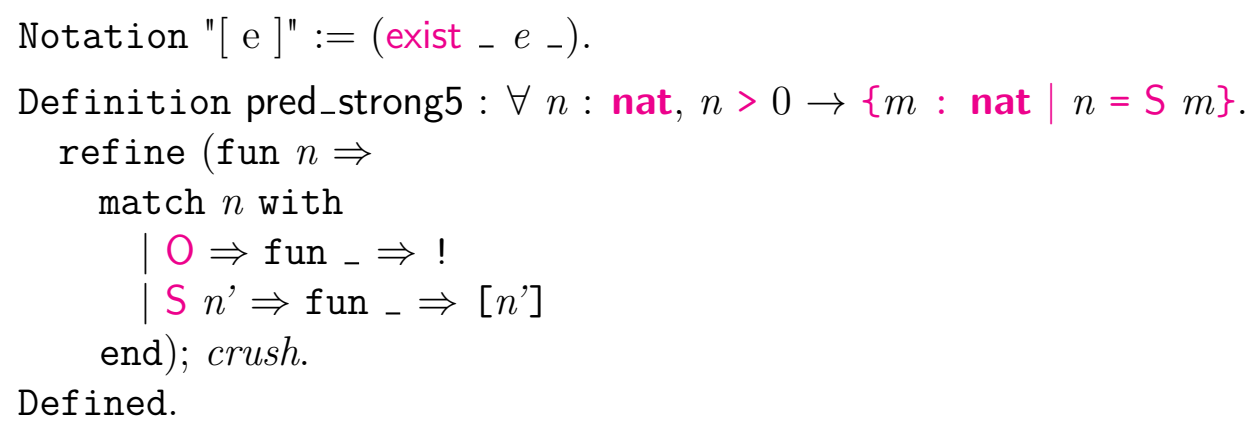

By default, notations are also used in pretty-printing terms, including results of evaluation.

Eval compute in pred_strong5 two_gt0.

$$
\begin{aligned}
& =[1] \\
& :\{m: \text { nat } \mid 2=\mathrm{S} m\}
\end{aligned}
$$

One other alternative is worth demonstrating. Recent Coq versions include a facility called Program that streamlines this style of definition. Here is a complete implementation using Program.

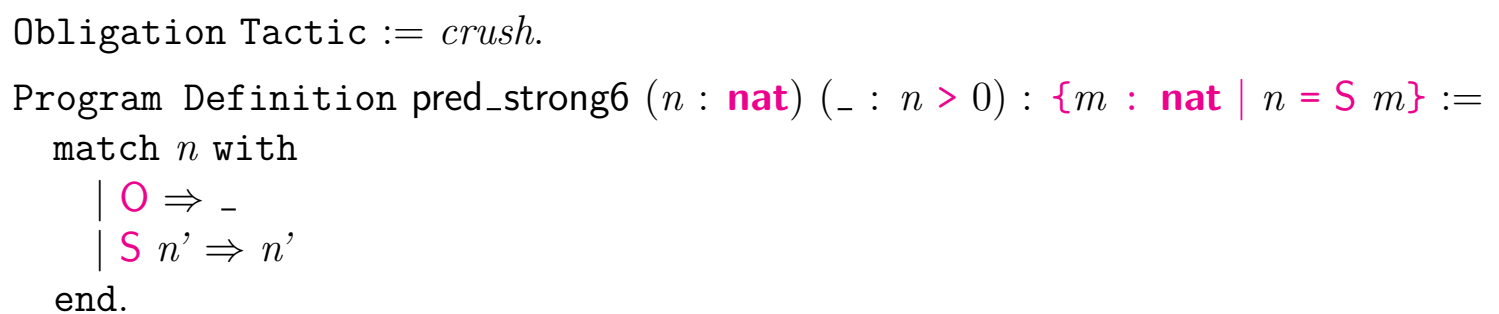

Printing the resulting definition of pred_strong6 yields a term very similar to what we built with refine. Program can save time in writing programs that use subset types. Nonetheless, ref ine is often just as effective, and refine gives more control over the form the final term takes, which can be useful when you want to prove additional theorems about your definition. Program will sometimes insert type casts that can complicate theorem proving.

Eval compute in pred_strong6 two_gt0.

$$
\begin{aligned}
& =[1] \\
& :\{m: \text { nat } \mid 2=\mathrm{S} m\}
\end{aligned}
$$

In this case, we see that the new definition yields the same computational behavior as before.

\subsection{Decidable Proposition Types}

There is another type in the standard library that captures the idea of program values that indicate which of two propositions is true.

Print sumbool. 
Inductive sumbool ( $A$ : Prop) ( $B$ : Prop) : Set $:=$

left $: A \rightarrow\{A\}+\{B\} \mid$ right $: B \rightarrow\{A\}+\{B\}$

Here, the constructors of sumbool have types written in terms of a registered notation for sumbool, such that the result type of each constructor desugars to sumbool $A B$. We can define some notations of our own to make working with sumbool more convenient.

Notation "'Yes'":=(left _ -).

Notation "No" := (right _ _).

Notation "'Reduce' $\mathrm{x} ":=$ (if $x$ then Yes else No) (at level 50).

The Reduce notation is notable because it demonstrates how if is overloaded in Coq. The if form actually works when the test expression has any two-constructor inductive type. Moreover, in the then and else branches, the appropriate constructor arguments are bound. This is important when working with sumbools, when we want to have the proof stored in the test expression available when proving the proof obligations generated in the appropriate branch.

Now we can write eq_nat_dec, which compares two natural numbers, returning either a proof of their equality or a proof of their inequality.

Definition eq_nat_dec : $\forall n m$ : nat, $\{n=m\}+\{n \neq m\}$.

refine $(\operatorname{fix} f(n m:$ nat $):\{n=m\}+\{n \neq m\}:=$

match $n, m$ with

$\mathrm{O}, \mathrm{O} \Rightarrow$ Yes

| S n', S m' $\Rightarrow$ Reduce ( $f n^{\prime} m^{\prime}$ )

${ }_{-},{ }_{-} \Rightarrow$ No

end); congruence.

Defined.

Eval compute in eq_nat_dec 22 .

$=Y e s$

$:\{2=2\}+\{2 \neq 2\}$

Eval compute in eq_nat_dec 23.

$=N_{O}$

$:\{2=3\}+\{2 \neq 3\}$

Note that the Yes and No notations are hiding proofs establishing the correctness of the outputs.

Our definition extracts to reasonable OCaml code.

Extraction eq_nat_dec.

(** val eq_nat_dec : nat $\rightarrow$ nat $\rightarrow$ sumbool $* *$ )

let $r e c$ eq_nat_dec $\mathrm{n} \mathrm{m}=$ 


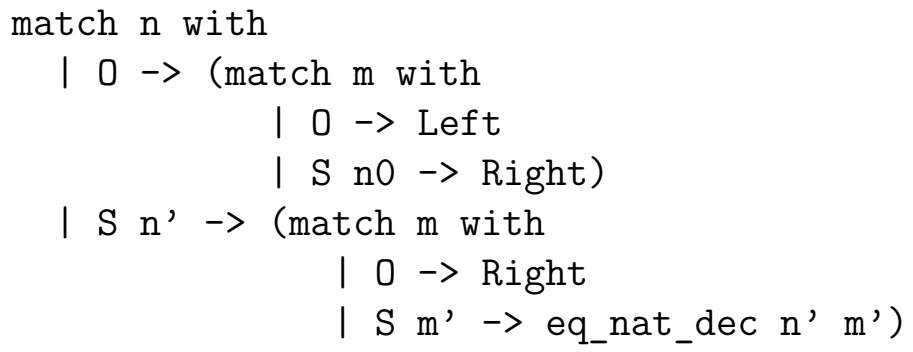

Proving this kind of decidable equality result is so common that Coq comes with a tactic for automating it.

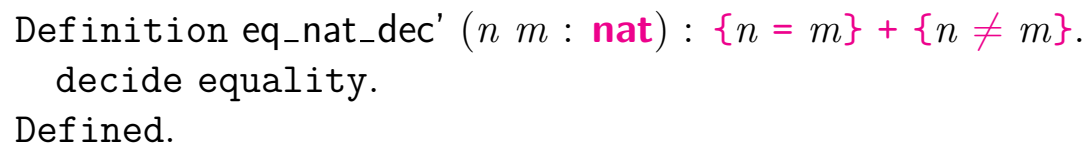

Curious readers can verify that the decide equality version extracts to the same OCaml code as our more manual version does. That OCaml code had one undesirable property, which is that it uses Left and Right constructors instead of the Boolean values built into OCaml. We can fix this, by using Coq's facility for mapping Coq inductive types to OCaml variant types.

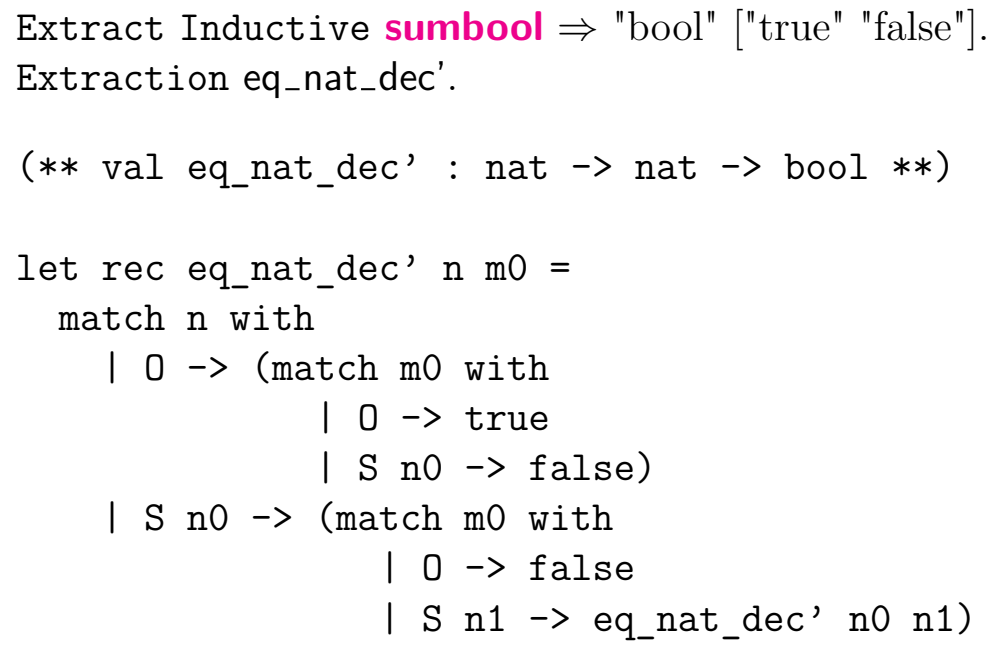

We can build "smart" versions of the usual Boolean operators and put them to good use in certified programming. For instance, here is a sumbool version of Boolean "or."

Notation "x $\| \mathrm{y}^{\text {" }}:=($ if $x$ then Yes else Reduce $y)$.

Let us use it for building a function that decides list membership. We need to assume the existence of an equality decision procedure for the type of list elements.

Section In_dec.

Variable $A$ : Set.

Variable $A_{-} e q_{-} d e c: \forall x y: A,\{x=y\}+\{x \neq y\}$.

The final function is easy to write using the techniques we have developed so far. 
Definition In_dec: $\forall(x: A)(l s:$ list $A),\{\ln x l s\}+\{\neg \ln x l s\}$. refine $(\operatorname{fix} f(x: A)(l s:$ list $A):\{\ln x l s\}+\{\neg \ln x l s\}:=$ match $l s$ with

$$
\begin{aligned}
& \mid \text { nil } \Rightarrow \text { No } \\
& x^{\prime}:: \text { ls } \Rightarrow s_{-} e q_{-} d e c x x^{\prime} \| f x l s^{\prime}
\end{aligned}
$$

end); crush.

Defined.

End In_dec.

Eval compute in In_dec eq_nat_dec 2 (1:: $2::$ nil).

$$
\begin{aligned}
& =\text { Yes } \\
& :\{\ln 2(1:: 2:: \text { nil })\}+\{\neg \ln 2(1:: 2:: \text { nil })\}
\end{aligned}
$$

Eval compute in In_dec eq_nat_dec 3 (1:: 2 : : nil).

$$
\begin{aligned}
& =N_{o} \\
& :\{\ln 3(1:: 2:: \text { nil })\}+\{\neg \ln 3(1:: 2:: \text { nil })\}
\end{aligned}
$$

The $I n_{-}$dec function has a reasonable extraction to OCaml.

Extraction In_dec.

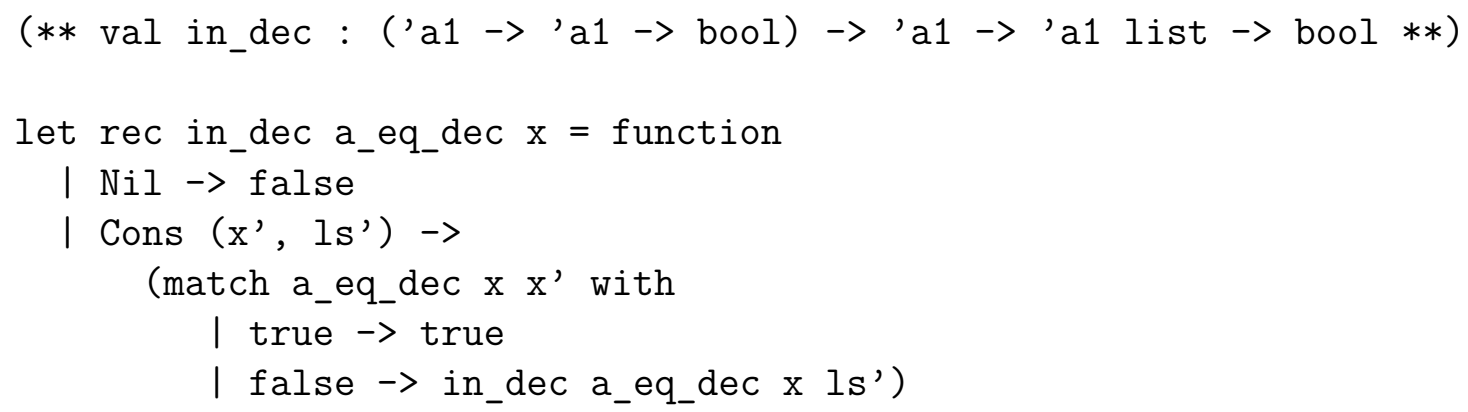

This is more or the less code for the corresponding function from the OCaml standard library.

\subsection{Partial Subset Types}

Our final implementation of dependent predecessor used a very specific argument type to ensure that execution could always complete normally. Sometimes we want to allow execution to fail, and we want a more principled way of signaling failure than returning a default value, as pred does for 0 . One approach is to define this type family maybe, which is a version of sig that allows obligation-free failure.

Inductive maybe $(A:$ Set $)(P: A \rightarrow$ Prop $):$ Set $:=$ | Unknown : maybe $P$

Found : $\forall x: A, P x \rightarrow$ maybe $P$. 
We can define some new notations, analogous to those we defined for subset types.

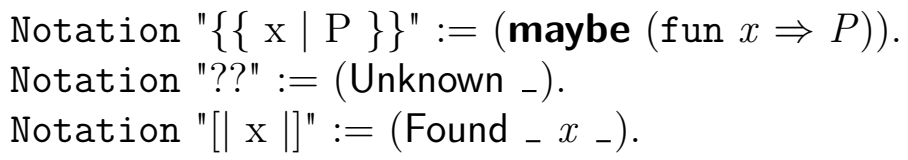

Now our next version of pred is trivial to write.

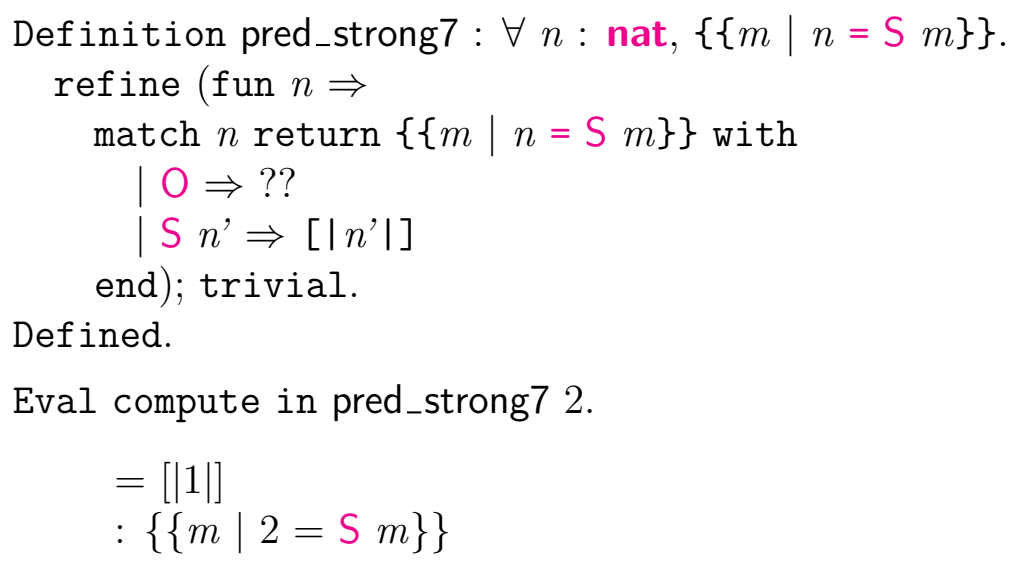

Eval compute in pred_strong7 0.

$$
\begin{aligned}
& =? ? \\
& :\{\{m \mid 0=\mathrm{S} m\}\}
\end{aligned}
$$

Because we used maybe, one valid implementation of the type we gave pred_strong7 would return ?? in every case. We can strengthen the type to rule out such vacuous implementations, and the type family sumor from the standard library provides the easiest starting point. For type $A$ and proposition $B, A+\{B\}$ desugars to sumor $A B$, whose values are either values of $A$ or proofs of $B$.

Print sumor.

Inductive sumor $(A$ : Type) $(B$ : Prop) : Type :=

inleft : $A \rightarrow A+\{B\} \mid$ inright $: B \rightarrow A+\{B\}$

We add notations for easy use of the sumor constructors. The second notation is specialized to sumors whose $A$ parameters are instantiated with regular subset types, since this is how we will use sumor below.

Notation "!!" := (inright _ -).

Notation "[|| x ||]" :=(inleft _ [x]).

Now we are ready to give the final version of possibly failing predecessor. The sumorbased type that we use is maximally expressive; any implementation of the type has the same input-output behavior.

Definition pred_strong8: $\forall n$ : nat, $\{m:$ nat $\mid n=\mathrm{S} m\}+\{n=0\}$.

refine (fun $n \Rightarrow$

match $n$ with 


$$
\begin{aligned}
& \quad \mid \mathrm{O} \Rightarrow ! ! \\
& \mid \mathrm{S} n^{\prime} \Rightarrow\left[|| n^{\prime}||\right] \\
& \text { end); trivial. } \\
& \text { Defined. } \\
& \text { Eval compute in pred_strong8 } 2 . \\
& =[|| 1||] \\
& \quad:\{m: \text { nat } \mid 2=\mathrm{S} m\}+\{2=0\} \\
& \text { Eval compute in pred_strong8 } 0 . \\
& \quad=! ! \\
& \quad:\{m: \text { nat } \mid 0=\mathrm{S} m\}+\{0=0\}
\end{aligned}
$$

As with our other maximally expressive pred function, we arrive at quite simple output values, thanks to notations.

\subsection{Monadic Notations}

We can treat maybe like a monad [45], in the same way that the Haskell Maybe type is interpreted as a failure monad. Our maybe has the wrong type to be a literal monad, but a "bind"-like notation will still be helpful. Note that the notation definition uses an ASCII $<-$, while later code uses (in this rendering) a nicer left arrow $\leftarrow$.

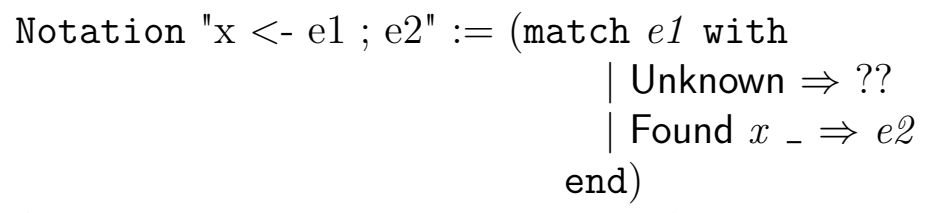

The meaning of $x \leftarrow e 1$; e2 is: First run $e 1$. If it fails to find an answer, then announce failure for our derived computation, too. If e1 does find an answer, pass that answer on to e2 to find the final result. The variable $x$ can be considered bound in $e \mathscr{2}$.

This notation is very helpful for composing richly typed procedures. For instance, here is a very simple implementation of a function to take the predecessors of two naturals at once.

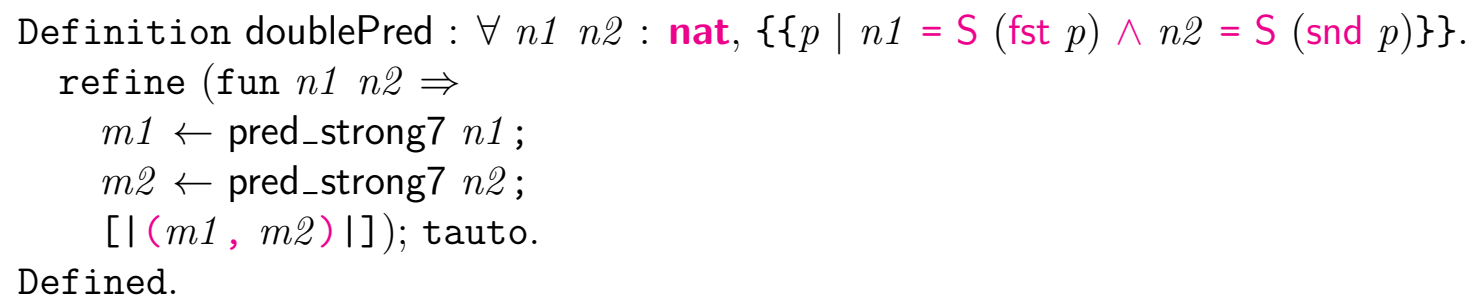

We can build a sumor version of the "bind" notation and use it to write a similarly straightforward version of this function. Again, the notation definition exposes the ASCII syntax with an operator $<--$, while the later code uses a nicer long left arrow $\longleftarrow$. 


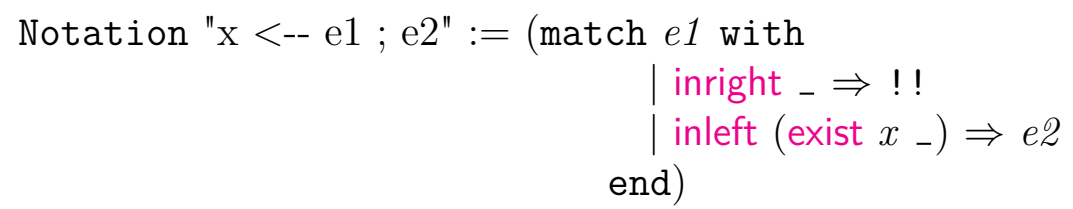

This example demonstrates how judicious selection of notations can hide complexities in the rich types of programs.

\subsection{A Type-Checking Example}

We can apply these specification types to build a certified type checker for a simple expression language.

Inductive exp : Set $:=$
$\mid$ Nat : nat $\rightarrow \exp$
$\mid$ Plus : exp $\rightarrow \exp \rightarrow \exp$
$\mid$ Bool : bool $\rightarrow \exp$
And : exp $\rightarrow \exp \rightarrow \exp$.

We define a simple language of types and its typing rules, in the style introduced in Chapter 4.

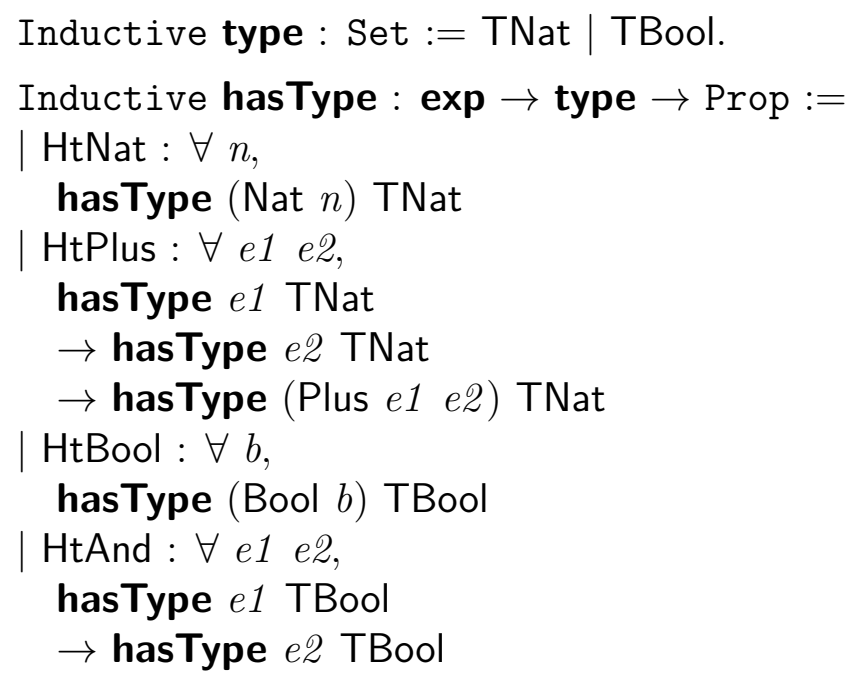


$\rightarrow$ hasType (And e1 e2) TBool.

It will be helpful to have a function for comparing two types. We build one using decide equality.

Definition eq_type_dec: $\forall t 1$ t2 : type, $\{t 1=t 2\}+\{t 1 \neq t 2\}$.

decide equality.

Defined.

Another notation complements the monadic notation for maybe that we defined earlier. Sometimes we want to include "assertions" in our procedures. That is, we want to run a decision procedure and fail if it fails; otherwise, we want to continue, with the proof that it produced made available to us. This infix notation captures that idea, for a procedure that returns an arbitrary two-constructor type.

Notation "e1 ;; e2" :=(if e1 then $e 2$ else ??)

(right associativity, at level 60).

With that notation defined, we can implement a typeCheck function, whose code is only more complex than what we would write in ML because it needs to include some extra type annotations. Every $[|e|]$ expression adds a hasType proof obligation, and crush makes short work of them when we add hasType's constructors as hints.

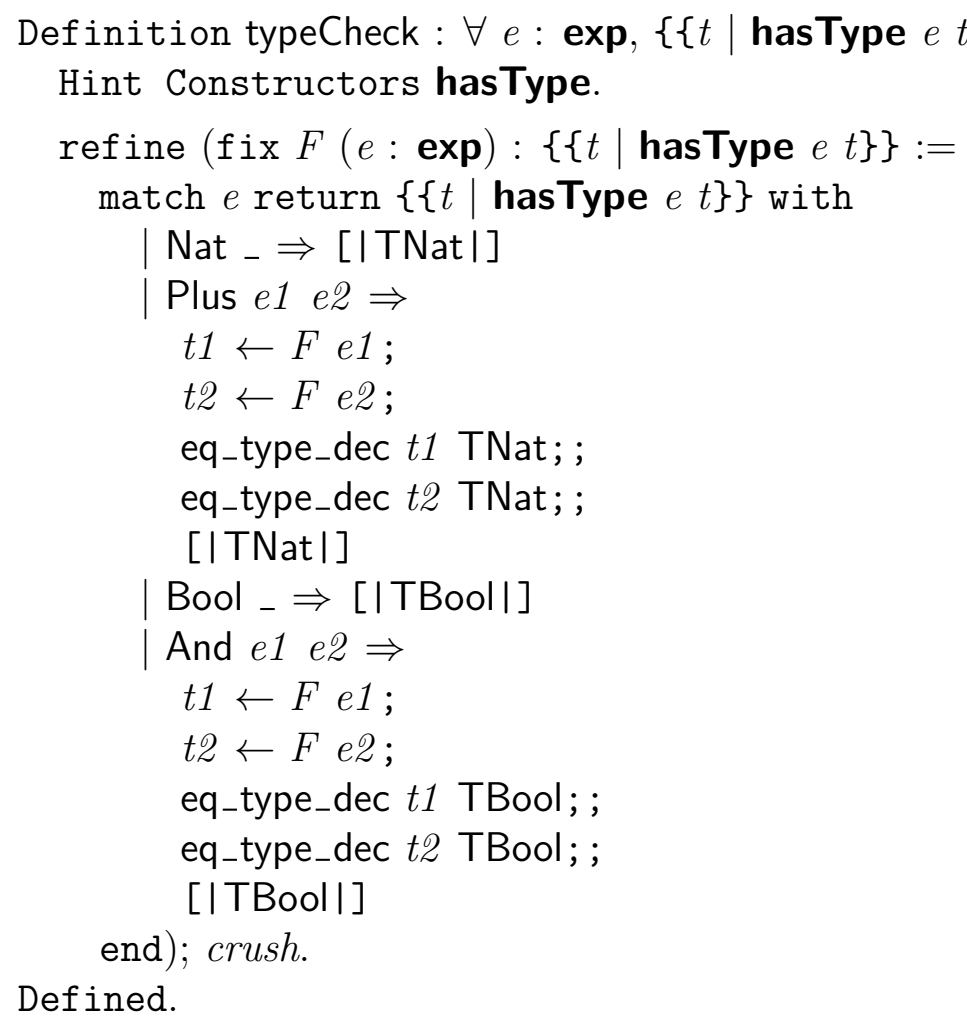

Despite manipulating proofs, our type checker is easy to run.

Eval simpl in typeCheck (Nat 0 ).

$$
=[|\mathrm{TNat}|]
$$


$:\{\{t \mid$ hasType (Nat 0$) t\}\}$

Eval simpl in typeCheck (Plus (Nat 1) (Nat 2)).

$=[|\mathrm{TNat}|]$

$:\{\{t \mid$ hasType (Plus (Nat 1) (Nat 2)) $t\}\}$

Eval simpl in typeCheck (Plus (Nat 1) (Bool false)).

$=? ?$

$:\{\{t \mid$ hasType (Plus (Nat 1) (Bool false)) $t\}\}$

The type checker also extracts to some reasonable OCaml code.

Extraction typeCheck.

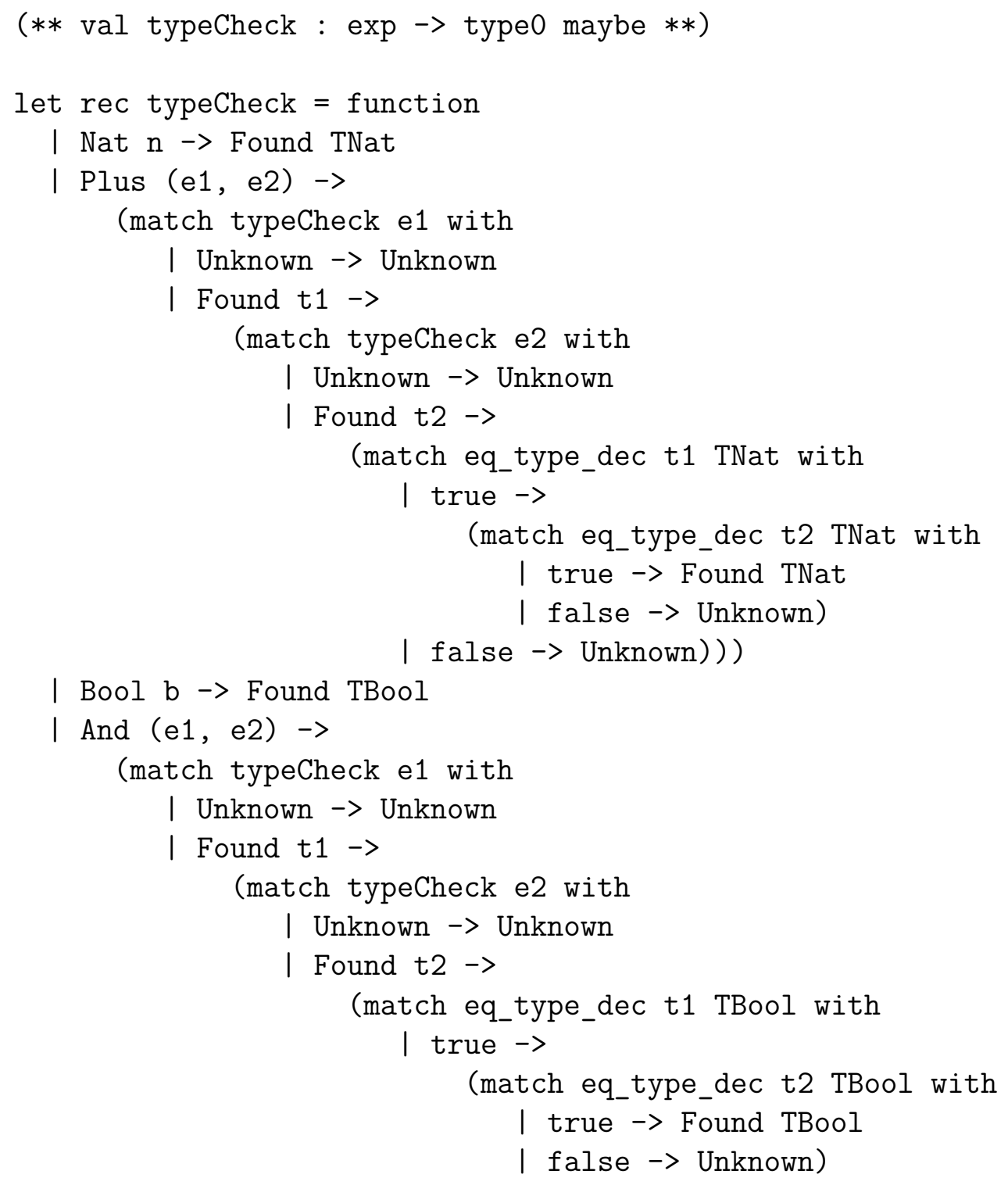




\section{( false $\rightarrow$ Unknown)))}

We can adapt this implementation to use sumor, so that we know our type-checker only fails on ill-typed inputs. First, we define an analogue to the "assertion" notation.

Notation "e1 ;; e2" :=(if e1 then e2 else !!)

(right associativity, at level 60).

Next, we prove a helpful lemma, which states that a given expression can have at most one type.

Lemma hasType_det : $\forall$ e $t 1$,

hasType $e$ t1

$\rightarrow \forall t$, hasType $e$ t2

$\rightarrow t 1=t 2$.

induction 1 ; inversion 1 ; crush.

Qed.

Now we can define the type-checker. Its type expresses that it only fails on untypable expressions.

Definition typeCheck' : $\forall e$ : exp, $\{t:$ type $\mid$ hasType $e t\}+\{\forall t, \neg$ hasType $e t\}$.

Hint Constructors hasType.

We register all of the typing rules as hints.

Hint Resolve hasType_det.

The lemma hasType_det will also be useful for proving proof obligations with contradictory contexts. Since its statement includes $\forall$-bound variables that do not appear in its conclusion, only eauto will apply this hint.

Finally, the implementation of typeCheck can be transcribed literally, simply switching notations as needed.

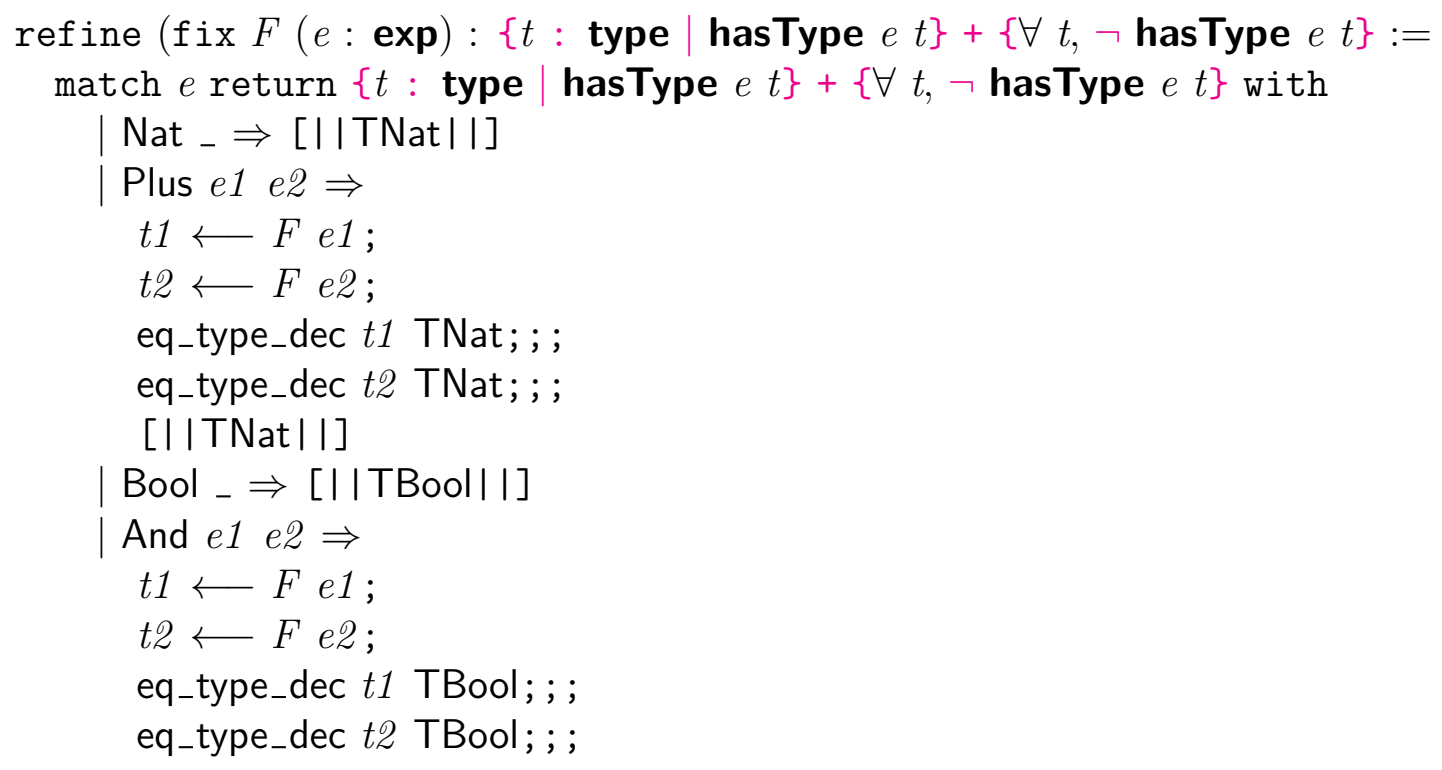




\section{[I|TBooll|]}

end); clear F; crush'tt hasType; eauto.

We clear $F$, the local name for the recursive function, to avoid strange proofs that refer to recursive calls that we never make. Such a step is usually warranted when defining a recursive function with refine. The crush variant crush' helps us by performing automatic inversion on instances of the predicates specified in its second argument. Once we throw in eauto to apply hasType_det for us, we have discharged all the subgoals.

\section{Defined.}

The short implementation here hides just how time-saving automation is. Every use of one of the notations adds a proof obligation, giving us 12 in total. Most of these obligations require multiple inversions and either uses of hasType_det or applications of hasType rules.

Our new function remains easy to test:

Eval simpl in typeCheck' (Nat 0).

$$
\begin{aligned}
= & {[\| \text { TNat } \|] } \\
: & \{t: \text { type } \mid \text { hasType }(\text { Nat } 0) t\}+ \\
& \{(\forall t: \text { type }, \neg \text { hasType }(\text { Nat } 0) t)\}
\end{aligned}
$$

Eval simpl in typeCheck' (Plus (Nat 1) (Nat 2)).

$$
\begin{aligned}
= & {[\| \text { TNat } \|] } \\
: & \{t: \text { type } \mid \text { hasType (Plus (Nat } 1)(\text { Nat } 2)) t\}+ \\
& \{(\forall t: \text { type }, \neg \text { hasType (Plus (Nat } 1)(\text { Nat } 2)) t)\}
\end{aligned}
$$

Eval simpl in typeCheck' (Plus (Nat 1) (Bool false)).

$$
\begin{aligned}
= & ! ! \\
: & \{t: \text { type } \mid \text { hasType (Plus (Nat } 1)(\text { Bool false) }) t\}+ \\
& \{(\forall t: \text { type, } \neg \text { hasType (Plus (Nat } 1)(\text { Bool false) }) t)\}
\end{aligned}
$$

The results of simplifying calls to typeCheck' look deceptively similar to the results for typeCheck, but now the types of the results provide more information. 


\section{Chapter 7}

\section{General Recursion}

Termination of all programs is a crucial property of Gallina. Non-terminating programs introduce logical inconsistency, where any theorem can be proved with an infinite loop. Coq uses a small set of conservative, syntactic criteria to check termination of all recursive definitions. These criteria are insufficient to support the natural encodings of a variety of important programming idioms. Further, since Coq makes it so convenient to encode mathematics computationally, with functional programs, we may find ourselves wanting to employ more complicated recursion in mathematical definitions.

What exactly are the conservative criteria that we run up against? For recursive definitions, recursive calls are only allowed on syntactic subterms of the original primary argument, a restriction known as primitive recursion. In fact, Coq's handling of reflexive inductive types (those defined in terms of functions returning the same type) gives a bit more flexibility than in traditional primitive recursion, but the term is still applied commonly. In Chapter 5, we saw how co-recursive definitions are checked against a syntactic guardedness condition that guarantees productivity.

Many natural recursion patterns satisfy neither condition. For instance, there is our simple running example in this chapter, merge sort. We will study three different approaches to more flexible recursion, and the latter two of the approaches will even support definitions that may fail to terminate on certain inputs, without any up-front characterization of which inputs those may be.

Before proceeding, it is important to note that the problem here is not as fundamental as it may appear. The final example of Chapter 5 demonstrated what is called a deep embedding of the syntax and semantics of a programming language. That is, we gave a mathematical definition of a language of programs and their meanings. This language clearly admitted non-termination, and we could think of writing all our sophisticated recursive functions with such explicit syntax types. However, in doing so, we forfeit our chance to take advantage of Coq's very good built-in support for reasoning about Gallina programs. We would rather use a shallow embedding, where we model informal constructs by encoding them as normal Gallina programs. Each of the three techniques of this chapter follows that style. 


\subsection{Well-Founded Recursion}

The essence of terminating recursion is that there are no infinite chains of nested recursive calls. This intuition is commonly mapped to the mathematical idea of a well-founded relation, and the associated standard technique in Coq is well-founded recursion. The syntacticsubterm relation that Coq applies by default is well-founded, but many cases demand alternate well-founded relations. To demonstrate, let us see where we get stuck on attempting a standard merge sort implementation.

Section mergeSort.

Variable $A$ : Type.

Variable le $: A \rightarrow A \rightarrow$ bool.

We have a set equipped with some "less-than-or-equal-to" test.

A standard function inserts an element into a sorted list, preserving sortedness.

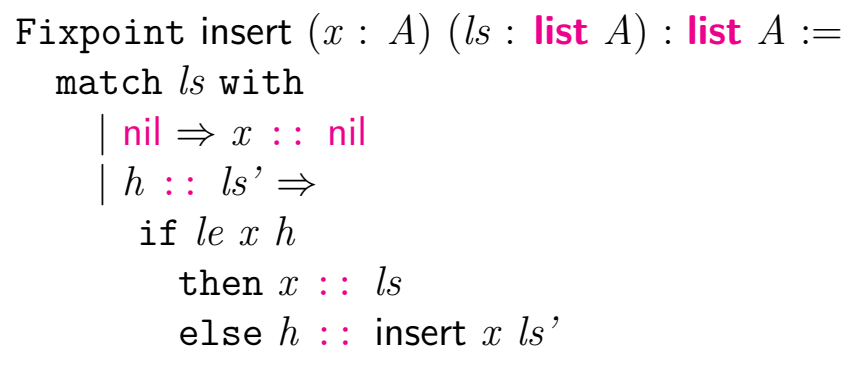

end.

We will also need a function to merge two sorted lists. (We use a less efficient implementation than usual, because the more efficient implementation already forces us to think about well-founded recursion, while here we are only interested in setting up the example of merge sort.)

Fixpoint merge $(l s 1$ lsQ : list $A)$ : list $A:=$ match $l s 1$ with

nil $\Rightarrow l s 2$

$h:: l s^{\prime} \Rightarrow$ insert $h$ (merge ls' ls2)

end.

The last helper function for classic merge sort is the one that follows, to split a list arbitrarily into two pieces of approximately equal length.

Fixpoint split $(l s:$ list $A):$ list $A \times$ list $A:=$ match $l s$ with

| nil $\Rightarrow$ (nil, nil)

$h::$ nil $\Rightarrow(h::$ nil, nil $)$

| h1 : : h2 : : ls' $\Rightarrow$

let $(l s 1, l s 2):=$ split $l s^{\prime}$ in

(h1:: ls1, h2 : : ls2)

end. 
Now, let us try to write the final sorting function, using a natural number " $\leq$ " test leb from the standard library.

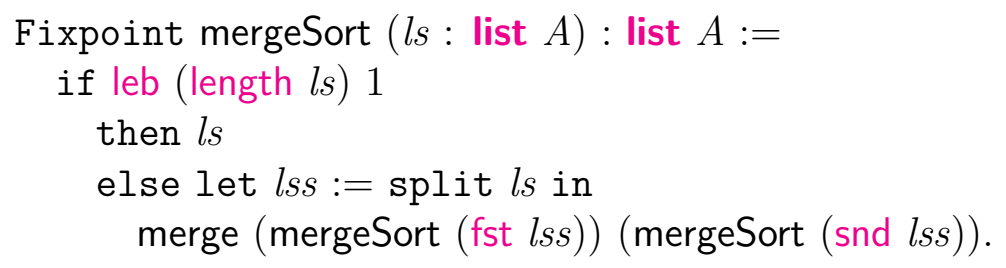

The definition is rejected for not following the simple primitive recursion criterion. In particular, it is not apparent that recursive calls to mergeSort are syntactic subterms of the original argument $l s$; indeed, they are not, yet we know this is a well-founded recursive definition.

To produce an acceptable definition, we need to choose a well-founded relation and prove that mergeSort respects it. A good starting point is an examination of how well-foundedness is formalized in the Coq standard library.

Print well_founded.

well_founded $=$

fun $(A:$ Type $)(R: A \rightarrow A \rightarrow$ Prop $) \Rightarrow \forall a: A$, Acc $R a$

The bulk of the definitional work devolves to the accessibility relation Acc, whose definition we may also examine.

Print Acc.

Inductive Acc ( $A:$ Type) $(R: A \rightarrow A \rightarrow \operatorname{Prop})(x: A):$ Prop $:=$

Acc_intro: $(\forall y: A, R y x \rightarrow$ Acc $R y) \rightarrow$ Acc $R x$

In prose, an element $x$ is accessible for a relation $R$ if every element "less than" $x$ according to $R$ is also accessible. Since Acc is defined inductively, we know that any accessibility proof involves a finite chain of invocations, in a certain sense that we can make formal. Building on Chapter 5's examples, let us define a co-inductive relation that is closer to the usual informal notion of "absence of infinite decreasing chains."

CoInductive infiniteDecreasingChain $A(R: A \rightarrow A \rightarrow$ Prop) : stream $A \rightarrow$ Prop $:=$

| ChainCons : $\forall x$ y $s$, infiniteDecreasingChain $R$ (Cons $y s$ )

$\rightarrow R$ y $x$

$\rightarrow$ infiniteDecreasingChain $R$ (Cons $x$ (Cons $y s)$ ).

We can now prove that any accessible element cannot be the beginning of any infinite decreasing chain.

Lemma noBadChains' : $\forall A(R: A \rightarrow A \rightarrow$ Prop) $x$, Acc $R x$

$\rightarrow \forall s, \neg$ infiniteDecreasingChain $R$ (Cons $x s$ ). 
induction 1 ; crush;

match goal with

$\mid\left[H\right.$ : infiniteDecreasingChain _ $\left.\vdash_{-}\right] \Rightarrow$ inversion $H$; eauto end.

Qed.

From here, the absence of infinite decreasing chains in well-founded sets is immediate.

Theorem noBadChains : $\forall A(R: A \rightarrow A \rightarrow$ Prop), well_founded $R$

$\rightarrow \forall s, \neg$ infiniteDecreasingChain $R s$.

destruct $s$; apply noBadChains'; auto.

Qed.

Absence of infinite decreasing chains implies absence of infinitely nested recursive calls, for any recursive definition that respects the well-founded relation. The Fix combinator from the standard library formalizes that intuition:

Check Fix.

Fix

$$
\begin{aligned}
: & \forall(A: \text { Type })(R: A \rightarrow A \rightarrow \text { Prop }), \\
& \text { well_founded } R \rightarrow \\
& \forall P: A \rightarrow \text { Type, } \\
& (\forall x: A,(\forall y: A, R y x \rightarrow P y) \rightarrow P x) \rightarrow \\
& \forall x: A, P x
\end{aligned}
$$

A call to Fix must present a relation $R$ and a proof of its well-foundedness. The next argument, $P$, is the possibly dependent range type of the function we build; the domain $A$ of $R$ is the function's domain. The following argument has this type:

$$
\forall x: A,(\forall y: A, R y x \rightarrow P y) \rightarrow P x
$$

This is an encoding of the function body. The input $x$ stands for the function argument, and the next input stands for the function we are defining. Recursive calls are encoded as calls to the second argument, whose type tells us it expects a value $y$ and a proof that $y$ is "less than" $x$, according to $R$. In this way, we enforce the well-foundedness restriction on recursive calls.

The rest of Fix's type tells us that it returns a function of exactly the type we expect, so we are now ready to use it to implement mergeSort. Careful readers may have noticed that Fix has a dependent type of the sort we met in the previous chapter.

Before writing mergeSort, we need to settle on a well-founded relation. The right one for this example is based on lengths of lists.

Definition lengthOrder $(l s 1$ ls2 : list $A):=$

length $l s 1<$ length $l s 2$.

We must prove that the relation is truly well-founded. To save some space in the rest of this chapter, we skip right to nice, automated proof scripts, though we postpone introducing the principles behind such scripts to Part III of the book. Curious readers may still replace semicolons with periods and newlines to step through these scripts interactively. 


\section{Hint Constructors Acc.}

Lemma lengthOrder_wf' $: \forall$ len, $\forall l s$, length $l s \leq l e n \rightarrow$ Acc lengthOrder $l s$. unfold lengthOrder; induction len; crush.

Defined.

Theorem lengthOrder_wf : well_founded lengthOrder. red; intro; eapply lengthOrder_wf'; eauto.

Defined.

Notice that we end these proofs with Defined, not Qed. Recall that Defined marks the theorems as transparent, so that the details of their proofs may be used during program execution. Why could such details possibly matter for computation? It turns out that Fix satisfies the primitive recursion restriction by declaring itself as recursive in the structure of Acc proofs. This is possible because Acc proofs follow a predictable inductive structure. We must do work, as in the last theorem's proof, to establish that all elements of a type belong to Acc, but the automatic unwinding of those proofs during recursion is straightforward. If we ended the proof with Qed, the proof details would be hidden from computation, in which case the unwinding process would get stuck.

To justify our two recursive mergeSort calls, we will also need to prove that split respects the lengthOrder relation. These proofs, too, must be kept transparent, to avoid stuckness of Fix evaluation. We use the syntax @ foo to reference identifier foo with its implicit argument behavior turned off. (The proof details below use Ltac features not introduced yet, and they are safe to skip for now.)

Lemma split_wf : $\forall$ len $l s, 2 \leq$ length $l s \leq l e n$

$\rightarrow$ let $(l s 1, l s 2):=$ split $l s$ in

lengthOrder ls1 ls $\wedge$ lengthOrder ls2 ls.

unfold lengthOrder; induction len; crush; do 2 (destruct ls; crush);

destruct (le_lt_dec 2 (length $l s)$ );

repeat (match goal with

$$
\begin{aligned}
& \mid\left[{ }_{-}: \text {length } ? E<2 \vdash{ }_{-}\right] \Rightarrow \text { destruct } E \\
& \left.\mid[-: \text { S (length } ? E)<2 \vdash{ }_{-}\right] \Rightarrow \text { destruct } E \\
& \mid\left[I H:{ }_{-} \vdash \text { context }[\text { split ? } L]\right] \Rightarrow \\
& \quad \text { specialize }(I H L) ; \text { destruct (split } L) \text {; destruct } I H \\
& \text { end; crush). }
\end{aligned}
$$

Defined.

Ltac split_wf := intros ls ?; intros; generalize (@split_wf (length ls) ls); destruct (split ls); destruct 1; crush.

Lemma split_wf1 : $\forall l s, 2 \leq$ length $l s$

$\rightarrow$ lengthOrder (fst (split $l s)$ ) $l s$.

split_wf.

Defined.

Lemma split_wf2 : $\forall l s, 2 \leq$ length $l s$

$\rightarrow$ lengthOrder (snd (split ls)) ls. 


\section{split_wf. \\ Defined.}

Hint Resolve split_wf1 split_wf2.

To write the function definition itself, we use the refine tactic as a convenient way to write a program that needs to manipulate proofs, without writing out those proofs manually. We also use a replacement le_lt_dec for leb that has a more interesting dependent type. (Note that we would not be able to complete the definition without this change, since refine will generate subgoals for the if branches based only on the type of the test expression, not its value.)

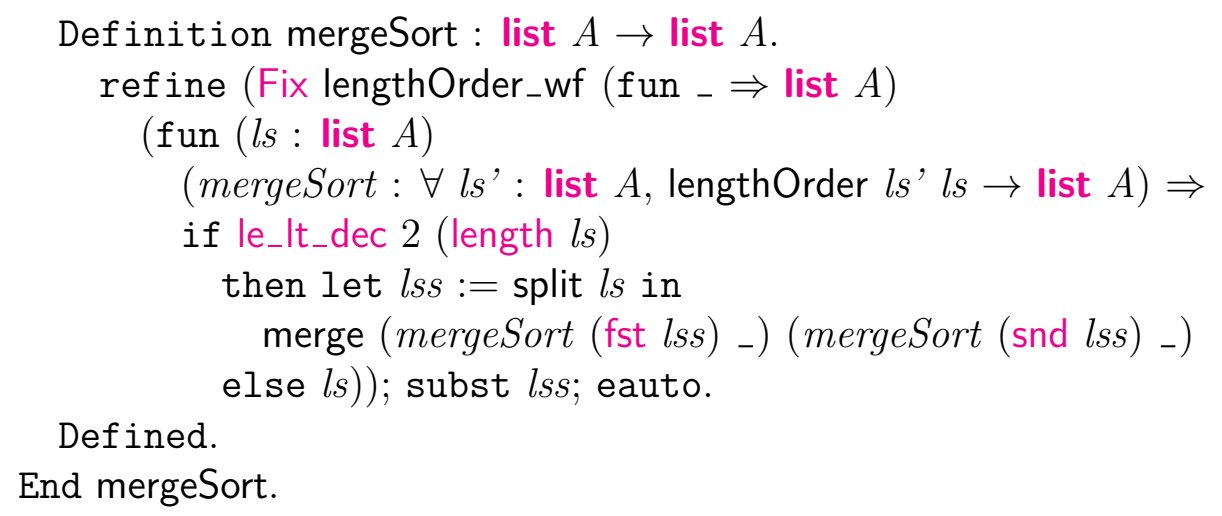

The important thing is that it is now easy to evaluate calls to mergeSort.

Eval compute in mergeSort leb $(1:: 2:: 36:: 8:: 19::$ nil).

$=1:: 2:: 8$ :: $19:: 36::$ nil

Since the subject of this chapter is merely how to define functions with unusual recursion structure, we will not prove any further correctness theorems about mergeSort. Instead, we stop at proving that mergeSort has the expected computational behavior, for all inputs, not merely the one we just tested.

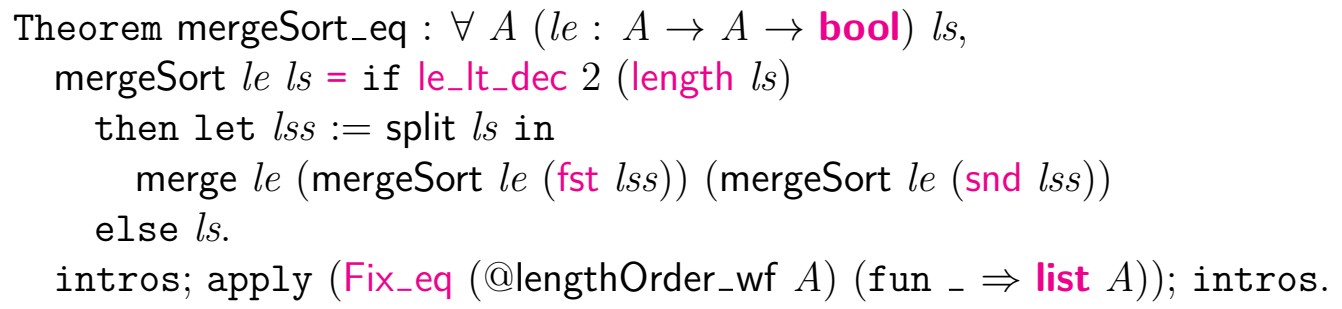

The library theorem Fix_eq imposes one more strange subgoal upon us. We must prove that the function body is unable to distinguish between "self" arguments that map equal inputs to equal outputs. One might think this should be true of any Gallina code, but in fact this general function extensionality property is neither provable nor disprovable within Coq. The type of Fix_eq makes clear what we must show manually:

Check Fix_eq.

Fix_eq

$: \forall(A:$ Type $)(R: A \rightarrow A \rightarrow$ Prop $)(R w f:$ well_founded $R)$ 


$$
\begin{aligned}
& \quad(P: A \rightarrow \text { Type }) \\
& \quad(F: \forall x: A,(\forall y: A, R y x \rightarrow P y) \rightarrow P x), \\
& (\forall(x: A)(f g: \forall y: A, R y x \rightarrow P y), \\
& \quad(\forall(y: A)(p: R y x), f y p=g y p) \rightarrow F x f=F x g) \rightarrow \\
& \forall x: A, \\
& \text { Fix Rwf } P F x=F x\left(\text { fun }(y: A)\left({ }_{-}: R y x\right) \Rightarrow \text { Fix Rwf } P F y\right)
\end{aligned}
$$

Most such obligations are dischargeable with straightforward proof automation, and this example is no exception.

match goal with

$\mid\left[\vdash\right.$ context [match ?E with left _ $\Rightarrow{ }_{-} \mid$right ${ }_{-} \Rightarrow_{-}$end] $] \Rightarrow \operatorname{destruct~} E$ end; simpl; f_equal; auto.

Qed.

As a final test of our definition's suitability, we can extract to OCaml.

Extraction mergeSort.

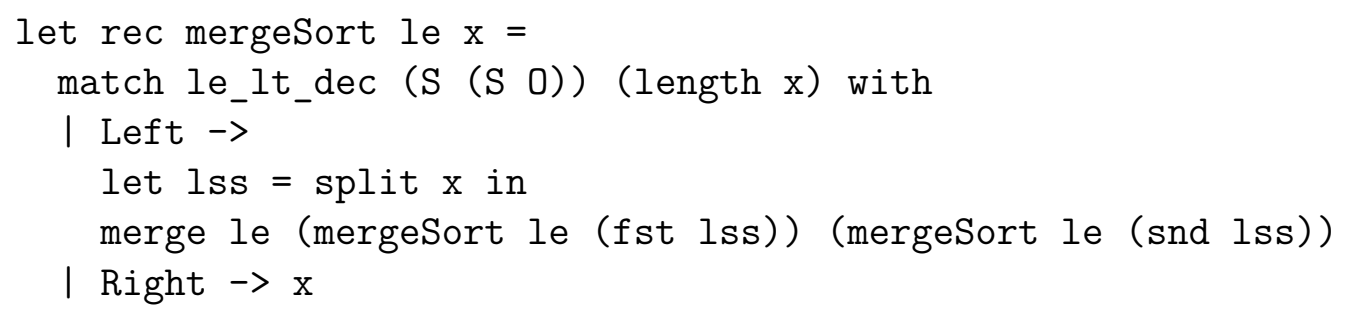

We see almost precisely the same definition we would have written manually in OCaml! It might be a good exercise for the reader to use the commands we saw in the previous chapter to clean up some remaining differences from idiomatic OCaml.

One more piece of the full picture is missing. To go on and prove correctness of mergeSort, we would need more than a way of unfolding its definition. We also need an appropriate induction principle matched to the well-founded relation. Such a principle is available in the standard library, though we will say no more about its details here.

Check well_founded_induction.

well_founded_induction

$$
\begin{aligned}
: & \forall(A: \text { Type })(R: A \rightarrow A \rightarrow \text { Prop }), \\
& \text { well_founded } R \rightarrow \\
& \forall P: A \rightarrow \text { Set }, \\
& (\forall x: A,(\forall y: A, R \text { y } x \rightarrow P y) \rightarrow P x) \rightarrow \\
& \forall a: A, P a
\end{aligned}
$$

Some more recent Coq features provide more convenient syntax for defining recursive functions. Interested readers can consult the Coq manual about the commands Function and Program Fixpoint. 


\subsection{A Non-Termination Monad Inspired by Domain Theory}

The key insights of domain theory [49] inspire the next approach to modeling non-termination. Domain theory is based on information orders that relate values representing computation results, according to how much information these values convey. For instance, a simple domain might include values "the program does not terminate" and "the program terminates with the answer 5." The former is considered to be an approximation of the latter, while the latter is not an approximation of "the program terminates with the answer 6." The details of domain theory will not be important in what follows; we merely borrow the notion of an approximation ordering on computation results.

Consider this definition of a type of computations.

Section computation.

Variable $A$ : Type.

The type $A$ describes the result a computation will yield, if it terminates.

We give a rich dependent type to computations themselves:

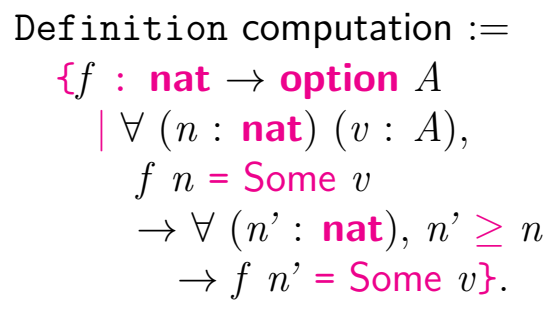

A computation is fundamentally a function $f$ from an approximation level $n$ to an optional result. Intuitively, higher $n$ values enable termination in more cases than lower values. A call to $f$ may return None to indicate that $n$ was not high enough to run the computation to completion; higher $n$ values may yield Some. Further, the proof obligation within the subset type asserts that $f$ is monotone in an appropriate sense: when some $n$ is sufficient to produce termination, so are all higher $n$ values, and they all yield the same program result $v$.

It is easy to define a relation characterizing when a computation runs to a particular result at a particular approximation level.

Definition runTo $(m:$ computation) $(n:$ nat $)(v: A):=$ proj1_sig $m n=$ Some $v$.

On top of runTo, we also define run, which is the most abstract notion of when a computation runs to a value.

Definition run $(m:$ computation) $(v: A):=$

$\exists n$, runTo $m n v$.

End computation.

The book source code contains at this point some tactics, lemma proofs, and hint commands, to be used in proving facts about computations. Since their details are orthogonal 
to the message of this chapter, I have omitted them in the rendered version.

Now, as a simple first example of a computation, we can define Bottom, which corresponds to an infinite loop. For any approximation level, it fails to terminate (returns None). Note the use of abstract to create a new opaque lemma for the proof found by the run tactic. In contrast to the previous section, opaque proofs are fine here, since the proof components of computations do not influence evaluation behavior. It is generally preferable to make proofs opaque when possible, as this enforces a kind of modularity in the code to follow, preventing it from depending on any details of the proof.

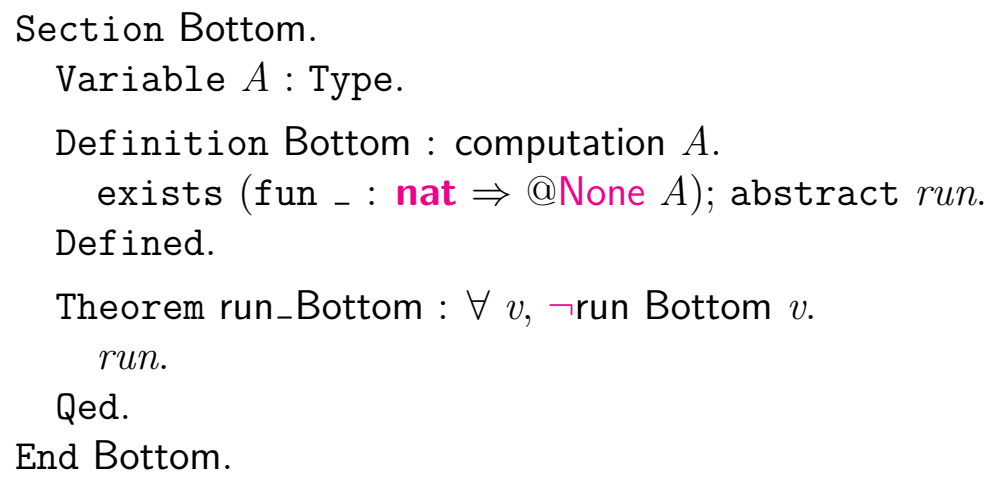

A slightly more complicated example is Return, which gives the same terminating answer at every approximation level.

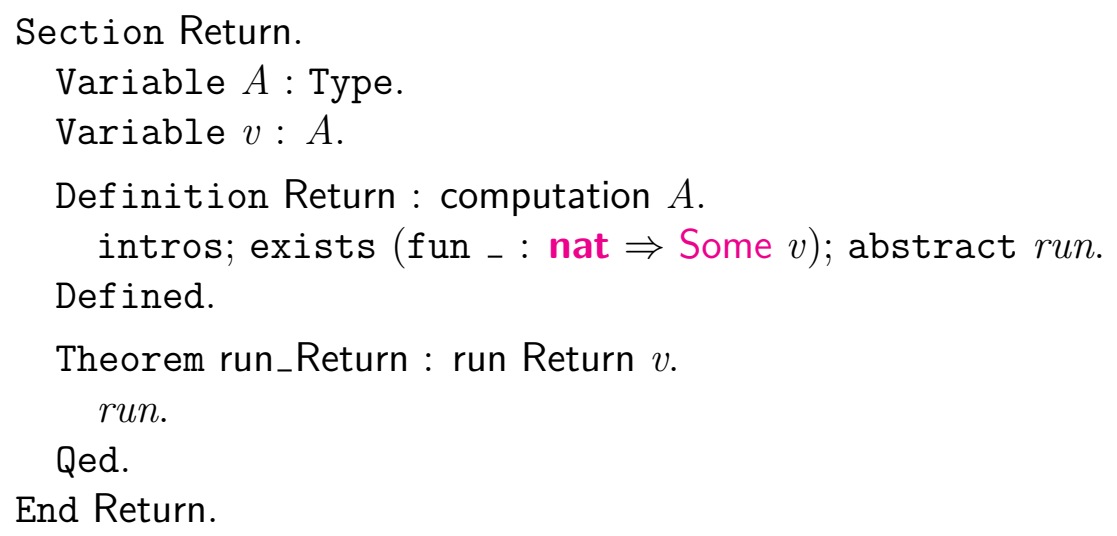

The name Return was meant to be suggestive of the standard operations of monads [45]. The other standard operation is Bind, which lets us run one computation and, if it terminates, pass its result off to another computation. We implement bind using the notation let $(x, y)$ $:=e 1$ in $e$ 2, for pulling apart the value $e 1$ which may be thought of as a pair. The second component of a computation is a proof, which we do not need to mention directly in the definition of Bind.

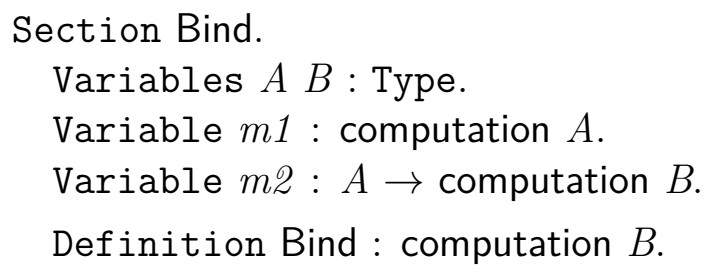




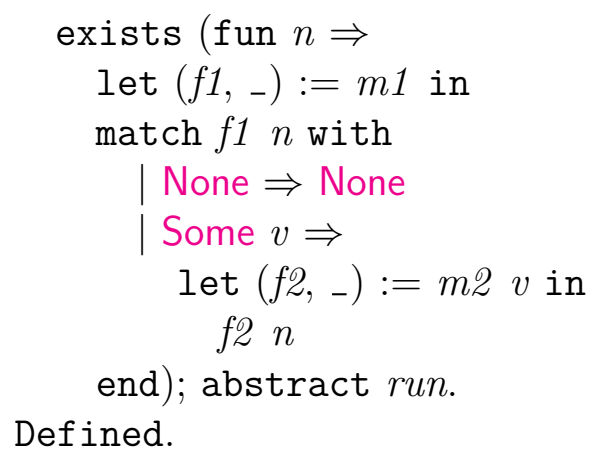

A simple notation lets us write Bind calls the way they appear in Haskell. Notation "x <- $\mathrm{m} 1 ; \mathrm{m} 2$ " :=

(Bind $m 1$ (fun $x \Rightarrow m 2)$ ) (right associativity, at level 70).

We can verify that we have indeed defined a monad, by proving the standard monad laws. Part of the exercise is choosing an appropriate notion of equality between computations. We use "equality at all approximation levels."

Definition meq $A(m 1 m 2$ : computation $A):=\forall n$, proj1_sig $m 1 n=$ proj1_sig $m 2 n$.

Theorem left_identity : $\forall A B(a: A)(f: A \rightarrow$ computation $B)$, meq (Bind (Return $a) f)(f a)$.

run.

Qed.

Theorem right_identity : $\forall A(m$ : computation $A)$, meq (Bind $m$ (@Return _)) $m$.

run.

Qed.

Theorem associativity : $\forall A B C(m$ : computation $A)$

$(f: A \rightarrow$ computation $B)(g: B \rightarrow$ computation $C)$,

meq (Bind (Bind $m f$ ) $g$ ) (Bind $m$ (fun $x \Rightarrow$ Bind $(f x) g)$ ).

run.

Qed.

Now we come to the piece most directly inspired by domain theory. We want to support general recursive function definitions, but domain theory tells us that not all definitions are 
reasonable; some fail to be continuous and thus represent unrealizable computations. To formalize an analogous notion of continuity for our non-termination monad, we write down the approximation relation on computation results that we have had in mind all along.

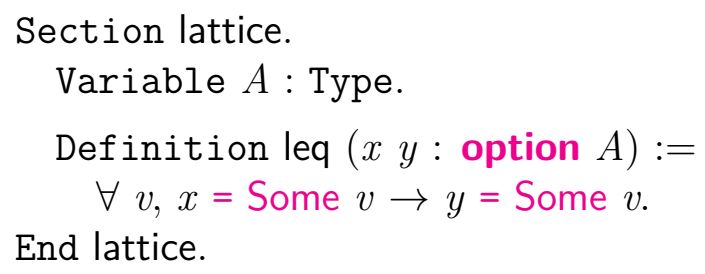

We now have the tools we need to define a new Fix combinator that, unlike the one we saw in the prior section, does not require a termination proof, and in fact admits recursive definition of functions that fail to terminate on some or all inputs.

Section Fix.

First, we have the function domain and range types.

Variables $A B$ : Type.

Next comes the function body, which is written as though it can be parameterized over itself, for recursive calls.

Variable $f:(A \rightarrow$ computation $B) \rightarrow(A \rightarrow$ computation $B)$.

Finally, we impose an obligation to prove that the body $f$ is continuous. That is, when $f$ terminates according to one recursive version of itself, it also terminates with the same result at the same approximation level when passed a recursive version that refines the original, according to leq.

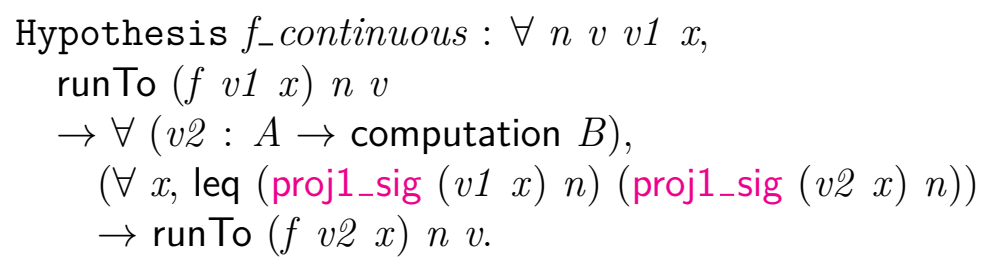

The computational part of the Fix combinator is easy to define. At approximation level 0, we diverge; at higher levels, we run the body with a functional argument drawn from the next lower level.

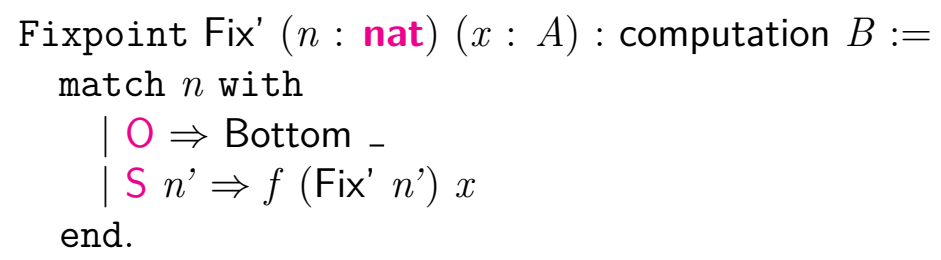

Now it is straightforward to package Fix' as a computation combinator Fix.

Hint Extern $1\left(_{-} \geq{ }_{-}\right) \Rightarrow$ omega.

Hint Unfold leq.

Lemma Fix'_ok : $\forall$ steps $n x v$, proj1_sig (Fix' $n x$ ) steps $=$ Some $v$ 
$\rightarrow \forall n^{\prime}, n^{\prime} \geq n$

$\rightarrow$ proj1_sig (Fix' n'x) steps $=$ Some $v$.

unfold runTo in *; induction $n$; crush;

match goal with

| [H: _ $\left.\geq_{-} \vdash_{-}\right] \Rightarrow$ inversion $H$; crush; eauto

end.

Qed.

Hint Resolve Fix'_ok.

Hint Extern 1 (proj1_sig _ _ = _) $\Rightarrow$ simpl;

match goal with

$\mid\left[\vdash\right.$ proj1_sig ? $\left.E_{-}=_{-}\right] \Rightarrow$ eapply (proj2_sig $E$ )

end.

Definition Fix : $A \rightarrow$ computation $B$.

intro $x$; exists (fun $n \Rightarrow$ proj1_sig (Fix' $n x) n$ ); abstract run.

Defined.

Finally, we can prove that Fix obeys the expected computation rule.

Theorem run_Fix : $\forall x v$,

run $(f$ Fix $x) v$

$\rightarrow \operatorname{run}($ Fix $x) v$.

run; match goal with

$\mid\left[n:\right.$ nat $\left.\vdash_{-}\right] \Rightarrow$ exists $(\mathrm{S} n)$; eauto

end.

Qed.

End Fix.

After all that work, it is now fairly painless to define a version of mergeSort that requires no proof of termination. We appeal to a program-specific tactic whose definition is hidden here but present in the book source.

Definition mergeSort' $: \forall A,(A \rightarrow A \rightarrow$ bool $) \rightarrow$ list $A \rightarrow$ computation (list $A)$.

refine (fun $A$ le $\Rightarrow$ Fix

(fun (mergeSort : list $A \rightarrow$ computation (list $A)$ )

$(l s:$ list $A) \Rightarrow$

if le_It_dec 2 (length $l s$ )

then let lss $:=$ split $l s$ in

ls1 $\leftarrow$ mergeSort (fst lss);

ls2 $\leftarrow$ mergeSort (snd lss);

Return (merge le ls1 ls2)

else Return ls) _); abstract mergeSort'.

\section{Defined.}

Furthermore, "running" mergeSort' on concrete inputs is as easy as choosing a sufficiently high approximation level and letting Coq's computation rules do the rest. Contrast this with 
the proof work that goes into deriving an evaluation fact for a deeply embedded language, with one explicit proof rule application per execution step.

Lemma test_mergeSort' : run (mergeSort' leb $(1:: 2:: 36:: 8:: 19::$ nil))

$(1:: 2:: 8:: 19:: 36::$ nil).

exists 4 ; reflexivity.

Qed.

There is another benefit of our new Fix compared with the one we used in the previous section: we can now write recursive functions that sometimes fail to terminate, without losing easy reasoning principles for the terminating cases. Consider this simple example, which appeals to another tactic whose definition we elide here.

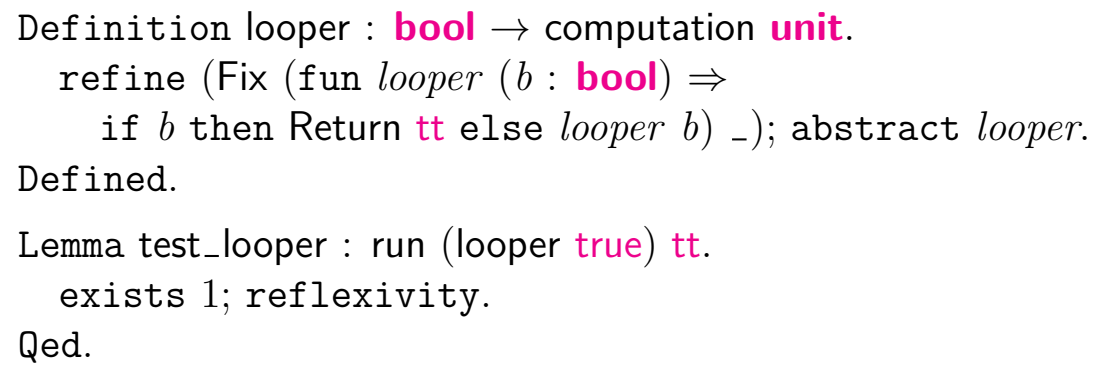

As before, proving outputs for specific inputs is as easy as demonstrating a high enough approximation level.

There are other theorems that are important to prove about combinators like Return, Bind, and Fix. In general, for a computation $c$, we sometimes have a hypothesis proving run $c v$ for some $v$, and we want to perform inversion to deduce what $v$ must be. Each combinator should ideally have a theorem of that kind, for $c$ built directly from that combinator. We have omitted such theorems here, but they are not hard to prove. In general, the domain theoryinspired approach avoids the type-theoretic "gotchas" that tend to show up in approaches that try to mix normal Coq computation with explicit syntax types. The next section of this chapter demonstrates two alternate approaches of that sort. In the final section of the chapter, we review the pros and cons of the different choices, coming to the conclusion that none of them is obviously better than any one of the others for all situations.

\subsection{Co-Inductive Non-Termination Monads}

There are two key downsides to both of the previous approaches: both require unusual syntax based on explicit calls to fixpoint combinators, and both generate immediate proof obligations about the bodies of recursive definitions. In Chapter 5 , we have already seen how co-inductive types support recursive definitions that exhibit certain well-behaved varieties of non-termination. It turns out that we can leverage that co-induction support for encoding of general recursive definitions, by adding layers of co-inductive syntax. In effect, we mix elements of shallow and deep embeddings.

Our first example of this kind, proposed by Capretta [4], defines a silly-looking type of 
thunks; that is, computations that may be forced to yield results, if they terminate.

CoInductive thunk $(A$ : Type) : Type :=

| Answer : $A \rightarrow$ thunk $A$

| Think: thunk $A \rightarrow$ thunk $A$.

A computation is either an immediate Answer or another computation wrapped inside Think. Since thunk is co-inductive, every thunk type is inhabited by an infinite nesting of Thinks, standing for non-termination. Terminating results are Answer wrapped inside some finite number of Thinks.

Why bother to write such a strange definition? The definition of thunk is motivated by the ability it gives us to define a "bind" operation, similar to the one we defined in the previous section.

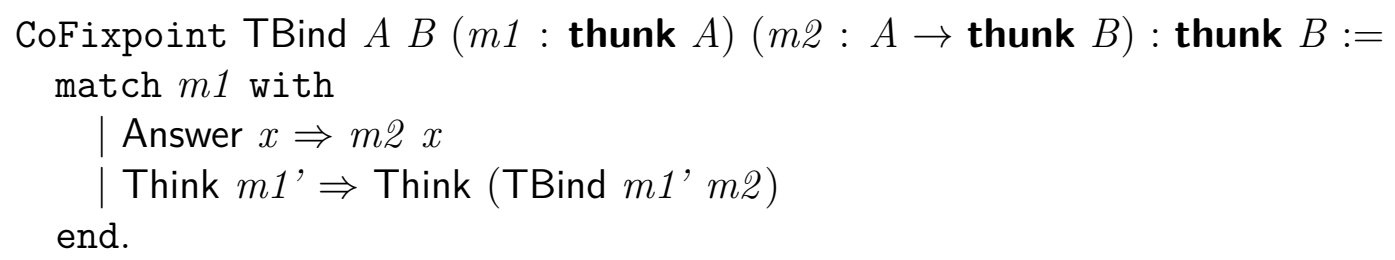

Note that the definition would violate the co-recursion guardedness restriction if we left out the seemingly superfluous Think on the righthand side of the second match branch.

We can prove that Answer and TBind form a monad for thunk. The proof is omitted here but present in the book source. As usual for this sort of proof, a key element is choosing an appropriate notion of equality for thunks.

In the proofs to follow, we will need a function similar to one we saw in Chapter 5, to pull apart and reassemble a thunk in a way that provokes reduction of co-recursive calls.

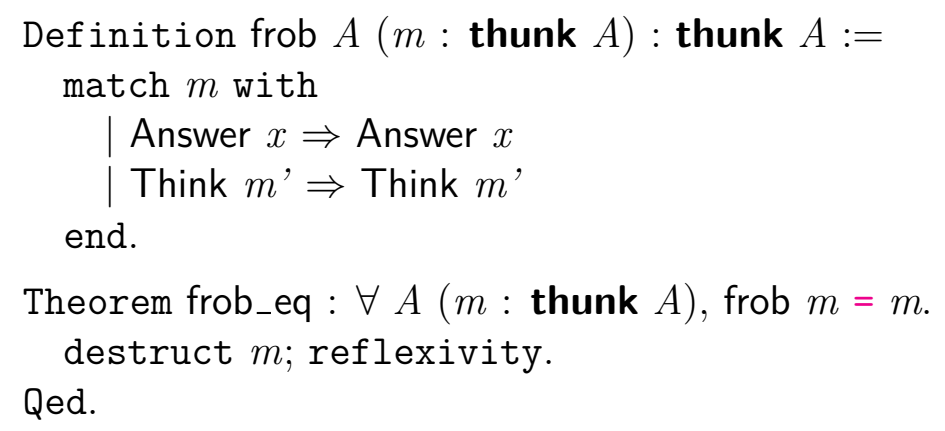

As a simple example, here is how we might define a tail-recursive factorial function.

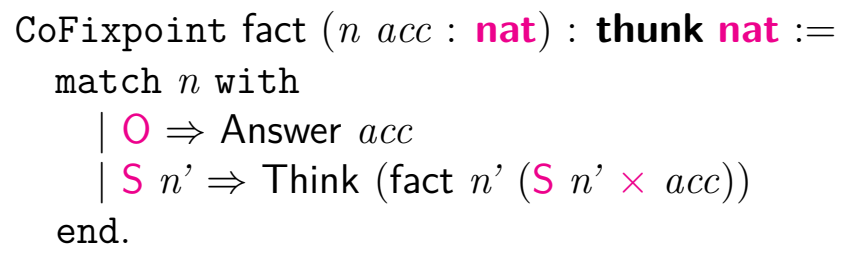

To test our definition, we need an evaluation relation that characterizes results of evaluating thunks. 
Inductive eval $A$ : thunk $A \rightarrow A \rightarrow$ Prop $:=$

| EvalAnswer : $\forall x$, eval (Answer $x$ ) $x$

| EvalThink : $\forall m x$, eval $m x \rightarrow$ eval (Think $m$ ) $x$.

Hint Rewrite frob_eq.

Lemma eval_frob : $\forall A(c:$ thunk $A) x$, eval (frob $c) x$

$\rightarrow$ eval $c x$.

crush.

Qed.

Theorem eval_fact : eval (fact 5 1) 120.

repeat (apply eval_frob; simpl; constructor). Qed.

We need to apply constructors of eval explicitly, but the process is easy to automate completely for concrete input programs.

Now consider another very similar definition, this time of a Fibonacci number function.

Notation "x $<-\mathrm{m} 1 ; \mathrm{m} 2$ " :=

(TBind $m 1$ (fun $x \Rightarrow m 2)$ ) (right associativity, at level 70).

CoFixpoint fib ( $n$ : nat) : thunk nat :=

match $n$ with

$0 \Rightarrow$ Answer 1

$1 \Rightarrow$ Answer 1

| $\Rightarrow n 1 \leftarrow$ fib $($ pred $n)$;

$n 2 \leftarrow$ fib $($ pred $($ pred $n))$

Answer $(n 1+n 2)$

end.

Coq complains that the guardedness condition is violated. The two recursive calls are immediate arguments to TBind, but TBind is not a constructor of thunk. Rather, it is a defined function. This example shows a very serious limitation of thunk for traditional functional programming: it is not, in general, possible to make recursive calls and then make further recursive calls, depending on the first call's result. The fact example succeeded because it was already tail recursive, meaning no further computation is needed after a recursive call.

I know no easy fix for this problem of thunk, but we can define an alternate co-inductive monad that avoids the problem, based on a proposal by Megacz [24]. We ran into trouble because TBind was not a constructor of thunk, so let us define a new type family where "bind" is a constructor.

CoInductive comp $(A:$ Type $):$ Type :=

| Ret : $A \rightarrow \operatorname{comp} A$

| Bnd : $\forall B, \operatorname{comp} B \rightarrow(B \rightarrow \operatorname{comp} A) \rightarrow \operatorname{comp} A$. 
This example shows off Coq's support for recursively non-uniform parameters, as in the case of the parameter $A$ declared above, where each constructor's type ends in comp $A$, but there is a recursive use of comp with a different parameter $B$. Beside that technical wrinkle, we see the simplest possible definition of a monad, via a type whose two constructors are precisely the monad operators.

It is easy to define the semantics of terminating comp computations.

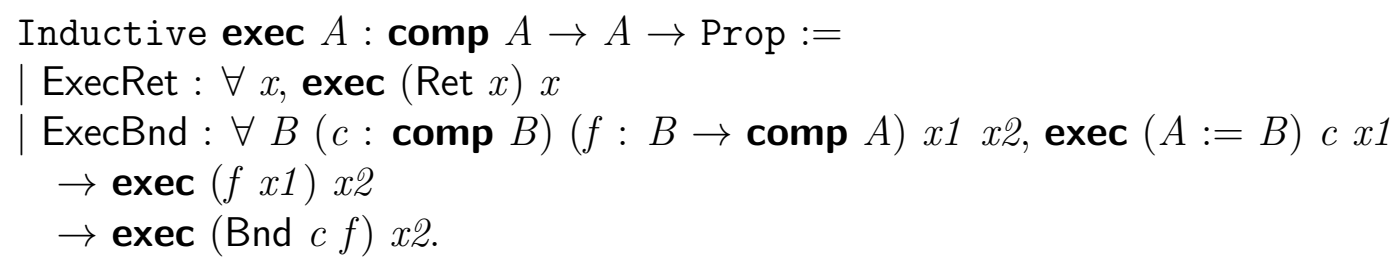

We can also prove that Ret and Bnd form a monad according to a notion of comp equality based on exec, but we omit details here; they are in the book source at this point.

Not only can we define the Fibonacci function with the new monad, but even our running example of merge sort becomes definable. By shadowing our previous notation for "bind," we can write almost exactly the same code as in our previous mergeSort' definition, but with less syntactic clutter.

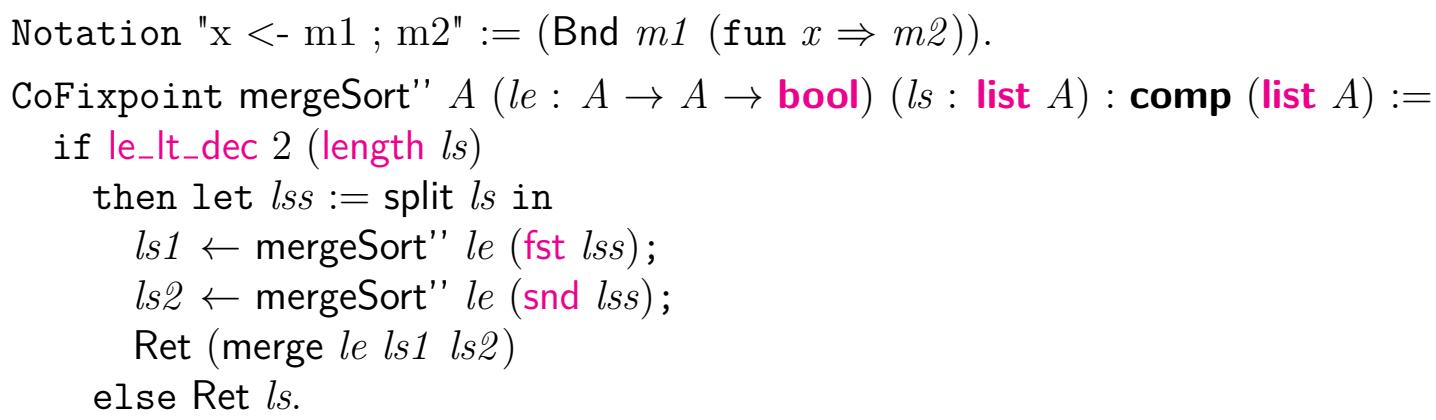

To execute this function, we go through the usual exercise of writing a function to catalyze evaluation of co-recursive calls.

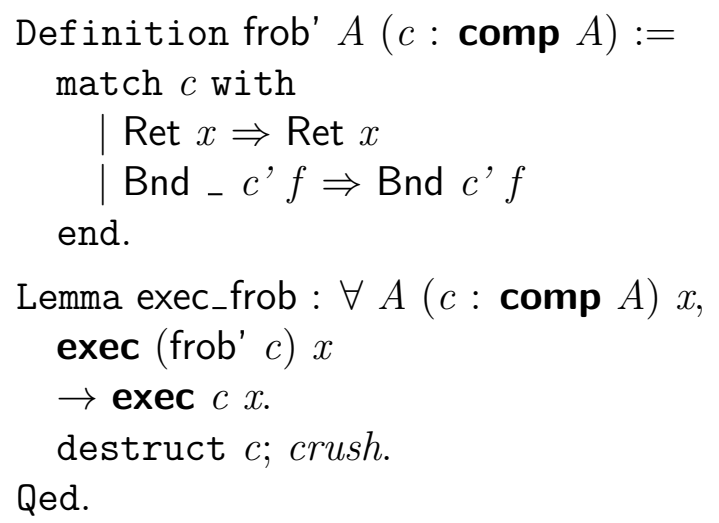

Now the same sort of proof script that we applied for testing thunks will get the job done.

Lemma test_mergeSort"' : exec (mergeSort" leb $(1:: 2:: 36:: 8:: 19::$ nil)) 
$(1:: 2:: 8:: 19:: 36::$ nil).

repeat (apply exec_frob; simpl; econstructor).

Qed.

Have we finally reached the ideal solution for encoding general recursive definitions, with minimal hassle in syntax and proof obligations? Unfortunately, we have not, as comp has a serious expressivity weakness. Consider the following definition of a curried addition function:

Definition curriedAdd ( $n$ : nat) $:=\operatorname{Ret}($ fun $m$ : nat $\Rightarrow \operatorname{Ret}(n+m)$ ).

This definition works fine, but we run into trouble when we try to apply it in a trivial way.

Definition testCurriedAdd := Bnd (curriedAdd 2) (fun $f \Rightarrow f 3$ ).

Error: Universe inconsistency.

The problem has to do with rules for inductive definitions that we will study in more detail in Chapter 12. Briefly, recall that the type of the constructor Bnd quantifies over a type $B$. To make testCurriedAdd work, we would need to instantiate $B$ as nat $\rightarrow$ comp nat. However, Coq enforces a predicativity restriction that (roughly) no quantifier in an inductive or co-inductive type's definition may ever be instantiated with a term that contains the type being defined. Chapter 12 presents the exact mechanism by which this restriction is enforced, but for now our conclusion is that comp is fatally flawed as a way of encoding interesting higher-order functional programs that use general recursion.

\subsection{Comparing the Alternatives}

We have seen four different approaches to encoding general recursive definitions in Coq. Among them there is no clear champion that dominates the others in every important way. Instead, we close the chapter by comparing the techniques along a number of dimensions. Every technique allows recursive definitions with termination arguments that go beyond Coq's built-in termination checking, so we must turn to subtler points to highlight differences.

One useful property is automatic integration with normal Coq programming. That is, we would like the type of a function to be the same, whether or not that function is defined using an interesting recursion pattern. Only the first of the four techniques, well-founded recursion, meets this criterion. It is also the only one of the four to meet the related criterion that evaluation of function calls can take place entirely inside Coq's built-in computation machinery. The monad inspired by domain theory occupies some middle ground in this dimension, since generally standard computation is enough to evaluate a term once a high enough approximation level is provided.

Another useful property is that a function and its termination argument may be developed separately. We may even want to define functions that fail to terminate on some or all inputs. The well-founded recursion technique does not have this property, but the other three do. 
One minor plus is the ability to write recursive definitions in natural syntax, rather than with calls to higher-order combinators. This downside of the first two techniques is actually rather easy to get around using Coq's notation mechanism, though we leave the details as an exercise for the reader. (For this and other details of notations, see Chapter 12 of the Coq 8.4 manual.)

The first two techniques impose proof obligations that are more basic than termination arguments, where well-founded recursion requires a proof of extensionality and domaintheoretic recursion requires a proof of continuity. A function may not be defined, and thus may not be computed with, until these obligations are proved. The co-inductive techniques avoid this problem, as recursive definitions may be made without any proof obligations.

We can also consider support for common idioms in functional programming. For instance, the thunk monad effectively only supports recursion that is tail recursion, while the others allow arbitrary recursion schemes.

On the other hand, the comp monad does not support the effective mixing of higherorder functions and general recursion, while all the other techniques do. For instance, we can finish the failed curriedAdd example in the domain-theoretic monad.

Definition curriedAdd' ( $n$ : nat) $:=\operatorname{Return~(fun~} m$ : nat $\Rightarrow \operatorname{Return}(n+m)$ ).

Definition testCurriedAdd := Bind (curriedAdd' 2) (fun $f \Rightarrow f 3$ ).

The same techniques also apply to more interesting higher-order functions like list map, and, as in all four techniques, we can mix primitive and general recursion, preferring the former when possible to avoid proof obligations.

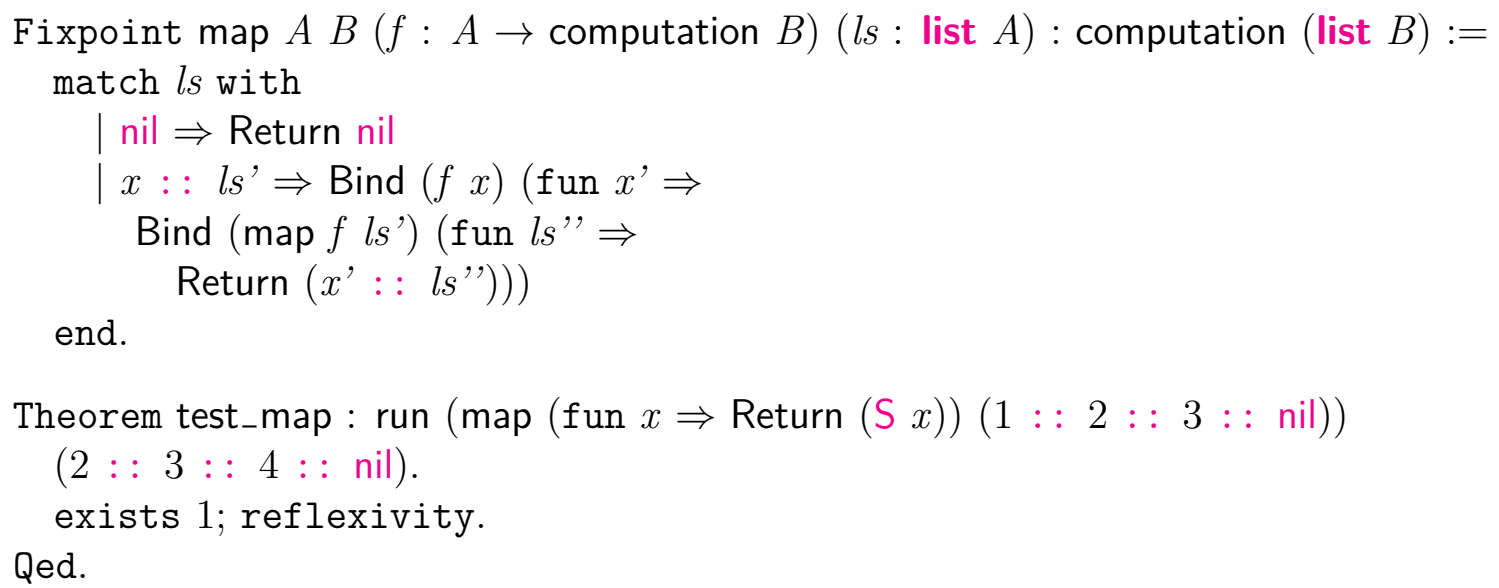

One further disadvantage of comp is that we cannot prove an inversion lemma for executions of Bind without appealing to an axiom, a logical complication that we discuss at more length in Chapter 12. The other three techniques allow proof of all the important theorems within the normal logic of Coq.

Perhaps one theme of our comparison is that one must trade off between, on one hand, functional programming expressiveness and compatibility with normal Coq types and computation; and, on the other hand, the level of proof obligations one is willing to handle at function definition time. 


\section{Chapter 8}

\section{More Dependent Types}

Subset types and their relatives help us integrate verification with programming. Though they reorganize the certified programmer's workflow, they tend not to have deep effects on proofs. We write largely the same proofs as we would for classical verification, with some of the structure moved into the programs themselves. It turns out that, when we use dependent types to their full potential, we warp the development and proving process even more than that, picking up "free theorems" to the extent that often a certified program is hardly more complex than its uncertified counterpart in Haskell or ML.

In particular, we have only scratched the tip of the iceberg that is Coq's inductive definition mechanism. The inductive types we have seen so far have their counterparts in the other proof assistants that we surveyed in Chapter 1. This chapter explores the strange new world of dependent inductive datatypes outside Prop, a possibility that sets Coq apart from all of the competition not based on type theory.

\subsection{Length-Indexed Lists}

Many introductions to dependent types start out by showing how to use them to eliminate array bounds checks. When the type of an array tells you how many elements it has, your compiler can detect out-of-bounds dereferences statically. Since we are working in a pure functional language, the next best thing is length-indexed lists, which the following code defines.

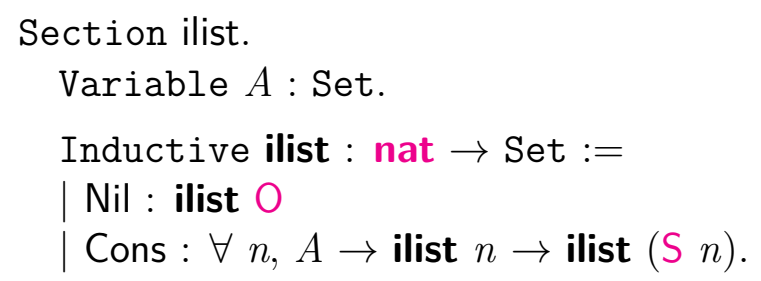

We see that, within its section, ilist is given type nat $\rightarrow$ Set. Previously, every inductive type we have seen has either had plain Set as its type or has been a predicate with some type ending in Prop. The full generality of inductive definitions lets us integrate the expressivity 
of predicates directly into our normal programming.

The nat argument to ilist tells us the length of the list. The types of ilist's constructors tell us that a Nil list has length $\mathrm{O}$ and that a Cons list has length one greater than the length of its tail. We may apply ilist to any natural number, even natural numbers that are only known at runtime. It is this breaking of the phase distinction that characterizes ilist as dependently typed.

In expositions of list types, we usually see the length function defined first, but here that would not be a very productive function to code. Instead, let us implement list concatenation.

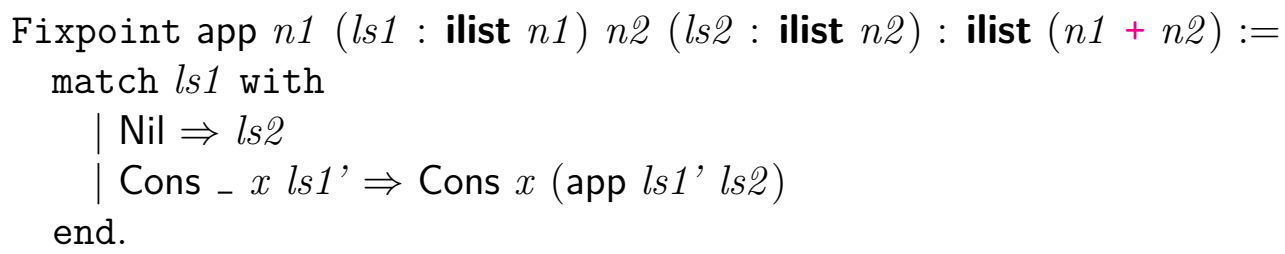

Past Coq versions signalled an error for this definition. The code is still invalid within Coq's core language, but current Coq versions automatically add annotations to the original program, producing a valid core program. These are the annotations on match discriminees that we began to study in the previous chapter. We can rewrite app to give the annotations explicitly.

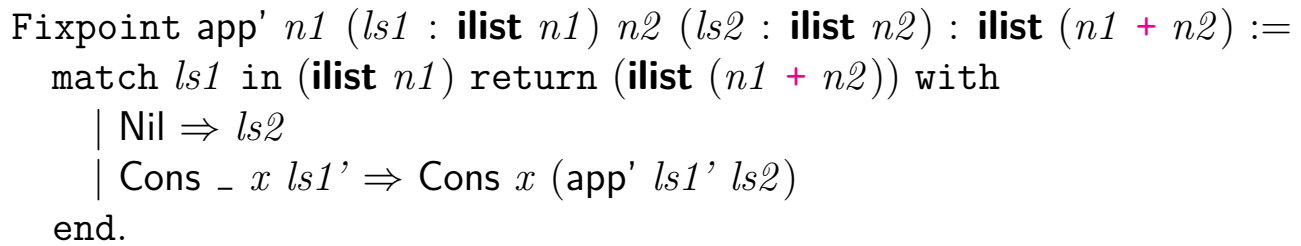

Using return alone allowed us to express a dependency of the match result type on the value of the discriminee. What in adds to our arsenal is a way of expressing a dependency on the type of the discriminee. Specifically, the $n 1$ in the in clause above is a binding occurrence whose scope is the return clause.

We may use in clauses only to bind names for the arguments of an inductive type family. That is, each in clause must be an inductive type family name applied to a sequence of underscores and variable names of the proper length. The positions for parameters to the type family must all be underscores. Parameters are those arguments declared with section variables or with entries to the left of the first colon in an inductive definition. They cannot vary depending on which constructor was used to build the discriminee, so Coq prohibits pointless matches on them. It is those arguments defined in the type to the right of the colon that we may name with in clauses.

Our app function could be typed in so-called stratified type systems, which avoid true dependency. That is, we could consider the length indices to lists to live in a separate, compile-time-only universe from the lists themselves. Compile-time data may be erased such that we can still execute a program. As an example where erasure would not work, consider an injection function from regular lists to length-indexed lists. Here the run-time 
computation actually depends on details of the compile-time argument, if we decide that the list to inject can be considered compile-time. More commonly, we think of lists as run-time data. Neither case will work with naïve erasure. (It is not too important to grasp the details of this run-time/compile-time distinction, since Coq's expressive power comes from avoiding such restrictions.)

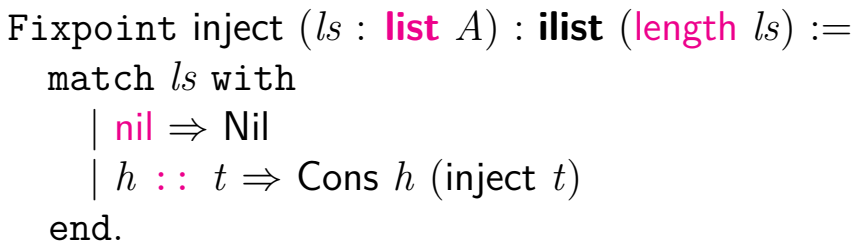

We can define an inverse conversion and prove that it really is an inverse.

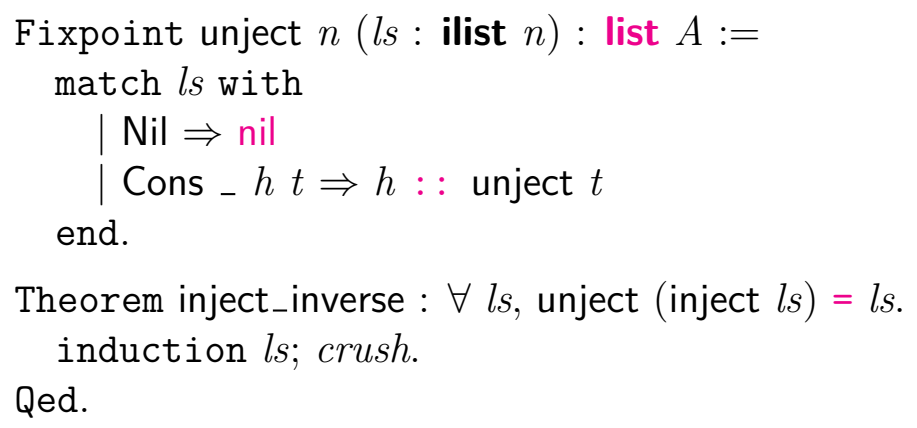

Now let us attempt a function that is surprisingly tricky to write. In ML, the list head function raises an exception when passed an empty list. With length-indexed lists, we can rule out such invalid calls statically, and here is a first attempt at doing so. We write ??? as a placeholder for a term that we do not know how to write, not for any real Coq notation like those introduced two chapters ago.

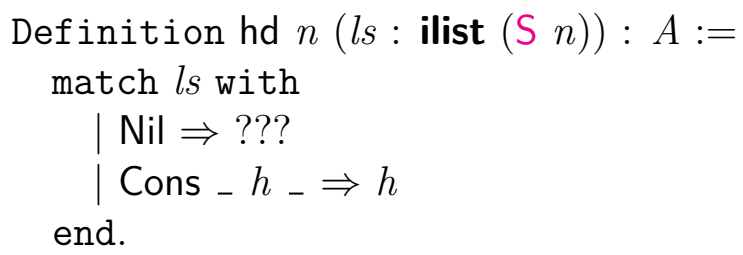

It is not clear what to write for the Nil case, so we are stuck before we even turn our function over to the type checker. We could try omitting the Nil case:

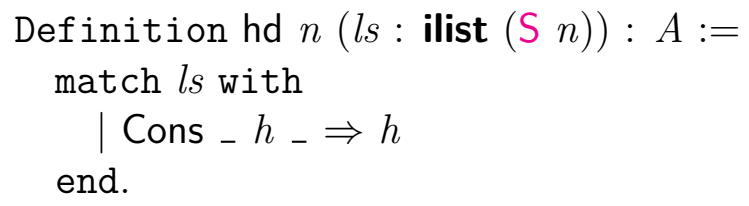

Error: Non exhaustive pattern-matching: no clause found for pattern Nil

Unlike in ML, we cannot use inexhaustive pattern matching, because there is no conception of a Match exception to be thrown. In fact, recent versions of Coq do allow this, by 
implicit translation to a match that considers all constructors; the error message above was generated by an older Coq version. It is educational to discover for ourselves the encoding that the most recent Coq versions use. We might try using an in clause somehow.

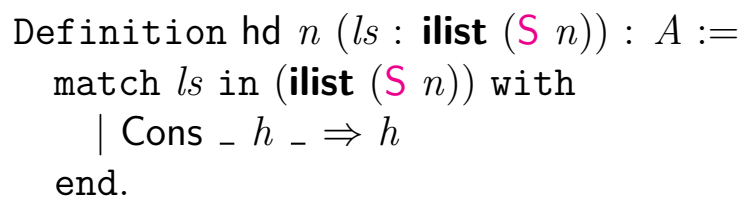

Error: The reference $\mathrm{n}$ was not found in the current environment

In this and other cases, we feel like we want in clauses with type family arguments that are not variables. Unfortunately, Coq only supports variables in those positions. A completely general mechanism could only be supported with a solution to the problem of higher-order unification [15], which is undecidable. There are useful heuristics for handling non-variable indices which are gradually making their way into Coq, but we will spend some time in this and the next few chapters on effective pattern matching on dependent types using only the primitive match annotations.

Our final, working attempt at hd uses an auxiliary function and a surprising return annotation.

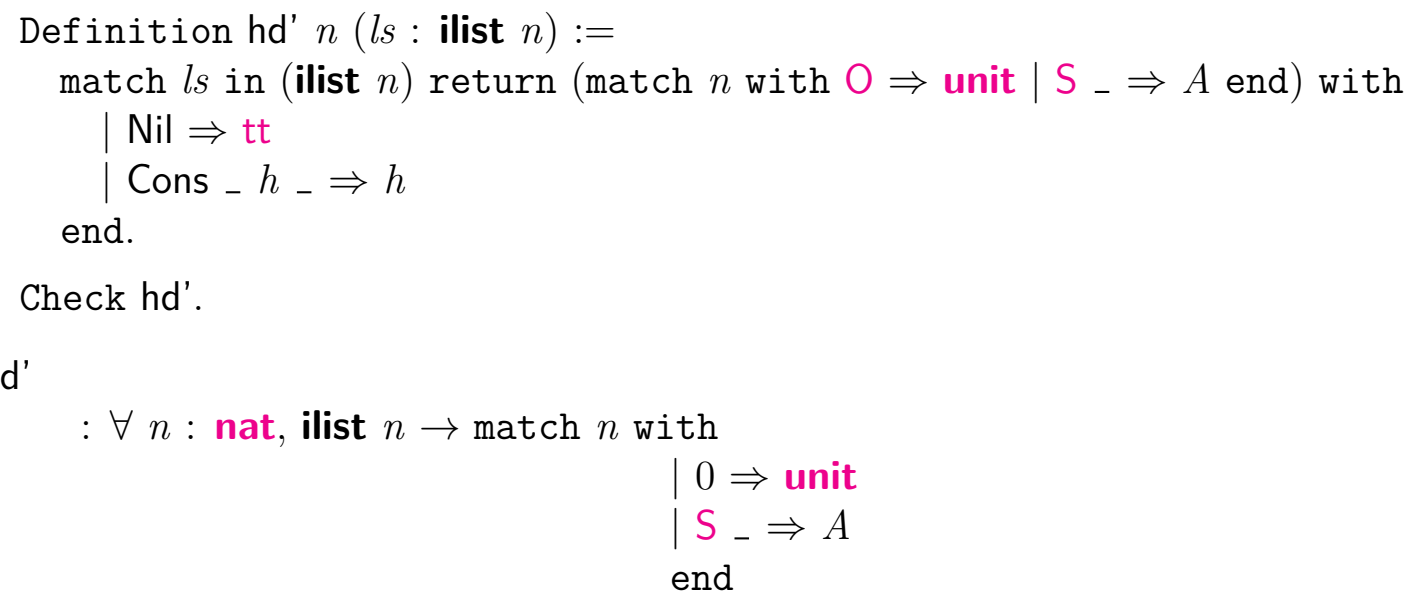

Definition hd $n(l s:$ ilist $(\mathrm{S} n)): A:=\mathrm{hd}^{\prime} l s$.

End ilist.

We annotate our main match with a type that is itself a match. We write that the function hd' returns unit when the list is empty and returns the carried type $A$ in all other cases. In the definition of hd, we just call hd'. Because the index of $l s$ is known to be nonzero, the type checker reduces the match in the type of hd' to $A$. 


\subsection{The One Rule of Dependent Pattern Matching in Coq}

The rest of this chapter will demonstrate a few other elegant applications of dependent types in Coq. Readers encountering such ideas for the first time often feel overwhelmed, concluding that there is some magic at work whereby Coq sometimes solves the halting problem for the programmer and sometimes does not, applying automated program understanding in a way far beyond what is found in conventional languages. The point of this section is to cut off that sort of thinking right now! Dependent type-checking in Coq follows just a few algorithmic rules. Chapters 10 and 12 introduce many of those rules more formally, and the main additional rule is centered on dependent pattern matching of the kind we met in the previous section.

A dependent pattern match is a match expression where the type of the overall match is a function of the value and/or the type of the discriminee, the value being matched on. In other words, the match type depends on the discriminee.

When exactly will Coq accept a dependent pattern match as well-typed? Some other dependently typed languages employ fancy decision procedures to determine when programs satisfy their very expressive types. The situation in Coq is just the opposite. Only very straightforward symbolic rules are applied. Such a design choice has its drawbacks, as it forces programmers to do more work to convince the type checker of program validity. However, the great advantage of a simple type checking algorithm is that its action on invalid programs is easier to understand!

We come now to the one rule of dependent pattern matching in Coq. A general dependent pattern match assumes this form (with unnecessary parentheses included to make the syntax easier to parse):

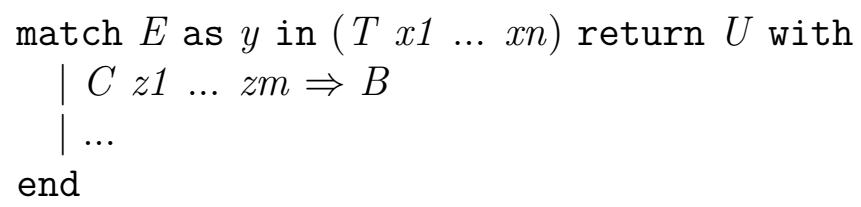

The discriminee is a term $E$, a value in some inductive type family $T$, which takes $n$ arguments. An as clause binds the name $y$ to refer to the discriminee $E$. An in clause binds an explicit name $x i$ for the $i$ th argument passed to $T$ in the type of $E$.

We bind these new variables $y$ and $x i$ so that they may be referred to in $U$, a type given in the return clause. The overall type of the match will be $U$, with $E$ substituted for $y$, and with each $x i$ substituted by the actual argument appearing in that position within $E$ 's type.

In general, each case of a match may have a pattern built up in several layers from the constructors of various inductive type families. To keep this exposition simple, we will focus on patterns that are just single applications of inductive type constructors to lists of variables. Coq actually compiles the more general kind of pattern matching into this more restricted kind automatically, so understanding the typing of match requires understanding the typing of matches lowered to match one constructor at a time.

The last piece of the typing rule tells how to type-check a match case. A generic con- 
structor application $C z 1 \ldots z m$ has some type $T x 1^{\prime} \ldots x n$, an application of the type family used in $E$ 's type, probably with occurrences of the $z i$ variables. From here, a simple recipe determines what type we will require for the case body $B$. The type of $B$ should be $U$ with the following two substitutions applied: we replace $y$ (the as clause variable) with $C z 1 \ldots z m$, and we replace each $x i$ (the in clause variables) with $x i$ '. In other words, we specialize the result type based on what we learn based on which pattern has matched the discriminee.

This is an exhaustive description of the ways to specify how to take advantage of which pattern has matched! No other mechanisms come into play. For instance, there is no way to specify that the types of certain free variables should be refined based on which pattern has matched. In the rest of the book, we will learn design patterns for achieving similar effects, where each technique leads to an encoding only in terms of in, as, and return clauses.

A few details have been omitted above. In Chapter 3, we learned that inductive type families may have both parameters and regular arguments. Within an in clause, a parameter position must have the wildcard _ written, instead of a variable. (In general, Coq uses wildcard _'s either to indicate pattern variables that will not be mentioned again or to indicate positions where we would like type inference to infer the appropriate terms.) Furthermore, recent Coq versions are adding more and more heuristics to infer dependent match annotations in certain conditions. The general annotation inference problem is undecidable, so there will always be serious limitations on how much work these heuristics can do. When in doubt about why a particular dependent match is failing to type-check, add an explicit return annotation! At that point, the mechanical rule sketched in this section will provide a complete account of "what the type checker is thinking." Be sure to avoid the common pitfall of writing a return annotation that does not mention any variables bound by in or as; such a match will never refine typing requirements based on which pattern has matched. (One simple exception to this rule is that, when the discriminee is a variable, that same variable may be treated as if it were repeated as an as clause.)

\subsection{A Tagless Interpreter}

A favorite example for motivating the power of functional programming is implementation of a simple expression language interpreter. In ML and Haskell, such interpreters are often implemented using an algebraic datatype of values, where at many points it is checked that a value was built with the right constructor of the value type. With dependent types, we can implement a tagless interpreter that both removes this source of runtime inefficiency and gives us more confidence that our implementation is correct.

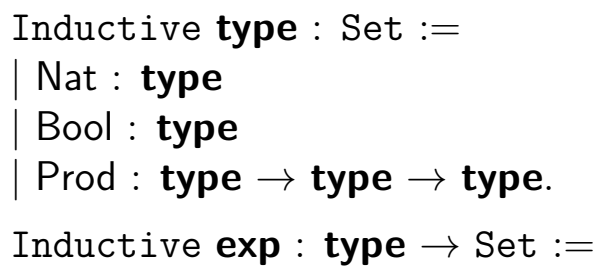




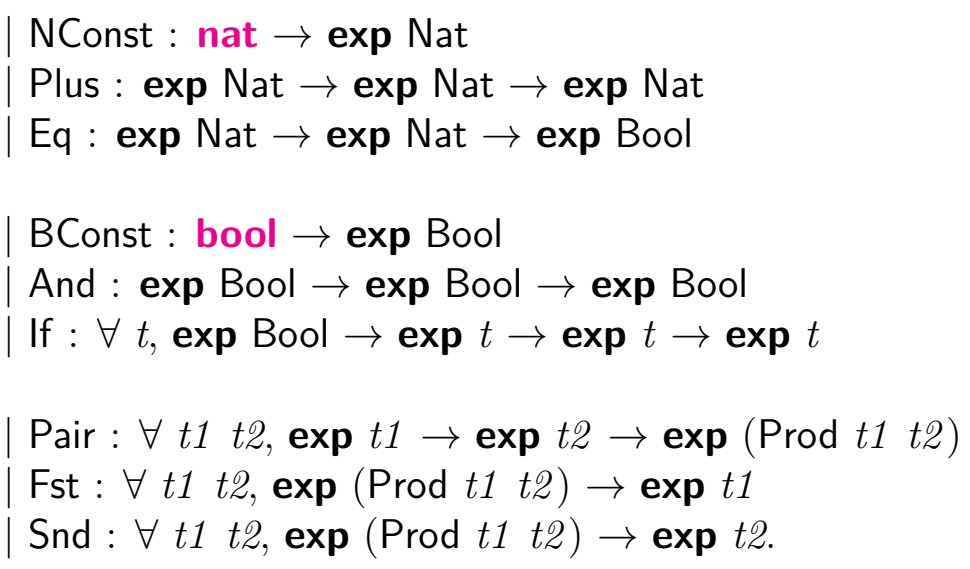

We have a standard algebraic datatype type, defining a type language of naturals, Booleans, and product (pair) types. Then we have the indexed inductive type exp, where the argument to exp tells us the encoded type of an expression. In effect, we are defining the typing rules for expressions simultaneously with the syntax.

We can give types and expressions semantics in a new style, based critically on the chance for type-level computation.

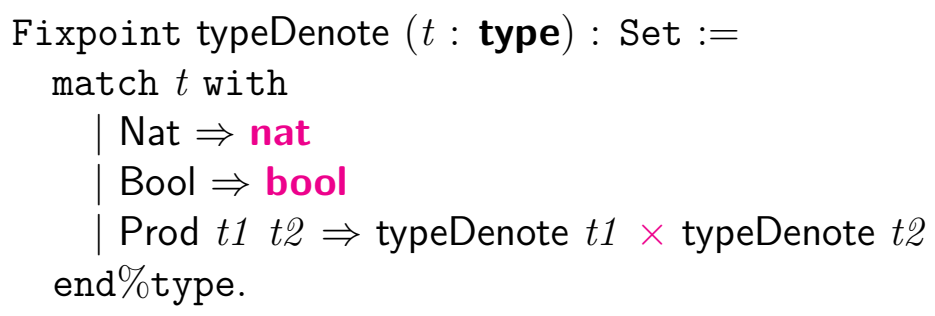

The typeDenote function compiles types of our object language into "native" Coq types. It is deceptively easy to implement. The only new thing we see is the \%type annotation, which tells Coq to parse the match expression using the notations associated with types. Without this annotation, the $\times$ would be interpreted as multiplication on naturals, rather than as the product type constructor. The token type is one example of an identifier bound to a notation scope delimiter. In this book, we will not go into more detail on notation scopes, but the Coq manual can be consulted for more information.

We can define a function expDenote that is typed in terms of typeDenote.

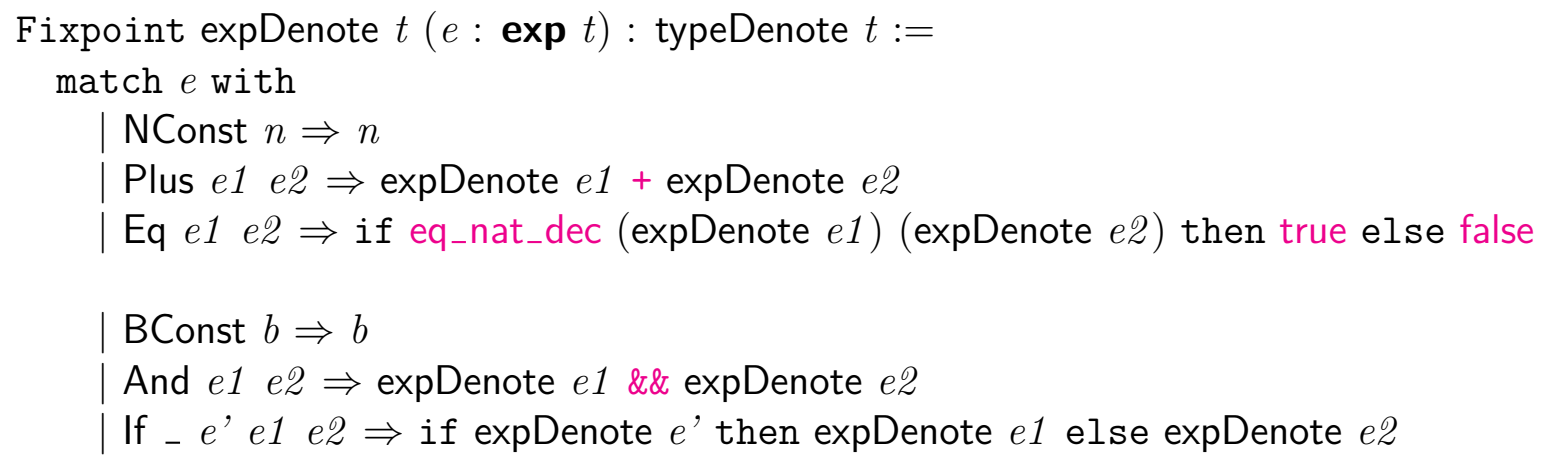




$$
\begin{aligned}
& \mid \text { Pair }-e 1 e^{2} \Rightarrow(\operatorname{expDenote} e 1, \text { expDenote } e 2) \\
& \left.\mid \text { Fst }-e^{\prime} \Rightarrow \text { fst (expDenote } e^{\prime}\right) \\
& \left.\mid \text { Snd } e^{\prime} \Rightarrow \text { snd (expDenote } e^{\prime}\right) \\
& \text { end. }
\end{aligned}
$$

Despite the fancy type, the function definition is routine. In fact, it is less complicated than what we would write in ML or Haskell 98, since we do not need to worry about pushing final values in and out of an algebraic datatype. The only unusual thing is the use of an expression of the form if $E$ then true else false in the Eq case. Remember that eq_nat_dec has a rich dependent type, rather than a simple Boolean type. Coq's native if is overloaded to work on a test of any two-constructor type, so we can use if to build a simple Boolean from the sumbool that eq_nat_dec returns.

We can implement our old favorite, a constant folding function, and prove it correct. It will be useful to write a function pairOut that checks if an exp of Prod type is a pair, returning its two components if so. Unsurprisingly, a first attempt leads to a type error.

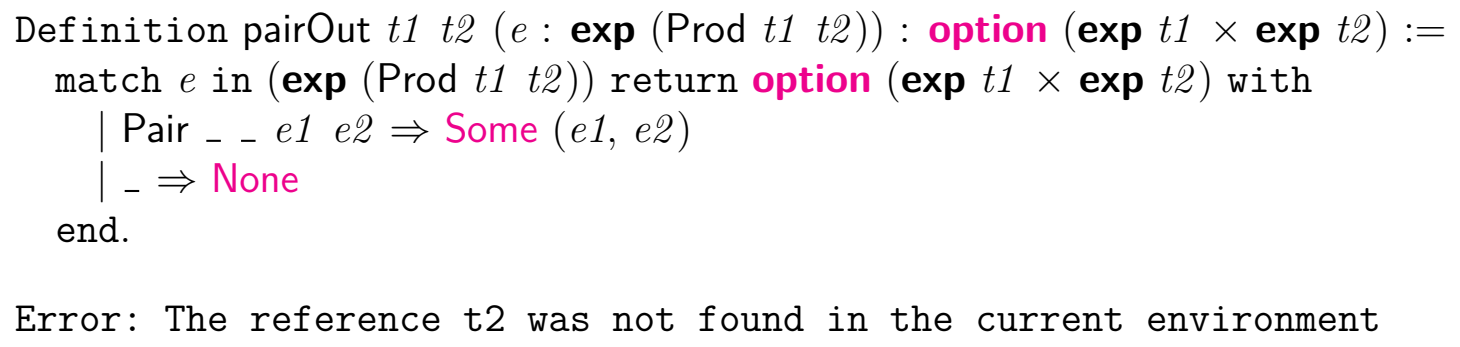

Error: The reference t2 was not found in the current environment

We run again into the problem of not being able to specify non-variable arguments in in clauses. The problem would just be hopeless without a use of an in clause, though, since the result type of the match depends on an argument to exp. Our solution will be to use a more general type, as we did for hd. First, we define a type-valued function to use in assigning a type to pairOut.

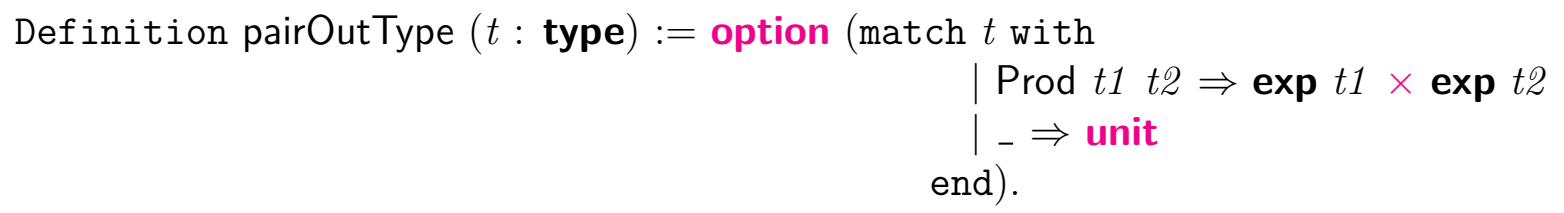

When passed a type that is a product, pairOutType returns our final desired type. On any other input type, pairOutType returns the harmless option unit, since we do not care about extracting components of non-pairs. Now pairOut is easy to write.

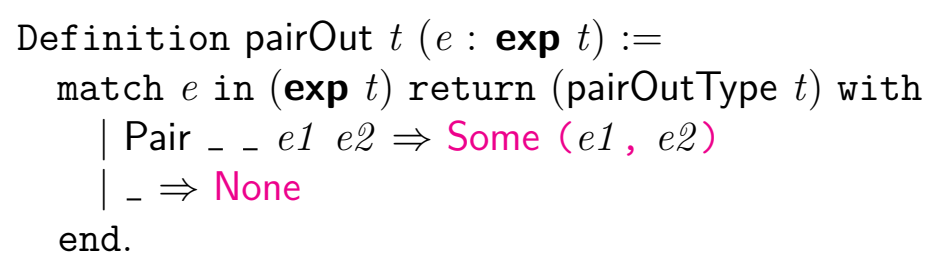

With pairOut available, we can write cfold in a straightforward way. There are really no surprises beyond that Coq verifies that this code has such an expressive type, given the 
small annotation burden. In some places, we see that Coq's match annotation inference is too smart for its own good, and we have to turn that inference off with explicit return clauses.

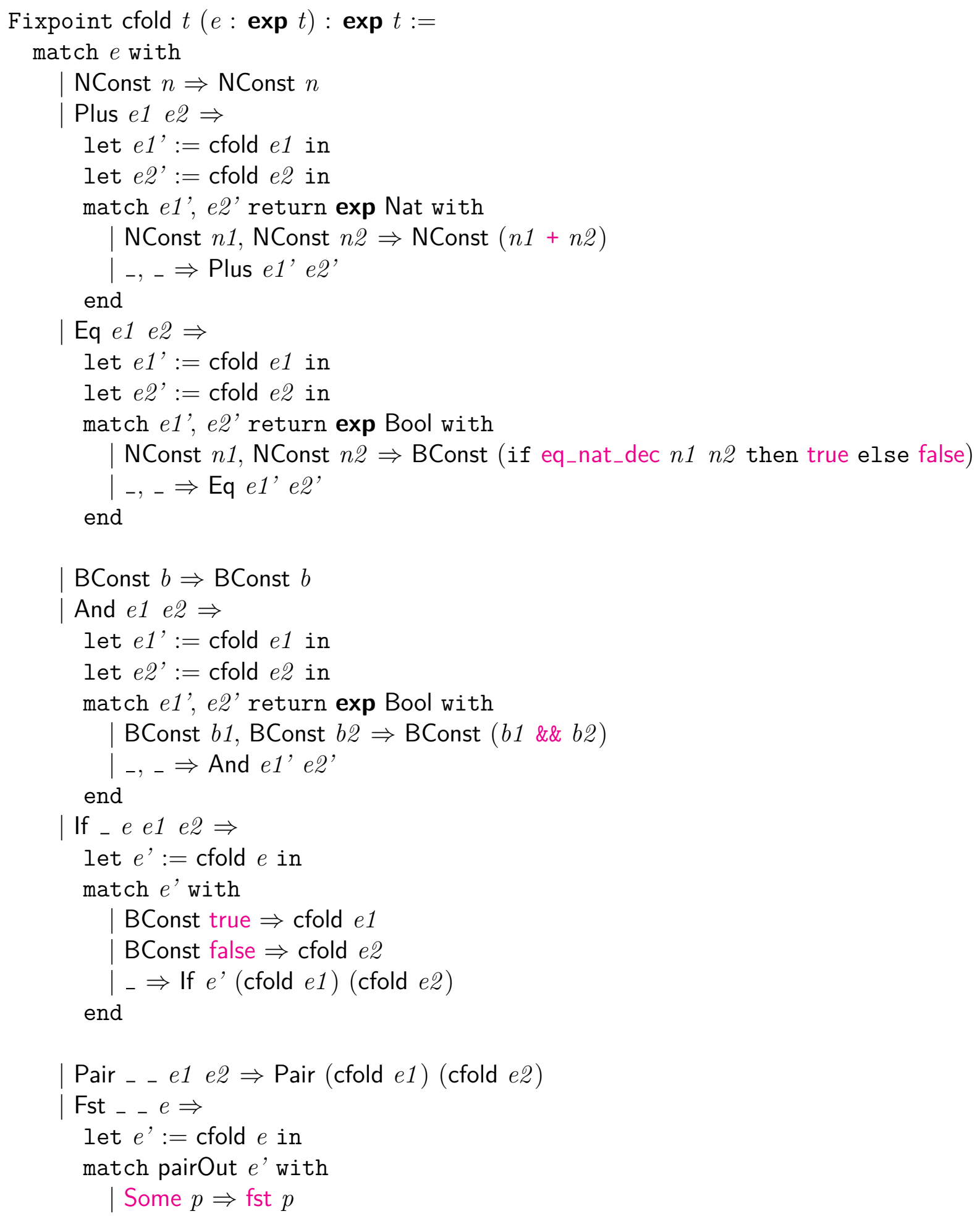




$$
\begin{aligned}
& \mid \text { None } \Rightarrow \text { Fst } e^{\prime} \\
& \text { end } \\
& \text { | Snd } \_-e \Rightarrow \\
& \text { let } e^{\prime}:=\text { cfold } e \text { in } \\
& \text { match pairOut } e^{\prime} \text { with } \\
& \text { | Some } p \Rightarrow \text { snd } p \\
& \mid \text { None } \Rightarrow \text { Snd } e \\
& \text { end }
\end{aligned}
$$

end.

The correctness theorem for cfold turns out to be easy to prove, once we get over one serious hurdle.

Theorem cfold_correct : $\forall t(e: \exp t)$, expDenote $e=\operatorname{expDenote}($ cfold $e)$. induction $e$; crush.

The first remaining subgoal is:

$$
\begin{aligned}
& \text { expDenote }(\text { cfold } e 1)+\operatorname{expDenote}(\text { cfold } e \mathscr{2})= \\
& \text { expDenote } \\
& \text { match cfold } e 1 \text { with } \\
& \text { | NConst } n 1 \Rightarrow \\
& \text { match cfold } e 2 \text { with } \\
& \text { NConst } n 2 \Rightarrow \text { NConst }(n 1+n 2) \\
& \text { | Plus _ _ } \Rightarrow \text { Plus (cfold e1) (cfold e2) } \\
& \text { Eq _ _ } \Rightarrow \text { Plus (cfold e1) (cfold e2) } \\
& \text { | BConst _ } \Rightarrow \text { Plus (cfold e1) (cfold e2) } \\
& \text { | And _ _ } \Rightarrow \text { Plus (cfold e1) (cfold e2) } \\
& \text { If _ _ _ _ } \Rightarrow \text { Plus (cfold } e 1 \text { ) (cfold e2) } \\
& \text { | Pair _ . _ } \Rightarrow \text { Plus (cfold e1) (cfold e2) } \\
& \text { | Fst _ _ _ } \Rightarrow \text { Plus (cfold e1) (cfold e2) } \\
& \text { Snd _ _ } \Rightarrow \text { Plus (cfold e1) (cfold e2) } \\
& \text { end } \\
& \text { | Plus _ _ } \Rightarrow \text { Plus (cfold e1) (cfold e2) } \\
& \text { | Eq _ _ P Plus (cfold e1) (cfold e2) } \\
& \text { | BConst _ } \Rightarrow \text { Plus (cfold e1) (cfold e2) } \\
& \text { | And _ _ } \Rightarrow \text { Plus (cfold e1) (cfold e2) } \\
& \text { | If _ _ _ _ } \Rightarrow \text { Plus (cfold e1) (cfold e2) } \\
& \text { | Pair _ . _ _ Plus (cfold e1) (cfold e2) } \\
& \text { | Fst _ _ } \Rightarrow \text { Plus (cfold e1) (cfold e2) } \\
& \text { | Snd _ _ } \Rightarrow \text { Plus (cfold e1) (cfold e2) } \\
& \text { end }
\end{aligned}
$$

We would like to do a case analysis on cfold $e 1$, and we attempt to do so in the way that 
has worked so far.

destruct (cfold e1).

User error: e1 is used in hypothesis e

Coq gives us another cryptic error message. Like so many others, this one basically means that Coq is not able to build some proof about dependent types. It is hard to generate helpful and specific error messages for problems like this, since that would require some kind of understanding of the dependency structure of a piece of code. We will encounter many examples of case-specific tricks for recovering from errors like this one.

For our current proof, we can use a tactic dep_destruct defined in the book's CpdtTactics module. General elimination/inversion of dependently typed hypotheses is undecidable, as witnessed by a simple reduction from the known-undecidable problem of higher-order unification, which has come up a few times already. The tactic dep_destruct makes a best effort to handle some common cases, relying upon the more primitive dependent destruction tactic that comes with Coq. In a future chapter, we will learn about the explicit manipulation of equality proofs that is behind dependent destruction's implementation, but for now, we treat it as a useful black box. (In Chapter 12, we will also see how dependent destruction forces us to make a larger philosophical commitment about our logic than we might like, and we will see some workarounds.)

dep_destruct (cfold e1).

This successfully breaks the subgoal into 5 new subgoals, one for each constructor of exp that could produce an exp Nat. Note that dep_destruct is successful in ruling out the other cases automatically, in effect automating some of the work that we have done manually in implementing functions like hd and pairOut.

This is the only new trick we need to learn to complete the proof. We can back up and give a short, automated proof (which again is safe to skip and uses Ltac features not introduced yet).

Restart.

induction $e$; crush;

repeat (match goal with

$\mid\left[\vdash\right.$ context [match cfold ?E with NConst _ $\Rightarrow_{-} \mid{ }_{-} \Rightarrow_{-}$end $\left.]\right] \Rightarrow$ dep_destruct (cfold $E$ )

$\mid\left[\vdash\right.$ context [match pairOut (cfold ?E) with Some _ $\Rightarrow_{-}$ | None $\Rightarrow$ _ end] ] $\Rightarrow$

dep_destruct (cfold $E$ )

$\mid\left[\vdash(\right.$ if ? E then _ else _ $\left.)=_{-}\right] \Rightarrow$ destruct $E$

end; (crush).

Qed.

With this example, we get a first taste of how to build automated proofs that adapt automatically to changes in function definitions. 


\subsection{Dependently Typed Red-Black Trees}

Red-black trees are a favorite purely functional data structure with an interesting invariant. We can use dependent types to guarantee that operations on red-black trees preserve the invariant. For simplicity, we specialize our red-black trees to represent sets of nats.

Inductive color: Set $:=$ Red $\mid$ Black.

Inductive rbtree : color $\rightarrow$ nat $\rightarrow$ Set $:=$

| Leaf : rbtree Black 0

RedNode : $\forall n$, rbtree Black $n \rightarrow$ nat $\rightarrow$ rbtree Black $n \rightarrow$ rbtree Red $n$ | BlackNode : $\forall c 1$ c2 $n$, rbtree $c 1 n \rightarrow$ nat $\rightarrow$ rbtree $c^{2} n \rightarrow$ rbtree Black (S $\left.n\right)$.

A value of type rbtree $c d$ is a red-black tree whose root has color $c$ and that has black depth $d$. The latter property means that there are exactly $d$ black-colored nodes on any path from the root to a leaf.

At first, it can be unclear that this choice of type indices tracks any useful property. To convince ourselves, we will prove that every red-black tree is balanced. We will phrase our theorem in terms of a depth calculating function that ignores the extra information in the types. It will be useful to parameterize this function over a combining operation, so that we can re-use the same code to calculate the minimum or maximum height among all paths from root to leaf.

Require Import Max Min.

Section depth.

Variable $f:$ nat $\rightarrow$ nat $\rightarrow$ nat.

Fixpoint depth $c n(t$ : rbtree $c n):$ nat := match $t$ with

Leaf $\Rightarrow 0$

RedNode $-t 1$ - t2 $\Rightarrow \mathrm{S}(f($ depth $t 1)$ (depth $t 2))$

| BlackNode _ _ t1 _ t2 $\Rightarrow \mathrm{S}(f($ depth $t 1)$ (depth $t 2))$

end.

End depth.

Our proof of balanced-ness decomposes naturally into a lower bound and an upper bound. We prove the lower bound first. Unsurprisingly, a tree's black depth provides such a bound on the minimum path length. We use the richly typed procedure min_dec to do case analysis on whether $\min X Y$ equals $X$ or $Y$.

Check min_dec.

min_dec

$: \forall n m:$ nat, $\{\min n m=n\}+\{\min n m=m\}$

Theorem depth_min : $\forall c n(t:$ rbtree $c n)$, depth $\min t \geq n$.

induction $t$; crush;

match goal with 


$$
\mid[\vdash \operatorname{context}[\min ? X ? Y]] \Rightarrow \operatorname{destruct}\left(\min \_d e c X Y\right)
$$

end; crush.

Qed.

There is an analogous upper-bound theorem based on black depth. Unfortunately, a symmetric proof script does not suffice to establish it.

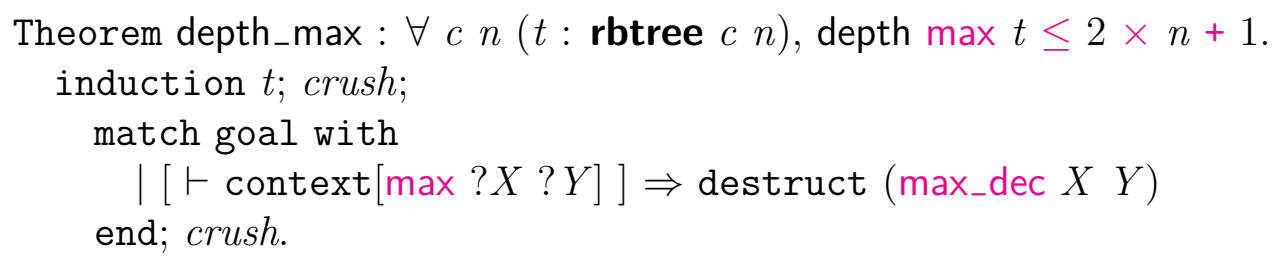

Two subgoals remain. One of them is:

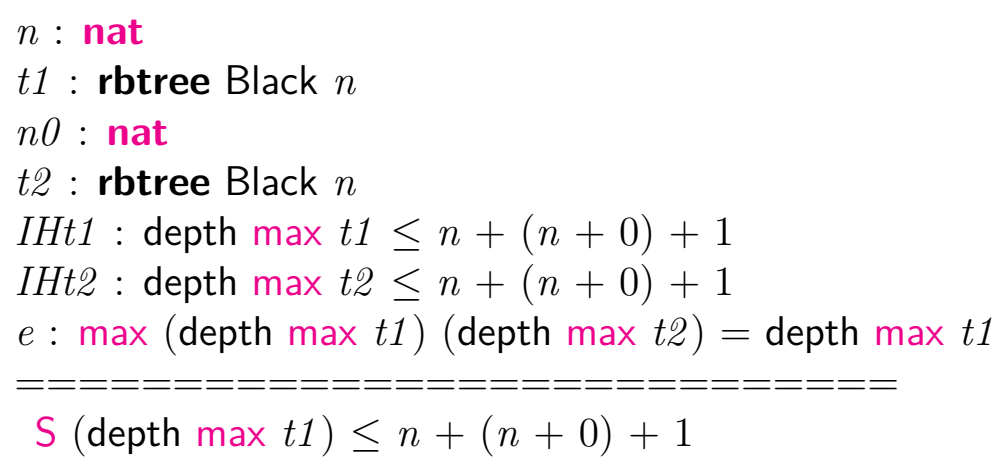

We see that IHt1 is almost the fact we need, but it is not quite strong enough. We will need to strengthen our induction hypothesis to get the proof to go through.

Abort.

In particular, we prove a lemma that provides a stronger upper bound for trees with black root nodes. We got stuck above in a case about a red root node. Since red nodes have only black children, our IH strengthening will enable us to finish the proof.

Lemma depth_max' $: \forall c n(t:$ rbtree $c n)$, match $c$ with

induction $t$; crush;

$$
\begin{aligned}
& \text { Red } \Rightarrow \text { depth max } t \leq 2 \times n+1 \\
& \text { Black } \Rightarrow \text { depth max } t \leq 2 \times n
\end{aligned}
$$
end.

match goal with

$\mid[\vdash \operatorname{context}[\max ? X ? Y]] \Rightarrow \operatorname{destruct}\left(\max _{-} \operatorname{dec} X Y\right)$

end; crush;

repeat (match goal with

$$
\begin{aligned}
& \left.\quad \mid H \text { : context }\left[\text { match } ? C \text { with } \operatorname{Red} \Rightarrow{ }_{-} \mid \text {Black } \Rightarrow{ }_{-} \text {end }\right] \vdash_{-}\right] \Rightarrow \\
& \quad \text { destruct } C \\
& \text { end; } \text { crush }) .
\end{aligned}
$$

Qed. 
The original theorem follows easily from the lemma. We use the tactic generalize $p f$, which, when $p f$ proves the proposition $P$, changes the goal from $Q$ to $P \rightarrow Q$. This transformation is useful because it makes the truth of $P$ manifest syntactically, so that automation machinery can rely on $P$, even if that machinery is not smart enough to establish $P$ on its own.

Theorem depth_max : $\forall c n(t:$ rbtree $c n)$, depth $\max t \leq 2 \times n+1$.

intros; generalize (depth_max' $t$ ); destruct $c$; crush. Qed.

The final balance theorem establishes that the minimum and maximum path lengths of any tree are within a factor of two of each other.

Theorem balanced: $\forall c n(t:$ rbtree $c n), 2 \times$ depth min $t+1 \geq$ depth max $t$.

intros; generalize (depth_min $t$ ); generalize (depth_max $t$ ); crush. Qed.

Now we are ready to implement an example operation on our trees, insertion. Insertion can be thought of as breaking the tree invariants locally but then rebalancing. In particular, in intermediate states we find red nodes that may have red children. The type rtree captures the idea of such a node, continuing to track black depth as a type index.

Inductive rtree : nat $\rightarrow$ Set $:=$

| RedNode' : $\forall c 1$ c2 $n$, rbtree $c 1 n \rightarrow$ nat $\rightarrow$ rbtree $c^{2} n \rightarrow$ rtree $n$.

Before starting to define insert, we define predicates capturing when a data value is in the set represented by a normal or possibly invalid tree.

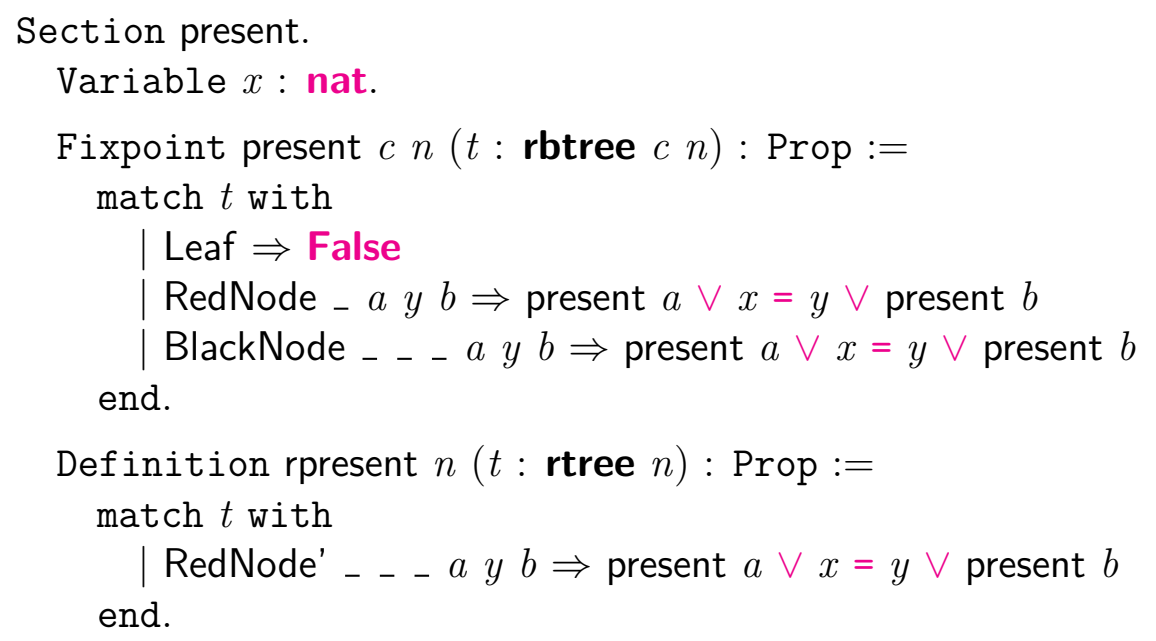

Insertion relies on two balancing operations. It will be useful to give types to these operations using a relative of the subset types from last chapter. While subset types let us pair a value with a proof about that value, here we want to pair a value with another non-proof dependently typed value. The sigT type fills this role.

Locate " $\{-:-\&-\}$ ". 
Notation Scope

" $\{\mathrm{x}: \mathrm{A} \& \mathrm{P}\} ":=\operatorname{sigT}($ fun $x: A \Rightarrow P)$

Print sigT.

Inductive sigT $(A:$ Type $)(P: A \rightarrow$ Type $):$ Type $:=$

existT $: \forall x: A, P x \rightarrow \operatorname{sigT} P$

It will be helpful to define a concise notation for the constructor of sigT.

Notation " $\{<\mathrm{x}\rangle\}$ " := (existT $\ldots x)$.

Each balance function is used to construct a new tree whose keys include the keys of two input trees, as well as a new key. One of the two input trees may violate the red-black alternation invariant (that is, it has an rtree type), while the other tree is known to be valid. Crucially, the two input trees have the same black depth.

A balance operation may return a tree whose root is of either color. Thus, we use a sigT type to package the result tree with the color of its root. Here is the definition of the first balance operation, which applies when the possibly invalid rtree belongs to the left of the valid rbtree.

A quick word of encouragement: After writing this code, even I do not understand the precise details of how balancing works! I consulted Chris Okasaki's paper "Red-Black Trees in a Functional Setting" [30] and transcribed the code to use dependent types. Luckily, the details are not so important here; types alone will tell us that insertion preserves balancedness, and we will prove that insertion produces trees containing the right keys.

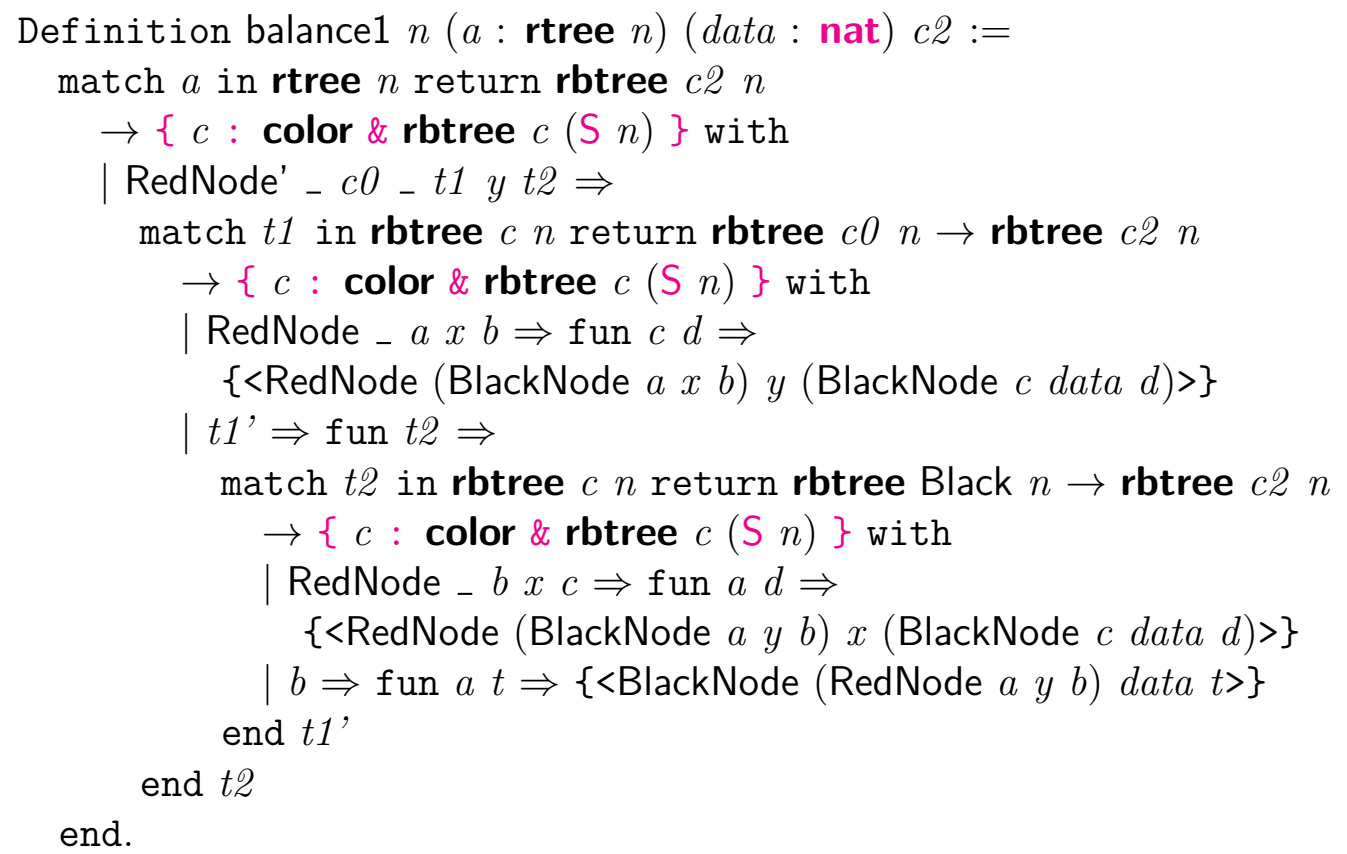

We apply a trick that I call the convoy pattern. Recall that match annotations only make it possible to describe a dependence of a match result type on the discriminee. There is no automatic refinement of the types of free variables. However, it is possible to effect such a 
refinement by finding a way to encode free variable type dependencies in the match result type, so that a return clause can express the connection.

In particular, we can extend the match to return functions over the free variables whose types we want to refine. In the case of balance1, we only find ourselves wanting to refine the type of one tree variable at a time. We match on one subtree of a node, and we want the type of the other subtree to be refined based on what we learn. We indicate this with a return clause starting like rbtree ${ }_{-} n \rightarrow \ldots$, where $n$ is bound in an in pattern. Such a match expression is applied immediately to the "old version" of the variable to be refined, and the type checker is happy.

Here is the symmetric function balance2, for cases where the possibly invalid tree appears on the right rather than on the left.

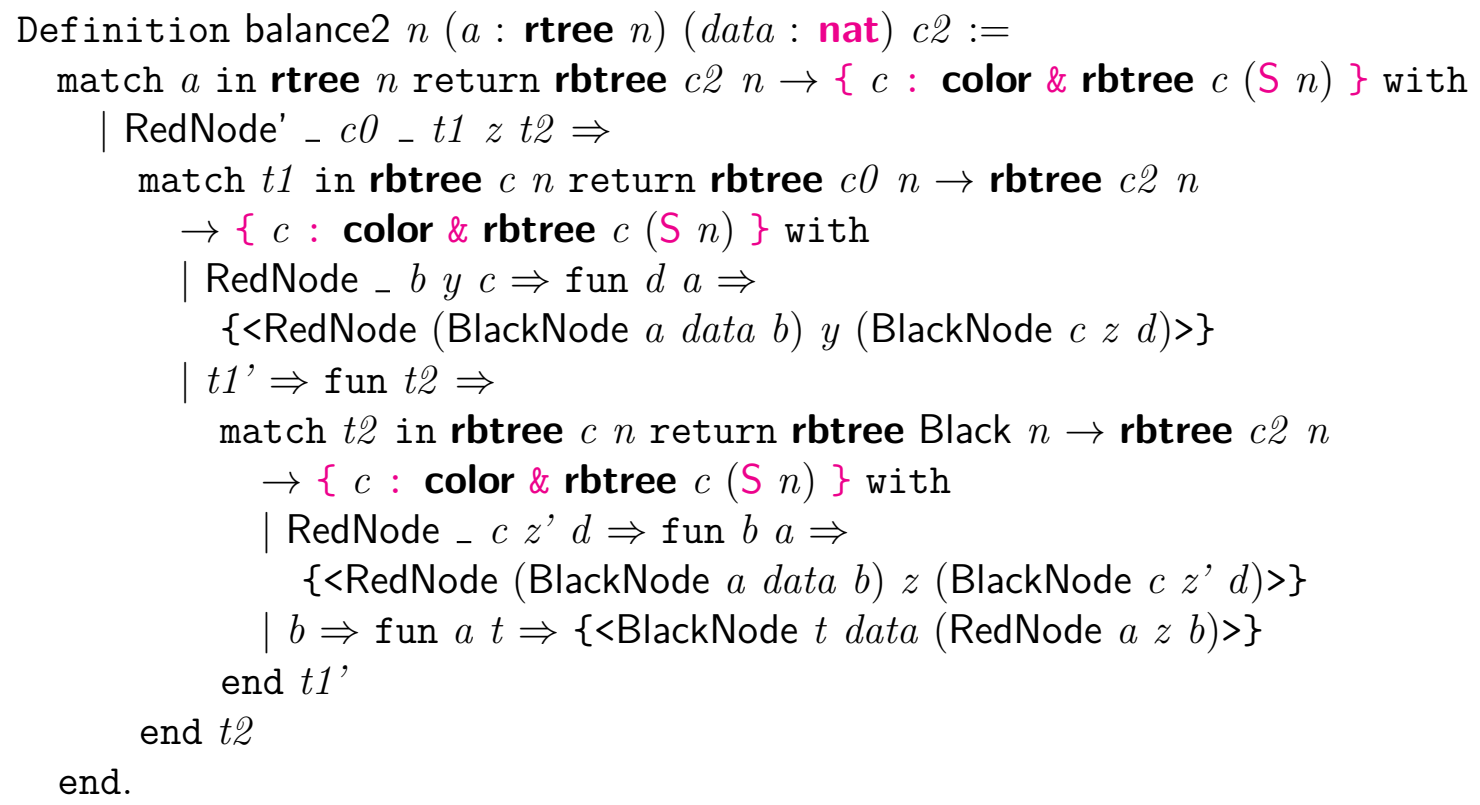

Now we are almost ready to get down to the business of writing an insert function. First, we enter a section that declares a variable $x$, for the key we want to insert.

Section insert.

Variable $x$ : nat.

Most of the work of insertion is done by a helper function ins, whose return types are expressed using a type-level function insResult.

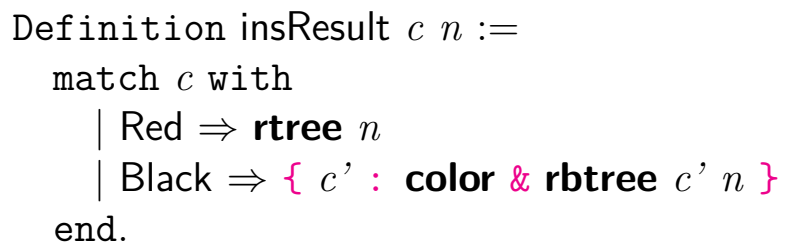

That is, inserting into a tree with root color $c$ and black depth $n$, the variety of tree we get out depends on $c$. If we started with a red root, then we get back a possibly invalid tree of depth $n$. If we started with a black root, we get back a valid tree of depth $n$ with a root 
node of an arbitrary color.

Here is the definition of ins. Again, we do not want to dwell on the functional details.

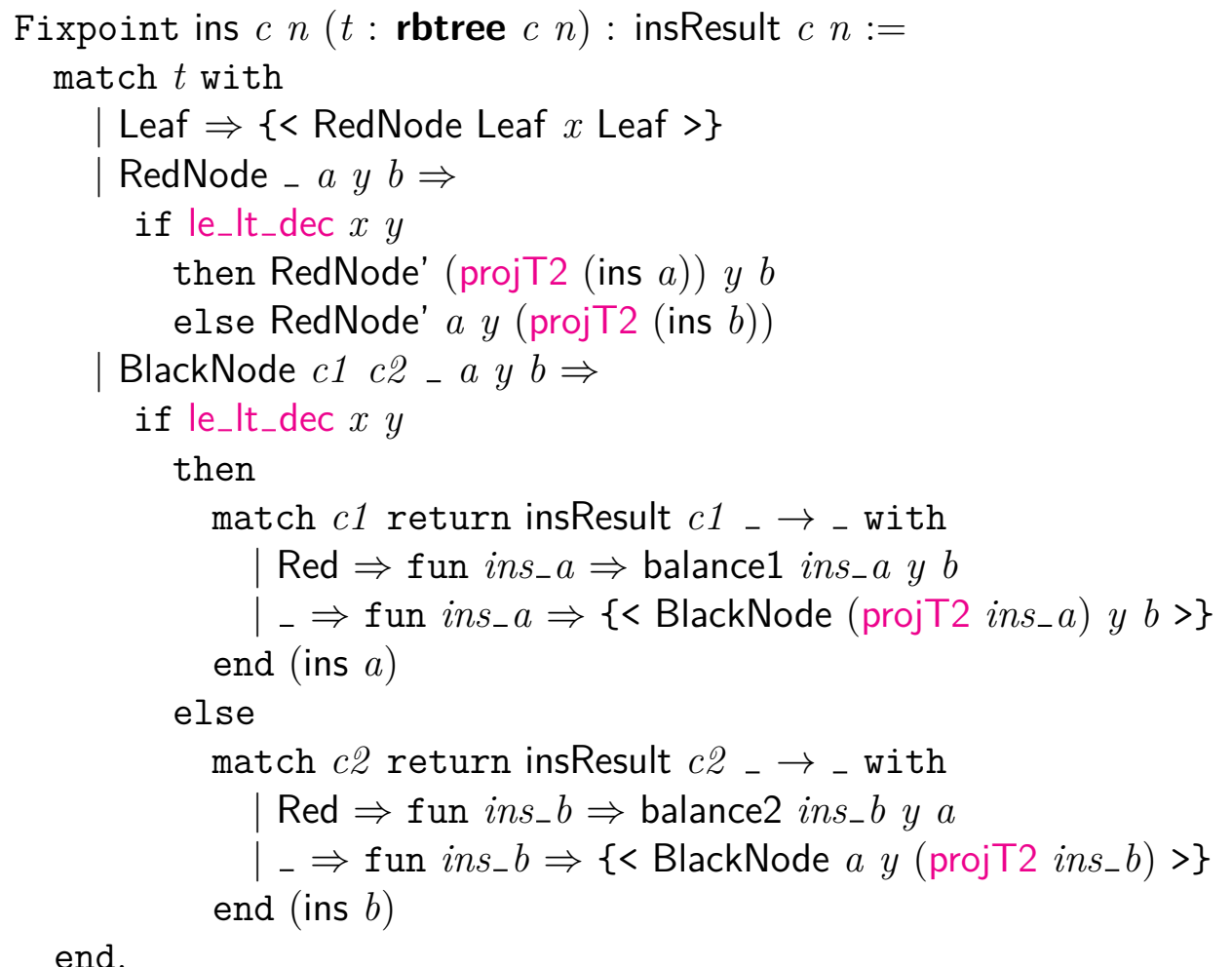

The one new trick is a variation of the convoy pattern. In each of the last two pattern matches, we want to take advantage of the typing connection between the trees $a$ and $b$. We might naïvely apply the convoy pattern directly on $a$ in the first match and on $b$ in the second. This satisfies the type checker per se, but it does not satisfy the termination checker. Inside each match, we would be calling ins recursively on a locally bound variable. The termination checker is not smart enough to trace the dataflow into that variable, so the checker does not know that this recursive argument is smaller than the original argument. We make this fact clearer by applying the convoy pattern on the result of a recursive call, rather than just on that call's argument.

Finally, we are in the home stretch of our effort to define insert. We just need a few more definitions of non-recursive functions. First, we need to give the final characterization of insert's return type. Inserting into a red-rooted tree gives a black-rooted tree where black depth has increased, and inserting into a black-rooted tree gives a tree where black depth has stayed the same and where the root is an arbitrary color.

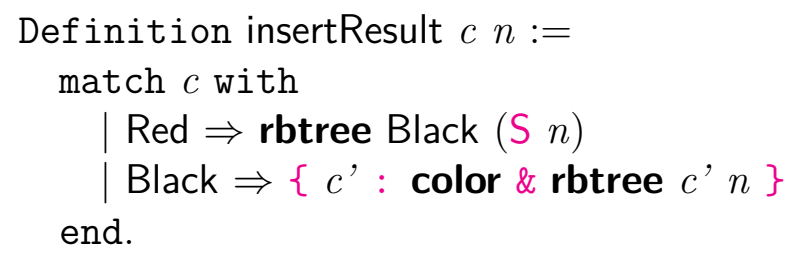


A simple clean-up procedure translates insResults into insertResults.

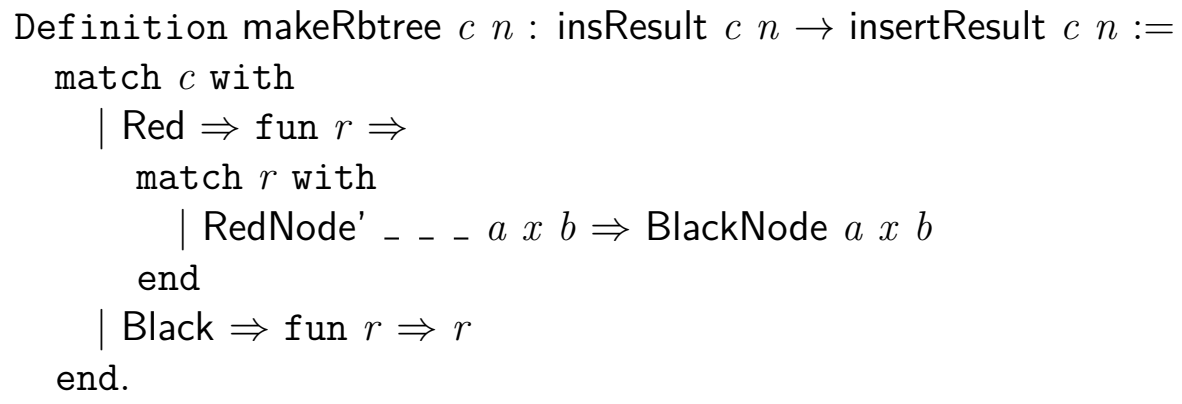

We modify Coq's default choice of implicit arguments for makeRbtree, so that we do not need to specify the $c$ and $n$ arguments explicitly in later calls.

\section{Arguments makeRbtree $\left[\begin{array}{ll}c & n\end{array}\right]$..}

Finally, we define insert as a simple composition of ins and makeRbtree.

\section{Definition insert $c n(t:$ rbtree $c n)$ : insertResult $c n:=$} makeRbtree (ins $t$ ).

As we noted earlier, the type of insert guarantees that it outputs balanced trees whose depths have not increased too much. We also want to know that insert operates correctly on trees interpreted as finite sets, so we finish this section with a proof of that fact.

Section present.

Variable $z$ : nat.

The variable $z$ stands for an arbitrary key. We will reason about $z$ 's presence in particular trees. As usual, outside the section the theorems we prove will quantify over all possible keys, giving us the facts we wanted.

We start by proving the correctness of the balance operations. It is useful to define a custom tactic present_balance that encapsulates the reasoning common to the two proofs. We use the keyword Ltac to assign a name to a proof script. This particular script just iterates between crush and identification of a tree that is being pattern-matched on and should be destructed.

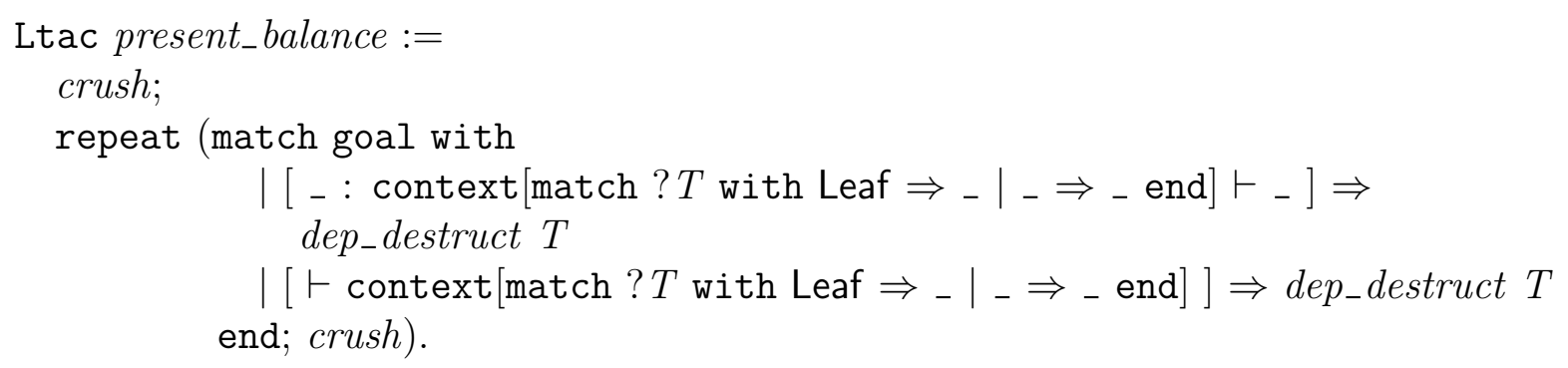

The balance correctness theorems are simple first-order logic equivalences, where we use the function projT2 to project the payload of a sigT value.

\footnotetext{
Lemma present_balance1 : $\forall n(a:$ rtree $n)(y:$ nat $)$ c2 $(b:$ rbtree $c \mathscr{2} n)$, present $z$ (projT2 (balance1 a y $b)$ )
} 
$\leftrightarrow$ rpresent $z a \vee z=y \vee$ present $z b$.

destruct $a$; present_balance.

Qed.

Lemma present_balance2 $: \forall n(a:$ rtree $n)(y:$ nat $) c 2(b:$ rbtree $c \mathscr{2} n)$, present $z($ projT2 (balance2 $a$ l $y$ b) )

$\leftrightarrow$ rpresent $z a \vee z=y \vee$ present $z b$.

destruct $a$; present_balance.

Qed.

To state the theorem for ins, it is useful to define a new type-level function, since ins returns different result types based on the type indices passed to it. Recall that $x$ is the section variable standing for the key we are inserting.

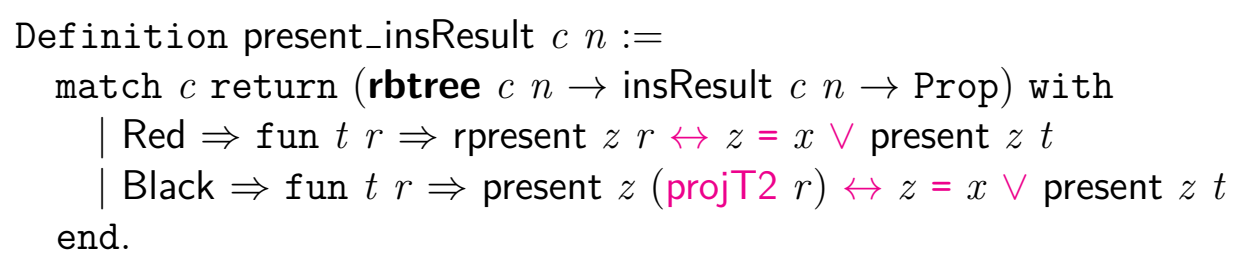

Now the statement and proof of the ins correctness theorem are straightforward, if verbose. We proceed by induction on the structure of a tree, followed by finding case analysis opportunities on expressions we see being analyzed in if or match expressions. After that, we pattern-match to find opportunities to use the theorems we proved about balancing. Finally, we identify two variables that are asserted by some hypothesis to be equal, and we use that hypothesis to replace one variable with the other everywhere.

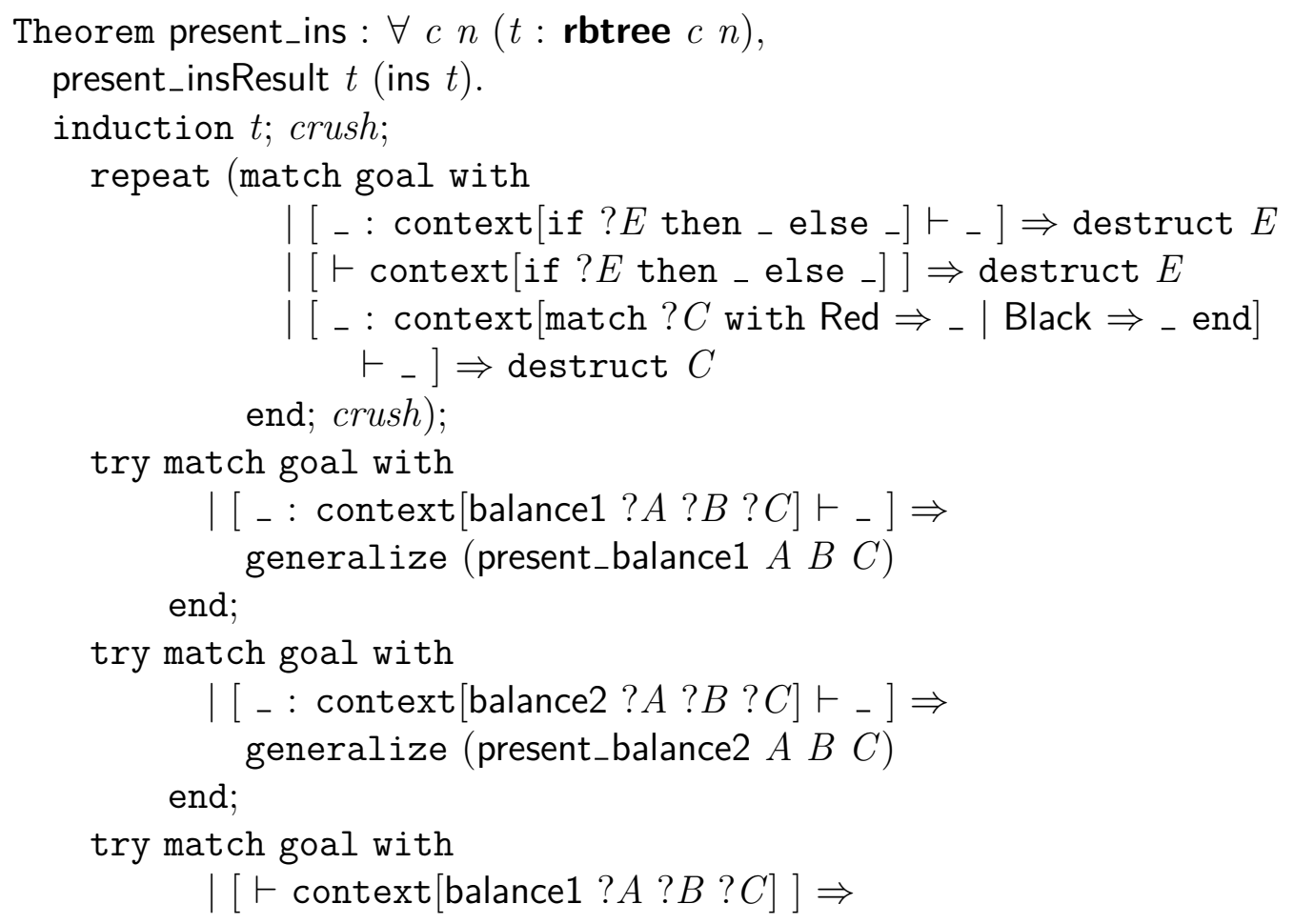




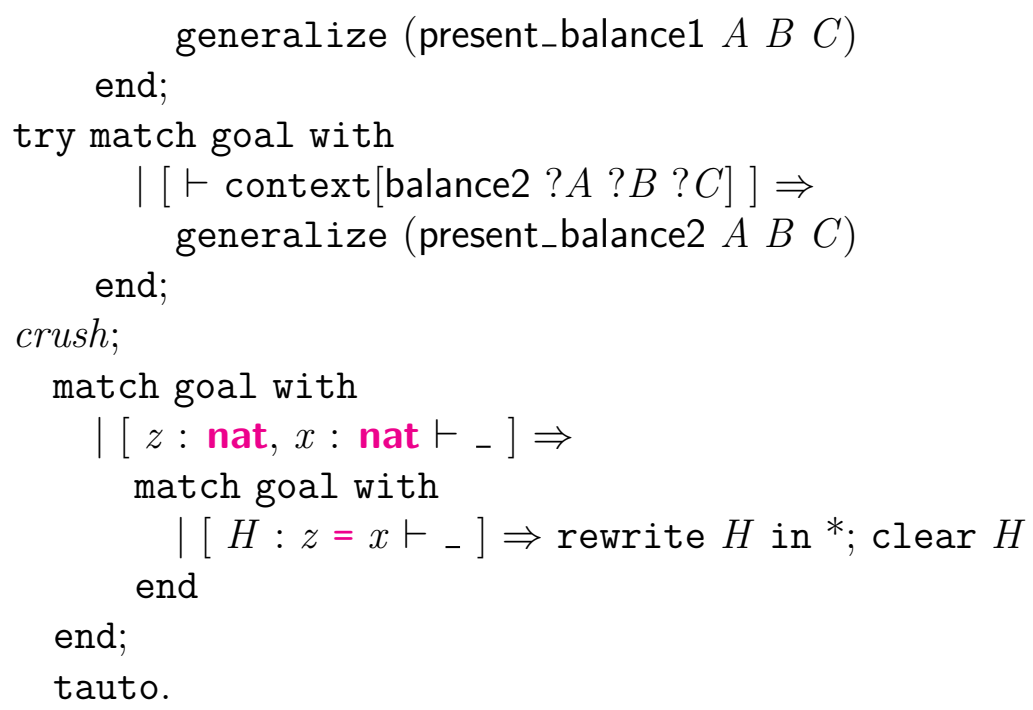

Qed.

The hard work is done. The most readable way to state correctness of insert involves splitting the property into two color-specific theorems. We write a tactic to encapsulate the reasoning steps that work to establish both facts.

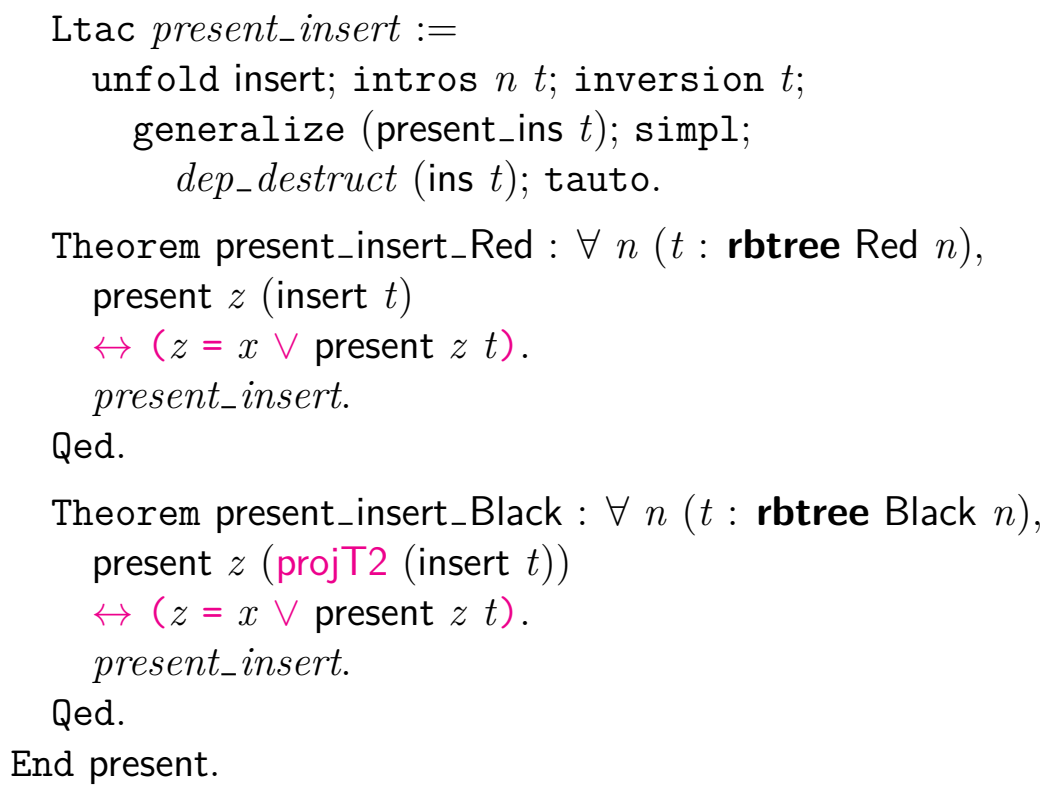

We can generate executable OCaml code with the command Recursive Extraction insert, which also automatically outputs the OCaml versions of all of insert's dependencies. In our previous extractions, we wound up with clean OCaml code. Here, we find uses of Obj.magic, OCaml's unsafe cast operator for tweaking the apparent type of an expression in an arbitrary way. Casts appear for this example because the return type of insert depends on the value of the function's argument, a pattern that OCaml cannot handle. Since Coq's type system is much more expressive than OCaml's, such casts are unavoidable in general. 
Since the OCaml type-checker is no longer checking full safety of programs, we must rely on Coq's extractor to use casts only in provably safe ways.

\subsection{A Certified Regular Expression Matcher}

Another interesting example is regular expressions with dependent types that express which predicates over strings particular regexps implement. We can then assign a dependent type to a regular expression matching function, guaranteeing that it always decides the string property that we expect it to decide.

Before defining the syntax of expressions, it is helpful to define an inductive type capturing the meaning of the Kleene star. That is, a string $s$ matches regular expression star $e$ if and only if $s$ can be decomposed into a sequence of substrings that all match $e$. We use Coq's string support, which comes through a combination of the String library and some parsing notations built into Coq. Operators like ++ and functions like length that we know from lists are defined again for strings. Notation scopes help us control which versions we want to use in particular contexts.

Require Import Ascii String.

Open Scope string_scope.

Section star.

Variable $P$ : string $\rightarrow$ Prop.

Inductive star : string $\rightarrow$ Prop :=

| Empty : star " "

| Iter : $\forall s 1$ s2,

$P$ s1

$\rightarrow$ star $s^{2}$

$\rightarrow \operatorname{star}(s 1++s 2)$.

End star.

Now we can make our first attempt at defining a regexp type that is indexed by predicates on strings, such that the index of a regexp tells us which language (string predicate) it recognizes. Here is a reasonable-looking definition that is restricted to constant characters and concatenation. We use the constructor String, which is the analogue of list cons for the type string, where " " is like list nil.

Inductive regexp : (string $\rightarrow$ Prop) $\rightarrow$ Set $:=$

| Char : $\forall$ ch : ascii,

regexp (fun $s \Rightarrow s=$ String $c^{\prime \prime \prime}$ )

| Concat : $\forall(P 1$ P2 : string $\rightarrow$ Prop $)(r 1: \operatorname{regexp} P 1)(r \mathscr{2}: \operatorname{regexp} P \mathscr{2})$,

regexp (fun $s \Rightarrow \exists s 1, \exists s 2, s=s 1++s 2 \wedge P 1 s 1 \wedge P 2$ s2).

User error: Large non-propositional inductive types must be in Type

What is a large inductive type? In Coq, it is an inductive type that has a constructor that quantifies over some type of type Type. We have not worked with Type very much to 
this point. Every term of CIC has a type, including Set and Prop, which are assigned type Type. The type string $\rightarrow$ Prop from the failed definition also has type Type.

It turns out that allowing large inductive types in Set leads to contradictions when combined with certain kinds of classical logic reasoning. Thus, by default, such types are ruled out. There is a simple fix for our regexp definition, which is to place our new type in Type. While fixing the problem, we also expand the list of constructors to cover the remaining regular expression operators.

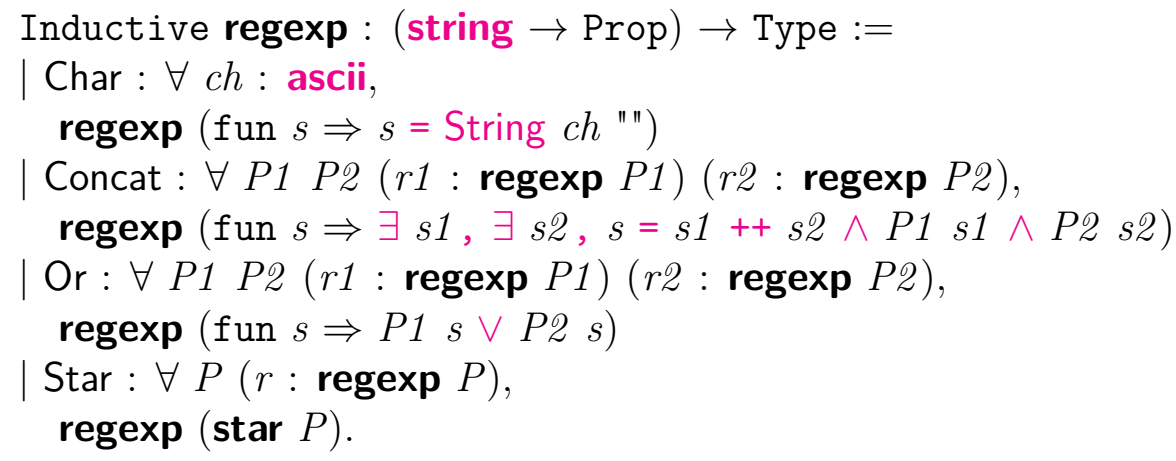

Many theorems about strings are useful for implementing a certified regexp matcher, and few of them are in the String library. The book source includes statements, proofs, and hint commands for a handful of such omitted theorems. Since they are orthogonal to our use of dependent types, we hide them in the rendered versions of this book.

A few auxiliary functions help us in our final matcher definition. The function split will be used to implement the regexp concatenation case.

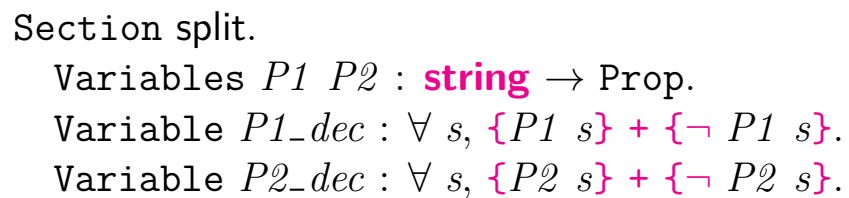

We require a choice of two arbitrary string predicates and functions for deciding them.

Variable $s$ : string.

Our computation will take place relative to a single fixed string, so it is easiest to make it a Variable, rather than an explicit argument to our functions.

The function split' is the workhorse behind split. It searches through the possible ways of splitting $s$ into two pieces, checking the two predicates against each such pair. The execution of split' progresses right-to-left, from splitting all of $s$ into the first piece to splitting all of $s$ into the second piece. It takes an extra argument, $n$, which specifies how far along we are in this search process.

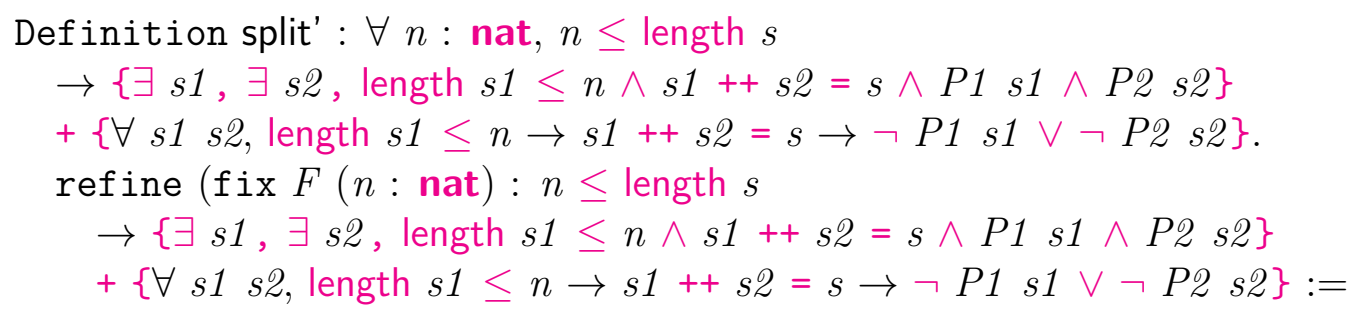


match $n$ with

$\mathrm{O} \Rightarrow$ fun _ $\Rightarrow$ Reduce (P1_dec " \&\& P2_dec $s)$

$\mathrm{S} n^{\prime} \Rightarrow$ fun $_{-} \Rightarrow$ (P1_dec (substring 0 (S n') $\left.s\right)$

\&\& P2_dec (substring (S $n^{\prime}$ ) (length $\left.s-\mathrm{S} n^{\prime}\right) s$ ))

II $F n^{\prime}$ -

end); clear F; crush; eauto 7;

match goal with

| [ - : length $\left.? \leq 0 \vdash_{-}\right] \Rightarrow$ destruct $S$

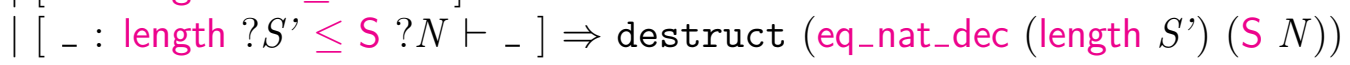

end; crush.

\section{Defined.}

There is one subtle point in the split' code that is worth mentioning. The main body of the function is a match on $n$. In the case where $n$ is known to be $\mathrm{S} n$, we write $\mathrm{S} n$ ' in several places where we might be tempted to write $n$. However, without further work to craft proper match annotations, the type-checker does not use the equality between $n$ and S $n$ '. Thus, it is common to see patterns repeated in match case bodies in dependently typed Coq code. We can at least use a let expression to avoid copying the pattern more than once, replacing the first case body with:

$$
\begin{aligned}
& \text { | S n' } \Rightarrow \text { fun _ } \Rightarrow \text { let } n:=\mathrm{S} n^{\prime} \text { in } \\
& \text { ( } P 1_{-} \text {dec (substring } 0 n s \text { ) } \\
& \text { \&\& P2_dec (substring } n \text { (length } s-n) s) \text { ) } \\
& \| F n^{\prime}
\end{aligned}
$$

The split function itself is trivial to implement in terms of split'. We just ask split' to begin its search with $n=$ length $s$.

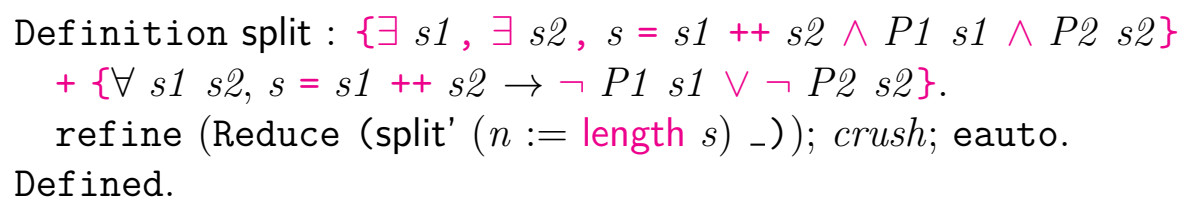

Arguments split $\left[\begin{array}{ll}P 1 & P \mathscr{2}\end{array}\right] P 1_{-}$dec P2_dec s.

One more helper function will come in handy: dec_star, for implementing another linear search through ways of splitting a string, this time for implementing the Kleene star.

\section{Section dec_star.}

Variable $P$ : string $\rightarrow$ Prop.

Variable $P_{-}$dec $: \forall s,\{P s\}+\{\neg P s\}$.

Some new lemmas and hints about the star type family are useful. We omit them here; they are included in the book source at this point.

The function dec_star" implements a single iteration of the star. That is, it tries to find a string prefix matching $P$, and it calls a parameter function on the remainder of the string. 


\section{Section dec_star'.}

Variable $n$ : nat.

Variable $n$ is the length of the prefix of $s$ that we have already processed.

$$
\begin{aligned}
& \text { Variable } P^{\prime} \text { : string } \rightarrow \text { Prop. } \\
& \text { Variable } P^{\prime} \text { _dec: } \forall n^{\prime}: \text { nat, } n^{\prime}>n \\
& \quad \rightarrow\left\{P^{\prime}\left(\text { substring } n^{\prime}\left(\text { length } s-n^{\prime}\right) s\right)\right\} \\
& \quad+\left\{\neg P^{\prime}\left(\text { substring } n^{\prime}\left(\text { length } s-n^{\prime}\right) s\right)\right\} .
\end{aligned}
$$

When we use dec_star", we will instantiate $P_{-}^{\prime} d e c$ with a function for continuing the search for more instances of $P$ in $s$.

Now we come to dec_star" itself. It takes as an input a natural $l$ that records how much of the string has been searched so far, as we did for split'. The return type expresses that dec_star" is looking for an index into $s$ that splits $s$ into a nonempty prefix and a suffix, such that the prefix satisfies $P$ and the suffix satisfies $P$ '.

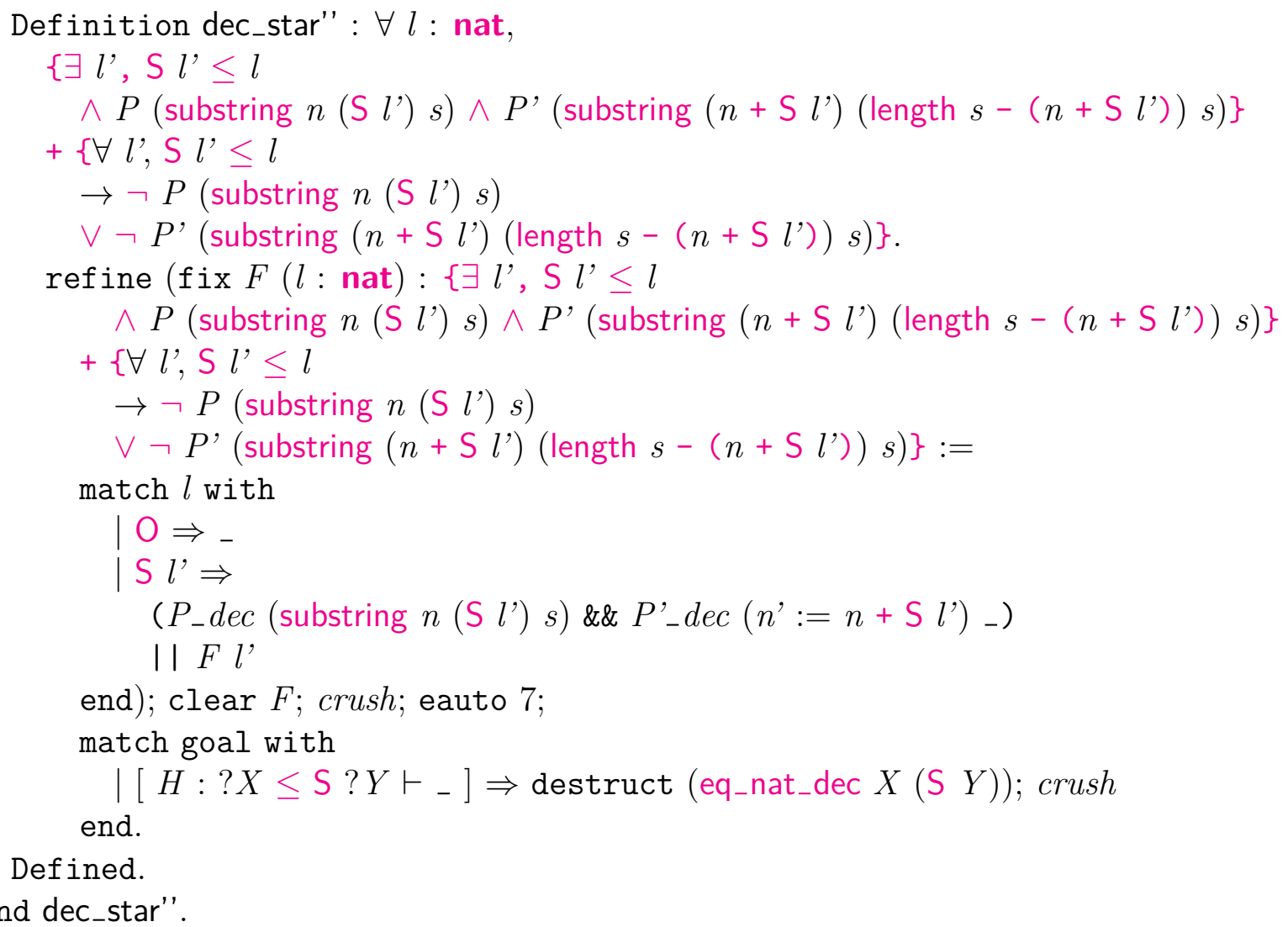

The work of dec_star" is nested inside another linear search by dec_star', which provides the final functionality we need, but for arbitrary suffixes of $s$, rather than just for $s$ overall.

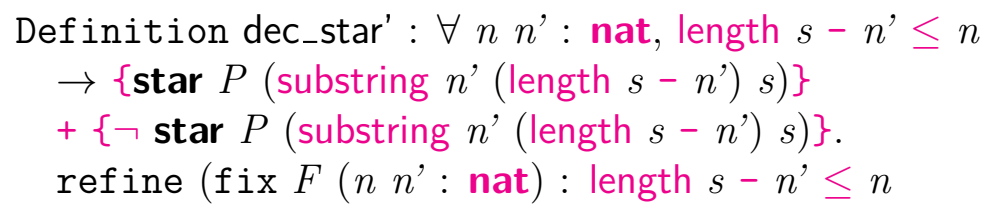




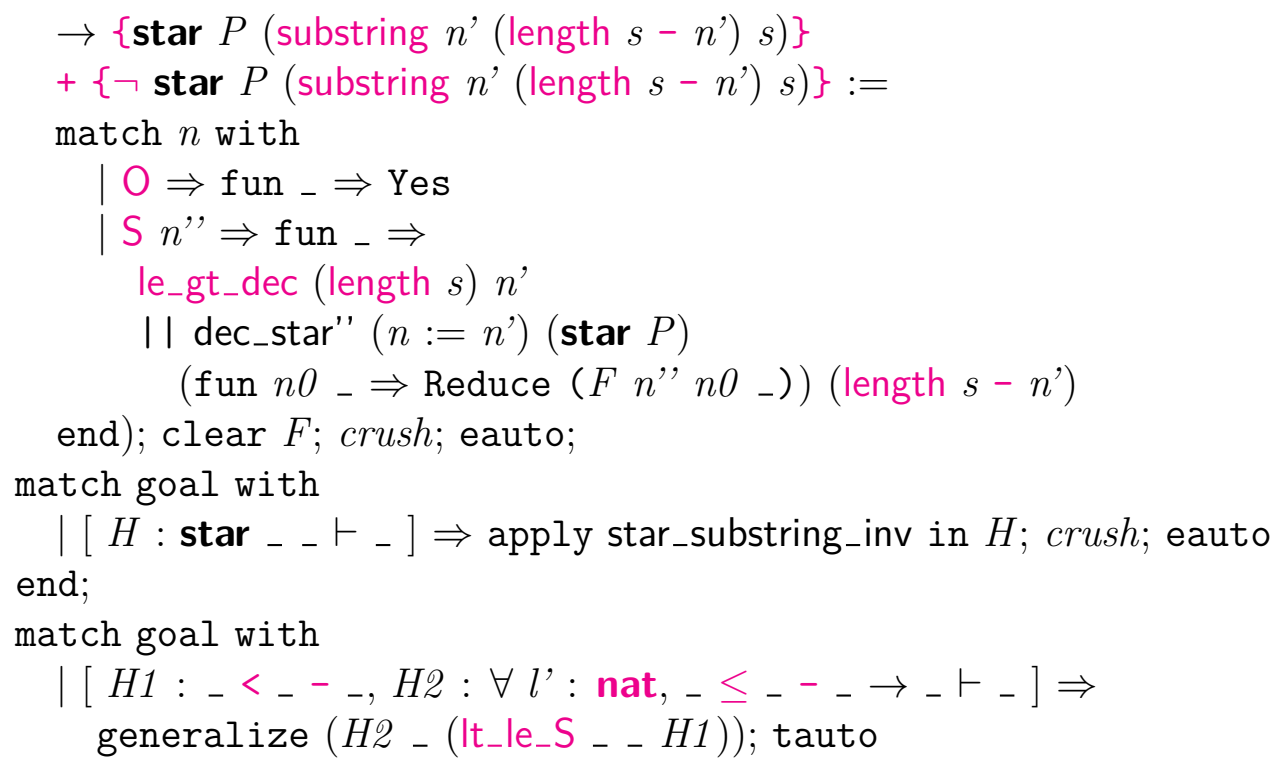

Finally, we have dec_star, defined by straightforward reduction from dec_star'.

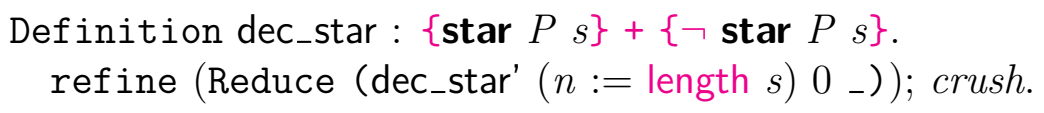

With these helper functions completed, the implementation of our matches function is refreshingly straightforward. We only need one small piece of specific tactic work beyond what crush does for us.

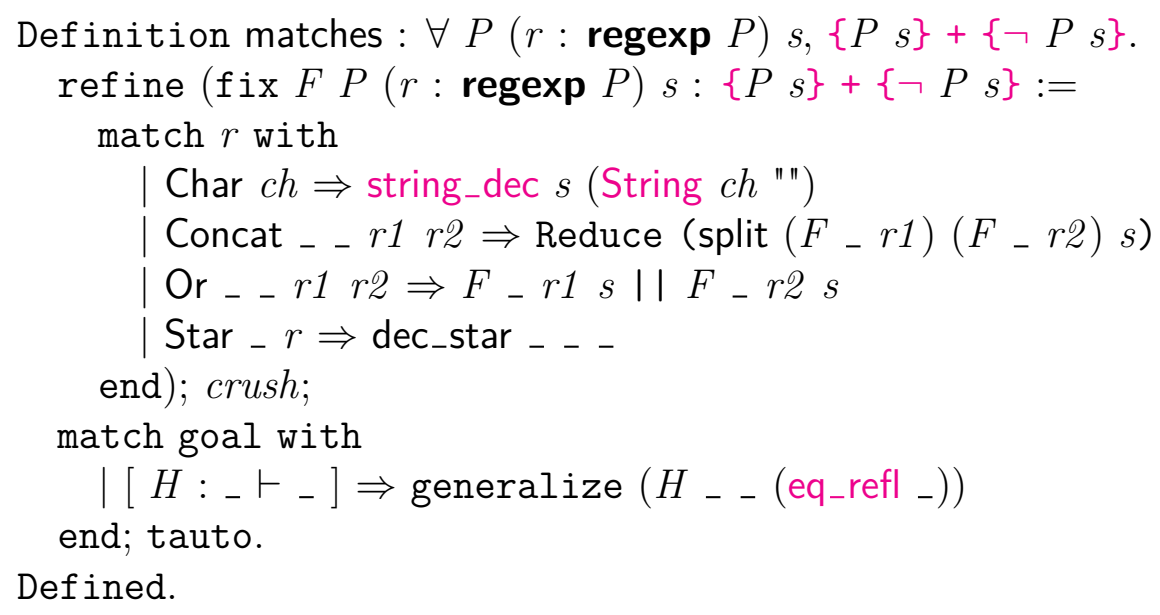

It is interesting to pause briefly to consider alternate implementations of matches. Dependent types give us much latitude in how specific correctness properties may be encoded with types. For instance, we could have made regexp a non-indexed inductive type, along the lines of what is possible in traditional ML and Haskell. We could then have implemented 
a recursive function to map regexps to their intended meanings, much as we have done with types and programs in other examples. That style is compatible with the refine-based approach that we have used here, and it might be an interesting exercise to redo the code from this subsection in that alternate style or some further encoding of the reader's choice. The main advantage of indexed inductive types is that they generally lead to the smallest amount of code.

Many regular expression matching problems are easy to test. The reader may run each of the following queries to verify that it gives the correct answer. We use evaluation strategy hnf to reduce each term to head-normal form, where the datatype constructor used to build its value is known. (Further reduction would involve wasteful simplification of proof terms justifying the answers of our procedures.)

Example a_star := Star (Char "a"\% char).

Eval hnf in matches a_star" ".

Eval hnf in matches a_star "a".

Eval hnf in matches a_star "b".

Eval hnf in matches a_star "aa".

Evaluation inside Coq does not scale very well, so it is easy to build other tests that run for hours or more. Such cases are better suited to execution with the extracted OCaml code. 


\section{Chapter 9}

\section{Dependent Data Structures}

Our red-black tree example from the last chapter illustrated how dependent types enable static enforcement of data structure invariants. To find interesting uses of dependent data structures, however, we need not look to the favorite examples of data structures and algorithms textbooks. More basic examples like length-indexed and heterogeneous lists come up again and again as the building blocks of dependent programs. There is a surprisingly large design space for this class of data structure, and we will spend this chapter exploring it.

\subsection{More Length-Indexed Lists}

We begin with a deeper look at the length-indexed lists that began the last chapter.

Section ilist.

Variable $A$ : Set.

Inductive ilist : nat $\rightarrow$ Set $:=$

Nil : ilist $\mathrm{O}$

Cons : $\forall n, A \rightarrow$ ilist $n \rightarrow$ ilist $(\mathrm{S} n)$.

We might like to have a certified function for selecting an element of an ilist by position. We could do this using subset types and explicit manipulation of proofs, but dependent types let us do it more directly. It is helpful to define a type family fin, where fin $n$ is isomorphic to $\{m$ : nat $\mid m<n\}$. The type family name stands for "finite."

Inductive fin : nat $\rightarrow$ Set $:=$

First : $\forall n$, fin $(S n)$

Next : $\forall n$, fin $n \rightarrow$ fin $(\mathrm{S} n)$.

An instance of fin is essentially a more richly typed copy of a prefix of the natural numbers. Every element is a First iterated through applying Next a number of times that indicates which number is being selected. For instance, the three values of type fin 3 are First 2, Next (First 1), and Next (Next (First 0)).

Now it is easy to pick a Prop-free type for a selection function. As usual, our first implementation attempt will not convince the type checker, and we will attack the deficiencies 
one at a time.

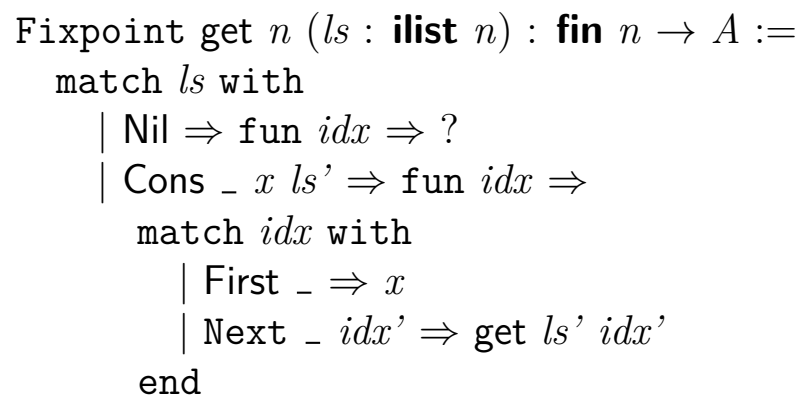

end.

We apply the usual wisdom of delaying arguments in Fixpoints so that they may be included in return clauses. This still leaves us with a quandary in each of the match cases. First, we need to figure out how to take advantage of the contradiction in the Nil case. Every fin has a type of the form $S n$, which cannot unify with the $O$ value that we learn for $n$ in the Nil case. The solution we adopt is another case of match-within-return, with the return clause chosen carefully so that it returns the proper type $A$ in case the fin index is $\mathrm{O}$, which we know is true here; and so that it returns an easy-to-inhabit type unit in the remaining, impossible cases, which nonetheless appear explicitly in the body of the match.

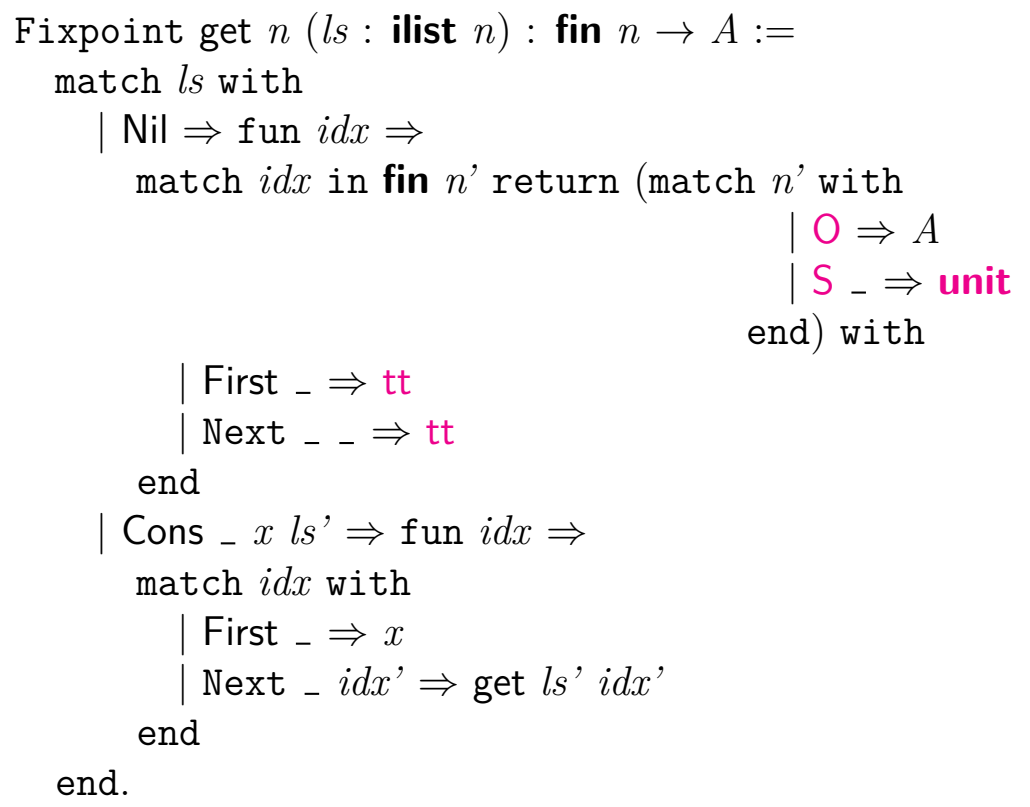

Now the first match case type-checks, and we see that the problem with the Cons case is that the pattern-bound variable $i d x^{\prime}$ does not have an apparent type compatible with $l s$ '. In fact, the error message Coq gives for this exact code can be confusing, thanks to an overenthusiastic type inference heuristic. We are told that the Nil case body has type match $X$ with $|\mathrm{O} \Rightarrow A| \mathrm{S}_{-} \Rightarrow$ unit end for a unification variable $X$, while it is expected to have type $A$. We can see that setting $X$ to $O$ resolves the conflict, but Coq is not yet smart enough to do this unification automatically. Repeating the function's type in a return annotation, 
used with an in annotation, leads us to a more informative error message, saying that $i d x$ ' has type fin $n 1$ while it is expected to have type fin $n 0$, where $n 0$ is bound by the Cons pattern and $n 1$ by the Next pattern. As the code is written above, nothing forces these two natural numbers to be equal, though we know intuitively that they must be.

We need to use match annotations to make the relationship explicit. Unfortunately, the usual trick of postponing argument binding will not help us here. We need to match on both $l s$ and $i d x$; one or the other must be matched first. To get around this, we apply the convoy pattern that we met last chapter. This application is a little more clever than those we saw before; we use the natural number predecessor function pred to express the relationship between the types of these variables.

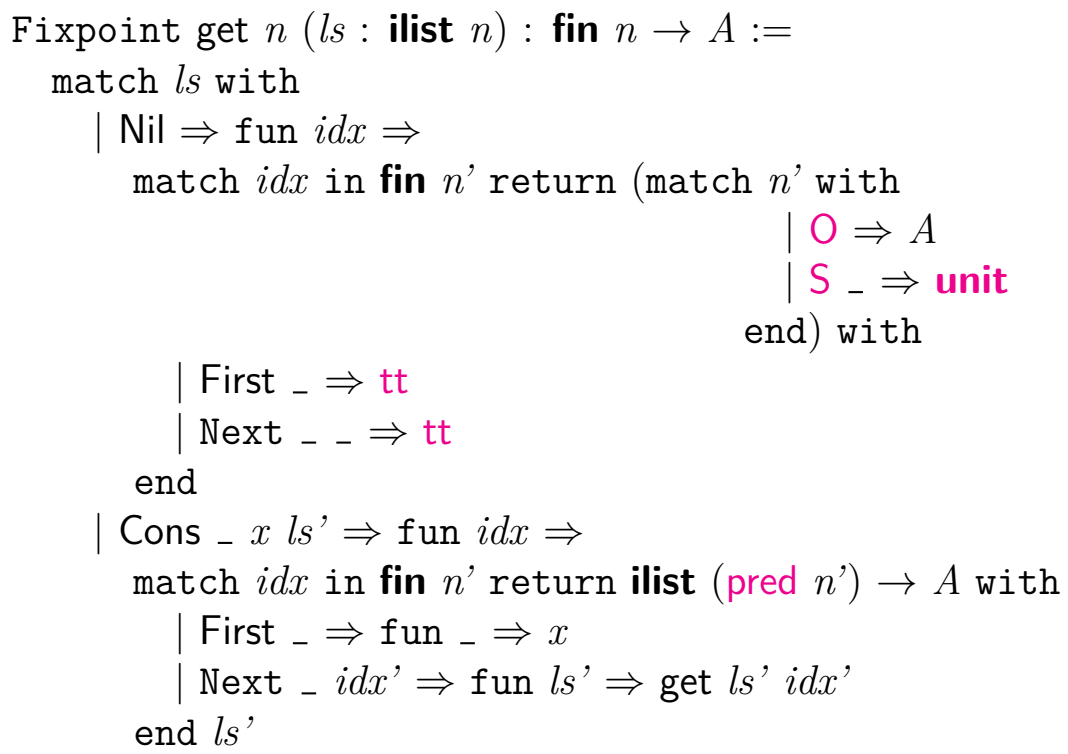

end.

There is just one problem left with this implementation. Though we know that the local $l s^{\prime}$ in the Next case is equal to the original $l s$ ', the type-checker is not satisfied that the recursive call to get does not introduce non-termination. We solve the problem by convoybinding the partial application of get to $l s^{\prime}$, rather than $l s^{\prime}$ by itself.

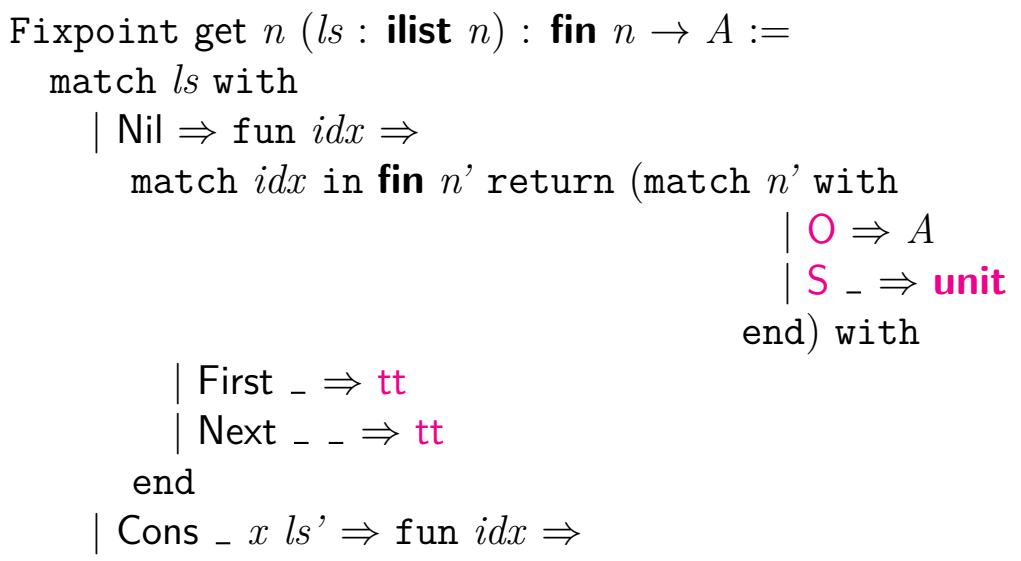


match $i d x$ in fin $n^{\prime}$ return $\left(\right.$ fin $\left(\right.$ pred $\left.\left.n^{\prime}\right) \rightarrow A\right) \rightarrow A$ with

| First _ $\Rightarrow$ fun _ $\Rightarrow x$

| Next $-i d x^{\prime} \Rightarrow$ fun get_ls' $\Rightarrow$ get_ls' $i d x^{\prime}$ end (get $l s^{\prime}$ )

end.

End ilist.

Arguments Nil $[A]$.

Arguments First $[n]$.

A few examples show how to make use of these definitions.

Check Cons 0 (Cons 1 (Cons $2 \mathrm{Nil})$ ).

Cons 0 (Cons 1 (Cons $2 \mathrm{Nil})$ )

\section{: ilist nat 3}

Eval simpl in get (Cons 0 (Cons 1 (Cons 2 Nil))) First.

$$
=0
$$

: nat

Eval simpl in get (Cons 0 (Cons 1 (Cons 2 Nil))) (Next First).

$$
\begin{aligned}
& =1 \\
& \text { : nat }
\end{aligned}
$$

Eval simpl in get (Cons 0 (Cons 1 (Cons 2 Nil))) (Next (Next First)).

$$
\begin{aligned}
& =2 \\
& \text { : nat }
\end{aligned}
$$

Our get function is also quite easy to reason about. We show how with a short example about an analogue to the list map function.

Section ilist_map.

Variables $A B$ : Set.

Variable $f: A \rightarrow B$.

Fixpoint imap $n(l s:$ ilist $A n)$ : ilist $B n:=$ match $l s$ with

$$
\begin{aligned}
& \mid \mathrm{Nil} \Rightarrow \mathrm{Nil} \\
& \mid \text { Cons }-x l s^{\prime} \Rightarrow \text { Cons }(f x)\left(\text { imap } l s^{\prime}\right)
\end{aligned}
$$

It is easy to prove that get "distributes over" imap calls.

Theorem get_imap : $\forall n(i d x:$ fin $n)(l s$ : ilist $A n)$, get $($ imap $l s) i d x=f($ get $l s i d x)$.

induction $l s$; dep_destruct $i d x$; crush. 
Qed.

End ilist_map.

The only tricky bit is remembering to use our dep_destruct tactic in place of plain destruct when faced with a baffling tactic error message.

\section{$9.2 \quad$ Heterogeneous Lists}

Programmers who move to statically typed functional languages from scripting languages often complain about the requirement that every element of a list have the same type. With fancy type systems, we can partially lift this requirement. We can index a list type with a "type-level" list that explains what type each element of the list should have. This has been done in a variety of ways in Haskell using type classes, and we can do it much more cleanly and directly in Coq.

Section hlist.

Variable $A$ : Type.

Variable $B: A \rightarrow$ Type.

We parameterize our heterogeneous lists by a type $A$ and an $A$-indexed type $B$.

Inductive hlist : list $A \rightarrow$ Type :=

| HNil : hlist nil

HCons : $\forall(x: A)$ (ls: list $A), B x \rightarrow$ hlist $l s \rightarrow$ hlist $(x:: l s)$.

We can implement a variant of the last section's get function for hlists. To get the dependent typing to work out, we will need to index our element selectors (in type family member) by the types of data that they point to.

Variable elm: $A$.

Inductive member : list $A \rightarrow$ Type $:=$

| HFirst : $\forall l s$, member $(e l m:: l s)$

| HNext : $\forall x l s$, member $l s \rightarrow$ member $(x:: l s)$.

Because the element elm that we are "searching for" in a list does not change across the constructors of member, we simplify our definitions by making elm a local variable. In the definition of member, we say that elm is found in any list that begins with elm, and, if removing the first element of a list leaves elm present, then elm is present in the original list, too. The form looks much like a predicate for list membership, but we purposely define member in Type so that we may decompose its values to guide computations.

We can use member to adapt our definition of get to hlists. The same basic match tricks apply. In the HCons case, we form a two-element convoy, passing both the data element $x$ and the recursor for the sublist $m l_{s}$ ' to the result of the inner match. We did not need to do that in get's definition because the types of list elements were not dependent there.

Fixpoint hget $l s(\mathrm{mls}:$ hlist $l s):$ member $l s \rightarrow B$ elm $:=$ match $\mathrm{mls}$ with 


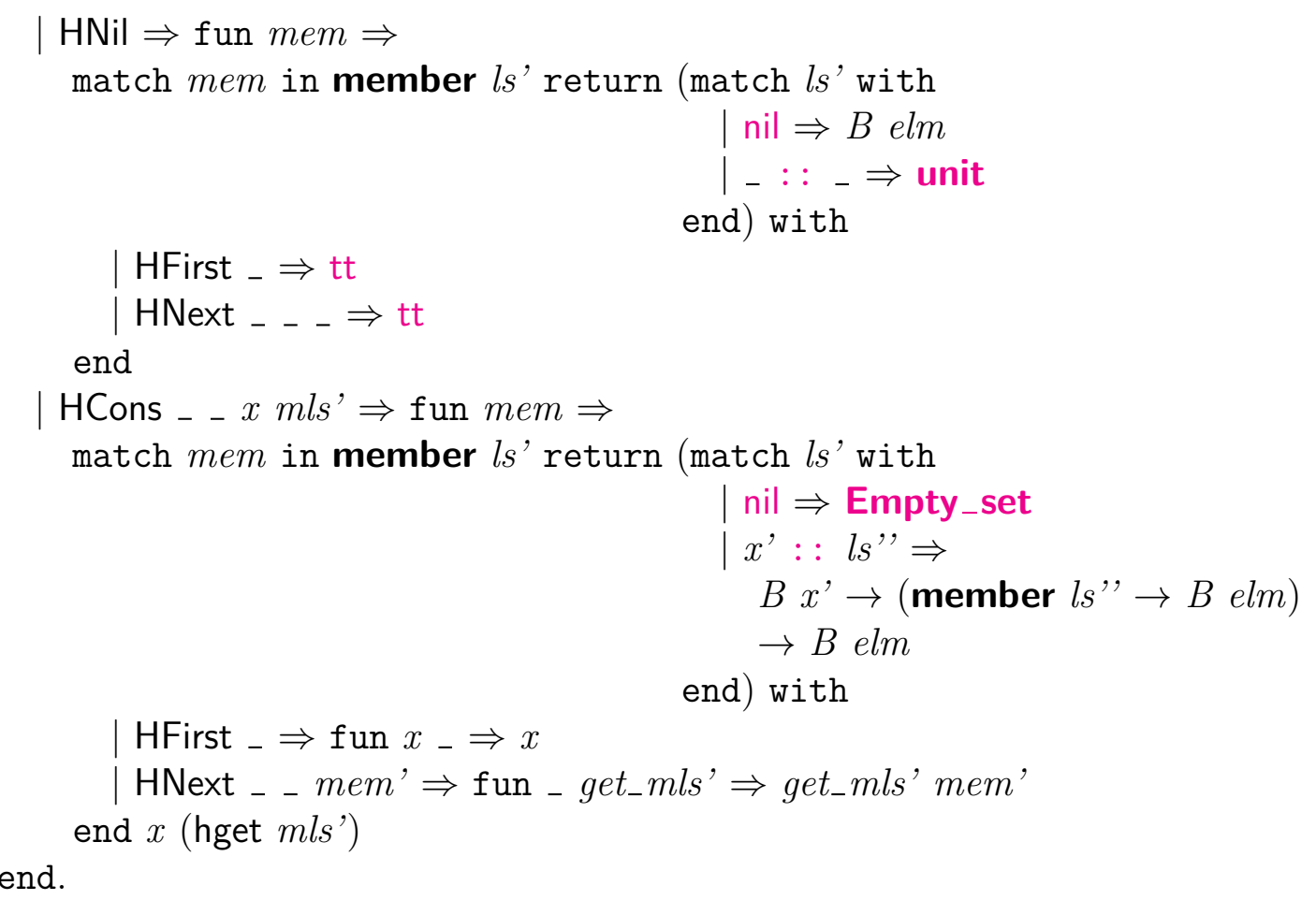

End hlist.

Arguments $\mathrm{HNil}\left[\begin{array}{ll}A & B\end{array}\right]$.

Arguments HCons $\left[\begin{array}{llll}A & B & x & l s\end{array}\right]_{-}$..

Arguments HFirst $[A$ elm ls].

Arguments HNext $\left[\begin{array}{llll}A & \text { elm } & x & l s\end{array}\right]_{-}$.

By putting the parameters $A$ and $B$ in Type, we enable fancier kinds of polymorphism than in mainstream functional languages. For instance, one use of hlist is for the simple heterogeneous lists that we referred to earlier.

Definition someTypes : list Set := nat : : bool : : nil.

Example someValues : hlist (fun $T$ : Set $\Rightarrow T$ ) someTypes :=

HCons 5 (HCons true HNil).

Eval simpl in hget someValues HFirst.

$$
\begin{aligned}
& =5 \\
& :(\text { fun } T: \text { Set } \Rightarrow T) \text { nat }
\end{aligned}
$$

Eval simpl in hget someValues (HNext HFirst).

$$
\begin{aligned}
& =\text { true } \\
& :(\text { fun } T: \text { Set } \Rightarrow T) \text { bool }
\end{aligned}
$$

We can also build indexed lists of pairs in this way.

Example somePairs : hlist (fun $T$ : Set $\Rightarrow T \times T$ )\%type someTypes := 
HCons (1, 2) (HCons (true, false) HNil).

There are many other useful applications of heterogeneous lists, based on different choices of the first argument to hlist.

\subsubsection{A Lambda Calculus Interpreter}

Heterogeneous lists are very useful in implementing interpreters for functional programming languages. Using the types and operations we have already defined, it is trivial to write an interpreter for simply typed lambda calculus. Our interpreter can alternatively be thought of as a denotational semantics (but worry not if you are not familiar with such terminology from semantics).

We start with an algebraic datatype for types.

Inductive type : Set :=

| Unit : type

Arrow : type $\rightarrow$ type $\rightarrow$ type.

Now we can define a type family for expressions. An exp $t s t$ will stand for an expression that has type $t$ and whose free variables have types in the list $t s$. We effectively use the de Bruijn index variable representation [11]. Variables are represented as member values; that is, a variable is more or less a constructive proof that a particular type is found in the type environment.

Inductive exp : list type $\rightarrow$ type $\rightarrow$ Set $:=$

| Const : $\forall t s, \exp t s$ Unit

Var : $\forall$ ts $t$, member $t$ ts $\rightarrow \exp t s t$

App : $\forall$ ts dom ran, $\exp t s$ (Arrow dom ran) $\rightarrow \exp t s d o m \rightarrow \exp t s$ ran

Abs : $\forall$ ts dom ran, $\exp (d o m:: t s)$ ran $\rightarrow \exp t s$ (Arrow dom ran).

Arguments Const $[t s]$.

We write a simple recursive function to translate types into Sets.

Fixpoint typeDenote $(t$ : type $)$ : Set $:=$

match $t$ with

Unit $\Rightarrow$ unit

Arrow $t 1$ t2 $\Rightarrow$ typeDenote $t 1 \rightarrow$ typeDenote $t \mathscr{2}$

end.

Now it is straightforward to write an expression interpreter. The type of the function, expDenote, tells us that we translate expressions into functions from properly typed environments to final values. An environment for a free variable list ts is simply an hlist typeDenote ts. That is, for each free variable, the heterogeneous list that is the environment must have a value of the variable's associated type. We use hget to implement the Var case, and we use HCons to extend the environment in the Abs case.

Fixpoint expDenote $t s t(e: \exp t s t):$ hlist typeDenote $t s \rightarrow$ typeDenote $t:=$ 


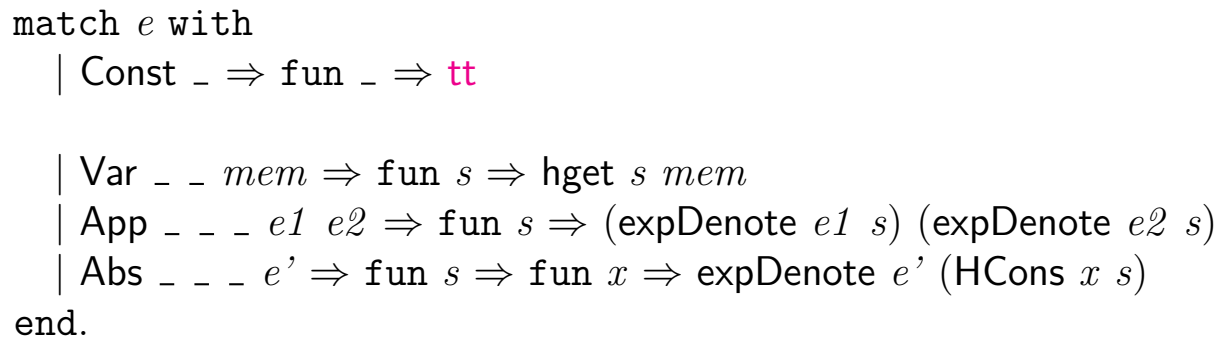

Like for previous examples, our interpreter is easy to run with simpl.

Eval simpl in expDenote Const HNil.

$$
\begin{aligned}
& =\mathrm{tt} \\
& : \text { typeDenote Unit }
\end{aligned}
$$

Eval simpl in expDenote (Abs (dom $:=$ Unit) (Var HFirst)) HNil.

$=$ fun $x$ : unit $\Rightarrow x$

: typeDenote (Arrow Unit Unit)

Eval simpl in expDenote (Abs (dom := Unit)

(Abs $($ dom $:=$ Unit) (Var (HNext HFirst) $)))$ HNil.

$=$ fun $x_{-}:$unit $\Rightarrow x$

: typeDenote (Arrow Unit (Arrow Unit Unit))

Eval simpl in expDenote (Abs (dom $:=$ Unit) (Abs (dom $:=$ Unit) (Var HFirst))) HNil.

$=$ fun $-x 0:$ unit $\Rightarrow x 0$

: typeDenote (Arrow Unit (Arrow Unit Unit))

Eval simpl in expDenote (App (Abs (Var HFirst)) Const) HNil.

$$
=\mathrm{tt}
$$

: typeDenote Unit

We are starting to develop the tools behind dependent typing's amazing advantage over alternative approaches in several important areas. Here, we have implemented complete syntax, typing rules, and evaluation semantics for simply typed lambda calculus without even needing to define a syntactic substitution operation. We did it all without a single line of proof, and our implementation is manifestly executable. Other, more common approaches to language formalization often state and prove explicit theorems about type safety of languages. In the above example, we got type safety, termination, and other meta-theorems for free, by reduction to CIC, which we know has those properties. 


\subsection{Recursive Type Definitions}

There is another style of datatype definition that leads to much simpler definitions of the get and hget definitions above. Because Coq supports "type-level computation," we can redo our inductive definitions as recursive definitions. Here we will preface type names with the letter $f$ to indicate that they are based on explicit recursive function definitions.

Section filist.

Variable $A$ : Set.

Fixpoint filist $(n:$ nat $):$ Set $:=$ match $n$ with

$\mathrm{O} \Rightarrow$ unit

$\mathrm{S} n^{\prime} \Rightarrow A \times$ filist $n^{\prime}$

end \%type.

We say that a list of length 0 has no contents, and a list of length $S n^{\prime}$ is a pair of a data value and a list of length $n$ '.

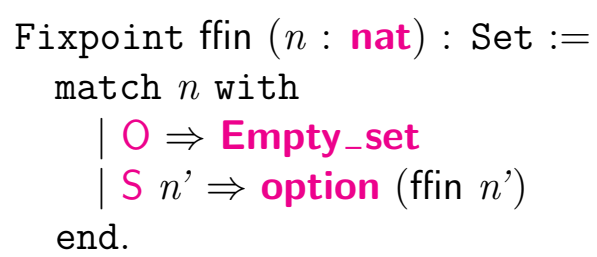

We express that there are no index values when $n=0$, by defining such indices as type Empty_set; and we express that, at $n=\mathrm{S} n$, there is a choice between picking the first element of the list (represented as None) or choosing a later element (represented by Some $i d x$, where $i d x$ is an index into the list tail). For instance, the three values of type ffin 3 are None, Some None, and Some (Some None).

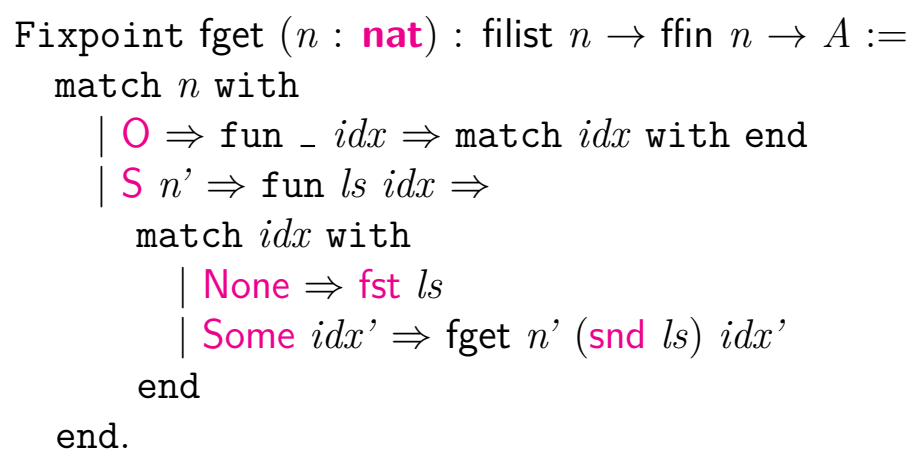

Our new get implementation needs only one dependent match, and its annotation is inferred for us. Our choices of data structure implementations lead to just the right typing behavior for this new definition to work out.

End filist.

Heterogeneous lists are a little trickier to define with recursion, but we then reap similar benefits in simplicity of use. 


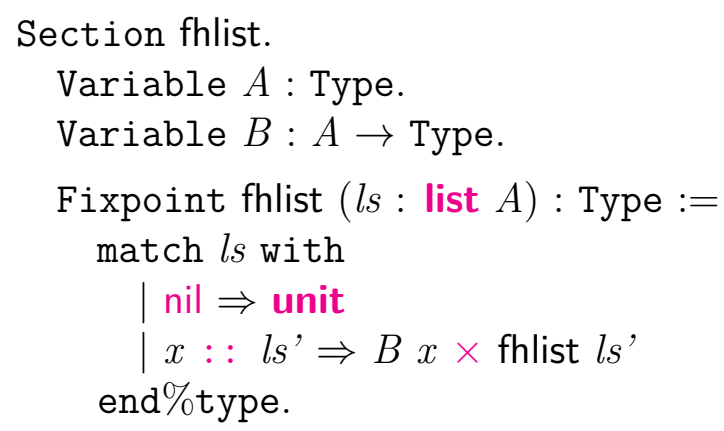

The definition of fhlist follows the definition of filist, with the added wrinkle of dependently typed data elements.

Variable elm: $A$.

Fixpoint fmember (ls: list $A)$ : Type := match $l s$ with

| nil $\Rightarrow$ Empty_set

$x:: l s^{\prime} \Rightarrow(x=e l m)+$ fmember $l s^{\prime}$

end \%type.

The definition of fmember follows the definition of ffin. Empty lists have no members, and member types for nonempty lists are built by adding one new option to the type of members of the list tail. While for ffin we needed no new information associated with the option that we add, here we need to know that the head of the list equals the element we are searching for. We express that idea with a sum type whose left branch is the appropriate equality proposition. Since we define fmember to live in Type, we can insert Prop types as needed, because Prop is a subtype of Type.

We know all of the tricks needed to write a first attempt at a get function for fhlists.

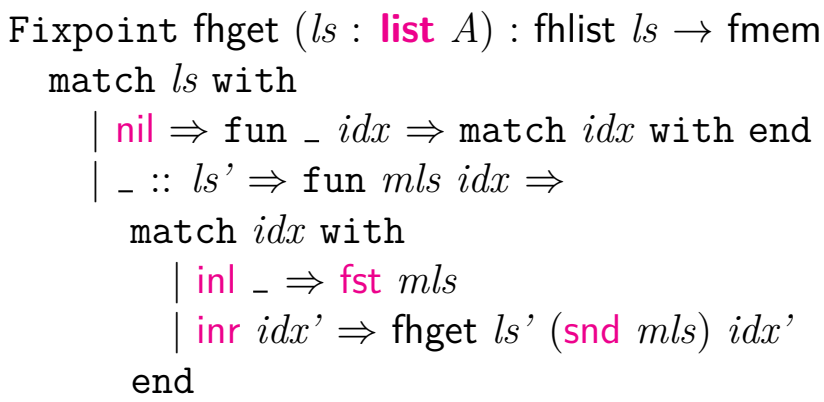

end.

Only one problem remains. The expression fst $m l s$ is not known to have the proper type. To demonstrate that it does, we need to use the proof available in the inl case of the inner match.

Fixpoint fhget $(l s:$ list $A):$ fhlist $l s \rightarrow$ fmember $l s \rightarrow B$ elm $:=$ match $l s$ with

| nil $\Rightarrow$ fun _ $i d x \Rightarrow$ match $i d x$ with end

। _ : : ls' $\Rightarrow$ fun $m l s$ idx $\Rightarrow$ 


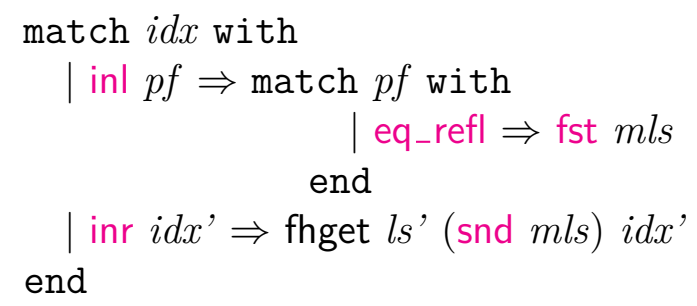

end.

By pattern-matching on the equality proof $p f$, we make that equality known to the typechecker. Exactly why this works can be seen by studying the definition of equality.

Print eq.

Inductive eq $(A:$ Type $)(x: A): A \rightarrow$ Prop $:=$ eq_refl $: x=x$

In a proposition $x=y$, we see that $x$ is a parameter and $y$ is a regular argument. The type of the constructor eq_refl shows that $y$ can only ever be instantiated to $x$. Thus, within a pattern-match with eq_refl, occurrences of $y$ can be replaced with occurrences of $x$ for typing purposes.

End fhlist.

Arguments fhget $\left[\begin{array}{lll}A & B & \text { elm } l s\end{array}\right]$ _..

How does one choose between the two data structure encoding strategies we have presented so far? Before answering that question in this chapter's final section, we introduce one further approach.

\subsection{Data Structures as Index Functions}

Indexed lists can be useful in defining other inductive types with constructors that take variable numbers of arguments. In this section, we consider parameterized trees with arbitrary branching factor.

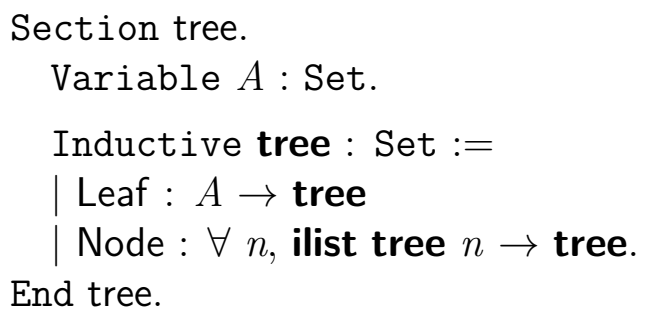

Every Node of a tree has a natural number argument, which gives the number of child trees in the second argument, typed with ilist. We can define two operations on trees of naturals: summing their elements and incrementing their elements. It is useful to define a generic fold function on ilists first.

Section ifoldr.

Variables $A B$ : Set. 


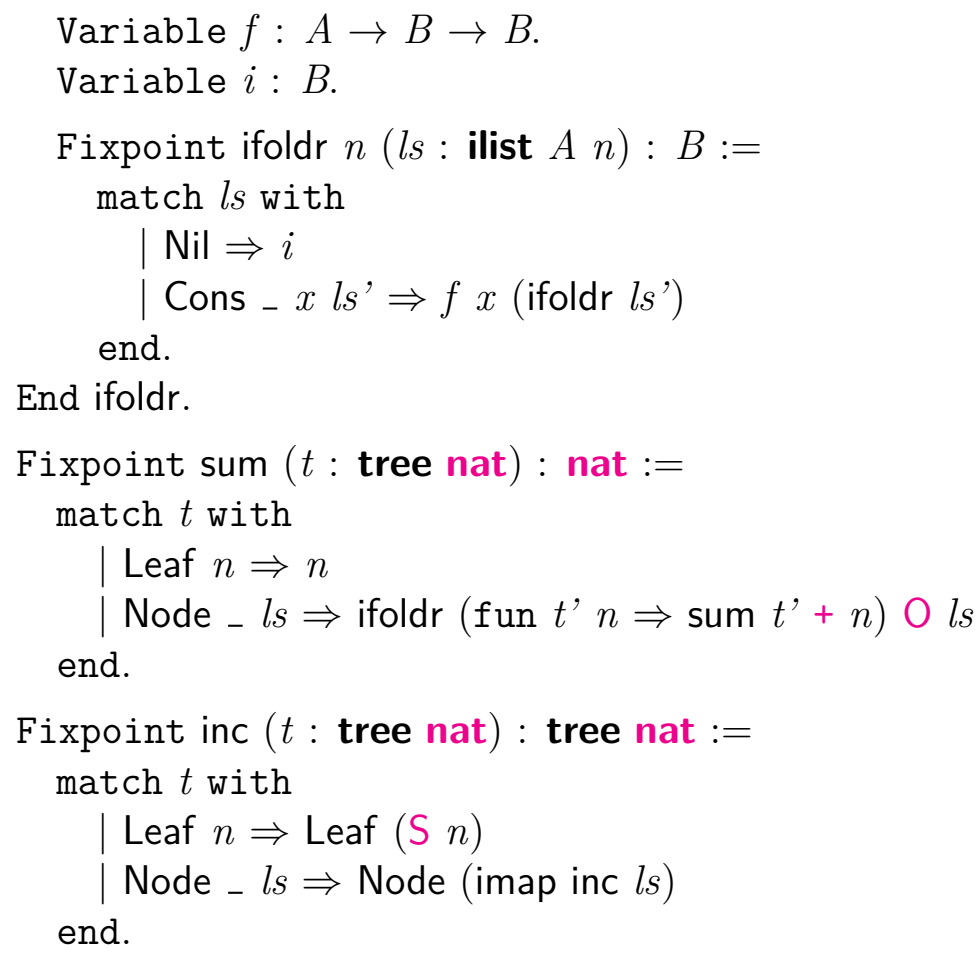

Now we might like to prove that inc does not decrease a tree's sum.

Theorem sum_inc : $\forall t$, sum (inc $t) \geq \operatorname{sum} t$.

induction $t$; crush.

$n$ : nat

$i$ : ilist (tree nat) $n$

We are left with a single subgoal which does not seem provable directly. This is the same problem that we met in Chapter 3 with other nested inductive types.

Check tree_ind.

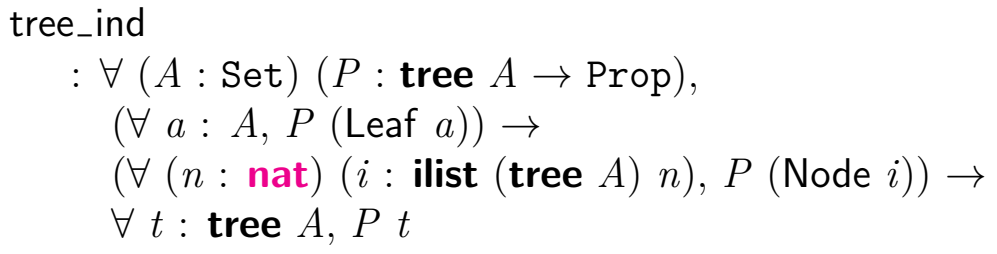

The automatically generated induction principle is too weak. For the Node case, it gives us no inductive hypothesis. We could write our own induction principle, as we did in Chapter 3 , but there is an easier way, if we are willing to alter the definition of tree. 
Abort.

Reset tree.

First, let us try using our recursive definition of ilists instead of the inductive version.

\section{Section tree.}

Variable $A$ : Set.

Inductive tree : Set :=

Leaf : $A \rightarrow$ tree

| Node : $\forall n$, filist tree $n \rightarrow$ tree.

Error: Non strictly positive occurrence of "tree" in

"forall $n$ : nat, filist tree $n \rightarrow$ tree"

The special-case rule for nested datatypes only works with nested uses of other inductive types, which could be replaced with uses of new mutually inductive types. We defined filist recursively, so it may not be used in nested inductive definitions.

Our final solution uses yet another of the inductive definition techniques introduced in Chapter 3, reflexive types. Instead of merely using fin to get elements out of ilist, we can define ilist in terms of fin. For the reasons outlined above, it turns out to be easier to work with ffin in place of fin.

Inductive tree : Set :=

| Leaf : $A \rightarrow$ tree

Node $: \forall n,($ ffin $n \rightarrow$ tree $) \rightarrow$ tree.

A Node is indexed by a natural number $n$, and the node's $n$ children are represented as a function from ffin $n$ to trees, which is isomorphic to the ilist-based representation that we used above.

End tree.

Arguments Node $\left[\begin{array}{ll}A & n\end{array}\right]$..

We can redefine sum and inc for our new tree type. Again, it is useful to define a generic fold function first. This time, it takes in a function whose domain is some ffin type, and it folds another function over the results of calling the first function at every possible ffin value.

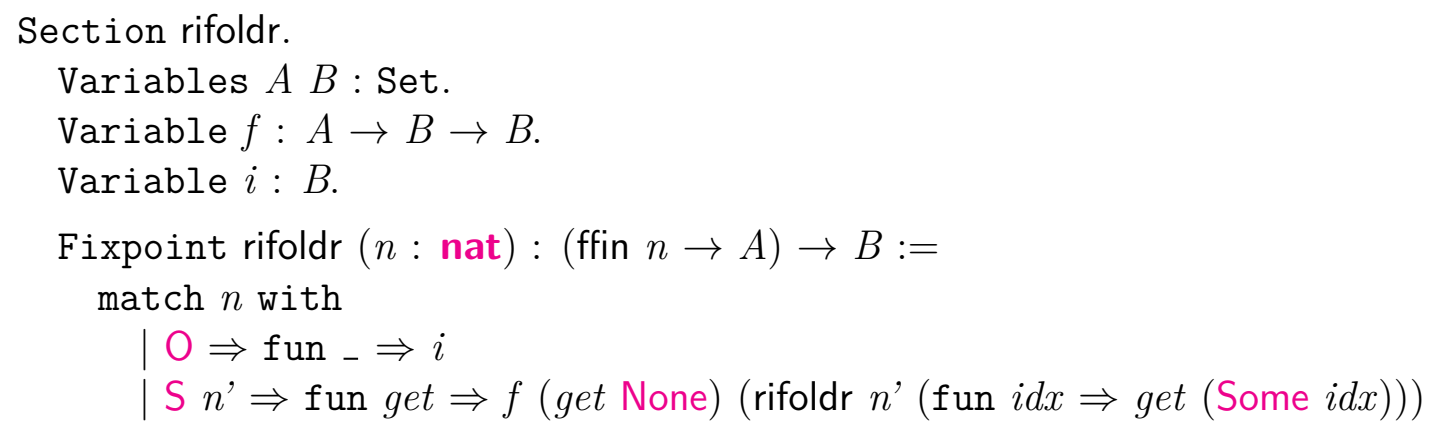


end.

End rifoldr.

Arguments rifoldr $\left[\begin{array}{ll}A B & B\end{array}\right] i[n]$-.

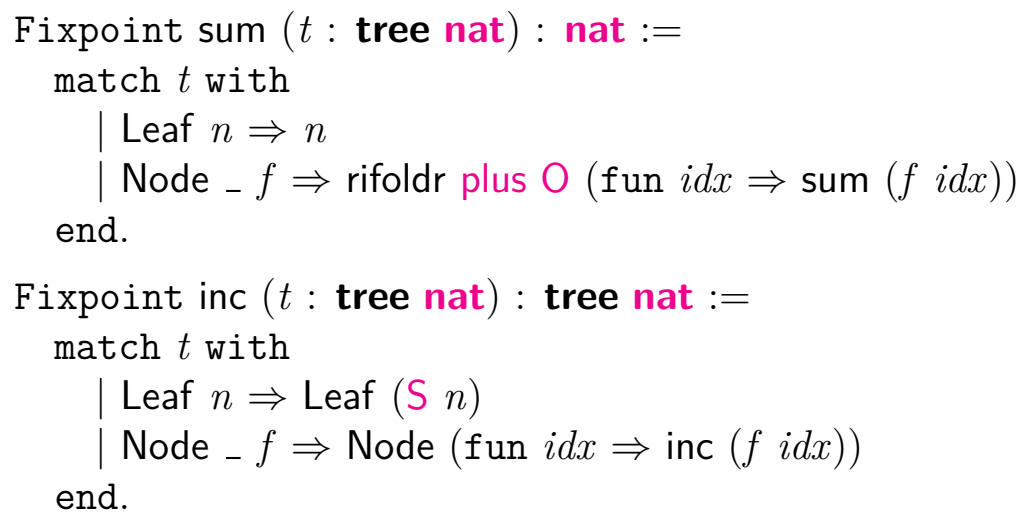

Now we are ready to prove the theorem where we got stuck before. We will not need to define any new induction principle, but it will be helpful to prove some lemmas.

Lemma plus_ge : $\forall x 1$ y1 $x 2$ y2,

$x 1 \geq x 2$

$\rightarrow y 1 \geq y^{2}$

$\rightarrow x 1+y 1 \geq x 2+y 2$.

crush.

Qed.

Lemma sum_inc' : $\forall n(f 1 f 2:$ ffin $n \rightarrow$ nat $)$,

$(\forall i d x, f 1 \quad i d x \geq f 2 i d x)$

$\rightarrow$ rifoldr plus $\bigcirc f 1 \geq$ rifoldr plus $\bigcirc$ f2.

Hint Resolve plus_ge.

induction $n$; crush.

Qed.

Theorem sum_inc : $\forall t$, sum (inc $t) \geq \operatorname{sum} t$.

Hint Resolve sum_inc'.

induction $t$; crush.

Qed.

Even if Coq would generate complete induction principles automatically for nested inductive definitions like the one we started with, there would still be advantages to using this style of reflexive encoding. We see one of those advantages in the definition of inc, where we did not need to use any kind of auxiliary function. In general, reflexive encodings often admit direct implementations of operations that would require recursion if performed with more traditional inductive data structures. 


\subsubsection{Another Interpreter Example}

We develop another example of variable-arity constructors, in the form of optimization of a small expression language with a construct like Scheme's cond. Each of our conditional expressions takes a list of pairs of boolean tests and bodies. The value of the conditional comes from the body of the first test in the list to evaluate to true. To simplify the interpreter we will write, we force each conditional to include a final, default case.

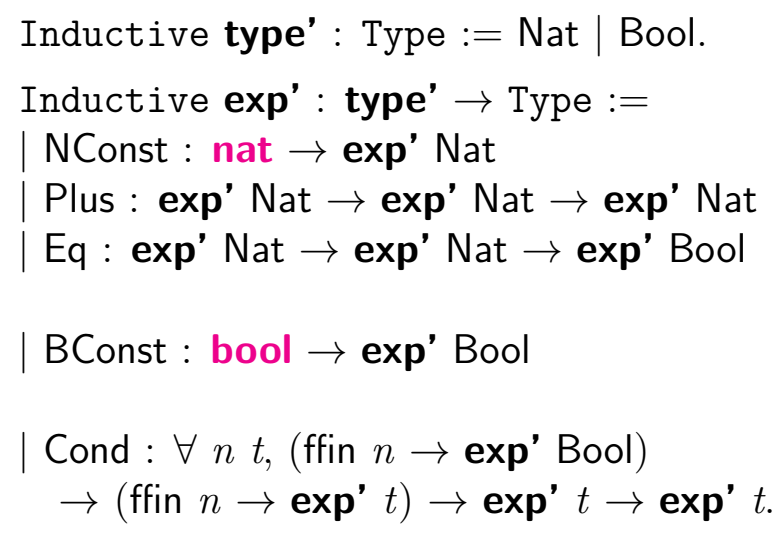

A Cond is parameterized by a natural $n$, which tells us how many cases this conditional has. The test expressions are represented with a function of type ffin $n \rightarrow$ exp' Bool, and the bodies are represented with a function of type ffin $n \rightarrow \operatorname{exp'} t$, where $t$ is the overall type. The final exp' $t$ argument is the default case. For example, here is an expression that successively checks whether $2+2=5$ (returning 0 if so) or if $1+1=2$ (returning 1 if so), returning 2 otherwise.

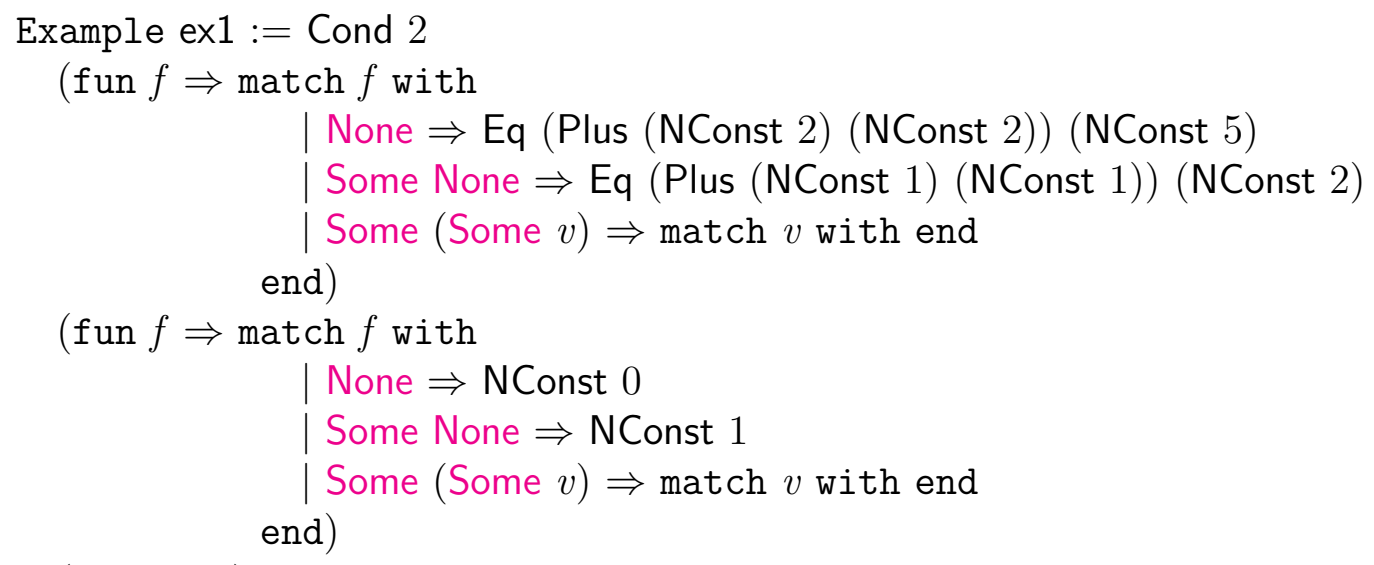

(NConst 2).

We start implementing our interpreter with a standard type denotation function.

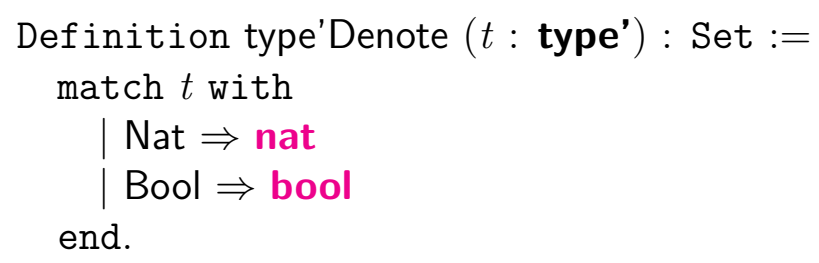


To implement the expression interpreter, it is useful to have the following function that implements the functionality of Cond without involving any syntax.

Section cond.

Variable $A$ : Set.

Variable default : A.

Fixpoint cond ( $n$ : nat) $:($ ffin $n \rightarrow$ bool $) \rightarrow($ ffin $n \rightarrow A) \rightarrow A:=$

match $n$ with

$\mathrm{O} \Rightarrow$ fun _ _ $\Rightarrow$ default

| $\mathrm{S} n^{\prime} \Rightarrow$ fun tests bodies $\Rightarrow$

if tests None

then bodies None

else cond $n$ '

(fun $i d x \Rightarrow$ tests (Some $i d x)$ )

(fun $i d x \Rightarrow$ bodies (Some $i d x)$ )

end.

End cond.

Arguments cond $[A]$ default $[n]$ _ ..

Now the expression interpreter is straightforward to write.

Fixpoint exp'Denote $t\left(e: \exp ^{\prime} t\right)$ : type'Denote $t:=$ match $e$ with

NConst $n \Rightarrow n$

| Plus $e 1$ e2 $\Rightarrow$ exp'Denote $e 1+\exp$ 'Denote $e^{2}$

| Eq $e 1$ e2 $\Rightarrow$ if eq_nat_dec (exp'Denote $e 1)$ (exp'Denote $e 2)$ then true else false

BConst $b \Rightarrow b$

| Cond _ _ tests bodies default $\Rightarrow$

cond

(exp'Denote default)

(fun $i d x \Rightarrow$ exp'Denote (tests $i d x)$ )

(fun $i d x \Rightarrow$ exp'Denote (bodies $i d x)$ )

end.

We will implement a constant-folding function that optimizes conditionals, removing cases with known-false tests and cases that come after known-true tests. A function cfoldCond implements the heart of this logic. The convoy pattern is used again near the end of the implementation.

Section cfoldCond.

Variable $t$ : type'.

Variable default : exp' $t$.

Fixpoint cfoldCond $(n:$ nat) 


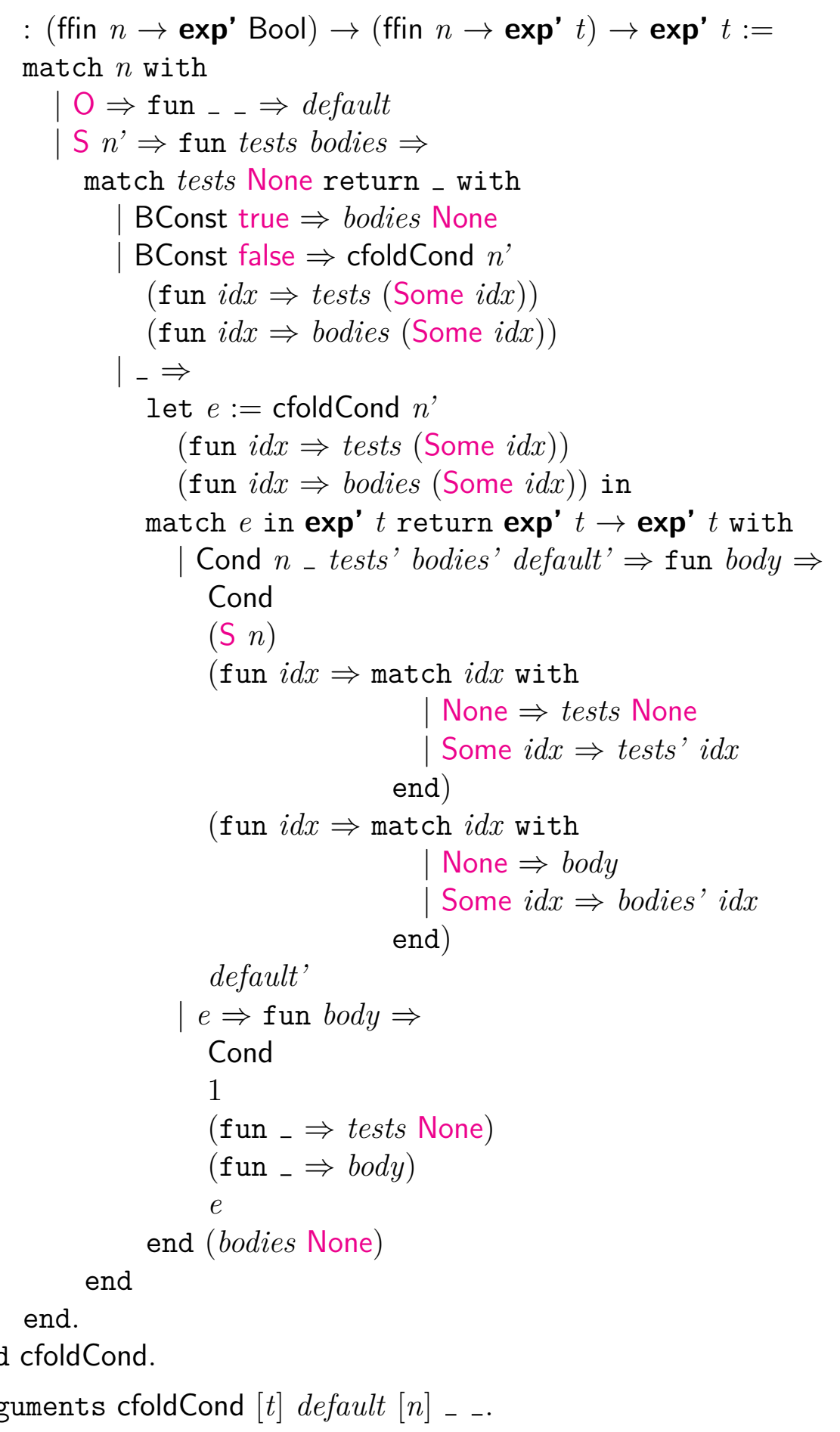

Like for the interpreters, most of the action was in this helper function, and cfold itself is easy to write.

Fixpoint cfold $t\left(e: \exp ^{\prime} t\right): \exp ^{\prime} t:=$ match $e$ with

| NConst $n \Rightarrow$ NConst $n$ 


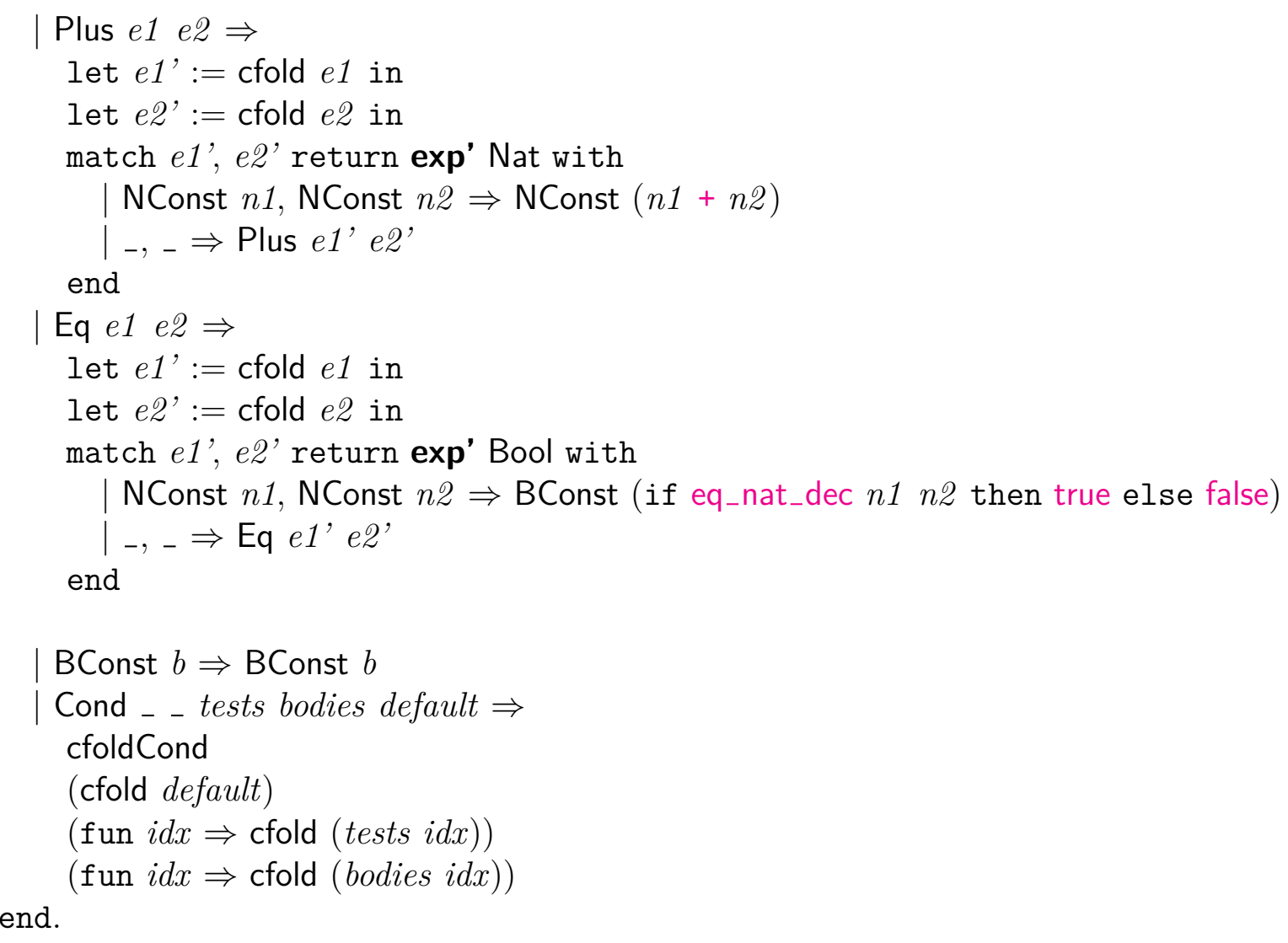

To prove our final correctness theorem, it is useful to know that cfoldCond preserves expression meanings. The following lemma formalizes that property. The proof is a standard mostly automated one, with the only wrinkle being a guided instantiation of the quantifiers in the induction hypothesis.

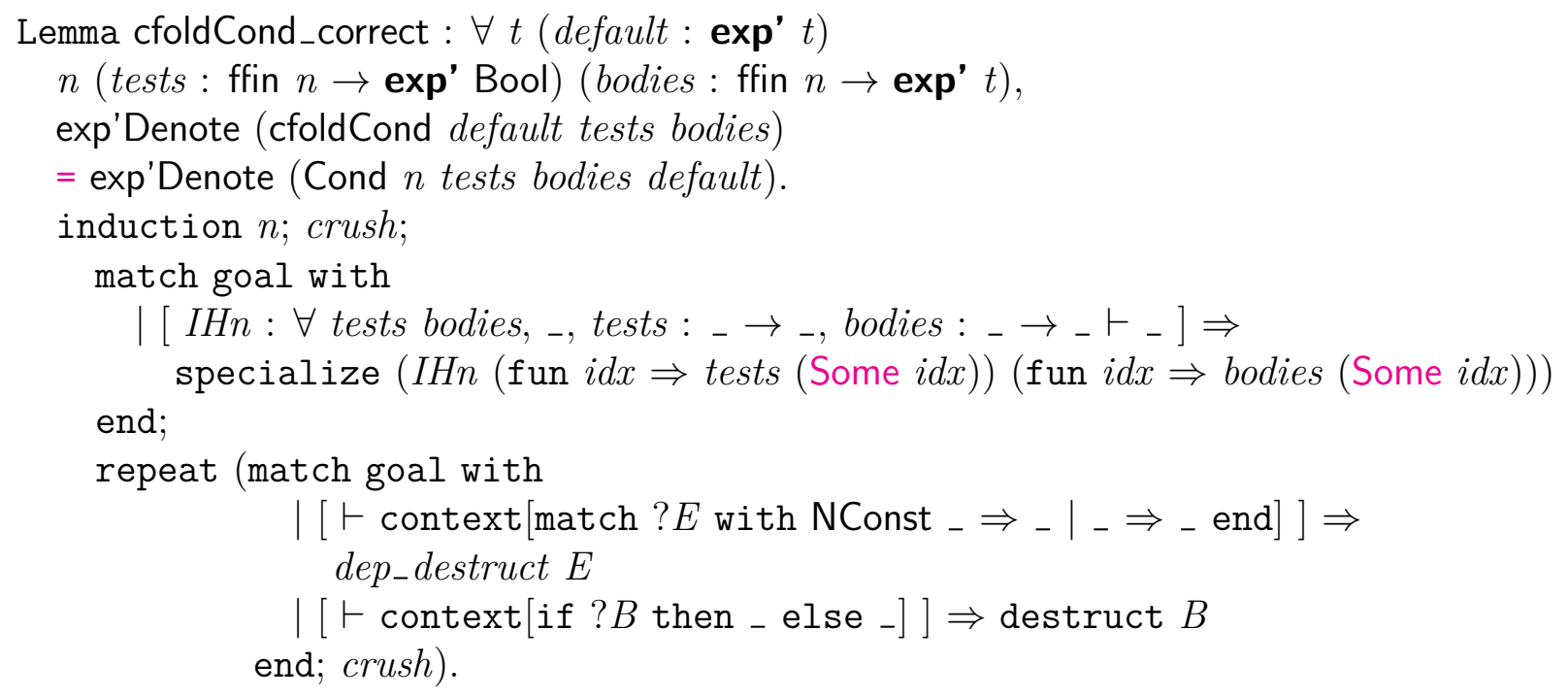

Qed.

It is also useful to know that the result of a call to cond is not changed by substituting 
new tests and bodies functions, so long as the new functions have the same input-output behavior as the old. It turns out that, in Coq, it is not possible to prove in general that functions related in this way are equal. We treat this issue with our discussion of axioms in a later chapter. For now, it suffices to prove that the particular function cond is extensional; that is, it is unaffected by substitution of functions with input-output equivalents.

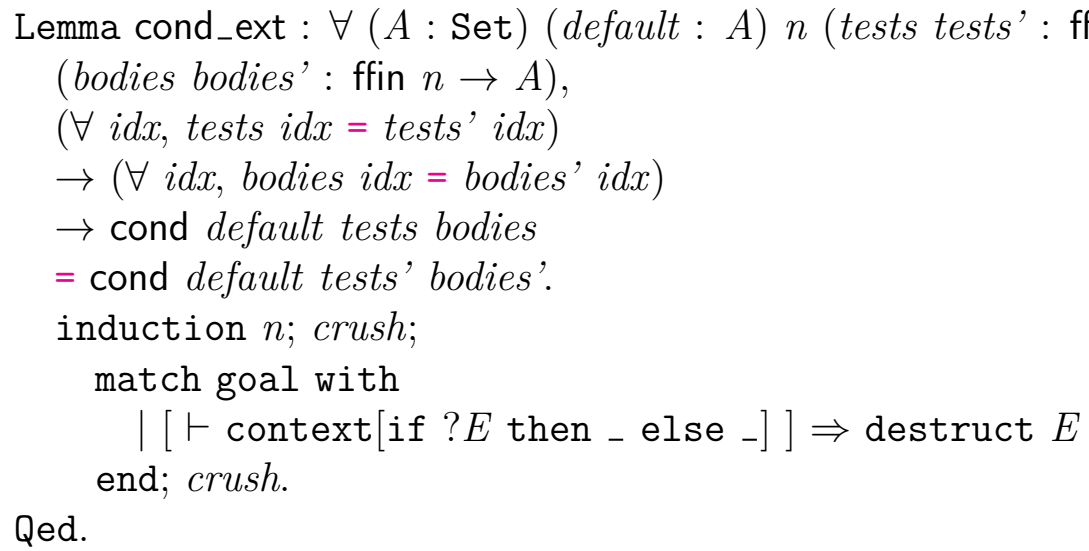

Now the final theorem is easy to prove.

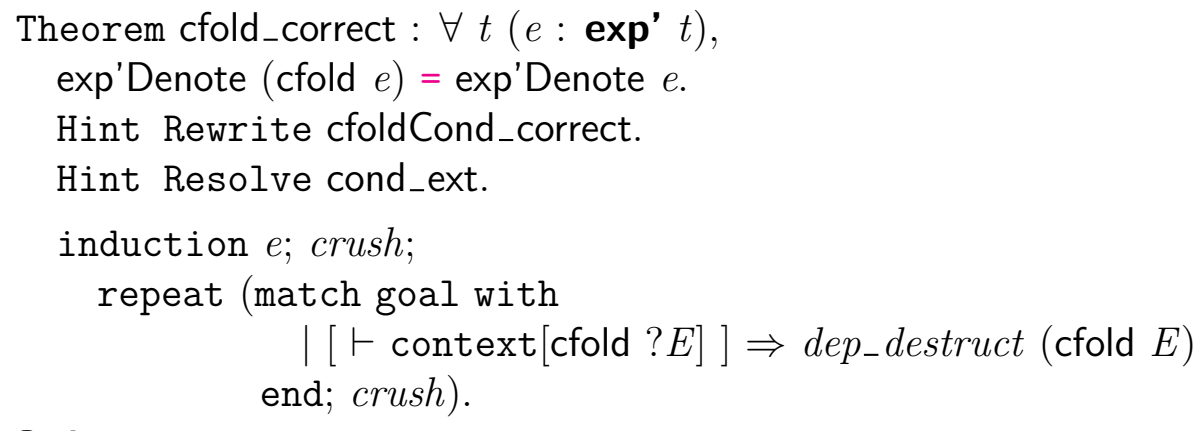

Qed.

We add our two lemmas as hints and perform standard automation with pattern-matching of subterms to destruct.

\subsection{Choosing Between Representations}

It is not always clear which of these representation techniques to apply in a particular situation, but I will try to summarize the pros and cons of each.

Inductive types are often the most pleasant to work with, after someone has spent the time implementing some basic library functions for them, using fancy match annotations. Many aspects of Coq's logic and tactic support are specialized to deal with inductive types, and you may miss out if you use alternate encodings.

Recursive types usually involve much less initial effort, but they can be less convenient to use with proof automation. For instance, the simpl tactic (which is among the ingredients in crush) will sometimes be overzealous in simplifying uses of functions over recursive types. Consider a call get $l f$, where variable $l$ has type filist $A(\mathrm{~S} n)$. The type of $l$ would be 
simplified to an explicit pair type. In a proof involving many recursive types, this kind of unhelpful "simplification" can lead to rapid bloat in the sizes of subgoals. Even worse, it can prevent syntactic pattern-matching, like in cases where filist is expected but a pair type is found in the "simplified" version. The same problem applies to applications of recursive functions to values in recursive types: the recursive function call may "simplify" when the top-level structure of the type index but not the recursive value is known, because such functions are generally defined by recursion on the index, not the value.

Another disadvantage of recursive types is that they only apply to type families whose indices determine their "skeletons." This is not true for all data structures; a good counterexample comes from the richly typed programming language syntax types we have used several times so far. The fact that a piece of syntax has type Nat tells us nothing about the tree structure of that syntax.

Finally, Coq type inference can be more helpful in constructing values in inductive types. Application of a particular constructor of that type tells Coq what to expect from the arguments, while, for instance, forming a generic pair does not make clear an intention to interpret the value as belonging to a particular recursive type. This downside can be mitigated to an extent by writing "constructor" functions for a recursive type, mirroring the definition of the corresponding inductive type.

Reflexive encodings of data types are seen relatively rarely. As our examples demonstrated, manipulating index values manually can lead to hard-to-read code. A normal inductive type is generally easier to work with, once someone has gone through the trouble of implementing an induction principle manually with the techniques we studied in Chapter 3. For small developments, avoiding that kind of coding can justify the use of reflexive data structures. There are also some useful instances of co-inductive definitions with nested data structures (e.g., lists of values in the co-inductive type) that can only be deconstructed effectively with reflexive encoding of the nested structures. 


\section{Chapter 10}

\section{Reasoning About Equality Proofs}

In traditional mathematics, the concept of equality is usually taken as a given. On the other hand, in type theory, equality is a very contentious subject. There are at least three different notions of equality that are important in Coq, and researchers are actively investigating new definitions of what it means for two terms to be equal. Even once we fix a notion of equality, there are inevitably tricky issues that arise in proving properties of programs that manipulate equality proofs explicitly. In this chapter, I will focus on design patterns for circumventing these tricky issues, and I will introduce the different notions of equality as they are germane.

\subsection{The Definitional Equality}

We have seen many examples so far where proof goals follow "by computation." That is, we apply computational reduction rules to reduce the goal to a normal form, at which point it follows trivially. Exactly when this works and when it does not depends on the details of Coq's definitional equality. This is an untyped binary relation appearing in the formal metatheory of CIC. CIC contains a typing rule allowing the conclusion $E$ : $T$ from the premise $E$ : $T^{\prime}$ and a proof that $T$ and $T^{\prime}$ are definitionally equal.

The cbv tactic will help us illustrate the rules of Coq's definitional equality. We redefine the natural number predecessor function in a somewhat convoluted way and construct a manual proof that it returns 0 when applied to 1 .

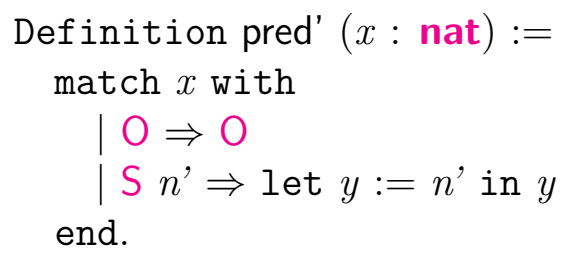

Theorem reduce_me : pred' $1=0$.

CIC follows the traditions of lambda calculus in associating reduction rules with Greek letters. Coq can certainly be said to support the familiar alpha reduction rule, which allows capture-avoiding renaming of bound variables, but we never need to apply alpha explicitly, 
since Coq uses a de Bruijn representation [11] that encodes terms canonically.

The delta rule is for unfolding global definitions. We can use it here to unfold the definition of pred'. We do this with the cbv tactic, which takes a list of reduction rules and makes as many call-by-value reduction steps as possible, using only those rules. There is an analogous tactic lazy for call-by-need reduction.

cbv delta.

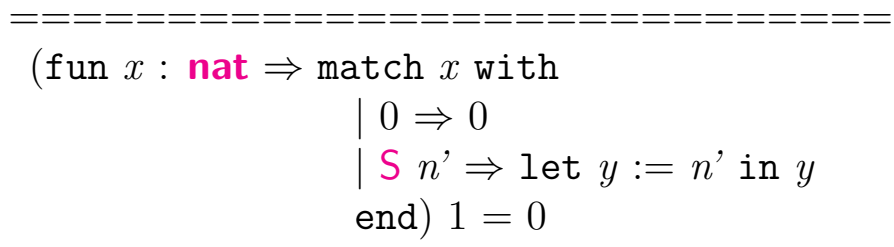

At this point, we want to apply the famous beta reduction of lambda calculus, to simplify the application of a known function abstraction.

cbv beta.

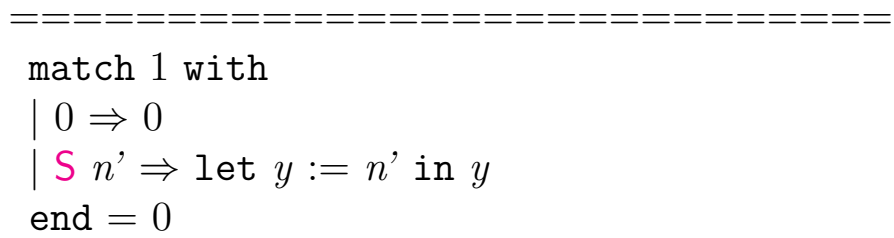

Next on the list is the iota reduction, which simplifies a single match term by determining which pattern matches.

cbv iota.

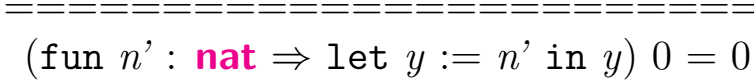

Now we need another beta reduction.

cbv beta.

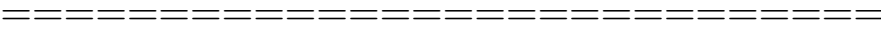

(let $y:=0$ in $y)=0$

The final reduction rule is zeta, which replaces a let expression by its body with the appropriate term substituted.

cbv zeta.

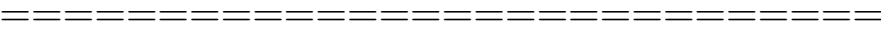

$$
\begin{aligned}
& 0=0
\end{aligned}
$$

reflexivity.

Qed.

The beta reduction rule applies to recursive functions as well, and its behavior may be 
surprising in some instances. For instance, we can run some simple tests using the reduction strategy compute, which applies all applicable rules of the definitional equality.

Definition id $(n:$ nat) $:=n$.

Eval compute in fun $x \Rightarrow$ id $x$.

$=$ fun $x:$ nat $\Rightarrow x$

Fixpoint id' ( $n:$ nat) $:=n$.

Eval compute in fun $x \Rightarrow$ id' $x$.

$=$ fun $x:$ nat $\Rightarrow($ fix id' $(n:$ nat $):$ nat $:=n) x$

By running compute, we ask Coq to run reduction steps until no more apply, so why do we see an application of a known function, where clearly no beta reduction has been performed? The answer has to do with ensuring termination of all Gallina programs. One candidate rule would say that we apply recursive definitions wherever possible. However, this would clearly lead to nonterminating reduction sequences, since the function may appear fully applied within its own definition, and we would naïvely "simplify" such applications immediately. Instead, Coq only applies the beta rule for a recursive function when the top-level structure of the recursive argument is known. For id' above, we have only one argument $n$, so clearly it is the recursive argument, and the top-level structure of $n$ is known when the function is applied to $\mathrm{O}$ or to some $\mathrm{S} e$ term. The variable $x$ is neither, so reduction is blocked.

What are recursive arguments in general? Every recursive function is compiled by Coq to a $\mathrm{fix}$ expression, for anonymous definition of recursive functions. Further, every $\mathrm{f}$ ix with multiple arguments has one designated as the recursive argument via a struct annotation. The recursive argument is the one that must decrease across recursive calls, to appease Coq's termination checker. Coq will generally infer which argument is recursive, though we may also specify it manually, if we want to tweak reduction behavior. For instance, consider this definition of a function to add two lists of nats elementwise:

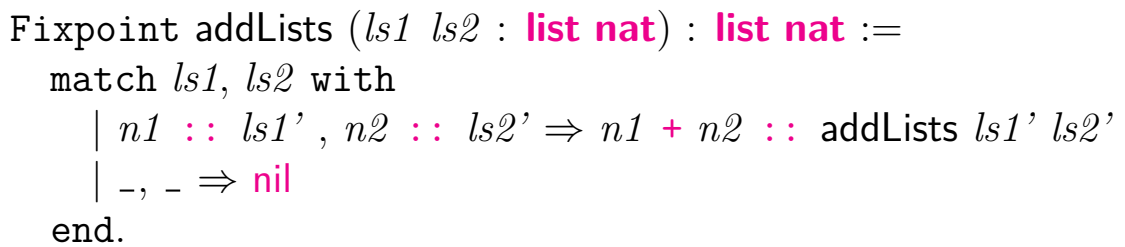

By default, Coq chooses $l s 1$ as the recursive argument. We can see that $l s 2$ would have been another valid choice. The choice has a critical effect on reduction behavior, as these two examples illustrate:

Eval compute in fun $l s \Rightarrow$ addLists nil $l s$.

$=$ fun $_{-}$: list nat $\Rightarrow$ nil

Eval compute in fun $l s \Rightarrow$ addLists $l s$ nil.

$=$ fun $l s:$ list nat $\Rightarrow$ 


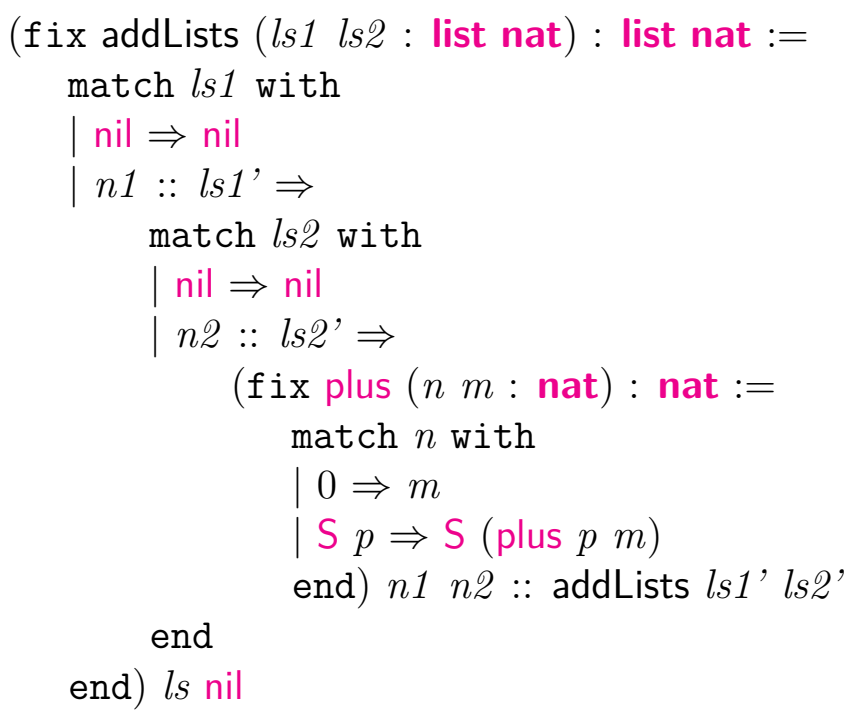

The outer application of the fix expression for addLists was only simplified in the first case, because in the second case the recursive argument is $l s$, whose top-level structure is not known.

The opposite behavior pertains to a version of addLists with $l s 2$ marked as recursive.
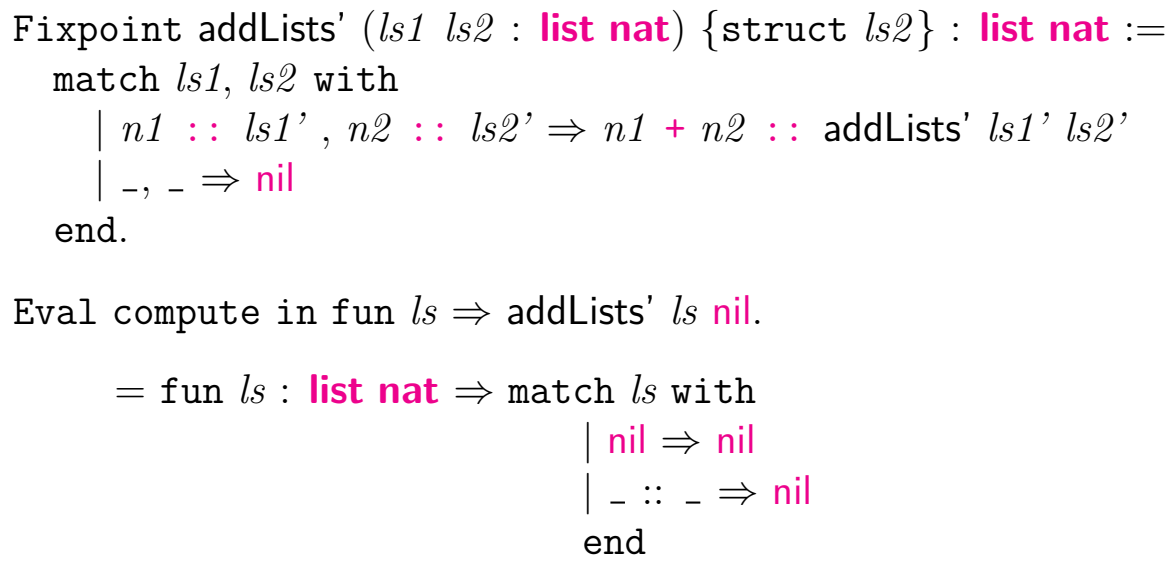

We see that all use of recursive functions has been eliminated, though the term has not quite simplified to nil. We could get it to do so by switching the order of the match discriminees in the definition of addLists'.

Recall that co-recursive definitions have a dual rule: a co-recursive call only simplifies when it is the discriminee of a match. This condition is built into the beta rule for cofix, the anonymous form of CoFixpoint.

The standard eq relation is critically dependent on the definitional equality. The relation eq is often called a propositional equality, because it reifies definitional equality as a proposition that may or may not hold. Standard axiomatizations of an equality predicate in first-order logic define equality in terms of properties it has, like reflexivity, symmetry, and transitivity. In contrast, for eq in Coq, those properties are implicit in the properties of the definitional equality, which are built into CIC's metatheory and the implementation of 
Gallina. We could add new rules to the definitional equality, and eq would keep its definition and methods of use.

This all may make it sound like the choice of eq's definition is unimportant. To the contrary, in this chapter, we will see examples where alternate definitions may simplify proofs. Before that point, I will introduce proof methods for goals that use proofs of the standard propositional equality "as data."

\subsection{Heterogeneous Lists Revisited}

One of our example dependent data structures from the last chapter (code repeated below) was the heterogeneous list and its associated "cursor" type. The recursive version poses some special challenges related to equality proofs, since it uses such proofs in its definition of fmember types.

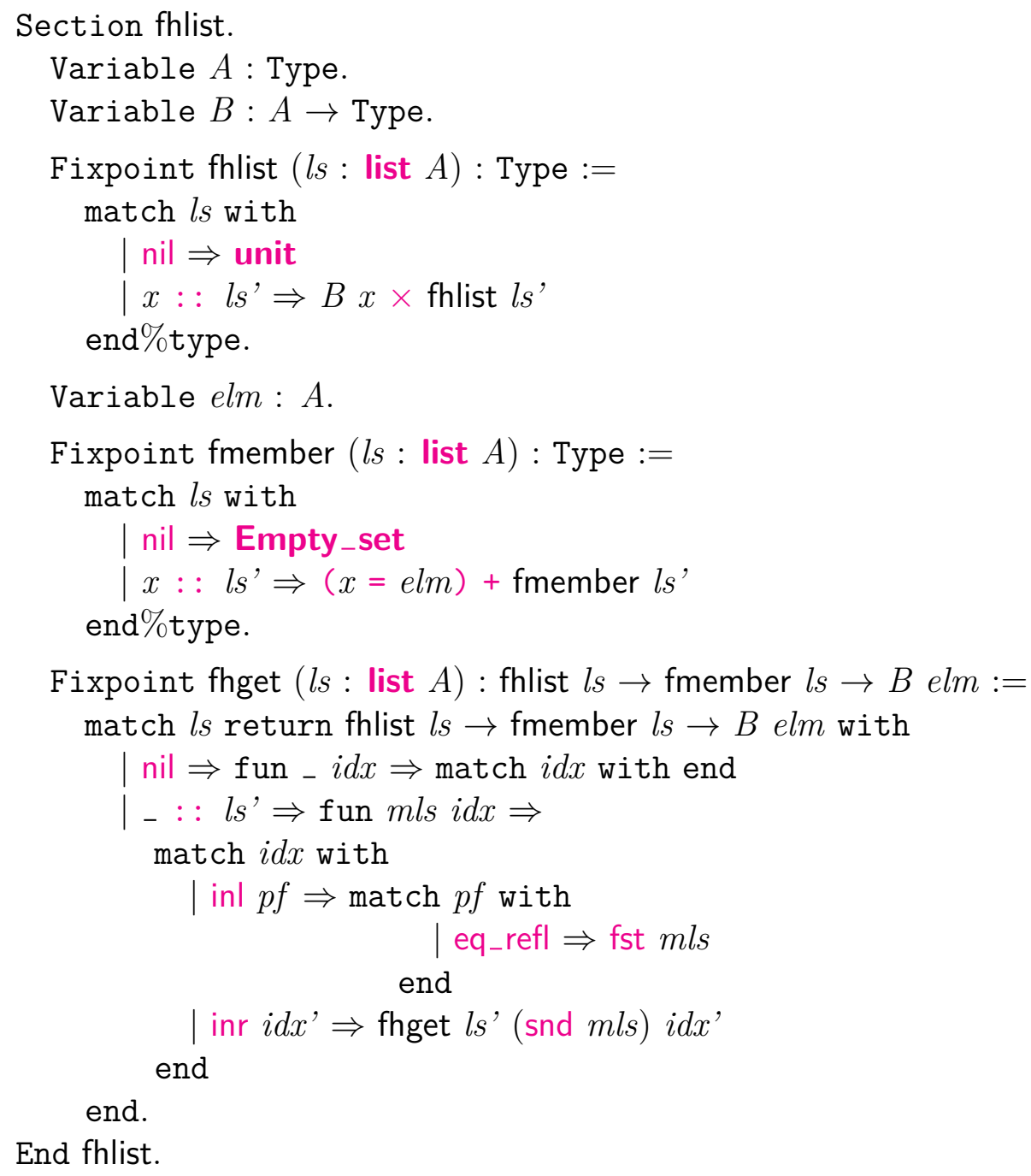

Arguments fhget $\left[\begin{array}{llll}A & B & \mathrm{elm} & \mathrm{ls}\end{array}\right] \ldots$ 
We can define a map-like function for fhlists.

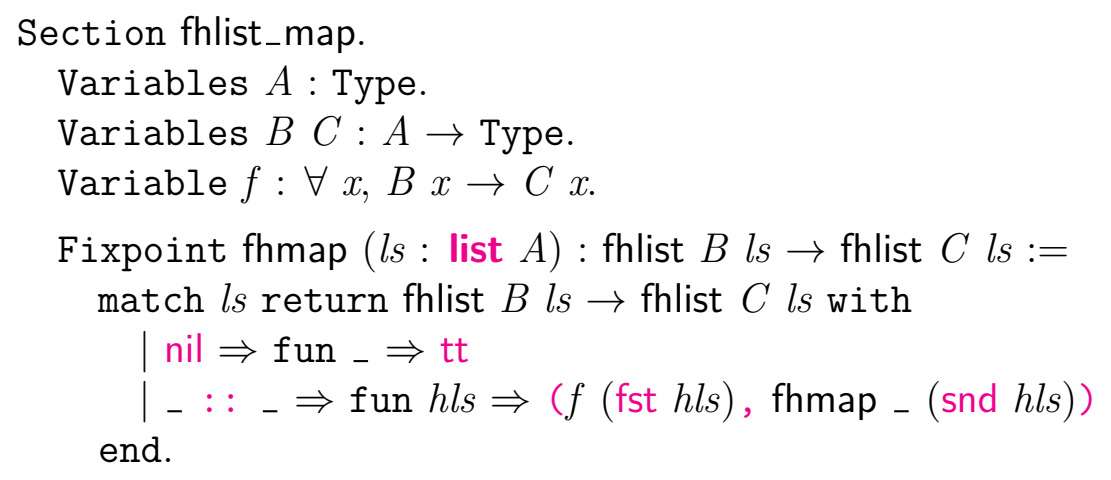

\section{Arguments fhmap $[l s]_{-}$.}

For the inductive versions of the ilist definitions, we proved a lemma about the interaction of get and imap. It was a strategic choice not to attempt such a proof for the definitions that we just gave, which sets us on a collision course with the problems that are the subject of this chapter.

Variable elm: $A$.

Theorem fhget_fhmap : $\forall l s$ (mem : fmember elm ls) (hls: fhlist $B l s$ ), fhget (fhmap hls) mem $=f$ (fhget hls mem).

induction $l s$; crush.

In Coq 8.2, one subgoal remains at this point. Coq 8.3 has added some tactic improvements that enable crush to complete all of both inductive cases. To introduce the basics of reasoning about equality, it will be useful to review what was necessary in Coq 8.2.

Part of our single remaining subgoal is:

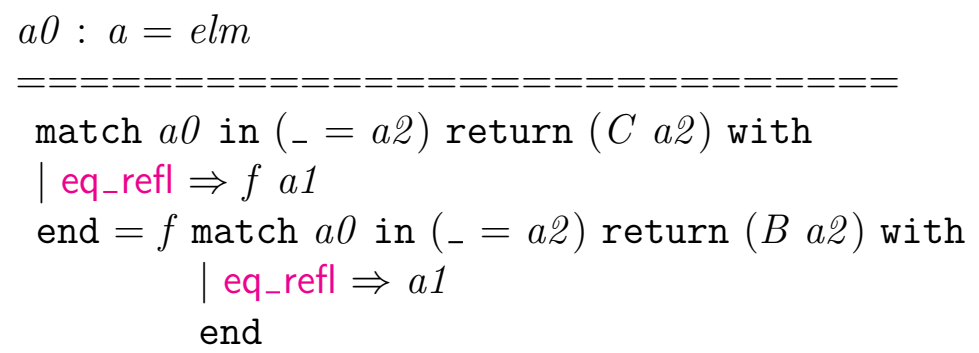

This seems like a trivial enough obligation. The equality proof a0 must be eq_refl, the only constructor of eq. Therefore, both the matches reduce to the point where the conclusion follows by reflexivity.

destruct $a 0$.

User error: Cannot solve a second-order unification problem

This is one of Coq's standard error messages for informing us of a failure in its heuristics for attempting an instance of an undecidable problem about dependent typing. We might 
try to nudge things in the right direction by stating the lemma that we believe makes the conclusion trivial.

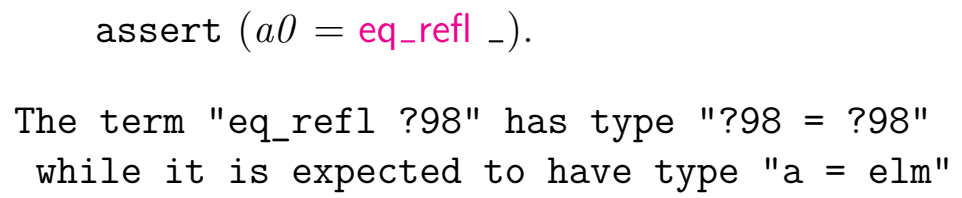

In retrospect, the problem is not so hard to see. Reflexivity proofs only show $x=x$ for particular values of $x$, whereas here we are thinking in terms of a proof of $a=e l m$, where the two sides of the equality are not equal syntactically. Thus, the essential lemma we need does not even type-check!

Is it time to throw in the towel? Luckily, the answer is "no." In this chapter, we will see several useful patterns for proving obligations like this.

For this particular example, the solution is surprisingly straightforward. The destruct tactic has a simpler sibling case which should behave identically for any inductive type with one constructor of no arguments.

case $a 0$.

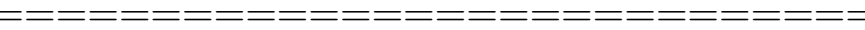

$$
\begin{aligned}
& f a 1=f a 1
\end{aligned}
$$

It seems that destruct was trying to be too smart for its own good.

$$
\text { reflexivity. }
$$

\section{Qed.}

It will be helpful to examine the proof terms generated by this sort of strategy. A simpler example illustrates what is going on.

Lemma lemma1 : $\forall x(p f: x=$ elm $), \mathrm{O}=$ match $p f$ with eq_refl $\Rightarrow \mathrm{O}$ end. simple destruct $p f$; reflexivity.

$$
\text { Qed. }
$$

The tactic simple destruct $p f$ is a convenient form for applying case. It runs intro to bring into scope all quantified variables up to its argument.

Print lemma1.

$$
\begin{aligned}
& \text { lemma1 }= \\
& \text { fun }(x: A)(p f: x=\text { elm }) \Rightarrow
\end{aligned}
$$$$
\text { match } p f \text { as } e \text { in }(-=y) \text { return }(0=\text { match } e \text { with }
$$$$
\text { | eq_refl } \Rightarrow 0
$$$$
\text { end) with }
$$

| eq_refl $\Rightarrow$ eq_refl 0

end

$$
\begin{aligned}
: \forall(x: A)(p f: x=e l m), 0=\text { match } p f \text { with } \\
\qquad \mid \text { eq_refl } \Rightarrow 0
\end{aligned}
$$


end

Using what we know about shorthands for match annotations, we can write this proof in shorter form manually.

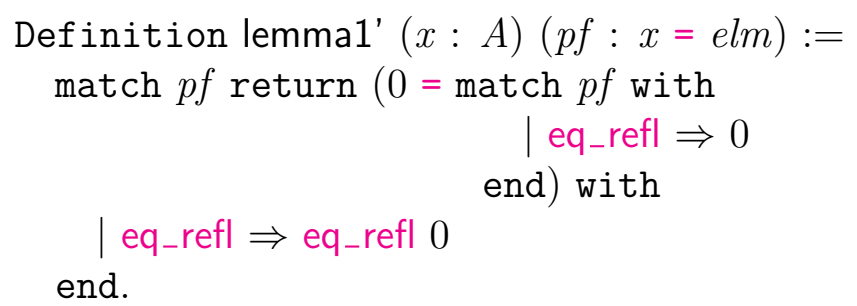

Surprisingly, what seems at first like a simpler lemma is harder to prove.

Lemma lemma2 $: \forall(x: A)(p f: x=x), \mathrm{O}=$ match $p f$ with eq_refl $\Rightarrow \mathrm{O}$ end. simple destruct $p f$.

User error: Cannot solve a second-order unification problem

Abort.

Nonetheless, we can adapt the last manual proof to handle this theorem.

Definition lemma2 :=

fun $(x: A)(p f: x=x) \Rightarrow$ match $p f$ return $(0=$ match $p f$ with

$$
\text { | eq_refl } \Rightarrow 0
$$

| eq_refl $\Rightarrow$ eq_refl 0

end) with

end.

We can try to prove a lemma that would simplify proofs of many facts like lemma2:

Lemma lemma3 $: \forall(x: A)(p f: x=x), p f=$ eq_refl $x$. simple destruct $p f$.

User error: Cannot solve a second-order unification problem

Abort.

This time, even our manual attempt fails.

'Definition lemma3':=

fun $(x: A)(p f: x=x) \Rightarrow$ match $p f$ as $p f^{\prime}$ in $\left({ }_{-}=x^{\prime}\right)$ return $\left(p f^{\prime}=\right.$ eq_refl $\left.x^{\prime}\right)$ with

| eq_refl $\Rightarrow$ eq_refl _ end.

The term "eq_refl $\mathrm{x}$ " has type " $\mathrm{x}$ " $=\mathrm{x}$ " "while it is expected to have type $" \mathrm{x}=\mathrm{x}$ " 
The type error comes from our return annotation. In that annotation, the as-bound variable $p f^{\prime}$ has type $x=x$ ', referring to the in-bound variable $x$ '. To do a dependent match, we must choose a fresh name for the second argument of eq. We are just as constrained to use the "real" value $x$ for the first argument. Thus, within the return clause, the proof we are matching on must equate two non-matching terms, which makes it impossible to equate that proof with reflexivity.

Nonetheless, it turns out that, with one catch, we can prove this lemma.

Lemma lemma3 : $\forall(x: A)(p f: x=x), p f=$ eq_refl $x$.

intros; apply UIP_refl.

Qed.

Check UIP_refl.

UIP_refl

$: \forall(U:$ Type $)(x: U)(p: x=x), p=$ eq_refl $x$

The theorem UIP_refl comes from the Eqdep module of the standard library. (Its name uses the acronym "UIP" for "unicity of identity proofs.") Do the Coq authors know of some clever trick for building such proofs that we have not seen yet? If they do, they did not use it for this proof. Rather, the proof is based on an axiom, the term eq_rect_eq below.

Print eq_rect_eq.

$* * *[$ eq_rect_eq :

$\forall(U:$ Type $)(p: U)(Q: U \rightarrow$ Type $)(x: Q p)(h: p=p)$, $x=$ eq_rect $p Q x p h]$

The axiom eq_rect_eq states a "fact" that seems like common sense, once the notation is deciphered. The term eq_rect is the automatically generated recursion principle for eq. Calling eq_rect is another way of matching on an equality proof. The proof we match on is the argument $h$, and $x$ is the body of the match. The statement of eq_rect_eq just says that matches on proofs of $p=p$, for any $p$, are superfluous and may be removed. We can see this intuition better in code by asking Coq to simplify the theorem statement with the compute reduction strategy.

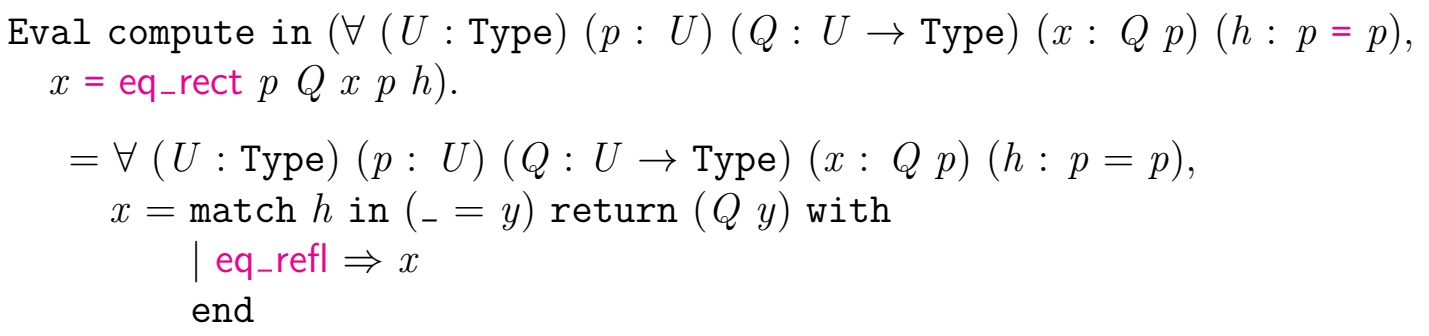

Perhaps surprisingly, we cannot prove eq_rect_eq from within Coq. This proposition is introduced as an axiom; that is, a proposition asserted as true without proof. We cannot assert just any statement without proof. Adding False as an axiom would allow us to prove any proposition, for instance, defeating the point of using a proof assistant. In general, we need to be sure that we never assert inconsistent sets of axioms. A set of axioms is 
inconsistent if its conjunction implies False. For the case of eq_rect_eq, consistency has been verified outside of Coq via "informal" metatheory [43], in a study that also established unprovability of the axiom in CIC.

This axiom is equivalent to another that is more commonly known and mentioned in type theory circles.

Check Streicher_K.

Streicher_K

$: \forall(U:$ Type $)(x: U)(P: x=x \rightarrow$ Prop $)$,

$P$ eq_refl $\rightarrow \forall p: x=x, P p$

This is the opaquely named "Streicher's axiom K," which says that a predicate on properly typed equality proofs holds of all such proofs if it holds of reflexivity.

End fhlist_map.

It is worth remarking that it is possible to avoid axioms altogether for equalities on types with decidable equality. The Eqdep_dec module of the standard library contains a parametric proof of UIP_refl for such cases. To simplify presentation, we will stick with the axiom version in the rest of this chapter.

\subsection{Type-Casts in Theorem Statements}

Sometimes we need to use tricks with equality just to state the theorems that we care about. To illustrate, we start by defining a concatenation function for fhlists.

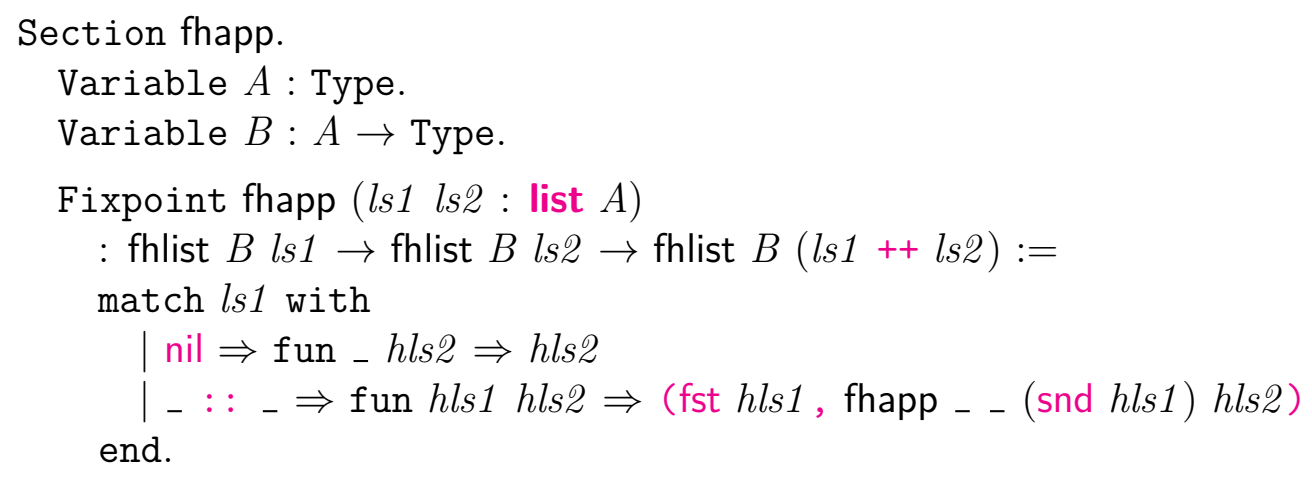

Arguments fhapp $\left[\begin{array}{ll}l s 1 & l s 2\end{array}\right]$ _..

We might like to prove that fhapp is associative.

Theorem fhapp_assoc : $\forall l s 1$ ls2 ls3

(hls1 : fhlist $B$ ls1) (hls2 : fhlist $B$ ls2) (hls3 : fhlist $B$ ls3),

fhapp hls1 (fhapp hls2 hls3) = fhapp (fhapp hls1 hls2) hls3.

The term

"fhapp (ls1:=ls1 ++ ls2) (ls2:=ls3) (fhapp (ls1:=ls1) (ls2:=ls2) hls1 hls2) 


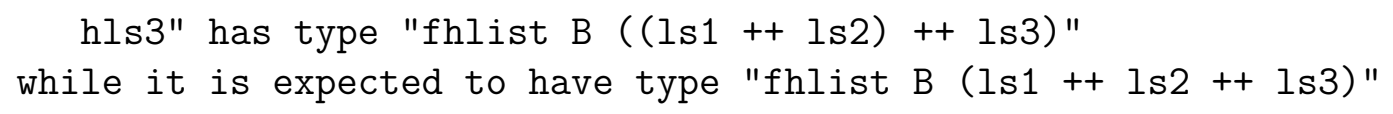

This first cut at the theorem statement does not even type-check. We know that the two fhlist types appearing in the error message are always equal, by associativity of normal list append, but this fact is not apparent to the type checker. This stems from the fact that Coq's equality is intensional, in the sense that type equality theorems can never be applied after the fact to get a term to type-check. Instead, we need to make use of equality explicitly in the theorem statement.

Theorem fhapp_assoc : $\forall l s 1$ ls2 ls3

$(p f:(l s 1++l s 2)++l s 3=l s 1++(l s 2++l s 3))$

(hls1 : fhlist $B$ ls1) (hls2 : fhlist $B$ ls2) (hls3 : fhlist $B$ ls3),

fhapp hls1 (fhapp hls2 hls3)

$=$ match $p f$ in $\left(_{-}=l s\right)$ return fhlist $\_l s$ with

| eq_refl $\Rightarrow$ fhapp (fhapp hls1 hls2) hls3

end.

induction $l s 1$; crush.

The first remaining subgoal looks trivial enough:

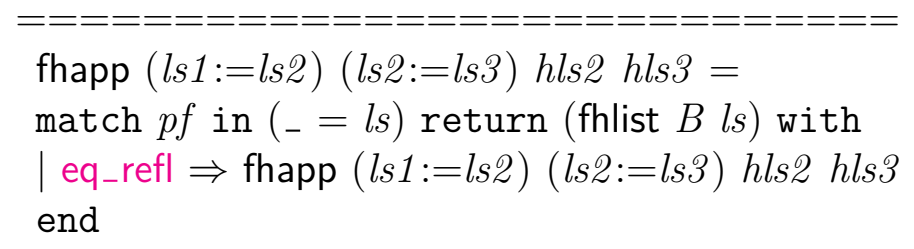

We can try what worked in previous examples.

case $p f$.

User error: Cannot solve a second-order unification problem

It seems we have reached another case where it is unclear how to use a dependent match to implement case analysis on our proof. The UIP_refl theorem can come to our rescue again.

$$
\text { rewrite (UIP_refl_ - pf). }
$$

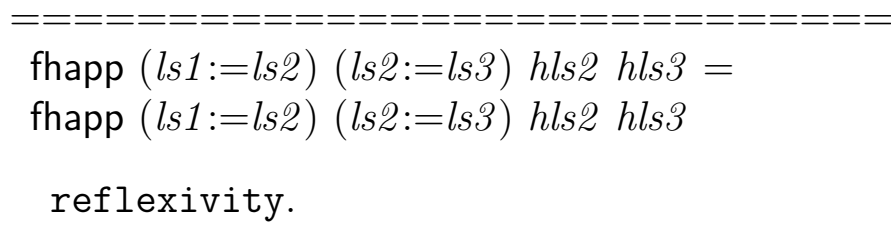

Our second subgoal is trickier.

$$
\begin{aligned}
& \text { pf: }: a::(l s 1++l s 2)++l s 3=a:: l s 1++l s 2++l s 3 \\
& =======================
\end{aligned}
$$




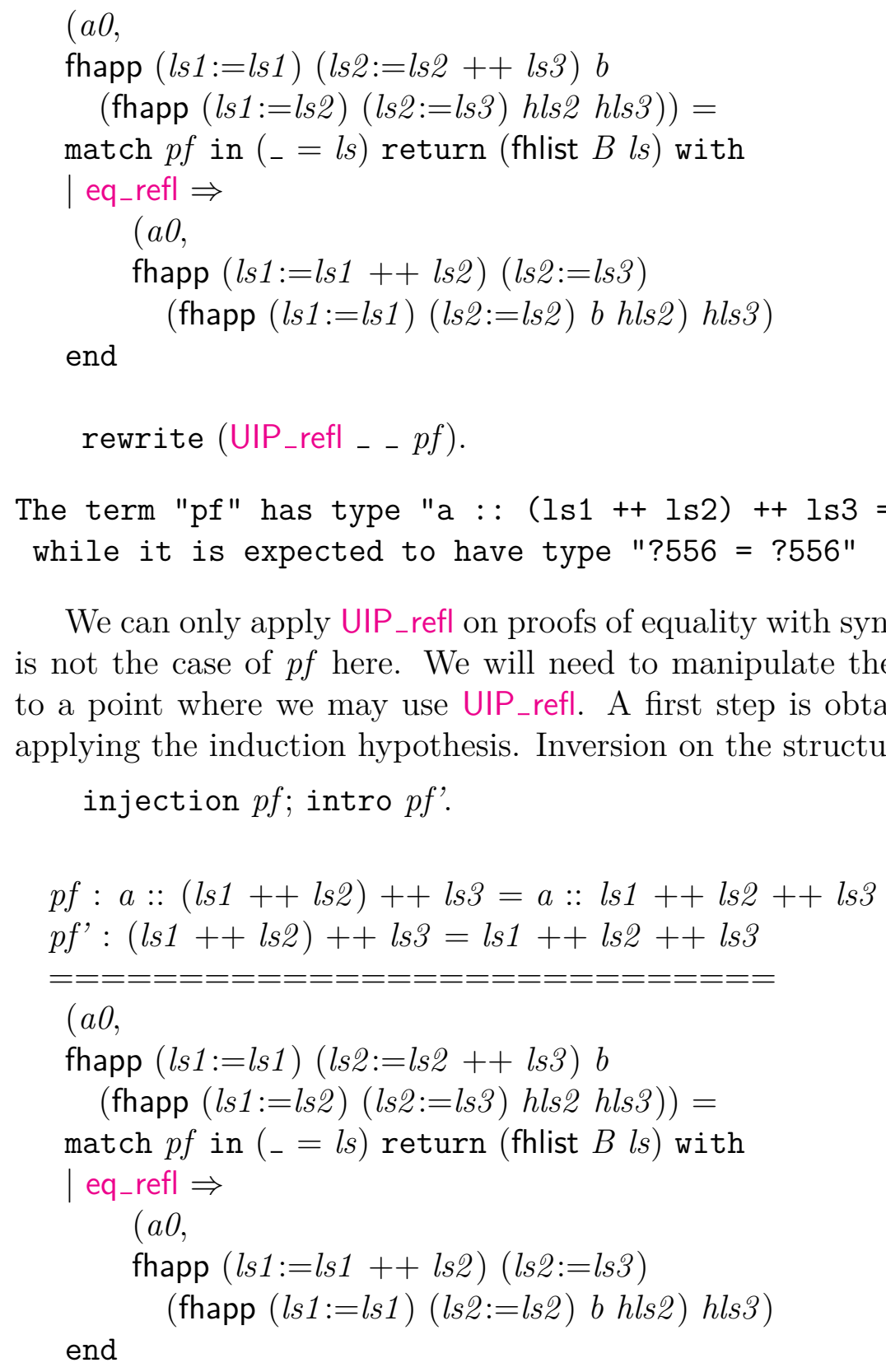

We can only apply UIP_refl on proofs of equality with syntactically equal operands, which is not the case of $p f$ here. We will need to manipulate the form of this subgoal to get us to a point where we may use UIP_refl. A first step is obtaining a proof suitable to use in applying the induction hypothesis. Inversion on the structure of $p f$ is sufficient for that.

Now we can rewrite using the inductive hypothesis.

rewrite (IHls1 - - pf').
$\begin{aligned}= & = \\ & (a 0,\end{aligned}$ match $p f^{\prime}$ in $\left(_{-}=l s\right)$ return (fhlist $\left.B l s\right)$ with | eq_refl $\Rightarrow$
fhapp $(l s 1:=l s 1++l s 2)(l s 2:=l s 3)$
(fhapp $(l s 1:=l s 1)(l s 2:=l s 2) b$ hls2) hls3




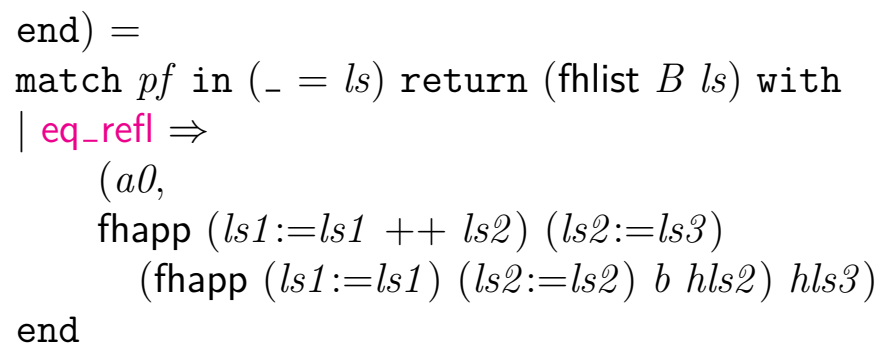

We have made an important bit of progress, as now only a single call to fhapp appears in the conclusion, repeated twice. Trying case analysis on our proofs still will not work, but there is a move we can make to enable it. Not only does just one call to fhapp matter to us now, but it also does not matter what the result of the call is. In other words, the subgoal should remain true if we replace this fhapp call with a fresh variable. The generalize tactic helps us do exactly that.

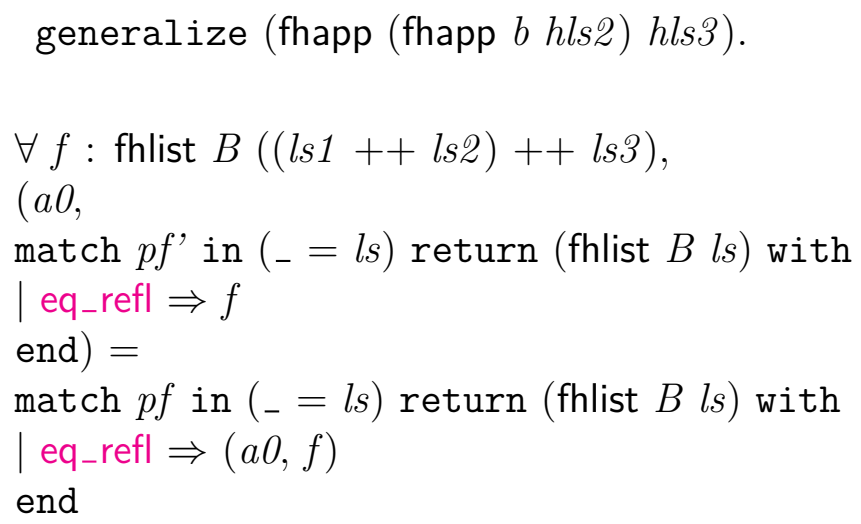

The conclusion has gotten markedly simpler. It seems counterintuitive that we can have an easier time of proving a more general theorem, but such a phenomenon applies to the case here and to many other proofs that use dependent types heavily. Speaking informally, the reason why this kind of activity helps is that match annotations contain some positions where only variables are allowed. By reducing more elements of a goal to variables, built-in tactics can have more success building match terms under the hood.

In this case, it is helpful to generalize over our two proofs as well.

$$
\begin{aligned}
& \text { generalize pf pf'. } \\
& \forall(p f 0: a::(l s 1++l s \mathscr{2})++l s 3=a:: l s 1++l s \mathscr{2}++l s 3) \\
& \quad\left(p f^{\prime} 0:(l s 1++l s \mathcal{2})++l s 3=l s 1++l s \mathscr{2}++l s 3\right) \\
& \quad(f: \text { fhlist } B((l s 1++l s \mathcal{2})++l s 3)), \\
& (a 0, \\
& \text { match } p f^{\prime} 0 \text { in }(=l s) \text { return }(\text { fhlist } B l s) \text { with } \\
& \text { | eq_refl } \Rightarrow f \\
& \text { end })= \\
& \text { match } p f 0 \text { in }(-=l s) \text { return (fhlist } B l s) \text { with } \\
& \text { | eq_refl } \Rightarrow(a 0, f)
\end{aligned}
$$


end

To an experienced dependent types hacker, the appearance of this goal term calls for a celebration. The formula has a critical property that indicates that our problems are over. To get our proofs into the right form to apply UIP_refl, we need to use associativity of list append to rewrite their types. We could not do so before because other parts of the goal require the proofs to retain their original types. In particular, the call to fhapp that we generalized must have type $(l s 1++l s 2)++l s 3$, for some values of the list variables. If we rewrite the type of the proof used to type-cast this value to something like $l s 1++l s 2++$ $l s 3=l s 1++l s 2++l s 3$, then the lefthand side of the equality would no longer match the type of the term we are trying to cast.

However, now that we have generalized over the fhapp call, the type of the term being type-cast appears explicitly in the goal and may be rewritten as well. In particular, the final masterstroke is rewriting everywhere in our goal using associativity of list append.

rewrite app_assoc.

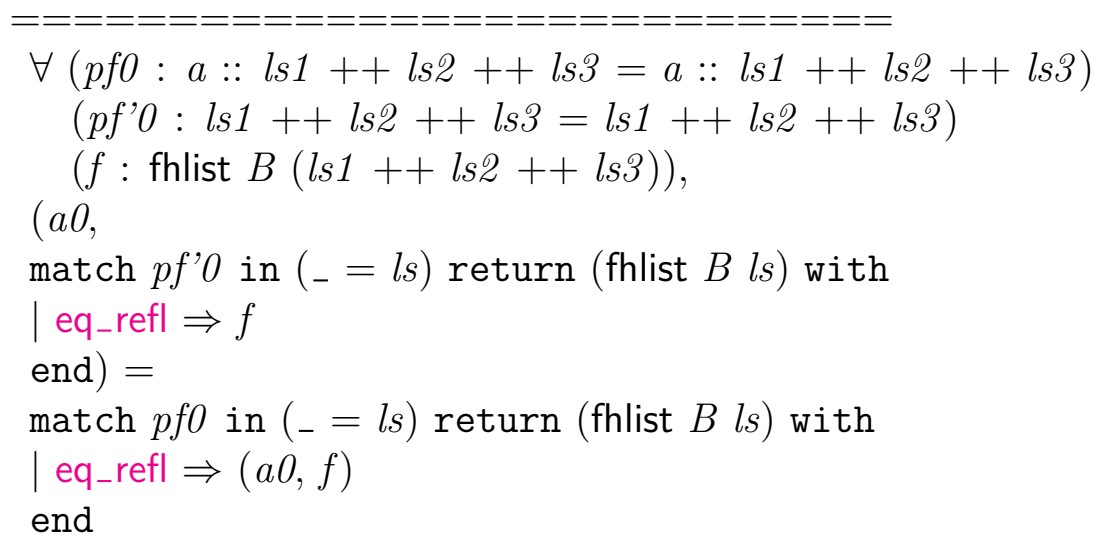

We can see that we have achieved the crucial property: the type of each generalized equality proof has syntactically equal operands. This makes it easy to finish the proof with UIP_refl.

intros.

rewrite (UIP_refl _ _ pfo).

rewrite (UIP_refl _ $-p f^{\prime} 0$ ).

reflexivity.

Qed.

End fhapp.

Arguments fhapp $\left[\begin{array}{lllll}A & B & l s 1 & l s 2\end{array}\right]$ _..

This proof strategy was cumbersome and unorthodox, from the perspective of mainstream mathematics. The next section explores an alternative that leads to simpler developments in some cases. 


\subsection{Heterogeneous Equality}

There is another equality predicate, defined in the JMeq module of the standard library, implementing heterogeneous equality.

Print JMeq.

Inductive JMeq ( $A$ : Type) $(x: A): \forall B:$ Type, $B \rightarrow$ Prop $:=$

JMeq_refl : JMeq $x x$

The identifier JMeq stands for "John Major equality," a name coined by Conor McBride [23] as an inside joke about British politics. The definition JMeq starts out looking a lot like the definition of eq. The crucial difference is that we may use JMeq on arguments of different types. For instance, a lemma that we failed to establish before is trivial with JMeq. It makes for prettier theorem statements to define some syntactic shorthand first.

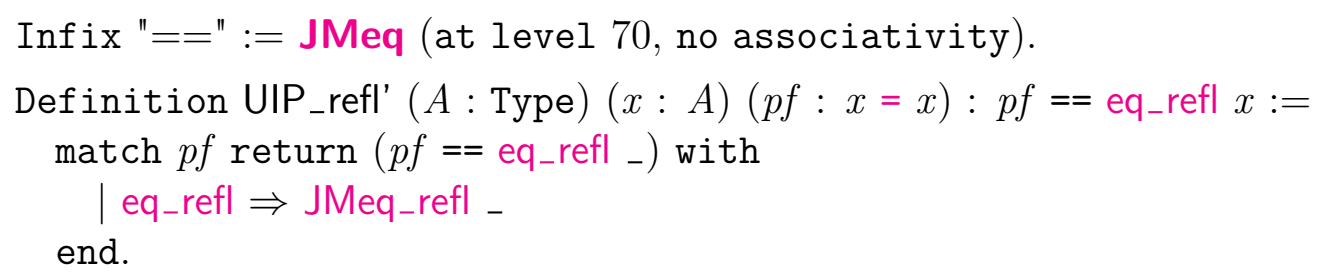

There is no quick way to write such a proof by tactics, but the underlying proof term that we want is trivial.

Suppose that we want to use UIP_refl' to establish another lemma of the kind we have run into several times so far.

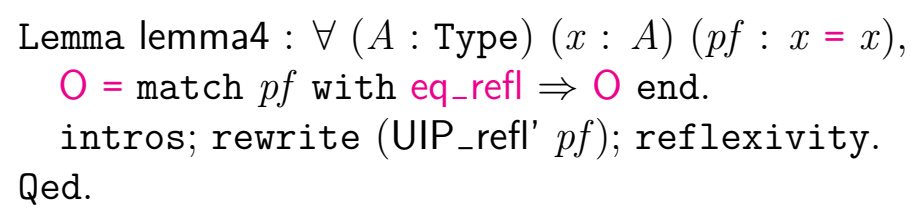

All in all, refreshingly straightforward, but there really is no such thing as a free lunch. The use of rewrite is implemented in terms of an axiom:

Check JMeq_eq.

JMeq_eq

$$
: \forall(A: \text { Type })(x y: A), x==y \rightarrow x=y
$$

It may be surprising that we cannot prove that heterogeneous equality implies normal equality. The difficulties are the same kind we have seen so far, based on limitations of match annotations. The JMeq_eq axiom has been proved on paper to be consistent, but asserting it may still be considered to complicate the logic we work in, so there is some motivation for avoiding it.

We can redo our fhapp associativity proof based around JMeq.

Section fhapp'.

Variable $A$ : Type.

Variable $B: A \rightarrow$ Type. 
This time, the naïve theorem statement type-checks.

Theorem fhapp_assoc' : $\forall$ ls1 ls2 ls3 (hls1 : fhlist $B$ ls1) (hls2 : fhlist $B$ ls2)

(hls3 : fhlist $B$ ls3),

fhapp hls1 (fhapp hls2 hls3) == fhapp (fhapp hls1 hls2) hls3.

induction $l s 1$; crush.

Even better, crush discharges the first subgoal automatically. The second subgoal is:

$==================$

$(a 0$, fhapp $b($ fhapp hls2 hls3) $)==(a 0$, fhapp (fhapp $b$ hls2 $) h l s 3)$

It looks like one rewrite with the inductive hypothesis should be enough to make the goal trivial. Here is what happens when we try that in Coq 8.2:

rewrite $I H l s 1$.

Error: Impossible to unify "fhlist B ((ls1 ++ ?1572) ++ ?1573)" with

"fhlist B (ls1 ++ ?1572 ++ ?1573)"

Coq 8.4 currently gives an error message about an uncaught exception. Perhaps that will be fixed soon. In any case, it is educational to consider a more explicit approach.

We see that JMeq is not a silver bullet. We can use it to simplify the statements of equality facts, but the Coq type-checker uses non-trivial heterogeneous equality facts no more readily than it uses standard equality facts. Here, the problem is that the form (e1, e2) is syntactic sugar for an explicit application of a constructor of an inductive type. That application mentions the type of each tuple element explicitly, and our rewrite tries to change one of those elements without updating the corresponding type argument.

We can get around this problem by another multiple use of generalize. We want to bring into the goal the proper instance of the inductive hypothesis, and we also want to generalize the two relevant uses of fhapp.

generalize (fhapp $b$ (fhapp hls2 hls3))

(fhapp (fhapp $b$ hls2) hls3)

(IHls1 _ $b$ hls2 hls3).

$======================$
$\forall(f:$ fhlist $B(l s 1++l s 2++l s 3))$
$\quad(f 0:$ fhlist $B((l s 1++l s 2)++l s 3)), f==f 0 \rightarrow(a 0, f)==(a 0, f 0)$

Now we can rewrite with append associativity, as before.

rewrite app_assoc.

$==\mathrm{=}=\mathrm{=}=\mathrm{=}=\mathrm{=}=\mathrm{=}=\mathrm{=}=\mathrm{=}=\mathrm{=}=\mathrm{=}=$

$\forall f f 0:$ fhlist $B(l s 1++l s 2++l s 3), f==f 0 \rightarrow(a 0, f)==(a 0, f 0)$

From this point, the goal is trivial.

intros $f$ fO $H$; rewrite $H$; reflexivity. 
Qed.

End fhapp'.

This example illustrates a general pattern: heterogeneous equality often simplifies theorem statements, but we still need to do some work to line up some dependent pattern matches that tactics will generate for us.

The proof we have found relies on the JMeq_eq axiom, which we can verify with a command that we will discuss more in two chapters.

Print Assumptions fhapp_assoc'.

Axioms:

JMeq_eq : $\forall(A:$ Type $)(x y: A), x==y \rightarrow x=y$

It was the rewrite $H$ tactic that implicitly appealed to the axiom. By restructuring the proof, we can avoid axiom dependence. A general lemma about pairs provides the key element. (Our use of generalize above can be thought of as reducing the proof to another, more complex and specialized lemma.)

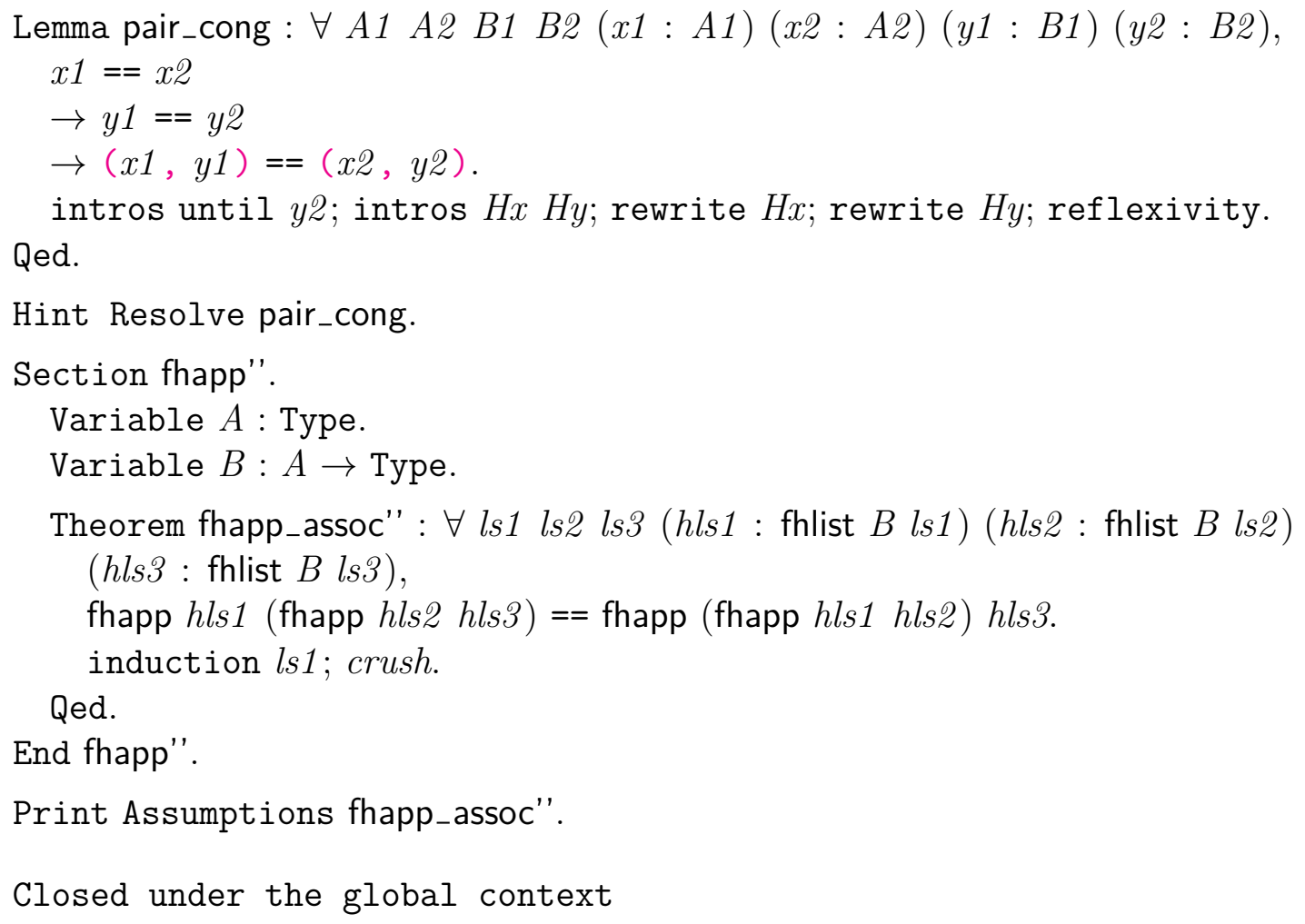

One might wonder exactly which elements of a proof involving JMeq imply that JMeq_eq must be used. For instance, above we noticed that rewrite had brought JMeq_eq into the proof of fhapp_assoc', yet here we have also used rewrite with JMeq hypotheses while avoiding axioms! One illuminating exercise is comparing the types of the lemmas that rewrite uses under the hood to implement the rewrites. Here is the normal lemma for eq rewriting: 
Check eq_ind_r.

eq_ind_r

$$
\begin{aligned}
& : \forall(A: \text { Type })(x: A)(P: A \rightarrow \text { Prop }), \\
& \quad P x \rightarrow \forall y: A, y=x \rightarrow P y
\end{aligned}
$$

The corresponding lemma used for JMeq in the proof of pair_cong is defined internally by rewrite as needed, but its type happens to be the following.

internal_JMeq_rew_r

$$
\begin{aligned}
& : \forall(A: \text { Type })(x: A)(B: \text { Type })(b: B) \\
& \quad(P: \forall B 0: \text { Type, } B 0 \rightarrow \text { Type }), P B b \rightarrow x==b \rightarrow P A x
\end{aligned}
$$

The key difference is that, where the eq lemma is parameterized on a predicate of type $A$ $\rightarrow$ Prop, the JMeq lemma is parameterized on a predicate of type more like $\forall A$ : Type, $A \rightarrow$ Prop. To apply eq_ind_r with a proof of $x=y$, it is only necessary to rearrange the goal into an application of a fun abstraction to $y$. In contrast, to apply the alternative principle, it is necessary to rearrange the goal to an application of a fun abstraction to both $y$ and its type. In other words, the predicate must be polymorphic in $y$ 's type; any type must make sense, from a type-checking standpoint. There may be cases where the former rearrangement is easy to do in a type-correct way, but the second rearrangement done naïvely leads to a type error.

When rewrite cannot figure out how to apply the alternative principle for $x==y$ where $x$ and $y$ have the same type, the tactic can instead use a different theorem, which is easy to prove as a composition of eq_ind_r and JMeq_eq.

Check JMeq_ind_r.

$$
\begin{aligned}
& \text { JMeq_ind_r } \\
& : \forall(A: \text { Type })(x: A)(P: A \rightarrow \text { Prop }), \\
& P x \rightarrow \forall y: A, y==x \rightarrow P y
\end{aligned}
$$

Ironically, where in the proof of fhapp_assoc' we used rewrite app_assoc to make it clear that a use of JMeq was actually homogeneously typed, we created a situation where rewrite applied the axiom-based JMeq_ind_r instead of the axiom-free principle!

For another simple example, consider this theorem that applies a heterogeneous equality to prove a congruence fact.

Theorem out_of_luck : $\forall n m$ : nat,

$$
\begin{aligned}
& n==m \\
& \rightarrow \mathrm{S} n==\mathrm{S} m . \\
& \text { intros } n m H .
\end{aligned}
$$

Applying JMeq_ind_r is easy, as the pattern tactic will transform the goal into an application of an appropriate fun to a term that we want to abstract. (In general, pattern abstracts over a term by introducing a new anonymous function taking that term as argument.)

pattern $n$. 


$$
\begin{aligned}
& n: \text { nat } \\
& m: \text { nat } \\
& H: n==m \\
& ===================== \\
& \text { ( } \text { f } u \text { n } n 0: \text { nat } \Rightarrow \mathrm{S} n 0==\mathrm{S} m) n \\
& \text { apply JMeq_ind_r with }(x:=m) ; \text { auto. }
\end{aligned}
$$

However, we run into trouble trying to get the goal into a form compatible with the alternative principle.

Undo 2.

pattern nat, $n$.

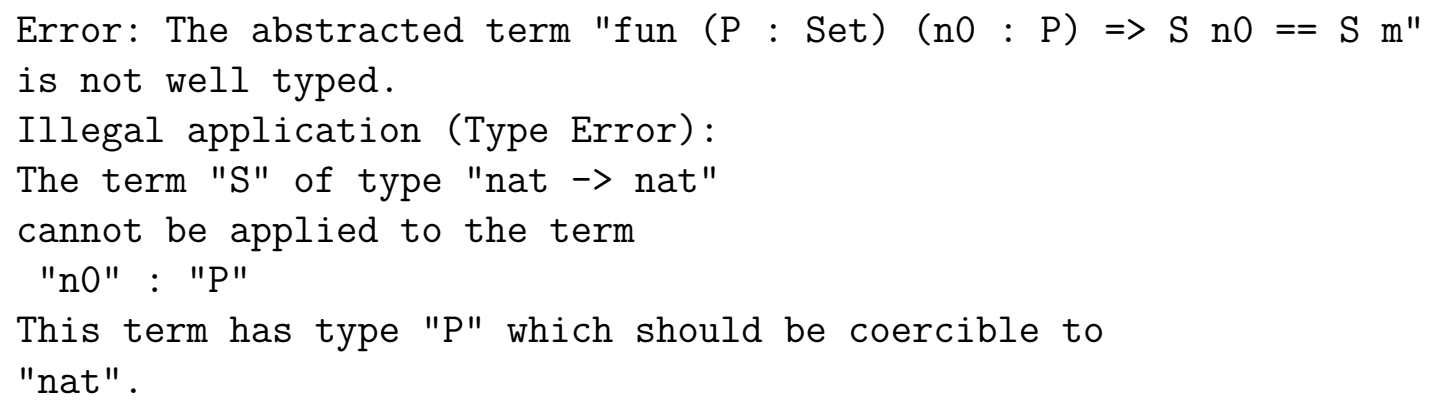

In other words, the successor function $S$ is insufficiently polymorphic. If we try to generalize over the type of $n$, we find that $\mathrm{S}$ is no longer legal to apply to $n$.

\section{Abort.}

Why did we not run into this problem in our proof of fhapp_assoc"'? The reason is that the pair constructor is polymorphic in the types of the pair components, while functions like $\mathrm{S}$ are not polymorphic at all. Use of such non-polymorphic functions with JMeq tends to push toward use of axioms. The example with nat here is a bit unrealistic; more likely cases would involve functions that have some polymorphism, but not enough to allow abstractions of the sort we attempted above with pattern. For instance, we might have an equality between two lists, where the goal only type-checks when the terms involved really are lists, though everything is polymorphic in the types of list data elements. The Heq ${ }^{1}$ library builds up a slightly different foundation to help avoid such problems.

\subsection{Equivalence of Equality Axioms}

Assuming axioms (like axiom $\mathrm{K}$ and $J M e q_{-} e q$ ) is a hazardous business. The due diligence associated with it is necessarily global in scope, since two axioms may be consistent alone

\footnotetext{
${ }^{1}$ http://www.mpi-sws.org/ gil/Heq/
} 
but inconsistent together. It turns out that all of the major axioms proposed for reasoning about equality in Coq are logically equivalent, so that we only need to pick one to assert without proof. In this section, we demonstrate by showing how each of the previous two sections' approaches reduces to the other logically.

To show that JMeq and its axiom let us prove UIP_refl, we start from the lemma UIP_refl' from the previous section. The rest of the proof is trivial.

Lemma UIP_refl" : $\forall(A$ : Type $)(x: A)(p f: x=x), p f=$ eq_refl $x$. intros; rewrite (UIP_refl' $p f$ ); reflexivity.

Qed.

The other direction is perhaps more interesting. Assume that we only have the axiom of the Eqdep module available. We can define JMeq in a way that satisfies the same interface as the combination of the JMeq module's inductive definition and axiom.

Definition JMeq' $(A$ : Type) $(x: A)(B:$ Type $)(y: B)$ : Prop :=

$\exists p f: B=A, x=$ match $p f$ with eq_refl $\Rightarrow y$ end.

Infix "===" := JMeq' (at level 70, no associativity).

We say that, by definition, $x$ and $y$ are equal if and only if there exists a proof $p f$ that their types are equal, such that $x$ equals the result of casting $y$ with $p f$. This statement can look strange from the standpoint of classical math, where we almost never mention proofs explicitly with quantifiers in formulas, but it is perfectly legal Coq code.

We can easily prove a theorem with the same type as that of the JMeq_refl constructor of JMeq.

Theorem JMeq_refl' : $\forall(A:$ Type $)(x: A), x===x$.

intros; unfold JMeq'; exists (eq_refl $A$ ); reflexivity. Qed.

The proof of an analogue to JMeq_eq is a little more interesting, but most of the action is in appealing to UIP_refl.

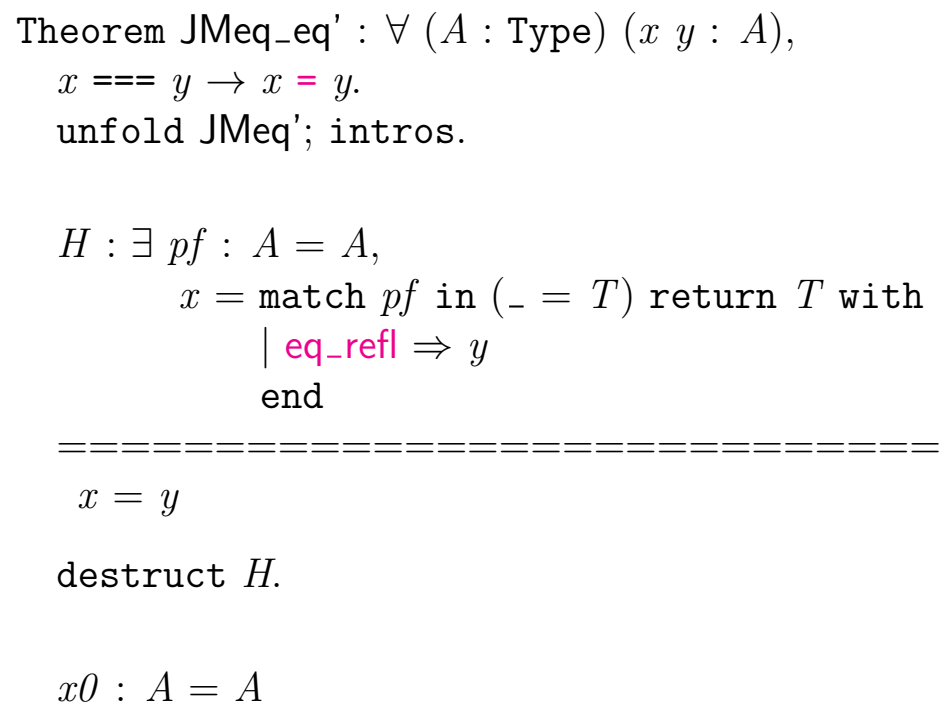




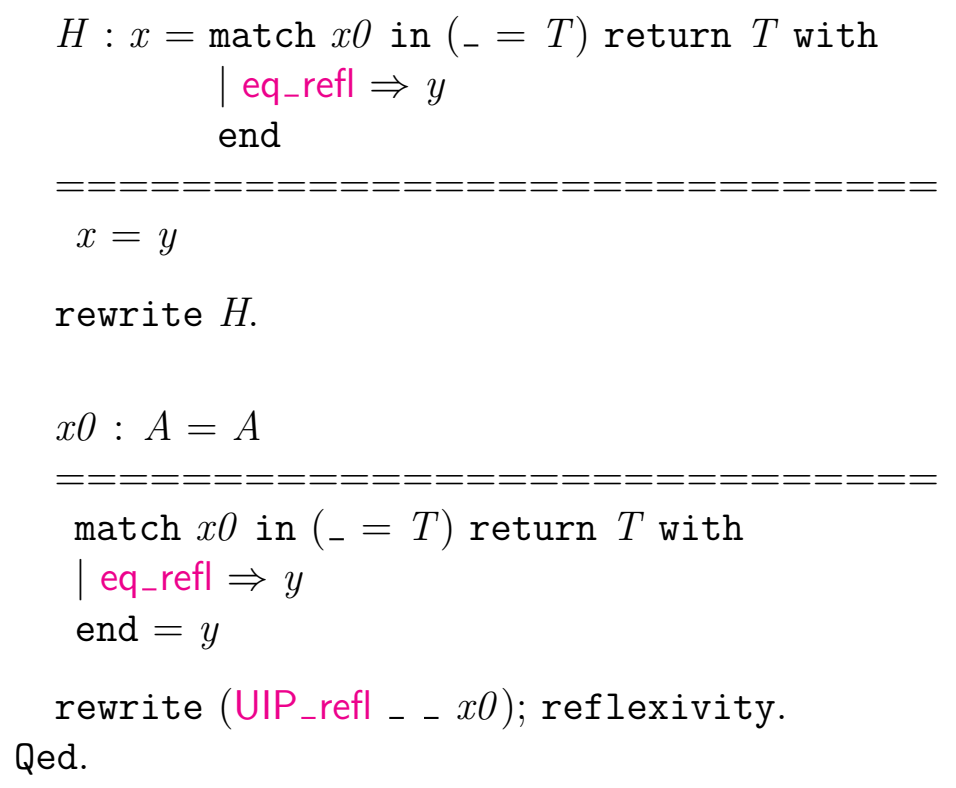

We see that, in a very formal sense, we are free to switch back and forth between the two styles of proofs about equality proofs. One style may be more convenient than the other for some proofs, but we can always interconvert between our results. The style that does not use heterogeneous equality may be preferable in cases where many results do not require the tricks of this chapter, since then the use of axioms is avoided altogether for the simple cases, and a wider audience will be able to follow those "simple" proofs. On the other hand, heterogeneous equality often makes for shorter and more readable theorem statements.

\subsection{Equality of Functions}

The following seems like a reasonable theorem to want to hold, and it does hold in set theory.

Theorem two_funs: ( f un $n \Rightarrow n)=($ fun $n \Rightarrow n+0)$.

Unfortunately, this theorem is not provable in CIC without additional axioms. None of the definitional equality rules force function equality to be extensional. That is, the fact that two functions return equal results on equal inputs does not imply that the functions are equal. We can assert function extensionality as an axiom, and indeed the standard library already contains that axiom.

Require Import FunctionalExtensionality.

About functional_extensionality.

functional_extensionality :

$\forall(A B:$ Type $)(f g: A \rightarrow B),(\forall x: A, f x=g x) \rightarrow f=g$

This axiom has been verified metatheoretically to be consistent with CIC and the two equality axioms we considered previously. With it, the proof of two_funs is trivial.

Theorem two_funs: ( fun $n \Rightarrow n)=($ fun $n \Rightarrow n+0)$. 
apply functional_extensionality; crush.

Qed.

The same axiom can help us prove equality of types, where we need to "reason under quantifiers."

Theorem forall_eq : $(\forall x:$ nat, match $x$ with

$$
\begin{aligned}
& \mid O_{\text {end }} \Rightarrow \text { True } \\
& \mid S_{-} \Rightarrow \text { True }
\end{aligned}
$$

$=(\forall-$ : nat, True $)$.

There are no immediate opportunities to apply functional_extensionality, but we can use change to fix that problem.

$$
\begin{aligned}
& \text { change ( } \forall x \text { : nat, (fun } x \Rightarrow \text { match } x \text { with } \\
& 0 \Rightarrow \text { True } \\
& \text { | } \mathrm{S}_{-} \Rightarrow \text { True } \\
& \text { end) } x)=(\text { nat } \rightarrow \text { True }) \text { ). } \\
& 0 \Rightarrow \text { True } \\
& \text { | } \mathrm{S}_{-} \Rightarrow \text { True } \\
& \text { end) (fun _ } \Rightarrow \text { True)). }
\end{aligned}
$$

2 subgoals

$$
\begin{aligned}
& =========== \\
& (\text { nat } \rightarrow \text { True })=(\text { nat } \rightarrow \text { True })
\end{aligned}
$$

subgoal 2 is:

$\forall x$ : nat, match $x$ with

$$
\begin{aligned}
& \mid 0 \Rightarrow \text { True } \\
& \mid S_{-} \Rightarrow \text { True } \\
& \text { end }=\text { True }
\end{aligned}
$$

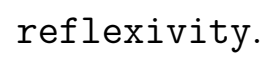

Qed.

Unlike in the case of eq_rect_eq, we have no way of deriving this axiom of functional extensionality for types with decidable equality. To allow equality reasoning without axioms, it may be worth rewriting a development to replace functions with alternate representations, such as finite map types for which extensionality is derivable in CIC. 


\section{Chapter 11}

\section{Generic Programming}

Generic programming makes it possible to write functions that operate over different types of data. Parametric polymorphism in ML and Haskell is one of the simplest examples. ML-style module systems [22] and Haskell type classes [44] are more flexible cases. These language features are often not as powerful as we would like. For instance, while Haskell includes a type class classifying those types whose values can be pretty-printed, per-type pretty-printing is usually either implemented manually or implemented via a deriving clause [33], which triggers ad-hoc code generation. Some clever encoding tricks have been used to achieve better within Haskell and other languages, but we can do datatype-generic programming much more cleanly with dependent types. Thanks to the expressive power of CIC, we need no special language support.

Generic programming can often be very useful in Coq developments, so we devote this chapter to studying it. In a proof assistant, there is the new possibility of generic proofs about generic programs, which we also devote some space to.

\subsection{Reifying Datatype Definitions}

The key to generic programming with dependent types is universe types. This concept should not be confused with the idea of universes from the metatheory of CIC and related languages, which we will study in more detail in the next chapter. Rather, the idea of universe types is to define inductive types that provide syntactic representations of Coq types. We cannot directly write CIC programs that do case analysis on types, but we can case analyze on reified syntactic versions of those types.

Thus, to begin, we must define a syntactic representation of some class of datatypes. In this chapter, our running example will have to do with basic algebraic datatypes, of the kind found in ML and Haskell, but without additional bells and whistles like type parameters and mutually recursive definitions.

The first step is to define a representation for constructors of our datatypes. We use the Record command as a shorthand for defining an inductive type with a single constructor, plus projection functions for pulling out any of the named arguments to that constructor. 


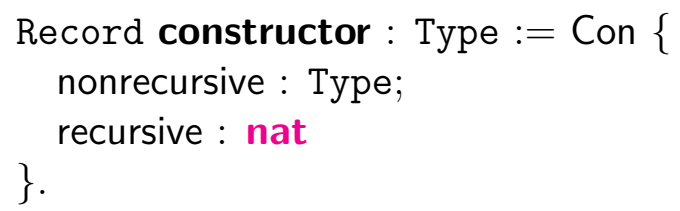

The idea is that a constructor represented as Con $T n$ has $n$ arguments of the type that we are defining. Additionally, all of the other, non-recursive arguments can be encoded in the type $T$. When there are no non-recursive arguments, $T$ can be unit. When there are two non-recursive arguments, of types $A$ and $B, T$ can be $A \times B$. We can generalize to any number of arguments via tupling.

With this definition, it is easy to define a datatype representation in terms of lists of constructors. The intended meaning is that the datatype came from an inductive definition including exactly the constructors in the list.

\section{Definition datatype := list constructor.}

Here are a few example encodings for some common types from the Coq standard library. While our syntax type does not support type parameters directly, we can implement them at the meta level, via functions from types to datatypes.

Definition Empty_set_dt : datatype $:=$ nil.

Definition unit_dt : datatype $:=$ Con unit $0::$ nil.

Definition bool_dt : datatype $:=$ Con unit $0::$ Con unit $0::$ nil.

Definition nat_dt : datatype $:=$ Con unit $0:$ : Con unit $1::$ nil.

Definition list_dt $(A:$ Type $)$ : datatype $:=$ Con unit $0::$ Con $A 1:$ : nil.

The type Empty_set has no constructors, so its representation is the empty list. The type unit has one constructor with no arguments, so its one reified constructor indicates no nonrecursive data and 0 recursive arguments. The representation for bool just duplicates this single argumentless constructor. We get from bool to nat by changing one of the constructors to indicate 1 recursive argument. We get from nat to list by adding a non-recursive argument of a parameter type $A$.

As a further example, we can do the same encoding for a generic binary tree type.

Section tree.

Variable $A$ : Type.

Inductive tree : Type :=

| Leaf : $A \rightarrow$ tree

Node : tree $\rightarrow$ tree $\rightarrow$ tree.

End tree.

Definition tree_dt $(A:$ Type $):$ datatype $:=$ Con $A 0::$ Con unit $2::$ nil.

Each datatype representation stands for a family of inductive types. For a specific real datatype and a reputed representation for it, it is useful to define a type of evidence that the datatype is compatible with the encoding.

Section denote. 
Variable $T$ : Type.

This variable stands for the concrete datatype that we are interested in.

Definition constructorDenote $(c$ : constructor $):=$ nonrecursive $c \rightarrow$ ilist $T$ (recursive $c) \rightarrow T$.

We write that a constructor is represented as a function returning a $T$. Such a function takes two arguments, which pack together the non-recursive and recursive arguments of the constructor. We represent a tuple of all recursive arguments using the length-indexed list type ilist that we met in Chapter 8.

Definition datatypeDenote $:=$ hlist constructorDenote.

Finally, the evidence for type $T$ is a heterogeneous list, including a constructor denotation for every constructor encoding in a datatype encoding. Recall that, since we are inside a section binding $T$ as a variable, constructorDenote is automatically parameterized by $T$.

End denote.

Some example pieces of evidence should help clarify the convention. First, we define a helpful notation for constructor denotations. The ASCII > from the notation will be rendered later as $\rightsquigarrow$.

Notation "[ $\left.\mathrm{v}, \mathrm{r}^{\sim}>\mathrm{x}\right] ":=(($ fun $v r \Rightarrow x)$ : constructorDenote _ (Con _ - $\left.)\right)$.

Definition Empty_set_den : datatypeDenote Empty_set Empty_set_dt :=

HNil.

Definition unit_den : datatypeDenote unit unit_dt :=

[_, $\rightsquigarrow$ tt] : : : HNil.

Definition bool_den : datatypeDenote bool bool_dt :=

[-, $\rightsquigarrow$ true] : : : [_, - $_{-}$false $]:::$HNil.

Definition nat_den : datatypeDenote nat nat_dt $:=$

$\left[{ }_{-}, \rightsquigarrow \mathrm{O}\right]:::\left[{ }_{-}, r \rightsquigarrow \mathrm{S}(\right.$ hd $\left.r)\right]:::$ HNil.

Definition list_den $(A$ : Type) $:$ datatypeDenote (list $A$ ) (list_dt $A):=$

$\left[{ }_{-},-\right.$nil $]:::[x, r \rightsquigarrow x::$ hd $r]:::$ HNil.

Definition tree_den $(A$ : Type) : datatypeDenote $($ tree $A)$ (tree_dt $A):=$

$[v,-\rightsquigarrow$ Leaf $v]:::\left[{ }_{-}, r \rightsquigarrow\right.$ Node $($ hd $r)$ (hd $\left.\left.(\mathrm{tl} r)\right)\right]:::$ HNil.

Recall that the hd and $\mathrm{t}$ calls above operate on richly typed lists, where type indices tell us the lengths of lists, guaranteeing the safety of operations like hd. The type annotation attached to each definition provides enough information for Coq to infer list lengths at appropriate points.

\subsection{Recursive Definitions}

We built these encodings of datatypes to help us write datatype-generic recursive functions. To do so, we will want a reified representation of a recursion scheme for each type, similar to the $T_{-}$rect principle generated automatically for an inductive definition of $T$. A clever reuse of datatypeDenote yields a short definition. 
Definition fixDenote ( $T$ : Type) $(d t:$ datatype $):=$

$\forall(R:$ Type $)$, datatypeDenote $R d t \rightarrow(T \rightarrow R)$.

The idea of a recursion scheme is parameterized by a type and a reputed encoding of it. The principle itself is polymorphic in a type $R$, which is the return type of the recursive function that we mean to write. The next argument is a heterogeneous list of one case of the recursive function definition for each datatype constructor. The datatypeDenote function turns out to have just the right definition to express the type we need; a set of function cases is just like an alternate set of constructors where we replace the original type $T$ with the function result type $R$. Given such a reified definition, a fixDenote invocation returns a function from $T$ to $R$, which is just what we wanted.

We are ready to write some example functions now. It will be useful to use one new function from the DepList library included in the book source.

Check hmake.

hmake

$$
\begin{aligned}
& : \forall(A: \text { Type })(B: A \rightarrow \text { Type }), \\
& \quad(\forall x: A, B x) \rightarrow \forall l s: \text { list } A, \text { hlist } B l s
\end{aligned}
$$

The function hmake is a kind of map alternative that goes from a regular list to an hlist. We can use it to define a generic size function that counts the number of constructors used to build a value in a datatype.

Definition size $T d t(f x:$ fixDenote $T d t): T \rightarrow$ nat $:=$ $f x$ nat (hmake $(B:=$ constructorDenote nat) (fun _ $r \Rightarrow$ foldr plus $1 r) d t$ ).

Our definition is parameterized over a recursion scheme $f x$. We instantiate $f x$ by passing it the function result type and a set of function cases, where we build the latter with hmake. The function argument to hmake takes three arguments: the representation of a constructor, its non-recursive arguments, and the results of recursive calls on all of its recursive arguments. We only need the recursive call results here, so we call them $r$ and bind the other two inputs with wildcards. The actual case body is simple: we add together the recursive call results and increment the result by one (to account for the current constructor). This foldr function is an ilist-specific version defined in the DepList module.

It is instructive to build fixDenote values for our example types and see what specialized size functions result from them.

Definition Empty_set_fix : fixDenote Empty_set Empty_set_dt :=

fun $R_{-} e m p \Rightarrow$ match $e m p$ with end.

Eval compute in size Empty_set_fix.

$$
=\text { fun } e m p: \text { Empty_set } \Rightarrow \text { match } e m p \text { return nat with }
$$$$
: \text { Empty_set } \rightarrow \text { nat }
$$

Despite all the fanciness of the generic size function, CIC's standard computation rules suffice to normalize the generic function specialization to exactly what we would have written manually. 
Definition unit_fix : fixDenote unit unit_dt :=

fun $R$ cases _ $\Rightarrow$ (hhd cases) tt INil.

Eval compute in size unit_fix.

$=$ fun $_{-}:$unit $\Rightarrow 1$

$:$ unit $\rightarrow$ nat

Again normalization gives us the natural function definition. We see this pattern repeated for our other example types.

Definition bool_fix : fixDenote bool bool_dt :=

fun $R$ cases $b \Rightarrow$ if $b$

then (hhd cases) tt INil

else (hhd (htl cases)) tt INil.

Eval compute in size bool_fix.

$=$ fun $b:$ bool $\Rightarrow$ if $b$ then 1 else 1

$:$ bool $\rightarrow$ nat

Definition nat_fix : fixDenote nat nat_dt $:=$

fun $R$ cases $\Rightarrow \operatorname{fix} F(n:$ nat $): R:=$

match $n$ with

$\mathrm{O} \Rightarrow$ (hhd cases) tt INil

I S n' $\Rightarrow($ hhd $($ htl cases $))$ tt (ICons ( $\left.F n^{\prime}\right)$ INil)

end.

To peek at the size function for nat, it is useful to avoid full computation, so that the recursive definition of addition is not expanded inline. We can accomplish this with proper flags for the cbv reduction strategy.

Eval cbv beta iota delta -[plus] in size nat_fix.

$$
\begin{aligned}
& =\operatorname{fix} F(n: \text { nat }): \text { nat }:=\operatorname{match} n \text { with } \\
& 0 \Rightarrow 1 \\
& \mathrm{~S} n^{\prime} \Rightarrow F n^{\prime}+1 \\
& \text { end }
\end{aligned}
$$

$:$ nat $\rightarrow$ nat

Definition list_fix $(A$ : Type) : fixDenote (list $A)$ (list_dt $A):=$

fun $R$ cases $\Rightarrow \operatorname{fix} F(l s:$ list $A): R:=$

match $l s$ with

| nil $\Rightarrow$ (hhd cases) tt INil

| $x:: l s^{\prime} \Rightarrow($ hhd $($ htl cases $)) x$ (ICons (F l $\left.l s^{\prime}\right)$ INil)

end.

Eval cbv beta iota delta -[plus] in fun $A \Rightarrow$ size (@list_fix $A$ ).

$$
\begin{aligned}
= & \text { fun } A: \text { Type } \Rightarrow \\
& \text { fix } F(l s: \text { list } A): \text { nat }:=
\end{aligned}
$$




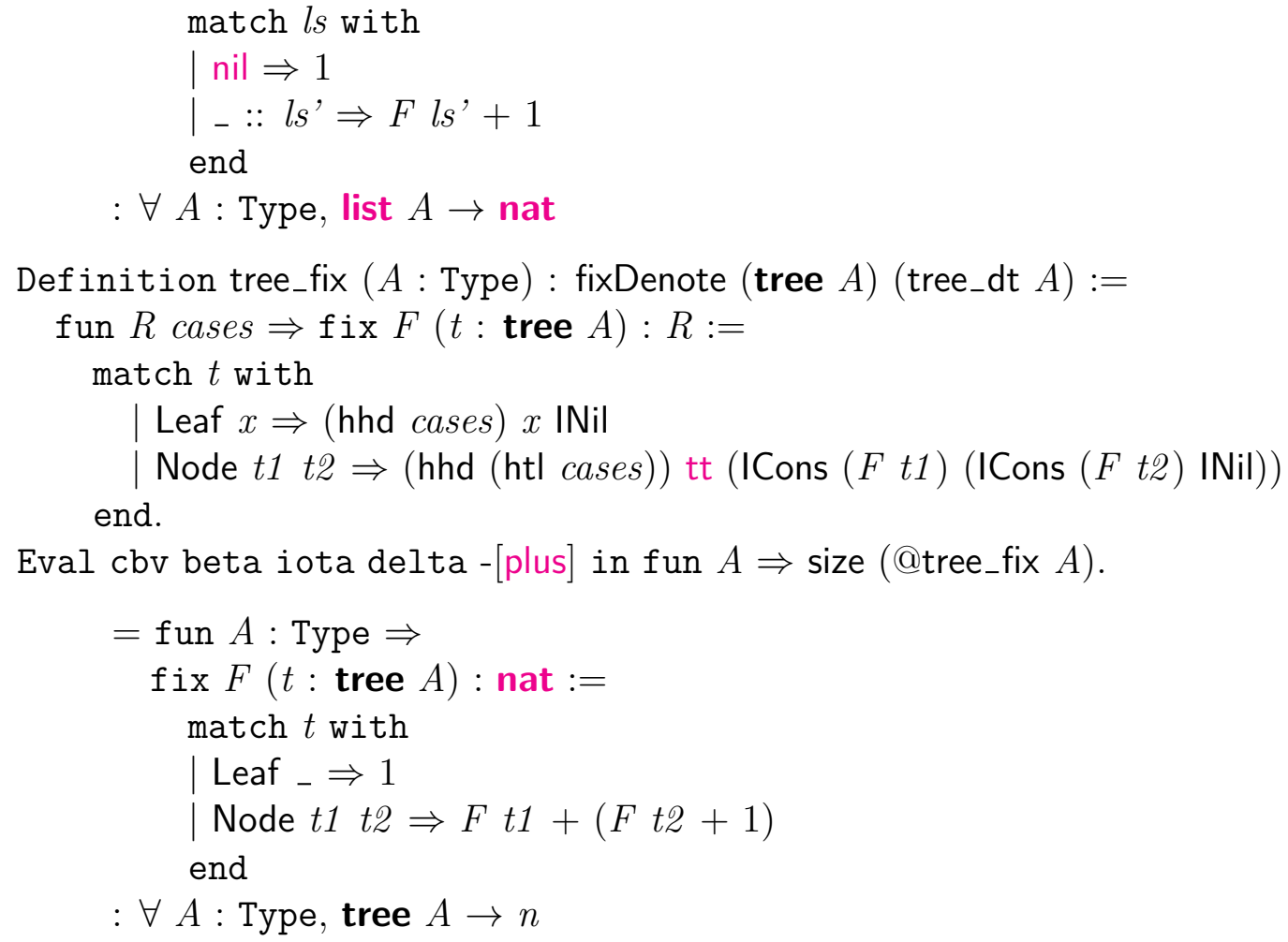

As our examples show, even recursive datatypes are mapped to normal-looking size functions.

\subsubsection{Pretty-Printing}

It is also useful to do generic pretty-printing of datatype values, rendering them as humanreadable strings. To do so, we will need a bit of metadata for each constructor. Specifically, we need the name to print for the constructor and the function to use to render its nonrecursive arguments. Everything else can be done generically.

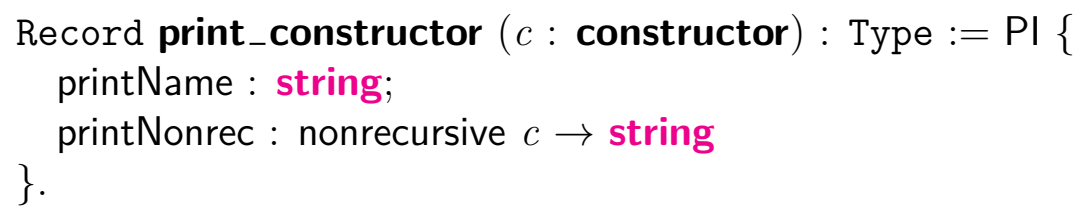

It is useful to define a shorthand for applying the constructor PI. By applying it explicitly to an unknown application of the constructor Con, we help type inference work.

Notation "^" :=(PI (Con _ _)).

As in earlier examples, we define the type of metadata for a datatype to be a heterogeneous list type collecting metadata for each constructor.

Definition print_datatype $:=$ hlist print_constructor.

We will be doing some string manipulation here, so we import the notations associated with strings. 
Local Open Scope string_scope.

Now it is easy to implement our generic printer, using another function from DepList. Check hmap.

hmap

$: \forall(A:$ Type $)\left(B 1 B^{2}: A \rightarrow\right.$ Type $)$,

$(\forall x: A, B 1 x \rightarrow B 2 x) \rightarrow$

$\forall l s:$ list $A$, hlist $B 1 l s \rightarrow$ hlist $B 2 l s$

Definition print $T d t(p r:$ print_datatype $d t)(f x:$ fixDenote $T d t): T \rightarrow$ string $:=$ $f x$ string (hmap $(B 1:=$ print_constructor) (B2 := constructorDenote string)

(fun _ $p c x r \Rightarrow$ printName $p c++"("++$ printNonrec $p c x$

++ foldr (fun $s$ acc $\Rightarrow$ ", " ++ $s++a c c$ ) ")" $r$ ) pr).

Some simple tests establish that print gets the job done.

Eval compute in print HNil Empty_set_fix.

$=$ fun $e m p:$ Empty_set $\Rightarrow$ match $e m p$ return string with

$:$ Empty_set $\rightarrow$ string

Eval compute in print ( " $t$ tt" (fun _ $\Rightarrow$ " ") : : : HNil) unit_fix.

$=$ fun $_{-}$: unit $\Rightarrow$ "tt ()$"$

$:$ unit $\rightarrow$ string

Eval compute in print ( "true" (fun _ $\Rightarrow$ " ")

$::$ "false" (fun _ $\Rightarrow$ " ")

: : : HNil) bool_fix.

$=$ fun $b:$ bool $\Rightarrow$ if $b$ then "true()" else "false()"

$:$ bool $\rightarrow$ string

Definition print_nat $:=$ print ( ${ }^{\text {"O" }}$ (fun _ $\Rightarrow$ " ")

$::$ $_{\text {"S" (fun _ }} \Rightarrow$ " ")

$::$ : HNil) nat_fix.

Eval cbv beta iota delta -[append] in print_nat.

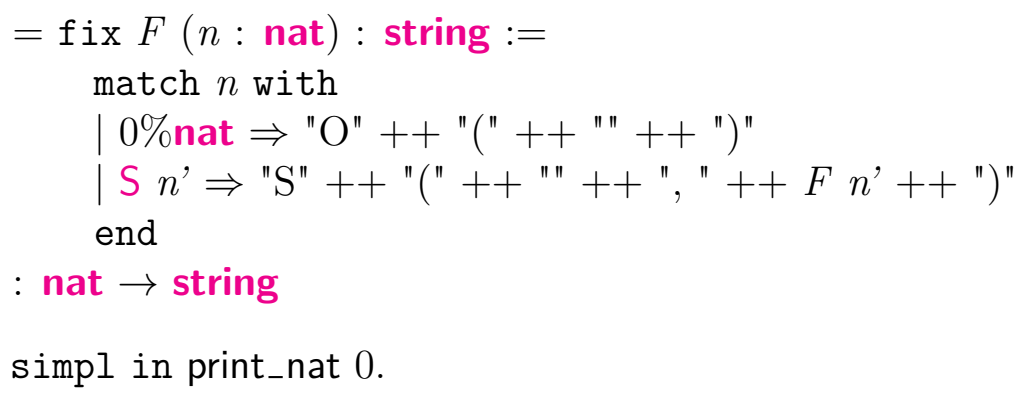


$=" \mathrm{O}()^{"}$
: string

Eval simpl in print_nat 1.

$=" \mathrm{~S}(, \mathrm{O}()) "$

: string

Eval simpl in print_nat 2.

$=" \mathrm{~S}(, \mathrm{~S}(, \mathrm{O}())) "$

: string

Eval cbv beta iota delta -[append] in fun $A(p r: A \rightarrow$ string $) \Rightarrow$ print ( "nil" (fun _ $\Rightarrow$ " ")

$::$ " $"$ cons" $p r$

: : : HNil) (@list_fix $A)$.

$=$ fun $(A:$ Type $)(p r: A \rightarrow$ string $) \Rightarrow$ fix $F(l s:$ list $A):$ string := match $l s$ with | nil $\Rightarrow$ "nil" ++ "(" ++ " " ++ " )" | $x:: l s^{\prime} \Rightarrow$ "cons" ++ "(" ++ pr $x++$ ", " ++ Fls' ++ " )" end

$: \forall A:$ Type, $(A \rightarrow$ string $) \rightarrow$ list $A \rightarrow$ string

Eval cbv beta iota delta -[append] in fun $A(p r: A \rightarrow$ string $) \Rightarrow$ print ( "Leaf" $p r$

$::$ "Node" (fun _ $\Rightarrow$ " ")

: : : HNil) (@tree_fix $A$ ).

$=\operatorname{fun}(A:$ Type $)(p r: A \rightarrow$ string $) \Rightarrow$ fix $F(t$ : tree $A)$ : string := match $t$ with | Leaf $x \Rightarrow$ "Leaf" ++ "(" ++ pr $x++$ ")" Node $t 1$ t2 $\Rightarrow$

"Node" ++ "(" ++ " " ++ ", " $++F t 1++$ ", " $++F$ t2 ++ " )" end

$: \forall A:$ Type, $(A \rightarrow$ string $) \rightarrow$ tree $A \rightarrow$ string

Some of these simplified terms seem overly complex because we have turned off simplification of calls to append, which is what uses of the ++ operator desugar to. Selective ++ simplification would combine adjacent string literals, yielding more or less the code we would write manually to implement this printing scheme. 


\subsubsection{Mapping}

By this point, we have developed enough machinery that it is old hat to define a generic function similar to the list map function.

Definition map $T d t(d d:$ datatypeDenote $T d t)(f x:$ fixDenote $T d t)(f: T \rightarrow T)$

$: T \rightarrow T:=$

fx $T$ (hmap $(B 1:=$ constructorDenote $T)(B 2:=$ constructorDenote $T)$

(fun _ $c x r \Rightarrow f(c x r)) d d$ ).

Eval compute in map Empty_set_den Empty_set_fix.

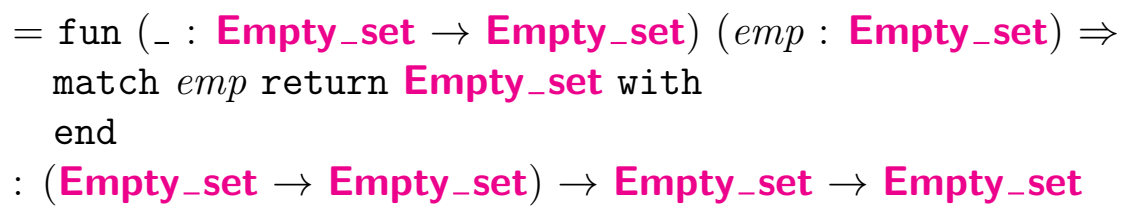

Eval compute in map unit_den unit_fix.

$=$ fun $(f:$ unit $\rightarrow$ unit $)($ - : unit $) \Rightarrow f$ tt

$:($ unit $\rightarrow$ unit $) \rightarrow$ unit $\rightarrow$ unit

Eval compute in map bool_den bool_fix.

$=$ fun $(f:$ bool $\rightarrow$ bool $)(b:$ bool $) \Rightarrow$ if $b$ then $f$ true else $f$ false

$:($ bool $\rightarrow$ bool $) \rightarrow$ bool $\rightarrow$ bool

Eval compute in map nat_den nat_fix.

$$
\begin{aligned}
& =\text { fun } f: \text { nat } \rightarrow \text { nat } \Rightarrow \\
& \text { fix } F(n: \text { nat }): \text { nat }:= \\
& \text { match } n \text { with } \\
& \mid 0 \% \text { nat } \Rightarrow f 0 \% \text { nat } \\
& \mid \mathrm{S} n^{\prime} \Rightarrow f\left(\mathrm{~S}\left(F n^{\prime}\right)\right) \\
& \quad \text { end } \\
& : \text { (nat } \rightarrow \text { nat }) \rightarrow \text { nat } \rightarrow \text { nat }
\end{aligned}
$$

Eval compute in fun $A \Rightarrow \operatorname{map}($ list_den $A$ ) (@list_fix $A$ ).

$$
\begin{aligned}
& =\operatorname{fun}(A: \text { Type })(f: \text { list } A \rightarrow \text { list } A) \Rightarrow \\
& \text { fix } F(l s: \text { list } A): \text { list } A:= \\
& \quad \text { match } l s \text { with } \\
& \quad \mid \text { nil } \Rightarrow f \text { nil } \\
& \mid x:: l s^{\prime} \Rightarrow f\left(x:: F l s^{\prime}\right) \\
& \quad \text { end }
\end{aligned}
$$

$: \forall A:$ Type, $($ list $A \rightarrow$ list $A) \rightarrow$ list $A \rightarrow$ list $A$

Eval compute in fun $A \Rightarrow \operatorname{map}(\operatorname{tree}$ den $A)$ (@tree_fix $A$ ). 


$$
\begin{aligned}
& =\text { fun }(A: \text { Type })(f: \operatorname{tree} A \rightarrow \text { tree } A) \Rightarrow \\
& \quad \text { fix } F(t: \operatorname{tree} A): \operatorname{tree} A:= \\
& \quad \text { match } t \text { with } \\
& \quad \mid \text { Leaf } x \Rightarrow f(\text { Leaf } x) \\
& \quad \text { Node } t 1 t 2 \Rightarrow f(\text { Node }(F t 1)(F t 2)) \\
& \quad \text { end } \\
& : \forall A: \text { Type, }(\text { tree } A \rightarrow \text { tree } A) \rightarrow \text { tree } A \rightarrow \text { tree } A
\end{aligned}
$$

These map functions are just as easy to use as those we write by hand. Can you figure out the input-output pattern that map_nat $S$ displays in these examples?

Definition map_nat $:=$ map nat_den nat_fix.

Eval simpl in map_nat $S 0$.

$=1 \%$ nat

: nat

Eval simpl in map_nat S 1.

$=3 \%$ nat

: nat

Eval simpl in map_nat $S 2$.

$$
=5 \% \text { nat }
$$

: nat

We get map_nat $S n=2 \times n+1$, because the mapping process adds an extra $\mathrm{S}$ at every level of the inductive tree that defines a natural, including at the last level, the $\mathrm{O}$ constructor.

\subsection{Proving Theorems about Recursive Definitions}

We would like to be able to prove theorems about our generic functions. To do so, we need to establish additional well-formedness properties that must hold of pieces of evidence.

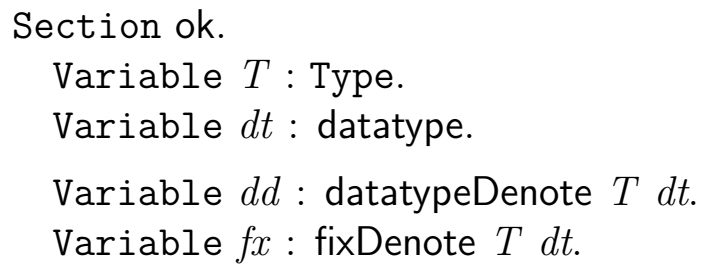

First, we characterize when a piece of evidence about a datatype is acceptable. The basic idea is that the type $T$ should really be an inductive type with the definition given by $d d$. Semantically, inductive types are characterized by the ability to do induction on them. Therefore, we require that the usual induction principle is true, with respect to the constructors given in the encoding $d d$. 


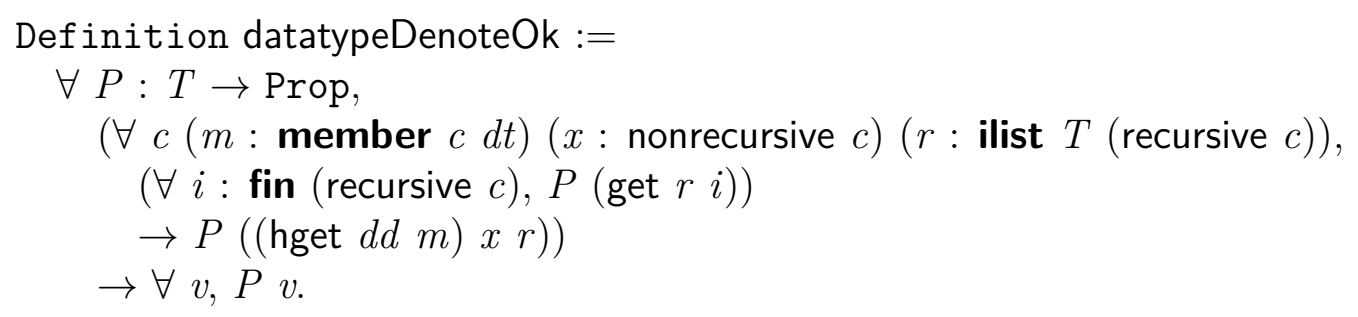

This definition can take a while to digest. The quantifier over $m:$ member $c d t$ is considering each constructor in turn; like in normal induction principles, each constructor has an associated proof case. The expression hget $d d m$ then names the constructor we have selected. After binding $m$, we quantify over all possible arguments (encoded with $x$ and $r$ ) to the constructor that $m$ selects. Within each specific case, we quantify further over $i$ : fin (recursive $c$ ) to consider all of our induction hypotheses, one for each recursive argument of the current constructor.

We have completed half the burden of defining side conditions. The other half comes in characterizing when a recursion scheme $f x$ is valid. The natural condition is that $f x$ behaves appropriately when applied to any constructor application.

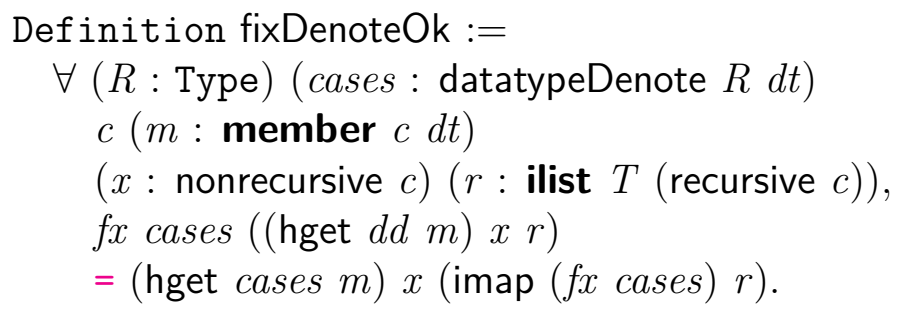

As for datatypeDenoteOk, we consider all constructors and all possible arguments to them by quantifying over $m, x$, and $r$. The lefthand side of the equality that follows shows a call to the recursive function on the specific constructor application that we selected. The righthand side shows an application of the function case associated with constructor $m$, applied to the non-recursive arguments and to appropriate recursive calls on the recursive arguments.

End ok.

We are now ready to prove that the size function we defined earlier always returns positive results. First, we establish a simple lemma.

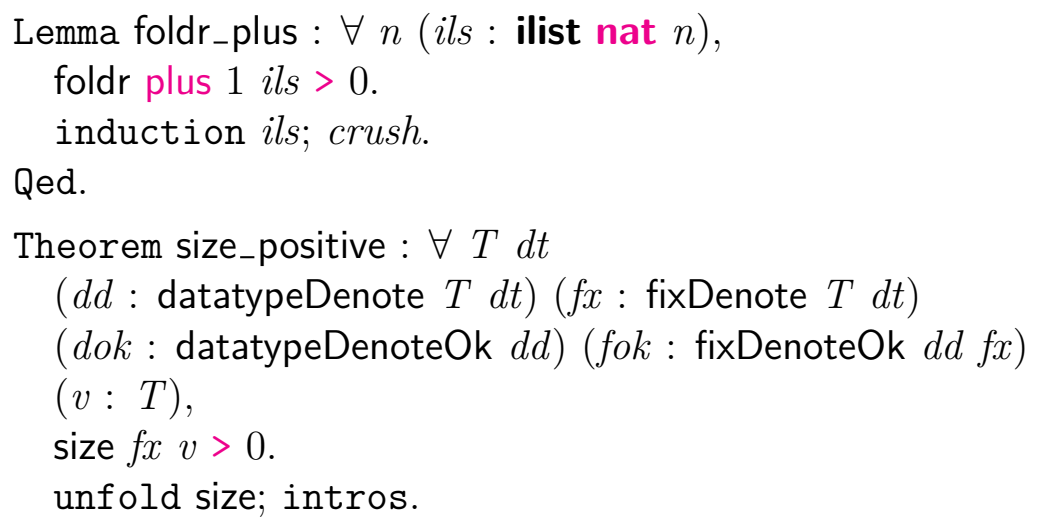




\section{$\begin{aligned}= & = \\ f x & \text { nat }\end{aligned}$ \\ (hmake \\ (fun ( $x$ : constructor) $(-:$ nonrecursive $x)$ \\ $(r$ : ilist nat (recursive $x)) \Rightarrow$ foldr plus $1 \%$ nat $r) d t) v>0$}

Our goal is an inequality over a particular call to size, with its definition expanded. How can we proceed here? We cannot use induction directly, because there is no way for Coq to know that $T$ is an inductive type. Instead, we need to use the induction principle encoded in our hypothesis dok of type datatypeDenoteOk $d d$. Let us try applying it directly.

apply dok.

Error: Impossible to unify "datatypeDenoteOk dd" with

"fx nat

(hmake

(fun ( $\mathrm{x}$ : constructor) (_ : nonrecursive $\mathrm{x}$ )

$(r$ : ilist nat (recursive $x)) \Rightarrow$ foldr plus $1 \%$ nat $r$ ) dt) $v>0 "$.

Matching the type of $d o k$ with the type of our conclusion requires more than simple first-order unification, so apply is not up to the challenge. We can use the pattern tactic to get our goal into a form that makes it apparent exactly what the induction hypothesis is.

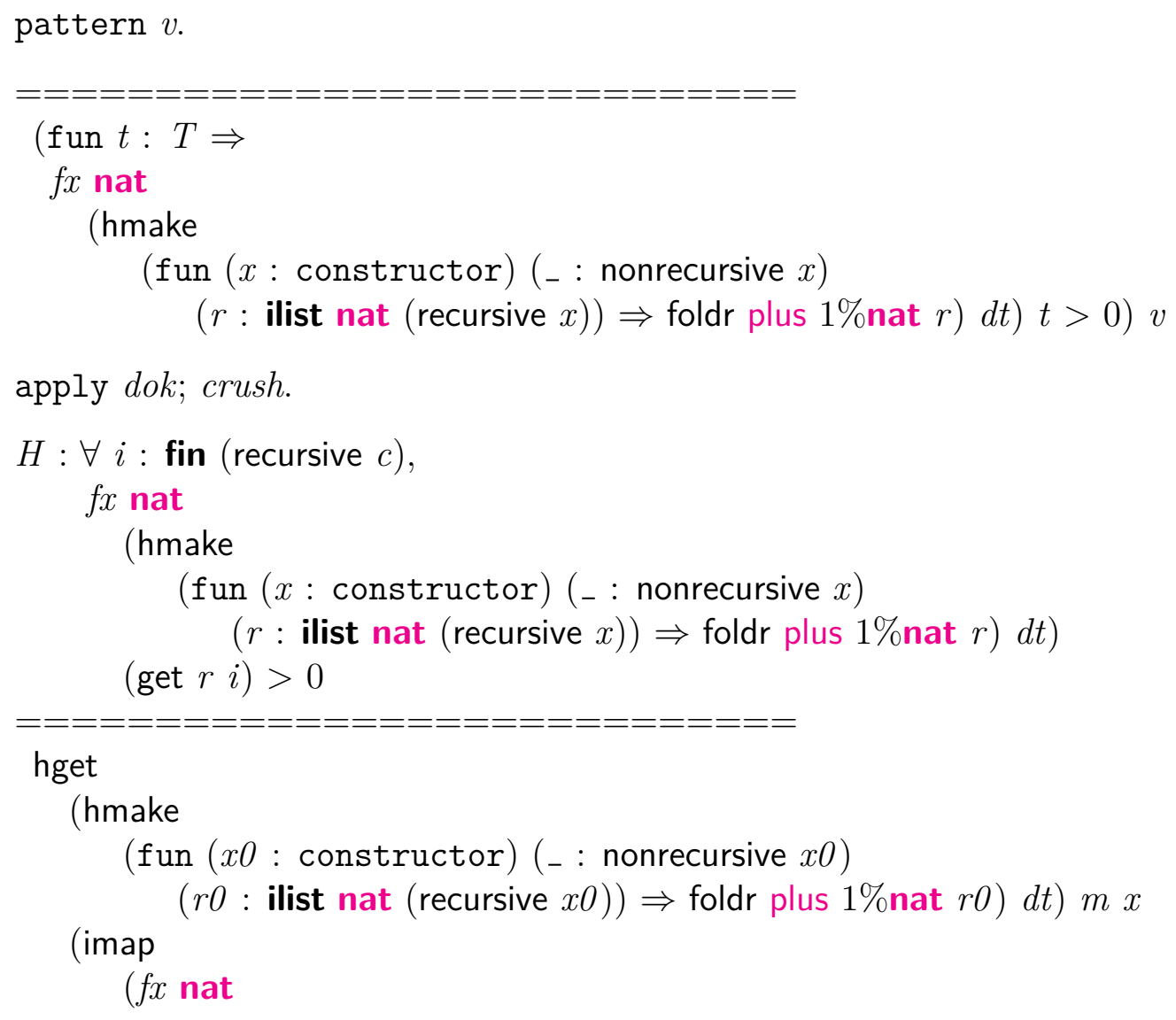




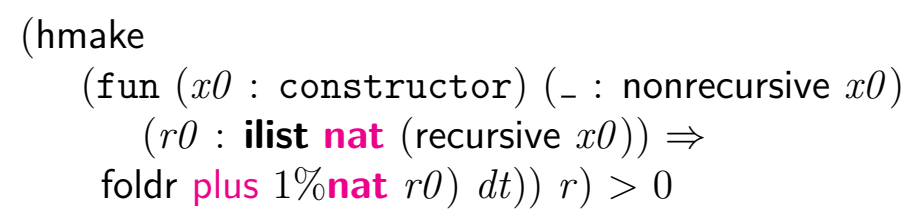

An induction hypothesis $H$ is generated, but we turn out not to need it for this example. We can simplify the goal using a library theorem about the composition of hget and hmake.

rewrite hget_hmake.

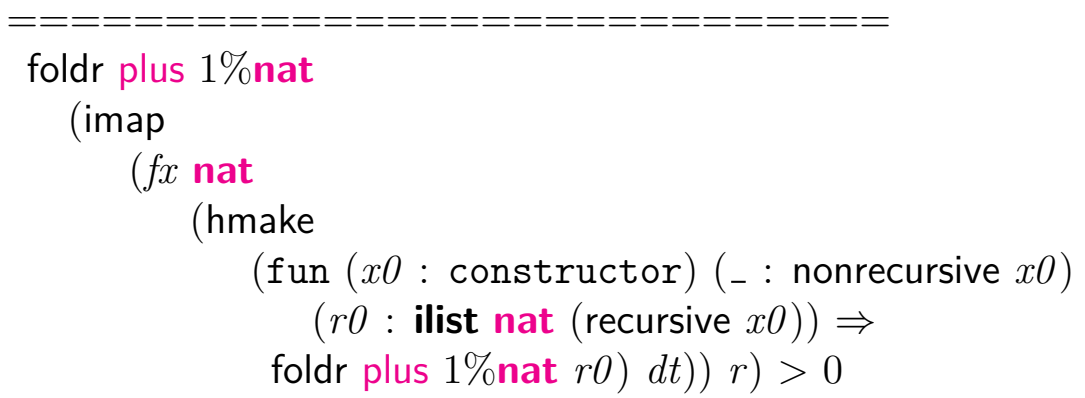

The lemma we proved earlier finishes the proof. apply foldr_plus.

Using hints, we can redo this proof in a nice automated form.

Restart.

Hint Rewrite hget_hmake.

Hint Resolve foldr_plus.

unfold size; intros; pattern $v$; apply dok; crush. Qed.

It turned out that, in this example, we only needed to use induction degenerately as case analysis. A more involved theorem may only be proved using induction hypotheses. We will give its proof only in unautomated form and leave effective automation as an exercise for the motivated reader.

In particular, it ought to be the case that generic map applied to an identity function is itself an identity function.

Theorem map_id : $\forall T d t$

( $d d$ : datatypeDenote $T d t)(f x$ : fixDenote $T d t)$

(dok : datatypeDenoteOk $d d$ ) (fok: fixDenoteOk $d d f x$ )

$(v: T)$,

map $d d f x($ fun $x \Rightarrow x) v=v$.

Let us begin as we did in the last theorem, after adding another useful library equality as a hint.

Hint Rewrite hget_hmap.

unfold map; intros; pattern $v$; apply dok; crush. 


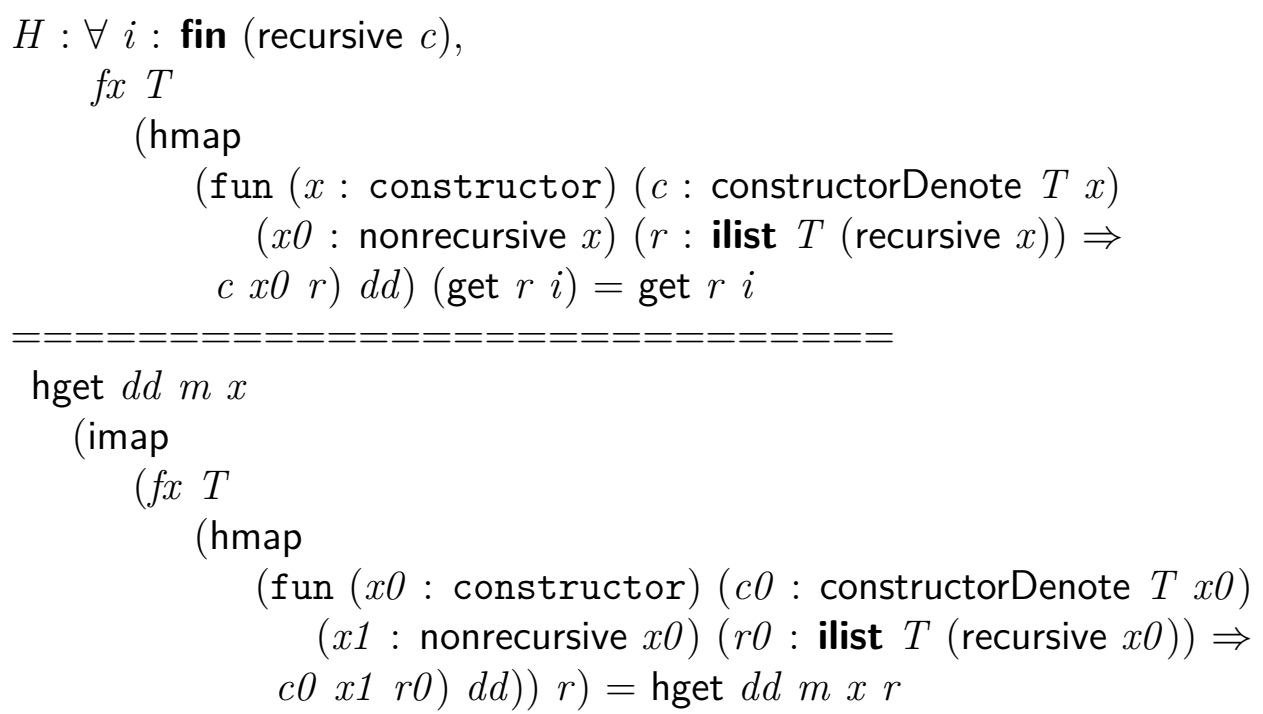

Our goal is an equality whose two sides begin with the same function call and initial arguments. We believe that the remaining arguments are in fact equal as well, and the $f_{-}$equal tactic applies this reasoning step for us formally.

\section{f_equal.}

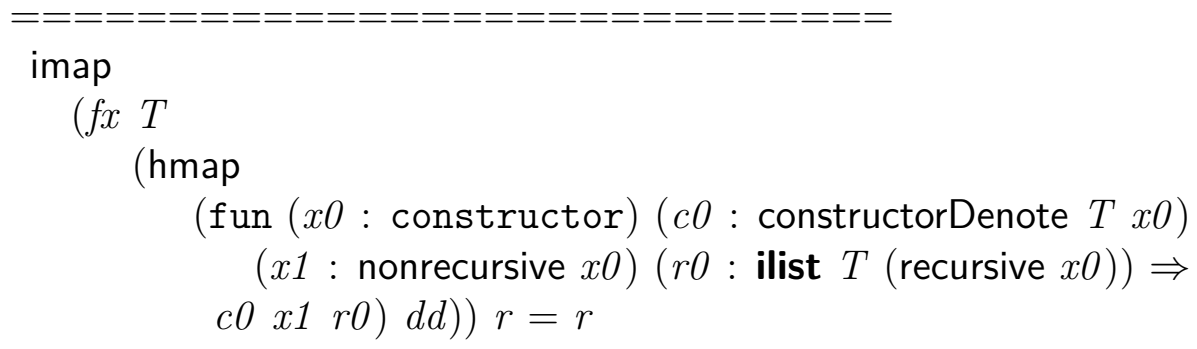

At this point, it is helpful to proceed by an inner induction on the heterogeneous list $r$ of recursive call results. We could arrive at a cleaner proof by breaking this step out into an explicit lemma, but here we will do the induction inline to save space.

induction $r$; crush.

The base case is discharged automatically, and the inductive case looks like this, where $H$ is the outer IH (for induction over $T$ values) and $I H r$ is the inner IH (for induction over the recursive arguments).

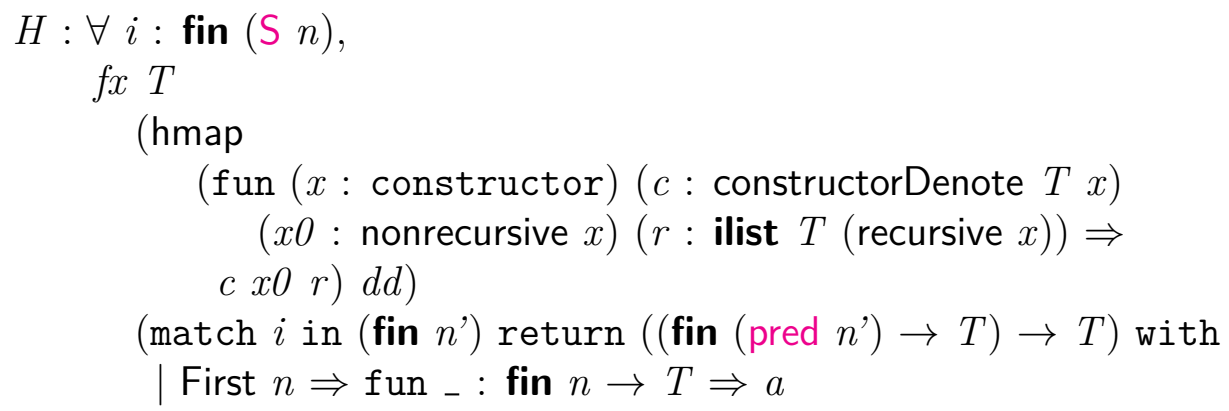




$$
\begin{aligned}
& \text { (fun }(x 0: \text { constructor })(c 0: \text { constructorDenote } T x 0) \\
& (x 1: \text { nonrecursive } x 0)(r 0: \text { ilist } T(\text { recursive } x 0)) \Rightarrow \\
& c 0 x 1 \quad r 0) d d)) r=r
\end{aligned}
$$

Now the goal matches the inner IH IHr.

apply IHr; crush.

$i$ : fin $n$

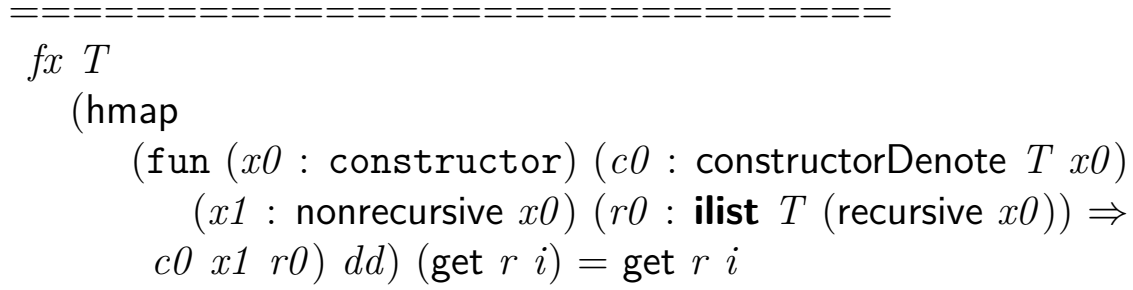

We can finish the proof by applying the outer IH again, specialized to a different fin value.

apply $(H($ Next $i))$.

Qed.

The proof involves complex subgoals, but, still, few steps are required, and then we may reuse our work across a variety of datatypes. 


\section{Chapter 12}

\section{Universes and Axioms}

Many traditional theorems can be proved in Coq without special knowledge of CIC, the logic behind the prover. A development just seems to be using a particular ASCII notation for standard formulas based on set theory. Nonetheless, as we saw in Chapter 4, CIC differs from set theory in starting from fewer orthogonal primitives. It is possible to define the usual logical connectives as derived notions. The foundation of it all is a dependently typed functional programming language, based on dependent function types and inductive type families. By using the facilities of this language directly, we can accomplish some things much more easily than in mainstream math.

Gallina, which adds features to the more theoretical CIC [31], is the logic implemented in Coq. It has a relatively simple foundation that can be defined rigorously in a page or two of formal proof rules. Still, there are some important subtleties that have practical ramifications. This chapter focuses on those subtleties, avoiding formal metatheory in favor of example code.

\subsection{The Type Hierarchy}

Every object in Gallina has a type.

Check 0 .

0

: nat

It is natural enough that zero be considered as a natural number.

Check nat.

nat

: Set

From a set theory perspective, it is unsurprising to consider the natural numbers as a "set."

Check Set. 
Set

: Type

The type Set may be considered as the set of all sets, a concept that set theory handles in terms of classes. In Coq, this more general notion is Type.

Check Type.

Type

: Type

Strangely enough, Type appears to be its own type. It is known that polymorphic languages with this property are inconsistent, via Girard's paradox [8]. That is, using such a language to encode proofs is unwise, because it is possible to "prove" any proposition. What is really going on here?

Let us repeat some of our queries after toggling a flag related to Coq's printing behavior.

Set Printing Universes.

Check nat.

nat

: Set

Check Set.

Set

: Type $(*(0)+1 *)$

Check Type.

Type $(*$ Top. $3 *)$

: Type $(*($ Top.3) $+1 *)$

Occurrences of Type are annotated with some additional information, inside comments. These annotations have to do with the secret behind Type: it really stands for an infinite hierarchy of types. The type of Set is Type(0), the type of Type(0) is Type(1), the type of Type(1) is Type(2), and so on. This is how we avoid the "Type: Type" paradox. As a convenience, the universe hierarchy drives Coq's one variety of subtyping. Any term whose type is Type at level $i$ is automatically also described by Type at level $j$ when $j>i$.

In the outputs of our first Check query, we see that the type level of Set's type is $(0)+1$. Here 0 stands for the level of Set, and we increment it to arrive at the level that classifies Set.

In the third query's output, we see that the occurrence of Type that we check is assigned a fresh universe variable Top.3. The output type increments Top.3 to move up a level in the universe hierarchy. As we write code that uses definitions whose types mention universe variables, unification may refine the values of those variables. Luckily, the user rarely has to worry about the details. 
Another crucial concept in CIC is predicativity. Consider these queries.

$$
\begin{aligned}
\text { Check } & \forall T \text { : nat, fin } T . \\
\forall T & : \text { nat, fin } T \\
& : \text { Set } \\
\text { Check } & \forall T: \text { Set, } T . \\
\forall T & : \text { Set, } T \\
& : \text { Type }\left({ }^{*} \max (0,(0)+1)^{*}\right) \\
\text { Check } & \forall T: \text { Type, } T . \\
\forall T & : \text { Type }\left({ }^{*} T o p .9 *\right), T \\
& : \text { Type }\left({ }^{*} \max (T o p .9,(T o p .9)+1) *\right)
\end{aligned}
$$

These outputs demonstrate the rule for determining which universe a $\forall$ type lives in. In particular, for a type $\forall x:$ T1, T2, we take the maximum of the universes of T1 and T2. In the first example query, both $T 1$ (nat) and T2 (fin $T$ ) are in Set, so the $\forall$ type is in Set, too. In the second query, $T 1$ is Set, which is at level $(0)+1$; and $T 2$ is $T$, which is at level 0 . Thus, the $\forall$ exists at the maximum of these two levels. The third example illustrates the same outcome, where we replace Set with an occurrence of Type that is assigned universe variable Top.9. This universe variable appears in the places where 0 appeared in the previous query.

The behind-the-scenes manipulation of universe variables gives us predicativity. Consider this simple definition of a polymorphic identity function, where the first argument $T$ will automatically be marked as implicit, since it can be inferred from the type of the second argument $x$.

Definition id $(T:$ Set) $(x: T): T:=x$.

Check id 0 .

id 0

: nat

Check id Set.

Error: Illegal application (Type Error):

...

The 1st term has type "Type (* (Top.15)+1*)"

which should be coercible to "Set".

The parameter $T$ of id must be instantiated with a Set. The type nat is a Set, but Set is not. We can try fixing the problem by generalizing our definition of id.

Reset id.

Definition id ( $T$ : Type) $(x: T): T:=x$. 
Check id 0 .

id 0

: nat

Check id Set.

id Set

$$
\text { : Type }(* \text { Top. } 17 *)
$$

Check id Type.

$$
\begin{aligned}
& \text { id Type }(* \text { Top. } 18 *) \\
& \text { : Type }(* \text { Top. } 19 *)
\end{aligned}
$$

So far so good. As we apply id to different $T$ values, the inferred index for $T$ 's Type occurrence automatically moves higher up the type hierarchy.

Check id id.

Error: Universe inconsistency (cannot enforce Top. $16<$ Top. 16).

This error message reminds us that the universe variable for $T$ still exists, even though it is usually hidden. To apply id to itself, that variable would need to be less than itself in the type hierarchy. Universe inconsistency error messages announce cases like this one where a term could only type-check by violating an implied constraint over universe variables. Such errors demonstrate that Type is predicative, where this word has a CIC meaning closely related to its usual mathematical meaning. A predicative system enforces the constraint that, when an object is defined using some sort of quantifier, none of the quantifiers may ever be instantiated with the object itself. Impredicativity is associated with popular paradoxes in set theory, involving inconsistent constructions like "the set of all sets that do not contain themselves" (Russell's paradox). Similar paradoxes would result from uncontrolled impredicativity in Coq.

\subsubsection{Inductive Definitions}

Predicativity restrictions also apply to inductive definitions. As an example, let us consider a type of expression trees that allows injection of any native Coq value. The idea is that an $\exp T$ stands for an encoded expression of type $T$.

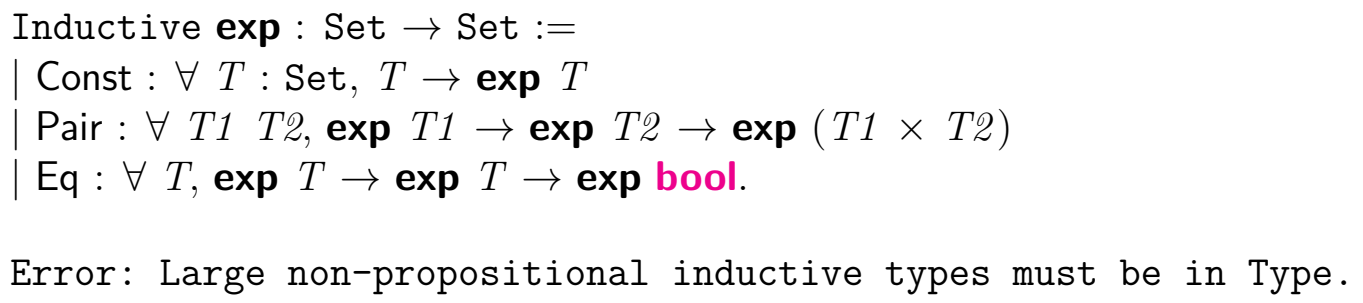


This definition is large in the sense that at least one of its constructors takes an argument whose type has type Type. Coq would be inconsistent if we allowed definitions like this one in their full generality. Instead, we must change exp to live in Type. We will go even further and move exp's index to Type as well.

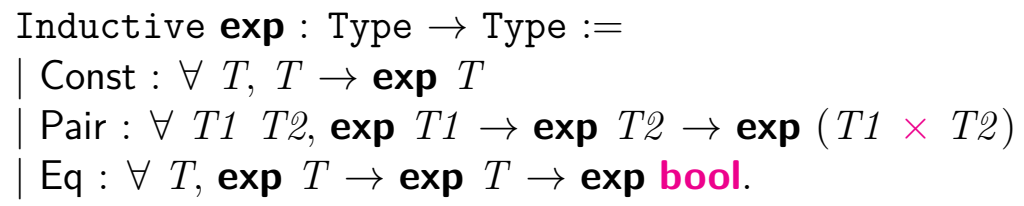

Note that before we had to include an annotation : Set for the variable $T$ in Const's type, but we need no annotation now. When the type of a variable is not known, and when that variable is used in a context where only types are allowed, Coq infers that the variable is of type Type, the right behavior here, though it was wrong for the Set version of exp.

Our new definition is accepted. We can build some sample expressions.

Check Const 0 .

\section{Const 0}

: exp nat

Check Pair (Const 0) (Const tt).

Pair (Const 0$)$ (Const tt)

: $\exp$ (nat $\times$ unit)

Check Eq (Const Set) (Const Type).

Eq (Const Set) (Const Type $(*$ Top.59*) )

: exp bool

We can check many expressions, including fancy expressions that include types. However, it is not hard to hit a type-checking wall.

Check Const (Const O).

Error: Universe inconsistency (cannot enforce Top.42< Top.42).

We are unable to instantiate the parameter $T$ of Const with an exp type. To see why, it is helpful to print the annotated version of exp's inductive definition.

Print exp.

Inductive exp

$$
\begin{aligned}
: & \text { Type }\left(* \text { Top. } 8^{*}\right) \rightarrow \\
& \text { Type } \\
& \left({ }^{*} \max (0,(\text { Top. } 11)+1,(\text { Top. } 14)+1,(\text { Top. } 15)+1,(\text { Top. } 19)+1) *\right):= \\
\text { Const }: \forall T: & \text { Type }\left({ }^{*} \text { Top. } 11^{*}\right), T \rightarrow \operatorname{exp~} T \\
\mid \text { Pair }: \forall(T 1: & \text { Type } \left.\left(* \text { Top. } 14^{*}\right)\right)\left(T 2: \text { Type }\left(* \text { Top. } 15^{*}\right)\right),
\end{aligned}
$$




\section{$\exp T 1 \rightarrow \exp T_{2} \rightarrow \exp \left(T 1 \times T_{2}\right)$ \\ | Eq : $\forall T:$ Type $\left(* T o p .19^{*}\right), \exp T \rightarrow \exp T \rightarrow \exp$ bool}

We see that the index type of exp has been assigned to universe level Top.8. In addition, each of the four occurrences of Type in the types of the constructors gets its own universe variable. Each of these variables appears explicitly in the type of exp. In particular, any type exp $T$ lives at a universe level found by incrementing by one the maximum of the four argument variables. Therefore, exp must live at a higher universe level than any type which may be passed to one of its constructors. This consequence led to the universe inconsistency.

Strangely, the universe variable Top. 8 only appears in one place. Is there no restriction imposed on which types are valid arguments to exp? In fact, there is a restriction, but it only appears in a global set of universe constraints that are maintained "off to the side," not appearing explicitly in types. We can print the current database.

Print Universes.

Top. $19<$ Top.9 $\leq$ Top. 8

Top. $15<$ Top. $9 \leq$ Top. $8 \leq$ Coq.Init.Datatypes. 38

Top. $14<$ Top. $9 \leq$ Top. $8 \leq$ Coq.Init.Datatypes. 37

Top. $11<$ Top.9 $\leq$ Top. 8

The command outputs many more constraints, but we have collected only those that mention Top variables. We see one constraint for each universe variable associated with a constructor argument from exp's definition. Universe variable Top.19 is the type argument to Eq. The constraint for Top.19 effectively says that Top.19 must be less than Top.8, the universe of exp's indices; an intermediate variable Top.9 appears as an artifact of the way the constraint was generated.

The next constraint, for Top.15, is more complicated. This is the universe of the second argument to the Pair constructor. Not only must Top.15 be less than Top.8, but it also comes out that Top. 8 must be less than Coq.Init.Datatypes.38. What is this new universe variable? It is from the definition of the prod inductive family, to which types of the form $A \times B$ are desugared.

Print prod.

Inductive prod $(A$ : Type $(*$ Coq.Init.Datatypes.37*) $)$

$$
\begin{aligned}
& \left(B \text { : Type }\left({ }^{*} \text { Coq.Init.Datatypes. } 38 *\right)\right) \\
& \quad: \text { Type }\left({ }^{*} \max \left(\text { Coq.Init.Datatypes.37, Coq.Init.Datatypes.38) }{ }^{*}\right):=\right.
\end{aligned}
$$

pair : $A \rightarrow B \rightarrow A \times B$

We see that the constraint is enforcing that indices to exp must not live in a higher universe level than $B$-indices to prod. The next constraint above establishes a symmetric condition for $A$.

Thus it is apparent that Coq maintains a tortuous set of universe variable inequalities behind the scenes. It may look like some functions are polymorphic in the universe levels of their arguments, but what is really happening is imperative updating of a system of constraints, such that all uses of a function are consistent with a global set of universe levels. 
When the constraint system may not be evolved soundly, we get a universe inconsistency error.

The annotated definition of prod reveals something interesting. A type prod $A B$ lives at a universe that is the maximum of the universes of $A$ and $B$. From our earlier experiments, we might expect that prod's universe would in fact need to be one higher than the maximum. The critical difference is that, in the definition of prod, $A$ and $B$ are defined as parameters; that is, they appear named to the left of the main colon, rather than appearing (possibly unnamed) to the right.

Parameters are not as flexible as normal inductive type arguments. The range types of all of the constructors of a parameterized type must share the same parameters. Nonetheless, when it is possible to define a polymorphic type in this way, we gain the ability to use the new type family in more ways, without triggering universe inconsistencies. For instance, nested pairs of types are perfectly legal.

Check (nat, (Type, Set)).

(nat, (Type $(*$ Top.44*), Set))

: Set $\times\left(\right.$ Type $\left(*\right.$ Top. $\left.45^{*}\right) \times$ Type $\left(*\right.$ Top. $\left.\left.46^{*}\right)\right)$

The same cannot be done with a counterpart to prod that does not use parameters.

Inductive prod' : Type $\rightarrow$ Type $\rightarrow$ Type $:=$

| pair' : $\forall A B$ : Type, $A \rightarrow B \rightarrow$ prod' $A B$.

Check (pair' nat (pair' Type Set)).

Error: Universe inconsistency (cannot enforce Top.51< Top.51).

The key benefit parameters bring us is the ability to avoid quantifying over types in the types of constructors. Such quantification induces less-than constraints, while parameters only introduce less-than-or-equal-to constraints.

Coq includes one more (potentially confusing) feature related to parameters. While Gallina does not support real universe polymorphism, there is a convenience facility that mimics universe polymorphism in some cases. We can illustrate what this means with a simple example.

Inductive foo $(A:$ Type $):$ Type :=

| Foo : $A \rightarrow$ foo $A$.

Check foo nat.

foo nat

: Set

Check foo Set.

foo Set

: Type 
Check foo True.

foo True

: Prop

The basic pattern here is that Coq is willing to automatically build a "copied-and-pasted" version of an inductive definition, where some occurrences of Type have been replaced by Set or Prop. In each context, the type-checker tries to find the valid replacements that are lowest in the type hierarchy. Automatic cloning of definitions can be much more convenient than manual cloning. We have already taken advantage of the fact that we may re-use the same families of tuple and list types to form values in Set and Type.

Imitation polymorphism can be confusing in some contexts. For instance, it is what is responsible for this weird behavior.

Inductive bar: Type := Bar : bar.

Check bar.

bar

: Prop

The type that Coq comes up with may be used in strictly more contexts than the type one might have expected.

\subsubsection{Deciphering Baffling Messages About Inability to Unify}

One of the most confusing sorts of Coq error messages arises from an interplay between universes, syntax notations, and implicit arguments. Consider the following innocuous lemma, which is symmetry of equality for the special case of types.

Theorem symmetry : $\forall A B$ : Type,

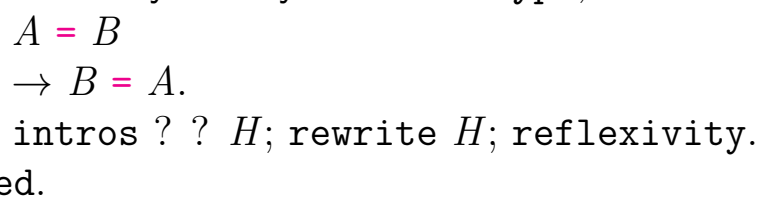

Let us attempt an admittedly silly proof of the following theorem.

Theorem illustrative_but_silly_detour : unit = unit.

apply symmetry.

Error: Impossible to unify "?35 = ?34" with "unit = unit".

Coq tells us that we cannot, in fact, apply our lemma symmetry here, but the error message seems defective. In particular, one might think that apply should unify ?35 and ?34 with unit to ensure that the unification goes through. In fact, the issue is in a part of the unification problem that is not shown to us in this error message!

The following command is the secret to getting better error messages in such cases:

Set Printing All. 
apply symmetry.

Error: Impossible to unify "@eq Type ?46 ?45" with "@eq Set unit unit".

Now we can see the problem: it is the first, implicit argument to the underlying equality function eq that disagrees across the two terms. The universe Set may be both an element and a subtype of Type, but the two are not definitionally equal.

Abort.

A variety of changes to the theorem statement would lead to use of Type as the implicit argument of eq. Here is one such change.

Theorem illustrative_but_silly_detour : (unit : Type) = unit.

apply symmetry; reflexivity. Qed.

There are many related issues that can come up with error messages, where one or both of notations and implicit arguments hide important details. The Set Printing All command turns off all such features and exposes underlying CIC terms.

For completeness, we mention one other class of confusing error message about inability to unify two terms that look obviously unifiable. Each unification variable has a scope; a unification variable instantiation may not mention variables that were not already defined within that scope, at the point in proof search where the unification variable was introduced. Consider this illustrative example:

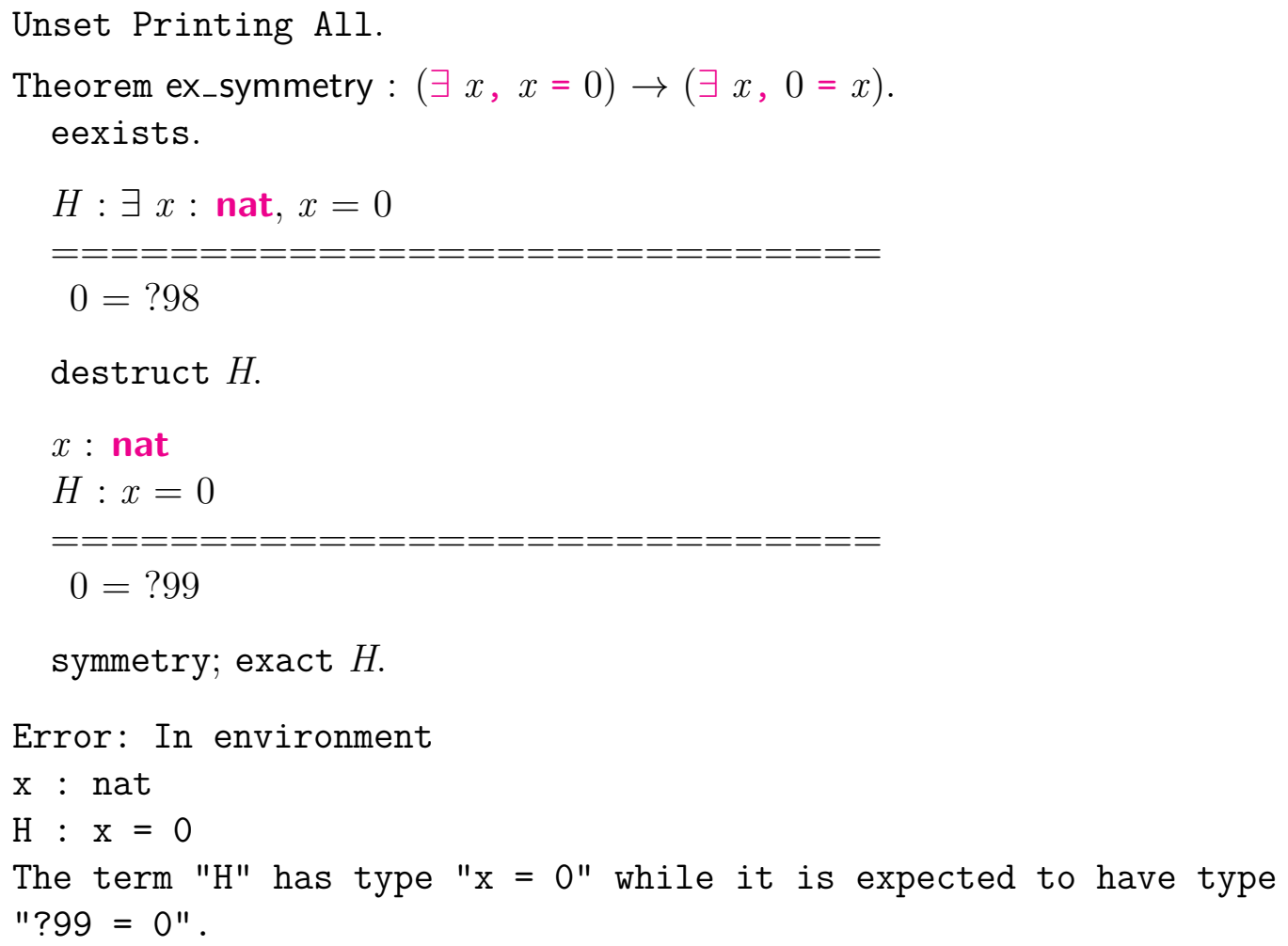


The problem here is that variable $x$ was introduced by destruct after we introduced ?99 with eexists, so the instantiation of ?99 may not mention $x$. A simple reordering of the proof solves the problem.

Restart.

destruct 1 as $[x]$; apply ex_intro with $x$; symmetry; assumption. Qed.

This restriction for unification variables may seem counterintuitive, but it follows from the fact that CIC contains no concept of unification variable. Rather, to construct the final proof term, at the point in a proof where the unification variable is introduced, we replace it with the instantiation we eventually find for it. It is simply syntactically illegal to refer there to variables that are not in scope. Without such a restriction, we could trivially "prove" such non-theorems as $\exists n$ : nat, $\forall m$ : nat, $n=m$ by econstructor; intro; reflexivity.

\subsection{The Prop Universe}

In Chapter 4, we saw parallel versions of useful datatypes for "programs" and "proofs." The convention was that programs live in Set, and proofs live in Prop. We gave little explanation for why it is useful to maintain this distinction. There is certainly documentation value from separating programs from proofs; in practice, different concerns apply to building the two types of objects. It turns out, however, that these concerns motivate formal differences between the two universes in Coq.

Recall the types sig and ex, which are the program and proof versions of existential quantification. Their definitions differ only in one place, where sig uses Type and ex uses Prop.

Print sig.

Inductive sig ( $A$ : Type) $(P: A \rightarrow$ Prop) : Type $:=$ exist : $\forall x: A, P x \rightarrow \operatorname{sig} P$

Print ex.

Inductive ex ( $A$ : Type) $(P: A \rightarrow$ Prop) $:$ Prop $:=$ ex_intro: $\forall x: A, P x \rightarrow$ ex $P$

It is natural to want a function to extract the first components of data structures like these. Doing so is easy enough for sig.

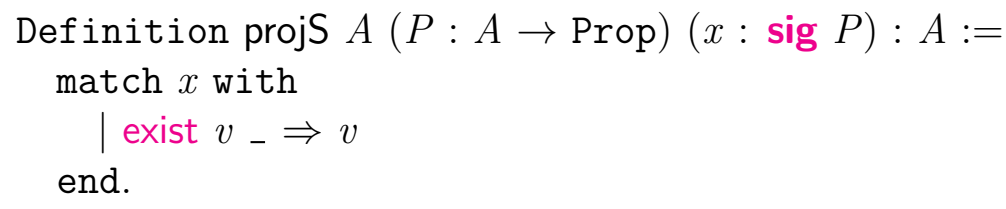

We run into trouble with a version that has been changed to work with ex. Definition projE $A(P: A \rightarrow$ Prop) $(x:$ ex $P): A:=$ 


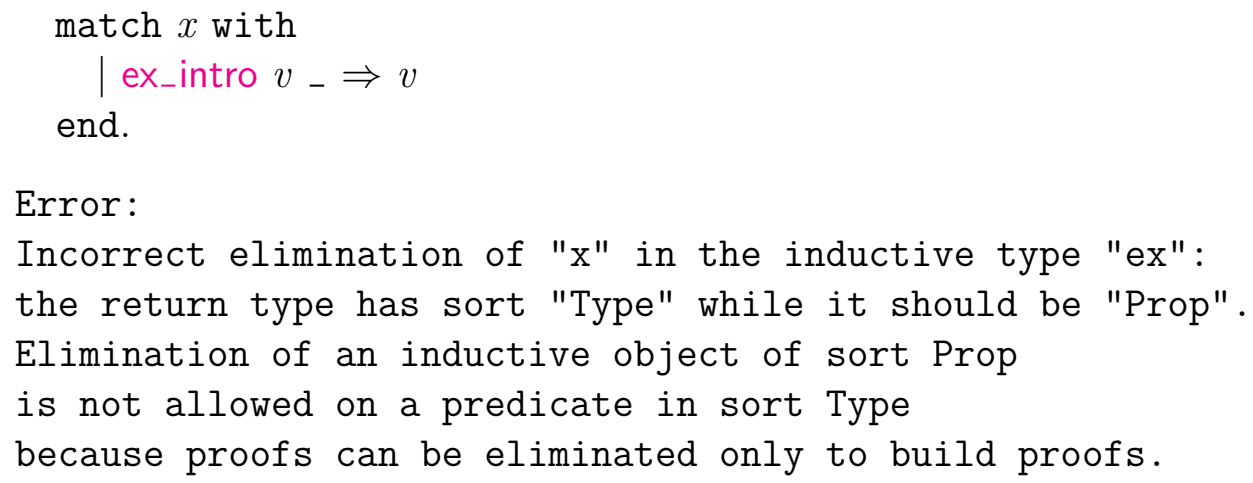

In formal Coq parlance, "elimination" means "pattern-matching." The typing rules of Gallina forbid us from pattern-matching on a discriminee whose type belongs to Prop, whenever the result type of the match has a type besides Prop. This is a sort of "information flow" policy, where the type system ensures that the details of proofs can never have any effect on parts of a development that are not also marked as proofs.

This restriction matches informal practice. We think of programs and proofs as clearly separated, and, outside of constructive logic, the idea of computing with proofs is ill-formed. The distinction also has practical importance in Coq, where it affects the behavior of extraction.

Recall that extraction is Coq's facility for translating Coq developments into programs in general-purpose programming languages like OCaml. Extraction erases proofs and leaves programs intact. A simple example with sig and ex demonstrates the distinction.

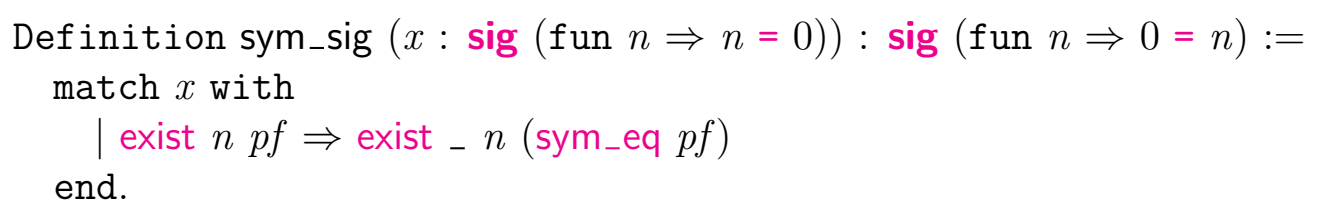

Extraction sym_sig.

(** val sym_sig : nat $\rightarrow$ nat $* *$ )

let sym_sig $\mathrm{x}=\mathrm{x}$

Since extraction erases proofs, the second components of sig values are elided, making sig a simple identity type family. The sym_sig operation is thus an identity function.

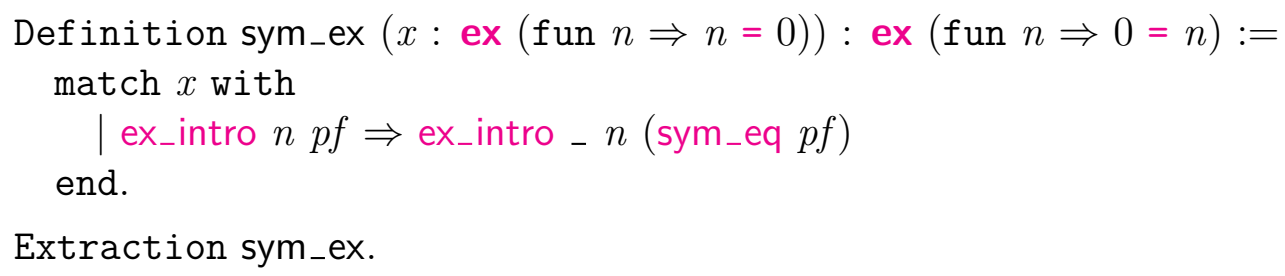

$(* *$ val sym_ex : _- $* *)$

let sym_ex $=$ 
In this example, the ex type itself is in Prop, so whole ex packages are erased. Coq extracts every proposition as the (Coq-specific) type _-, whose single constructor is _.. Not only are proofs replaced by _-_, but proof arguments to functions are also removed completely, as we see here.

Extraction is very helpful as an optimization over programs that contain proofs. In languages like Haskell, advanced features make it possible to program with proofs, as a way of convincing the type checker to accept particular definitions. Unfortunately, when proofs are encoded as values in GADTs [50], these proofs exist at runtime and consume resources. In contrast, with Coq, as long as all proofs are kept within Prop, extraction is guaranteed to erase them.

Many fans of the Curry-Howard correspondence support the idea of extracting programs from proofs. In reality, few users of Coq and related tools do any such thing. Instead, extraction is better thought of as an optimization that reduces the runtime costs of expressive typing.

We have seen two of the differences between proofs and programs: proofs are subject to an elimination restriction and are elided by extraction. The remaining difference is that Prop is impredicative, as this example shows.

Check $\forall P Q:$ Prop, $P \vee Q \rightarrow Q \vee P$.

$$
\forall P Q: \text { Prop, } P \vee Q \rightarrow Q \vee P
$$

: Prop

We see that it is possible to define a Prop that quantifies over other Props. This is fortunate, as we start wanting that ability even for such basic purposes as stating propositional tautologies. In the next section of this chapter, we will see some reasons why unrestricted impredicativity is undesirable. The impredicativity of Prop interacts crucially with the elimination restriction to avoid those pitfalls.

Impredicativity also allows us to implement a version of our earlier exp type that does not suffer from the weakness that we found.

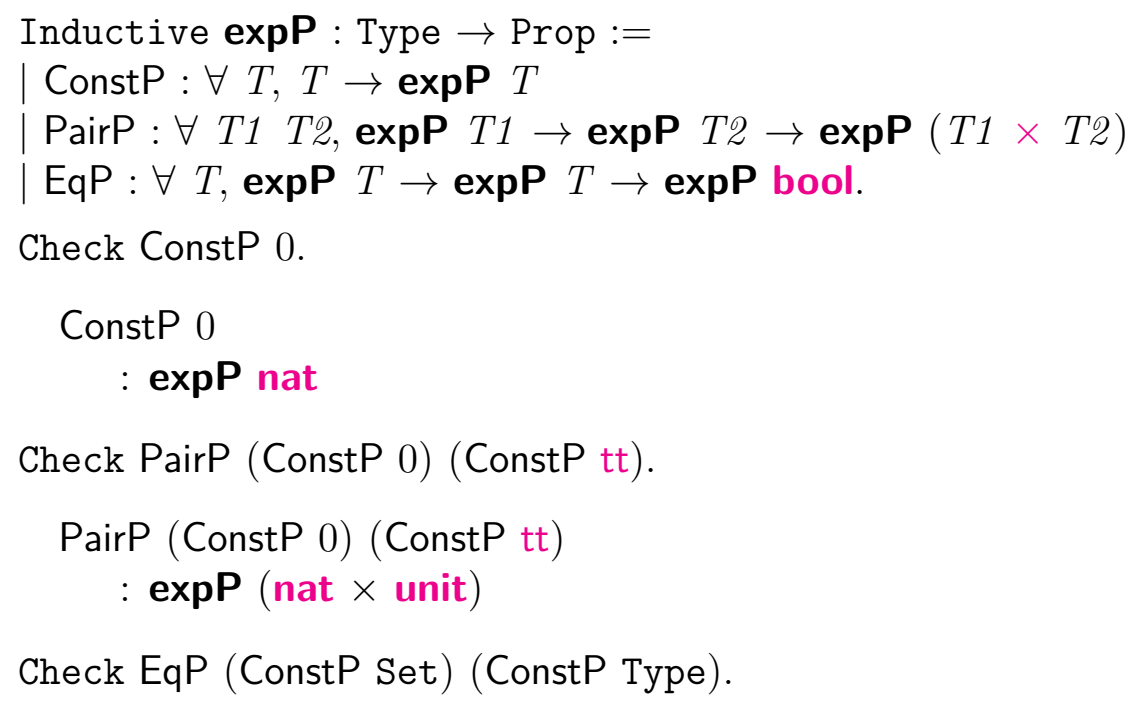




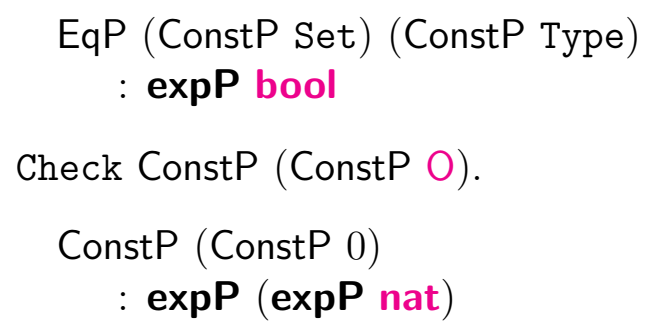

In this case, our victory is really a shallow one. As we have marked expP as a family of proofs, we cannot deconstruct our expressions in the usual programmatic ways, which makes them almost useless for the usual purposes. Impredicative quantification is much more useful in defining inductive families that we really think of as judgments. For instance, this code defines a notion of equality that is strictly more permissive than the base equality $=$.

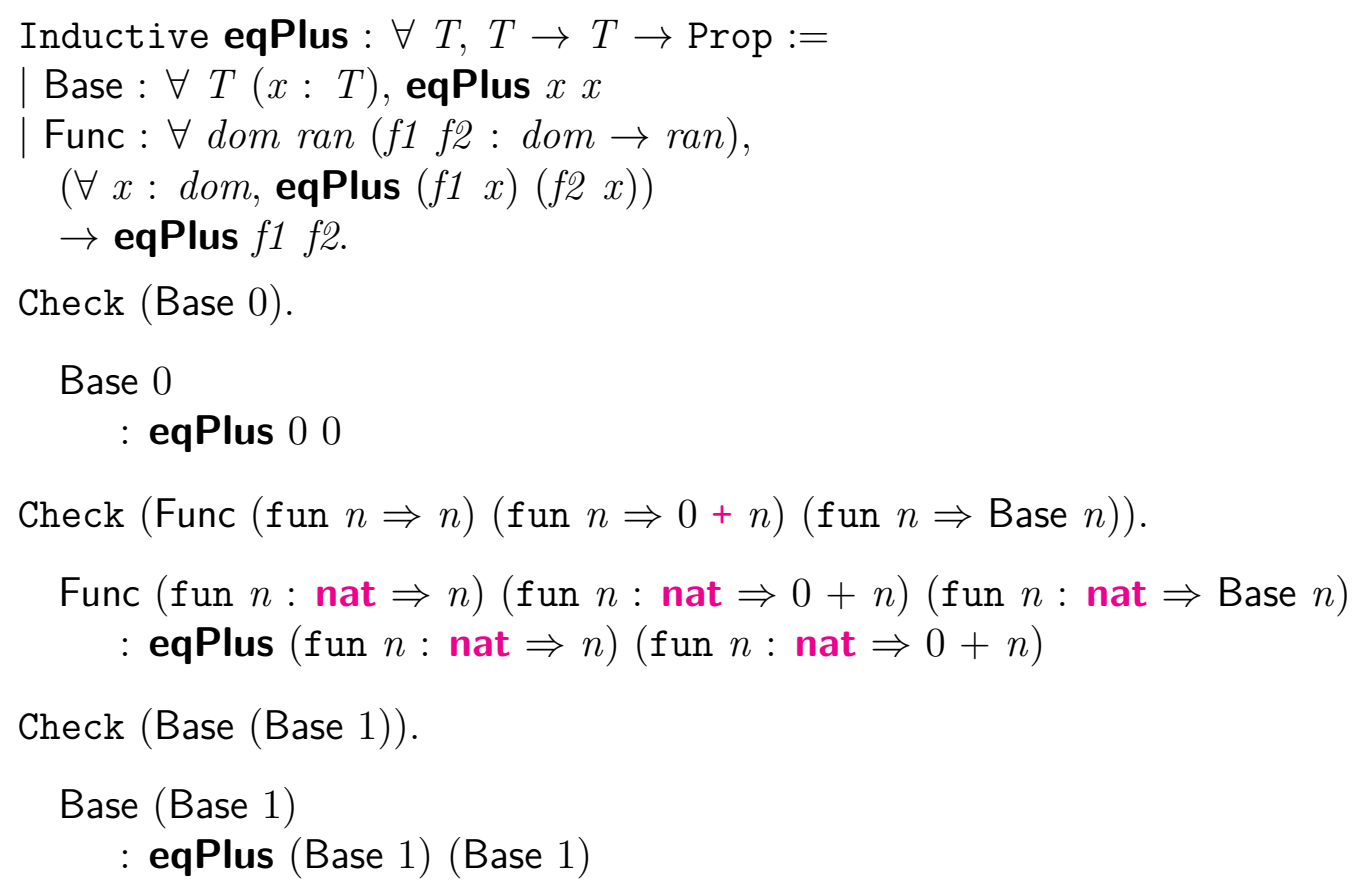

Stating equality facts about proofs may seem baroque, but we have already seen its utility in the chapter on reasoning about equality proofs.

\subsection{Axioms}

While the specific logic Gallina is hardcoded into Coq's implementation, it is possible to add certain logical rules in a controlled way. In other words, Coq may be used to reason about many different refinements of Gallina where strictly more theorems are provable. We achieve this by asserting axioms without proof.

We will motivate the idea by touring through some standard axioms, as enumerated in Coq's online FAQ. I will add additional commentary as appropriate. 


\subsubsection{The Basics}

One simple example of a useful axiom is the law of the excluded middle.

Require Import Classical_Prop.

Print classic.

*** [ classic : $\forall P$ : Prop, $P \vee \neg P$ ]

In the implementation of module Classical_Prop, this axiom was defined with the command

Axiom classic : $\forall P$ : Prop, $P \vee \neg P$.

An Axiom may be declared with any type, in any of the universes. There is a synonym Parameter for Axiom, and that synonym is often clearer for assertions not of type Prop. For instance, we can assert the existence of objects with certain properties.

Parameter num : nat.

Axiom positive : num $>0$.

Reset num.

This kind of "axiomatic presentation" of a theory is very common outside of higher-order logic. However, in Coq, it is almost always preferable to stick to defining your objects, functions, and predicates via inductive definitions and functional programming.

In general, there is a significant burden associated with any use of axioms. It is easy to assert a set of axioms that together is inconsistent. That is, a set of axioms may imply False, which allows any theorem to be proved, which defeats the purpose of a proof assistant. For example, we could assert the following axiom, which is consistent by itself but inconsistent when combined with classic.

Axiom not_classic : $\neg \forall P$ : Prop, $P \vee \neg P$.

Theorem uhoh : False.

generalize classic not_classic; tauto.

Qed.

Theorem uhoh_again : $1+1=3$.

destruct uhoh.

Qed.

Reset not_classic.

On the subject of the law of the excluded middle itself, this axiom is usually quite harmless, and many practical Coq developments assume it. It has been proved metatheoretically to be consistent with CIC. Here, "proved metatheoretically" means that someone proved on paper that excluded middle holds in a model of CIC in set theory [48]. All of the other axioms that we will survey in this section hold in the same model, so they are all consistent together.

Recall that Coq implements constructive logic by default, where the law of the excluded middle is not provable. Proofs in constructive logic can be thought of as programs. A $\forall$ 
quantifier denotes a dependent function type, and a disjunction denotes a variant type. In such a setting, excluded middle could be interpreted as a decision procedure for arbitrary propositions, which computability theory tells us cannot exist. Thus, constructive logic with excluded middle can no longer be associated with our usual notion of programming.

Given all this, why is it all right to assert excluded middle as an axiom? The intuitive justification is that the elimination restriction for Prop prevents us from treating proofs as programs. An excluded middle axiom that quantified over Set instead of Prop would be problematic. If a development used that axiom, we would not be able to extract the code to OCaml (soundly) without implementing a genuine universal decision procedure. In contrast, values whose types belong to Prop are always erased by extraction, so we sidestep the axiom's algorithmic consequences.

Because the proper use of axioms is so precarious, there are helpful commands for determining which axioms a theorem relies on.

Theorem t1 : $\forall P:$ Prop, $P \rightarrow \neg \neg P$.

tauto.

Qed.

Print Assumptions t1.

Closed under the global context

Theorem t2 : $\forall P:$ Prop, $\neg \neg P \rightarrow P$.

tauto.

Error: tauto failed.

intro $P$; destruct (classic $P$ ); tauto.

Qed.

Print Assumptions t2.

Axioms:

classic : $\forall P$ : Prop, $P \vee \neg P$

It is possible to avoid this dependence in some specific cases, where excluded middle $i s$ provable, for decidable families of propositions.

Theorem nat_eq_dec : $\forall n m$ : nat, $n=m \vee n \neq m$.

induction $n$; destruct $m$; intuition; generalize (IHn $\mathrm{m})$; intuition. Qed.

Theorem t2' $: \forall n m:$ nat, $\neg \neg(n=m) \rightarrow n=m$.

intros $n m$; destruct (nat_eq_dec $n m$ ); tauto.

Qed.

Print Assumptions t2'.

Closed under the global context 
Mainstream mathematical practice assumes excluded middle, so it can be useful to have it available in Coq developments, though it is also nice to know that a theorem is proved in a simpler formal system than classical logic. There is a similar story for proof irrelevance, which simplifies proof issues that would not even arise in mainstream math.

Require Import Prooflrrelevance.

Print proof_irrelevance.

*** [ proof_irrelevance : $\forall(P$ : Prop $)(p 1 p 2: P), p 1=p 2]$

This axiom asserts that any two proofs of the same proposition are equal. Recall this example function from Chapter 6 .

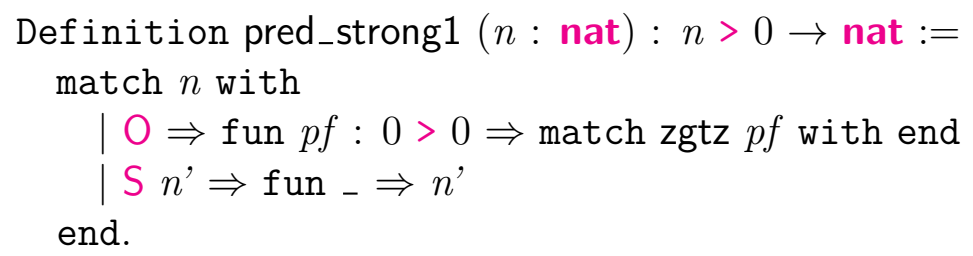

We might want to prove that different proofs of $n>0$ do not lead to different results from our richly typed predecessor function.

Theorem pred_strong1_irrel : $\forall n(p f 1 p f 2: n>0)$, pred_strong1 pf1 = pred_strong1 $p f 2$.

destruct $n$; crush.

Qed.

The proof script is simple, but it involved peeking into the definition of pred_strong1. For more complicated function definitions, it can be considerably more work to prove that they do not discriminate on details of proof arguments. This can seem like a shame, since the Prop elimination restriction makes it impossible to write any function that does otherwise. Unfortunately, this fact is only true metatheoretically, unless we assert an axiom like proof_irrelevance. With that axiom, we can prove our theorem without consulting the definition of pred_strong1.

Theorem pred_strong1_irrel' : $\forall n(p f 1 p f 2: n>0)$, pred_strong1 $p f 1=$ pred_strong1 $p f 2$. intros; f_equal; apply proof_irrelevance. Qed.

In the chapter on equality, we already discussed some axioms that are related to proof irrelevance. In particular, Coq's standard library includes this axiom:

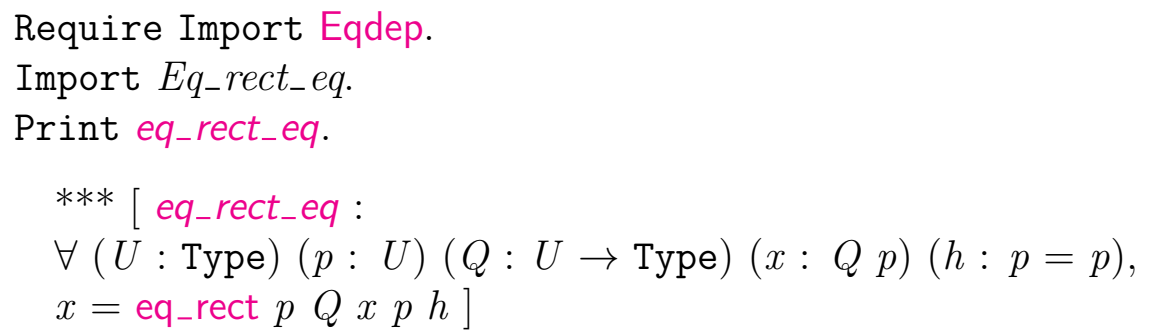


This axiom says that it is permissible to simplify pattern matches over proofs of equalities like $e=e$. The axiom is logically equivalent to some simpler corollaries. In the theorem names, "UIP" stands for "unicity of identity proofs", where "identity" is a synonym for "equality."

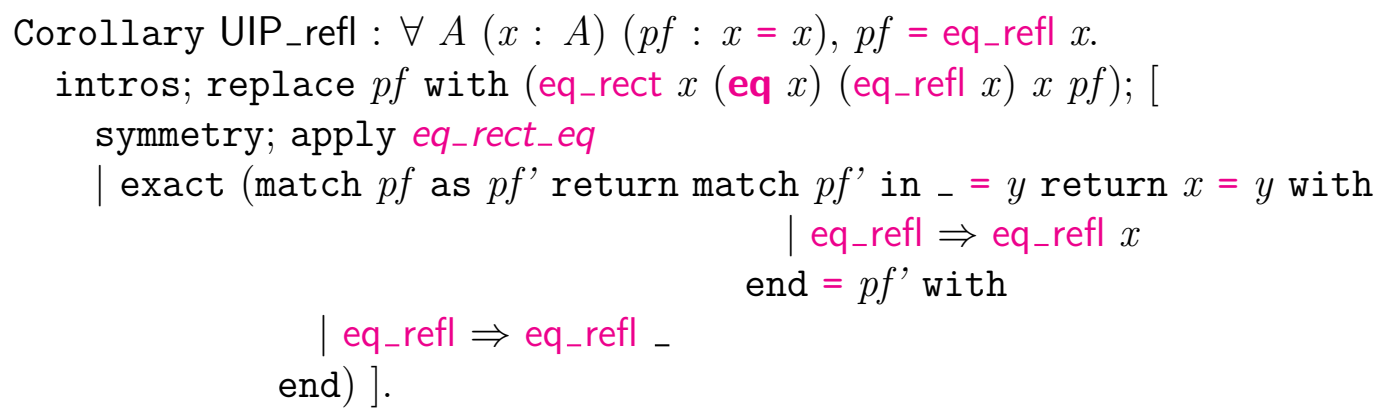

Qed.

Corollary UIP : $\forall A(x y: A)(p f 1 p f 2: x=y), p f 1=p f 2$.

intros; generalize pf1 pf2; subst; intros;

match goal with

$\mid[\vdash ? p f 1=$ ?pf2 $] \Rightarrow$ rewrite (UIP_refl $p f 1$ ); rewrite (UIP_refl pf2); reflexivity end.

Qed.

These corollaries are special cases of proof irrelevance. In developments that only need proof irrelevance for equality, there is no need to assert full irrelevance.

Another facet of proof irrelevance is that, like excluded middle, it is often provable for specific propositions. For instance, UIP is provable whenever the type $A$ has a decidable equality operation. The module Eqdep_dec of the standard library contains a proof. A similar phenomenon applies to other notable cases, including less-than proofs. Thus, it is often possible to use proof irrelevance without asserting axioms.

There are two more basic axioms that are often assumed, to avoid complications that do not arise in set theory.

Require Import FunctionalExtensionality.

Print functional_extensionality_dep.

*** [ functional_extensionality_dep :

$\forall(A:$ Type $)(B: A \rightarrow$ Type $)(f g: \forall x: A, B x)$,

$(\forall x: A, f x=g x) \rightarrow f=g]$

This axiom says that two functions are equal if they map equal inputs to equal outputs. Such facts are not provable in general in CIC, but it is consistent to assume that they are.

A simple corollary shows that the same property applies to predicates.

Corollary predicate_extensionality : $\forall$ (A: Type) $(B: A \rightarrow$ Prop) $(f g: \forall x: A, B x)$,

$(\forall x: A, f x=g x) \rightarrow f=g$. 
intros; apply functional_extensionality_dep; assumption.

Qed.

In some cases, one might prefer to assert this corollary as the axiom, to restrict the consequences to proofs and not programs.

\subsubsection{Axioms of Choice}

Some Coq axioms are also points of contention in mainstream math. The most prominent example is the axiom of choice. In fact, there are multiple versions that we might consider, and, considered in isolation, none of these versions means quite what it means in classical set theory.

First, it is possible to implement a choice operator without axioms in some potentially surprising cases.

Require Import ConstructiveEpsilon.

Check constructive_definite_description.

constructive_definite_description

$: \forall(A:$ Set $)(f: A \rightarrow$ nat $)(g:$ nat $\rightarrow A)$,

$(\forall x: A, g(f x)=x) \rightarrow$

$\forall P: A \rightarrow$ Prop,

$(\forall x: A,\{P x\}+\{\neg P x\}) \rightarrow$

$(\exists ! x: A, P x) \rightarrow\{x: A \mid P x\}$

Print Assumptions constructive_definite_description.

Closed under the global context

This function transforms a decidable predicate $P$ into a function that produces an element satisfying $P$ from a proof that such an element exists. The functions $f$ and $g$, in conjunction with an associated injectivity property, are used to express the idea that the set $A$ is countable. Under these conditions, a simple brute force algorithm gets the job done: we just enumerate all elements of $A$, stopping when we find one satisfying $P$. The existence proof, specified in terms of unique existence $\exists$ !, guarantees termination. The definition of this operator in Coq uses some interesting techniques, as seen in the implementation of the ConstructiveEpsilon module.

Countable choice is provable in set theory without appealing to the general axiom of choice. To support the more general principle in Coq, we must also add an axiom. Here is a functional version of the axiom of unique choice.

Require Import ClassicalUniqueChoice.

Check dependent_unique_choice.

dependent_unique_choice

$: \forall(A:$ Type $)(B: A \rightarrow$ Type $)(R: \forall x: A, B x \rightarrow$ Prop $)$, 


$$
\begin{aligned}
& (\forall x: A, \exists ! y: B x, R x y) \rightarrow \\
& \exists f: \forall x: A, B x \\
& \quad \forall x: A, R x(f x)
\end{aligned}
$$

This axiom lets us convert a relational specification $R$ into a function implementing that specification. We need only prove that $R$ is truly a function. An alternate, stronger formulation applies to cases where $R$ maps each input to one or more outputs. We also simplify the statement of the theorem by considering only non-dependent function types.

Require Import ClassicalChoice.

Check choice.

choice

$$
\begin{aligned}
& : \forall(A B: \text { Type })(R: A \rightarrow B \rightarrow \text { Prop }), \\
& \quad(\forall x: A, \exists y: B, R x y) \rightarrow \\
& \quad \exists f: A \rightarrow B, \forall x: A, R x(f x)
\end{aligned}
$$

This principle is proved as a theorem, based on the unique choice axiom and an additional axiom of relational choice from the RelationalChoice module.

In set theory, the axiom of choice is a fundamental philosophical commitment one makes about the universe of sets. In Coq, the choice axioms say something weaker. For instance, consider the simple restatement of the choice axiom where we replace existential quantification by its Curry-Howard analogue, subset types.

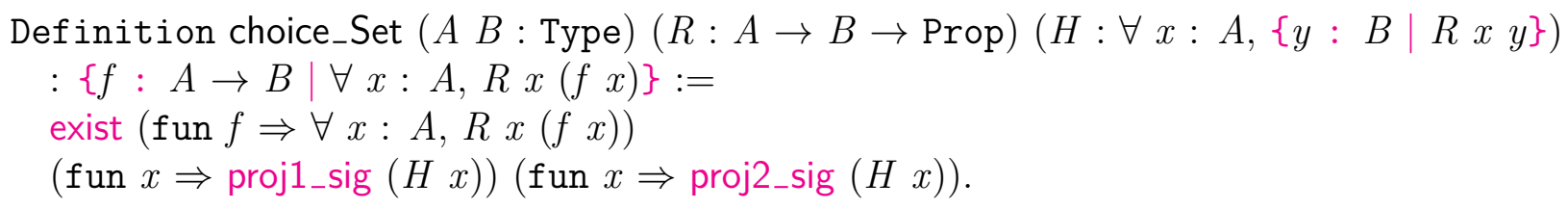

Via the Curry-Howard correspondence, this "axiom" can be taken to have the same meaning as the original. It is implemented trivially as a transformation not much deeper than uncurrying. Thus, we see that the utility of the axioms that we mentioned earlier comes in their usage to build programs from proofs. Normal set theory has no explicit proofs, so the meaning of the usual axiom of choice is subtly different. In Gallina, the axioms implement a controlled relaxation of the restrictions on information flow from proofs to programs.

However, when we combine an axiom of choice with the law of the excluded middle, the idea of "choice" becomes more interesting. Excluded middle gives us a highly noncomputational way of constructing proofs, but it does not change the computational nature of programs. Thus, the axiom of choice is still giving us a way of translating between two different sorts of "programs," but the input programs (which are proofs) may be written in a rich language that goes beyond normal computability. This combination truly is more than repackaging a function with a different type.

The Coq tools support a command-line flag-impredicative-set, which modifies Gallina in a more fundamental way by making Set impredicative. A term like $\forall T$ : Set, $T$ has type Set, and inductive definitions in Set may have constructors that quantify over arguments 
of any types. To maintain consistency, an elimination restriction must be imposed, similarly to the restriction for Prop. The restriction only applies to large inductive types, where some constructor quantifies over a type of type Type. In such cases, a value in this inductive type may only be pattern-matched over to yield a result type whose type is Set or Prop. This rule contrasts with the rule for Prop, where the restriction applies even to non-large inductive types, and where the result type may only have type Prop.

In old versions of Coq, Set was impredicative by default. Later versions make Set predicative to avoid inconsistency with some classical axioms. In particular, one should watch out when using impredicative Set with axioms of choice. In combination with excluded middle or predicate extensionality, inconsistency can result. Impredicative Set can be useful for modeling inherently impredicative mathematical concepts, but almost all Coq developments get by fine without it.

\subsubsection{Axioms and Computation}

One additional axiom-related wrinkle arises from an aspect of Gallina that is very different from set theory: a notion of computational equivalence is central to the definition of the formal system. Axioms tend not to play well with computation. Consider this example. We start by implementing a function that uses a type equality proof to perform a safe type-cast.

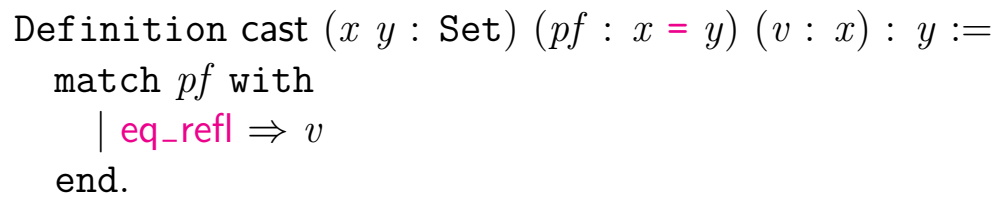

Computation over programs that use cast can proceed smoothly.

Eval compute in (cast (eq_refl (nat $\rightarrow$ nat)) (fun $n \Rightarrow \mathrm{S} n)) 12$.

$$
\begin{aligned}
& =13 \\
& \text { : nat }
\end{aligned}
$$

Things do not go as smoothly when we use cast with proofs that rely on axioms.

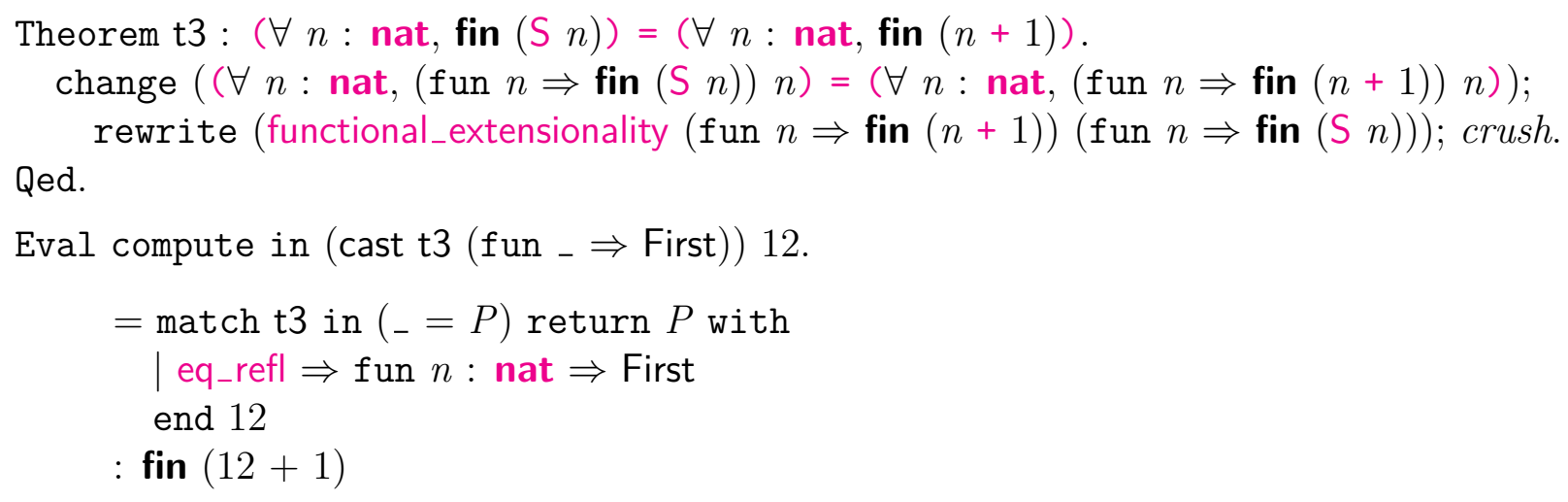

Computation gets stuck in a pattern-match on the proof $t 3$. The structure of $\mathrm{t} 3$ is not known, so the match cannot proceed. It turns out a more basic problem leads to this 
particular situation. We ended the proof of $\mathrm{t} 3$ with Qed, so the definition of $\mathrm{t} 3$ is not available to computation. That mistake is easily fixed.

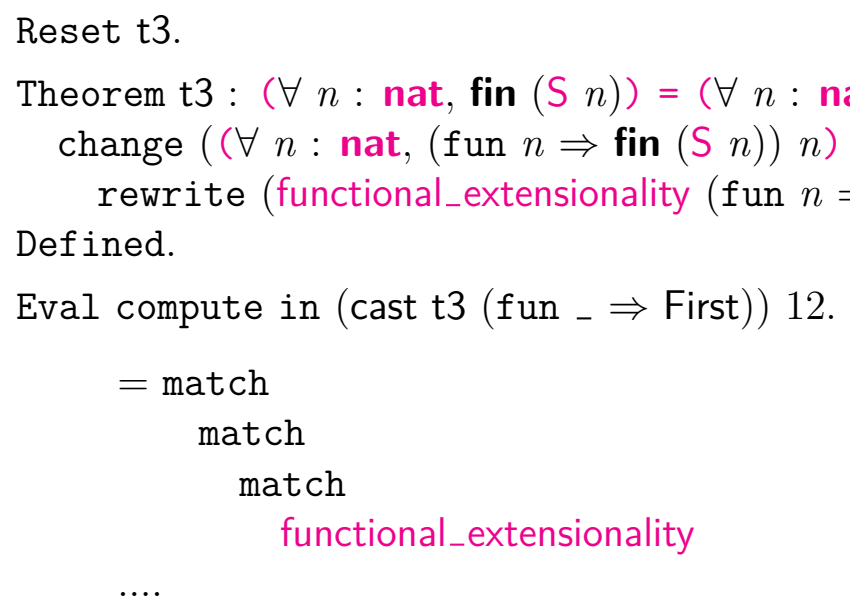

We elide most of the details. A very unwieldy tree of nested matches on equality proofs appears. This time evaluation really is stuck on a use of an axiom.

If we are careful in using tactics to prove an equality, we can still compute with casts over the proof.

Lemma plus1: $\forall n, \mathrm{~S} n=n+1$.

induction $n$; simpl; intuition.

Defined.

Theorem t4: $\forall n$, fin $(\mathrm{S} n)=$ fin $(n+1)$.

intro; f_equal; apply plus1.

Defined.

Eval compute in cast (t4 13) First.

$=$ First

: fin $(13+1)$

This simple computational reduction hides the use of a recursive function to produce a suitable eq_refl proof term. The recursion originates in our use of induction in t4's proof.

\subsubsection{Methods for Avoiding Axioms}

The last section demonstrated one reason to avoid axioms: they interfere with computational behavior of terms. A further reason is to reduce the philosophical commitment of a theorem. The more axioms one assumes, the harder it becomes to convince oneself that the formal system corresponds appropriately to one's intuitions. A refinement of this last point, in applications like proof-carrying code [27] in computer security, has to do with minimizing the size of a trusted code base. To convince ourselves that a theorem is true, we must convince ourselves of the correctness of the program that checks the theorem. Axioms effectively become new source code for the checking program, increasing the effort required to perform a correctness audit. 
An earlier section gave one example of avoiding an axiom. We proved that pred_strong1 is agnostic to details of the proofs passed to it as arguments, by unfolding the definition of the function. A "simpler" proof keeps the function definition opaque and instead applies a proof irrelevance axiom. By accepting a more complex proof, we reduce our philosophical commitment and trusted base. (By the way, the less-than relation that the proofs in question here prove turns out to admit proof irrelevance as a theorem provable within normal Gallina!)

One dark secret of the dep_destruct tactic that we have used several times is reliance on an axiom. Consider this simple case analysis principle for fin values:

Theorem fin_cases : $\forall n(f$ : fin $(\mathrm{S} n)), f=$ First $\vee \exists f^{\prime}, f=\operatorname{Next} f^{\prime}$.

intros; dep_destruct $f$; eauto.

Qed.

Print Assumptions fin_cases.

Axioms:

JMeq_eq : $\forall$ (A: Type) $(x y: A)$, JMeq $x y \rightarrow x=y$

The proof depends on the JMeq_eq axiom that we met in the chapter on equality proofs. However, a smarter tactic could have avoided an axiom dependence. Here is an alternate proof via a slightly strange looking lemma.

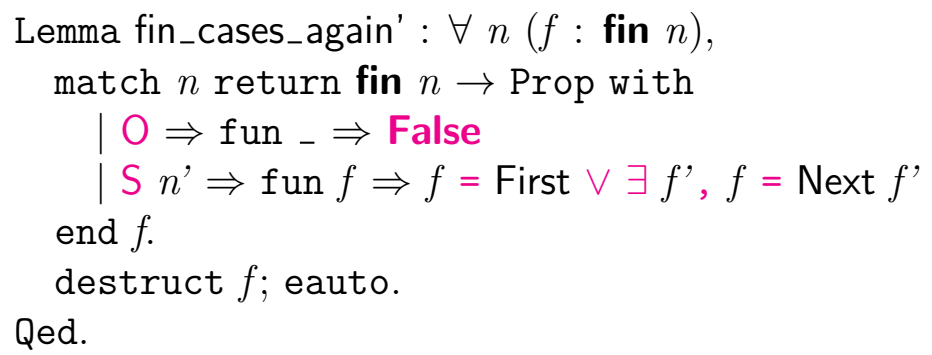

We apply a variant of the convoy pattern, which we are used to seeing in function implementations. Here, the pattern helps us state a lemma in a form where the argument to fin is a variable. Recall that, thanks to basic typing rules for pattern-matching, destruct will only work effectively on types whose non-parameter arguments are variables. The exact tactic, which takes as argument a literal proof term, now gives us an easy way of proving the original theorem.

Theorem fin_cases_again : $\forall n(f:$ fin $(\mathrm{S} n)), f=$ First $\vee \exists f^{\prime}, f=$ Next $f^{\prime}$. intros; exact (fin_cases_again' $f$ ).

Qed.

Print Assumptions fin_cases_again.

Closed under the global context

As the Curry-Howard correspondence might lead us to expect, the same pattern may be applied in programming as in proving. Axioms are relevant in programming, too, because, while Coq includes useful extensions like Program that make dependently typed programming 
more straightforward, in general these extensions generate code that relies on axioms about equality. We can use clever pattern matching to write our code axiom-free.

As an example, consider a Set version of fin_cases. We use Set types instead of Prop types, so that return values have computational content and may be used to guide the behavior of algorithms. Beside that, we are essentially writing the same "proof" in a more explicit way.

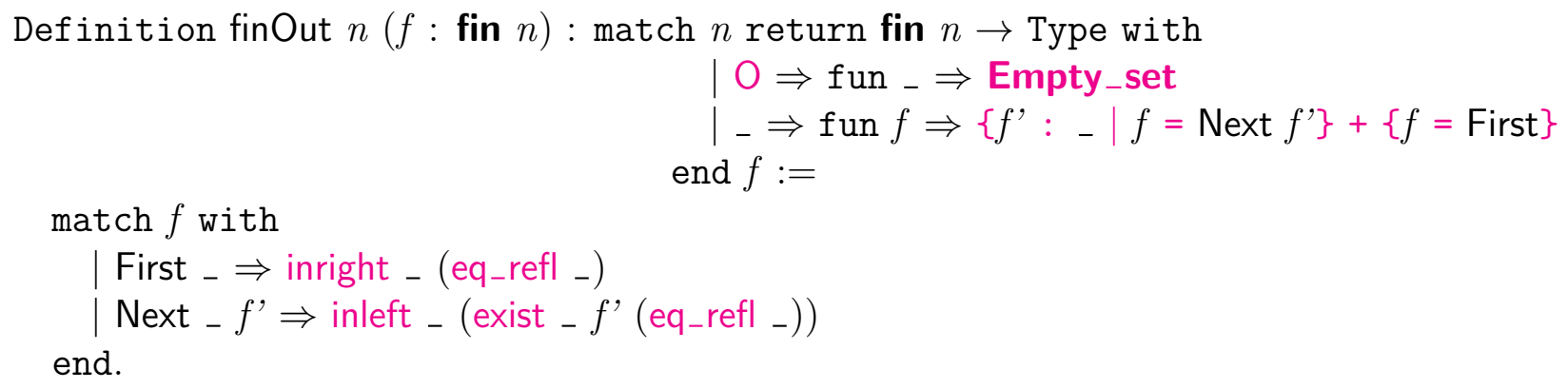

As another example, consider the following type of formulas in first-order logic. The intent of the type definition will not be important in what follows, but we give a quick intuition for the curious reader. Our formulas may include $\forall$ quantification over arbitrary Types, and we index formulas by environments telling which variables are in scope and what their types are; such an environment is a list Type. A constructor Inject lets us include any Coq Prop as a formula, and VarEq and Lift can be used for variable references, in what is essentially the de Bruijn index convention. (Again, the detail in this paragraph is not important to understand the discussion that follows!)

Inductive formula : list Type $\rightarrow$ Type $:=$

| Inject : $\forall T s$, Prop $\rightarrow$ formula $T s$

VarEq : $\forall T T s, T \rightarrow$ formula $(T:: T s)$

Lift : $\forall T T s$, formula $T s \rightarrow$ formula $(T:: T s)$

Forall : $\forall T T s$, formula $(T:: T s) \rightarrow$ formula $T s$

And : $\forall T s$, formula $T s \rightarrow$ formula $T s \rightarrow$ formula $T s$.

This example is based on my own experiences implementing variants of a program logic called XCAP [28], which also includes an inductive predicate for characterizing which formulas are provable. Here I include a pared-down version of such a predicate, with only two constructors, which is sufficient to illustrate certain tricky issues.

Inductive proof : formula nil $\rightarrow$ Prop $:=$

PInject : $\forall$ (P: Prop), $P \rightarrow$ proof (Inject nil $P)$

PAnd : $\forall p q$, proof $p \rightarrow$ proof $q \rightarrow$ proof (And $p q)$.

Let us prove a lemma showing that a " $P \wedge Q \rightarrow P$ " rule is derivable within the rules of proof.

Theorem proj1 : $\forall p q$, proof (And $p q) \rightarrow$ proof $p$.

destruct 1 .

$p:$ formula nil 


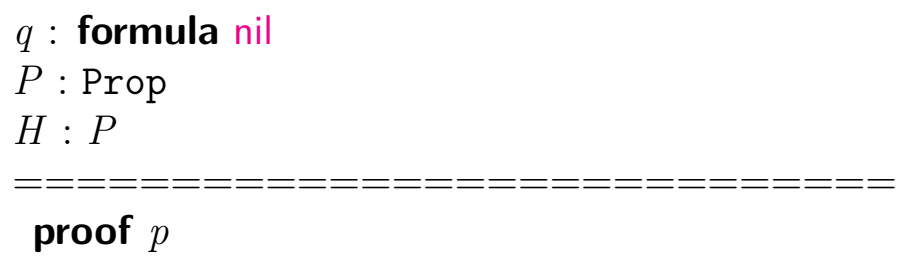

We are reminded that induction and destruct do not work effectively on types with non-variable arguments. The first subgoal, shown above, is clearly unprovable. (Consider the case where $p=$ Inject nil False.)

An application of the dependent destruction tactic (the basis for dep_destruct) solves the problem handily. We use a shorthand with the intros tactic that lets us use question marks for variable names that do not matter.

Restart.

Require Import Program.

intros ? ? $H$; dependent destruction $H$; auto.

Qed.

Print Assumptions proj1.

Axioms:

eq_rect_eq $: \forall(U:$ Type $)(p: U)(Q: U \rightarrow$ Type $)(x: Q p)(h: p=p)$, $x=$ eq_rect $p Q x p h$

Unfortunately, that built-in tactic appeals to an axiom. It is still possible to avoid axioms by giving the proof via another odd-looking lemma. Here is a first attempt that fails at remaining axiom-free, using a common equality-based trick for supporting induction on non-variable arguments to type families. The trick works fine without axioms for datatypes more traditional than formula, but we run into trouble with our current type.

Lemma proj1_again' : $\forall r$, proof $r$

$\rightarrow \forall p q, r=$ And $p q \rightarrow$ proof $p$.

destruct 1; crush.

$H 0$ : Inject [] $P=$ And $p q$

$====================$

proof $p$

The first goal looks reasonable. Hypothesis $H O$ is clearly contradictory, as discriminate can show.

try discriminate.

$H 1$ : And $p q=$ And $p 0 q 0$

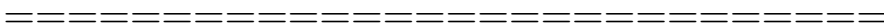

\section{proof $p 0$}

It looks like we are almost done. Hypothesis $H 1$ gives $p=p 0$ by injectivity of constructors, and then $H$ finishes the case.

injection $H 1$; intros. 

form:

Unfortunately, the "equality" that we expected between $p$ and $p 0$ comes in a strange

$$
\begin{aligned}
& H 3: \text { exist } T \text { (fun } T s: \text { list Type } \Rightarrow \text { formula } T s)[] \% \text { list } p= \\
& \quad \text { exist } T \text { (fun } T s: \text { list Type } \Rightarrow \text { formula } T s)[] \% \text { list } p 0 \\
& ======================= \\
& \text { proof } p 0
\end{aligned}
$$

It may take a bit of tinkering, but, reviewing Chapter 3's discussion of writing injection principles manually, it makes sense that an exist $T$ type is the most direct way to express the output of injection on a dependently typed constructor. The constructor And is dependently typed, since it takes a parameter Ts upon which the types of $p$ and $q$ depend. Let us not dwell further here on why this goal appears; the reader may like to attempt the (impossible) exercise of building a better injection lemma for And, without using axioms.

How exactly does an axiom come into the picture here? Let us ask crush to finish the proof.

crush.

Qed.

Print Assumptions proj1_again'.

Axioms:

eq_rect_eq $: \forall(U:$ Type $)(p: U)(Q: U \rightarrow$ Type $)(x: Q p)(h: p=p)$, $x=$ eq_rect $p Q x p h$

It turns out that this familiar axiom about equality (or some other axiom) is required to deduce $p=p 0$ from the hypothesis $H 3$ above. The soundness of that proof step is neither provable nor disprovable in Gallina.

Hope is not lost, however. We can produce an even stranger looking lemma, which gives us the theorem without axioms. As always when we want to do case analysis on a term with a tricky dependent type, the key is to refactor the theorem statement so that every term we match on has variables as its type indices; so instead of talking about proofs of And $p q$, we talk about proofs of an arbitrary $r$, but we only conclude anything interesting when $r$ is an And.

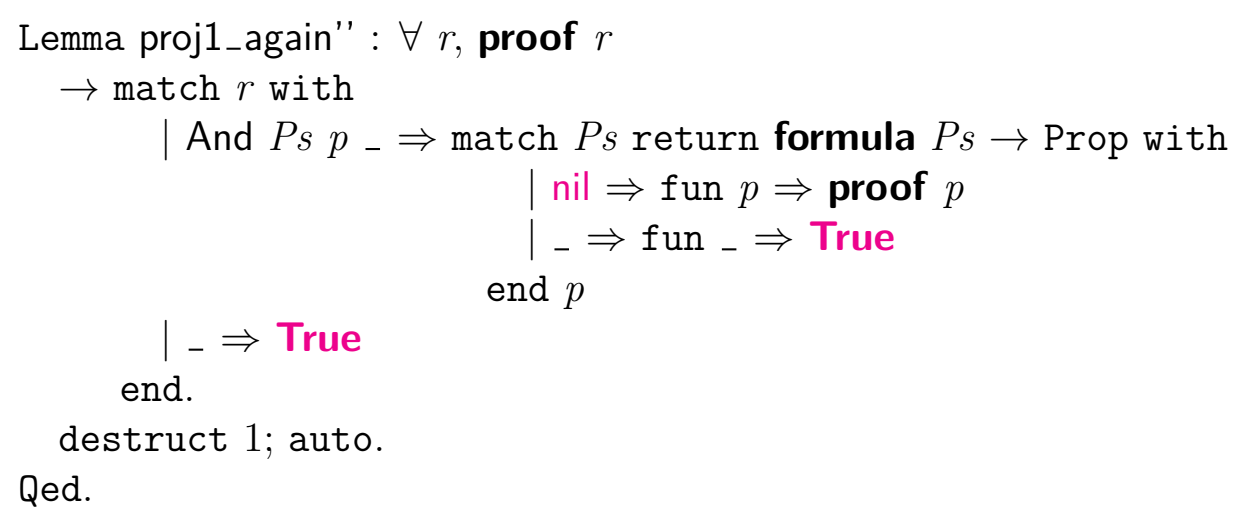


Theorem proj1_again : $\forall p q$, proof (And $p q) \rightarrow$ proof $p$.

intros? ? $H$; exact (proj1_again" $H$ ).

Qed.

Print Assumptions proj1_again.

Closed under the global context

This example illustrates again how some of the same design patterns we learned for dependently typed programming can be used fruitfully in theorem statements.

To close the chapter, we consider one final way to avoid dependence on axioms. Often this task is equivalent to writing definitions such that they compute. That is, we want Coq's normal reduction to be able to run certain programs to completion. Here is a simple example where such computation can get stuck. In proving properties of such functions, we would need to apply axioms like $\mathrm{K}$ manually to make progress.

Imagine we are working with deeply embedded syntax of some programming language, where each term is considered to be in the scope of a number of free variables that hold normal Coq values. To enforce proper typing, we will need to model a Coq typing environment somehow. One natural choice is as a list of types, where variable number $i$ will be treated as a reference to the $i$ th element of the list.

Section withTypes.

Variable types : list Set.

To give the semantics of terms, we will need to represent value environments, which assign each variable a term of the proper type.

Variable values : hlist (fun $x$ : Set $\Rightarrow x$ ) types.

Now imagine that we are writing some procedure that operates on a distinguished variable of type nat. A hypothesis formalizes this assumption, using the standard library function nth_error for looking up list elements by position.

Variable natIndex : nat.

Variable natIndex_ok: nth_error types natIndex = Some nat.

It is not hard to use this hypothesis to write a function for extracting the nat value in position natIndex of values, starting with two helpful lemmas, each of which we finish with Defined to mark the lemma as transparent, so that its definition may be expanded during evaluation.

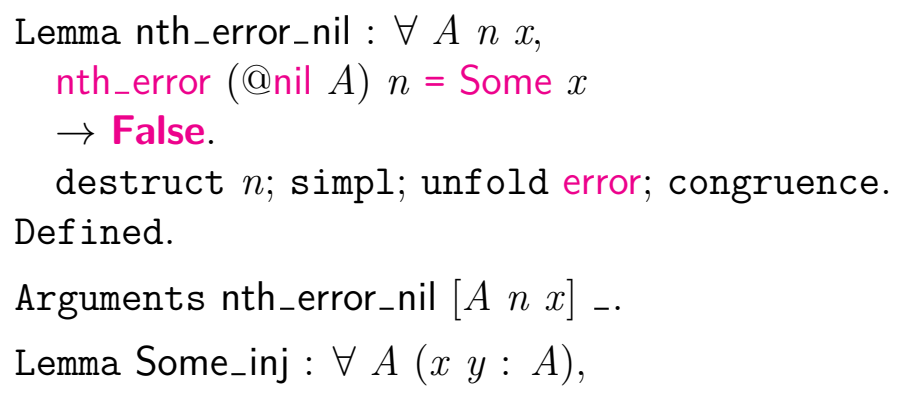


Some $x=$ Some $y$

$\rightarrow x=y$.

congruence.

Defined.

Fixpoint getNat (types' : list Set) (values': hlist (fun $x$ : Set $\Rightarrow x$ ) types')

(natIndex : nat) : (nth_error types' natIndex $=$ Some nat $) \rightarrow$ nat $:=$

match values' with

| $\mathrm{HNil} \Rightarrow$ fun $p f \Rightarrow$ match nth_error_nil $p f$ with end

| HCons $t$ ts $x$ values" $\Rightarrow$

match natIndex return nth_error $(t:: t s)$ natIndex $=$ Some nat $\rightarrow$ nat with

$\mathrm{O} \Rightarrow$ fun $p f \Rightarrow$

match Some_inj $p f$ in $\_=T$ return $T$ with

| eq_refl $\Rightarrow x$

end

I S natIndex' $\Rightarrow$ getNat values" natIndex'

end

end.

End withTypes.

The problem becomes apparent when we experiment with running getNat on a concrete types list.

Definition myTypes := unit : : nat : : bool : : nil.

Definition myValues : hlist ( fun $x$ : Set $\Rightarrow x$ ) myTypes :=

tt $::: 3:::$ false $:::$ HNil.

Definition myNatlndex $:=1$.

Theorem myNatIndex_ok : nth_error myTypes myNatIndex = Some nat.

reflexivity.

Defined.

Eval compute in getNat myValues myNatIndex myNatIndex_ok.

$=3$

We have not hit the problem yet, since we proceeded with a concrete equality proof for myNatIndex_ok. However, consider a case where we want to reason about the behavior of getNat independently of a specific proof.

Theorem getNat_is_reasonable : $\forall p f$, getNat myValues myNatIndex $p f=3$.

intro; compute.

1 subgoal

pf : nth_error myTypes myNatIndex = Some nat

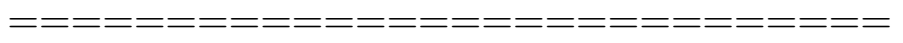

match 


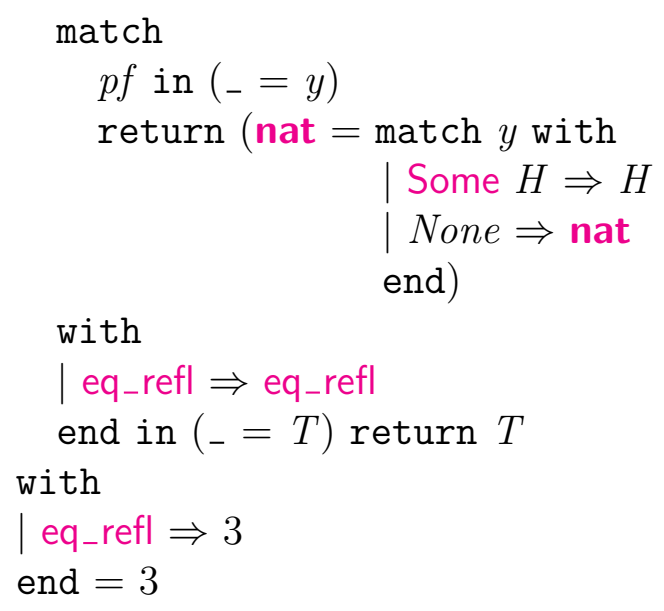

Since the details of the equality proof $p f$ are not known, computation can proceed no further. A rewrite with axiom $\mathrm{K}$ would allow us to make progress, but we can rethink the definitions a bit to avoid depending on axioms.

Abort.

Here is a definition of a function that turns out to be useful, though no doubt its purpose will be mysterious for now. A call update $l s n x$ overwrites the $n$th position of the list $l s$ with the value $x$, padding the end of the list with extra $x$ values as needed to ensure sufficient length.

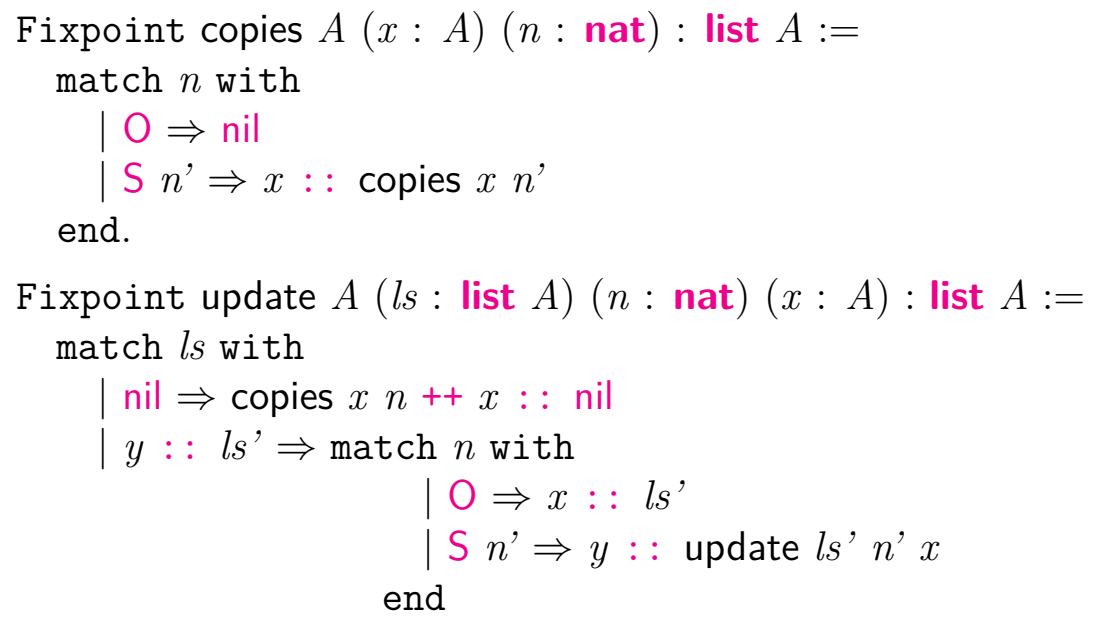

end.

Now let us revisit the definition of getNat.

\section{Section withTypes'.}

Variable types : list Set.

Variable natIndex : nat.

Here is the trick: instead of asserting properties about the list types, we build a "new" list that is guaranteed by construction to have those properties.

Definition types' $:=$ update types natIndex nat. 
Variable values: hlist (fun $x$ : Set $\Rightarrow x$ ) types'.

Now a bit of dependent pattern matching helps us rewrite getNat in a way that avoids any use of equality proofs.

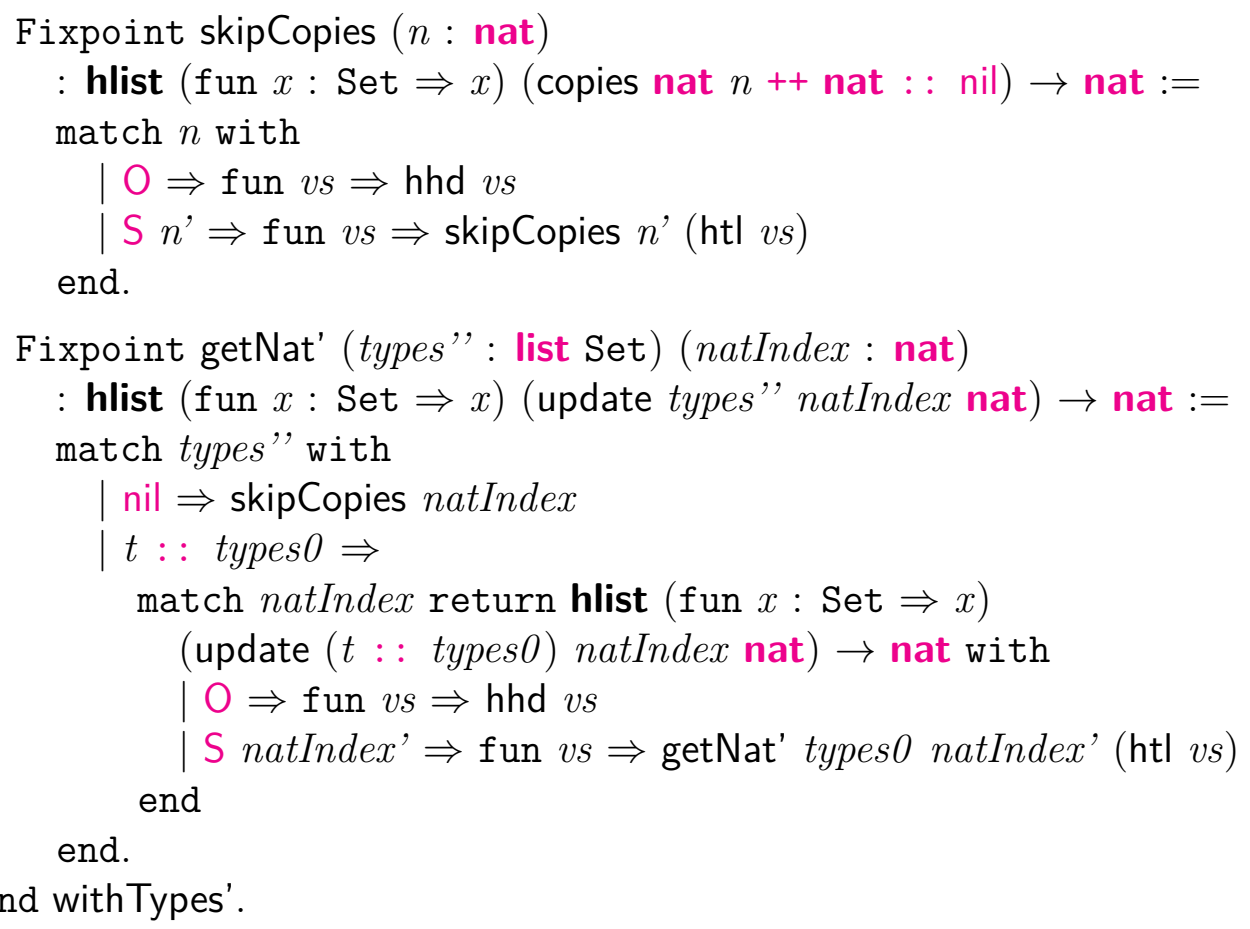

Now the surprise comes in how easy it is to use getNat'. While typing works by modification of a types list, we can choose parameters so that the modification has no effect.

Theorem getNat_is_reasonable : getNat' myTypes myNatIndex myValues $=3$.

reflexivity.

Qed.

The same parameters as before work without alteration, and we avoid use of axioms. 


\section{Part III}

\section{Proof Engineering}




\section{Chapter 13}

\section{Proof Search by Logic Programming}

The Curry-Howard correspondence tells us that proving is "just" programming, but the pragmatics of the two activities are very different. Generally we care about properties of a program besides its type, but the same is not true about proofs. Any proof of a theorem will do just as well. As a result, automated proof search is conceptually simpler than automated programming.

The paradigm of logic programming [21], as embodied in languages like Prolog [42], is a good match for proof search in higher-order logic. This chapter introduces the details, attempting to avoid any dependence on past logic programming experience.

\subsection{Introducing Logic Programming}

Recall the definition of addition from the standard library.

Print plus.

plus $=$

fix plus $(n m:$ nat $):$ nat $:=$ match $n$ with

$$
\begin{aligned}
& \mid 0 \Rightarrow m \\
& \mid \mathrm{S} p \Rightarrow \mathrm{S} \text { (plus } p m \text { ) } \\
& \text { end }
\end{aligned}
$$

This is a recursive definition, in the style of functional programming. We might also follow the style of logic programming, which corresponds to the inductive relations we have defined in previous chapters.

Inductive plusR : nat $\rightarrow$ nat $\rightarrow$ nat $\rightarrow$ Prop $:=$

| PlusO : $\forall m$, plusR $\bigcirc m m$

| PlusS : $\forall n m r$, plusR $n m r$

$\rightarrow$ plusR $(\mathrm{S} n) m(\mathrm{~S} r)$.

Intuitively, a fact plusR $n m r$ only holds when plus $n m=r$. It is not hard to prove this correspondence formally. 


\section{Hint Constructors plusR.}

Theorem plus_plusR : $\forall n m$,

plusR $n m(n+m)$.

induction $n$; crush.

Qed.

Theorem plusR_plus : $\forall n m r$,

plusR $n m r$

$\rightarrow r=n+m$.

induction 1 ; crush.

Qed.

With the functional definition of plus, simple equalities about arithmetic follow by computation.

Example four_plus_three : $4+3=7$.

reflexivity.

Qed.

Print four_plus_three.

four_plus_three $=$ eq_refl

With the relational definition, the same equalities take more steps to prove, but the process is completely mechanical. For example, consider this simple-minded manual proof search strategy. The steps with error messages shown afterward will be omitted from the final script.

Example four_plus_three' : plusR 437.

apply PlusO.

Error: Impossible to unify "plusR 0 ?24 ?24" with "plusR 43 7".

apply PlusS.

apply PlusO.

Error: Impossible to unify "plusR 0 ?25 ?25" with "plusR 33 6".

apply PlusS.

apply PlusO.

Error: Impossible to unify "plusR 0 ?26 ?26" with "plusR 23 5". apply PlusS.

apply PlusO.

Error: Impossible to unify "plusR 0 ?27 ?27" with "plusR 134 ". 


\section{apply PlusS.}

apply PlusO.

At this point the proof is completed. It is no doubt clear that a simple procedure could find all proofs of this kind for us. We are just exploring all possible proof trees, built from the two candidate steps apply PlusO and apply PlusS. The built-in tactic auto follows exactly this strategy, since above we used Hint Constructors to register the two candidate proof steps as hints.

Restart.

auto.

Qed.

Print four_plus_three'.

four_plus_three' $=$ PlusS $($ PlusS $($ PlusS $($ PlusS $($ PlusO 3)) ))

Let us try the same approach on a slightly more complex goal.

Example five_plus_three : plusR 538 .

auto.

This time, auto is not enough to make any progress. Since even a single candidate step may lead to an infinite space of possible proof trees, auto is parameterized on the maximum depth of trees to consider. The default depth is 5 , and it turns out that we need depth 6 to prove the goal.

auto 6 .

Sometimes it is useful to see a description of the proof tree that auto finds, with the info tactical. (This tactical is not available in Coq 8.4 as of this writing, but I hope it reappears soon. The special case info_auto tactic is provided as a chatty replacement for auto.)

Restart.

info auto 6.

$==$ apply PlusS; apply PlusS; apply PlusS; apply PlusS; apply PlusS; apply PlusO.

Qed.

The two key components of logic programming are backtracking and unification. To see these techniques in action, consider this further silly example. Here our candidate proof steps will be reflexivity and quantifier instantiation.

Example seven_minus_three : $\exists x, x+3=7$.

For explanatory purposes, let us simulate a user with minimal understanding of arithmetic. We start by choosing an instantiation for the quantifier. Recall that ex_intro is the constructor for existentially quantified formulas.

apply ex_intro with 0 .

reflexivity.

Error: Impossible to unify "7" with "0 + 3". 
This seems to be a dead end. Let us backtrack to the point where we ran apply and make a better alternate choice.

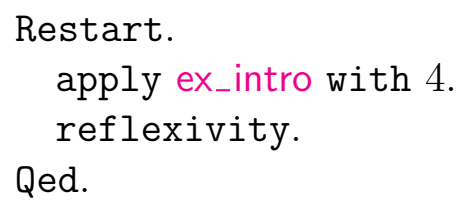

The above was a fairly tame example of backtracking. In general, any node in an underconstruction proof tree may be the destination of backtracking an arbitrarily large number of times, as different candidate proof steps are found not to lead to full proof trees, within the depth bound passed to auto.

Next we demonstrate unification, which will be easier when we switch to the relational formulation of addition.

Example seven_minus_three' : $\exists x$, plusR $x 37$.

We could attempt to guess the quantifier instantiation manually as before, but here there is no need. Instead of apply, we use eapply, which proceeds with placeholder unification variables standing in for those parameters we wish to postpone guessing.

eapply ex_intro.

1 subgoal

\section{plusR ?70 37}

Now we can finish the proof with the right applications of plusR's constructors. Note that new unification variables are being generated to stand for new unknowns.

apply PlusS.

\section{$====================$ \\ plusR ?71 36 \\ apply PlusS. apply PlusS. apply PlusS.}

\section{plusR ?74 33}

apply PlusO.

The auto tactic will not perform these sorts of steps that introduce unification variables, but the eauto tactic will. It is helpful to work with two separate tactics, because proof search in the eauto style can uncover many more potential proof trees and hence take much longer to run.

Restart.

info eauto 6 . 
$==$ eapply ex_intro; apply PlusS; apply PlusS;

apply PlusS; apply PlusS; apply PlusO.

Qed.

This proof gives us our first example where logic programming simplifies proof search compared to functional programming. In general, functional programs are only meant to be run in a single direction; a function has disjoint sets of inputs and outputs. In the last example, we effectively ran a logic program backwards, deducing an input that gives rise to a certain output. The same works for deducing an unknown value of the other input.

Example seven_minus_four' : $\exists x$, plusR $4 x 7$.

eauto 6.

Qed.

By proving the right auxiliary facts, we can reason about specific functional programs in the same way as we did above for a logic program. Let us prove that the constructors of plusR have natural interpretations as lemmas about plus. We can find the first such lemma already proved in the standard library, using the SearchRewrite command to find a library function proving an equality whose lefthand or righthand side matches a pattern with wildcards.

SearchRewrite $\left(\mathrm{O}+_{-}\right)$.

plus_O_n: $\forall n:$ nat, $0+n=n$

The command Hint Immediate asks auto and eauto to consider this lemma as a candidate step for any leaf of a proof tree.

Hint Immediate plus_O_n.

The counterpart to PlusS we will prove ourselves.

Lemma plusS : $\forall n m r$,

$n+m=r$

$\rightarrow \mathrm{S} n+m=\mathrm{S} r$.

crush.

Qed.

The command Hint Resolve adds a new candidate proof step, to be attempted at any level of a proof tree, not just at leaves.

Hint Resolve plusS.

Now that we have registered the proper hints, we can replicate our previous examples with the normal, functional addition plus.

Example seven_minus_three" : $\exists x, x+3=7$.

eauto 6.

Qed.

Example seven_minus_four: $\exists x, 4+x=7$. 
eauto 6 .

Qed.

This new hint database is far from a complete decision procedure, as we see in a further example that eauto does not finish.

Example seven_minus_four_zero: $\exists x, 4+x+0=7$.

eauto 6 .

Abort.

A further lemma will be helpful.

Lemma plusO : $\forall n m$,

$n=m$

$\rightarrow n+0=m$.

crush.

Qed.

Hint Resolve plusO.

Note that, if we consider the inputs to plus as the inputs of a corresponding logic program, the new rule plusO introduces an ambiguity. For instance, a sum $0+0$ would match both of plus_O_n and plusO, depending on which operand we focus on. This ambiguity may increase the number of potential search trees, slowing proof search, but semantically it presents no problems, and in fact it leads to an automated proof of the present example.

Example seven_minus_four_zero: $\exists x, 4+x+0=7$.

eauto 7.

Qed.

Just how much damage can be done by adding hints that grow the space of possible proof trees? A classic gotcha comes from unrestricted use of transitivity, as embodied in this library theorem about equality:

Check eq_trans.

eq_trans

$: \forall(A:$ Type) $(x y z: A), x=y \rightarrow y=z \rightarrow x=z$

Hints are scoped over sections, so let us enter a section to contain the effects of an unfortunate hint choice.

Section slow.

Hint Resolve eq_trans.

The following fact is false, but that does not stop eauto from taking a very long time to search for proofs of it. We use the handy Time command to measure how long a proof step takes to run. None of the following steps make any progress.

Example zero_minus_one : $\exists x, 1+x=0$.

Time eauto 1 .

Finished transaction in $0 . \operatorname{secs}(0 . u, 0 . s)$ 
Time eauto 2.

Finished transaction in $0 . \operatorname{secs}(0 . u, 0 . s)$

Time eauto 3.

Finished transaction in $0 . \operatorname{secs}(0.008 \mathrm{u}, 0 . \mathrm{s})$

Time eauto 4.

Finished transaction in $0 . \operatorname{secs}(0.068005 \mathrm{u}, 0.004 \mathrm{~s})$

Time eauto 5 .

Finished transaction in 2 . secs $(1.92012 \mathrm{u}, 0.044003 \mathrm{~s})$

We see worrying exponential growth in running time, and the debug tactical helps us see where eauto is wasting its time, outputting a trace of every proof step that is attempted. The rule eq_trans applies at every node of a proof tree, and eauto tries all such positions.

debug eauto 3 .

1 depth=3

1.1 depth=2 eapply ex_intro

1.1 .1 depth=1 apply plusO

1.1.1.1 depth=0 eapply eq_trans

1.1.2 depth=1 eapply eq_trans

1.1.2.1 depth=1 apply plus_n_O

1.1.2.1.1 depth=0 apply plusO

1.1.2.1.2 depth=0 eapply eq_trans

1.1.2.2 depth=1 apply @eq_refl

1.1.2.2.1 depth=0 apply plusO

1.1.2.2.2 depth=0 eapply eq_trans

1.1.2.3 depth=1 apply eq_add_S ; trivial

1.1.2.3.1 depth=0 apply plusO

1.1.2.3.2 depth=0 eapply eq_trans

1.1.2.4 depth=1 apply eq_sym ; trivial

1.1.2.4.1 depth=0 eapply eq_trans

1.1.2.5 depth $=0$ apply plusO

1.1.2.6 depth $=0$ apply plusS

1.1.2.7 depth $=0$ apply f_equal $(A:=$ nat $)$

1.1.2.8 depth=0 apply f_equal2 $(A 1:=$ nat $)(A 2:=$ nat $)$

1.1.2.9 depth $=0$ eapply eq_trans

Abort.

End slow. 
Sometimes, though, transitivity is just what is needed to get a proof to go through automatically with eauto. For those cases, we can use named hint databases to segregate hints into different groups that may be called on as needed. Here we put eq_trans into the database slow.

Hint Resolve eq_trans: slow.

Example from_one_to_zero : $\exists x, 1+x=0$.

Time eauto.

Finished transaction in $0 . \operatorname{secs}(0.004 \mathrm{u}, 0 . \mathrm{s})$

This eauto fails to prove the goal, but at least it takes substantially less than the 2 seconds required above!

Abort.

One simple example from before runs in the same amount of time, avoiding pollution by transitivity.

Example seven_minus_three_again : $\exists x, x+3=7$.

Time eauto 6 .

Finished transaction in $0 . \operatorname{secs}(0.004001 \mathrm{u}, 0 . \mathrm{s})$

Qed.

When we do need transitivity, we ask for it explicitly.

Example needs_trans: $\forall x y, 1+x=y$

$\rightarrow y=2$

$\rightarrow \exists z, z+x=3$.

info eauto with slow.

$==$ intro $x$; intro $y$; intro $H$; intro $H 0$; simple eapply ex_intro;

apply plusS; simple eapply eq_trans.

exact $H$.

exact $H O$.

Qed.

The info trace shows that eq_trans was used in just the position where it is needed to complete the proof. We also see that auto and eauto always perform intro steps without counting them toward the bound on proof tree depth.

\subsection{Searching for Underconstrained Values}

Recall the definition of the list length function.

Print length.

length $=$ 


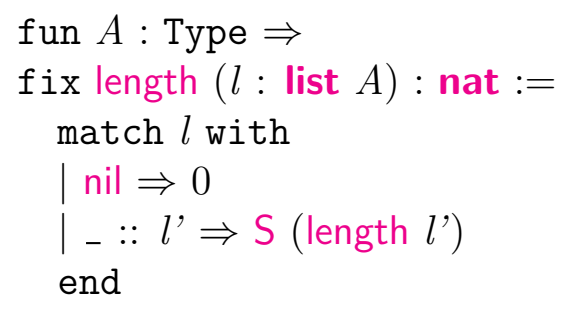

This function is easy to reason about in the forward direction, computing output from input.

Example length_1_2 : length $(1:: 2::$ nil $)=2$.

auto.

Qed.

Print length_1_2.

length_1_2 = eq_refl

As in the last section, we will prove some lemmas to recast length in logic programming style, to help us compute inputs from outputs.

Theorem length_O $: \forall A$, length (nil $(A:=A))=\mathrm{O}$.

crush.

Qed.

Theorem length_S : $\forall A(h: A) t n$, length $t=n$

$\rightarrow$ length $(h:: t)=\mathrm{S} n$.

crush.

Qed.

Hint Resolve length_O length_S.

Let us apply these hints to prove that a list nat of length 2 exists. (Here we register length_O with Hint Resolve instead of Hint Immediate merely as a convenience to use the same command as for length_S; Resolve and Immediate have the same meaning for a premise-free hint.)

Example length_is_2: $\exists l s$ : list nat, length $l s=2$.

eauto.

No more subgoals but non-instantiated existential variables:

Existential 1 = ?20249: [ |- nat]

Existential $2=? 20252$ : [ |- nat]

Coq complains that we finished the proof without determining the values of some unification variables created during proof search. The error message may seem a bit silly, since any value of type nat (for instance, 0) can be plugged in for either variable! However, for more complex types, finding their inhabitants may be as complex as theorem-proving in general. 
The Show Proof command shows exactly which proof term eauto has found, with the undetermined unification variables appearing explicitly where they are used.

Show Proof.

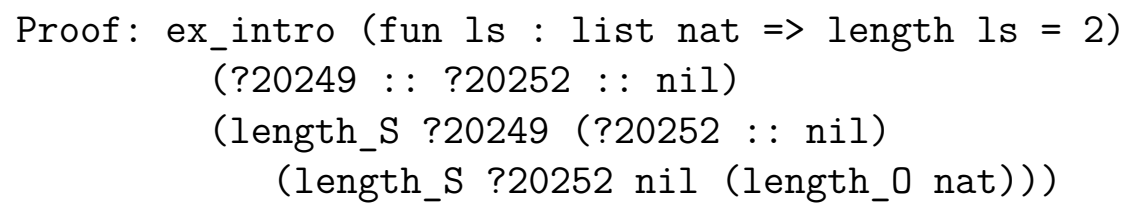

Abort.

We see that the two unification variables stand for the two elements of the list. Indeed, list length is independent of data values. Paradoxically, we can make the proof search process easier by constraining the list further, so that proof search naturally locates appropriate data elements by unification. The library predicate Forall will be helpful.

\section{Print Forall.}

Inductive Forall ( $A:$ Type) $(P: A \rightarrow$ Prop) $:$ list $A \rightarrow$ Prop $:=$ Forall_nil : Forall $P$ nil

| Forall_cons : $\forall(x: A)(l:$ list $A)$, $P x \rightarrow$ Forall $P l \rightarrow$ Forall $P(x:: l)$

Example length_is_2 : $\exists l s:$ list nat, length $l s=2$

$\wedge$ Forall (fun $n \Rightarrow n \geq 1$ ) ls.

eauto 9 .

Qed.

We can see which list eauto found by printing the proof term.

Print length_is_2.

length_is_2 $=$

ex_intro

(fun $l s:$ list nat $\Rightarrow$ length $l s=2 \wedge$ Forall (fun $n:$ nat $\Rightarrow n \geq 1) l s$ )

$(1:: 1::$ nil $)$

(conj (length_S 1 (1 :: nil) (length_S 1 nil (length_O nat)))

(Forall_cons 1 (le_n 1$)$

(Forall_cons 1 (le_n 1) (Forall_nil (fun $n$ : nat $\Rightarrow n \geq 1)))$ ))

Let us try one more, fancier example. First, we use a standard higher-order function to define a function for summing all data elements of a list.

Definition sum := fold_right plus $O$.

Another basic lemma will be helpful to guide proof search.

Lemma plusO' : $\forall n m$,

$n=m$

$\rightarrow 0+n=m$. 
crush.

Qed.

Hint Resolve plusO'.

Finally, we meet Hint Extern, the command to register a custom hint. That is, we provide a pattern to match against goals during proof search. Whenever the pattern matches, a tactic (given to the right of an arrow $\Rightarrow$ ) is attempted. Below, the number 1 gives a priority for this step. Lower priorities are tried before higher priorities, which can have a significant effect on proof search time.

Hint Extern 1 (sum _ ${ }_{-}$) $\Rightarrow$ simpl.

Now we can find a length-2 list whose sum is 0 .

Example length_and_sum : $\exists l s$ : list nat, length $l s=2$

$\wedge$ sum $l s=0$.

eauto 7 .

Qed.

Printing the proof term shows the unsurprising list that is found. Here is an example where it is less obvious which list will be used. Can you guess which list eauto will choose?

Example length_and_sum' : $\exists l s$ : list nat, length $l s=5$

$\wedge$ sum $l s=42$.

eauto 15 .

Qed.

We will give away part of the answer and say that the above list is less interesting than we would like, because it contains too many zeroes. A further constraint forces a different solution for a smaller instance of the problem.

Example length_and_sum" : $\exists l s$ : list nat, length $l s=2$

$\wedge$ sum $l s=3$

$\wedge$ Forall (fun $n \Rightarrow n \neq 0$ ) ls.

eauto 11.

Qed.

We could continue through exercises of this kind, but even more interesting than finding lists automatically is finding programs automatically.

\subsection{Synthesizing Programs}

Here is a simple syntax type for arithmetic expressions, similar to those we have used several times before in the book. In this case, we allow expressions to mention exactly one distinguished variable.

Inductive exp : Set :=

| Const : nat $\rightarrow$ exp 


$$
\begin{aligned}
& \text { Var : } \exp \\
& \mid \text { Plus }: \exp \rightarrow \exp \rightarrow \exp .
\end{aligned}
$$

An inductive relation specifies the semantics of an expression, relating a variable value and an expression to the expression value.

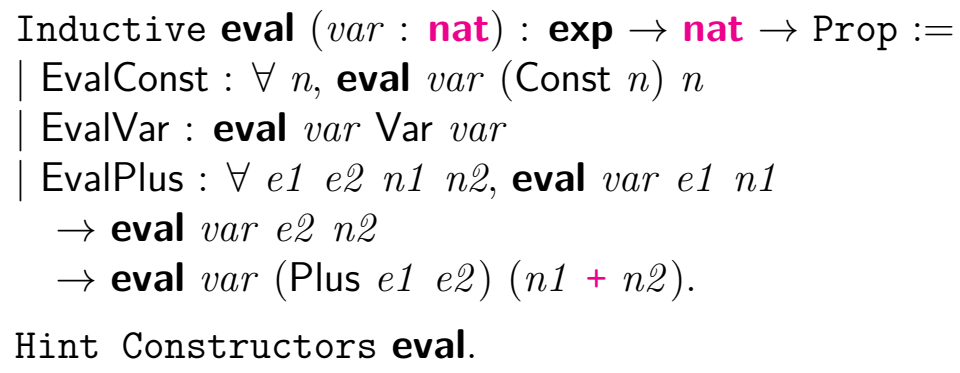

Hint Constructors eval.

We can use auto to execute the semantics for specific expressions.

Example eval1 : $\forall \operatorname{var}$, eval var (Plus Var (Plus (Const 8) Var)) $(\operatorname{var}+(8+\operatorname{var}))$ auto.

Qed.

Unfortunately, just the constructors of eval are not enough to prove theorems like the following, which depends on an arithmetic identity.

Example eval1' : $\forall$ var, eval var (Plus Var (Plus (Const 8) Var) $(2 \times \operatorname{var}+8)$. eauto.

Abort.

To help prove eval1', we prove an alternate version of EvalPlus that inserts an extra equality premise. This sort of staging is helpful to get around limitations of eauto's unification: EvalPlus as a direct hint will only match goals whose results are already expressed as additions, rather than as constants. With the alternate version below, to prove the first two premises, eauto is given free reign in deciding the values of $n 1$ and $n 2$, while the third premise can then be proved by reflexivity, no matter how each of its sides is decomposed as a tree of additions.

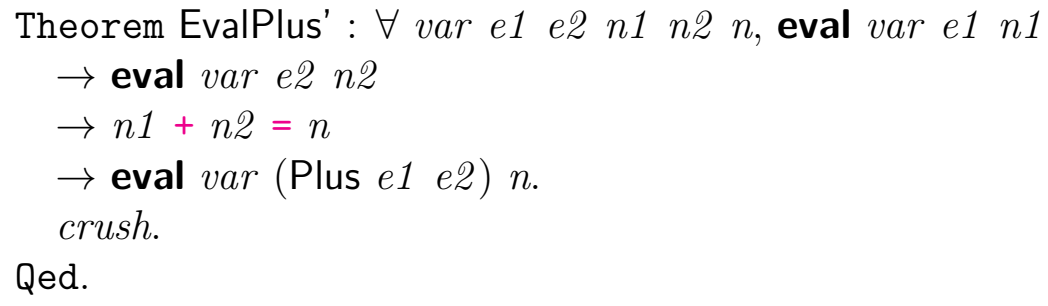

Further, we instruct eauto to apply omega, a standard tactic that provides a complete decision procedure for quantifier-free linear arithmetic. Via Hint Extern, we ask for use of omega on any equality goal. The abstract tactical generates a new lemma for every such successful proof, so that, in the final proof term, the lemma may be referenced in place of dropping in the full proof of the arithmetic equality. 
Hint Extern $1\left(_{-}=_{-}\right) \Rightarrow$ abstract omega.

Now we can return to eval1' and prove it automatically.

Example eval1' : $\forall$ var, eval var (Plus Var (Plus (Const 8) Var) $(2 \times \operatorname{var}+8)$.

eauto.

Qed.

Print eval1'.

eval1' $=$

fun var: nat $\Rightarrow$

EvalPlus' (EvalVar var) (EvalPlus (EvalConst var 8) (EvalVar var))

(eval1'_subproof var)

$: \forall$ var : nat,

eval var (Plus Var (Plus (Const 8) Var)) $(2 \times \operatorname{var}+8)$

The lemma eval1'_subproof was generated by abstract omega.

Now we are ready to take advantage of logic programming's flexibility by searching for a program (arithmetic expression) that always evaluates to a particular symbolic value.

Example synthesize1: $\exists e, \forall$ var, eval var $e(\operatorname{var}+7)$.

eauto.

Qed.

Print synthesize1.

synthesize $1=$

ex_intro (fun $e$ : $\exp \Rightarrow \forall$ var : nat, eval var $e(v a r+7)$ )

(Plus Var (Const 7))

(fun var : nat $\Rightarrow$ EvalPlus (EvalVar var) (EvalConst var 7 ))

Here are two more examples showing off our program synthesis abilities.

Example synthesize2: $\exists e, \forall$ var, eval var $e(2 \times \operatorname{var}+8)$.

eauto.

Qed.

Example synthesize3 : $\exists e, \forall$ var, eval var $e(3 \times$ var +42$)$. eauto.

Qed.

These examples show linear expressions over the variable var. Any such expression is equivalent to $k \times \operatorname{var}+n$ for some $k$ and $n$. It is probably not so surprising that we can prove that any expression's semantics is equivalent to some such linear expression, but it is tedious to prove such a fact manually. To finish this section, we will use eauto to complete the proof, finding $k$ and $n$ values automatically.

We prove a series of lemmas and add them as hints. We have alternate eval constructor lemmas and some facts about arithmetic.

Theorem EvalConst' : $\forall$ var $n m, n=m$ 
$\rightarrow$ eval $\operatorname{var}($ Const $n) m$.

crush.

Qed.

Hint Resolve EvalConst'.

Theorem zero_times : $\forall n m r$,

$r=m$

$\rightarrow r=0 \times n+m$.

crush.

Qed.

Hint Resolve zero_times.

Theorem EvalVar' : $\forall$ var $n$,

var $=n$

$\rightarrow$ eval var Var $n$.

crush.

Qed.

Hint Resolve EvalVar'.

Theorem plus_0 : $\forall n r$,

$r=n$

$\rightarrow r=n+0$.

crush.

Qed.

Theorem times_1 : $\forall n, n=1 \times n$.

crush.

Qed.

Hint Resolve plus_0 times_1.

We finish with one more arithmetic lemma that is particularly specialized to this theorem. This fact happens to follow by the axioms of the semiring algebraic structure, so, since the naturals form a semiring, we can use the built-in tactic ring.

Require Import Arith Ring.

Theorem combine : $\forall x k 1 k 2 n 1$ n2,

$(k 1 \times x+n 1)+(k 2 \times x+n 2)=(k 1+k 2) \times x+(n 1+n 2)$.

intros; ring.

Qed.

Hint Resolve combine.

Our choice of hints is cheating, to an extent, by telegraphing the procedure for choosing values of $k$ and $n$. Nonetheless, with these lemmas in place, we achieve an automated proof without explicitly orchestrating the lemmas' composition.

Theorem linear : $\forall e, \exists k, \exists n$, 
$\forall \operatorname{var}$, eval $\operatorname{var} e(k \times \operatorname{var}+n)$.

induction $e$; crush; eauto.

Qed.

By printing the proof term, it is possible to see the procedure that is used to choose the constants for each input term.

\subsection{More on auto Hints}

Let us stop at this point and take stock of the possibilities for auto and eauto hints. Hints are contained within hint databases, which we have seen extended in many examples so far. When no hint database is specified, a default database is used. Hints in the default database are always used by auto or eauto. The chance to extend hint databases imperatively is important, because, in Ltac programming, we cannot create "global variables" whose values can be extended seamlessly by different modules in different source files. We have seen the advantages of hints so far, where crush can be defined once and for all, while still automatically applying the hints we add throughout developments. In fact, crush is defined in terms of auto, which explains how we achieve this extensibility. Other user-defined tactics can take similar advantage of auto and eauto.

The basic hints for auto and eauto are: Hint Immediate lemma, asking to try solving a goal immediately by applying a lemma and discharging any hypotheses with a single proof step each; Resolve lemma, which does the same but may add new premises that are themselves to be subjects of nested proof search; Constructors type, which acts like Resolve applied to every constructor of an inductive type; and Unfold ident, which tries unfolding $i d e n t$ when it appears at the head of a proof goal. Each of these Hint commands may be used with a suffix, as in Hint Resolve lemma : my_db, to add the hint only to the specified database, so that it would only be used by, for instance, auto with $m y_{-} d b$. An additional argument to auto specifies the maximum depth of proof trees to search in depth-first order, as in auto 8 or auto 8 with $m y_{-} d b$. The default depth is 5 .

All of these Hint commands can be expressed with a more primitive hint kind, Extern. A few more examples of Hint Extern should illustrate more of the possibilities.

Theorem bool_neq : true $\neq$ false.

auto.

A call to crush would have discharged this goal, but the default hint database for auto contains no hint that applies.

Abort.

It is hard to come up with a bool-specific hint that is not just a restatement of the theorem we mean to prove. Luckily, a simpler form suffices, by appealing to the built-in tactic congruence, a complete procedure for the theory of equality, uninterpreted functions, and datatype constructors.

Hint Extern $1\left({ }_{-} \neq_{-}\right) \Rightarrow$ congruence. 
Theorem bool_neq : true $\neq$ false.

auto.

Qed.

A Hint Extern may be implemented with the full Ltac language. This example shows a case where a hint uses a match.

Section forall_and.

Variable $A$ : Set.

Variables $P Q: A \rightarrow$ Prop.

Hypothesis both: $\forall x, P x \wedge Q x$.

Theorem forall_and : $\forall z, P z$.

crush.

The crush invocation makes no progress beyond what intros would have accomplished. An auto invocation will not apply the hypothesis both to prove the goal, because the conclusion of both does not unify with the conclusion of the goal. However, we can teach auto to handle this kind of goal.

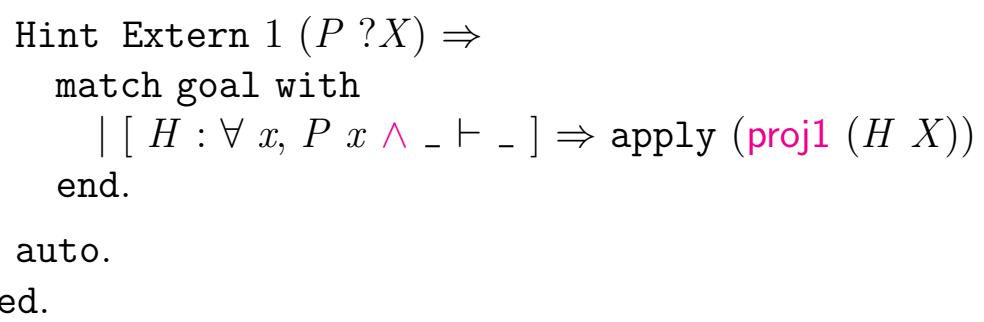

We see that an Extern pattern may bind unification variables that we use in the associated tactic. The function proj1 is from the standard library, for extracting a proof of $U$ from a proof of $U \wedge V$.

End forall_and.

After our success on this example, we might get more ambitious and seek to generalize the hint to all possible predicates $P$.

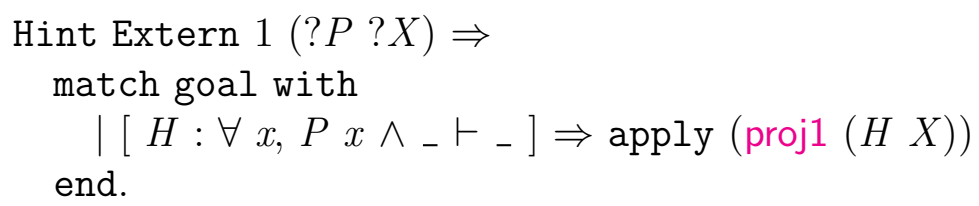

\section{User error: Bound head variable}

Coq's auto hint databases work as tables mapping head symbols to lists of tactics to try. Because of this, the constant head of an Extern pattern must be determinable statically. In our first Extern hint, the head symbol was not, since $x \neq y$ desugars to not (eq $x y$ ); and, in the second example, the head symbol was $P$.

Fortunately, a more basic form of Hint Extern also applies. We may simply leave out the pattern to the left of the $\Rightarrow$, incorporating the corresponding logic into the Ltac script. 


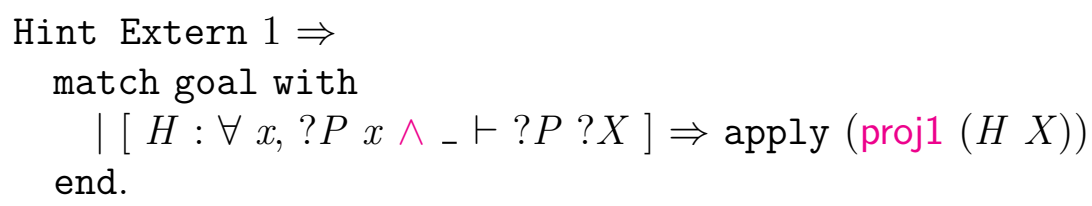

Be forewarned that a Hint Extern of this kind will be applied at every node of a proof tree, so an expensive Ltac script may slow proof search significantly.

\subsection{Rewrite Hints}

Another dimension of extensibility with hints is rewriting with quantified equalities. We have used the associated command Hint Rewrite in many examples so far. The crush tactic uses these hints by calling the built-in tactic autorewrite. Our rewrite hints have taken the form Hint Rewrite lemma, which by default adds them to the default hint database core; but alternate hint databases may also be specified just as with, e.g., Hint Resolve.

The next example shows a direct use of autorewrite. Note that, while Hint Rewrite uses a default database, autorewrite requires that a database be named.

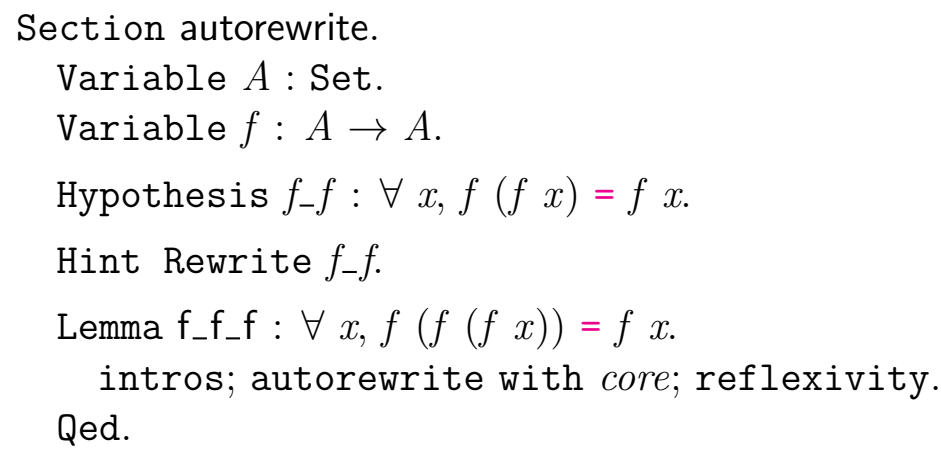

There are a few ways in which autorewrite can lead to trouble when insufficient care is taken in choosing hints. First, the set of hints may define a nonterminating rewrite system, in which case invocations to autorewrite may not terminate. Second, we may add hints that "lead autorewrite down the wrong path." For instance:

Section garden_path.

Variable $g: A \rightarrow A$.

Hypothesis $f_{-} g: \forall x, f x=g x$.

Hint Rewrite $f_{-} g$.

Lemma $\mathrm{f}_{-} \mathrm{f}_{-} \mathrm{f}^{\prime}: \forall x, f(f(f x))=f x$. intros; autorewrite with core.

$\begin{aligned}= & == \\ & g(g(g x))= \\ & =1 x\end{aligned}$

Abort. 
Our new hint was used to rewrite the goal into a form where the old hint could no longer be applied. This "non-monotonicity" of rewrite hints contrasts with the situation for auto, where new hints may slow down proof search but can never "break" old proofs. The key difference is that auto either solves a goal or makes no changes to it, while autorewrite may change goals without solving them. The situation for eauto is slightly more complicated, as changes to hint databases may change the proof found for a particular goal, and that proof may influence the settings of unification variables that appear elsewhere in the proof state.

Reset garden_path.

The autorewrite tactic also works with quantified equalities that include additional premises, but we must be careful to avoid similar incorrect rewritings.

Section garden_path.

Variable $P: A \rightarrow$ Prop.

Variable $g: A \rightarrow A$.

Hypothesis $f_{-} g: \forall x, P x \rightarrow f x=g x$.

Hint Rewrite $f_{-} g$.

Lemma $\mathbf{f}_{-} \mathrm{f}_{-} \mathrm{f}^{\prime}: \forall x, f(f(f x))=f x$.

intros; autorewrite with core.

$$
\begin{aligned}
& ==\overline{=}=\mathrm{=}=\mathrm{=}=\mathrm{=}=\mathrm{=}=\mathrm{=}=\mathrm{=}=\mathrm{=}=\mathrm{=}=\mathrm{=} \\
& g(g(g x))=g x
\end{aligned}
$$

subgoal 2 is:

$P x$

subgoal 3 is:

$P(f x)$

subgoal 4 is:

$P(f x)$

\section{Abort.}

The inappropriate rule fired the same three times as before, even though we know we will not be able to prove the premises.

\section{Reset garden_path.}

Our final, successful, attempt uses an extra argument to Hint Rewrite that specifies a tactic to apply to generated premises. Such a hint is only used when the tactic succeeds for all premises, possibly leaving further subgoals for some premises.

Section garden_path.

Variable $P: A \rightarrow$ Prop.

Variable $g: A \rightarrow A$.

Hypothesis $f_{-} g: \forall x, P x \rightarrow f x=g x$.

Hint Rewrite $f_{-} g$ using assumption. 
Lemma f_f_f' : $\forall x, f(f(f x))=f x$.

intros; autorewrite with core; reflexivity.

Qed.

We may still use autorewrite to apply $f_{-} g$ when the generated premise is among our assumptions.

Lemma f_f_f_f $_{-}: \forall x, P x \rightarrow f(f x)=g x$. intros; autorewrite with core; reflexivity.

Qed.

End garden_path.

It can also be useful to apply the autorewrite with $d b$ in $*$ form, which does rewriting in hypotheses, as well as in the conclusion.

Lemma in_star : $\forall x y, f(f(f(f x)))=f(f y)$

$\rightarrow f x=f(f(f y))$.

intros; autorewrite with core in *; assumption.

Qed.

End autorewrite.

Many proofs can be automated in pleasantly modular ways with deft combinations of auto and autorewrite. 


\section{Chapter 14}

\section{Proof Search in Ltac}

We have seen many examples of proof automation so far, some with tantalizing code snippets from Ltac, Coq's domain-specific language for proof search procedures. This chapter aims to give a bottom-up presentation of the features of Ltac, focusing in particular on the Ltac match construct, which supports a novel approach to backtracking search. First, though, we will run through some useful automation tactics that are built into Coq. They are described in detail in the manual, so we only outline what is possible.

\subsection{Some Built-In Automation Tactics}

A number of tactics are called repeatedly by crush. The intuition tactic simplifies propositional structure of goals. The congruence tactic applies the rules of equality and congruence closure, plus properties of constructors of inductive types. The omega tactic provides

a complete decision procedure for a theory that is called quantifier-free linear arithmetic or Presburger arithmetic, depending on whom you ask. That is, omega proves any goal that follows from looking only at parts of that goal that can be interpreted as propositional formulas whose atomic formulas are basic comparison operations on natural numbers or integers, with operands built from constants, variables, addition, and subtraction (with multiplication by a constant available as a shorthand for addition or subtraction).

The ring tactic solves goals by appealing to the axioms of rings or semi-rings (as in algebra), depending on the type involved. Coq developments may declare new types to be parts of rings and semi-rings by proving the associated axioms. There is a similar tactic field for simplifying values in fields by conversion to fractions over rings. Both ring and field can only solve goals that are equalities. The fourier tactic uses Fourier's method to prove inequalities over real numbers, which are axiomatized in the Coq standard library.

The setoid facility makes it possible to register new equivalence relations to be understood by tactics like rewrite. For instance, Prop is registered as a setoid with the equivalence relation "if and only if." The ability to register new setoids can be very useful in proofs of a kind common in math, where all reasoning is done after "modding out by a relation."

There are several other built-in "black box" automation tactics, which one can learn 
about by perusing the Coq manual. The real promise of Coq, though, is in the coding of problem-specific tactics with Ltac.

\subsection{Ltac Programming Basics}

We have already seen many examples of Ltac programs. In the rest of this chapter, we attempt to give a thorough introduction to the important features and design patterns.

One common use for match tactics is identification of subjects for case analysis, as we see in this tactic definition.

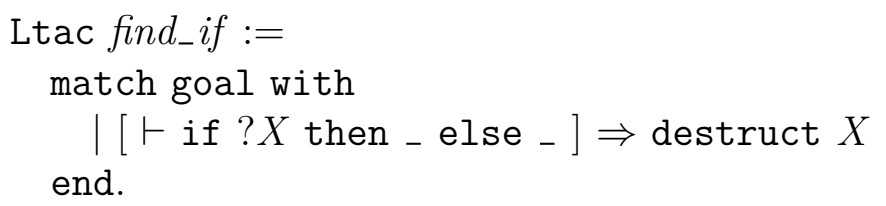

The tactic checks if the conclusion is an if, destructing the test expression if so. Certain classes of theorem are trivial to prove automatically with such a tactic.

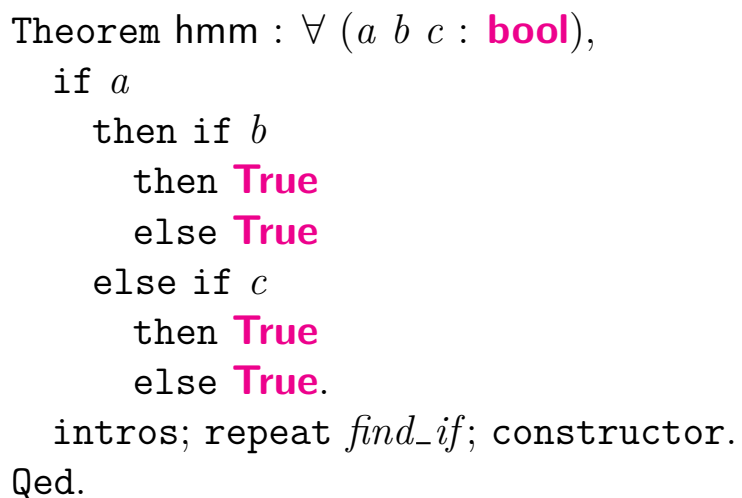

The repeat that we use here is called a tactical, or tactic combinator. The behavior of repeat $t$ is to loop through running $t$, running $t$ on all generated subgoals, running $t$ on their generated subgoals, and so on. When $t$ fails at any point in this search tree, that particular subgoal is left to be handled by later tactics. Thus, it is important never to use repeat with a tactic that always succeeds.

Another very useful Ltac building block is context patterns.

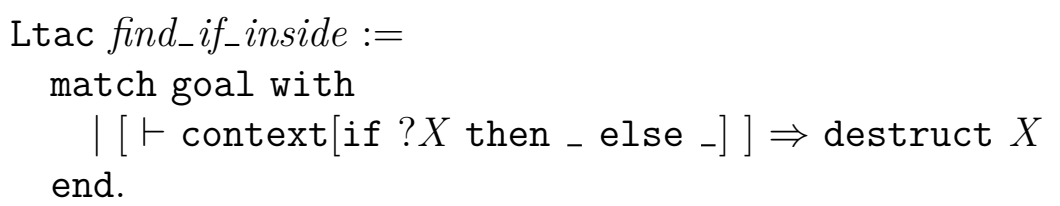

The behavior of this tactic is to find any subterm of the conclusion that is an if and then destruct the test expression. This version subsumes find_if.

Theorem hmm' : $\forall\left(\begin{array}{lll}a & b & c\end{array}\right.$ : bool),

if $a$

then if $b$ 


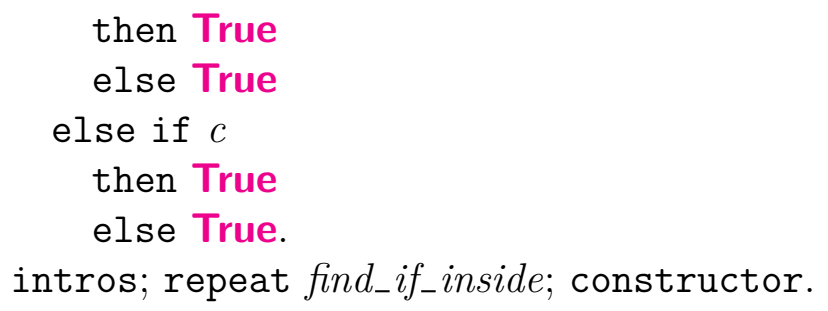

We can also use find_if_inside to prove goals that find_if does not simplify sufficiently. Theorem hmm2 : $\forall(a b:$ bool $)$,

(if $a$ then 42 else 42 ) = (if $b$ then 42 else 42 ).

intros; repeat find_if_inside; reflexivity.

Qed.

Many decision procedures can be coded in Ltac via "repeat match loops." For instance, we can implement a subset of the functionality of tauto.

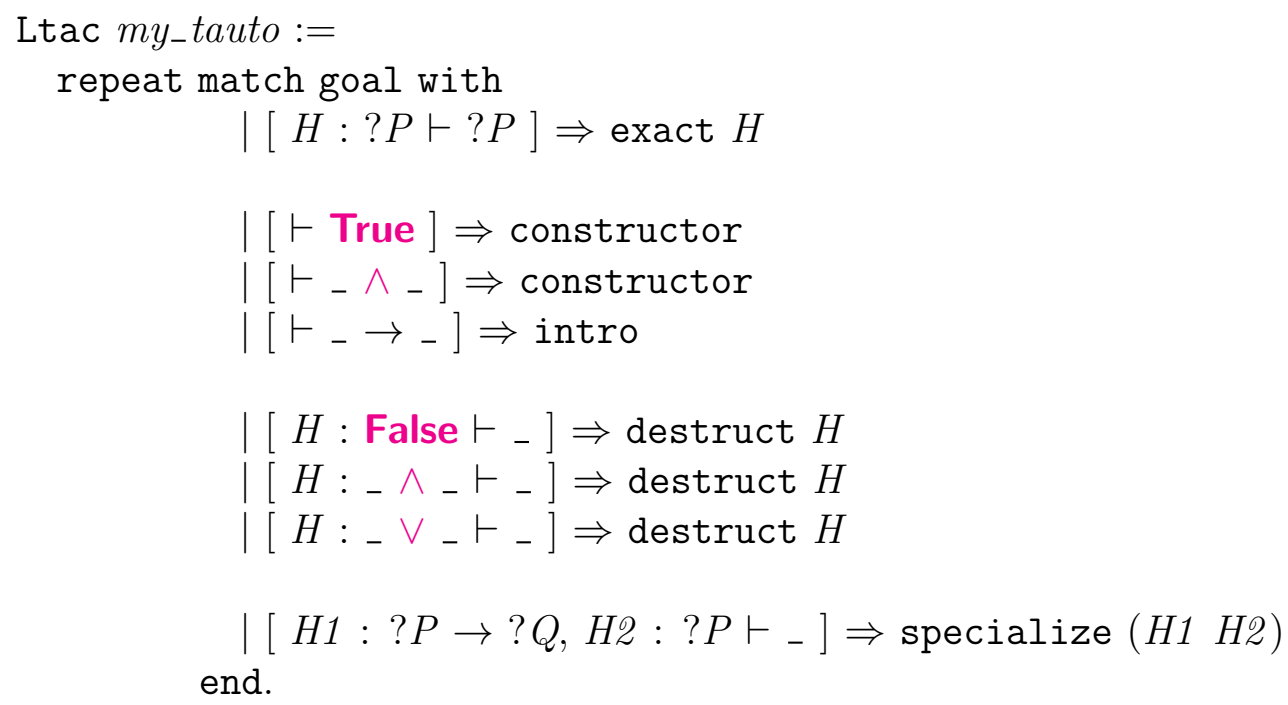

Since match patterns can share unification variables between hypothesis and conclusion patterns, it is easy to figure out when the conclusion matches a hypothesis. The exact tactic solves a goal completely when given a proof term of the proper type.

It is also trivial to implement the introduction rules (in the sense of natural deduction [37]) for a few of the connectives. Implementing elimination rules is only a little more work, since we must give a name for a hypothesis to destruct.

The last rule implements modus ponens, using a tactic specialize which will replace a hypothesis with a version that is specialized to a provided set of arguments (for quantified variables or local hypotheses from implications). By convention, when the argument to specialize is an application of a hypothesis $H$ to a set of arguments, the result of the specialization replaces $H$. For other terms, the outcome is the same as with generalize.

Section propositional.

Variables $P Q R$ : Prop. 
Theorem propositional : $(P \vee Q \vee$ False $) \wedge(P \rightarrow Q) \rightarrow$ True $\wedge Q$.

my_tauto.

Qed.

End propositional.

It was relatively easy to implement modus ponens, because we do not lose information by clearing every implication that we use. If we want to implement a similarly complete procedure for quantifier instantiation, we need a way to ensure that a particular proposition is not already included among our hypotheses. To do that effectively, we first need to learn a bit more about the semantics of match.

It is tempting to assume that match works like it does in ML. In fact, there are a few critical differences in its behavior. One is that we may include arbitrary expressions in patterns, instead of being restricted to variables and constructors. Another is that the same variable may appear multiple times, inducing an implicit equality constraint.

There is a related pair of two other differences that are much more important than the others. The match construct has a backtracking semantics for failure. In ML, pattern matching works by finding the first pattern to match and then executing its body. If the body raises an exception, then the overall match raises the same exception. In Coq, failures in case bodies instead trigger continued search through the list of cases.

For instance, this (unnecessarily verbose) proof script works:

Theorem $\mathrm{m} 1$ : True.

match goal with

$\left.\mid \vdash_{-}\right] \Rightarrow$ intro

| $[\vdash$ True $] \Rightarrow$ constructor

end.

Qed.

The first case matches trivially, but its body tactic fails, since the conclusion does not begin with a quantifier or implication. In a similar ML match, the whole pattern-match would fail. In Coq, we backtrack and try the next pattern, which also matches. Its body tactic succeeds, so the overall tactic succeeds as well.

The example shows how failure can move to a different pattern within a match. Failure can also trigger an attempt to find a different way of matching a single pattern. Consider another example:

Theorem m2: $\forall P Q R:$ Prop, $P \rightarrow Q \rightarrow R \rightarrow Q$.

intros; match goal with

| [H: $\left.\vdash_{-}\right] \Rightarrow$ idtac $H$

end.

Coq prints "H1". By applying idtac with an argument, a convenient debugging tool for "leaking information out of matches," we see that this match first tries binding $H$ to $H 1$, which cannot be used to prove $Q$. Nonetheless, the following variation on the tactic succeeds at proving the goal:

match goal with 


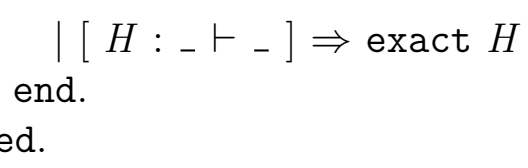

The tactic first unifies $H$ with $H 1$, as before, but exact $H$ fails in that case, so the tactic engine searches for more possible values of $H$. Eventually, it arrives at the correct value, so that exact $H$ and the overall tactic succeed.

Now we are equipped to implement a tactic for checking that a proposition is not among our hypotheses:

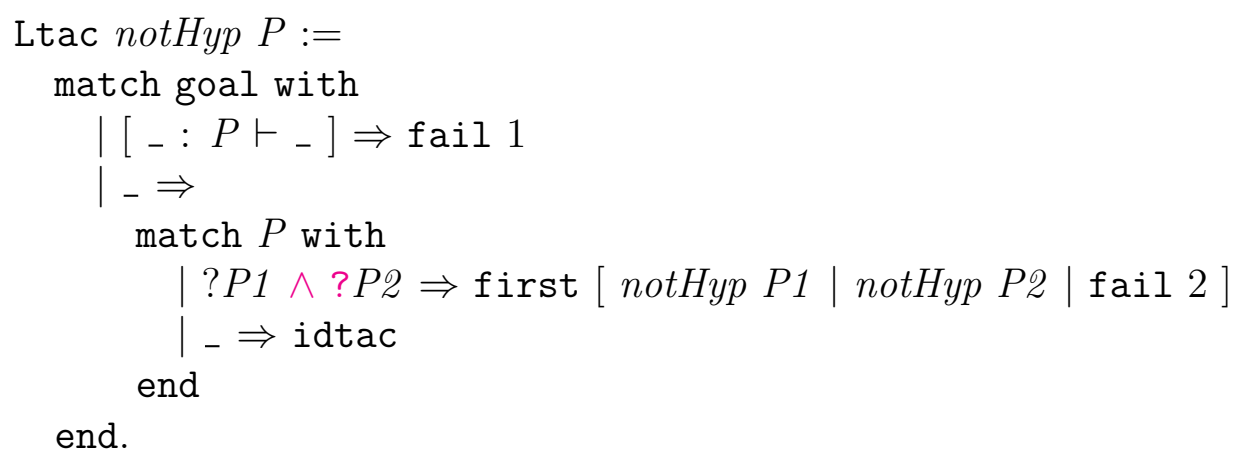

We use the equality checking that is built into pattern-matching to see if there is a hypothesis that matches the proposition exactly. If so, we use the fail tactic. Without arguments, fail signals normal tactic failure, as you might expect. When fail is passed an argument $n, n$ is used to count outwards through the enclosing cases of backtracking search. In this case, fail 1 says "fail not just in this pattern-matching branch, but for the whole match." The second case will never be tried when the fail 1 is reached.

This second case, used when $P$ matches no hypothesis, checks if $P$ is a conjunction. Other simplifications may have split conjunctions into their component formulas, so we need to check that at least one of those components is also not represented. To achieve this, we apply the first tactical, which takes a list of tactics and continues down the list until one of them does not fail. The fail 2 at the end says to fail both the first and the match wrapped around it.

The body of the ?P1 $\wedge$ ?P2 case guarantees that, if it is reached, we either succeed completely or fail completely. Thus, if we reach the wildcard case, $P$ is not a conjunction. We use idtac, a tactic that would be silly to apply on its own, since its effect is to succeed at doing nothing. Nonetheless, idtac is a useful placeholder for cases like what we see here.

With the non-presence check implemented, it is easy to build a tactic that takes as input a proof term and adds its conclusion as a new hypothesis, only if that conclusion is not already present, failing otherwise.

Ltac extend $p f:=$

let $t:=$ type of $p f$ in

notHyp $t$; generalize $p f$; intro.

We see the useful type of operator of Ltac. This operator could not be implemented in Gallina, but it is easy to support in Ltac. We end up with $t$ bound to the type of $p f$. We 
check that $t$ is not already present. If so, we use a generalize/intro combo to add a new hypothesis proved by $p f$. The tactic generalize takes as input a term $t$ (for instance, a proof of some proposition) and then changes the conclusion from $G$ to $T \rightarrow G$, where $T$ is the type of $t$ (for instance, the proposition proved by the proof $t$ ).

With these tactics defined, we can write a tactic completer for, among other things, adding to the context all consequences of a set of simple first-order formulas.

Ltac completer $:=$

repeat match goal with

$$
\begin{aligned}
& \mid\left[\vdash \wedge_{-}\right] \Rightarrow \text { constructor } \\
& \mid\left[H: \wedge_{-} \vdash_{-}\right] \Rightarrow \text { destruct } H \\
& \quad\left[H: ? P \rightarrow ? Q, H^{\prime}: ? P \vdash_{-}\right] \Rightarrow \text { specialize }\left(H H^{\prime}\right) \\
& \mid\left[\vdash \forall x,{ }_{-}\right] \Rightarrow \text { intro } \\
& \quad \mid\left[H: \forall x, ? P x \rightarrow{ }_{-}, H^{\prime}: ? P ? X \vdash_{-}\right] \Rightarrow \operatorname{extend}\left(H X H^{\prime}\right) \\
& \text { end. }
\end{aligned}
$$

We use the same kind of conjunction and implication handling as previously. Note that, since $\rightarrow$ is the special non-dependent case of $\forall$, the fourth rule handles intro for implications, too.

In the fifth rule, when we find a $\forall$ fact $H$ with a premise matching one of our hypotheses, we add the appropriate instantiation of $H$ 's conclusion, if we have not already added it.

We can check that completer is working properly, with a theorem that introduces a spurious variable whose didactic purpose we will come to shortly.

\section{Section firstorder.}

Variable $A$ : Set.

Variables $P Q R S: A \rightarrow$ Prop.

Hypothesis $H 1: \forall x, P x \rightarrow Q x \wedge R x$.

Hypothesis H2 : $\forall x, R x \rightarrow S x$.

Theorem fo: $\forall(y x: A), P x \rightarrow S x$.

completer.

$y: A$

$x: A$

$H: P x$

$H 0: Q x$

Н3 : $R x$

$H_{4}: S x$

$$
\begin{aligned}
= & = \\
& x
\end{aligned}
$$

assumption.

Qed. 


\section{End firstorder.}

We narrowly avoided a subtle pitfall in our definition of completer. Let us try another definition that even seems preferable to the original, to the untrained eye. (We change the second match case a bit to make the tactic smart enough to handle some subtleties of Ltac behavior that had not been exercised previously.)

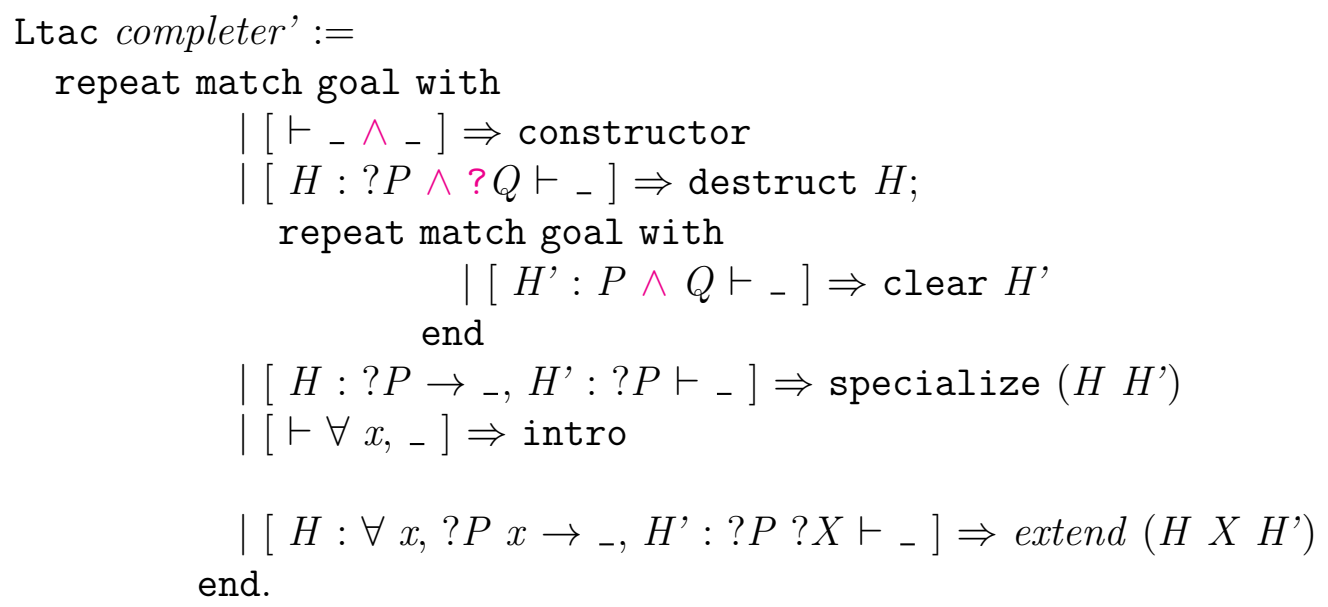

The only other difference is in the modus ponens rule, where we have replaced an unused unification variable ? $Q$ with a wildcard. Let us try our example again with this version:

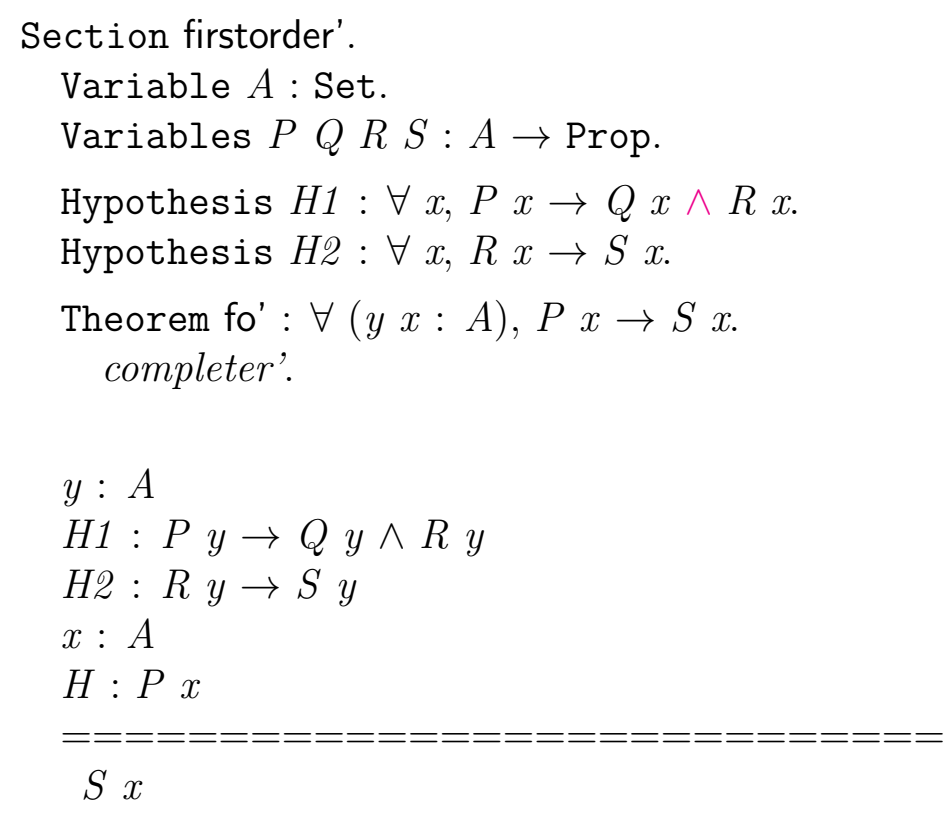

The quantified theorems have been instantiated with $y$ instead of $x$, reducing a provable goal to one that is unprovable. Our code in the last match case for completer' is careful only to instantiate quantifiers along with suitable hypotheses, so why were incorrect choices made?

Abort.

End firstorder'. 
A few examples should illustrate the issue. Here we see a match-based proof that works fine:

Theorem t1: $\forall x:$ nat, $x=x$.

match goal with

$\mid[\vdash \forall x,-] \Rightarrow$ trivial

end.

Qed.

This one fails.

Theorem t1': $\forall x$ : nat, $x=x$.

match goal with

$\mid[\vdash \forall x, ? P] \Rightarrow$ trivial

end.

User error: No matching clauses for match goal

Abort.

The problem is that unification variables may not contain locally bound variables. In this case, ?P would need to be bound to $x=x$, which contains the local quantified variable $x$. By using a wildcard in the earlier version, we avoided this restriction. To understand why this restriction affects the behavior of the completer tactic, recall that, in Coq, implication is shorthand for degenerate universal quantification where the quantified variable is not used. Nonetheless, in an Ltac pattern, Coq is happy to match a wildcard implication against a universal quantification.

The Coq 8.2 release includes a special pattern form for a unification variable with an explicit set of free variables. That unification variable is then bound to a function from the free variables to the "real" value. In Coq 8.1 and earlier, there is no such workaround. We will see an example of this fancier binding form in Section 15.5.

No matter which Coq version you use, it is important to be aware of this restriction. As we have alluded to, the restriction is the culprit behind the surprising behavior of completer'. We unintentionally match quantified facts with the modus ponens rule, circumventing the check that a suitably matching hypothesis is available and leading to different behavior, where wrong quantifier instantiations are chosen. Our earlier completer tactic uses a modus ponens rule that matches the implication conclusion with a variable, which blocks matching against non-trivial universal quantifiers.

Actually, the behavior demonstrated here applies to Coq version 8.4, but not 8.4pl1. The latter version will allow regular Ltac pattern variables to match terms that contain locally bound variables, but a tactic failure occurs if that variable is later used as a Gallina term.

\subsection{Functional Programming in Ltac}

Ltac supports quite convenient functional programming, with a Lisp-with-syntax kind of flavor. However, there are a few syntactic conventions involved in getting programs to be 
accepted. The Ltac syntax is optimized for tactic-writing, so one has to deal with some inconveniences in writing more standard functional programs.

To illustrate, let us try to write a simple list length function. We start out writing it just as in Gallina, simply replacing Fixpoint (and its annotations) with Ltac.

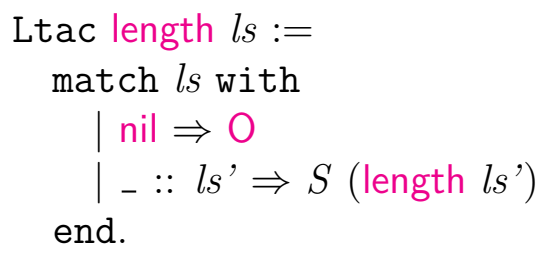

Error: The reference 1s' was not found in the current environment

At this point, we hopefully remember that pattern variable names must be prefixed by question marks in Ltac.

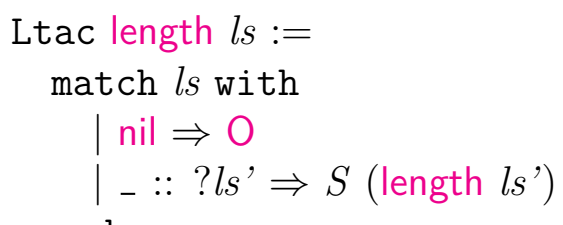

end.

Error: The reference $\mathrm{S}$ was not found in the current environment

The problem is that Ltac treats the expression $S$ (length $l s^{\prime}$ ) as an invocation of a tactic $S$ with argument length $l s$ '. We need to use a special annotation to "escape into" the Gallina parsing nonterminal.

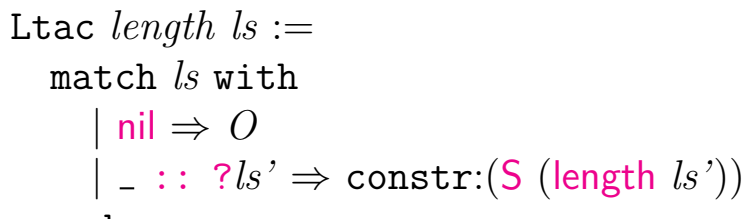

end.

This definition is accepted. It can be a little awkward to test Ltac definitions like this one. Here is one method.

Goal False.

let $n:=$ length $(1:: 2:: 3::$ nil) in pose $n$.

$n:=S$ (length $(2:: 3::$ nil) $):$ nat

\section{False}

We use the pose tactic, which extends the proof context with a new variable that is set equal to a particular term. We could also have used idtac $n$ in place of pose $n$, which would have printed the result without changing the context. 
The value of $n$ only has the length calculation unrolled one step. What has happened here is that, by escaping into the constr nonterminal, we referred to the length function of Gallina, rather than the length Ltac function that we are defining.

\section{Abort.}

Reset length.

The thing to remember is that Gallina terms built by tactics must be bound explicitly via let or a similar technique, rather than inserting Ltac calls directly in other Gallina terms.

Ltac length ls:=

match $l s$ with

| nil $\Rightarrow O$

- $::$ ? ls' $\Rightarrow$

let $l s "=$ length $l s$ ' in

constr: (S ls"')

end.

Goal False.

let $n:=$ length $(1:: 2:: 3::$ nil $)$ in

pose $n$.

$n:=3$ : nat

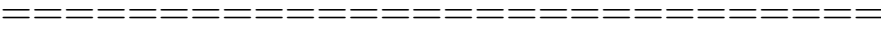

False

Abort.

We can also use anonymous function expressions and local function definitions in Ltac, as this example of a standard list map function shows.

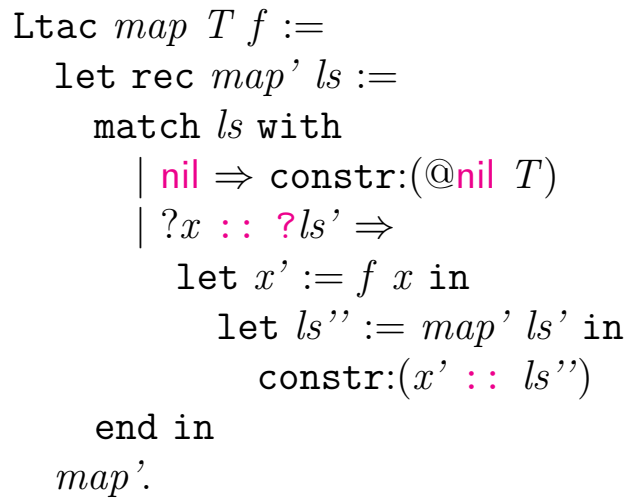

Ltac functions can have no implicit arguments. It may seem surprising that we need to pass $T$, the carried type of the output list, explicitly. We cannot just use type of $f$, because $f$ is an Ltac term, not a Gallina term, and Ltac programs are dynamically typed. The function $f$ could use very syntactic methods to decide to return differently typed terms for different inputs. We also could not replace constr:(@nil T) with constr:nil, because we 
have no strongly typed context to use to infer the parameter to nil. Luckily, we do have sufficient context within constr: $\left(x^{\prime}:: l s^{\prime \prime}\right)$.

Sometimes we need to employ the opposite direction of "nonterminal escape," when we want to pass a complicated tactic expression as an argument to another tactic, as we might want to do in invoking map.

Goal False.

let $l s:=\operatorname{map}($ nat $\times$ nat)\%type ltac:(fun $x \Rightarrow$ constr: $((x, x)))(1:: 2:: 3::$ nil) in

pose $l s$.

$l:=(1,1)::(2,2)::(3,3)::$ nil : list $($ nat $\times$ nat $)$

False

Abort.

Each position within an Ltac script has a default applicable non-terminal, where constr and Itac are the main options worth thinking about, standing respectively for terms of Gallina and Ltac. The explicit colon notation can always be used to override the default non-terminal choice, though code being parsed as Gallina can no longer use such overrides. Within the Itac non-terminal, top-level function applications are treated as applications in Ltac, not Gallina; but the arguments to such functions are parsed with constr by default. This choice may seem strange, until we realize that we have been relying on it all along in all the proof scripts we write! For instance, the apply tactic is an Ltac function, and it is natural to interpret its argument as a term of Gallina, not Ltac. We use an ltac prefix to parse Ltac function arguments as Ltac terms themselves, as in the call to map above. For some simple cases, Ltac terms may be passed without an extra prefix. For instance, an identifier that has an Ltac meaning but no Gallina meaning will be interpreted in Ltac automatically.

One other gotcha shows up when we want to debug our Ltac functional programs. We might expect the following code to work, to give us a version of length that prints a debug trace of the arguments it is called with.

Reset length.

Ltac length $l s:=$

idtac $l s$;

match $l s$ with

| nil $\Rightarrow O$

| - : : ?ls' $\Rightarrow$

let $l s "=$ length $l s^{\prime}$ in

constr: (S ls ',

end.

Coq accepts the tactic definition, but the code is fatally flawed and will always lead to dynamic type errors. 


\section{Goal False.}

let $n:=$ length $(1:: 2:: 3::$ nil $)$ in

pose $n$.

Error: variable $\mathrm{n}$ should be bound to a term.

Abort.

What is going wrong here? The answer has to do with the dual status of Ltac as both a purely functional and an imperative programming language. The basic programming language is purely functional, but tactic scripts are one "datatype" that can be returned by such programs, and Coq will run such a script using an imperative semantics that mutates proof states. Readers familiar with monadic programming in Haskell [45, 34] may recognize a similarity. Haskell programs with side effects can be thought of as pure programs that return the code of programs in an imperative language, where some out-of-band mechanism takes responsibility for running these derived programs. In this way, Haskell remains pure, while supporting usual input-output side effects and more. Ltac uses the same basic mechanism, but in a dynamically typed setting. Here the embedded imperative language includes all the tactics we have been applying so far.

Even basic idtac is an embedded imperative program, so we may not automatically mix it with purely functional code. In fact, a semicolon operator alone marks a span of Ltac code as an embedded tactic script. This makes some amount of sense, since pure functional languages have no need for sequencing: since they lack side effects, there is no reason to run an expression and then just throw away its value and move on to another expression.

An alternate explanation that avoids an analogy to Haskell monads (admittedly a tricky concept in its own right) is: An Ltac tactic program returns a function that, when run later, will perform the desired proof modification. These functions are distinct from other types of data, like numbers or Gallina terms. The prior, correctly working version of length computed solely with Gallina terms, but the new one is implicitly returning a tactic function, as indicated by the use of idtac and semicolon. However, the new version's recursive call to length is structured to expect a Gallina term, not a tactic function, as output. As a result, we have a basic dynamic type error, perhaps obscured by the involvement of first-class tactic scripts.

The solution is like in Haskell: we must "monadify" our pure program to give it access to side effects. The trouble is that the embedded tactic language has no return construct. Proof scripts are about proving theorems, not calculating results. We can apply a somewhat awkward workaround that requires translating our program into continuation-passing style [39], a program structuring idea popular in functional programming.

Reset length.

Ltac length ls $k:=$

idtac $l s$;

match ls with

| nil $\Rightarrow k O$ 


$$
\begin{aligned}
& \mid \text { _ : : ?ls' } \Rightarrow \text { length ls' Itac: }(\text { fun } n \Rightarrow k(\mathrm{~S} n)) \\
& \text { end. }
\end{aligned}
$$

The new length takes a new input: a continuation $k$, which is a function to be called to continue whatever proving process we were in the middle of when we called length. The argument passed to $k$ may be thought of as the return value of length.

Goal False.

length (1:: $2:: 3::$ nil) ltac:(fun $n \Rightarrow$ pose $n)$.

$(1:: 2 \quad:: 3::$ nil)

$(2:: 3::$ nil $)$

$(3::$ nil $)$

nil

Abort.

We see exactly the trace of function arguments that we expected initially, and an examination of the proof state afterward would show that variable $n$ has been added with value 3 .

Considering the comparison with Haskell's IO monad, there is an important subtlety that deserves to be mentioned. A Haskell IO computation represents (theoretically speaking, at least) a transformer from one state of the real world to another, plus a pure value to return. Some of the state can be very specific to the program, as in the case of heap-allocated mutable references, but some can be along the lines of the favorite example "launch missile," where the program has a side effect on the real world that is not possible to undo.

In contrast, Ltac scripts can be thought of as controlling just two simple kinds of mutable state. First, there is the current sequence of proof subgoals. Second, there is a partial assignment of discovered values to unification variables introduced by proof search (for instance, by eauto, as we saw in the previous chapter). Crucially, every mutation of this state can be undone during backtracking introduced by match, auto, and other built-in Ltac constructs. Ltac proof scripts have state, but it is purely local, and all changes to it are reversible, which is a very useful semantics for proof search.

\subsection{Recursive Proof Search}

Deciding how to instantiate quantifiers is one of the hardest parts of automated first-order theorem proving. For a given problem, we can consider all possible bounded-length sequences of quantifier instantiations, applying only propositional reasoning at the end. This is probably a bad idea for almost all goals, but it makes for a nice example of recursive proof search procedures in Ltac.

We can consider the maximum "dependency chain" length for a first-order proof. We define the chain length for a hypothesis to be 0 , and the chain length for an instantiation of a quantified fact to be one greater than the length for that fact. The tactic inster $n$ is meant to try all possible proofs with chain length at most $n$. 


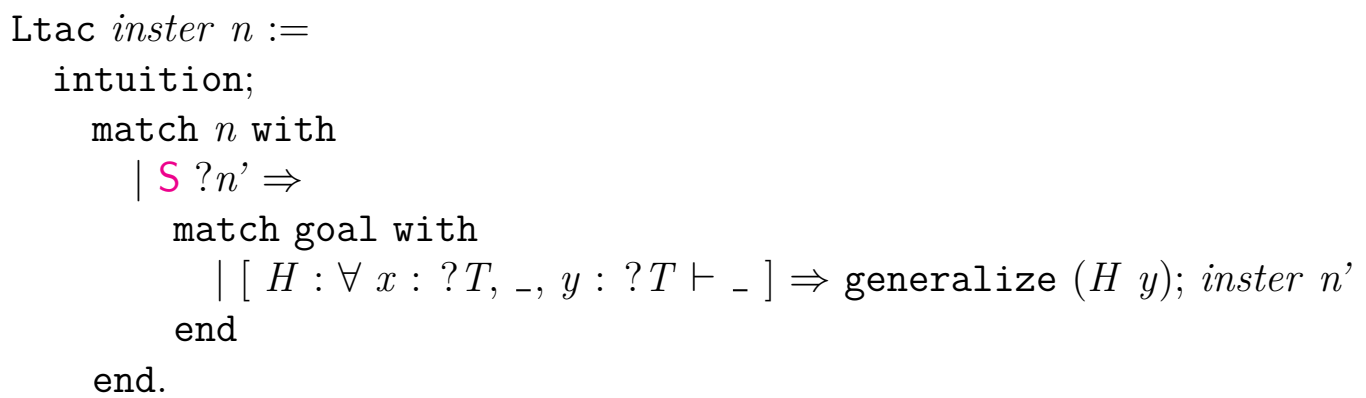

The tactic begins by applying propositional simplification. Next, it checks if any chain length remains, failing if not. Otherwise, it tries all possible ways of instantiating quantified hypotheses with properly typed local variables. It is critical to realize that, if the recursive call inster $n$ ' fails, then the match goal just seeks out another way of unifying its pattern against proof state. Thus, this small amount of code provides an elegant demonstration of how backtracking match enables exhaustive search.

We can verify the efficacy of inster with two short examples. The built-in firstorder tactic (with no extra arguments) is able to prove the first but not the second.

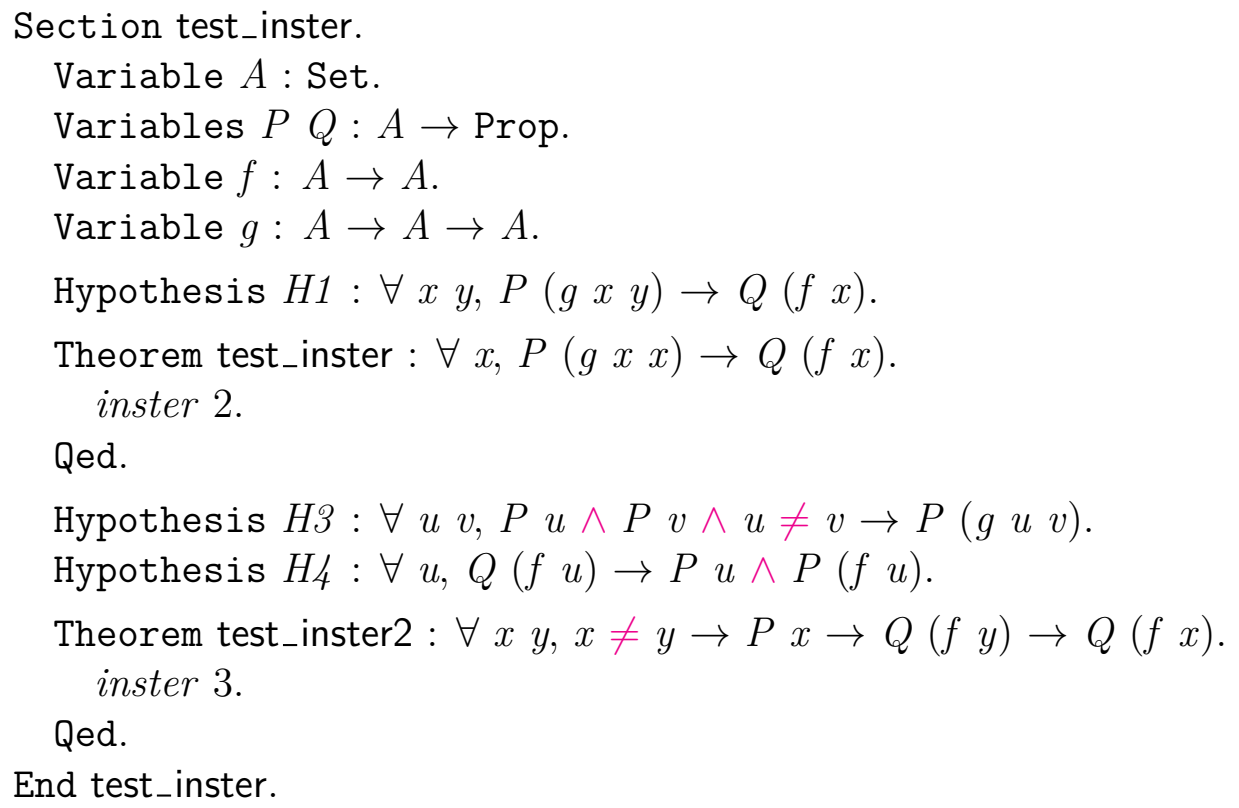

The style employed in the definition of inster can seem very counterintuitive to functional programmers. Usually, functional programs accumulate state changes in explicit arguments to recursive functions. In Ltac, the state of the current subgoal is always implicit. Nonetheless, recalling the discussion at the end of the last section, in contrast to general imperative programming, it is easy to undo any changes to this state, and indeed such "undoing" happens automatically at failures within matches. In this way, Ltac programming is similar to programming in Haskell with a stateful failure monad that supports a composition operator along the lines of the first tactical.

Functional programming purists may react indignantly to the suggestion of programming this way. Nonetheless, as with other kinds of "monadic programming," many problems are 
much simpler to solve with Ltac than they would be with explicit, pure proof manipulation in ML or Haskell. To demonstrate, we will write a basic simplification procedure for logical implications.

This procedure is inspired by one for separation logic [40], where conjuncts in formulas are thought of as "resources," such that we lose no completeness by "crossing out" equal conjuncts on the two sides of an implication. This process is complicated by the fact that, for reasons of modularity, our formulas can have arbitrary nested tree structure (branching at conjunctions) and may include existential quantifiers. It is helpful for the matching process to "go under" quantifiers and in fact decide how to instantiate existential quantifiers in the conclusion.

To distinguish the implications that our tactic handles from the implications that will show up as "plumbing" in various lemmas, we define a wrapper definition, a notation, and a tactic.

Definition imp (P1 P2 : Prop) $:=P 1 \rightarrow P 2$.

Infix " $->":=\operatorname{imp}$ (no associativity, at level 95).

Ltac imp $:=$ unfold imp; firstorder.

These lemmas about imp will be useful in the tactic that we will write.

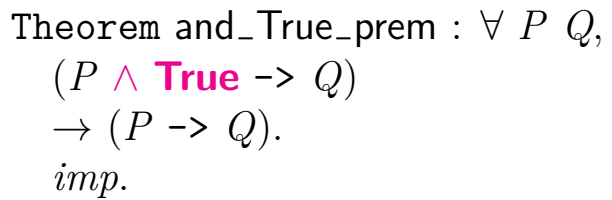

\section{Qed.}

Theorem and_True_conc : $\forall P Q$,

$(P \rightarrow Q \wedge$ True $)$

$\rightarrow(P \rightarrow Q)$.

$i m p$.

Qed.

Theorem pick_prem1 : $\forall P Q R S$, $(P \wedge(Q \wedge R) \rightarrow S)$

$\rightarrow((P \wedge Q) \wedge R \rightarrow S)$.

imp.

Qed.

Theorem pick_prem2 : $\forall P Q R S$,

$(Q \wedge(P \wedge R) \rightarrow S)$

$\rightarrow((P \wedge Q) \wedge R \rightarrow S)$.

imp.

Qed.

Theorem comm_prem : $\forall P Q R$,

$(P \wedge Q \rightarrow R)$

$\rightarrow(Q \wedge P \rightarrow R)$.

imp. 
Qed.

Theorem pick_conc1: $\forall P Q R S$,

$(S \rightarrow P \wedge(Q \wedge R))$

$\rightarrow(S \rightarrow(P \wedge Q) \wedge R)$.

imp.

Qed.

Theorem pick_conc2 : $\forall P Q R S$,

$(S \rightarrow Q \wedge(P \wedge R))$

$\rightarrow(S \rightarrow(P \wedge Q) \wedge R)$.

imp.

Qed.

Theorem comm_conc : $\forall P Q R$,

$(R \rightarrow P \wedge Q)$

$\rightarrow(R \rightarrow Q \wedge P)$.

$i m p$.

Qed.

The first order of business in crafting our matcher tactic will be auxiliary support for searching through formula trees. The search_prem tactic implements running its tactic argument tac on every subformula of an imp premise. As it traverses a tree, search_prem applies some of the above lemmas to rewrite the goal to bring different subformulas to the head of the goal. That is, for every subformula $P$ of the implication premise, we want $P$ to "have a turn," where the premise is rearranged into the form $P \wedge Q$ for some $Q$. The tactic tac should expect to see a goal in this form and focus its attention on the first conjunct of the premise.

Ltac search_prem tac :=

let rec search $P:=$

tac

II (apply and_True_prem; tac)

$\|$ match $P$ with

| ?P1 $\wedge$ ? $2 \Rightarrow$

(apply pick_prem1; search $P 1$ )

II (apply pick_prem2; search P2)

end

in match goal with

$\mid\left[\vdash ? P \wedge_{-}>_{-}\right] \Rightarrow \operatorname{search} P$

$\mid\left[\vdash{ }_{-} \wedge ? P \rightarrow_{-}\right] \Rightarrow$ apply comm_prem; search $P$

$\mid\left[\vdash{ }_{-}>_{-}\right] \Rightarrow$ progress (tac $\|$ (apply and_True_prem; tac))

end.

To understand how search_prem works, we turn first to the final match. If the premise begins with a conjunction, we call the search procedure on each of the conjuncts, or only the first conjunct, if that already yields a case where tac does not fail. The call search $P$ expects 
and maintains the invariant that the premise is of the form $P \wedge Q$ for some $Q$. We pass $P$ explicitly as a kind of decreasing induction measure, to avoid looping forever when tac always fails. The second match case calls a commutativity lemma to realize this invariant, before passing control to search. The final match case tries applying tac directly and then, if that fails, changes the form of the goal by adding an extraneous True conjunct and calls tac again. The progress tactical fails when its argument tactic succeeds without changing the current subgoal.

The search function itself tries the same tricks as in the last case of the final match, using the || operator as a shorthand for trying one tactic and then, if the first fails, trying another. Additionally, if neither works, it checks if $P$ is a conjunction. If so, it calls itself recursively on each conjunct, first applying associativity/commutativity lemmas to maintain the goal-form invariant.

We will also want a dual function search_conc, which does tree search through an imp conclusion.

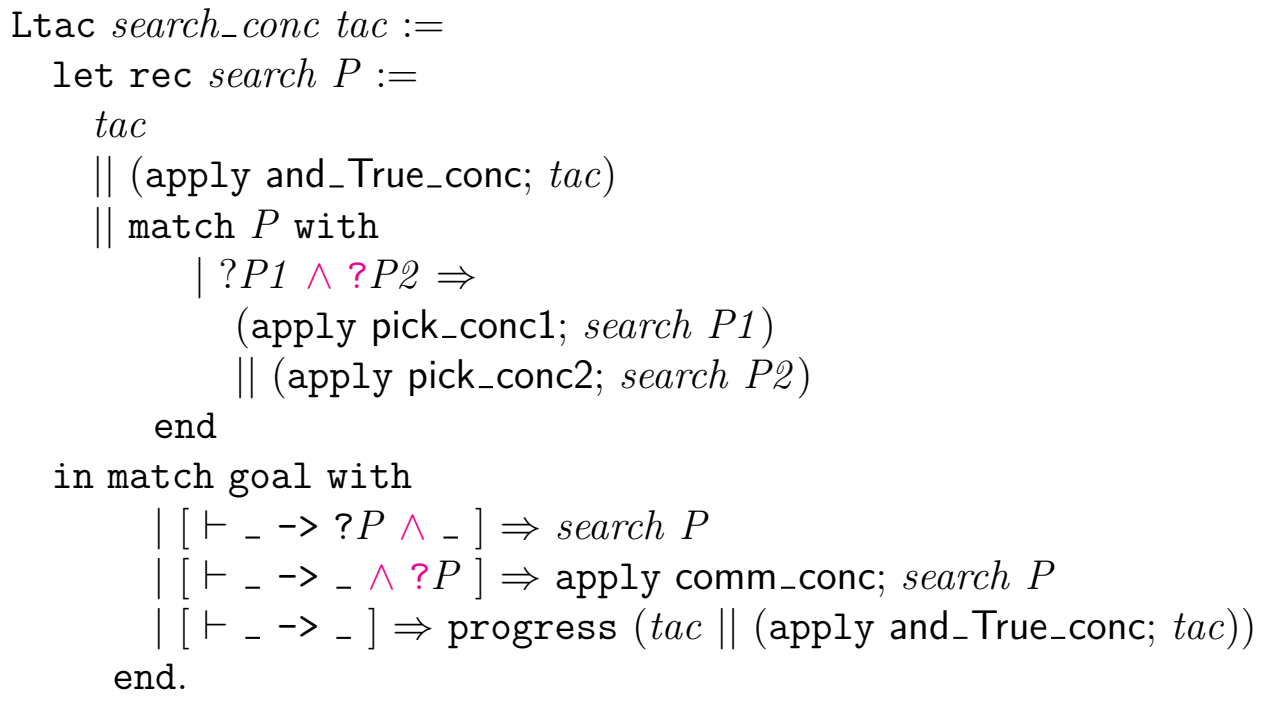

Now we can prove a number of lemmas that are suitable for application by our search tactics. A lemma that is meant to handle a premise should have the form $P \wedge Q->R$ for some interesting $P$, and a lemma that is meant to handle a conclusion should have the form $P \rightarrow Q \wedge R$ for some interesting $Q$.

Theorem False_prem : $\forall P Q$,

False $\wedge P \rightarrow Q$.

imp.

Qed.

Theorem True_conc : $\forall P Q$ : Prop,

$(P \rightarrow Q)$

$\rightarrow(P \rightarrow$ True $\wedge Q)$.

imp.

Qed. 
Theorem Match : $\forall P Q R$ : Prop,

$$
\begin{aligned}
& (Q \rightarrow R) \\
& \rightarrow(P \wedge Q \rightarrow P \wedge R) . \\
& \text { imp. }
\end{aligned}
$$

Qed.

Theorem ex_prem : $\forall$ ( $T:$ Type) $(P: T \rightarrow$ Prop) ( $Q R:$ Prop),

$(\forall x, P x \wedge Q \rightarrow R)$

$\rightarrow(\operatorname{ex} P \wedge Q \rightarrow R)$.

$i m p$.

Qed.

Theorem ex_conc $: \forall$ ( $T:$ Type $)(P: T \rightarrow$ Prop) $(Q R:$ Prop) $x$,

$(Q \rightarrow P x \wedge R)$

$\rightarrow(Q \rightarrow$ ex $P \wedge R)$.

imp.

Qed.

We will also want a "base case" lemma for finishing proofs where cancellation has removed every constituent of the conclusion.

Theorem imp_True : $\forall P$,

$P \rightarrow$ True.

imp.

Qed.

Our final matcher tactic is now straightforward. First, we intros all variables into scope. Then we attempt simple premise simplifications, finishing the proof upon finding False and eliminating any existential quantifiers that we find. After that, we search through the conclusion. We remove True conjuncts, remove existential quantifiers by introducing unification variables for their bound variables, and search for matching premises to cancel. Finally, when no more progress is made, we see if the goal has become trivial and can be solved by imp_True. In each case, we use the tactic simple apply in place of apply to use a simpler, less expensive unification algorithm.

Ltac matcher $:=$

intros;

repeat search_prem ltac:(simple apply False_prem || (simple apply ex_prem; intro)); repeat search_conc ltac:(simple apply True_conc || simple eapply ex_conc

|| search_prem ltac:(simple apply Match));

try simple apply imp_True.

Our tactic succeeds at proving a simple example.

Theorem t2 : $\forall P Q:$ Prop,

$Q \wedge(P \wedge$ False $) \wedge P \rightarrow P \wedge Q$.

matcher.

Qed. 
In the generated proof, we find a trace of the workings of the search tactics.

Print t2.

$\mathrm{t} 2=$

fun $P Q:$ Prop $\Rightarrow$

comm_prem (pick_prem1 (pick_prem2 (False_prem $(P:=P \wedge P \wedge Q)(P \wedge Q)))$ )

: $\forall P Q:$ Prop, $Q \wedge(P \wedge$ False $) \wedge P \rightarrow P \wedge Q$

We can also see that matcher is well-suited for cases where some human intervention is needed after the automation finishes.

Theorem t3: $\forall P Q R$ : Prop,

$P \wedge Q \rightarrow Q \wedge R \wedge P$.

matcher.

True $\rightarrow R$

Our tactic canceled those conjuncts that it was able to cancel, leaving a simplified subgoal for us, much as intuition does.

Abort.

The matcher tactic even succeeds at guessing quantifier instantiations. It is the unification that occurs in uses of the Match lemma that does the real work here.

Theorem t4: $\forall(P:$ nat $\rightarrow$ Prop) $Q,(\exists x, P x \wedge Q) \rightarrow Q \wedge(\exists x, P x)$.

matcher.

Qed.

Print t4.

$\mathrm{t} 4=$

fun $(P:$ nat $\rightarrow$ Prop $)(Q:$ Prop $) \Rightarrow$

and_True_prem

(ex_prem $(P:=$ fun $x:$ nat $\Rightarrow P x \wedge Q)$

(fun $x:$ nat $\Rightarrow$

pick_prem2

(Match $(P:=Q)$

(and_True_conc

(ex_conc (fun $x 0$ : nat $\Rightarrow P x 0) x$

$\left(\right.$ Match $(P:=P x)\left(\right.$ imp_True $_{-}(P:=$ True $\left.\left.\left.\left.\left.\left.)\right)\right)\right)\right)\right)\right)$

$: \forall(P:$ nat $\rightarrow$ Prop) $(Q:$ Prop),

$(\exists x:$ nat, $P x \wedge Q) \rightarrow Q \wedge(\exists x:$ nat, $P x)$

This proof term is a mouthful, and we can be glad that we did not build it manually! 


\subsection{Creating Unification Variables}

A final useful ingredient in tactic crafting is the ability to allocate new unification variables explicitly. Tactics like eauto introduce unification variables internally to support flexible proof search. While eauto and its relatives do backward reasoning, we often want to do similar forward reasoning, where unification variables can be useful for similar reasons.

For example, we can write a tactic that instantiates the quantifiers of a universally quantified hypothesis. The tactic should not need to know what the appropriate instantiations are; rather, we want these choices filled with placeholders. We hope that, when we apply the specialized hypothesis later, syntactic unification will determine concrete values.

Before we are ready to write a tactic, we can try out its ingredients one at a time.

Theorem t5: $(\forall x:$ nat, $\mathrm{S} x>x) \rightarrow 2>1$.

intros.

$H: \forall x:$ nat, $S x>x$

$====================$

$2>1$

To instantiate $H$ generically, we first need to name the value to be used for $x$.

evar ( $y$ : nat).

$H: \forall x:$ nat, $S x>x$

$y:=? 279:$ nat

$====================$

$2>1$

The proof context is extended with a new variable $y$, which has been assigned to be equal to a fresh unification variable ?279. We want to instantiate $H$ with ?279. To get ahold of the new unification variable, rather than just its alias $y$, we perform a trivial unfolding in the expression $y$, using the eval Ltac construct, which works with the same reduction strategies that we have seen in tactics (e.g., simpl, compute, etc.).

let $y^{\prime}:=$ eval unfold $y$ in $y$ in

clear $y$; specialize ( $\left.H y^{\prime}\right)$.

$H: S ? 279>? 279$

$===================$

$2>1$

Our instantiation was successful. We can finish the proof by using apply's unification to figure out the proper value of ?279. 
apply $H$.

Qed.

Now we can write a tactic that encapsulates the pattern we just employed, instantiating all quantifiers of a particular hypothesis.

Ltac inster $U H:=$

repeat match type of $H$ with

$\forall x: ? T,_{-} \Rightarrow$

let $x:=$ fresh "x" in

evar $(x: T)$;

let $x^{\prime}:=$ eval unfold $x$ in $x$ in

clear $x$; specialize $\left(\begin{array}{ll}H & \left.x^{\prime}\right)\end{array}\right.$

end.

Theorem t5' : $(\forall x:$ nat, $\mathrm{S} x>x) \rightarrow 2>1$.

intro $H$; inster $U$; apply $H$.

Qed.

This particular example is somewhat silly, since apply by itself would have solved the goal originally. Separate forward reasoning is more useful on hypotheses that end in existential quantifications. Before we go through an example, it is useful to define a variant of inster $U$ that does not clear the base hypothesis we pass to it. We use the Ltac construct fresh to generate a hypothesis name that is not already used, based on a string suggesting a good name.

Ltac insterKeep $H:=$

let $H^{\prime}:=$ fresh "H'" in generalize $H$; intro $H^{\prime}$; inster $U H^{\prime}$.

Section t6.

Variables $A B$ : Type.

Variable $P: A \rightarrow B \rightarrow$ Prop.

Variable $f: A \rightarrow A \rightarrow A$.

Variable $g: B \rightarrow B \rightarrow B$.

Hypothesis $H 1: \forall v, \exists u, P v u$.

Hypothesis H2: $\forall v 1$ u1 v2 u2,

$P$ v1 $u 1$

$\rightarrow P$ v2 u2

$\rightarrow P(f v 1$ v2) $(g u 1$ u2).

Theorem t6: $\forall$ v1 v2, $\exists u 1, \exists u 2, P(f v 1$ v2) $(g u 1$ u2).

intros.

Neither eauto nor firstorder is clever enough to prove this goal. We can help out by doing some of the work with quantifiers ourselves, abbreviating the proof with the do tactical for repetition of a tactic a set number of times.

do 2 insterKeep $H 1$. 
Our proof state is extended with two generic instances of $H 1$.

$H^{\prime}: \exists u: B, P ? 4289 u$

$H^{\prime} 0: \exists u: B, P ? 4288 u$

$==================$

$\exists u 1: B, \exists u 2: B, P(f$ v1 v2) $(g u 1$ u2)

Normal eauto still cannot prove the goal, so we eliminate the two new existential quantifiers. (Recall that ex is the underlying type family to which uses of the $\exists$ syntax are compiled.)

repeat match goal with

| $\left[H: e_{-} \vdash_{-}\right] \Rightarrow$ destruct $H$

end.

Now the goal is simple enough to solve by logic programming.

eauto.

Qed.

End t6.

Our inster $U$ tactic does not fare so well with quantified hypotheses that also contain implications. We can see the problem in a slight modification of the last example. We introduce a new unary predicate $Q$ and use it to state an additional requirement of our hypothesis $H 1$.

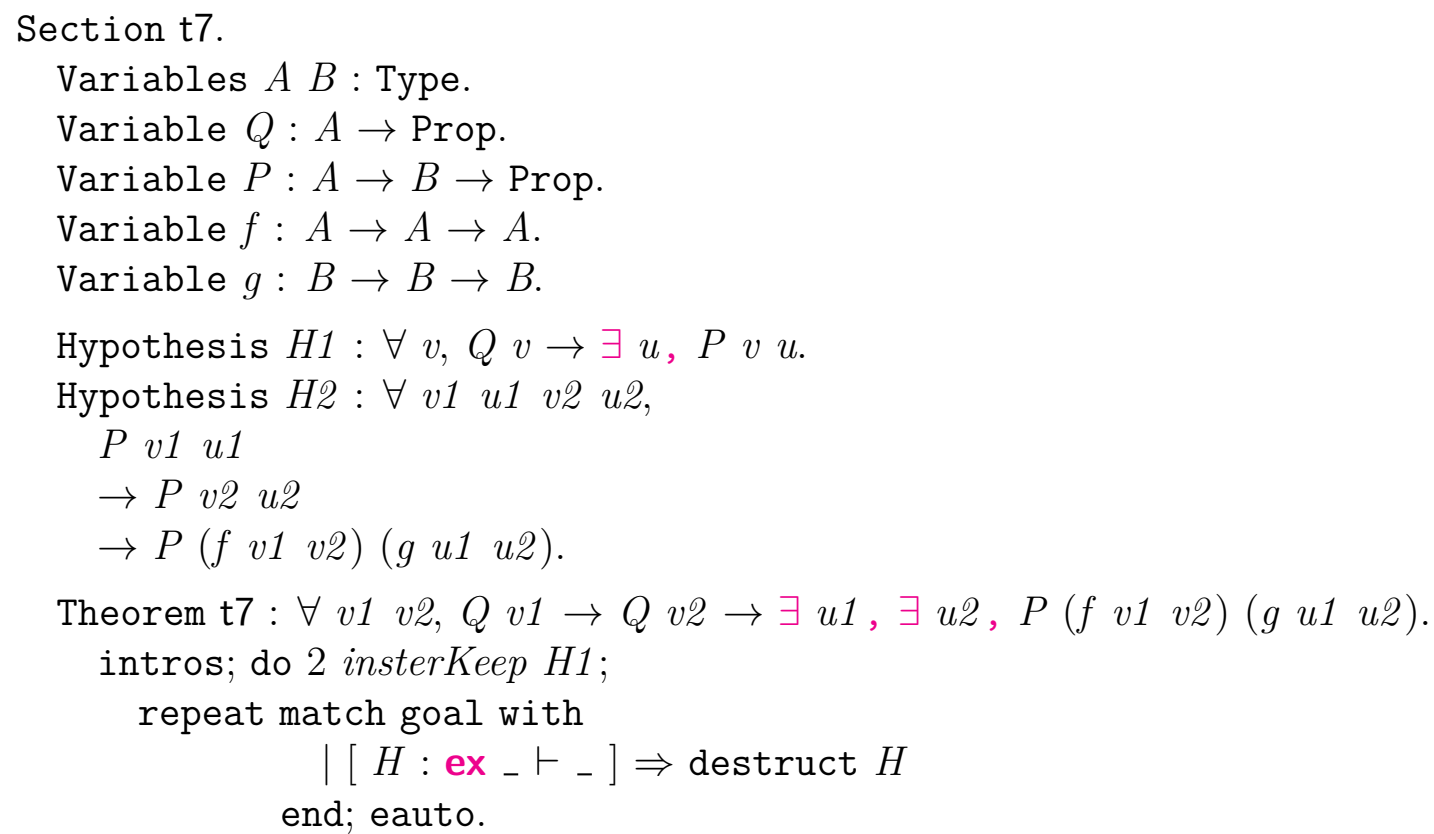

This proof script does not hit any errors until the very end, when an error message like this one is displayed. 


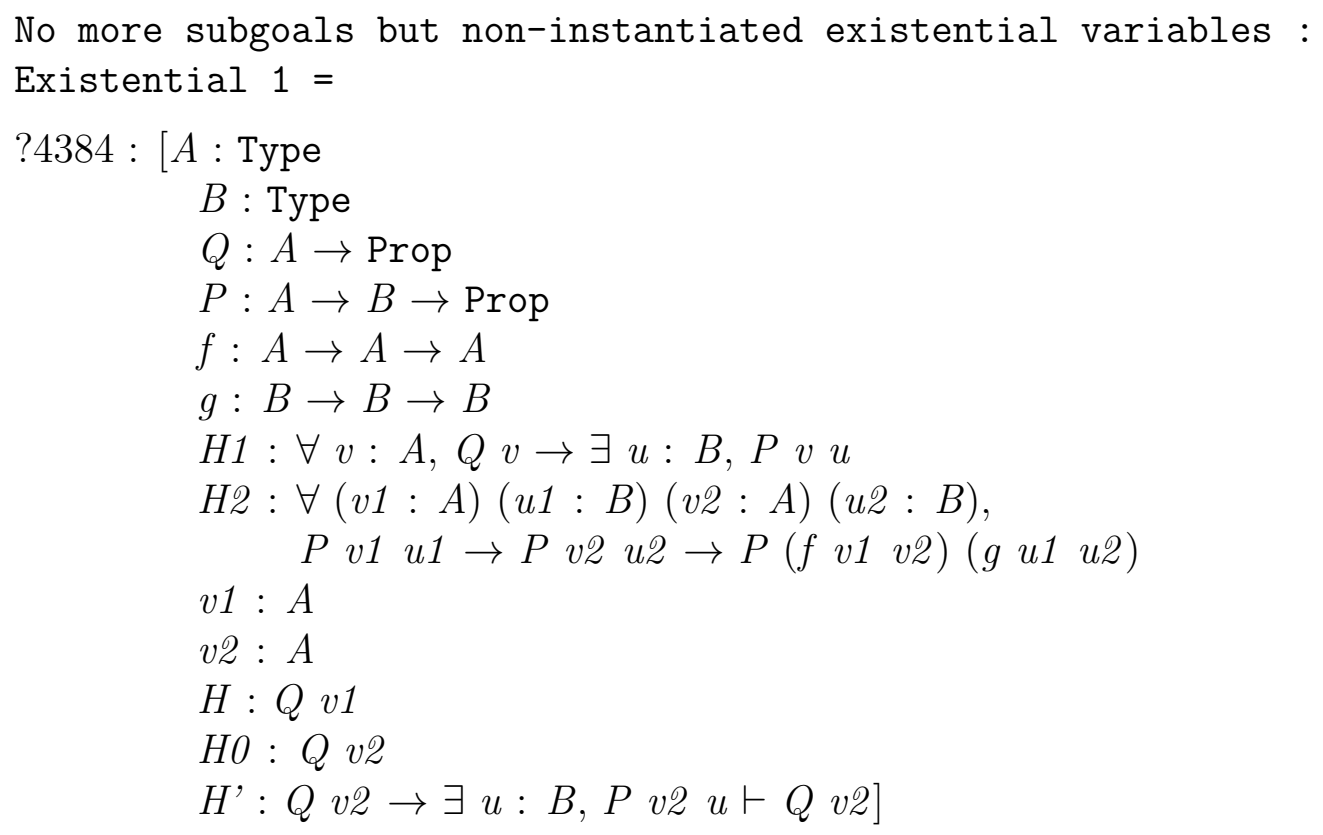

There is another similar line about a different existential variable. Here, "existential variable" means what we have also called "unification variable." In the course of the proof, some unification variable?4384 was introduced but never unified. Unification variables are just a device to structure proof search; the language of Gallina proof terms does not include them. Thus, we cannot produce a proof term without instantiating the variable.

The error message shows that ?4384 is meant to be a proof of $Q$ v2 in a particular proof state, whose variables and hypotheses are displayed. It turns out that?4384 was created by inster $U$, as the value of a proof to pass to H1. Recall that, in Gallina, implication is just a degenerate case of $\forall$ quantification, so the inster $U$ code to match against $\forall$ also matched the implication. Since any proof of $Q v 2$ is as good as any other in this context, there was never any opportunity to use unification to determine exactly which proof is appropriate. We expect similar problems with any implications in arguments to inster $U$.

Abort.

End $\mathrm{t} 7$.

Reset inster $U$.

We can redefine inster $U$ to treat implications differently. In particular, we pattern-match on the type of the type $T$ in $\forall x: ? T, \ldots$ If $T$ has type Prop, then $x$ 's instantiation should be thought of as a proof. Thus, instead of picking a new unification variable for it, we instead apply a user-supplied tactic tac. It is important that we end this special Prop case with || fail 1, so that, if tac fails to prove $T$, we abort the instantiation, rather than continuing on to the default quantifier handling. Also recall that the tactic form solve $[t]$ fails if $t$ does not completely solve the goal.

Ltac inster $U$ tac $H:=$

repeat match type of $H$ with

$\mid \forall x: ? T,_{-} \Rightarrow$ 


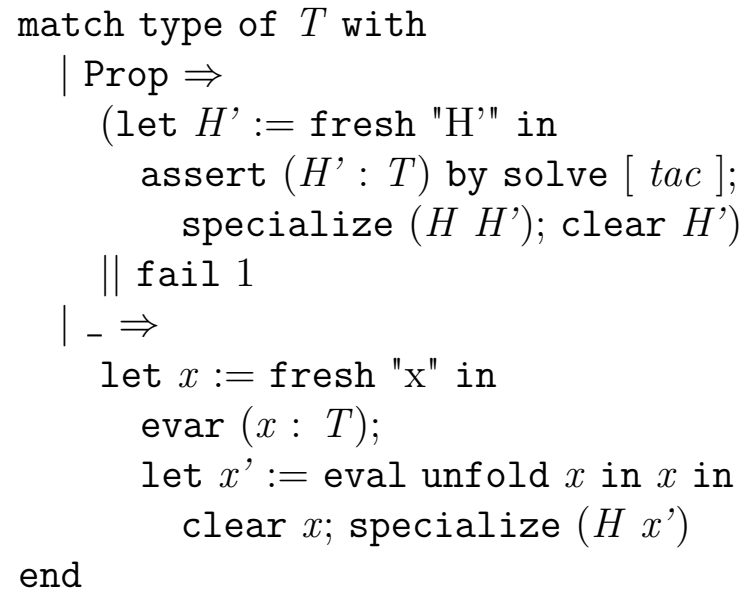

Ltac insterKeep tac $H:=$

let $H^{\prime}:=$ fresh " $H^{\prime \prime}$ in

generalize $H$; intro $H^{\prime}$; inster $U$ tac $H^{\prime}$.

\section{Section t7.}

Variables $A B$ : Type.

Variable $Q: A \rightarrow$ Prop.

Variable $P: A \rightarrow B \rightarrow$ Prop.

Variable $f: A \rightarrow A \rightarrow A$.

Variable $g: B \rightarrow B \rightarrow B$.

Hypothesis $H 1: \forall v, Q v \rightarrow \exists u, P v u$.

Hypothesis H2: $\forall v 1$ u1 v2 u2,

$P$ v1 $u 1$

$\rightarrow P$ v2 u2

$\rightarrow P(f v 1$ v2) $(g u 1$ u2).

Theorem $\mathrm{t} 7: \forall v 1$ v2, $Q v 1 \rightarrow Q$ v2 $\rightarrow \exists u 1, \exists u 2, P(f v 1$ v2) $(g$ u1 u2).

We can prove the goal by calling insterKeep with a tactic that tries to find and apply a $Q$ hypothesis over a variable about which we do not yet know any $P$ facts. We need to begin this tactic code with idtac; to get around a strange limitation in Coq's proof engine, where a first-class tactic argument may not begin with a match.

intros; do 2 insterKeep ltac:(idtac; match goal with

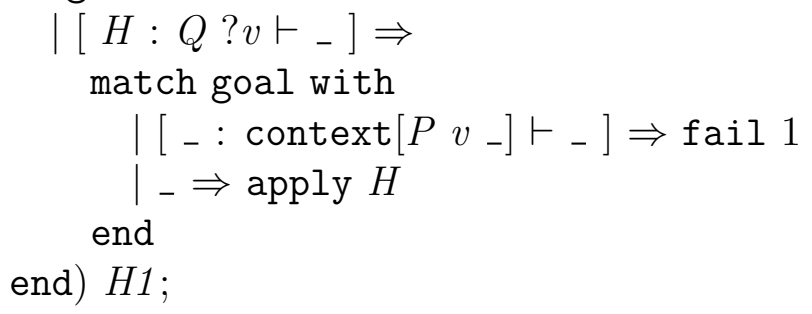

repeat match goal with

| [H: ex _ $\left.\vdash_{-}\right] \Rightarrow$ destruct $H$ 
end; eauto.

Qed.

End $\mathrm{t} 7$.

It is often useful to instantiate existential variables explicitly. A built-in tactic provides one way of doing so.

Theorem t8: $\exists p$ : nat $\times$ nat, fst $p=3$.

econstructor; instantiate $(1:=(3,2))$; reflexivity. Qed.

The 1 above is identifying an existential variable appearing in the current goal, with the last existential appearing assigned number 1, the second-last assigned number 2, and so on. The named existential is replaced everywhere by the term to the right of the $:=$.

The instantiate tactic can be convenient for exploratory proving, but it leads to very brittle proof scripts that are unlikely to adapt to changing theorem statements. It is often more helpful to have a tactic that can be used to assign a value to a term that is known to be an existential. By employing a roundabout implementation technique, we can build a tactic that generalizes this functionality. In particular, our tactic equate will assert that two terms are equal. If one of the terms happens to be an existential, then it will be replaced everywhere with the other term.

Ltac equate $x$ y $:=$

let dummy := constr:(eq_refl $x: x=y)$ in idtac.

This tactic fails if it is not possible to prove $x=y$ by eq_refl. We check the proof only for its unification side effects, ignoring the associated variable dummy. With equate, we can build a less brittle version of the prior example.

Theorem t9: $\exists p$ : nat $\times$ nat, fst $p=3$.

econstructor; match goal with

$$
\mid[\vdash \mathrm{fst} ? x=3] \Rightarrow \text { equate } x(3,2)
$$

end; reflexivity.

Qed.

This technique is even more useful within recursive and iterative tactics that are meant to solve broad classes of goals. 


\section{Chapter 15}

\section{Proof by Reflection}

The last chapter highlighted a very heuristic approach to proving. In this chapter, we will study an alternative technique, proof by reflection [2]. We will write, in Gallina, decision procedures with proofs of correctness, and we will appeal to these procedures in writing very short proofs. Such a proof is checked by running the decision procedure. The term reflection applies because we will need to translate Gallina propositions into values of inductive types representing syntax, so that Gallina programs may analyze them, and translating such a term back to the original form is called reflecting it.

\subsection{Proving Evenness}

Proving that particular natural number constants are even is certainly something we would rather have happen automatically. The Ltac-programming techniques that we learned in the last chapter make it easy to implement such a procedure.

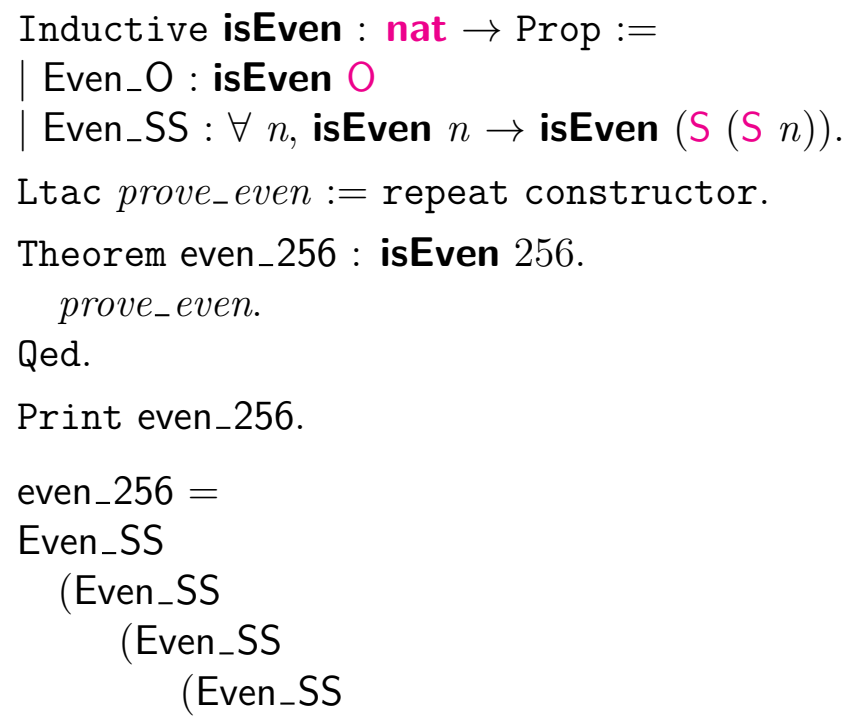

...and so on. This procedure always works (at least on machines with infinite resources), but it has a serious drawback, which we see when we print the proof it generates that 256 is even. 
The final proof term has length super-linear in the input value. Coq's implicit arguments mechanism is hiding the values given for parameter $n$ of Even_SS, which is why the proof term only appears linear here. Also, proof terms are represented internally as syntax trees, with opportunity for sharing of node representations, but in this chapter we will measure proof term size as simple textual length or as the number of nodes in the term's syntax tree, two measures that are approximately equivalent. Sometimes apparently large proof terms have enough internal sharing that they take up less memory than we expect, but one avoids having to reason about such sharing by ensuring that the size of a sharing-free version of a term is low enough.

Superlinear evenness proof terms seem like a shame, since we could write a trivial and trustworthy program to verify evenness of constants. The proof checker could simply call our program where needed.

It is also unfortunate not to have static typing guarantees that our tactic always behaves appropriately. Other invocations of similar tactics might fail with dynamic type errors, and we would not know about the bugs behind these errors until we happened to attempt to prove complex enough goals.

The techniques of proof by reflection address both complaints. We will be able to write proofs like in the example above with constant size overhead beyond the size of the input, and we will do it with verified decision procedures written in Gallina.

For this example, we begin by using a type from the MoreSpecif module (included in the book source) to write a certified evenness checker.

\section{Print partial.}

Inductive partial $(P:$ Prop $):$ Set $:=$ Proved $: P \rightarrow[P] \mid$ Uncertain $:[P]$

A partial $P$ value is an optional proof of $P$. The notation $[P]$ stands for partial $P$.

Local Open Scope partial_scope.

We bring into scope some notations for the partial type. These overlap with some of the notations we have seen previously for specification types, so they were placed in a separate scope that needs separate opening.

Definition check_even : $\forall n$ : nat, [isEven $n$ ].

Hint Constructors isEven.

refine (fix $F(n:$ nat) : [isEven $n]:=$

match $n$ with

$0 \Rightarrow$ Yes

$1 \Rightarrow$ No

| S (S $\left.n^{\prime}\right) \Rightarrow$ Reduce $\left(F n^{\prime}\right)$

end); auto.

Defined.

The function check_even may be viewed as a verified decision procedure, because its type guarantees that it never returns Yes for inputs that are not even. 
Now we can use dependent pattern-matching to write a function that performs a surprising feat. When given a partial $P$, this function partialOut returns a proof of $P$ if the partial value contains a proof, and it returns a (useless) proof of True otherwise. From the standpoint of ML and Haskell programming, it seems impossible to write such a type, but it is trivial with a return annotation.

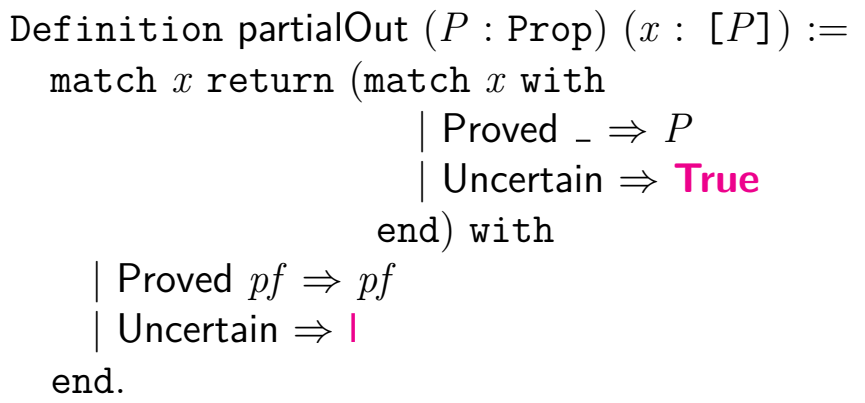

It may seem strange to define a function like this. However, it turns out to be very useful in writing a reflective version of our earlier prove_even tactic:

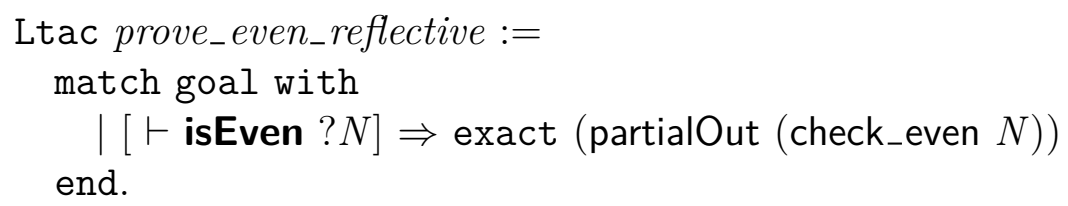

We identify which natural number we are considering, and we "prove" its evenness by pulling the proof out of the appropriate check_even call. Recall that the exact tactic proves a proposition $P$ when given a proof term of precisely type $P$.

Theorem even_256' : isEven 256.

prove_even_reflective.

Qed.

Print even_256'.

even_256' = partialOut (check_even 256)

\section{: isEven 256}

We can see a constant wrapper around the object of the proof. For any even number, this form of proof will suffice. The size of the proof term is now linear in the number being checked, containing two repetitions of the unary form of that number, one of which is hidden above within the implicit argument to partialOut.

What happens if we try the tactic with an odd number?

Theorem even_255: isEven 255.

prove_even_reflective.

User error: No matching clauses for match goal

Thankfully, the tactic fails. To see more precisely what goes wrong, we can run manually the body of the match.

exact (partialOut (check_even 255)). 


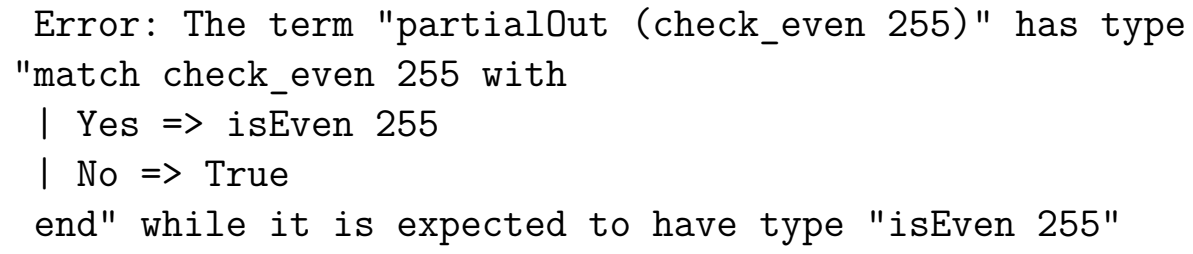

As usual, the type checker performs no reductions to simplify error messages. If we reduced the first term ourselves, we would see that check_even 255 reduces to a No, so that the first term is equivalent to True, which certainly does not unify with isEven 255.

Abort.

Our tactic prove_even_reflective is reflective because it performs a proof search process (a trivial one, in this case) wholly within Gallina, where the only use of Ltac is to translate a goal into an appropriate use of check_even.

\subsection{Reifying the Syntax of a Trivial Tautology Lan- guage}

We might also like to have reflective proofs of trivial tautologies like this one:

Theorem true_galore $:($ True $\wedge$ True $) \rightarrow($ True $\vee($ True $\wedge($ True $\rightarrow$ True $)))$.

tauto.

Qed.

Print true_galore.

true_galore $=$

fun $H$ : True $\wedge$ True $\Rightarrow$

and_ind (fun _ _ : True $\Rightarrow$ or_introl (True $\wedge($ True $\rightarrow$ True) $)$ I) $H$

$:$ True $\wedge$ True $\rightarrow$ True $\vee$ True $\wedge($ True $\rightarrow$ True $)$

As we might expect, the proof that tauto builds contains explicit applications of natural deduction rules. For large formulas, this can add a linear amount of proof size overhead, beyond the size of the input.

To write a reflective procedure for this class of goals, we will need to get into the actual "reflection" part of "proof by reflection." It is impossible to case-analyze a Prop in any way in Gallina. We must reify Prop into some type that we can analyze. This inductive type is a good candidate:

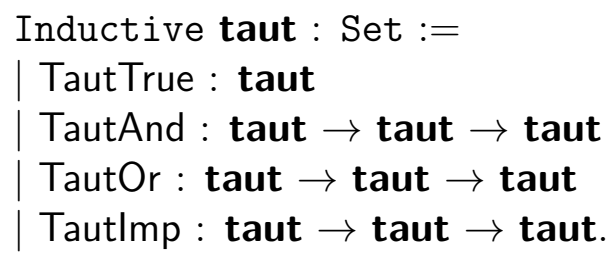


We write a recursive function to reflect this syntax back to Prop. Such functions are also called interpretation functions, and we have used them in previous examples to give semantics to small programming languages.

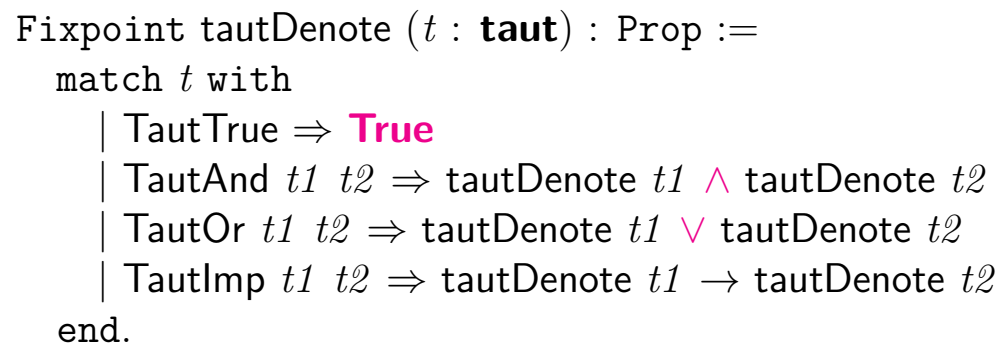

It is easy to prove that every formula in the range of tautDenote is true.

Theorem tautTrue : $\forall t$, tautDenote $t$.

induction $t$; crush.

Qed.

To use taut True to prove particular formulas, we need to implement the syntax reification process. A recursive Ltac function does the job.

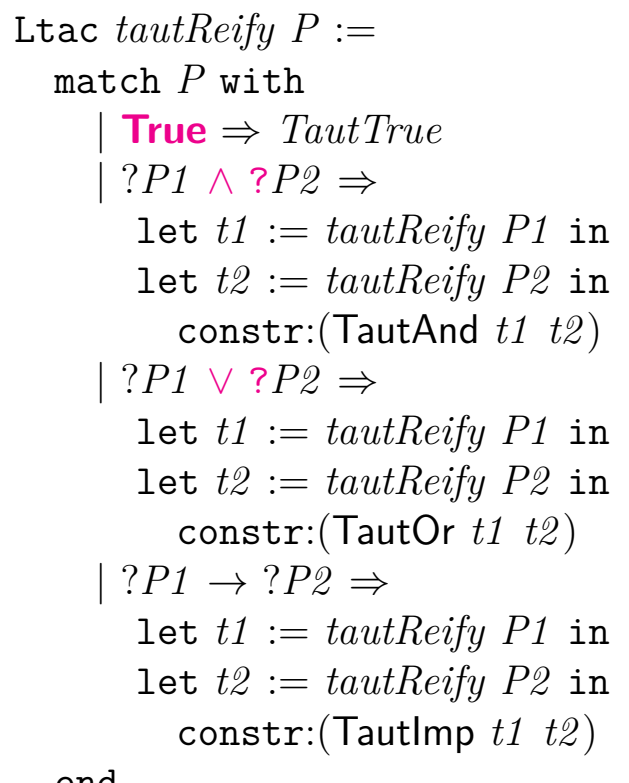

end.

With tautReify available, it is easy to finish our reflective tactic. We look at the goal formula, reify it, and apply tautTrue to the reified formula.

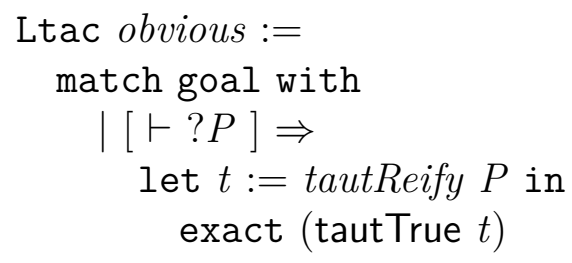

end. 
We can verify that obvious solves our original example, with a proof term that does not mention details of the proof.

Theorem true_galore' $:($ True $\wedge$ True $) \rightarrow($ True $\vee($ True $\wedge($ True $\rightarrow$ True $)))$. obvious.

Qed.

Print true_galore'.

true_galore' $=$

tautTrue

(Tautlmp (TautAnd TautTrue TautTrue)

(TautOr TautTrue (TautAnd TautTrue (Tautlmp TautTrue TautTrue))))

$:$ True $\wedge$ True $\rightarrow$ True $\vee$ True $\wedge$ (True $\rightarrow$ True $)$

It is worth considering how the reflective tactic improves on a pure-Ltac implementation. The formula reification process is just as ad-hoc as before, so we gain little there. In general, proofs will be more complicated than formula translation, and the "generic proof rule" that we apply here $i s$ on much better formal footing than a recursive Ltac function. The dependent type of the proof guarantees that it "works" on any input formula. This benefit is in addition to the proof-size improvement that we have already seen.

It may also be worth pointing out that our previous example of evenness testing used a function partialOut for sound handling of input goals that the verified decision procedure fails to prove. Here, we prove that our procedure tautTrue (recall that an inductive proof may be viewed as a recursive procedure) is able to prove any goal representable in taut, so no extra step is necessary.

\subsection{A Monoid Expression Simplifier}

Proof by reflection does not require encoding of all of the syntax in a goal. We can insert "variables" in our syntax types to allow injection of arbitrary pieces, even if we cannot apply specialized reasoning to them. In this section, we explore that possibility by writing a tactic for normalizing monoid equations.

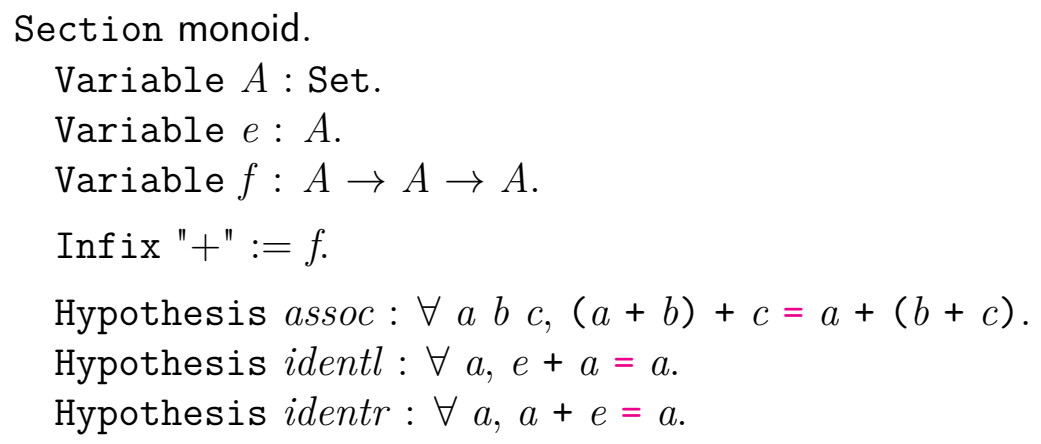

We add variables and hypotheses characterizing an arbitrary instance of the algebraic structure of monoids. We have an associative binary operator and an identity element for it. 
It is easy to define an expression tree type for monoid expressions. A Var constructor is a "catch-all" case for subexpressions that we cannot model. These subexpressions could be actual Gallina variables, or they could just use functions that our tactic is unable to understand.

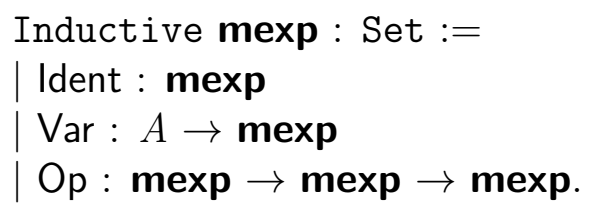

Next, we write an interpretation function.

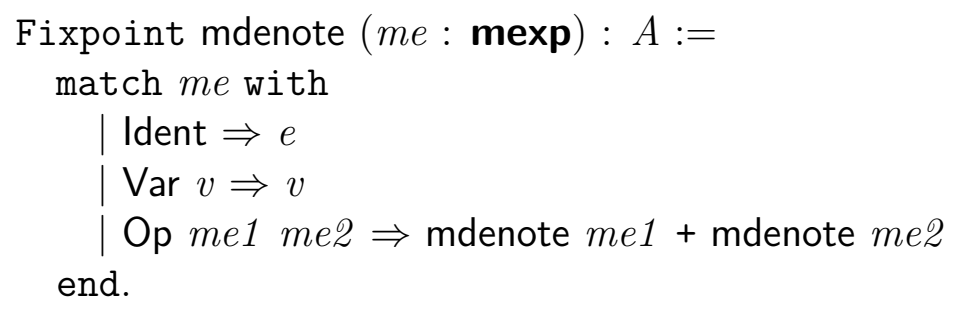

We will normalize expressions by flattening them into lists, via associativity, so it is helpful to have a denotation function for lists of monoid values.

Fixpoint mldenote $(l s:$ list $A): A:=$ match $l s$ with

| nil $\Rightarrow e$

$x:: l s^{\prime} \Rightarrow x+$ mldenote $l s^{\prime}$

end.

The flattening function itself is easy to implement.

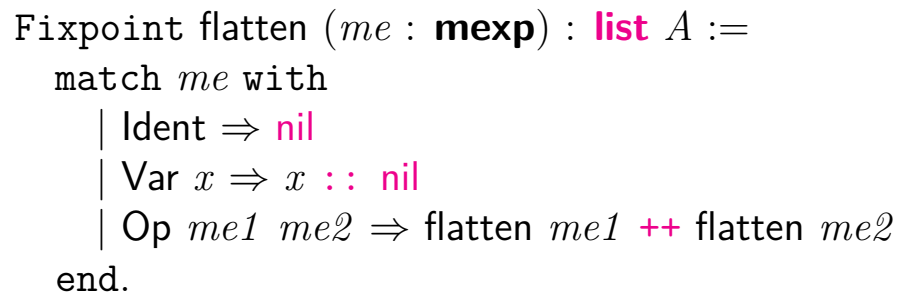

This function has a straightforward correctness proof in terms of our denote functions.

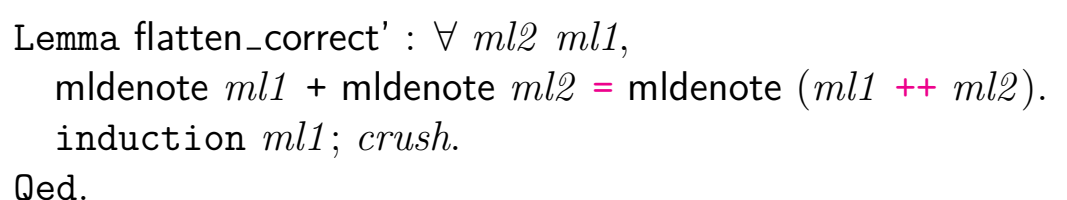

Theorem flatten_correct : $\forall m e$, mdenote $m e=$ mldenote (flatten $m e$ ).

Hint Resolve flatten_correct'.

induction me; crush.

Qed.

Now it is easy to prove a theorem that will be the main tool behind our simplification tactic. 
Theorem monoid_reflect : $\forall$ me1 me2,

mldenote $($ flatten $m e 1)=$ mldenote $($ flatten med $)$

$\rightarrow$ mdenote $m e 1=$ mdenote med.

intros; repeat rewrite flatten_correct; assumption.

Qed.

We implement reification into the mexp type.

Ltac reify $m e:=$

match me with

$e \Rightarrow$ Ident

? me1 + ?me2 $\Rightarrow$

let $r 1:=$ reify me1 in

let $r 2:=$ reify med in

constr:(Op r1 r2)

I _ constr:(Var me)

end.

The final monoid tactic works on goals that equate two monoid terms. We reify each and change the goal to refer to the reified versions, finishing off by applying monoid_reflect and simplifying uses of mldenote. Recall that the change tactic replaces a conclusion formula with another that is definitionally equal to it.

Ltac monoid :=

match goal with

$\mid[\vdash$ ?me1 $=$ ? me2 $] \Rightarrow$

let $r 1:=$ reify me1 in

let $r \mathscr{2}:=$ reify med in

change (mdenote $r 1=$ mdenote $r 2)$;

apply monoid_reflect; simpl

end.

We can make short work of theorems like this one:

Theorem t1: $\forall a b c d, a+b+c+d=a+(b+c)+d$. intros; monoid.

$==\overline{=}=\overline{=}=\overline{=}=\overline{=}=\overline{=}=\overline{=}=\overline{=}=$

$a+(b+(c+(d+e)))=a+(b+(c+(d+e)))$

Our tactic has canonicalized both sides of the equality, such that we can finish the proof by reflexivity.

reflexivity.

Qed.

It is interesting to look at the form of the proof. 
Print t1.

$\mathrm{t} 1=$

fun $a b c d: A \Rightarrow$

monoid_reflect $(\mathrm{Op}(\mathrm{Op}(\mathrm{Op}(\operatorname{Var} a)(\operatorname{Var} b))(\operatorname{Var} c))(\operatorname{Var} d))$

$(\mathrm{Op}(\mathrm{Op}(\operatorname{Var} a)(\mathrm{Op}(\operatorname{Var} b)(\operatorname{Var} c)))(\operatorname{Var} d))$

$($ eq_refl $(a+(b+(c+(d+e)))))$

$: \forall a b c d: A, a+b+c+d=a+(b+c)+d$

The proof term contains only restatements of the equality operands in reified form, followed by a use of reflexivity on the shared canonical form.

End monoid.

Extensions of this basic approach are used in the implementations of the ring and field tactics that come packaged with Coq.

\subsection{A Smarter Tautology Solver}

Now we are ready to revisit our earlier tautology solver example. We want to broaden the scope of the tactic to include formulas whose truth is not syntactically apparent. We will want to allow injection of arbitrary formulas, like we allowed arbitrary monoid expressions in the last example. Since we are working in a richer theory, it is important to be able to use equalities between different injected formulas. For instance, we cannot prove $P \rightarrow P$ by translating the formula into a value like Imp $(\operatorname{Var} P)(\operatorname{Var} P)$, because a Gallina function has no way of comparing the two $P$ s for equality.

To arrive at a nice implementation satisfying these criteria, we introduce the quote tactic and its associated library.

Require Import Quote.

Inductive formula : Set :=

| Atomic : index $\rightarrow$ formula

| Truth : formula

Falsehood : formula

And : formula $\rightarrow$ formula $\rightarrow$ formula

Or : formula $\rightarrow$ formula $\rightarrow$ formula

Imp : formula $\rightarrow$ formula $\rightarrow$ formula.

The type index comes from the Quote library and represents a countable variable type. The rest of formula's definition should be old hat by now.

The quote tactic will implement injection from Prop into formula for us, but it is not quite as smart as we might like. In particular, it wants to treat function types specially, so it gets confused if function types are part of the structure we want to encode syntactically. To trick quote into not noticing our uses of function types to express logical implication, we will need to declare a wrapper definition for implication, as we did in the last chapter.

Definition imp $(P 1$ P2 $:$ Prop $):=P 1 \rightarrow P \mathscr{2}$ 
Infix "->":= imp (no associativity, at level 95).

Now we can define our denotation function.

Definition asgn := varmap Prop.

Fixpoint formulaDenote (atomics : asgn) $(f$ : formula) : Prop $:=$ match $f$ with

| Atomic $v \Rightarrow$ varmap_find False $v$ atomics

Truth $\Rightarrow$ True

Falsehood $\Rightarrow$ False

And $f 1 f 2 \Rightarrow$ formulaDenote atomics $f 1 \wedge$ formulaDenote atomics f2

Or $f 1 f 2 \Rightarrow$ formulaDenote atomics $f 1 \vee$ formulaDenote atomics $f 2$

| Imp $f 1$ f2 $\Rightarrow$ formulaDenote atomics $f 1 \rightarrow$ formulaDenote atomics f2 end.

The varmap type family implements maps from index values. In this case, we define an assignment as a map from variables to Props. Our interpretation function formulaDenote works with an assignment, and we use the varmap_find function to consult the assignment in the Atomic case. The first argument to varmap_find is a default value, in case the variable is not found.

Section my_tauto.

Variable atomics : asgn.

Definition holds $(v$ : index) $:=$ varmap_find False $v$ atomics.

We define some shorthand for a particular variable being true, and now we are ready to define some helpful functions based on the ListSet module of the standard library, which (unsurprisingly) presents a view of lists as sets.

Require Import ListSet.

Definition index_eq : $\forall x y$ : index, $\{x=y\}+\{x \neq y\}$.

decide equality.

Defined.

Definition add $(s:$ set index) $(v$ : index $):=$ set_add index_eq $v s$.

Definition In_dec: $\forall v(s$ : set index), $\{\ln v s\}+\{\neg \ln v s\}$.

Local Open Scope specif_scope.

intro; refine (fix $F(s:$ set index) : $\{\ln v s\}+\{\neg \ln v s\}:=$

match $s$ with

| nil $\Rightarrow$ No

| $v^{\prime}:: s^{\prime} \Rightarrow$ index_eq $v^{\prime} v|| F s^{\prime}$

end); crush.

Defined.

We define what it means for all members of an index set to represent true propositions, and we prove some lemmas about this notion.

Fixpoint allTrue $(s$ : set index) : Prop := 


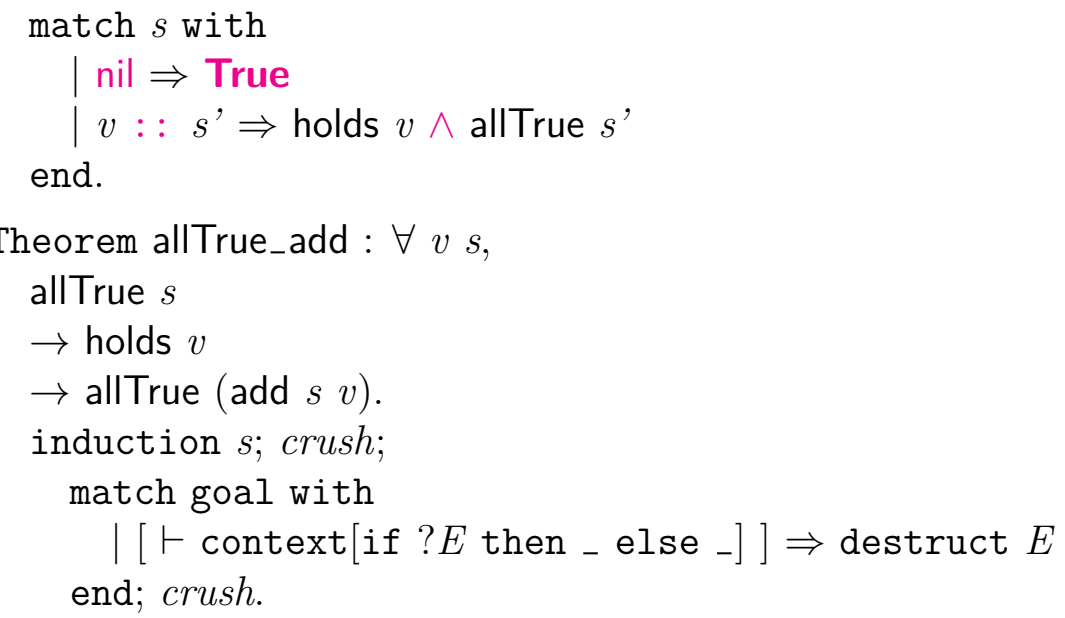

Qed.

Theorem allTrue_In : $\forall v s$, allTrue $s$

$\rightarrow$ set_ln $v s$

$\rightarrow$ varmap_find False $v$ atomics. induction $s$; crush.

Qed.

Hint Resolve allTrue_add allTrue_In.

Local Open Scope partial_scope.

Now we can write a function forward that implements deconstruction of hypotheses, expanding a compound formula into a set of sets of atomic formulas covering all possible cases introduced with use of Or. To handle consideration of multiple cases, the function takes in a continuation argument, which will be called once for each case.

The forward function has a dependent type, in the style of Chapter 6, guaranteeing correctness. The arguments to forward are a goal formula $f$, a set known of atomic formulas that we may assume are true, a hypothesis formula hyp, and a success continuation cont that we call when we have extended known to hold new truths implied by hyp.

Definition forward : $\forall$ ( $f$ : formula) (known : set index) (hyp : formula)

(cont : $\forall$ known', [allTrue known' $\rightarrow$ formulaDenote atomics $f$ ] ),

[allTrue known $\rightarrow$ formulaDenote atomics hyp $\rightarrow$ formulaDenote atomics $f$ ] .

refine (fix $F$ ( $f$ : formula) (known : set index) (hyp : formula)

(cont : $\forall$ known', [allTrue known' $\rightarrow$ formulaDenote atomics $f$ ] )

$:$ [allTrue known $\rightarrow$ formulaDenote atomics hyp $\rightarrow$ formulaDenote atomics $f$ ] := match hyp with

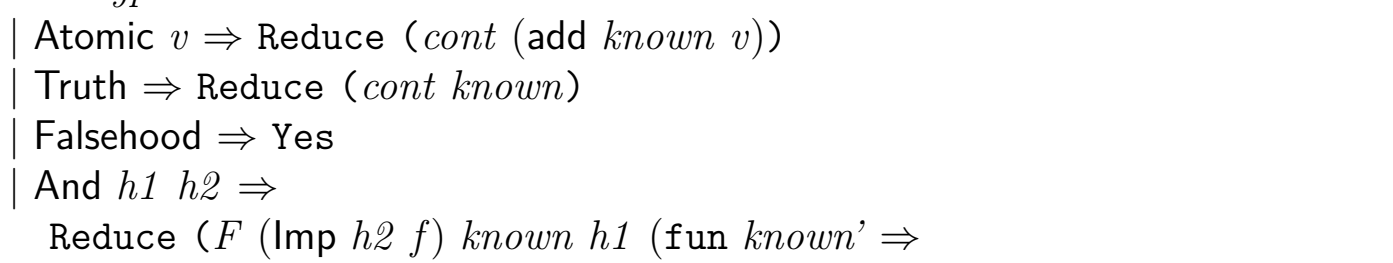


Reduce ( $F$ f known' h2 cont) ))

| Or h1 h2 $\Rightarrow F f$ known h1 cont \&\& $F f$ known h2 cont

| Imp _ _ Reduce (cont known)

end); crush.

Defined.

A backward function implements analysis of the final goal. It calls forward to handle implications.

Definition backward : $\forall$ (known : set index) $(f$ : formula),

[allTrue known $\rightarrow$ formulaDenote atomics $f$ ].

refine (fix $F$ (known : set index) ( $f$ : formula)

$:$ [allTrue known $\rightarrow$ formulaDenote atomics $f]:=$ match $f$ with

| Atomic $v \Rightarrow$ Reduce (In_dec $v$ known)

Truth $\Rightarrow$ Yes

Falsehood $\Rightarrow$ No

And $f 1 f \mathscr{2} \Rightarrow F$ known $f 1$ \&\& $F$ known $f 2$

| Or $f 1$ f2 $\Rightarrow F$ known $f 1|| F$ known f2

I Imp $f 1$ f2 $\Rightarrow$ forward f2 known f1 (fun known' $\Rightarrow F$ known' f2)

end); crush; eauto.

Defined.

A simple wrapper around backward gives us the usual type of a partial decision procedure.

Definition my_tauto: $\forall f$ : formula, [formulaDenote atomics $f$ ].

intro; refine (Reduce (backward nil f)); crush.

Defined.

End my_tauto.

Our final tactic implementation is now fairly straightforward. First, we intro all quantifiers that do not bind Props. Then we call the quote tactic, which implements the reification for us. Finally, we are able to construct an exact proof via partialOut and the my_tauto Gallina function.

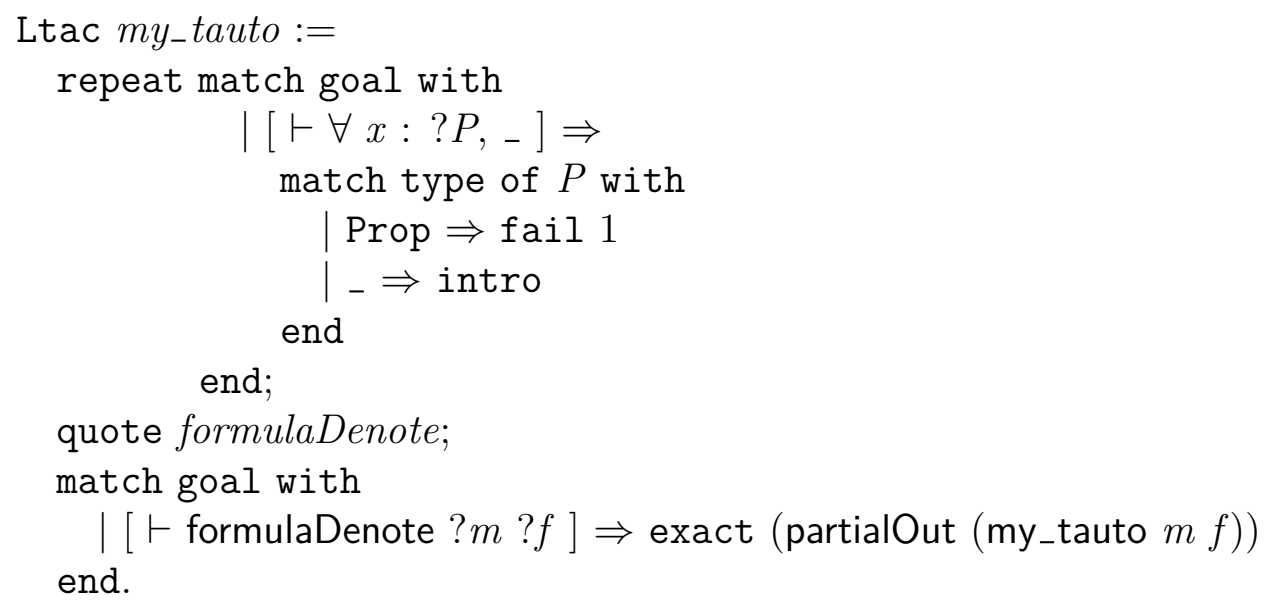


A few examples demonstrate how the tactic works.

Theorem mt1: True.

my_tauto.

Qed.

Print mt1.

$\mathrm{mt1}=$ partialOut (my_tauto (Empty_vm Prop) Truth)

: True

We see my_tauto applied with an empty varmap, since every subformula is handled by formulaDenote.

Theorem mt2: $\forall x y:$ nat, $x=y \rightarrow x=y$.

my_tauto.

Qed.

Print mt2.

$\mathrm{mt} 2=$

fun $x y:$ nat $\Rightarrow$

partialOut

(my_tauto (Node_vm $(x=y)$ (Empty_vm Prop) (Empty_vm Prop))

$(\operatorname{Imp}($ Atomic End_idx) (Atomic End_idx)))

$: \forall x y:$ nat, $x=y \rightarrow x=y$

Crucially, both instances of $x=y$ are represented with the same index, End_idx. The value of this index only needs to appear once in the varmap, whose form reveals that varmaps are represented as binary trees, where index values denote paths from tree roots to leaves.

Theorem mt3: $\forall x y z$,

$(x<y \wedge y>z) \vee(y>z \wedge x<\mathrm{S} y)$

$\rightarrow y>z \wedge(x<y \vee x<\mathrm{S} y)$.

my_tauto.

Qed.

Print mt3.

fun $x y z:$ nat $\Rightarrow$

partialOut

(my_tauto

(Node_vm $(x<\mathrm{S} y)$ (Node_vm $(x<y)$ (Empty_vm Prop) (Empty_vm Prop))

(Node_vm $(y>z)$ (Empty_vm Prop) (Empty_vm Prop)))

(Imp

(Or (And (Atomic (Left_idx End_idx)) (Atomic (Right_idx End_idx)))

(And (Atomic (Right_idx End_idx)) (Atomic End_idx)))

(And (Atomic (Right_idx End_idx))

(Or (Atomic (Left_idx End_idx)) (Atomic End_idx))))) 


$$
\begin{aligned}
& : \forall x y z: \text { nat, } \\
& \quad x<y \wedge y>z \vee y>z \wedge x<\mathrm{S} y->y>z \wedge(x<y \vee x<\mathrm{S} y)
\end{aligned}
$$

Our goal contained three distinct atomic formulas, and we see that a three-element varmap is generated.

It can be interesting to observe differences between the level of repetition in proof terms generated by my_tauto and tauto for especially trivial theorems.

Theorem mt4 : True $\wedge$ True $\wedge$ True $\wedge$ True $\wedge$ True $\wedge$ True $\wedge$ False $\rightarrow$ False.

my_tauto.

Qed.

Print mt4.

$\mathrm{mt} 4=$

partialOut

(my_tauto (Empty_vm Prop)

$(\operatorname{Imp}$

(And Truth

(And Truth

(And Truth (And Truth (And Truth (And Truth Falsehood))))))

Falsehood))

$:$ True $\wedge$ True $\wedge$ True $\wedge$ True $\wedge$ True $\wedge$ True $\wedge$ False $->$ False

Theorem mt4' $:$ True $\wedge$ True $\wedge$ True $\wedge$ True $\wedge$ True $\wedge$ True $\wedge$ False $\rightarrow$ False.

tauto.

Qed.

Print mt4'.

$\mathrm{mt}^{\prime}=$

fun $H:$ True $\wedge$ True $\wedge$ True $\wedge$ True $\wedge$ True $\wedge$ True $\wedge$ False $\Rightarrow$ and_ind

(fun $(-:$ True $)(H 1:$ True $\wedge$ True $\wedge$ True $\wedge$ True $\wedge$ True $\wedge$ False $) \Rightarrow$ and_ind

(fun $(-:$ True $)(H 3:$ True $\wedge$ True $\wedge$ True $\wedge$ True $\wedge$ False $) \Rightarrow$ and_ind

(fun (- : True) $(H 5:$ True $\wedge$ True $\wedge$ True $\wedge$ False $) \Rightarrow$ and_ind

(fun (_: True) $\left(H^{7} 7:\right.$ True $\wedge$ True $\wedge$ False $) \Rightarrow$ and_ind

(fun $($ _ : True $)(H 9:$ True $\wedge$ False $) \Rightarrow$

and_ind (fun (_ : True) (H11 : False) $\Rightarrow$ False_ind False H11)

H9) $H^{\text {r }}$ ) H5) H3) $\left.H 1\right) H$

$:$ True $\wedge$ True $\wedge$ True $\wedge$ True $\wedge$ True $\wedge$ True $\wedge$ False $\rightarrow$ False

The traditional tauto tactic introduces a quadratic blow-up in the size of the proof term, whereas proofs produced by my_tauto always have linear size. 


\subsubsection{Manual Reification of Terms with Variables}

The action of the quote tactic above may seem like magic. Somehow it performs equality comparison between subterms of arbitrary types, so that these subterms may be represented with the same reified variable. While quote is implemented in OCaml, we can code the reification process completely in Ltac, as well. To make our job simpler, we will represent variables as nats, indexing into a simple list of variable values that may be referenced.

Step one of the process is to crawl over a term, building a duplicate-free list of all values that appear in positions we will encode as variables. A useful helper function adds an element to a list, preventing duplicates. Note how we use Ltac pattern matching to implement an equality test on Gallina terms; this is simple syntactic equality, not even the richer definitional equality. We also represent lists as nested tuples, to allow different list elements to have different Gallina types.

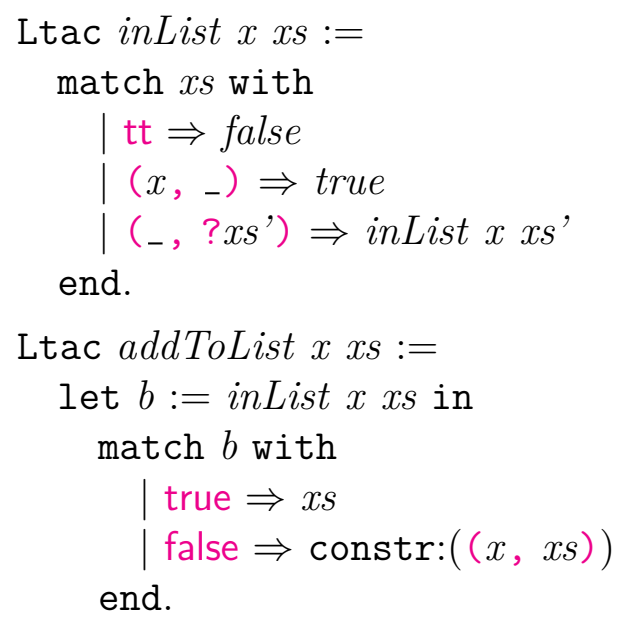

Now we can write our recursive function to calculate the list of variable values we will want to use to represent a term.

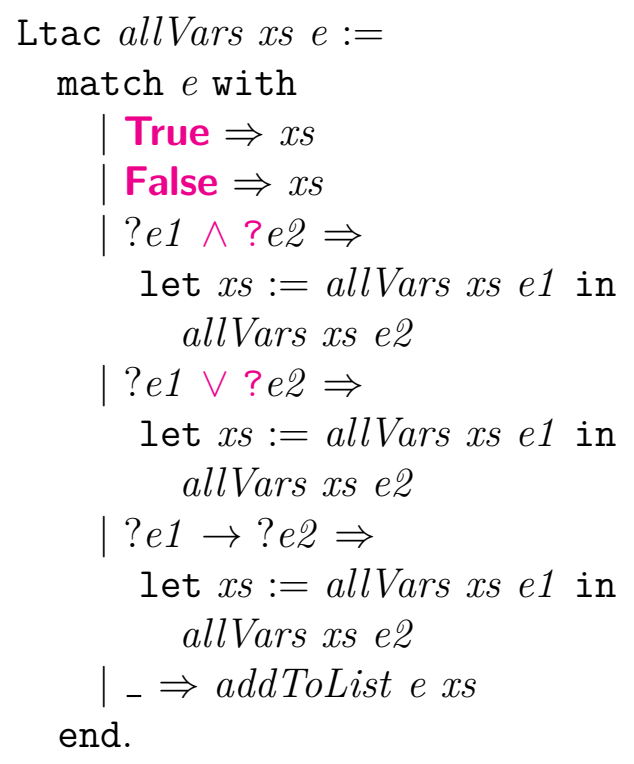


We will also need a way to map a value to its position in a list.

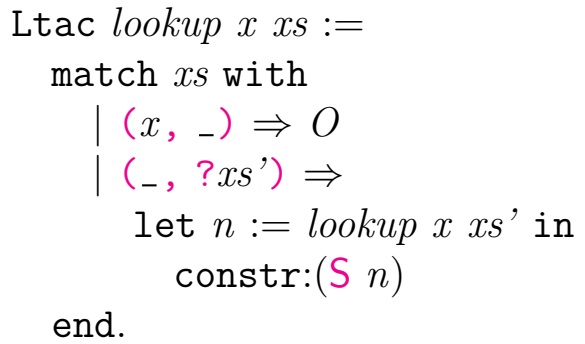

The next building block is a procedure for reifying a term, given a list of all allowed variable values. We are free to make this procedure partial, where tactic failure may be triggered upon attempting to reify a term containing subterms not included in the list of variables. The type of the output term is a copy of formula where index is replaced by nat, in the type of the constructor for atomic formulas.

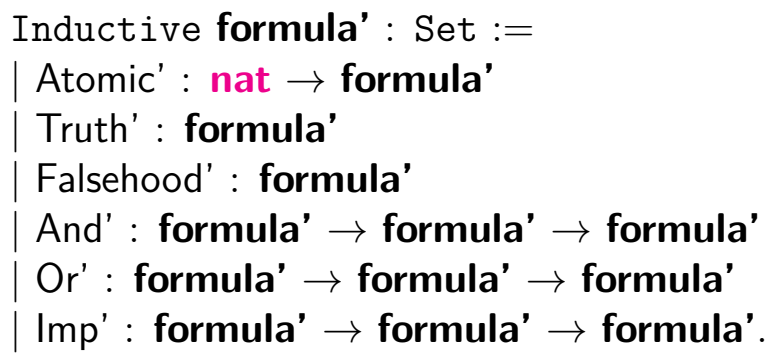

Note that, when we write our own Ltac procedure, we can work directly with the normal $\rightarrow$ operator, rather than needing to introduce a wrapper for it.

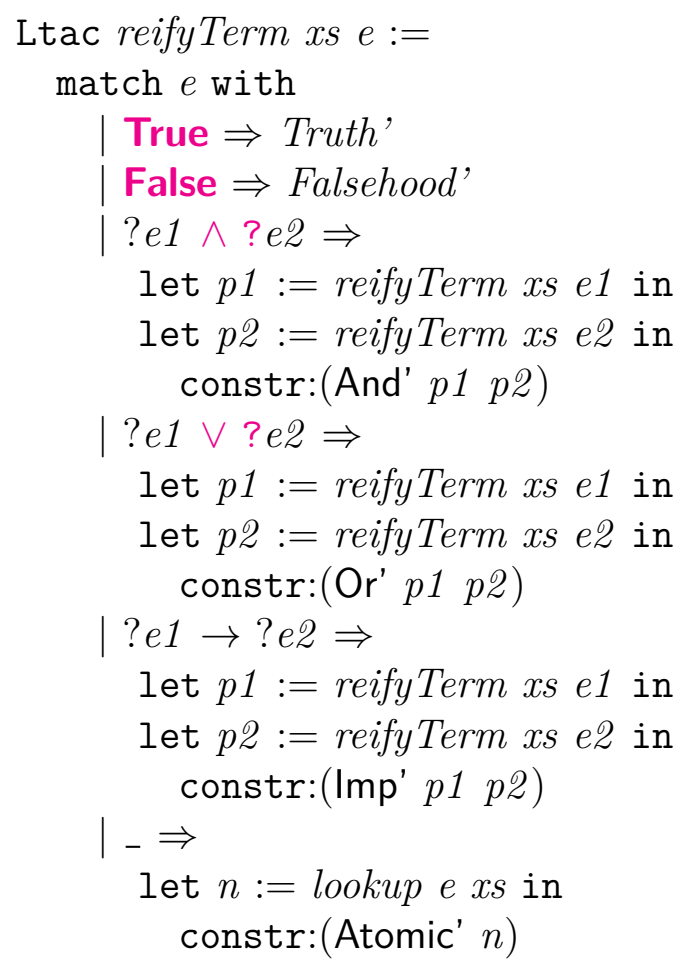


end.

Finally, we bring all the pieces together.

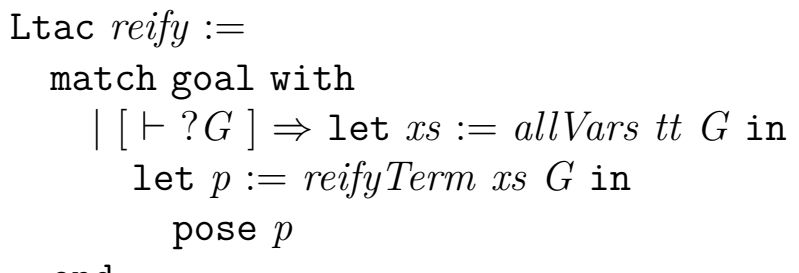

end.

A quick test verifies that we are doing reification correctly.

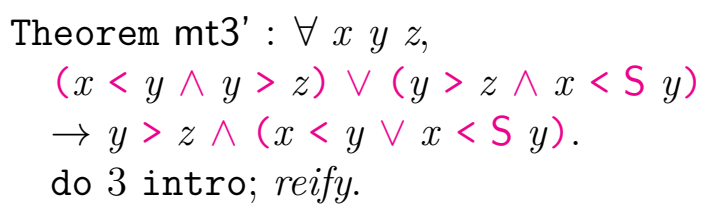

Our simple tactic adds the translated term as a new variable:

$f:=\operatorname{Imp}$

(Or' (And' (Atomic' 2) (Atomic' 1)) (And' (Atomic' 1) (Atomic' 0)))

(And' (Atomic' 1) (Or' (Atomic' 2) (Atomic' 0))) : formula'

Abort.

More work would be needed to complete the reflective tactic, as we must connect our new syntax type with the real meanings of formulas, but the details are the same as in our prior implementation with quote.

\subsection{Building a Reification Tactic that Recurses Under Binders}

All of our examples so far have stayed away from reifying the syntax of terms that use such features as quantifiers and fun function abstractions. Such cases are complicated by the fact that different subterms may be allowed to reference different sets of free variables. Some cleverness is needed to clear this hurdle, but a few simple patterns will suffice. Consider this example of a simple dependently typed term language, where a function abstraction body is represented conveniently with a Coq function.

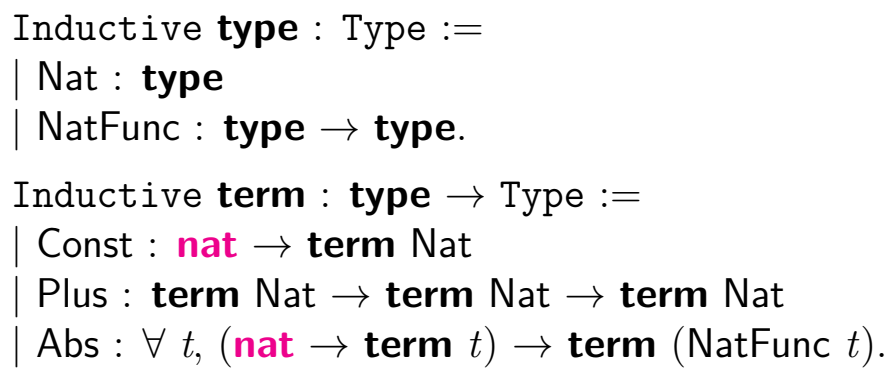




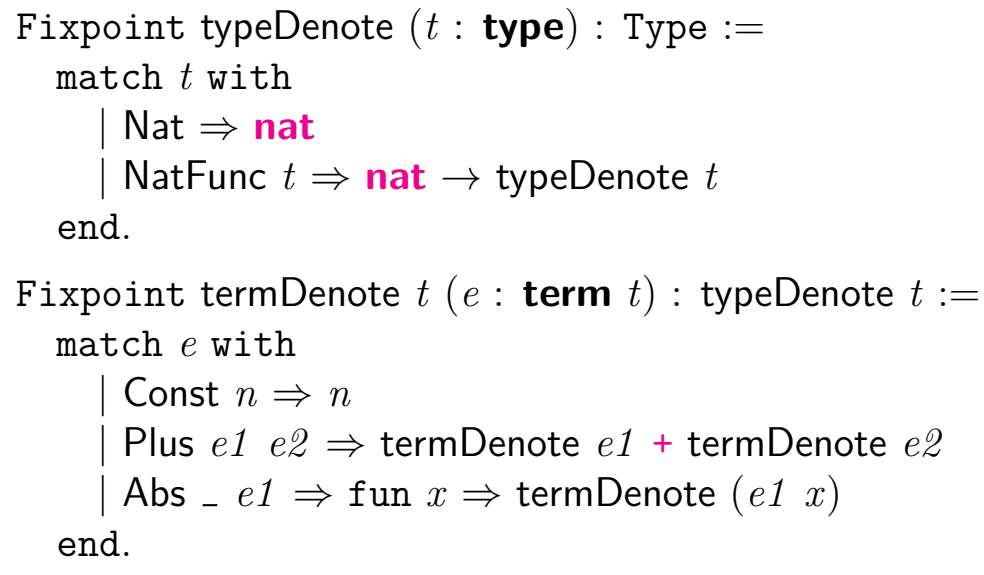

Here is a naïve first attempt at a reification tactic.

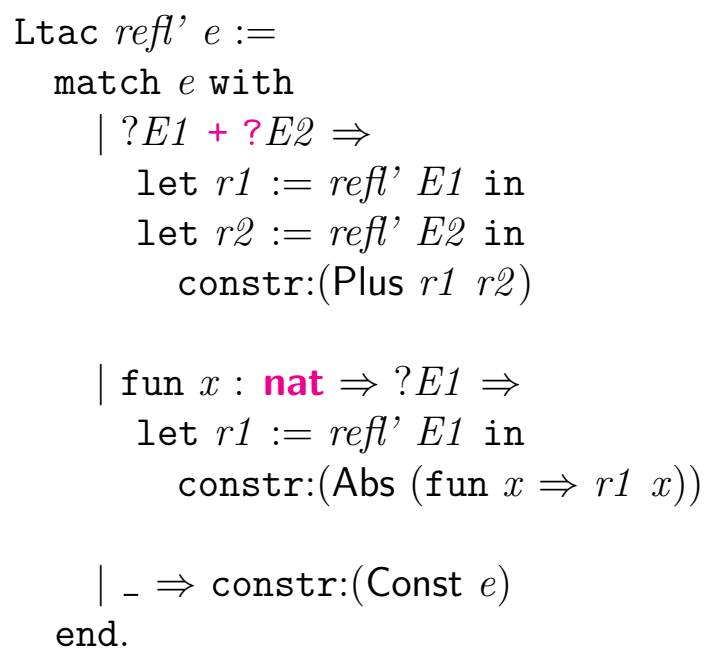

Recall that a regular Ltac pattern variable ?X only matches terms that do not mention new variables introduced within the pattern. In our naïve implementation, the case for matching function abstractions matches the function body in a way that prevents it from mentioning the function argument! Our code above plays fast and loose with the function body in a way that leads to independent problems, but we could change the code so that it indeed handles function abstractions that ignore their arguments.

To handle functions in general, we will use the pattern variable form @? $X$, which allows $X$ to mention newly introduced variables that are declared explicitly. A use of @? $X$ must be followed by a list of the local variables that may be mentioned. The variable $X$ then comes to stand for a Gallina function over the values of those variables. For instance:

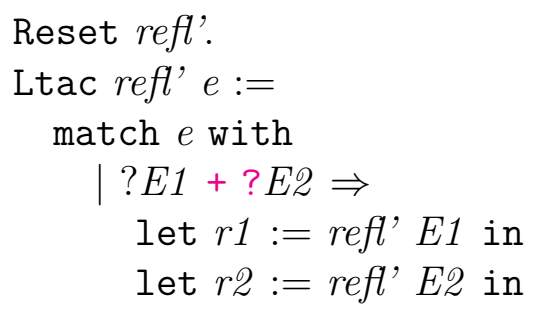




$$
\begin{aligned}
& \text { constr:(Plus } r 1 \text { r2) } \\
& \mid \text { fun } x: \text { nat } \Rightarrow @ ? E 1 x \Rightarrow \\
& \text { let } r 1:=\text { refl' } E 1 \text { in } \\
& \text { constr:(Abs } r 1) \\
& \left.\mid{ }_{-} \Rightarrow \text { constr:(Const } e\right) \\
& \text { end. }
\end{aligned}
$$

Now, in the abstraction case, we bind $E 1$ as a function from an $x$ value to the value of the abstraction body. Unfortunately, our recursive call there is not destined for success. It will match the same abstraction pattern and trigger another recursive call, and so on through infinite recursion. One last refactoring yields a working procedure. The key idea is to consider every input to refl' as a function over the values of variables introduced during recursion.

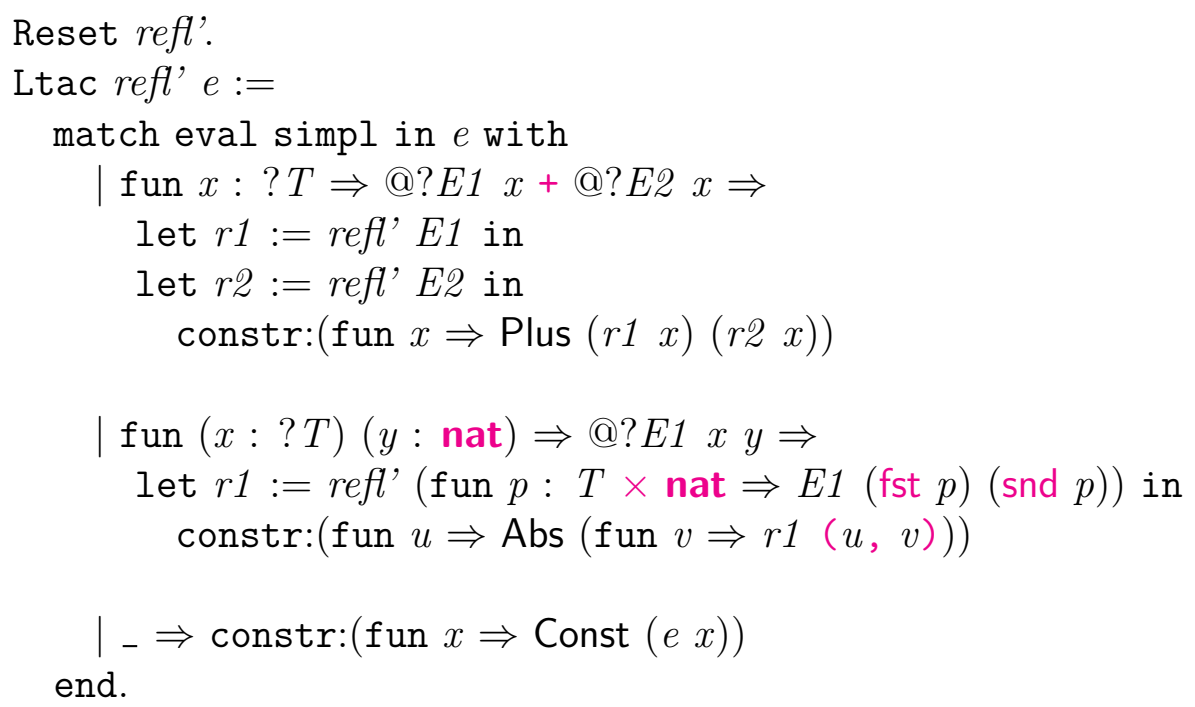

Note how now even the addition case works in terms of functions, with @? $X$ patterns. The abstraction case introduces a new variable by extending the type used to represent the free variables. In particular, the argument to refl' used type $T$ to represent all free variables. We extend the type to $T \times$ nat for the type representing free variable values within the abstraction body. A bit of bookkeeping with pairs and their projections produces an appropriate version of the abstraction body to pass in a recursive call. To ensure that all this repackaging of terms does not interfere with pattern matching, we add an extra simpl reduction on the function argument, in the first line of the body of refl'.

Now one more tactic provides an example of how to apply reification. Let us consider goals that are equalities between terms that can be reified. We want to change such goals into equalities between appropriate calls to termDenote.

Ltac refl :=

match goal with 


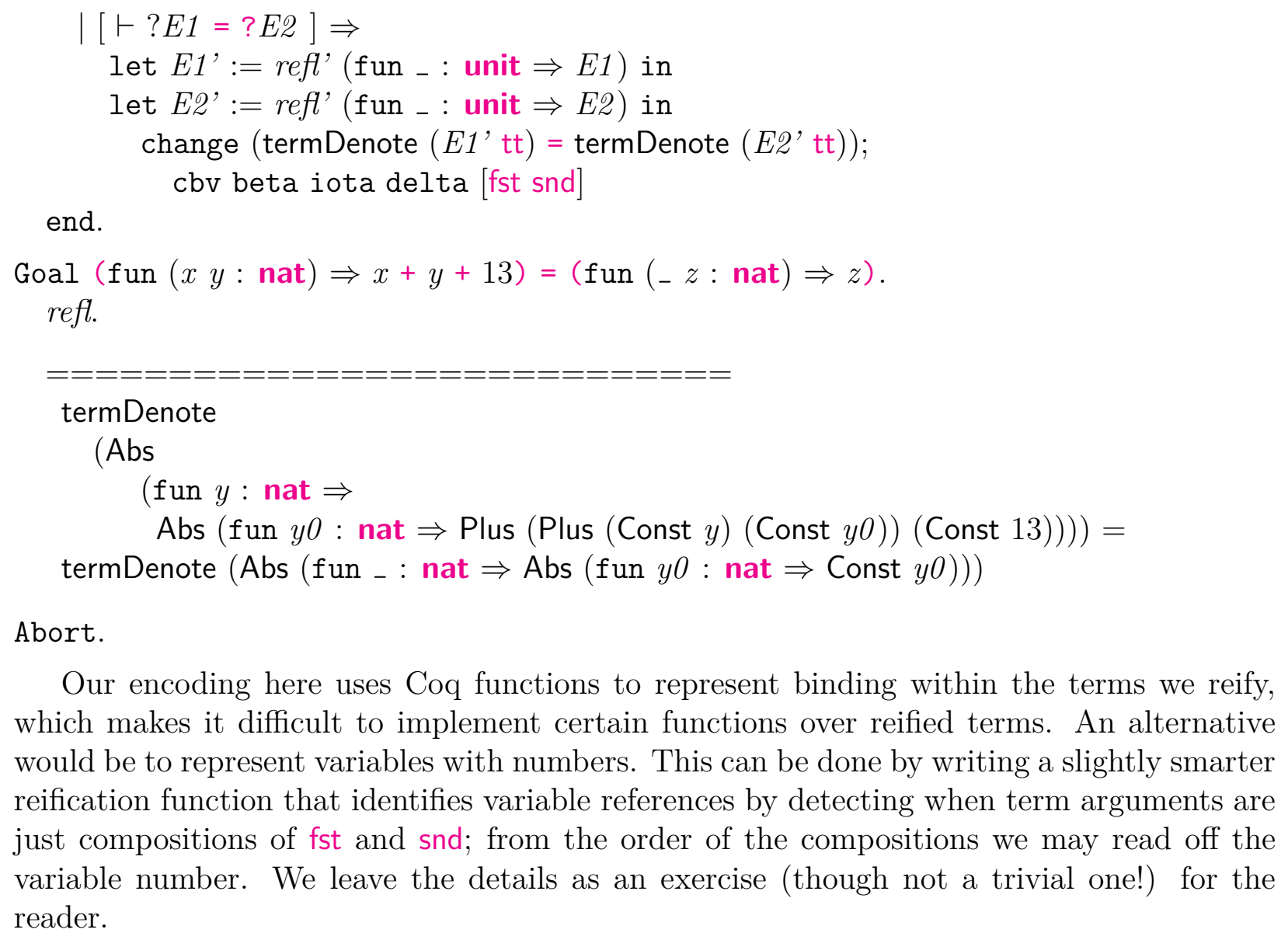

Our encoding here uses Coq functions to represent binding within the terms we reify, which makes it difficult to implement certain functions over reified terms. An alternative would be to represent variables with numbers. This can be done by writing a slightly smarter reification function that identifies variable references by detecting when term arguments are just compositions of fst and snd; from the order of the compositions we may read off the variable number. We leave the details as an exercise (though not a trivial one!) for the reader. 


\section{Part IV}

\section{The Big Picture}




\section{Chapter 16}

\section{Proving in the Large}

It is somewhat unfortunate that the term "theorem proving" looks so much like the word "theory." Most researchers and practitioners in software assume that mechanized theorem proving is profoundly impractical. Indeed, until recently, most advances in theorem proving for higher-order logics have been largely theoretical. However, starting around the beginning of the 21st century, there was a surge in the use of proof assistants in serious verification efforts. That line of work is still quite new, but I believe it is not too soon to distill some lessons on how to work effectively with large formal proofs.

Thus, this chapter gives some tips for structuring and maintaining large Coq developments.

\subsection{Ltac Anti-Patterns}

In this book, I have been following an unusual style, where proofs are not considered finished until they are "fully automated," in a certain sense. Each such theorem is proved by a single tactic. Since Ltac is a Turing-complete programming language, it is not hard to squeeze arbitrary heuristics into single tactics, using operators like the semicolon to combine steps. In contrast, most Ltac proofs "in the wild" consist of many steps, performed by individual tactics followed by periods. Is it really worth drawing a distinction between proof steps terminated by semicolons and steps terminated by periods?

I argue that this is, in fact, a very important distinction, with serious consequences for a majority of important verification domains. The more uninteresting drudge work a proof domain involves, the more important it is to work to prove theorems with single tactics. From an automation standpoint, single-tactic proofs can be extremely effective, and automation becomes more and more critical as proofs are populated by more uninteresting detail. In this section, I will give some examples of the consequences of more common proof styles.

As a running example, consider a basic language of arithmetic expressions, an interpreter for it, and a transformation that scales up every constant in an expression.

Inductive exp : Set :=

| Const : nat $\rightarrow$ exp 


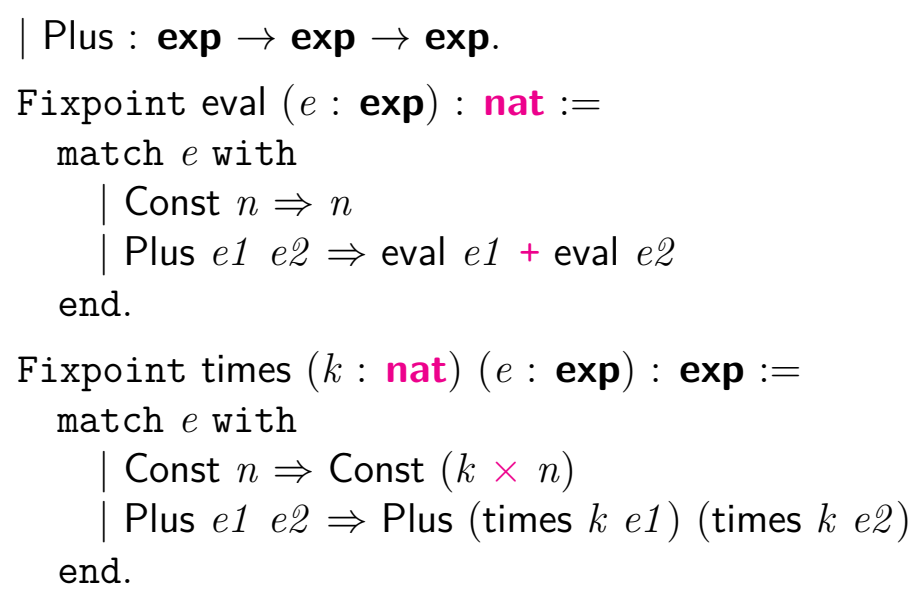

We can write a very manual proof that times really implements multiplication.

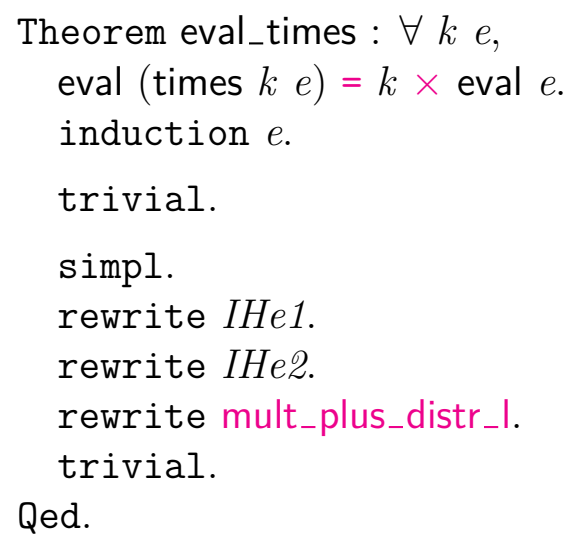

We use spaces to separate the two inductive cases, but note that these spaces have no real semantic content; Coq does not enforce that our spacing matches the real case structure of a proof. The second case mentions automatically generated hypothesis names explicitly. As a result, innocuous changes to the theorem statement can invalidate the proof.

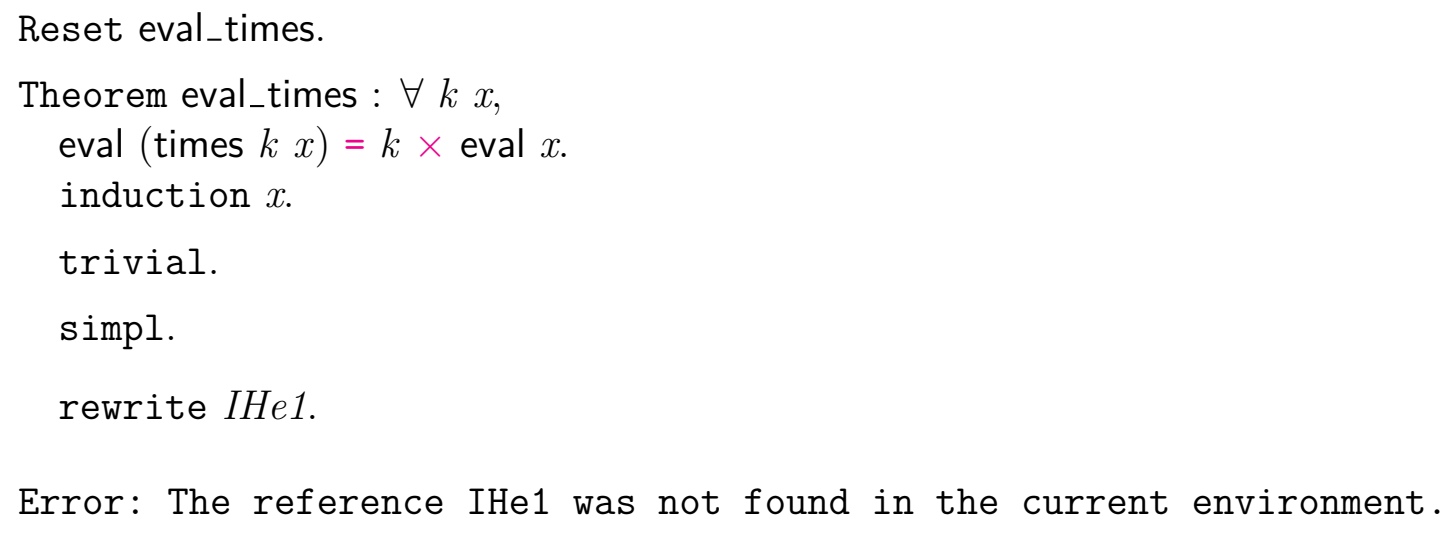

The inductive hypotheses are named IHxI and IHx2 now, not IHe1 and IHe2. Abort. 
We might decide to use a more explicit invocation of induction to give explicit binders for all of the names that we will reference later in the proof.

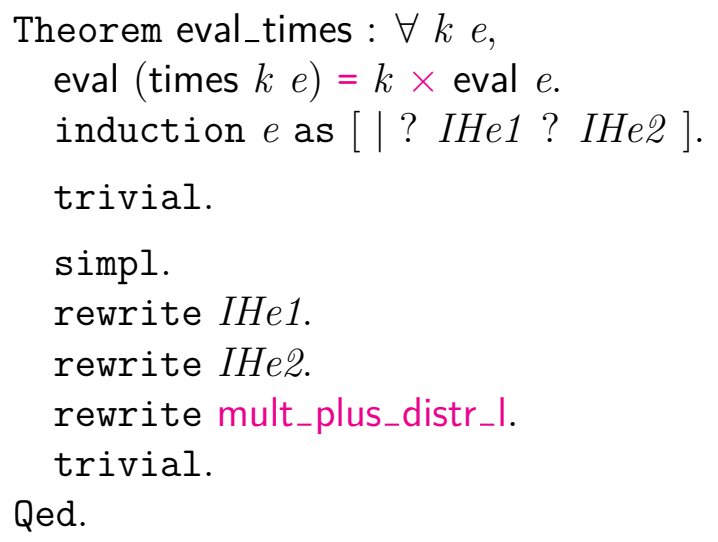

We pass induction an intro pattern, using a / character to separate instructions for the different inductive cases. Within a case, we write ? to ask Coq to generate a name automatically, and we write an explicit name to assign that name to the corresponding new variable. It is apparent that, to use intro patterns to avoid proof brittleness, one needs to keep track of the seemingly unimportant facts of the orders in which variables are introduced. Thus, the script keeps working if we replace $e$ by $x$, but it has become more cluttered. Arguably, neither proof is particularly easy to follow.

That category of complaint has to do with understanding proofs as static artifacts. As with programming in general, with serious projects, it tends to be much more important to be able to support evolution of proofs as specifications change. Unstructured proofs like the above examples can be very hard to update in concert with theorem statements. For instance, consider how the last proof script plays out when we modify times to introduce a bug.

Reset times.

Fixpoint times ( $k:$ nat) $(e: \exp ): \exp :=$ match $e$ with

| Const $n \Rightarrow$ Const $(1+k \times n)$

| Plus $e 1$ e2 $\Rightarrow$ Plus (times $k$ e1) (times $k$ e2) end.

Theorem eval_times : $\forall k e$, eval $($ times $k e)=k \times$ eval $e$. induction $e$ as [|? IHe1? IHe2 ].

trivial.

simpl.

rewrite $I H e 1$.

Error: The reference IHe1 was not found in the current environment. 
Abort.

Can you spot what went wrong, without stepping through the script step-by-step? The problem is that trivial never fails. Originally, trivial had been succeeding in proving an equality that follows by reflexivity. Our change to times leads to a case where that equality is no longer true. The invocation trivial happily leaves the false equality in place, and we continue on to the span of tactics intended for the second inductive case. Unfortunately, those tactics end up being applied to the first case instead.

The problem with trivial could be "solved" by writing, e.g., solve [ trivial ] instead, so that an error is signaled early on if something unexpected happens. However, the root problem is that the syntax of a tactic invocation does not imply how many subgoals it produces. Much more confusing instances of this problem are possible. For example, if a lemma $L$ is modified to take an extra hypothesis, then uses of apply $L$ will generate more subgoals than before. Old unstructured proof scripts will become hopelessly jumbled, with tactics applied to inappropriate subgoals. Because of the lack of structure, there is usually relatively little to be gleaned from knowledge of the precise point in a proof script where an error is raised.

Reset times.

Fixpoint times $(k:$ nat $)(e: \exp ): \exp :=$

match $e$ with

| Const $n \Rightarrow$ Const $(k \times n)$

| Plus $e 1$ e2 $\Rightarrow$ Plus (times $k$ e1) (times $k$ e2)

end.

Many real developments try to make essentially unstructured proofs look structured by applying careful indentation conventions, idempotent case-marker tactics included solely to serve as documentation, and so on. All of these strategies suffer from the same kind of failure of abstraction that was just demonstrated. I like to say that if you find yourself caring about indentation in a proof script, it is a sign that the script is structured poorly.

We can rewrite the current proof with a single tactic.

Theorem eval_times : $\forall k e$,

eval (times $k e)=k \times$ eval $e$.

induction $e$ as [|? IHe1? IHe2 ]; [

trivial

| simpl; rewrite $I H e 1$; rewrite $I H e 2$; rewrite mult_plus_distr_l; trivial ].

Qed.

We use the form of the semicolon operator that allows a different tactic to be specified for each generated subgoal. This change improves the robustness of the script: we no longer need to worry about tactics from one case being applied to a different case. Still, the proof script is not especially readable. Probably most readers would not find it helpful in explaining why the theorem is true. The same could be said for scripts using the bullets or curly braces provided by Coq 8.4, which allow code like the above to be stepped through interactively, with periods in place of the semicolons, while representing proof structure in a way that is 
enforced by Coq. Interactive replay of scripts becomes easier, but readability is not really helped.

The situation gets worse in considering extensions to the theorem we want to prove. Let us add multiplication nodes to our exp type and see how the proof fares.

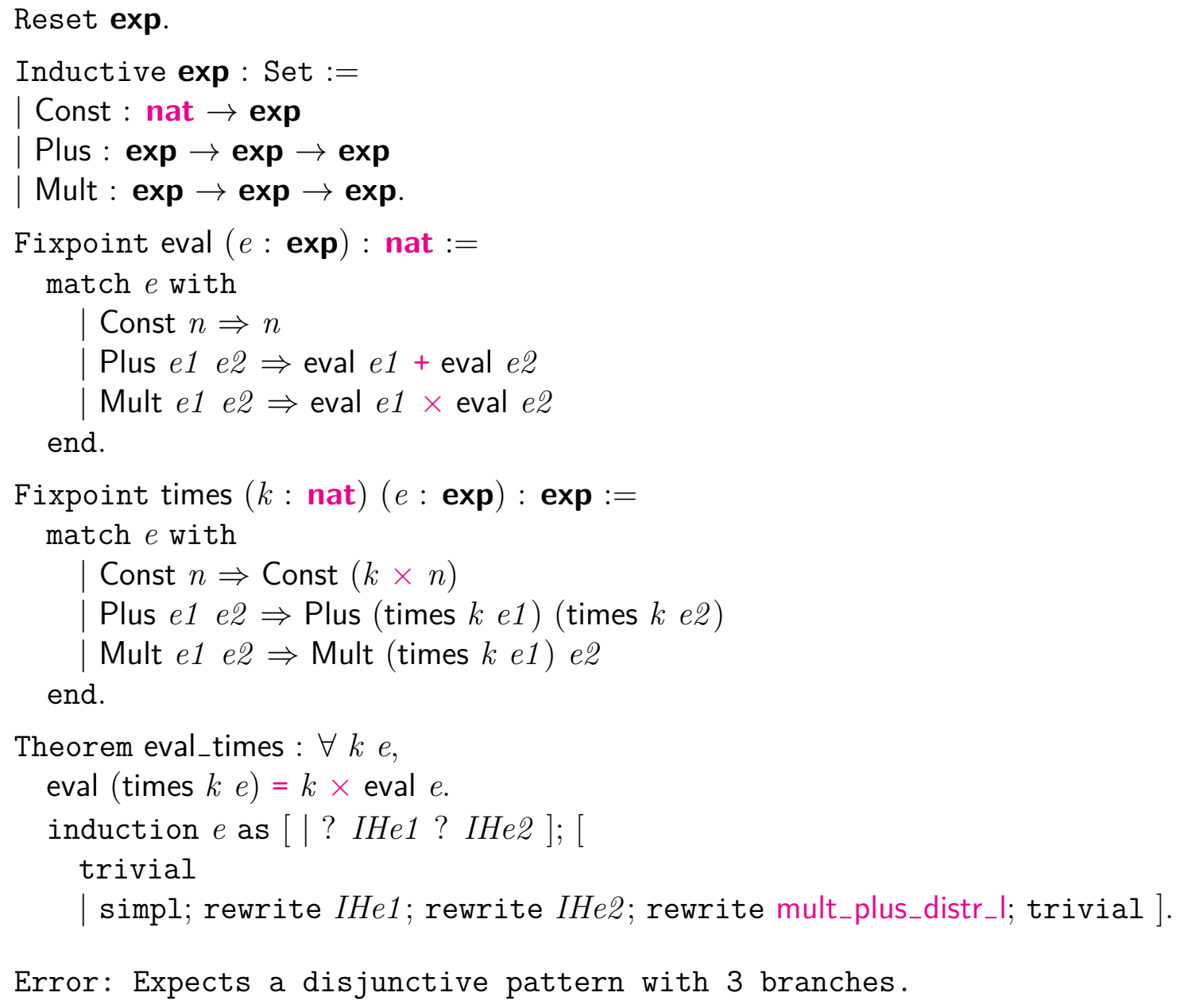

Abort.

Unsurprisingly, the old proof fails, because it explicitly says that there are two inductive cases. To update the script, we must, at a minimum, remember the order in which the inductive cases are generated, so that we can insert the new case in the appropriate place. Even then, it will be painful to add the case, because we cannot walk through proof steps interactively when they occur inside an explicit set of cases.

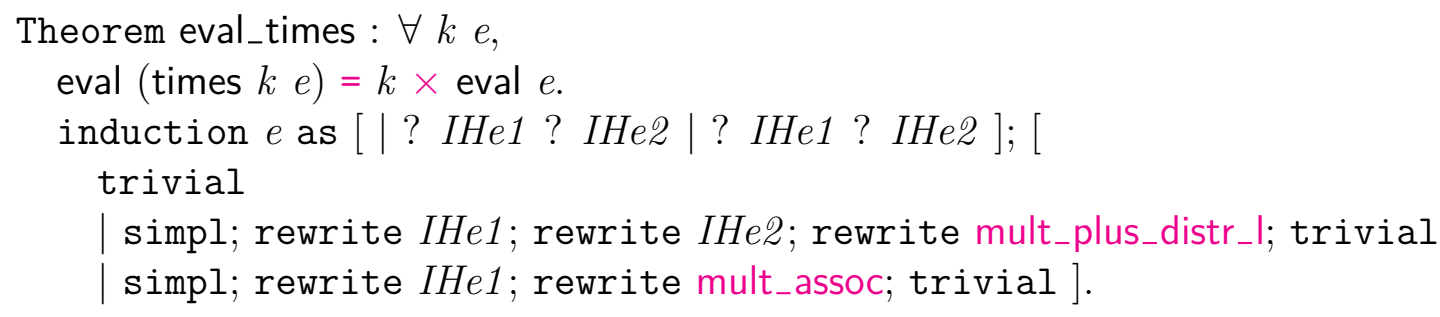


Qed.

Now we are in a position to see how much nicer is the style of proof that we have followed in most of this book.

Reset eval_times.

Hint Rewrite mult_plus_distr_l.

Theorem eval_times : $\forall k e$, eval $($ times $k e)=k \times$ eval $e$.

induction $e$; crush.

Qed.

This style is motivated by a hard truth: one person's manual proof script is almost always mostly inscrutable to most everyone else. I claim that step-by-step formal proofs are a poor way of conveying information. Thus, we might as well cut out the steps and automate as much as possible.

What about the illustrative value of proofs? Most informal proofs are read to convey the big ideas of proofs. How can reading induction e; crush convey any big ideas? My position is that any ideas that standard automation can find are not very big after all, and the real big ideas should be expressed through lemmas that are added as hints.

An example should help illustrate what I mean. Consider this function, which rewrites an expression using associativity of addition and multiplication.

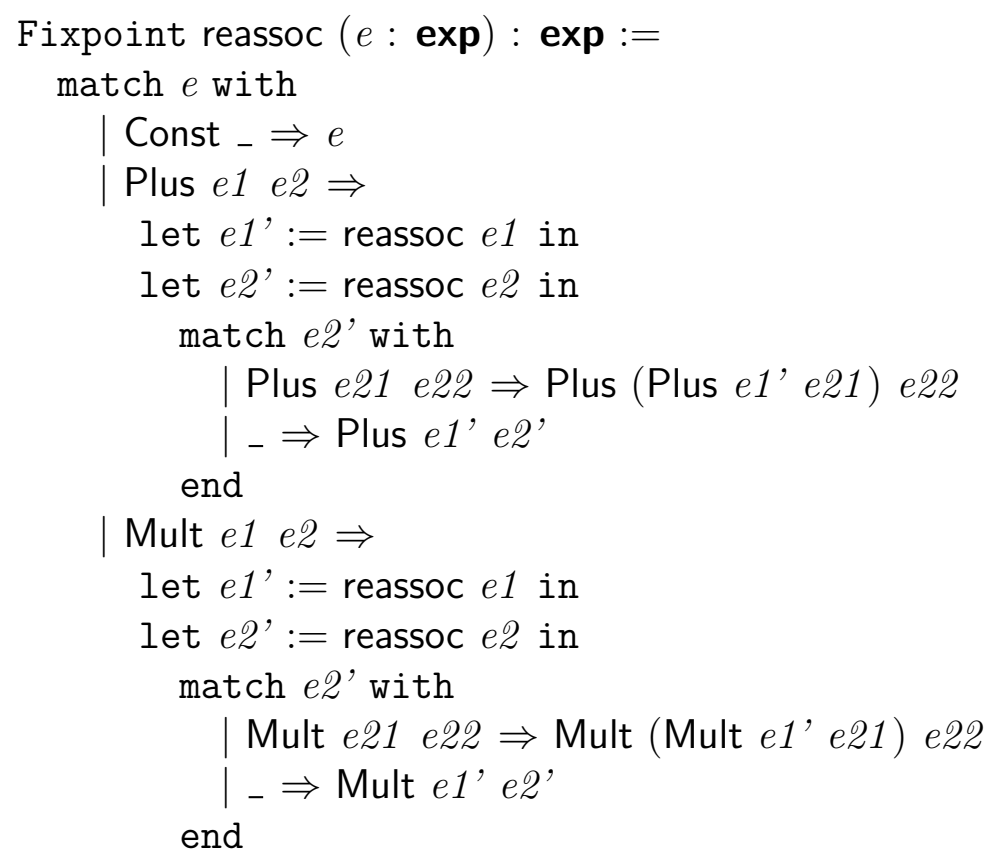

end.

Theorem reassoc_correct : $\forall e$, eval $($ reassoc $e)=$ eval $e$.

induction $e$; crush;

match goal with

$\mid\left[\vdash\right.$ context $\left[\right.$ match ?E with Const _ $\Rightarrow_{-} \mid{ }_{-} \Rightarrow{ }_{-}$end $\left.]\right] \Rightarrow$ 
destruct $E$; crush

end.

One subgoal remains:

IHe2 : eval e3 $\times$ eval $e_{4}=$ eval $e_{2}^{2}$

$==============-===$

eval e1 $\times$ eval e3 $\times$ eval $e 4=$ eval $e 1 \times$ eval $e 2$

The crush tactic does not know how to finish this goal. We could finish the proof manually.

rewrite $\leftarrow$ IHe2; crush

However, the proof would be easier to understand and maintain if we separated this insight into a separate lemma.

Abort.

Lemma rewr : $\forall a b c d, b \times c=d \rightarrow a \times b \times c=a \times d$.

crush.

Qed.

Hint Resolve rewr.

Theorem reassoc_correct : $\forall e$, eval $($ reassoc $e)=$ eval $e$.

induction $e$; crush;

match goal with

$\mid\left[\vdash\right.$ context $\left[\right.$ match ?E with Const _ $\Rightarrow_{-} \mid{ }_{-} \Rightarrow_{-}$end $\left.]\right] \Rightarrow$ destruct $E$; crush

end.

Qed.

In the limit, a complicated inductive proof might rely on one hint for each inductive case. The lemma for each hint could restate the associated case. Compared to manual proof scripts, we arrive at more readable results. Scripts no longer need to depend on the order in which cases are generated. The lemmas are easier to digest separately than are fragments of tactic code, since lemma statements include complete proof contexts. Such contexts can only be extracted from monolithic manual proofs by stepping through scripts interactively.

The more common situation is that a large induction has several easy cases that automation makes short work of. In the remaining cases, automation performs some standard simplification. Among these cases, some may require quite involved proofs; such a case may deserve a hint lemma of its own, where the lemma statement may copy the simplified version of the case. Alternatively, the proof script for the main theorem may be extended with some automation code targeted at the specific case. Even such targeted scripting is more desirable than manual proving, because it may be read and understood without knowledge of a proof's hierarchical structure, case ordering, or name binding structure.

A competing alternative to the common style of Coq tactics is the declarative style, most frequently associated today with the Isar [47] language. A declarative proof script is very explicit about subgoal structure and introduction of local names, aiming for human 
readability. The coding of proof automation is taken to be outside the scope of the proof language, an assumption related to the idea that it is not worth building new automation for each serious theorem. I have shown in this book many examples of theorem-specific automation, which I believe is crucial for scaling to significant results. Declarative proof scripts make it easier to read scripts to modify them for theorem statement changes, but the alternate adaptive style from this book allows use of the same scripts for many versions of a theorem.

Perhaps I am a pessimist for thinking that fully formal proofs will inevitably consist of details that are uninteresting to people, but it is my preference to focus on conveying proof-specific details through choice of lemmas. Additionally, adaptive Ltac scripts contain bits of automation that can be understood in isolation. For instance, in a big repeat match loop, each case can generally be digested separately, which is a big contrast from trying to understand the hierarchical structure of a script in a more common style. Adaptive scripts rely on variable binding, but generally only over very small scopes, whereas understanding a traditional script requires tracking the identities of local variables potentially across pages of code.

One might also wonder why it makes sense to prove all theorems automatically (in the sense of adaptive proof scripts) but not construct all programs automatically. My view there is that program synthesis is a very useful idea that deserves broader application! In practice, there are difficult obstacles in the way of finding a program automatically from its specification. A typical specification is not exhaustive in its description of program properties. For instance, details of performance on particular machine architectures are often omitted. As a result, a synthesized program may be correct in some sense while suffering from deficiencies in other senses. Program synthesis research will continue to come up with ways of dealing with this problem, but the situation for theorem proving is fundamentally different. Following mathematical practice, the only property of a formal proof that we care about is which theorem it proves, and it is trivial to check this property automatically. In other words, with a simple criterion for what makes a proof acceptable, automatic search is straightforward. Of course, in practice we also care about understandability of proofs to facilitate long-term maintenance, which is just what motivates the techniques outlined above, and the next section gives some related advice.

\subsection{Debugging and Maintaining Automation}

Fully automated proofs are desirable because they open up possibilities for automatic adaptation to changes of specification. A well-engineered script within a narrow domain can survive many changes to the formulation of the problem it solves. Still, as we are working with higher-order logic, most theorems fall within no obvious decidable theories. It is inevitable that most long-lived automated proofs will need updating.

Before we are ready to update our proofs, we need to write them in the first place. While fully automated scripts are most robust to changes of specification, it is hard to write every new proof directly in that form. Instead, it is useful to begin a theorem with exploratory 
proving and then gradually refine it into a suitable automated form.

Consider this theorem from Chapter 8, which we begin by proving in a mostly manual way, invoking crush after each step to discharge any low-hanging fruit. Our manual effort involves choosing which expressions to case-analyze on.

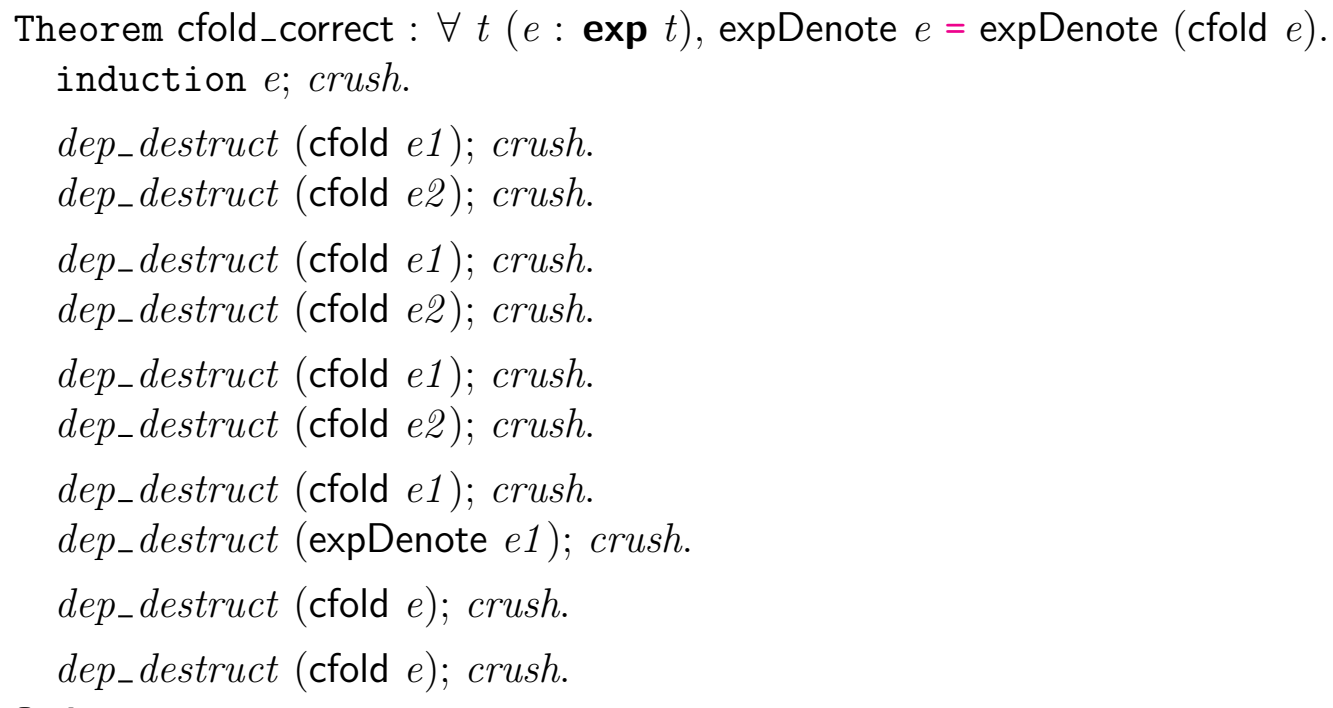

Qed.

In this complete proof, it is hard to avoid noticing a pattern. We rework the proof, abstracting over the patterns we find.

Reset cfold_correct.

Theorem cfold_correct : $\forall t(e: \exp t)$, expDenote $e=\operatorname{expDenote}($ cfold $e$ ).

induction $e$; crush.

The expression we want to destruct here turns out to be the discriminee of a match, and we can easily enough write a tactic that destructs all such expressions.

Ltac $t:=$

repeat (match goal with

$\mid\left[\vdash\right.$ context $\left[\right.$ match ? E with NConst _ $\Rightarrow_{-} \mid{ }_{-} \Rightarrow_{-}$end $\left.]\right] \Rightarrow$ dep_destruct $E$

end; crush).

$t$.

This tactic invocation discharges the whole case. It does the same on the next two cases, but it gets stuck on the fourth case.

$t$.

$t$.

$t$.

The subgoal's conclusion is: 
(if expDenote $e 1$ then expDenote (cfold $e 2)$ else expDenote (cfold $e 3)$ ) $=$ expDenote (if expDenote $e 1$ then cfold $e 2$ else cfold $e 3$ )

We need to expand our $t$ tactic to handle this case.

Ltac $t^{\prime}:=$

repeat (match goal with

$$
\begin{aligned}
& \mid\left[\vdash \text { context [match ?E with NConst _ }\left.\Rightarrow_{-}\right|_{-} \Rightarrow_{-} \text {end] }\right] \Rightarrow \\
& \text { dep_destruct } E \\
& \mid\left[\vdash(\text { if ? } E \text { then _ else _ })=_{-}\right] \Rightarrow \operatorname{destruct~} E \\
& \text { end; crush). }
\end{aligned}
$$

$t^{\prime}$

Now the goal is discharged, but $t^{\prime}$ has no effect on the next subgoal.

$t^{\prime}$.

A final revision of $t$ finishes the proof.

Ltac $t ":=$

repeat (match goal with

$\mid\left[\vdash\right.$ context[match ?E with NConst _ $\Rightarrow_{-}||_{-} \Rightarrow_{-}$end] $] \Rightarrow$ dep_destruct $E$

$\mid\left[\vdash(\right.$ if ? $E$ then _ else _ $\left.)=_{-}\right] \Rightarrow$ destruct $E$

| $\left[\vdash\right.$ context[match pairOut ? $E$ with Some ${ }_{-}{ }_{-}$

dep_destruct $E$

| None $\Rightarrow$ - end] ] $\Rightarrow$

end; crush).

$t^{\prime \prime}$

$t^{\prime \prime}$.

Qed.

We can take the final tactic and move it into the initial part of the proof script, arriving at a nicely automated proof.

Reset cfold_correct.

Theorem cfold_correct : $\forall t(e: \exp t)$, expDenote $e=\operatorname{expDenote}($ cfold $e$ ).

induction $e$; crush;

repeat (match goal with

$\mid\left[\vdash\right.$ context [match ? $E$ with NConst _ $\Rightarrow_{-} \mid{ }_{-} \Rightarrow_{-}$end] $] \Rightarrow$ dep_destruct $E$

$\mid\left[\vdash(\right.$ if ? $E$ then _ else _ $\left.)=_{-}\right] \Rightarrow$ destruct $E$

| $\left[\vdash\right.$ context[match pairOut ? $E$ with Some $\Rightarrow_{-}$

dep_destruct $E$

| None $\Rightarrow$ _ end] ] $\Rightarrow$

end; crush).

Qed. 
Even after we put together nice automated proofs, we must deal with specification changes that can invalidate them. It is not generally possible to step through single-tactic proofs interactively. There is a command Debug On that lets us step through points in tactic execution, but the debugger tends to make counterintuitive choices of which points we would like to stop at, and per-point output is quite verbose, so most Coq users do not find this debugging mode very helpful. How are we to understand what has broken in a script that used to work?

An example helps demonstrate a useful approach. Consider what would have happened in our proof of reassoc_correct if we had first added an unfortunate rewriting hint.

Reset reassoc_correct.

Theorem confounder : $\forall$ e1 $e^{2}$ e3,

eval $e 1 \times$ eval $e 2 \times$ eval $e 3=$ eval $e 1 \times($ eval $e 2+1-1) \times$ eval $e 3$.

crush.

Qed.

Hint Rewrite confounder.

Theorem reassoc_correct : $\forall e$, eval $($ reassoc $e)=$ eval $e$.

induction $e$; crush;

match goal with

$\mid\left[\vdash\right.$ context $\left[\right.$ match ?E with Const _ $\Rightarrow_{-} \mid{ }_{-} \Rightarrow{ }_{-}$end $\left.]\right] \Rightarrow$ destruct $E$; crush

end.

One subgoal remains:

$==\overline{=}=\mathrm{=}=\mathrm{=}=\mathrm{=}=\mathrm{=}=\mathrm{=}=\mathrm{=}=\mathrm{=}=$

eval $e 1 \times($ eval $e 3+1-1) \times$ eval $e 4=$ eval $e 1 \times$ eval $e^{2}$

The poorly chosen rewrite rule fired, changing the goal to a form where another hint no longer applies. Imagine that we are in the middle of a large development with many hints. How would we diagnose the problem? First, we might not be sure which case of the inductive proof has gone wrong. It is useful to separate out our automation procedure and apply it manually.

Restart.

Ltac $t:=$ crush; match goal with

$\mid\left[\vdash\right.$ context $\left[\right.$ match ?E with Const _ $\Rightarrow_{-} \mid{ }_{-} \Rightarrow_{-}$end $\left.]\right] \Rightarrow$ destruct $E$; crush

end.

induction $e$.

Since we see the subgoals before any simplification occurs, it is clear that we are looking at the case for constants. Our $t$ makes short work of it.

$t$. 
The next subgoal, for addition, is also discharged without trouble.

$t$.

The final subgoal is for multiplication, and it is here that we get stuck in the proof state summarized above.

$t$.

What is $t$ doing to get us to this point? The info command can help us answer this kind of question. (As of this writing, info is no longer functioning in the most recent Coq release, but I hope it returns.)

Undo.

info $t$.

$==\operatorname{simpl}$ in $*$ intuition; subst; autorewrite with core in *;

simpl in *; intuition; subst; autorewrite with core in *;

simpl in *; intuition; subst; destruct (reassoc e2).

simpl in *; intuition.

simpl in *; intuition.

simpl in *; intuition; subst; autorewrite with core in *;

refine (eq_ind_r

(fun $n$ : nat $\Rightarrow$

$n \times($ eval e3 $+1-1) \times$ eval $e 4=$ eval $e 1 \times$ eval $e 2)-I H e 1) ;$

autorewrite with core in *; simpl in *; intuition;

subst; autorewrite with core in *; simpl in *;

intuition; subst.

A detailed trace of $t$ 's execution appears. Since we are using the very general crush tactic, many of these steps have no effect and only occur as instances of a more general strategy. We can copy-and-paste the details to see where things go wrong.

Undo.

We arbitrarily split the script into chunks. The first few seem not to do any harm.

simpl in *; intuition; subst; autorewrite with core in *.

simpl in *; intuition; subst; autorewrite with core in *.

simpl in *; intuition; subst; destruct (reassoc e2).

simpl in *; intuition.

simpl in *; intuition.

The next step is revealed as the culprit, bringing us to the final unproved subgoal.

simpl in *; intuition; subst; autorewrite with core in *.

We can split the steps further to assign blame. 
Undo.

simpl in *.

intuition.

subst.

autorewrite with core in *.

It was the final of these four tactics that made the rewrite. We can find out exactly what happened. The info command presents hierarchical views of proof steps, and we can zoom down to a lower level of detail by applying info to one of the steps that appeared in the original trace.

Undo.

info autorewrite with core in *.

$==$ refine (eq_ind_r $($ fun $n:$ nat $\Rightarrow n=$ eval $e 1 \times$ eval $e \mathscr{2})$

(confounder (reassoc e1) e3 e4)).

The way a rewrite is displayed is somewhat baroque, but we can see that theorem confounder is the final culprit. At this point, we could remove that hint, prove an alternate version of the key lemma rewr, or come up with some other remedy. Fixing this kind of problem tends to be relatively easy once the problem is revealed.

Abort.

Sometimes a change to a development has undesirable performance consequences, even if it does not prevent any old proof scripts from completing. If the performance consequences are severe enough, the proof scripts can be considered broken for practical purposes.

Here is one example of a performance surprise.

\section{Section slow.}

Hint Resolve trans_eq.

The central element of the problem is the addition of transitivity as a hint. With transitivity available, it is easy for proof search to wind up exploring exponential search spaces. We also add a few other arbitrary variables and hypotheses, designed to lead to trouble later.

Variable $A$ : Set.

Variables $P Q R S: A \rightarrow A \rightarrow$ Prop.

Variable $f: A \rightarrow A$.

Hypothesis $H 1: \forall x y, P x y \rightarrow Q x y \rightarrow R x y \rightarrow f x=f y$.

Hypothesis H2: $\forall x y, S x y \rightarrow R x y$.

We prove a simple lemma very quickly, using the Time command to measure exactly how quickly.

Lemma slow : $\forall x y, P x y \rightarrow Q x y \rightarrow S x y \rightarrow f x=f y$.

Time eauto 6 . 
Finished transaction in $0 . \operatorname{secs}(0.068004 \mathrm{u}, 0 . \mathrm{s})$

Qed.

Now we add a different hypothesis, which is innocent enough; in fact, it is even provable as a theorem.

Hypothesis H3: $\forall x y, x=y \rightarrow f x=f y$.

Lemma slow' $: \forall x y, P x y \rightarrow Q x y \rightarrow S x y \rightarrow f x=f y$.

Time eauto 6 .

Finished transaction in $2 . \operatorname{secs}(1.264079 \mathrm{u}, 0 . \mathrm{s})$

Why has the search time gone up so much? The info command is not much help, since it only shows the result of search, not all of the paths that turned out to be worthless.

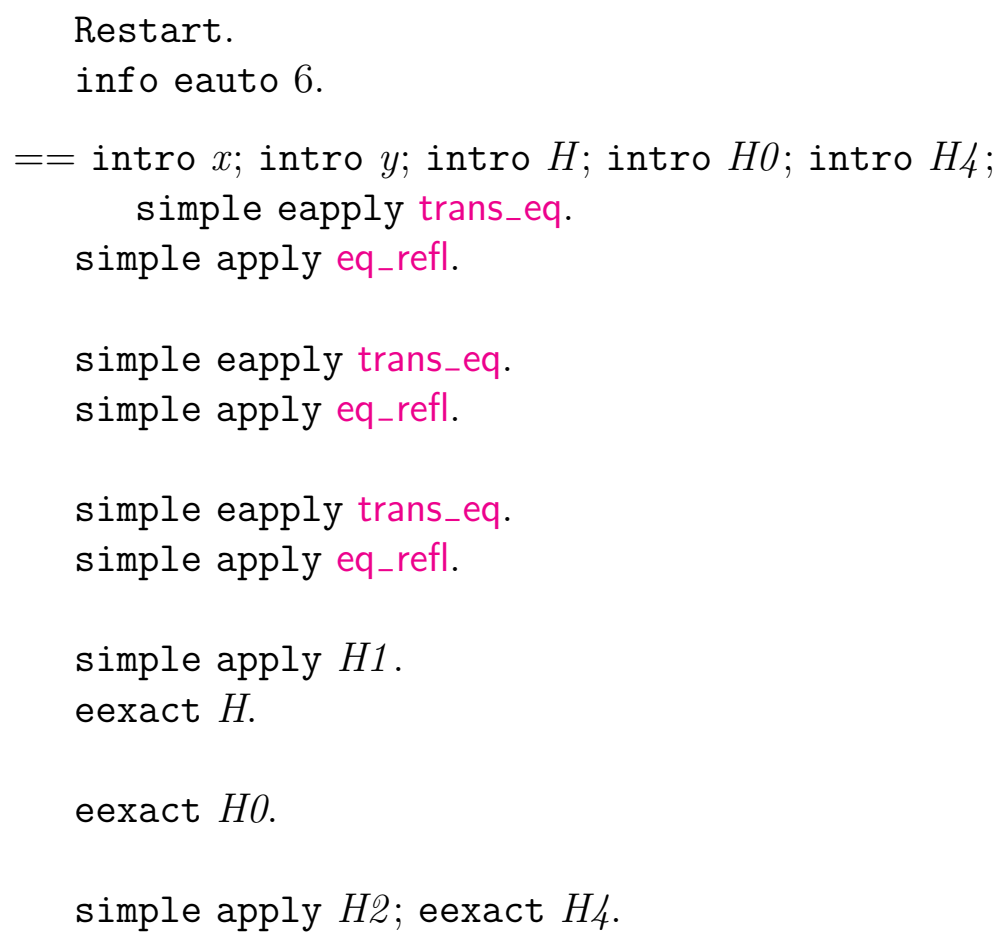

This output does not tell us why proof search takes so long, but it does provide a clue that would be useful if we had forgotten that we added transitivity as a hint. The eauto tactic is applying depth-first search, and the proof script where the real action is ends up buried inside a chain of pointless invocations of transitivity, where each invocation uses reflexivity to discharge one subgoal. Each increment to the depth argument to eauto adds another silly use of transitivity. This wasted proof effort only adds linear time overhead, as long as proof search never makes false steps. No false steps were made before we added the new hypothesis, but somehow the addition made possible a new faulty path. To understand which paths we enabled, we can use the debug command. 
Restart.

debug eauto 6 .

The output is a large proof tree. The beginning of the tree is enough to reveal what is happening:

1 depth $=6$

1.1 depth $=6$ intro

1.1.1 depth $=6$ intro

1.1.1.1 depth $=6$ intro

1.1.1.1.1 depth $=6$ intro

1.1.1.1.1.1 depth $=6$ intro

1.1.1.1.1.1.1 depth=5 apply $H_{3}$

1.1.1.1.1.1.1.1 depth=4 eapply trans_eq

1.1.1.1.1.1.1.1.1 depth=4 apply eq_refl

1.1.1.1.1.1.1.1.1.1 depth=3 eapply trans_eq

1.1.1.1.1.1.1.1.1.1.1 depth=3 apply eq_refl

1.1.1.1.1.1.1.1.1.1.1.1 depth=2 eapply trans_eq

1.1.1.1.1.1.1.1.1.1.1.1.1 depth=2 apply eq_refl

1.1.1.1.1.1.1.1.1.1.1.1.1.1 depth=1 eapply trans_eq

1.1.1.1.1.1.1.1.1.1.1.1.1.1.1 depth=1 apply eq_refl

1.1.1.1.1.1.1.1.1.1.1.1.1.1.1.1 depth=0 eapply trans_eq

1.1.1.1.1.1.1.1.1.1.1.1.1.1.2 depth=1 apply sym_eq ; trivial

1.1.1.1.1.1.1.1.1.1.1.1.1.1.2.1 depth=0 eapply trans_eq

1.1.1.1.1.1.1.1.1.1.1.1.1.1.3 depth=0 eapply trans_eq

1.1.1.1.1.1.1.1.1.1.1.1.2 depth=2 apply sym_eq ; trivial

1.1.1.1.1.1.1.1.1.1.1.1.2.1 depth=1 eapply trans_eq

1.1.1.1.1.1.1.1.1.1.1.1.2.1.1 depth=1 apply eq_refl

1.1.1.1.1.1.1.1.1.1.1.1.2.1.1.1 depth $=0$ eapply trans_eq

1.1.1.1.1.1.1.1.1.1.1.1.2.1.2 depth=1 apply sym_eq ; trivial

1.1.1.1.1.1.1.1.1.1.1.1.2.1.2.1 depth $=0$ eapply trans_eq

1.1.1.1.1.1.1.1.1.1.1.1.2.1.3 depth=0 eapply trans_eq

The first choice eauto makes is to apply H3, since H3 has the fewest hypotheses of all of the hypotheses and hints that match. However, it turns out that the single hypothesis generated is unprovable. That does not stop eauto from trying to prove it with an exponentially sized tree of applications of transitivity, reflexivity, and symmetry of equality. It is the children of the initial apply $H 3$ that account for all of the noticeable time in proof execution. In a more realistic development, we might use this output of debug to realize that adding transitivity as a hint was a bad idea.

Qed.

End slow.

As aggravating as the above situation may be, there is greater aggravation to be had from importing library modules with commands like Require Import. Such a command 
imports not just the Gallina terms from a module, but also all the hints for auto, eauto, and autorewrite. Some very recent versions of Coq include mechanisms for removing hints from databases, but the proper solution is to be very conservative in exporting hints from modules. Consider putting hints in named databases, so that they may be used only when called upon explicitly, as demonstrated in Chapter 13.

It is also easy to end up with a proof script that uses too much memory. As tactics run, they avoid generating proof terms, since serious proof search will consider many possible avenues, and we do not want to build proof terms for subproofs that end up unused. Instead, tactic execution maintains thunks (suspended computations, represented with closures), such that a tactic's proof-producing thunk is only executed when we run Qed. These thunks can use up large amounts of space, such that a proof script exhausts available memory, even when we know that we could have used much less memory by forcing some thunks earlier.

The abstract tactical helps us force thunks by proving some subgoals as their own lemmas. For instance, a proof induction $x$; crush can in many cases be made to use significantly less peak memory by changing it to induction $x$; abstract crush. The main limitation of abstract is that it can only be applied to subgoals that are proved completely, with no undetermined unification variables in their initial states. Still, many large automated proofs can realize vast memory savings via abstract.

\subsection{Modules}

Last chapter's examples of proof by reflection demonstrate opportunities for implementing abstract proof strategies with stronger formal guarantees than can be had with Ltac scripting. Coq's module system provides another tool for more rigorous development of generic theorems. This feature is inspired by the module systems found in Standard ML [22] and OCaml, and the discussion that follows assumes familiarity with the basics of one of those systems.

ML modules facilitate the grouping of abstract types with operations over those types. Moreover, there is support for functors, which are functions from modules to modules. A canonical example of a functor is one that builds a data structure implementation from a module that describes a domain of keys and its associated comparison operations.

When we add modules to a base language with dependent types, it becomes possible to use modules and functors to formalize kinds of reasoning that are common in algebra. For instance, the following module signature captures the essence of the algebraic structure known as a group. A group consists of a carrier set $G$, an associative binary operation $f$, a left identity element id for $f$, and an operation $i$ that is a left inverse for $f$.

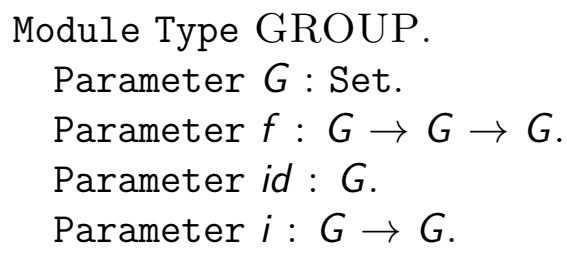




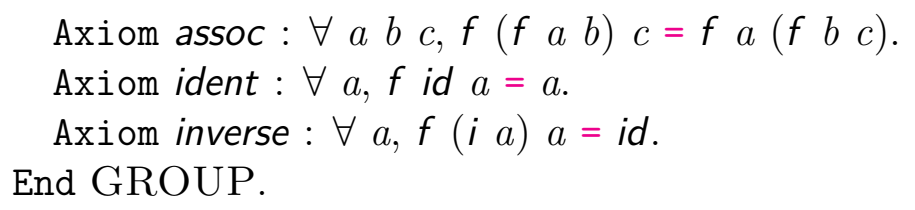

Many useful theorems hold of arbitrary groups. We capture some such theorem statements in another module signature.

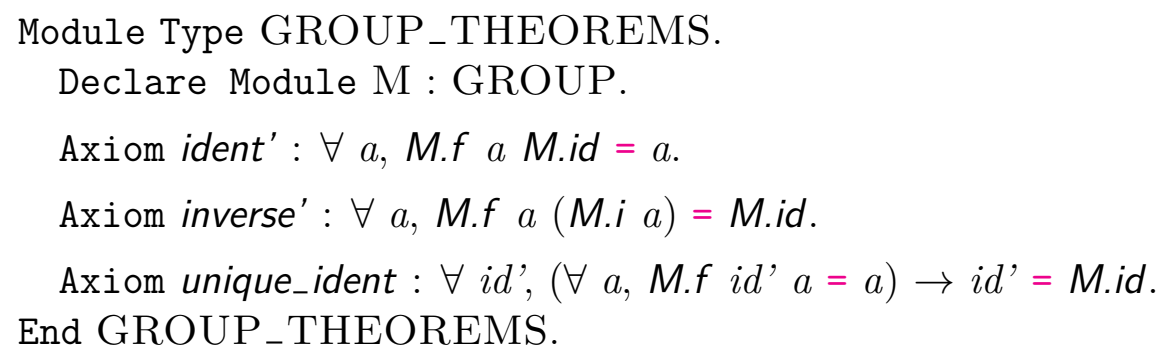

We implement generic proofs of these theorems with a functor, whose input is an arbitrary group $M$.

Module GroupProofs ( $M$ : GROUP) : GROUP_THEOREMS with Module $M:=$ M.

As in ML, Coq provides multiple options for ascribing signatures to modules. Here we use just the colon operator, which implements opaque ascription, hiding all details of the module not exposed by the signature. Another option is transparent ascription via the $<$ : operator, which checks for signature compatibility without hiding implementation details. Here we stick with opaque ascription but employ the with operation to add more detail to a signature, exposing just those implementation details that we need to. For instance, here we expose the underlying group representation set and operator definitions. Without such a refinement, we would get an output module proving theorems about some unknown group, which is not very useful. Also note that opaque ascription can in Coq have some undesirable consequences without analogues in ML, since not just the types but also the definitions of identifiers have significance in type checking and theorem proving.

Module $\mathrm{M}:=\mathrm{M}$.

To ensure that the module we are building meets the GROUP_THEOREMS signature, we add an extra local name for $M$, the functor argument.

\section{Import $M$.}

It would be inconvenient to repeat the prefix $M$. everywhere in our theorem statements and proofs, so we bring all the identifiers of $M$ into the local scope unqualified.

Now we are ready to prove the three theorems. The proofs are completely manual, which may seem ironic given the content of the previous sections! This illustrates another lesson, which is that short proof scripts that change infrequently may be worth leaving unautomated. It would take some effort to build suitable generic automation for these theorems about groups, so I stick with manual proof scripts to avoid distracting us from the main message of the section. We take the proofs from the Wikipedia page on elementary group theory.

Theorem inverse' : $\forall a, f a(i a)=i d$. 


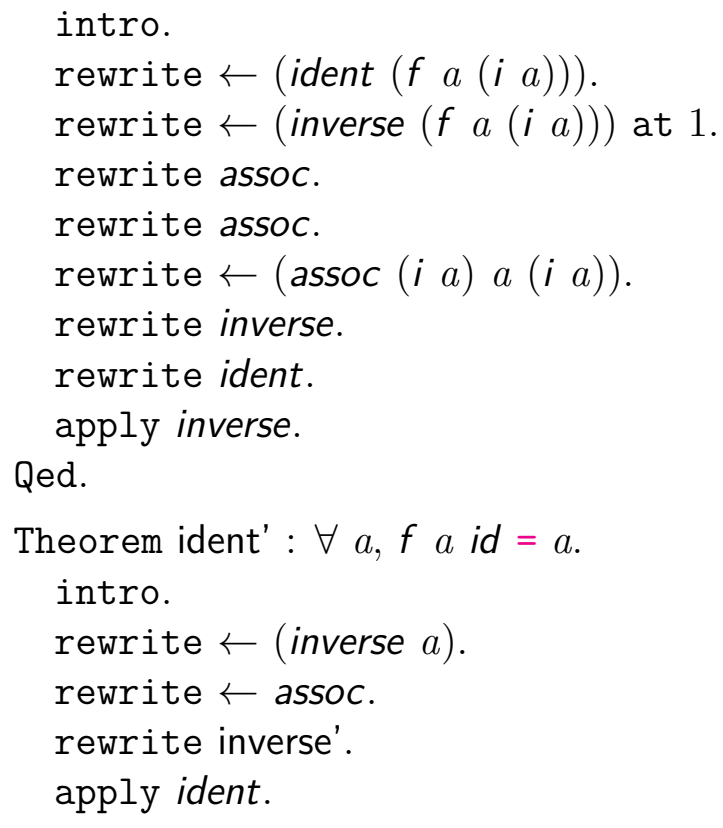

Theorem unique_ident : $\forall i d^{\prime},\left(\forall a\right.$, M.f $\left.i d^{\prime} a=a\right) \rightarrow i d^{\prime}=$ M.id. intros.

rewrite $\leftarrow$ ( $H$ id $)$.

symmetry.

apply ident'.

Qed.

\section{End GroupProofs.}

We can show that the integers with + form a group.

Require Import ZArith.

Open Scope Z_scope.

Module INT.

Definition $\mathrm{G}:=\mathbf{Z}$.

Definition $\mathrm{f} x y:=x+y$.

Definition id $:=0$.

Definition i $x:=-x$.

Theorem assoc : $\forall a b c, \mathrm{f}(\mathrm{f} a b) c=\mathrm{f} a(\mathrm{f} b c)$. unfold f; crush.

Qed.

Theorem ident : $\forall a, \mathrm{f}$ id $a=a$. unfold $\mathrm{f}$, id; crush.

Qed.

Theorem inverse : $\forall a, \mathrm{f}(\mathrm{i} a) a=\mathrm{id}$. unfold $\mathrm{f}$, i, id; crush. Qed. 
End INT.

Next, we can produce integer-specific versions of the generic group theorems.

Module IntProofs := GroupProofs(Int).

Check IntProofs.unique_ident.

IntProofs.unique_ident

$: \forall e^{\prime}:$ Int.G, $\left(\forall a:\right.$ Int. $G$, Int.f $\left.e^{\prime} a=a\right) \rightarrow e^{\prime}=$ Int.e

Projections like Int. $G$ are known to be definitionally equal to the concrete values we have assigned to them, so the above theorem yields as a trivial corollary the following more natural restatement:

Theorem unique_ident : $\forall i d^{\prime},\left(\forall a, i d^{\prime}+a=a\right) \rightarrow i d^{\prime}=0$.

exact IntProofs.unique_ident.

Qed.

As in ML, the module system provides an effective way to structure large developments. Unlike in ML, Coq modules add no expressiveness; we can implement any module as an inhabitant of a dependent record type. It is the second-class nature of modules that makes them easier to use than dependent records in many cases. Because modules may only be used in quite restricted ways, it is easier to support convenient module coding through special commands and editing modes, as the above example demonstrates. An isomorphic implementation with records would have suffered from lack of such conveniences as module subtyping and importation of the fields of a module. On the other hand, all module values must be determined statically, so modules may not be computed, e.g., within the definitions of normal functions, based on particular function parameters.

\subsection{Build Processes}

As in software development, large Coq projects are much more manageable when split across multiple files and when decomposed into libraries. Coq and Proof General provide very good support for these activities.

Consider a library that we will name LIB, housed in directory LIB and split between files A.v, B.v, and C.v. A simple Makefile will compile the library, relying on the standard Coq tool coq_makefile to do the hard work.

MODULES $:=A$ B C

VS $\quad:=\$($ MODULES $: \%=\%$ v)

.PHONY: coq clean

coq: Makefile.coq

$\$($ MAKE) -f Makefile.coq 


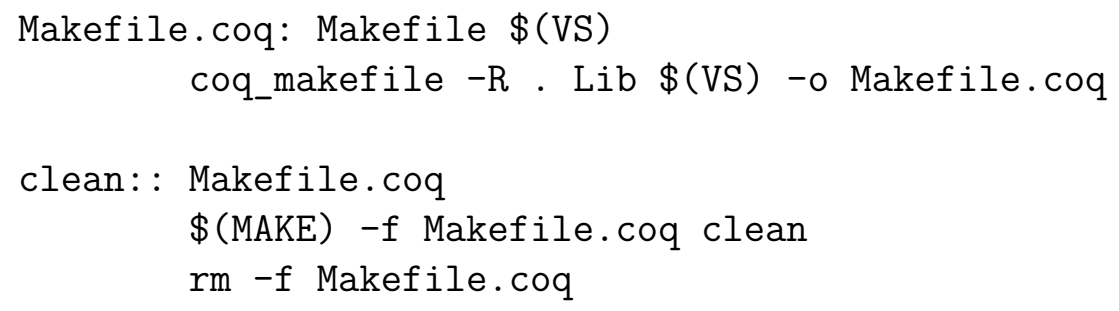

The Makefile begins by defining a variable vS holding the list of filenames to be included in the project. The primary target is coq, which depends on the construction of an auxiliary Makefile called Makefile.coq. Another rule explains how to build that file. We call coq_makefile, using the -R flag to specify that files in the current directory should be considered to belong to the library LIB. This Makefile will build a compiled version of each module, such that $\mathrm{X} . \mathrm{V}$ is compiled into $\mathrm{X}$. vo.

Now code in B.v may refer to definitions in A.v after running

Require Import LIB.A.

Library LIB is presented as a module, containing a submodule $A$, which contains the definitions from A.v. These are genuine modules in the sense of Coq's module system, and they may be passed to functors and so on.

The command Require Import is a convenient combination of two more primitive commands. The Require command finds the . vo file containing the named module, ensuring that the module is loaded into memory. The Import command loads all top-level definitions of the named module into the current namespace, and it may be used with local modules that do not have corresponding . vo files. Another command, Load, is for inserting the contents of a named file verbatim. It is generally better to use the module-based commands, since they avoid rerunning proof scripts, and they facilitate reorganization of directory structure without the need to change code.

Now we would like to use our library from a different development, called CLIENT and found in directory CLIENT, which has its own Makefile.

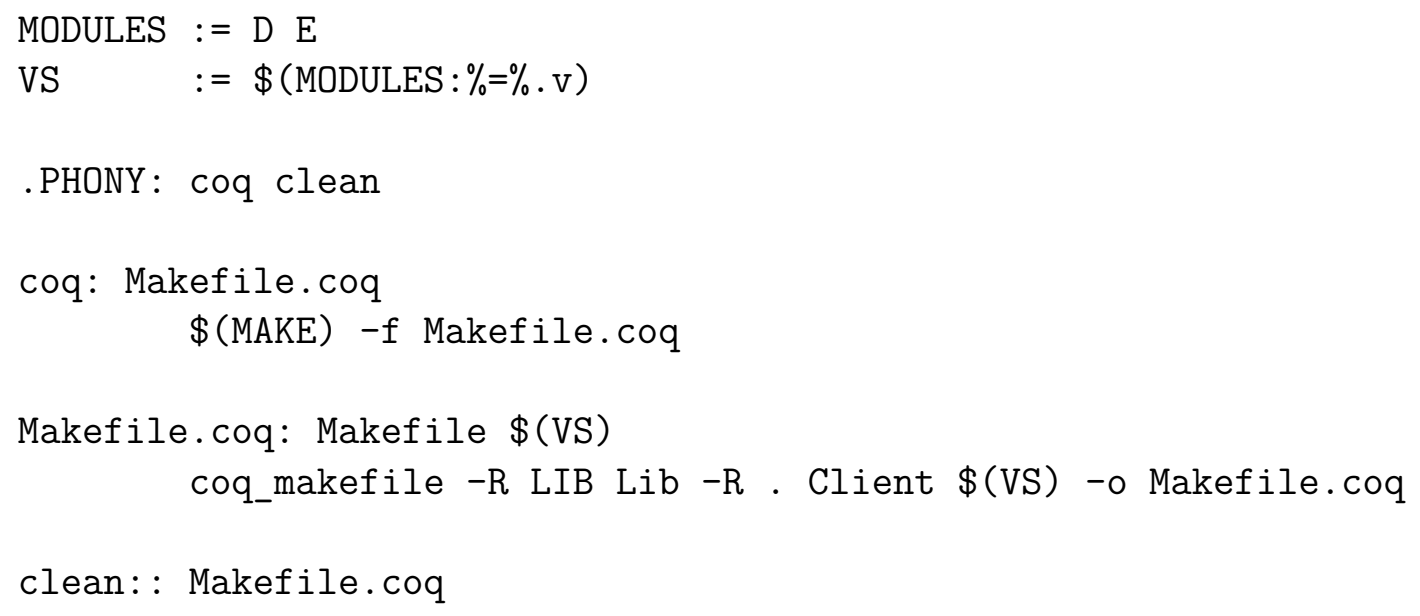




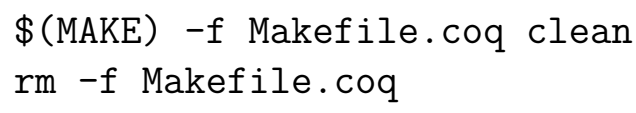

We change the coq_makefile call to indicate where the library LIB is found. Now D.v and E.v can refer to definitions from LIB module $A$ after running

Require Import LIB.A.

and E.v can refer to definitions from D.v by running

Require Import CLIENT.D.

It can be useful to split a library into several files, but it is also inconvenient for client code to import library modules individually. We can get the best of both worlds by, for example, adding an extra source file Lib.v to LIB's directory and Makefile, where that file contains just this line:

Require Export LiB.A LiB.B LiB.C.

Now client code can import all definitions from all of LiB's modules simply by running Require Import LIB.

The two Makefiles above share a lot of code, so, in practice, it is useful to define a common Makefile that is included by multiple library-specific Makefiles.

The remaining ingredient is the proper way of editing library code files in Proof General. Recall this snippet of .emacs code from Chapter 2, which tells Proof General where to find the library associated with this book.

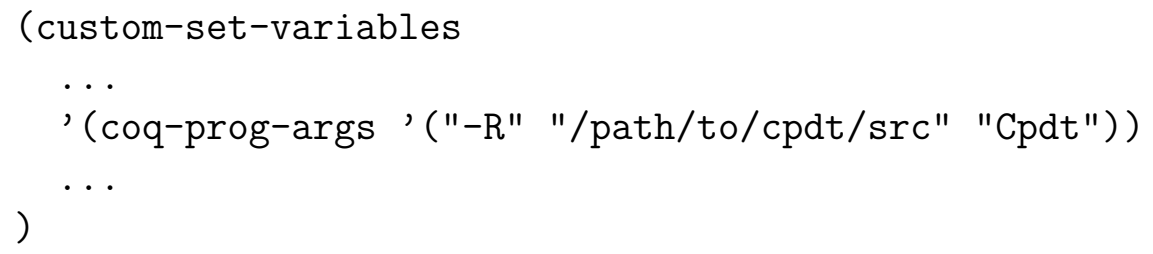

To do interactive editing of our current example, we just need to change the flags to point to the right places.

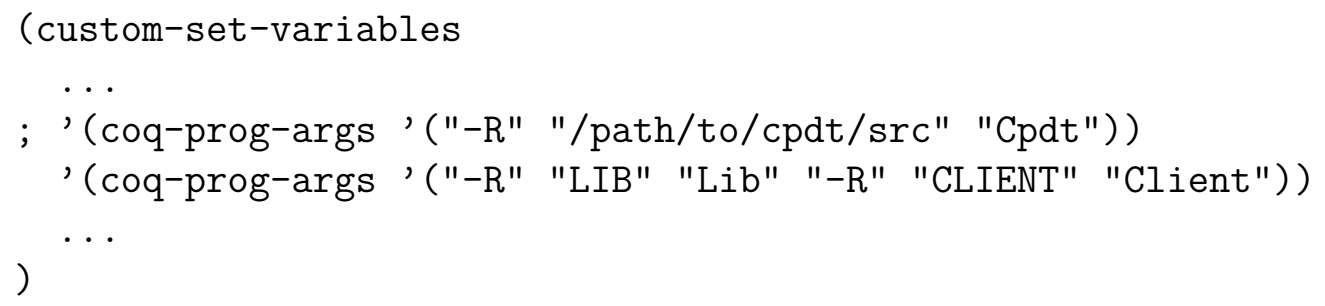

When working on multiple projects, it is useful to leave multiple versions of this setting in your . emacs file, commenting out all but one of them at any moment in time. To switch between projects, change the commenting structure and restart Emacs.

Alternatively, we can revisit the directory-local settings approach and write the following into a file .dir-locals.el in CLIENT: 
((coq-mode . ((coq-prog-args .

("-emacs-U" "-R" "LIB" "Lib" "-R" "CLIENT" "Client")))))

A downside of this approach is that users of your code may not want to trust the arbitrary Emacs Lisp programs that you are allowed to place in such files, so that they prefer to add mappings manually.

Relatively recent versions of Coq support another, more principled approach to all this. A project's list of settings and source files may be saved in a single file named _CoqProject, which is processed uniformly by recent enough versions of coq_makefile, Proof General, and CoqIDE. For details, see the Coq manual. 


\section{Chapter 17}

\section{A Taste of Reasoning About Programming Language Syntax}

Reasoning about the syntax and semantics of programming languages is a popular application of proof assistants. Before proving the first theorem of this kind, it is necessary to choose a formal encoding of the informal notions of syntax, dealing with such issues as variable binding conventions. I believe the pragmatic questions in this domain are far from settled and remain as important open research problems. However, in this chapter, I will demonstrate two underused encoding approaches. Note that I am not recommending either approach as a silver bullet! Mileage will vary across concrete problems, and I expect there to be significant future advances in our knowledge of encoding techniques. For a broader introduction to programming language formalization, using more elementary techniques, see Software Foundations ${ }^{1}$ by Pierce et al.

This chapter is also meant as a case study, bringing together what we have learned in the previous chapters. We will see a concrete example of the importance of representation choices; translating mathematics from paper to Coq is not a deterministic process, and different creative choices can have big impacts. We will also see dependent types and scripted proof automation in action, applied to solve a particular problem as well as possible, rather than to demonstrate new Coq concepts.

I apologize in advance to those readers not familiar with the theory of programming language semantics. I will make a few remarks intended to relate the material here with common ideas in semantics, but these remarks should be safe for others to skip.

We will define a small programming language and reason about its semantics, expressed as an interpreter into Coq terms, much as we have done in examples throughout the book. It will be helpful to build a slight extension of crush that tries to apply functional extensionality, an axiom we met in Chapter 12, which says that two functions are equal if they map equal inputs to equal outputs. We also use $f_{-}$equal to simplify goals of a particular form that will come up with the term denotation function that we define shortly.

Ltac ext $:=$ let $x:=$ fresh " $\mathrm{x}$ " in extensionality $x$.

\footnotetext{
${ }^{1}$ http://www.cis.upenn.edu/ bcpierce/sf /
} 
Ltac $p l:=$ crush; repeat (match goal with

$$
\begin{aligned}
& \mid\left[\vdash\left(\text { fun } x \Rightarrow_{-}\right)=\left(\text {fun } y \Rightarrow_{-}\right)\right] \Rightarrow \operatorname{ext} \\
& \mid\left[\vdash \ldots{ }_{-} ? E_{-}=_{-}{ }_{-} ? E_{-}\right] \Rightarrow \mathrm{f}_{-} \text {equal } \\
& \mid\left[\vdash ? E:::_{-}=? E:::_{-}\right] \Rightarrow f_{-} \text {equal } \\
& \mid[\vdash \text { hmap _ } ? E=\text { hmap _ } \text { E }] \Rightarrow f_{-} \text {equal } \\
& \text { end; crush). }
\end{aligned}
$$

At this point in the book source, some auxiliary proofs also appear.

Here is a definition of the type system we will use throughout the chapter. It is for simply typed lambda calculus with natural numbers as the base type.

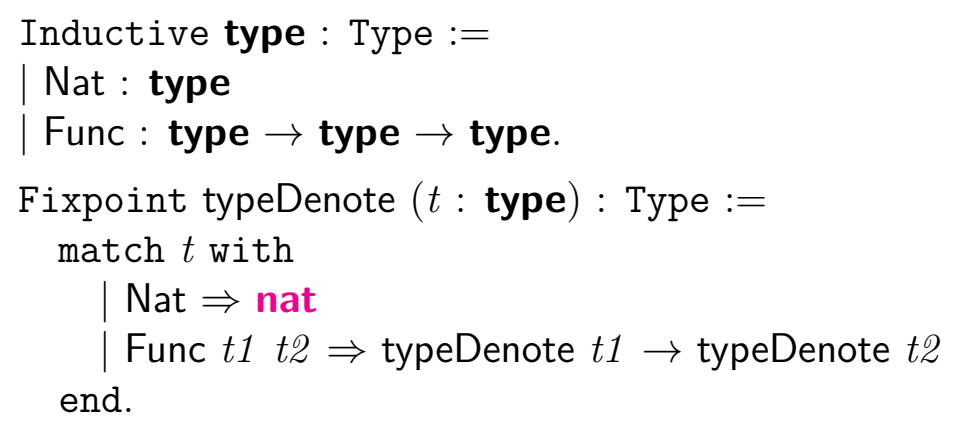

Now we have some choices as to how we represent the syntax of programs. The two sections of the chapter explore two such choices, demonstrating the effect the choice has on proof complexity.

\subsection{Dependent de Bruijn Indices}

The first encoding is one we met first in Chapter 9, the dependent de Bruijn index encoding. We represent program syntax terms in a type family parameterized by a list of types, representing the typing context, or information on which free variables are in scope and what their types are. Variables are represented in a way isomorphic to the natural numbers, where number 0 represents the first element in the context, number 1 the second element, and so on. Actually, instead of numbers, we use the member dependent type family from Chapter 9.

\section{Module FirstORDER.}

Here is the definition of the term type, including variables, constants, addition, function abstraction and application, and let binding of local variables.

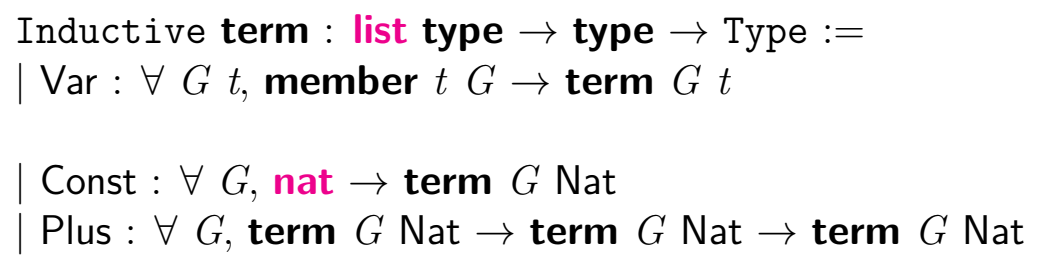




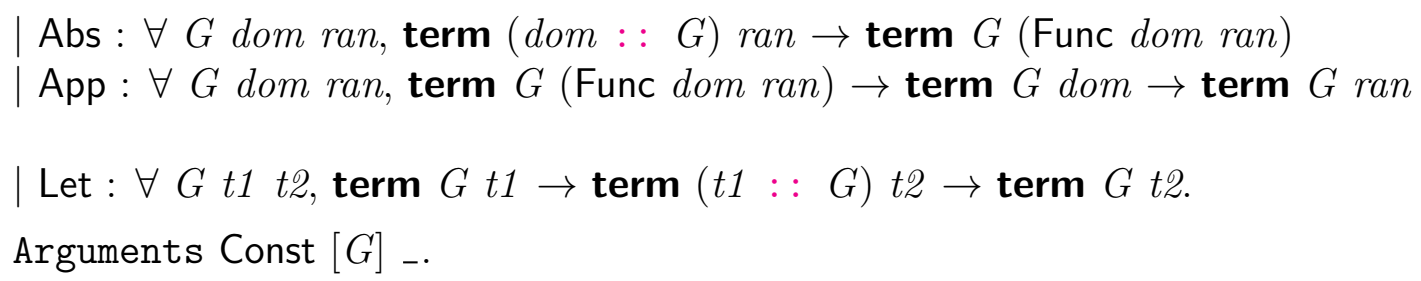

Here are two example term encodings, the first of addition packaged as a two-argument curried function, and the second of a sample application of addition to constants.

Example add : term nil (Func Nat (Func Nat Nat)) := Abs (Abs (Plus (Var (HNext HFirst)) (Var HFirst))).

Example three_the_hard_way : term nil Nat := App (App add (Const 1)) (Const 2).

Since dependent typing ensures that any term is well-formed in its context and has a particular type, it is easy to translate syntactic terms into Coq values.

Fixpoint termDenote $G t(e: \operatorname{term} G t):$ hlist typeDenote $G \rightarrow$ typeDenote $t:=$ match $e$ with

$$
\begin{aligned}
& \text { Var } \_-x \Rightarrow \text { fun } s \Rightarrow \text { hget } s x \\
& \text { | Const _ } n \Rightarrow \text { fun _ } \Rightarrow n \\
& \text { | Plus _ e1 } e 2 \Rightarrow \text { fun } s \Rightarrow \text { termDenote } e 1 s+\text { termDenote } e 2 s \\
& \text { | Abs _ - } e 1 \Rightarrow \text { fun } s \Rightarrow \text { fun } x \Rightarrow \text { termDenote } e 1(x::: s) \\
& \mid \text { App _ _ e1 e2 } \Rightarrow \text { fun } s \Rightarrow(\text { termDenote } e 1 s) \text { (termDenote } e \mathscr{2} s \text { ) } \\
& \text { | Let _ _ } e 1 \text { ed } \Rightarrow \text { fun } s \Rightarrow \text { termDenote } e \mathscr{2} \text { (termDenote } e 1 s::: s \text { ) }
\end{aligned}
$$

With this term representation, some program transformations are easy to implement and prove correct. Certainly we would be worried if this were not the the case for the identity transformation, which takes a term apart and reassembles it.

Fixpoint ident $G t(e: \operatorname{term} G t): \operatorname{term} G t:=$ match $e$ with

$$
\begin{aligned}
& \text { | Var _ }-x \Rightarrow \operatorname{Var} x \\
& \text { Const } \_n \Rightarrow \text { Const } n \\
& \text { | Plus _ e1 } e \mathscr{2} \Rightarrow \text { Plus (ident e1) (ident e2) } \\
& \text { | Abs _ _ } e 1 \Rightarrow \text { Abs (ident } e 1 \text { ) }
\end{aligned}
$$

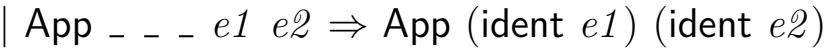

$$
\begin{aligned}
& \text { | Let _ _ } e 1 \text { e2 } \Rightarrow \text { Let (ident } e 1) \text { (ident } e \mathscr{2} \text { ) }
\end{aligned}
$$


Theorem identSound : $\forall G t(e: \operatorname{term} G t) s$, termDenote (ident $e$ ) $s=$ termDenote $e s$.

induction $e ; p l$.

Qed.

A slightly more ambitious transformation belongs to the family of constant folding optimizations we have used as examples in other chapters.

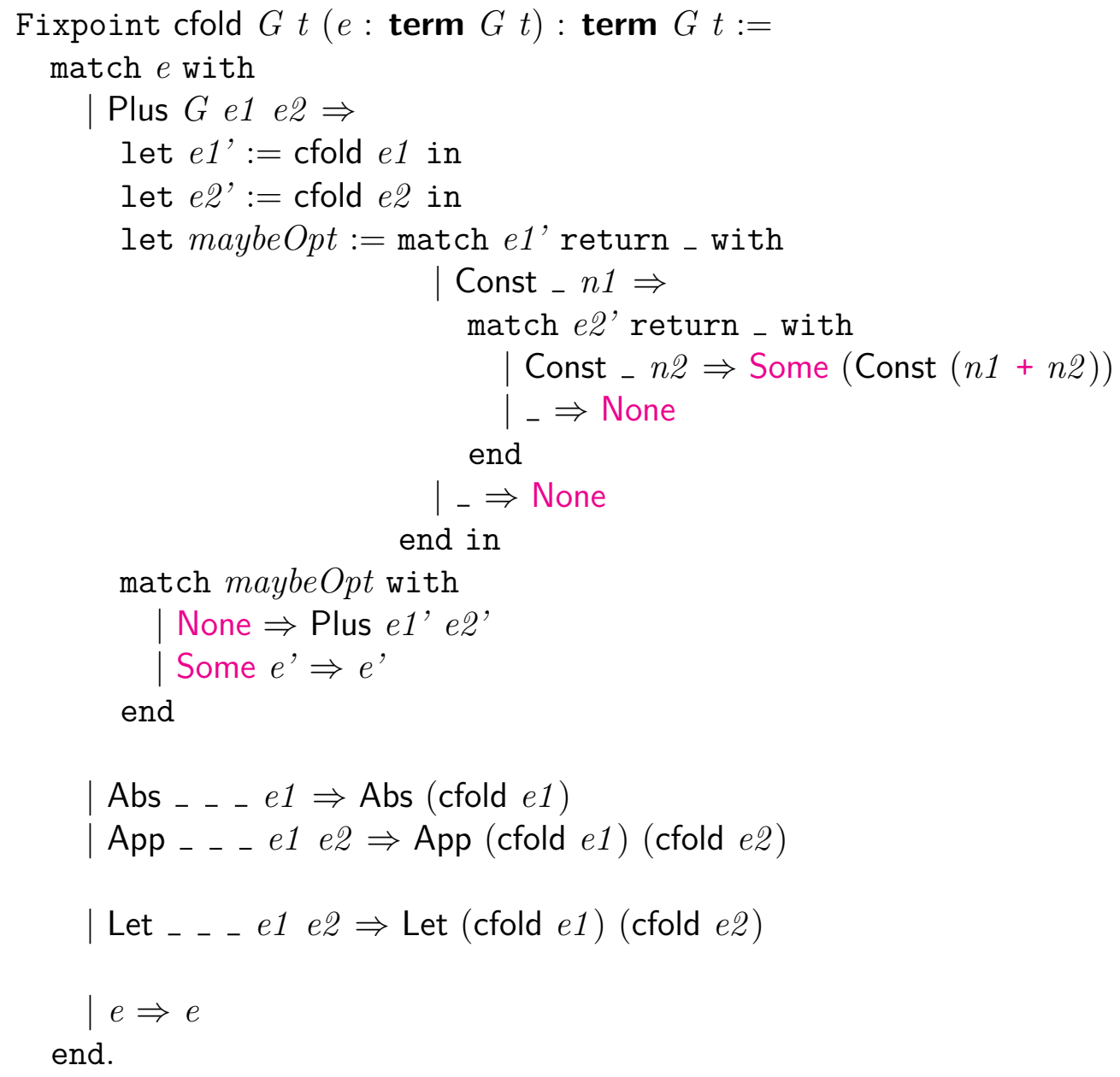

The correctness proof is more complex, but only slightly so.

Theorem cfoldSound : $\forall G t(e: \operatorname{term} G t) s$, termDenote (cfold $e$ ) $s=$ termDenote $e s$.

induction $e ; p l$;

repeat (match goal with

$\mid\left[\vdash\right.$ context $\left[\right.$ match ?E with $V_{\text {ar }}{ }_{-}{ }_{-}{ }_{-} \mid{ }_{-} \Rightarrow{ }_{-}$end $\left.]\right] \Rightarrow$ dep_destruct $E$

end; $p l$ ).

Qed.

The transformations we have tried so far have been straightforward because they do 
not have interesting effects on the variable binding structure of terms. The dependent de Bruijn representation is called first-order because it encodes variable identity explicitly; all such representations incur bookkeeping overheads in transformations that rearrange binding structure.

As an example of a tricky transformation, consider one that removes all uses of "let $x$ $=e 1$ in $e^{2}$ " by substituting $e 1$ for $x$ in e2. We will implement the translation by pairing the "compile-time" typing environment with a "run-time" value environment or substitution, mapping each variable to a value to be substituted for it. Such a substitute term may be placed within a program in a position with a larger typing environment than applied at the point where the substitute term was chosen. To support such context transplantation, we need lifting, a standard de Bruijn indices operation. With dependent typing, lifting corresponds to weakening for typing judgments.

The fundamental goal of lifting is to add a new variable to a typing context, maintaining the validity of a term in the expanded context. To express the operation of adding a type to a context, we use a helper function insertAt.

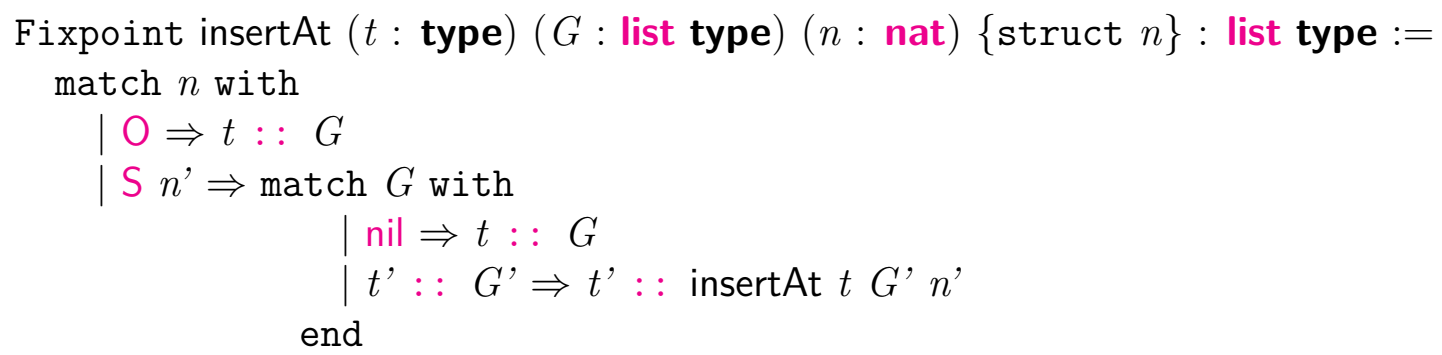

end.

Another function lifts bound variable instances, which we represent with member values.

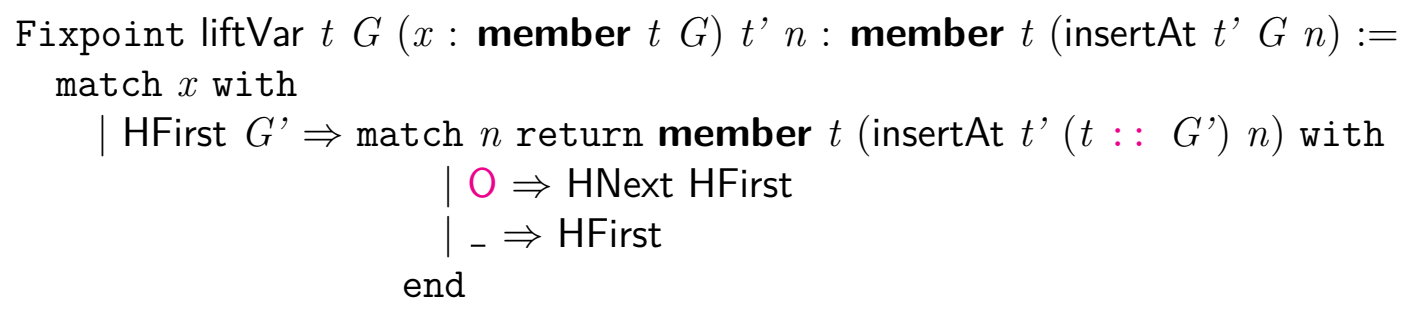

| HNext $t^{\prime \prime} G^{\prime} x^{\prime} \Rightarrow$ match $n$ return member $t$ (insertAt $t^{\prime}(t$ ' : : G') $n)$ with

$$
\begin{aligned}
& \mid \mathrm{O} \Rightarrow \mathrm{HNext}\left(\text { HNext } x^{\prime}\right) \\
& \text { end } \left.\mathrm{S} n^{\prime} \Rightarrow \text { HNext (liftVar } x^{\prime} t^{\prime} n^{\prime}\right) \\
& \text { end }
\end{aligned}
$$

end.

The final helper function for lifting allows us to insert a new variable anywhere in a typing context.

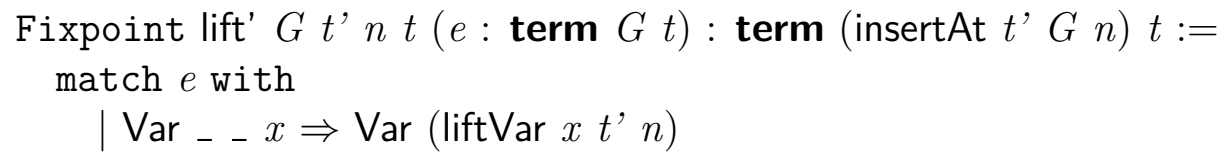




$$
\begin{aligned}
& \text { Const }-n \Rightarrow \text { Const } n \\
& \text { | Plus _ e1 } e \mathscr{2} \Rightarrow \text { Plus (lift' t' } n \text { e1) (lift' t' } n \text { e2) } \\
& \text { | Abs _ _ } \left.e 1 \Rightarrow \text { Abs (lift' } t^{\prime}(\mathrm{S} n) e 1\right)
\end{aligned}
$$

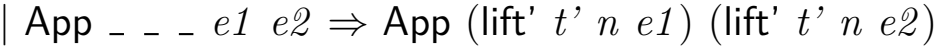

$$
\begin{aligned}
& \text { | Let _ - } e 1 \text { e2 } \Rightarrow \text { Let (lift' t' } n \text { e1) (lift' t' (S n) e2) }
\end{aligned}
$$

In the Let removal transformation, we only need to apply lifting to add a new variable at the beginning of a typing context, so we package lifting into this final, simplified form.

Definition lift $G t^{\prime} t(e: \operatorname{term} G t): \operatorname{term}\left(t^{\prime}:: G\right) t:=$ lift' $t^{\prime} \mathrm{O} e$.

Finally, we can implement Let removal. The argument of type hlist (term $G^{\prime}$ ) $G$ represents a substitution mapping each variable from context $G$ into a term that is valid in context $G$ '. Note how the Abs case (1) extends via lifting the substitution $s$ to hold in the broader context of the abstraction body $e 1$ and (2) maps the new first variable to itself. It is only the Let case that maps a variable to any substitute beside itself.

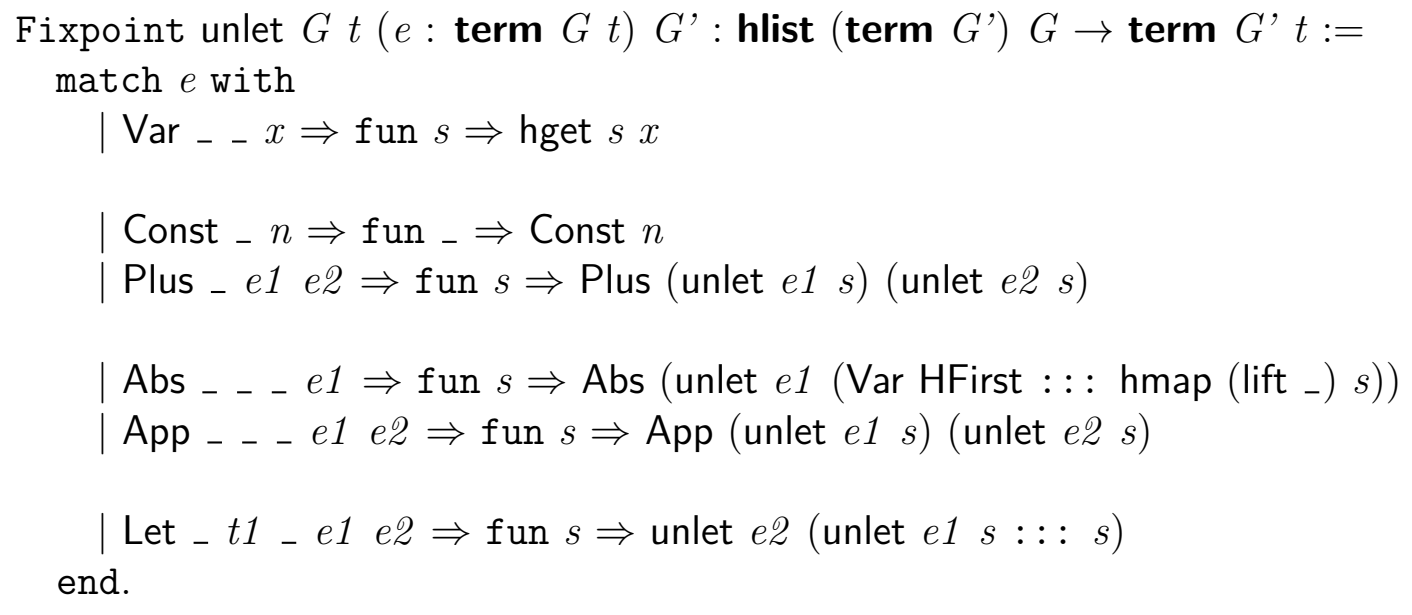

We have finished defining the transformation, but the parade of helper functions is not over. To prove correctness, we will use one more helper function and a few lemmas. First, we need an operation to insert a new value into a substitution at a particular position.

Fixpoint insertAtS $(t$ : type) $(x:$ typeDenote $t)(G:$ list type $)(n:$ nat $)$ struct $n\}$

$:$ hlist typeDenote $G \rightarrow$ hlist typeDenote (insertAt $t G n$ ) :=

match $n$ with

$\mathrm{O} \Rightarrow$ fun $s \Rightarrow x::: s$

I S $n^{\prime} \Rightarrow \operatorname{match} G$ return hlist typeDenote $G$

$\rightarrow$ hlist typeDenote (insertAt $t G\left(\mathrm{~S} n^{\prime}\right)$ ) with

$$
\begin{aligned}
& \mid \text { nil } \Rightarrow \text { fun } s \Rightarrow x::: s \\
& \qquad t^{\prime}:: G^{\prime} \Rightarrow \text { fun } s \Rightarrow \text { hhd } s:: \text { insertAtS } t x n^{\prime}(\text { htl } s) \\
& \text { end }
\end{aligned}
$$


end.

Arguments insertAtS $[t] x[G] n_{-}$.

Next we prove that liftVar is correct. That is, a lifted variable retains its value with respect to a substitution when we perform an analogue to lifting by inserting a new mapping into the substitution.

Lemma liftVarSound : $\forall t^{\prime}\left(x\right.$ : typeDenote $\left.t^{\prime}\right) t G(m:$ member $t G) s n$, hget $s m$ = hget (insertAtS $x n s$ ) (liftVar $m t^{\prime} n$ ). induction $m$; destruct $n$; dep_destruct $s$; $p l$.

Qed.

Hint Resolve liftVarSound.

An analogous lemma establishes correctness of lift'.

Lemma lift'Sound : $\forall G t^{\prime}\left(x\right.$ : typeDenote $\left.t^{\prime}\right) t(e:$ term $G t) n s$, termDenote $e s=$ termDenote (lift' $t^{\prime} n e$ ) (insertAtS $x n s$ ). induction $e ; p l$;

repeat match goal with | [IH: $\forall n s,{ }_{-}=$termDenote (lift' $\left.-n ? E\right)_{-}$

$\vdash$ context[lift' $-(\mathrm{S} ? N) ? E]] \Rightarrow$ specialize $(I H(\mathrm{~S} N))$ end; $p l$.

Qed.

Correctness of lift itself is an easy corollary.

Lemma liftSound : $\forall G t^{\prime}\left(x\right.$ : typeDenote $\left.t^{\prime}\right) t(e:$ term $G t) s$, termDenote (lift $\left.t^{\prime} e\right)(x::: s)=$ termDenote $e s$.

unfold lift; intros; rewrite (lift'Sound $-x e 0)$; trivial. Qed.

Hint Rewrite hget_hmap hmap_hmap liftSound.

Finally, we can prove correctness of unletSound for terms in arbitrary typing environments.

Lemma unletSound' : $\forall G t(e: \operatorname{term} G t) G^{\prime}\left(s:\right.$ hlist $\left.\left(\operatorname{term} G^{\prime}\right) G\right) s 1$, termDenote (unlet $e s) s 1$

$=$ termDenote $e\left(\operatorname{hmap}\left(\right.\right.$ fun $t^{\prime}\left(e^{\prime}:\right.$ term $\left.G^{\prime} t^{\prime}\right) \Rightarrow$ termDenote $\left.\left.e^{\prime} s 1\right) s\right)$. induction $e ; p l$.

Qed.

The lemma statement is a mouthful, with all its details of typing contexts and substitutions. It is usually prudent to state a final theorem in as simple a way as possible, to help your readers believe that you have proved what they expect. We follow that advice here for the simple case of terms with empty typing contexts.

Theorem unletSound : $\forall t(e:$ term nil $t)$, termDenote (unlet $e \mathrm{HNil}$ ) HNil = termDenote $e \mathrm{HNil}$. intros; apply unletSound'. 
Qed.

End FIRSTORDER.

The Let removal optimization is a good case study of a simple transformation that may turn out to be much more work than expected, based on representation choices. In the second part of this chapter, we consider an alternate choice that produces a more pleasant experience.

\subsection{Parametric Higher-Order Abstract Syntax}

In contrast to first-order encodings, higher-order encodings avoid explicit modeling of variable identity. Instead, the binding constructs of an object language (the language being formalized) can be represented using the binding constructs of the meta language (the language in which the formalization is done). The best known higher-order encoding is called higher-order abstract syntax (HOAS) [35], and we can start by attempting to apply it directly in Coq.

Module HigherORDER.

With HOAS, each object language binding construct is represented with a function of the meta language. Here is what we get if we apply that idea within an inductive definition of term syntax.

Inductive term : type $\rightarrow$ Type $:=$

| Const : nat $\rightarrow$ term Nat

| Plus : term Nat $\rightarrow$ term Nat $\rightarrow$ term Nat

Abs : $\forall$ dom ran, (term dom $\rightarrow$ term ran) $\rightarrow$ term (Func dom ran)

| App : $\forall$ dom ran, term (Func dom ran) $\rightarrow$ term $d o m \rightarrow$ term ran

| Let $: \forall t 1$ t2, term $t 1 \rightarrow($ term $t 1 \rightarrow$ term $t 2) \rightarrow$ term $t 2$.

However, Coq rejects this definition for failing to meet the strict positivity restriction. For instance, the constructor Abs takes an argument that is a function over the same type family term that we are defining. Inductive definitions of this kind can be used to write non-terminating Gallina programs, which breaks the consistency of Coq's logic.

An alternate higher-order encoding is parametric $H O A S$, as introduced by Washburn and Weirich [46] for Haskell and tweaked by me [5] for use in Coq. Here the idea is to parameterize the syntax type by a type family standing for a representation of variables.

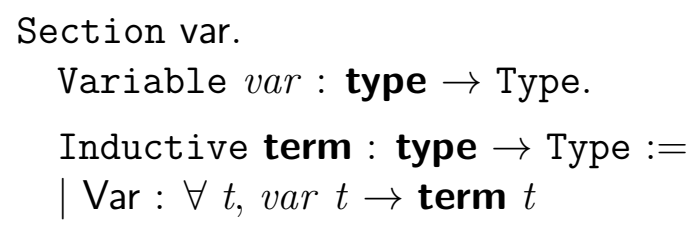




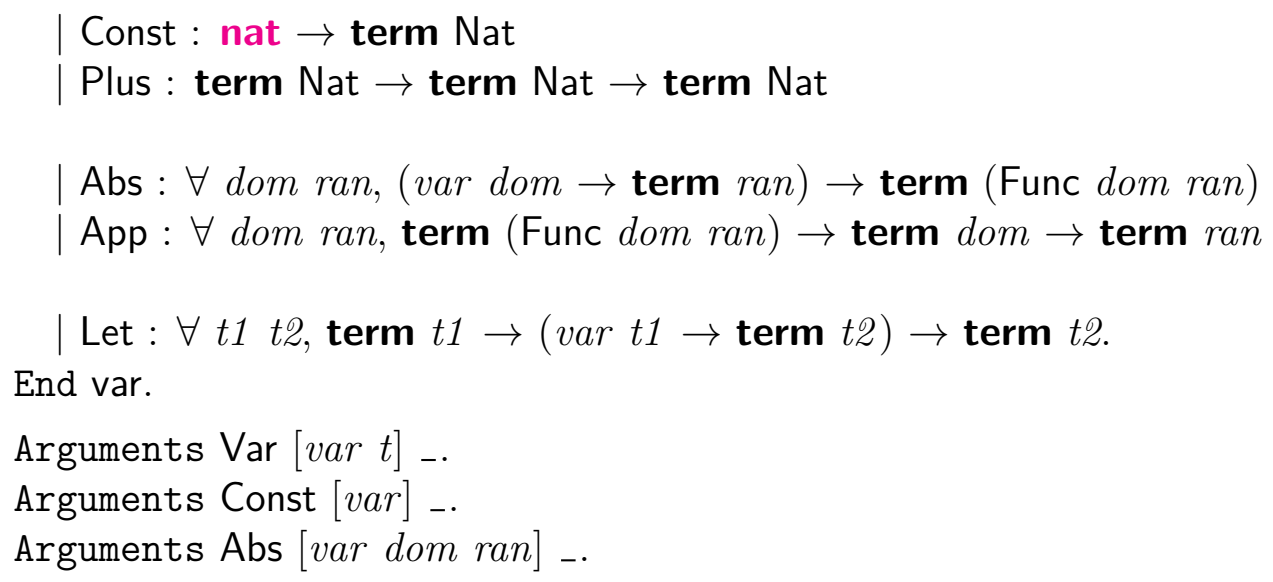

Coq accepts this definition because our embedded functions now merely take variables as arguments, instead of arbitrary terms. One might wonder whether there is an easy loophole to exploit here, instantiating the parameter var as term itself. However, to do that, we would need to choose a variable representation for this nested mention of term, and so on through an infinite descent into term arguments.

We write the final type of a closed term using polymorphic quantification over all possible choices of var type family.

Definition Term $t:=\forall$ var, term var $t$.

Here are the new representations of the example terms from the last section. Note how each is written as a function over a var choice, such that the specific choice has no impact on the structure of the term.

Example add : Term (Func Nat (Func Nat Nat)) := fun var $\Rightarrow$

Abs (fun $x \Rightarrow$ Abs (fun $y \Rightarrow$ Plus $(\operatorname{Var} x)(\operatorname{Var} y))$ ).

Example three_the_hard_way : Term Nat := fun var $\Rightarrow$

App (App (add var) (Const 1)) (Const 2).

The argument var does not even appear in the function body for add. How can that be? By giving our terms expressive types, we allow Coq to infer many arguments for us. In fact, we do not even need to name the var argument!

Example add' : Term (Func Nat (Func Nat Nat)) := fun _ $\Rightarrow$

Abs (fun $x \Rightarrow$ Abs (fun $y \Rightarrow$ Plus $(\operatorname{Var} x)(\operatorname{Var} y))$ ).

Example three_the_hard_way' : Term Nat := fun _ $\Rightarrow$

$\operatorname{App}\left(\operatorname{App}\left(\right.\right.$ add' $\left._{-}\right)$(Const 1)) (Const 2).

Even though the var formal parameters appear as underscores, they are mentioned in the function bodies that type inference calculates.

\subsubsection{Functional Programming with PHOAS}

It may not be at all obvious that the PHOAS representation admits the crucial computable operations. The key to effective deconstruction of PHOAS terms is one principle: treat 
the var parameter as an unconstrained choice of which data should be annotated on each variable. We will begin with a simple example, that of counting how many variable nodes appear in a PHOAS term. This operation requires no data annotated on variables, so we simply annotate variables with unit values. Note that, when we go under binders in the cases for Abs and Let, we must provide the data value to annotate on the new variable we pass beneath. For our current choice of unit data, we always pass tt.

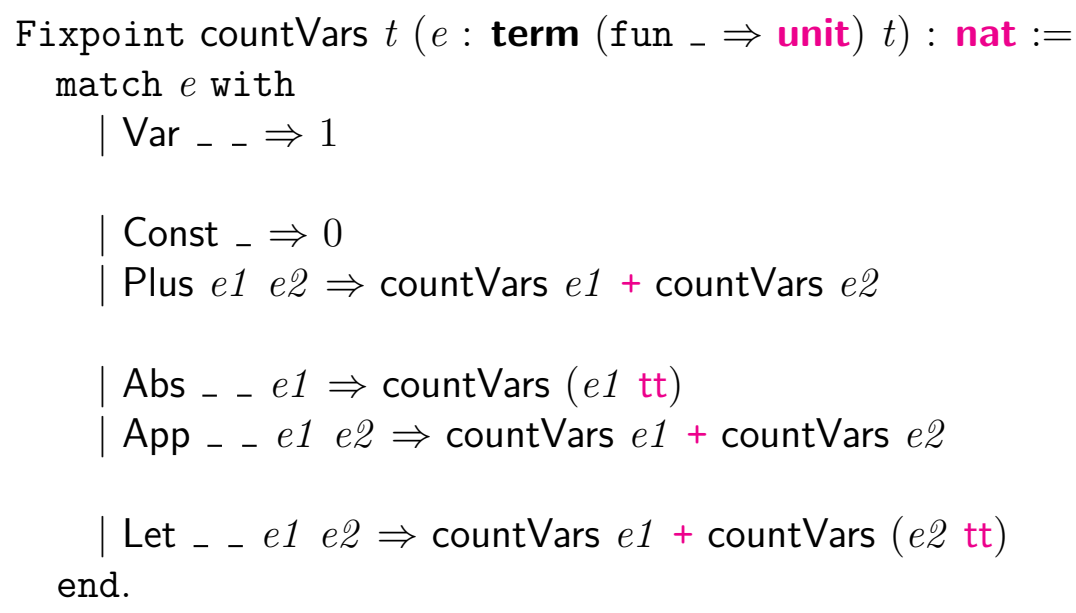

The above definition may seem a bit peculiar. What gave us the right to represent variables as unit values? Recall that our final representation of closed terms is as polymorphic functions. We merely specialize a closed term to exactly the right variable representation for the transformation we wish to perform.

Definition CountVars $t(E$ : Term $t):=$ countVars $\left(E\right.$ (fun $\_$unit)).

It is easy to test that CountVars operates properly.

Eval compute in CountVars three_the_hard_way. $=2$

In fact, PHOAS can be used anywhere that first-order representations can. We will not go into all the details here, but the intuition is that it is possible to interconvert between PHOAS and any reasonable first-order representation. Here is a suggestive example, translating PHOAS terms into strings giving a first-order rendering. To implement this translation, the key insight is to tag variables with strings, giving their names. The function takes as an additional input a string giving the name to be assigned to the next variable introduced. We evolve this name by adding a prime to its end. To avoid getting bogged down in orthogonal details, we render all constants as the string " $N$ ".

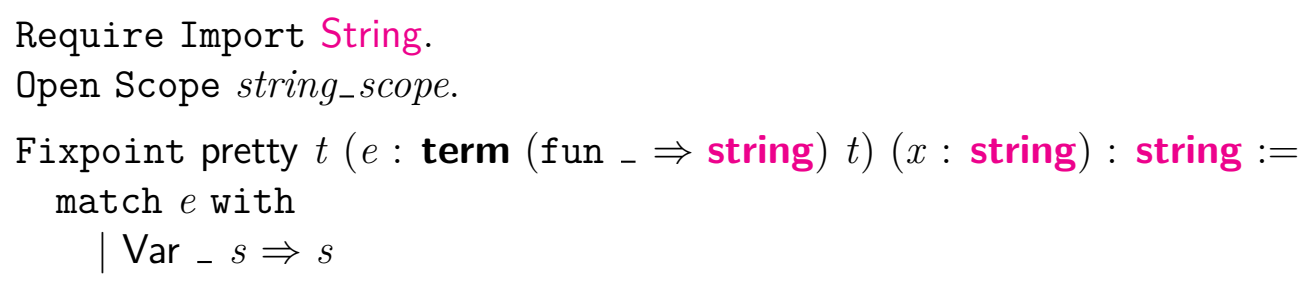




$$
\begin{aligned}
& \text { | Const _ } \Rightarrow \text { "N" }
\end{aligned}
$$

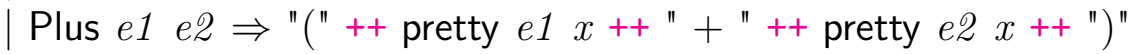

$$
\begin{aligned}
& \text { | Abs _ } e 1 \Rightarrow \text { "(fun " ++ } x++"=>\text { " ++ pretty }(e 1 x)(x++" \text { " " })++ \text { ")" }
\end{aligned}
$$

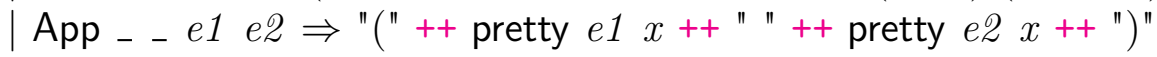

$$
\begin{aligned}
& \text { | Let _ } e 1 \text { e2 } \Rightarrow \text { "(let " }++x++"="++ \text { pretty } e 1 x++ \text { " in " } \\
& ++ \text { pretty }(\text { e2 } x)(x++" \text { " ") ++ ")" } \\
& ="\left(\left(\left(\text { fun } \mathrm{x}=>\left(\text { fun } \mathrm{x}^{\prime}=>\left(\mathrm{x}+\mathrm{x}^{\prime}\right)\right)\right) \mathrm{N}\right) \mathrm{N}\right) "
\end{aligned}
$$

However, it is not necessary to convert to first-order form to support many common operations on terms. For instance, we can implement substitution of terms for variables. The key insight here is to tag variables with terms, so that, on encountering a variable, we can simply replace it by the term in its tag. We will call this function initially on a term with exactly one free variable, tagged with the appropriate substitute. During recursion, new variables are added, but they are only tagged with their own term equivalents. Note that this function squash is parameterized over a specific var choice.

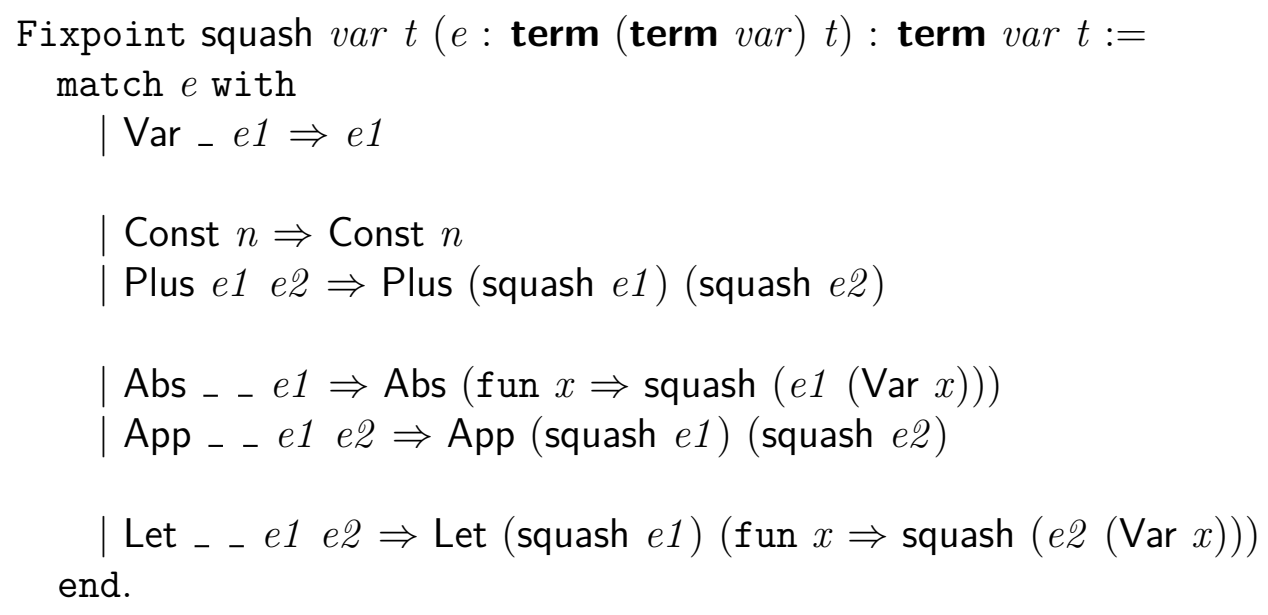

To define the final substitution function over terms with single free variables, we define Term1, an analogue to Term that we defined before for closed terms.

Definition Term1 $(t 1$ t2 $:$ type $):=\forall$ var, var $t 1 \rightarrow$ term var $t$ 2.

Substitution is defined by (1) instantiating a Term1 to tag variables with terms and (2) applying the result to a specific term to be substituted. Note how the parameter var of squash is instantiated: the body of Subst is itself a polymorphic quantification over var, standing for a variable tag choice in the output term; and we use that input to compute a tag choice for the input term.

Definition Subst $\left(t 1\right.$ t2 : type) $(E:$ Term1 t1 $t 2)\left(E^{\prime}:\right.$ Term $\left.t 1\right)$ : Term $t 2:=$ 


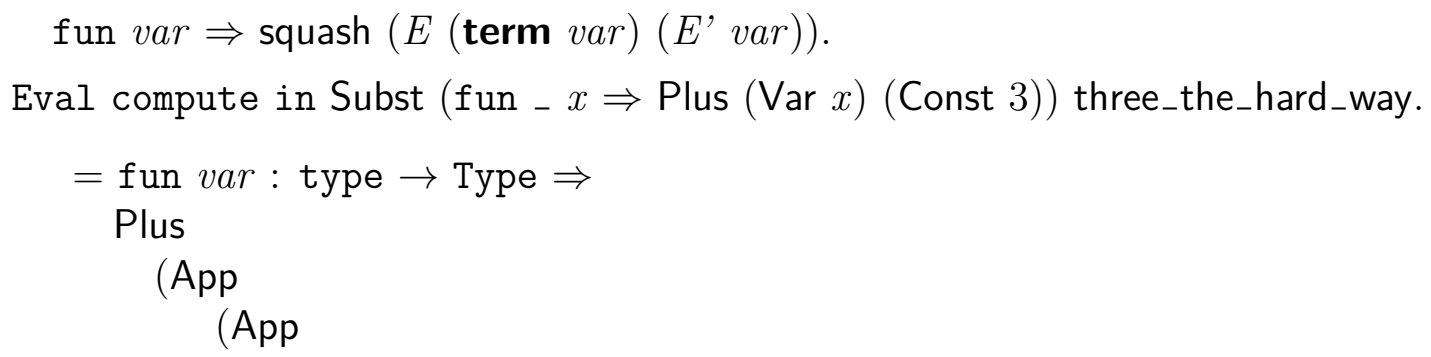

(Abs

(fun $x: \operatorname{var}$ Nat $\Rightarrow$

Abs (fun $y: \operatorname{var}$ Nat $\Rightarrow$ Plus $(\operatorname{Var} x)(\operatorname{Var} y)))$ )

(Const 1)) (Const 2)) (Const 3)

One further development, which may seem surprising at first, is that we can also implement a usual term denotation function, when we tag variables with their denotations.

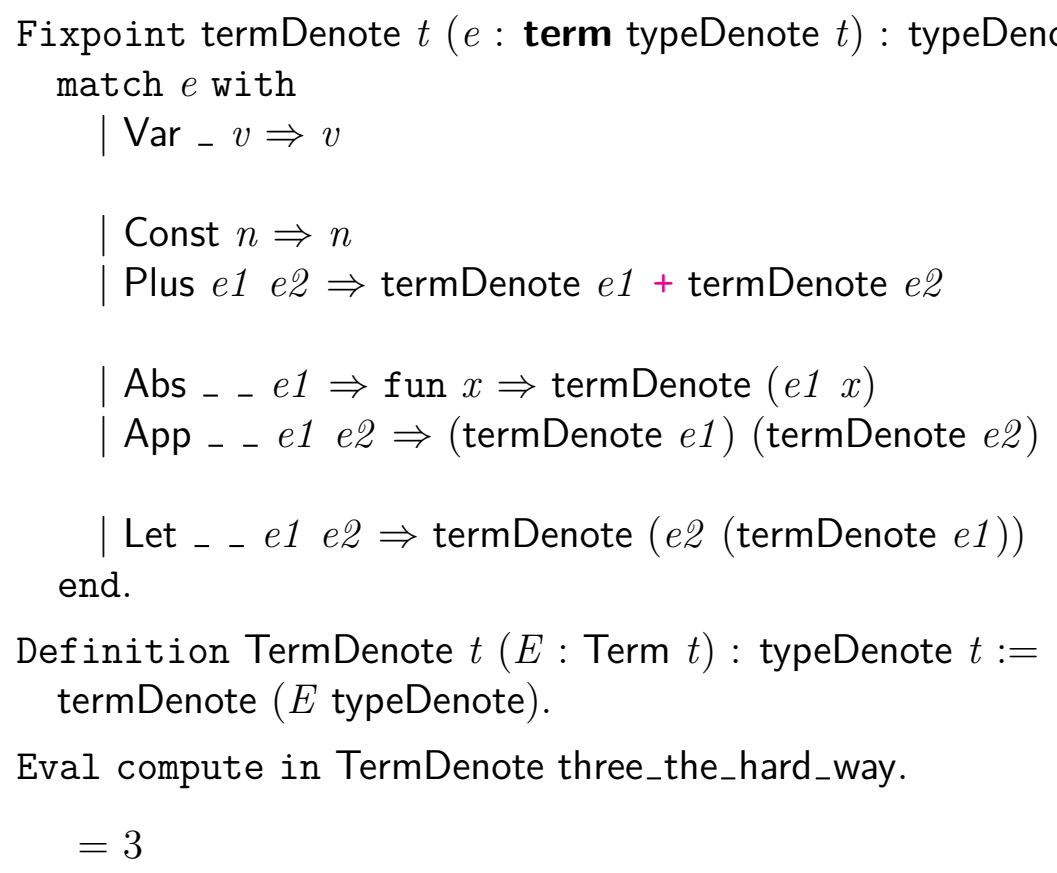

To summarize, the PHOAS representation has all the expressive power of more standard first-order encodings, and a variety of translations are actually much more pleasant to implement than usual, thanks to the novel ability to tag variables with data.

\subsubsection{Verifying Program Transformations}

Let us now revisit the three example program transformations from the last section. Each is easy to implement with PHOAS, and the last is substantially easier than with first-order representations.

First, we have the recursive identity function, following the same pattern as in the previous subsection, with a helper function, polymorphic in a tag choice; and a final function that instantiates the choice appropriately. 


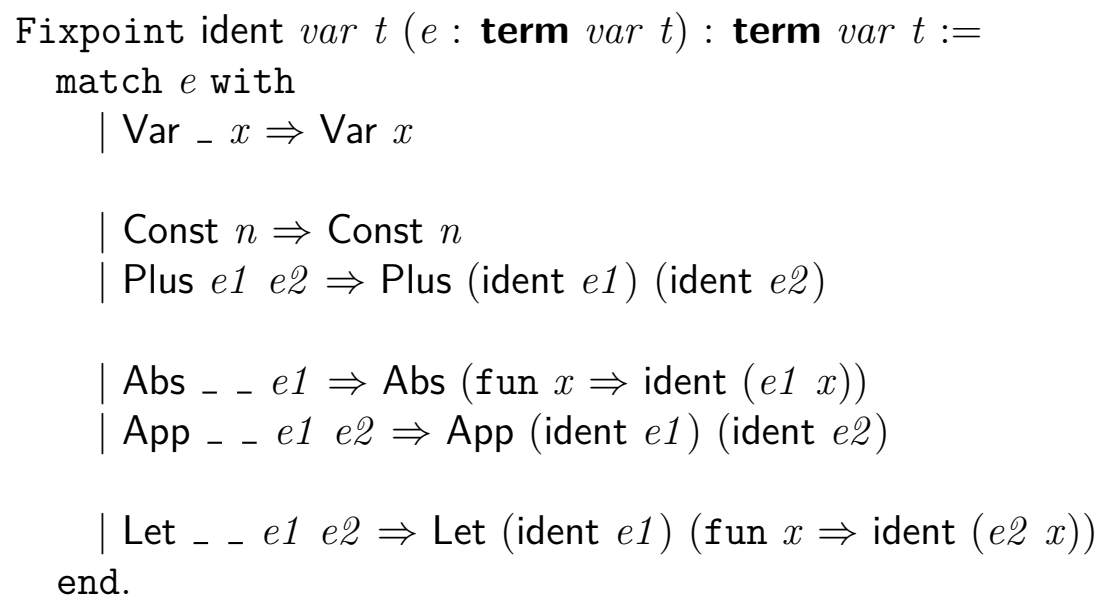

Definition Ident $t(E:$ Term $t):$ Term $t:=$ fun var $\Rightarrow$ ident ( $E$ var).

Proving correctness is both easier and harder than in the last section, easier because we do not need to manipulate substitutions, and harder because we do the induction in an extra lemma about ident, to establish the correctness theorem for Ident.

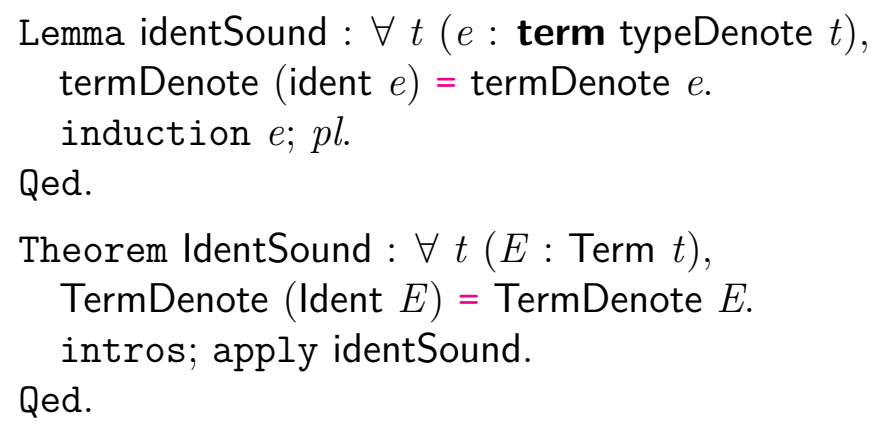

The translation of the constant-folding function and its proof work more or less the same way.

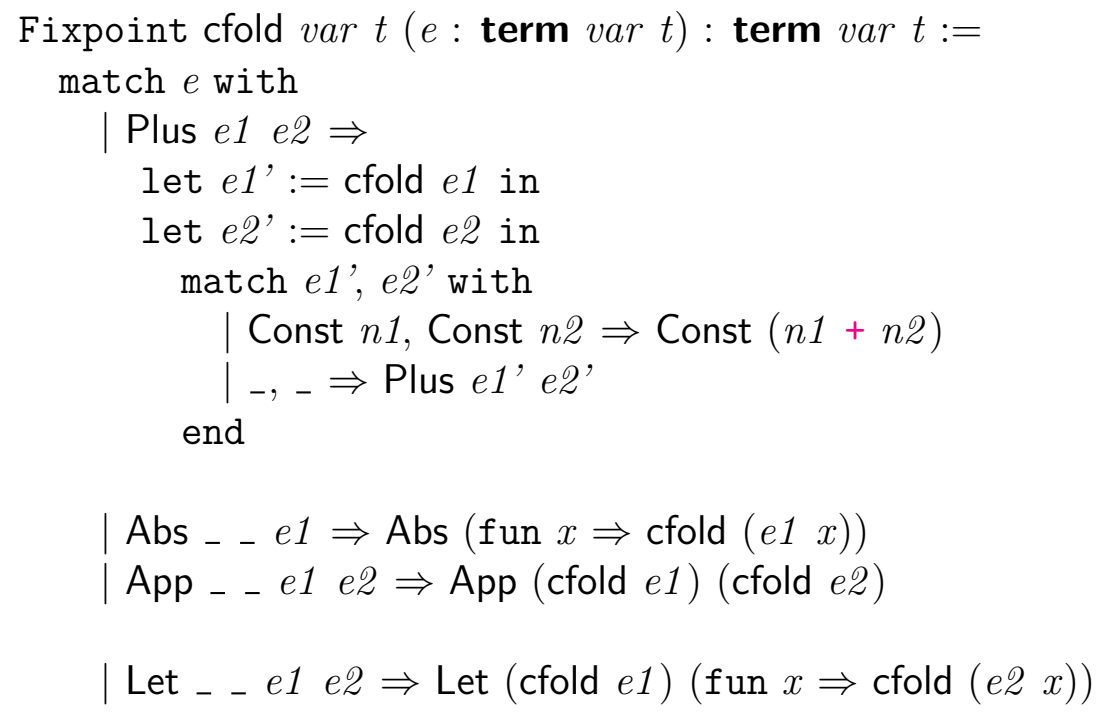




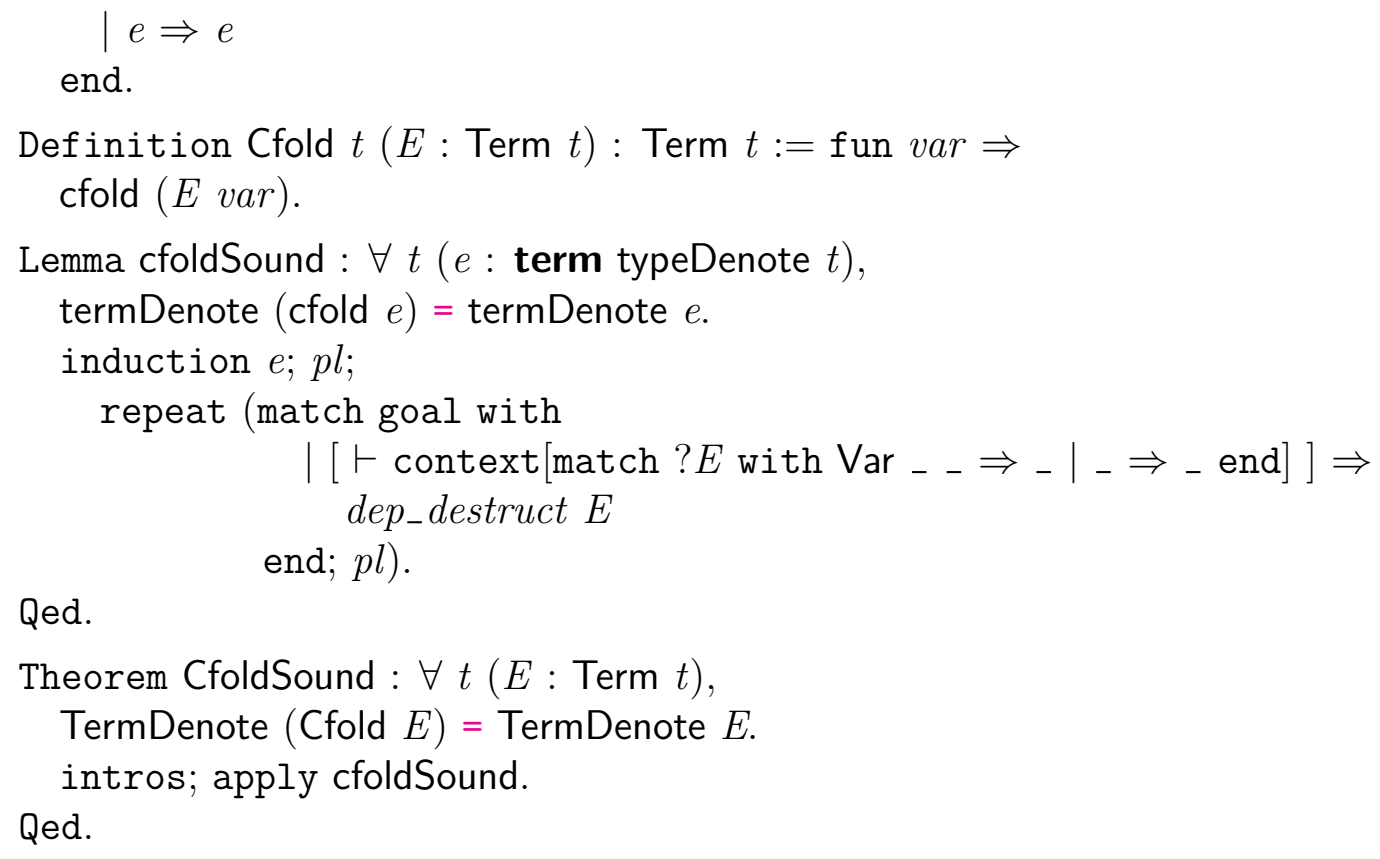

Qed.

Theorem CfoldSound : $\forall t(E$ : Term $t)$,

TermDenote $($ Cfold $E)=$ TermDenote $E$.

intros; apply cfoldSound.

\section{Qed.}

Things get more interesting in the Let-removal optimization. Our recursive helper function adapts the key idea from our earlier definitions of squash and Subst: tag variables with terms. We have a straightforward generalization of squash, where only the Let case has changed, to tag the new variable with the term it is bound to, rather than just tagging the variable with itself as a term.

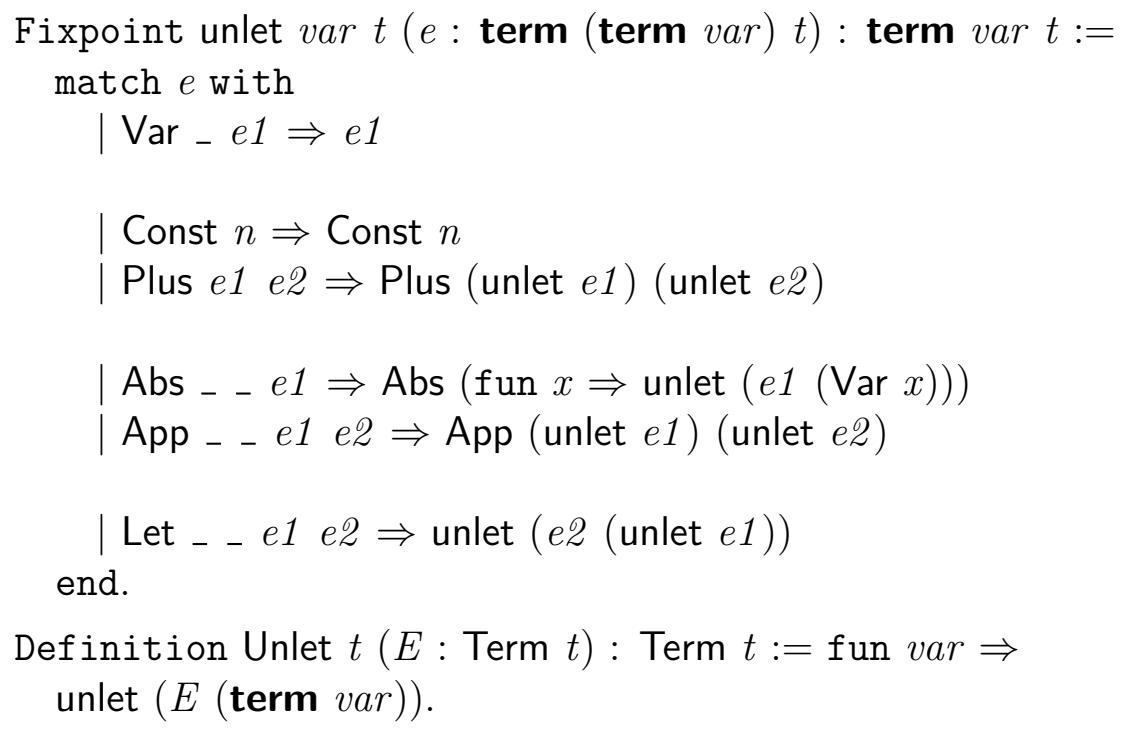

We can test Unlet first on an uninteresting example, three_the_hard_way, which does not use Let.

Eval compute in Unlet three_the_hard_way.

$$
=\text { fun var : type } \rightarrow \text { Type } \Rightarrow
$$


App

(App

(Abs

(fun $x:$ var Nat $\Rightarrow$

Abs $($ fun $x 0$ : var Nat $\Rightarrow$ Plus $(\operatorname{Var} x)(\operatorname{Var} x 0))))$

(Const 1)) (Const 2)

Next, we try a more interesting example, with some extra Lets introduced in three_the_hard_way.

Definition three_a_harder_way : Term Nat $:=$ fun $\_\Rightarrow$

Let (Const 1) (fun $x \Rightarrow$ Let (Const 2) (fun $y \Rightarrow \operatorname{App}\left(\operatorname{App}\left(\operatorname{add} \_\right)(\operatorname{Var} x)\right.$ ) (Var $\left.\left.y\right)\right)$ ).

Eval compute in Unlet three_a_harder_way.

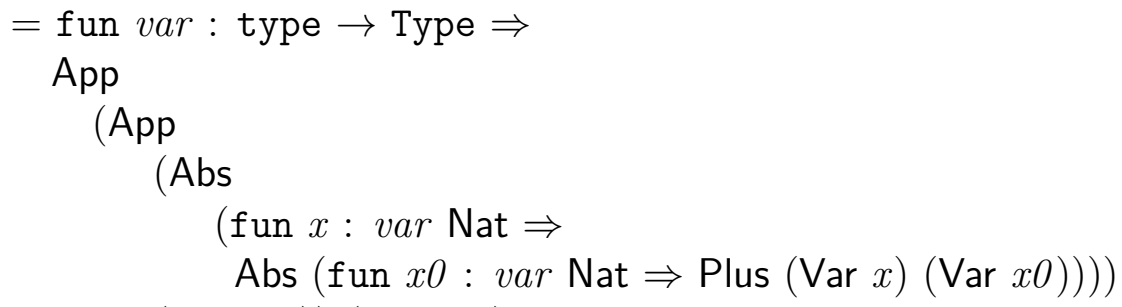

The output is the same as in the previous test, confirming that Unlet operates properly here.

Now we need to state a correctness theorem for Unlet, based on an inductively proved lemma about unlet. It is not at all obvious how to arrive at a proper induction principle for the lemma. The problem is that we want to relate two instantiations of the same Term, in a way where we know they share the same structure. Note that, while Unlet is defined to consider all possible var choices in the output term, the correctness proof conveniently only depends on the case of $v a r:=$ typeDenote. Thus, one parallel instantiation will set var $:=$ typeDenote, to take the denotation of the original term. The other parallel instantiation will set $\operatorname{var}:=$ term typeDenote, to perform the unlet transformation in the original term.

Here is a relation formalizing the idea that two terms are structurally the same, differing only by replacing the variable data of one with another isomorphic set of variable data in some possibly different type family.

Section wf.

Variables var1 var2 : type $\rightarrow$ Type.

To formalize the tag isomorphism, we will use lists of values with the following record type. Each entry has an object language type and an appropriate tag for that type, in each of the two tag families var1 and var2.

\footnotetext{
Record varEntry $:=\{$

Ty : type;

First : $\operatorname{var} 1 \mathrm{Ty}$;

Second : var2 Ty
} 
\} .

Here is the inductive relation definition. An instance wf $G$ e1 ed asserts that terms $e 1$ and $e^{2}$ are equivalent up to the variable tag isomorphism $G$. Note how the Var rule looks up an entry in $G$, and the Abs and Let rules include recursive wf invocations inside the scopes of quantifiers to introduce parallel tag values to be considered as isomorphic.

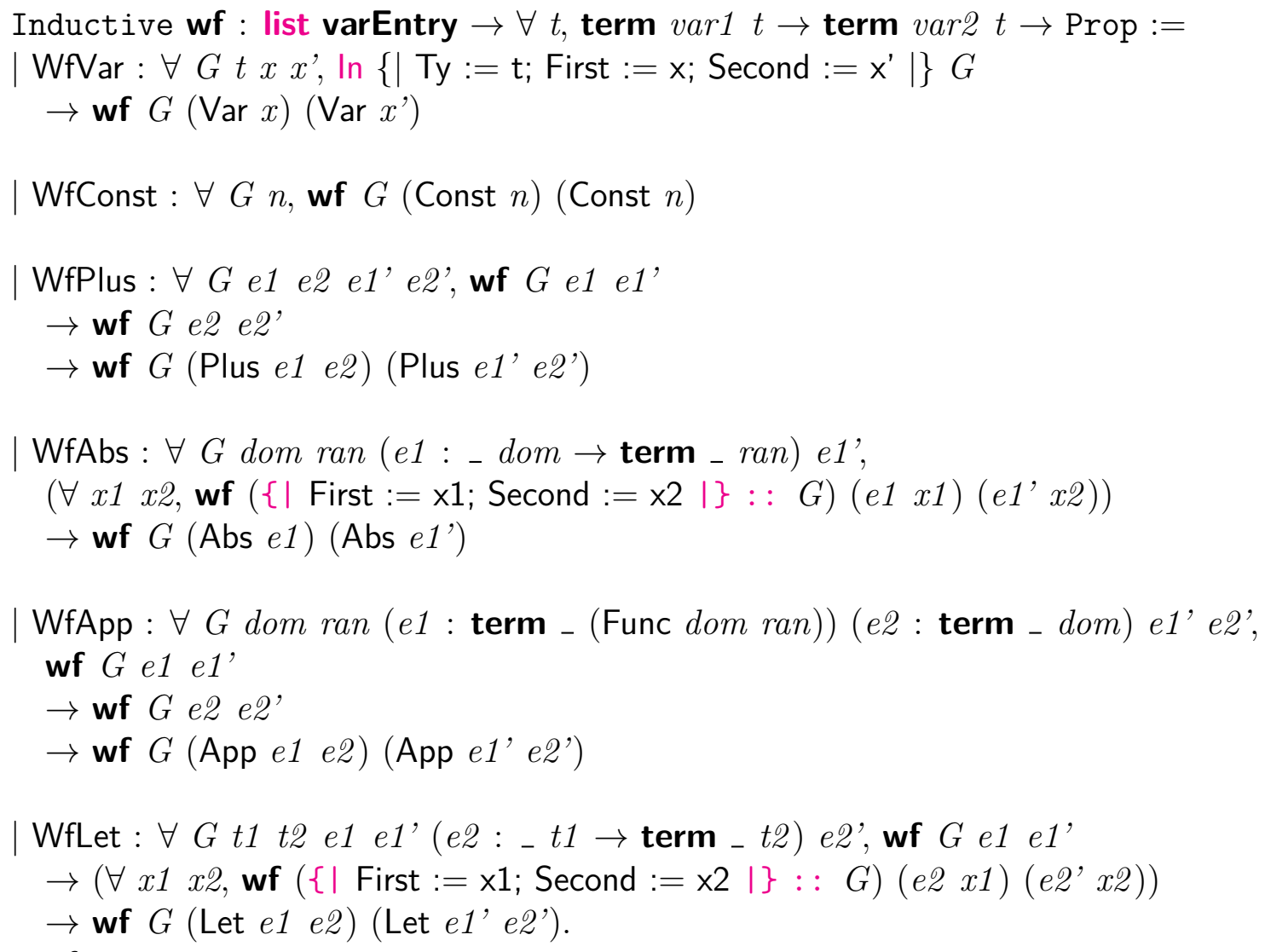

We can state a well-formedness condition for closed terms: for any two choices of tag type families, the parallel instantiations belong to the wf relation, starting from an empty variable isomorphism.

Definition Wf $t(E:$ Term $t):=\forall \operatorname{var1}$ vard, wf nil $(E \operatorname{var} 1)(E \operatorname{var} 2)$.

After digesting the syntactic details of $\mathrm{Wf}$, it is probably not hard to see that reasonable term encodings will satisfy it. For example:

Theorem three_the_hard_way_Wf : Wf three_the_hard_way.

red; intros; repeat match goal with

$$
\mid[\vdash \mathbf{w f} \ldots
$$

end; intuition.

Qed.

Now we are ready to give a nice simple proof of correctness for unlet. First, we add one hint to apply a small variant of a standard library theorem connecting Forall, a higher- 
order predicate asserting that every element of a list satisfies some property; and In, the list membership predicate.

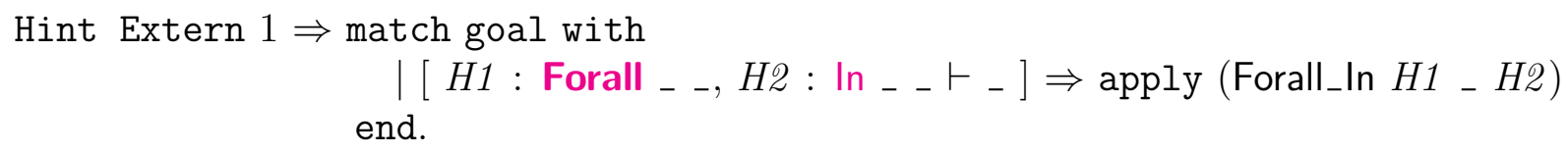

The rest of the proof is about as automated as we could hope for.

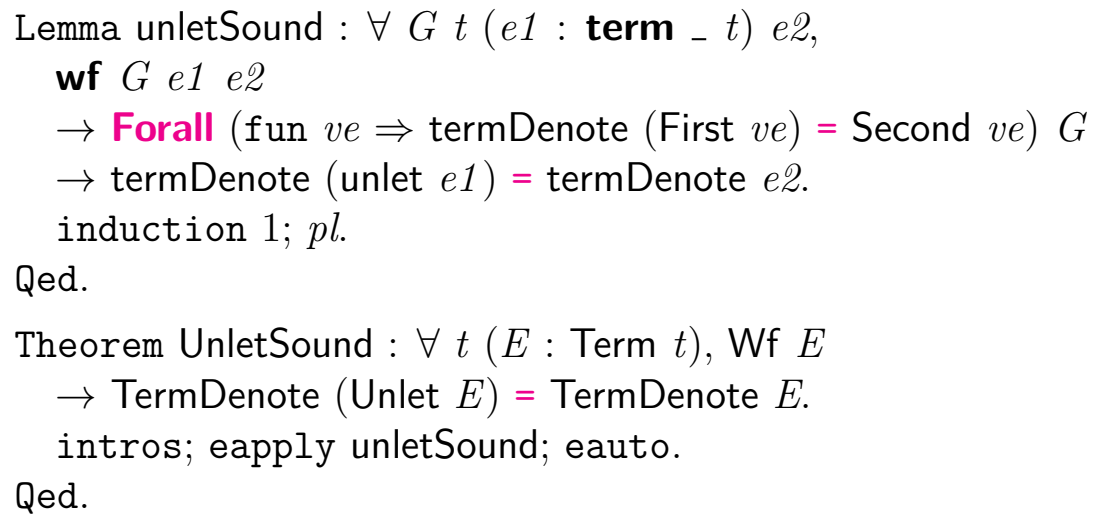

With this example, it is not obvious that the PHOAS encoding is more tractable than dependent de Bruijn. Where the de Bruijn version had lift and its helper functions, here we have $\mathrm{Wf}$ and its auxiliary definitions. In practice, $\mathrm{Wf}$ is defined once per object language, while such operations as lift often need to operate differently for different examples, forcing new implementations for new transformations.

The reader may also have come up with another objection: via Curry-Howard, wf proofs may be thought of as first-order encodings of term syntax! For instance, the In hypothesis of rule WfVar is equivalent to a member value. There is some merit to this objection. However, as the proofs above show, we are able to reason about transformations using first-order representation only for their inputs, not their outputs. Furthermore, explicit numbering of variables remains absent from the proofs.

Have we really avoided first-order reasoning about the output terms of translations? The answer depends on some subtle issues, which deserve a subsection of their own.

\subsubsection{Establishing Term Well-Formedness}

Can there be values of type Term $t$ that are not well-formed according to Wf? We expect that Gallina satisfies key parametricity [38] properties, which indicate how polymorphic types may only be inhabited by specific values. We omit details of parametricity theorems here, but $\forall t(E:$ Term $t)$, Wf $E$ follows the flavor of such theorems. One option would be to assert that fact as an axiom, "proving" that any output of any of our translations is wellformed. We could even prove the soundness of the theorem on paper meta-theoretically, say by considering some particular model of CIC.

To be more cautious, we could prove $\mathrm{Wf}$ for every term that interests us, threading such proofs through all transformations. Here is an example exercise of that kind, for Unlet. 
First, we prove that wf is monotone, in that a given instance continues to hold as we add new variable pairs to the variable isomorphism.

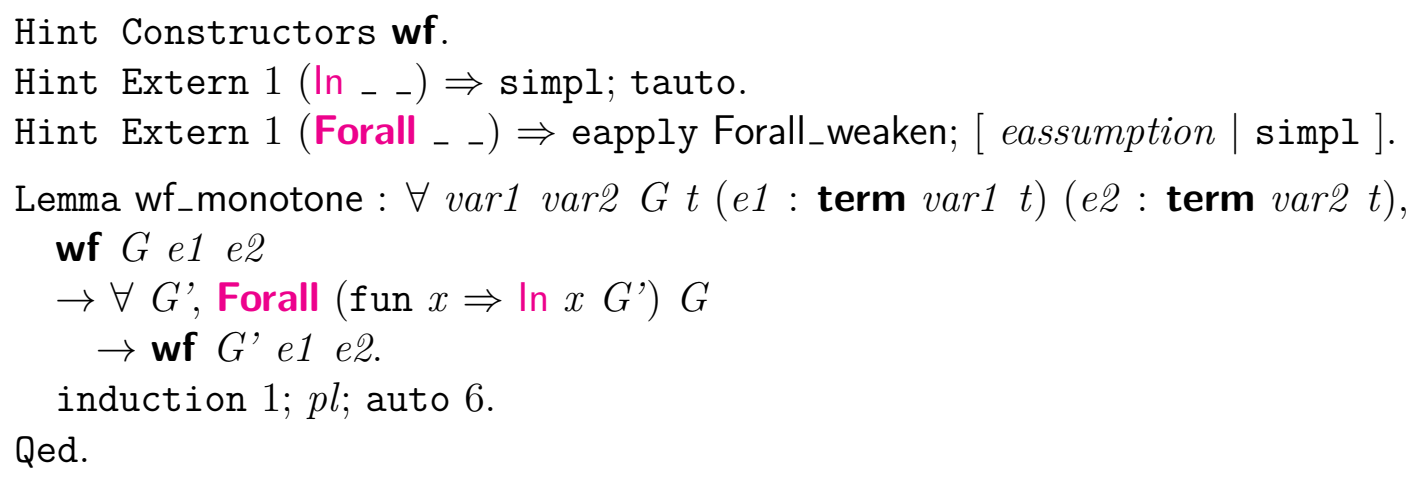

Hint Resolve wf_monotone Forall_In'.

Now we are ready to prove that unlet preserves any wf instance. The key invariant has to do with the parallel execution of unlet on two different var instantiations of a particular term. Since unlet uses term as the type of variable data, our variable isomorphism context $G$ contains pairs of terms, which, conveniently enough, allows us to state the invariant that any pair of terms in the context is also related by wf.

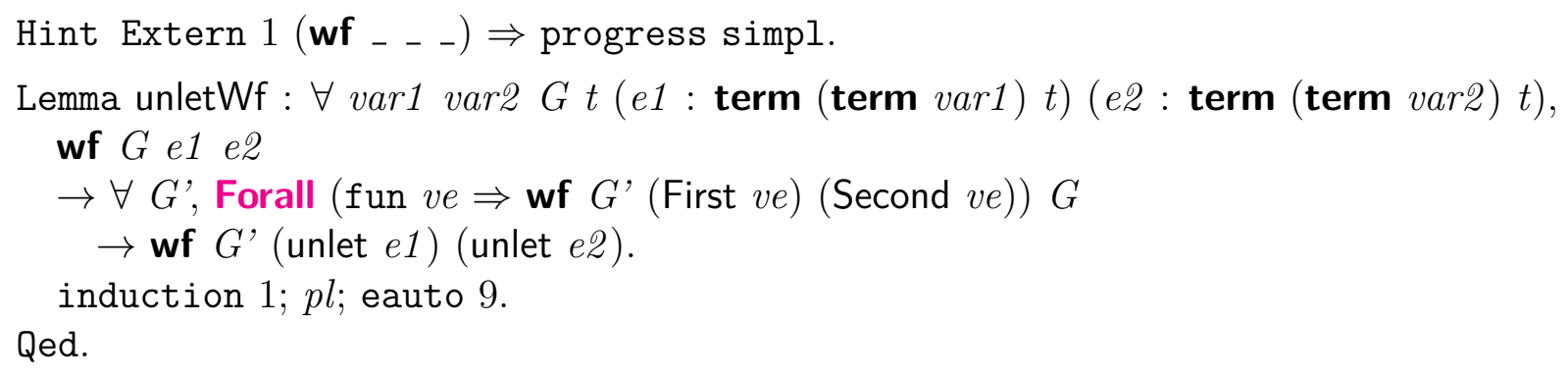

Repackaging unletWf into a theorem about Wf and Unlet is straightforward.

Theorem UnletWf : $\forall t(E:$ Term $t)$, Wf $E$

$\rightarrow \mathrm{Wf}($ Unlet $E)$.

red; intros; apply unletWf with nil; auto. Qed.

This example demonstrates how we may need to use reasoning reminiscent of that associated with first-order representations, though the bookkeeping details are generally easier to manage, and bookkeeping theorems may generally be proved separately from the independently interesting theorems about program transformations.

\subsubsection{A Few More Remarks}

Higher-order encodings derive their strength from reuse of the meta language's binding constructs. As a result, we can write encoded terms so that they look very similar to their informal counterparts, without variable numbering schemes like for de Bruijn indices. The example encodings above have demonstrated this fact, but modulo the clunkiness of explicit 
use of the constructors of term. After defining a few new Coq syntax notations, we can work with terms in an even more standard form.

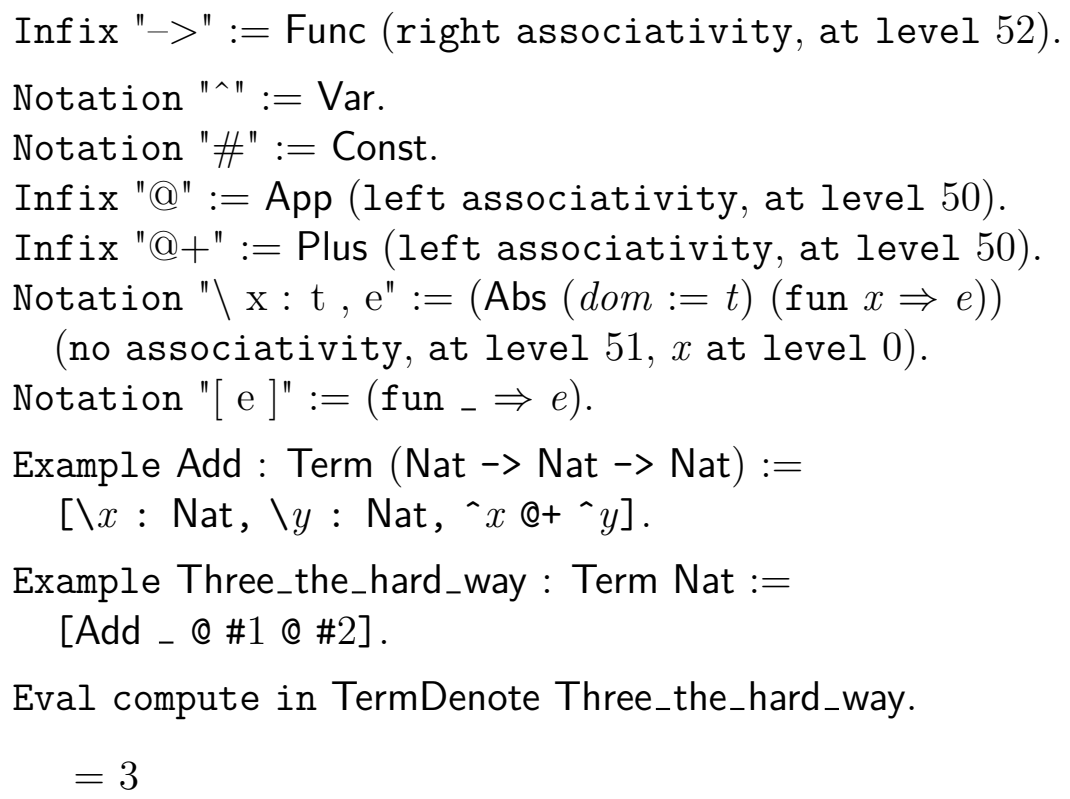

\section{End HigherORDER.}

The PHOAS approach shines here because we are working with an object language that has an easy embedding into Coq. That is, there is a straightforward recursive function translating object terms into terms of Gallina. All Gallina programs terminate, so clearly we cannot hope to find such embeddings for Turing-complete languages; and non-Turingcomplete languages may still require much more involved translations. I have some work [6] on modeling semantics of Turing-complete languages with PHOAS, but my impression is that there are many more advances left to be made in this field, possibly with completely new term representations that we have not yet been clever enough to think up. 


\section{Conclusion}

I have designed this book to present the key ideas needed to get started with productive use of Coq. Many people have learned to use Coq through a variety of resources, yet there is a distinct lack of agreement on structuring principles and techniques for easing the evolution of Coq developments over time. Here I have emphasized two unusual techniques: programming with dependent types and proving with scripted proof automation. I have also tried to present other material following my own take on how to keep Coq code beautiful and scalable.

Part of the attraction of Coq and similar tools is that their logical foundations are small. A few pages of LTEX code suffice to define CIC, Coq's logic, yet there do not seem to be any practical limits on which mathematical concepts may be encoded on top of this modest base. At the same time, the pragmatic foundation of Coq is vast, encompassing tactics, libraries, and design patterns for programs, theorem statements, and proof scripts. I hope the preceding chapters have given a sense of just how much there is to learn before it is possible to drive Coq with the same ease with which many readers write informal proofs! The pay-off of this learning process is that many proofs, especially those with many details to check, become much easier to write than they are on paper. Further, the truth of such theorems may be established with much greater confidence, even without reading proof details.

As Coq has so many moving parts to catalogue mentally, I have not attempted to describe most of them here; nor have I attempted to give exhaustive descriptions of the few I devote space to. To those readers who have made it this far through the book, my advice is: read through the Coq manual, front to back, at some level of detail. Get a sense for which bits of functionality are available. Dig more into those categories that sound relevant to the developments you want to build, and keep the rest in mind in case they come in handy later.

In a domain as rich as this one, the learning process never ends. The Coq Club mailing list (linked from the Coq home page) is a great place to get involved in discussions of the latest improvements, or to ask questions about stumbling blocks that you encounter. (I hope that this book will save you from needing to ask some of the most common questions!) I believe the best way to learn is to get started using Coq to build some development that interests you.

Good luck! 


\section{Bibliography}

[1] Yves Bertot and Pierre Castéran. Interactive Theorem Proving and Program Development. Coq'Art: The Calculus of Inductive Constructions. Texts in Theoretical Computer Science. Springer Verlag, 2004.

[2] Samuel Boutin. Using reflection to build efficient and certified decision procedures. In Proceedings of the Third International Symposium on Theoretical Aspects of Computer Software, pages 515-529, 1997.

[3] Robert S. Boyer, Matt Kaufmann, and J Strother Moore. The Boyer-Moore theorem prover and its interactive enhancement. Computers and Mathematics with Applications, 29(2):27-62, 1995.

[4] Venanzio Capretta. General recursion via coinductive types. Logical Methods in Computer Science, 1(2):1-18, 2005.

[5] Adam Chlipala. Parametric higher-order abstract syntax for mechanized semantics. In Proceedings of the 13th ACM SIGPLAN International Conference on Functional Programming, pages 143-156, 2008.

[6] Adam Chlipala. A verified compiler for an impure functional language. In Proceedings of the 37th ACM SIGPLAN-SIGACT Symposium on Principles of Programming Languages, pages 93-106, 2010.

[7] Coq Development Team. The Coq proof assistant reference manual, version 8.4. 2012.

[8] Thierry Coquand. An analysis of Girard's paradox. In Proceedings of the Symposium on Logic in Computer Science, pages 227-236, 1986.

[9] Thierry Coquand and Gérard Huet. The Calculus of Constructions. Information and Computation, 76(2-3), 1988.

[10] H. B. Curry. Functionality in combinatory logic. Proceedings of the National Academy of Sciences of the United States of America, 20(11):584-590, 1934.

[11] Nicolas G. de Bruijn. Lambda-calculus notation with nameless dummies: a tool for automatic formal manipulation with application to the Church-Rosser theorem. Indag. Math., 34(5):381-392, 1972. 
[12] Eduardo Giménez. A tutorial on recursive types in Coq. Technical Report 0221, INRIA, May 1998.

[13] Georges Gonthier. Formal proof-the four-color theorem. Notices of the American Mathematical Society, 55(11):1382-1393, 2008.

[14] William A. Howard. The formulae-as-types notion of construction. In Jonathan P. Seldin and J. Roger Hindley, editors, To H.B. Curry: Essays on Combinatory Logic, Lambda Calculus and Formalism, pages 479-490. Academic Press, 1980. Original paper manuscript from 1969.

[15] Gérard Huet. The undecidability of unification in third order logic. Information and Control, pages 257-267, 1973.

[16] John Hughes. Why functional programming matters. The Computer Journal, 32:98-107, 1984.

[17] Matt Kaufmann, Panagiotis Manolios, and J Strother Moore. Computer-Aided Reasoning: An Approach. Kluwer Academic Publishers, 2000.

[18] Gerwin Klein, Kevin Elphinstone, Gernot Heiser, June Andronick, David Cock, Philip Derrin, Dhammika Elkaduwe, Kai Engelhardt, Rafal Kolanski, Michael Norrish, Thomas Sewell, Harvey Tuch, and Simon Winwood. seL4: Formal verification of an OS kernel. In Proceedings of the 22nd ACM Symposium on Operating Systems Principles, pages 207-220, 2009.

[19] Xavier Leroy. A formally verified compiler back-end. Journal of Automated Reasoning, 43(4):363-446, 2009.

[20] Xavier Leroy and Hervé Grall. Coinductive big-step operational semantics. In Proceedings of the 15th European Symposium on Programming, pages 54-68, 2006.

[21] John W. Lloyd. Foundations of Logic Programming, 2nd Edition. Springer, 1987.

[22] David MacQueen. Modules for Standard ML. In Proceedings of the 1984 ACM Symposium on LISP and Functional Programming, pages 198-207, 1984.

[23] Conor McBride. Elimination with a motive. In Proceedings of the International Workshop on Types for Proofs and Programs, pages 197-216, 2000.

[24] Adam Megacz. A coinductive monad for prop-bounded recursion. In Proceedings of the ACM Workshop Programming Languages meets Program Verification, pages 11-20, 2007.

[25] J Strother Moore. Piton: A Mechanically Verified Assembly-Level Language. Automated Reasoning Series. Kluwer Academic Publishers, 1996. 
[26] J Strother Moore, Tom Lynch, and Matt Kaufmann. A mechanically checked proof of the correctness of the kernel of the AMD5k86 floating-point division algorithm. IEEE Transactions on Computers, 47(9):913-926, 1998.

[27] George C. Necula. Proof-carrying code. In Proceedings of the 24th ACM SIGPLANSIGACT Symposium on Principles of Programming Languages, pages 106-119, 1997.

[28] Zhaozhong Ni and Zhong Shao. Certified assembly programming with embedded code pointers. In Proceedings of the 33rd ACM SIGPLAN-SIGACT Symposium on Principles of Programming Languages, pages 320-333, 2006.

[29] Tobias Nipkow, Lawrence C. Paulson, and Markus Wenzel. Isabelle/HOL - A Proof Assistant for Higher-Order Logic, volume 2283 of Lecture Notes in Computer Science. Springer, 2002.

[30] Chris Okasaki. Red-black trees in a functional setting. J. Funct. Program., 9:471-477, 1999.

[31] Christine Paulin-Mohring. Inductive definitions in the system Coq - rules and properties. In Proceedings of the International Conference on Typed Lambda Calculi and Applications, pages 328-345, 1993.

[32] Lawrence C. Paulson. Isabelle: A Generic Theorem Prover, volume 828 of Lecture Notes in Computer Science. Springer, 1994.

[33] Simon Peyton Jones, Lennart Augustsson, Dave Barton, Brian Boutel, Warren Burton, Joseph Fasel, Kevin Hammond, Ralf Hinze, Paul Hudak, John Hughes, Thomas Johnsson, Mark Jones, John Launchbury, Erik Meijer, John Peterson, Alastair Reid, Colin Runciman, and Philip Wadler. Haskell 98 Language and Libraries: The Revised Report, chapter 4.3.3. 1998.

[34] Simon L. Peyton Jones and Philip Wadler. Imperative functional programming. In Proceedings of the 20th ACM SIGPLAN-SIGACT Symposium on Principles of Programming Languages, pages 71-84, 1993.

[35] F. Pfenning and C. Elliot. Higher-order abstract syntax. In Proceedings of the ACM SIGPLAN 1988 Conference on Programming Language Design and Implementation, pages 199-208, 1988.

[36] Benjamin C. Pierce. Types and Programming Languages. MIT Press, 2002.

[37] Benjamin C. Pierce. Types and Programming Languages, chapter 9.4. MIT Press, 2002.

[38] J.C. Reynolds. Types, abstraction, and parametric polymorphism. Information Processing, pages 513-523, 1983. 
[39] John C. Reynolds. The discoveries of continuations. Lisp Symb. Comput., 6(3-4):233248, November 1993.

[40] John C. Reynolds. Separation logic: A logic for shared mutable data structures. In Proceedings of the IEEE Symposium on Logic in Computer Science, pages 55-74, 2002.

[41] J. A. Robinson. A machine-oriented logic based on the resolution principle. J. ACM, 12(1):23-41, January 1965.

[42] Leon Sterling and Ehud Shapiro. The Art of Prolog, 2nd Edition. MIT Press, 1994.

[43] Thomas Streicher. Semantical Investigations into Intensional Type Theory. Habilitationsschrift, LMU München, 1993.

[44] P. Wadler and S. Blott. How to make ad-hoc polymorphism less ad hoc. In Proceedings of the 16th ACM SIGPLAN-SIGACT Symposium on Principles of Programming Languages, pages 60-76, 1989.

[45] Philip Wadler. The essence of functional programming. In Proceedings of the 19th ACM SIGPLAN-SIGACT Symposium on Principles of Programming Languages, pages 1-14, 1992.

[46] Geoffrey Washburn and Stephanie Weirich. Boxes go bananas: Encoding higher-order abstract syntax with parametric polymorphism. J. Funct. Program., 18(1):87-140, 2008.

[47] Markus Wenzel. Isar - a generic interpretative approach to readable formal proof documents. In Proceedings of the 12th International Conference on Theorem Proving in Higher Order Logics, pages 167-184, 1999.

[48] Benjamin Werner. Sets in types, types in sets. In Proceedings of the Third International Symposium on Theoretical Aspects of Computer Software, pages 530-546, 1997.

[49] Glynn Winskel. The Formal Semantics of Programming Languages, chapter 8. MIT Press, 1993.

[50] Hongwei Xi, Chiyan Chen, and Gang Chen. Guarded recursive datatype constructors. In Proceedings of the 30th ACM SIGPLAN-SIGACT Symposium on Principles of Programming Languages, pages 224-235, 2003. 


\section{Index}

.dir-locals.el file, 15

.emacs file, 15

abstract type, 333

accessibility relation, 123

ACL2, 6, 8, 9

adaptive proof scripts, 325

Agda, 10

algebraic datatypes, 18, 42

array bounds checks, 139

as clause, 143

axiom K, 194, 248

axiom of choice, 240

axioms, 193, 235

backtracking, 255

beta reduction, 186

bisimulation, 95

bullets, 321

Calculus of Constructions, 19

Calculus of Inductive Constructions, 19

certified program, 7

certifying program, 7

CIC, see Calculus of Inductive Constructions

class (in set theory), 224

classical logic, 74

co-induction principles, 94

co-inductive big-step operational semantics, 98

co-inductive predicates, 91

co-inductive types, 86, 184

co-recursive definitions, 87

CoC, see Calculus of Constructions

computability, see decidability

conclusion, 23 constructive logic, 43, 74, 236

context patterns, 273

continuation-passing style, 283

convoy pattern, 153, 244

coq_makefile, 336

coqdoc, 18

CoqIDE, 13

Curry-Howard correspondence, 40, 68, 86, 234

datatype-generic programming, 207

de Bruijn criterion, 9

decidability, 74

declarative proof scripts, 324

deep embedding, 121, 248

definitional equality, 185

delta reduction, 186

dependent pair, 107

dependent pattern matching, 32, 58, 143

dependent types, 103

deriving clauses, 207

discriminee, 143

domain theory, 128

elimination, 233

Epigram, 10

existential quantification, 75

extensionality, 126

extensionality of function equality, 205

extraction, see program extraction

failure monad, 115

first-order syntax, 344

free variable, 23

fully automated proofs, 318

Function, 127

functional extensionality, 206, 340 
functor, 333

GADTs, see generalized algebraic datatypes

Gallina, 19, 223

Gallina operators

$$
++, 21
$$

$\wedge, 54$

::, 21

Gallina terms

Acc, 123

and, 54, 62

app, 49

as, 58

bool, 44

cofix, 188

Cons, 49

Empty_set, 43

eq, 77

eq_ind_r, 201

eq_rect_eq, 193

ex, 75

exists, 75

False, 41

false, 44

fin, 165

Fix, 124

fix, 58, 187

for, 59

forall, 75

hlist, 169, 209

I, 41

if, 44

ilist, 165, 209

in, 140

index, 305

JMeq, 199

length, 49

list, 49

maybe, 113

member, 169

nat, 45

negb, 44

Nil, 49
O, 45

option, 21

or, 72

plus, 46

pred, 45

prod, 54, 71

proj1_sig, 107

Prop, 43

refl_equal, 77

return, 58

S, 45

Set, 18, 43

sig, 105

sigT, 152

sum, 72

sumbool, 110

sumor, 114

True, 41

true, 44

tt, 42

UIP_refl, 193

unit, 42

varmap, 306

with, 59

generalized algebraic datatypes, 31, 42

generic programming, 207

GHC Haskell, 31

Girard's paradox, 224

graphical interfaces to Coq, 12

guardedness condition, 88

Haskell, 31, 42, 55, 86, 283

head symbol, 268

head-normal form, 164

heterogeneous equality, 199

higher-order abstract syntax, 55, 347

higher-order syntax, 347

higher-order unification, 58, 105

higher-order vs. first-order languages, 8 hint databases, 260

HOAS, see higher-order abstract syntax HOL, 8

hypotheses, 23,26 
implicit arguments, 62, 230

impredicative Set, 241

impredicativity, 226, 234

in clause, 143

inconsistent axioms, 236

index function, 175

induction principles, 43, 56

inference rules, 76

intensional type theory, 195

interactive proof-editing mode, 22

interpretation function, 301

interpreters, 18, 171, 179

intro pattern, 99, 320

intuitionistic logic, see constructive logic

iota reduction, 186

Isabelle/HOL, 7, 9

Isar, 324

John Major equality, 199

judgment, 76

lambda calculus, 55, 171

large inductive types, 159, 227

law of the excluded middle, 74, 236

laziness, 86

length-indexed lists, 139

linear arithmetic, 272

logic programming, 253

Ltac, 9, 19

Makefile, 336

meta language, 31, 347

ML, 42, 55

module systems, 207

monad, 115, 129, 283

natural deduction, 76, 274

nested inductive type, 61, 176

notation scope delimiter, 145

notation scopes, 33

Nqthm, 6

Obj.magic, 158

object language, 31,347
OCaml, 103

opaque, 108

opaque ascription, 334

parameters, 144

parametric higher-order abstract syntax, 347

parametric polymorphism, 207

parametricity, 356

Park's principle, 94

phase distinction, 140

PHOAS, see parametric higher-order abstract syntax

polymorphism, 49

positivity requirement, 56

predicativity, 225

Presburger arithmetic, 272

primitive recursion, 121

principal types, 19

productivity, 90

Program, 110

program extraction, 37, 74, 103, 233

Program Fixpoint, 127

Prolog, 77, 253

proof by reflection, 10, 297

Proof General, 12, 14-15

proof irrelevance, 68, 238

proof term, 41

proof-carrying code, 243

propositional equality, 188

PVS, 8, 9

recursion principles, 57

recursion schemes, 209

recursive type definition, 173

recursively non-uniform parameters, 136

reduction strategy, 20

reflection, 10

reflexive inductive type, 177

reification, 300

relative consistency, 19

return clause, 143

rule induction, 81

Russell's paradox, 226 
sections, 50

set theory, 223

setoids, 272

shallow embedding, 121

sigma type, 107

stratified type systems, 140

strengthening the induction hypothesis, 22

strict positivity requirement, 56, 347

strong normalization, 19

structural induction, 23

subgoals, 23

subset types, 105

tactical, 273

tactics, 23

abstract, 109, 333

apply, 53, 84

assumption, 71

auto, 80, 255

autorewrite, 269

cbv, 185

change, 66, 206, 304

congruence, 47, 267, 272

constr, 280

constructor, 69

crush, 14, 28

crush', 120

debug, 259, 331

decide equality, 112

dep_destruct, 149

dependent destruction, 149, 246

destruct, 42, 99

discriminate, 45, 65

do, 292

eapply, 256

eauto, 84, 256

elimtype, 70

eval, 291

evar, 291

exact, 244, 274, 299

exists, 75

fail, 276

field, 305 first, 276

firstorder, 76

fold, 25, 26, 28

fourier, 272

fresh, 292

generalize, 152, 197, 277

hnf, 164

idtac, 275, 276

induction, 23, 28, 42, 320

info, 255, 329

info_auto, 255

injection, 47, 65

instantiate, 296

intro, 65

intros, 23, 26, 29, 246

intuition, 73, 272

inversion, 78

lazy, 186

left, 72

match, 84, 272

omega, 264, 272

pattern, 202, 218

pose, 280

progress, 288

quote, 305

red, 65

refine, 107, 126

reflexivity, 26, 28, 29

repeat, 273

rewrite, 27, 29, 66

right, 72

ring, 266, 272, 305

semicolon, 28

simpl, 25, 28, 46

simple apply, 289

simple destruct, 191

solve, 294

specialize, 274

split, 71

tauto, 72

trivial, 47

type of, 276 
unfold, 24, 26, 28

using, 64

with, 84

tagless interpreters, 144

termination checking, 20, 56

theory of equality and uninterpreted functions, 47

thunks, 333

transparent, 108

transparent ascription, 334

trusted code base, 243

Twelf, 8, 9, 55

type classes, 207

type hierarchy, 223

type inference, 19, 58

unicity of identity proofs, 239

unification, 255

unification variable, 256

universe inconsistency, 226

universe polymorphism, 229

universe types, 207

universe variable, 224

variable binding, 340

Vernacular commands, 19

Abort, 28

Arguments, 50

Axiom, 236

Check, 27

CoFixpoint, 87

CoInductive, 87

Debug On, 328

Declare Module, 334

Defined, 108, 125

Definition, 18

Eval, 20

Example, 54

Extract Inductive, 112

Extraction, 38, 103

Fixpoint, 19

Guarded, 92

Hint Constructors, 255, 267
Hint Extern, 64, 263

Hint Immediate, 257, 267

Hint Resolve, 257, 267

Hint Rewrite, 37, 269

Hint Unfold, 267

Hypothesis, 58

Import, 337

Inductive, 18

Lemma, 22

Load, 337

Locate, 62

Ltac, 156

Module, 334

Module Type, 333

Obligation Tactic, 110

Open Scope, 159

Parameter, 236

Print Assumptions, 201, 237

Print Universes, 228

Program Definition, 110

Qed, 28, 333

Recursive Extraction, 158

Require, 337

Require Export, 338

Require Import, 332

Restart, 45

Scheme, 52

SearchRewrite, 27, 257

Section, 50

Set Implicit Arguments, 50

Set Printing All, 230

Set Printing Universes, 224

Show Proof, 262

Theorem, 22

Time, 258, 330

Variable, 50

well-founded recursion, 122

well-founded relation, 122

Zermelo-Fraenkel set theory, 19

zeta reduction, 186 